University of Louisville

ThinkIR: The University of Louisville's Institutional Repository

$12-2019$

\title{
Oxygen deficient perovskites: effect of structure on electrical conductivity, magnetism and electrocatalytic activity.
}

\author{
Ram Krishna Hona \\ University of Louisville
}

Follow this and additional works at: https://ir.library.louisville.edu/etd

Part of the Inorganic Chemistry Commons

\section{Recommended Citation}

Hona, Ram Krishna, "Oxygen deficient perovskites: effect of structure on electrical conductivity, magnetism and electrocatalytic activity." (2019). Electronic Theses and Dissertations. Paper 3336.

https://doi.org/10.18297/etd/3336

This Doctoral Dissertation is brought to you for free and open access by ThinkIR: The University of Louisville's Institutional Repository. It has been accepted for inclusion in Electronic Theses and Dissertations by an authorized administrator of ThinkIR: The University of Louisville's Institutional Repository. This title appears here courtesy of the author, who has retained all other copyrights. For more information, please contact thinkir@louisville.edu. 
OXYGEN DEFICIENT PEROVSKITES:

EFFECT OF STRUCTURE ON ELECTRICAL CONDUCTIVITY, MAGNETISM AND ELECTROCATALYTIC ACTIVITY

\author{
By \\ Ram Krishna Hona \\ M.S., New Mexico State University, 2014

\begin{abstract}
A Dissertation
Submitted to the Faculty of the

College of Arts and Sciences of the University of Louisville in Partial Fulfilment of the Requirements

for the Degree of
\end{abstract}

Doctor of Philosophy in Chemistry

Department of Chemistry

University of Louisville

Louisville, Kentucky

December 2019 
(C) Copyright 2019 by Ram Krishna Hona

All rights reserved 



\title{
OXYGEN DEFICIENT PEROVSKITES:
}

\section{EFFECT OF VACANCY ORDER ON ELECTRICAL CONDUCTIVITY, MAGNETISM AND ELECTROLYTIC ACTIVITY}

\author{
By \\ Ram Krishna Hona \\ A Dissertation Approved on \\ November $20^{\text {th }}, 2019$ \\ by the following Dissertation Committee: \\ Dissertation Director - Dr. Farshid Ramezanipour \\ Dr. Mark Noble \\ Dr. Muriel Maurer \\ Dr. Ming Yu
}




\section{DEDICATION}

This dissertation is dedicated to my wife and my late mother. 


\section{ACKNOWLEDGEMENTS}

I would like to express my sincere gratitude to my research advisor Prof. Dr. Farshid Ramezanipour for his continuous support in my Ph.D. study and for motivation and enthusiasm toward intense research. His amiable personality with all-time happy behavior and proper guidance kept me happy in all the time of research and during writing this thesis. I could not have imagined having a better advisor and mentor for my Ph.D. study.

I would like to thank my research and dissertation committee members, Dr. Robert. B. Buchanon, Dr. Mark Nobel, Dr. Muriel Maurer and Dr. Ming Yu. I would like to thank Dr. Arjun Kumar Thapa who guided me for collaborative work.

I would like to thank my TA supervisors, Dr. Richad Balwin, Dr. Aleeta M. Powe, Dr. Neal Stolowich for their guidance in my teaching assistantship during my entire $\mathrm{PhD}$ study.

I would like to thank my fellow doctoral students for their feedback, cooperation and of course friendship. In addition, I would like to express my gratitude to the staff of chemistry department for their cooperation in my study.

Last but not the least, I am grateful to my wife who has provided me through moral and emotional support in my life. I am also grateful to my other family members and friends who have supported me along the way.

Thanks for all your encouragement! 


\begin{abstract}
OXYGEN DEFICIENT PEROVSKITES:

EFFECT OF VACANCY ORDER ON ELECTRICAL CONDUCTIVITY, MAGNETISM AND ELECTROLYTIC ACTIVITY.

Ram Krishna Hona
\end{abstract}

November 20, 2019

The present thesis deals with the synthesis and study of the physico-chemical properties of perovskite based oxide materials. Several novel oxygen deficient perovskites (ODP) have been synthesized by conventional solid state synthesis method. The novel compounds are $\mathrm{CaSrFe}_{2} \mathrm{O}_{6-\delta}, \quad \mathrm{CaSrFeCoO}_{6-\delta}, \quad \mathrm{Ca}_{2} \mathrm{Fe}_{1.5} \mathrm{Ga}_{0.5} \mathrm{O}_{5}, \quad \mathrm{CaSrFeGaO}_{5}$ and $\mathrm{BaSrFe}_{2} \mathrm{O}_{5}$. Their magnetic, charge transport and electrocatalytic properties have been studied. Structural effect on electrical conductivity, magnetic and electrocatalytic properties have been studied in some series of ODPs.

$\mathrm{CaSrFe}_{2} \mathrm{O}_{6-\delta}, \mathrm{CaSrFeCoO}_{6-\delta}, \mathrm{Ca}_{2} \mathrm{Fe}_{1.5} \mathrm{Ga}_{0.5} \mathrm{O}_{5}$ and $\mathrm{CaSrFeGaO}_{5}$ have brownmillerite type orthorhombic structures with layered structure having alternate tetrahedral and octahedral layers which are connected to one another by corner sharing. These are vacancy ordered compounds. $\mathrm{BaSrFe}_{2} \mathrm{O}_{5}$ is vacancy disordered compound with cubic structure. Most of the studied materials exhibited G-type long range antiferromagnetic arrangement of magnetic moments. 
During the study of charge transport property, compounds with structural order in a particular series show relatively less conductivity at room temperature and semiconductive nature and transition to metallic conductivity during temperature dependent conductivity measurement. Vacancy disordered compounds show relatively higher conductivity at room temperature and show mixed (semiconductive and metallic) conductivity during temperature dependent conductivity measurement.

The study of electrocatalytic properties revealed the relation with the conductivity and the structural order. The electrocatalytic activity toward oxygen evolution reaction is highly efficient if the material is highly conductive or highly ordered. 


\section{TABLE OF CONTENTS}

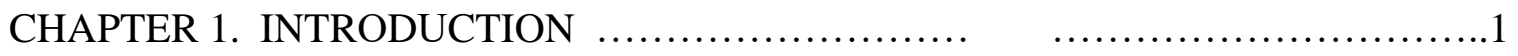

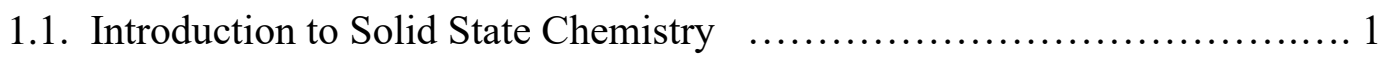

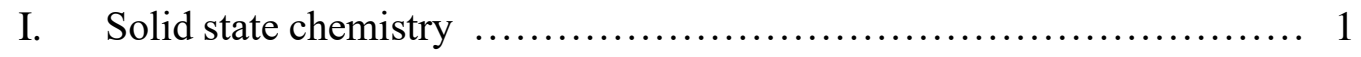

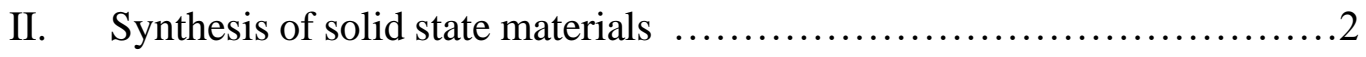

III. Characterization methods of solid-state materials....................... 3

i. Structure and phase purity identification $\quad$...................... 3

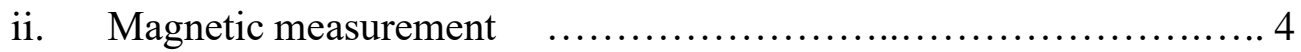

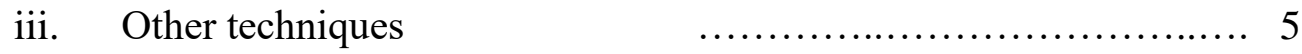

iv. Physico-chemical property measurement $\quad$................... 5

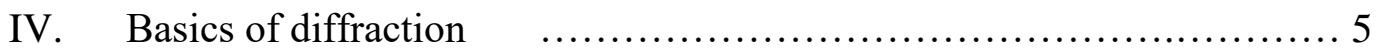

1.2. Introduction to Oxygen-Deficient Perovskites............................. 10

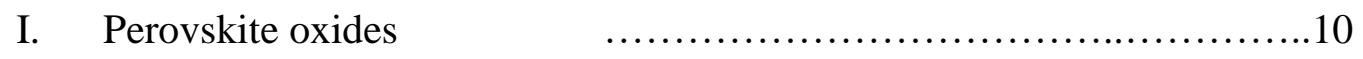


II. Oxygen deficient perovskites (ODPs)

III. Physical properties and possible applications $\quad \ldots \ldots \ldots \ldots \ldots \ldots \ldots \ldots . \ldots 14$

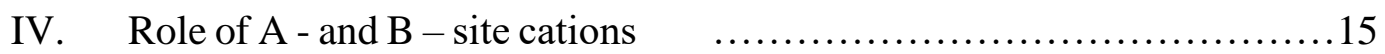

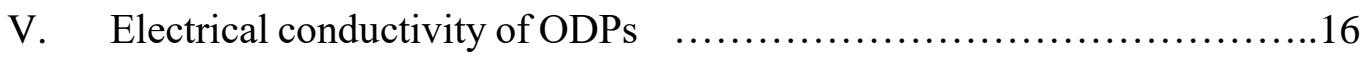

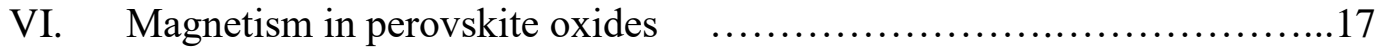

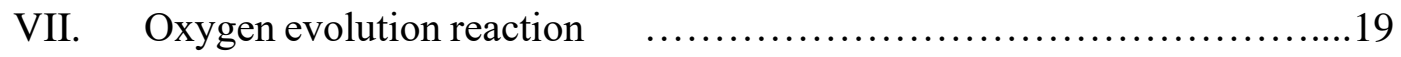

CHAPTER 2. TRANSFORMATION OF STRUCTURE, ELECTRICAL CONDUCTIVITY AND MAGNETISM IN AA' $\mathrm{Fe}_{2} \mathrm{O}_{6-\delta}, \mathrm{A}=\mathrm{Sr}, \mathrm{Ca}$ and $\mathrm{A}^{\prime}=\mathrm{Sr} . \ldots \ldots . .21$

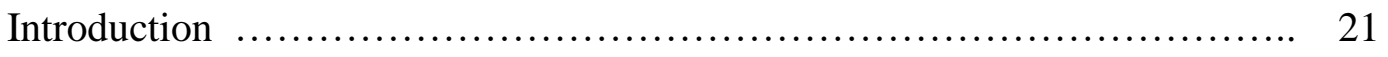

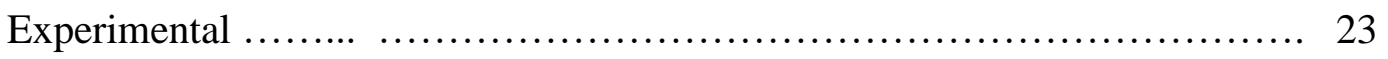

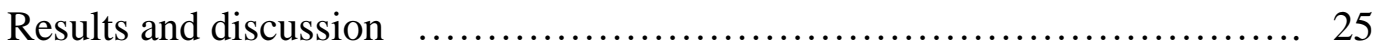

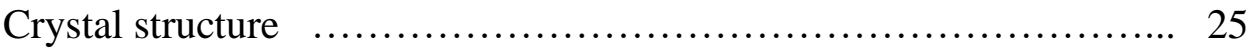

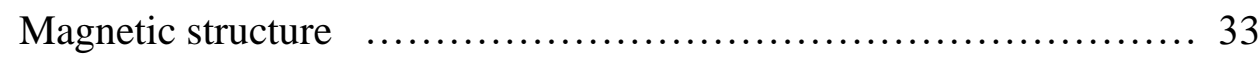

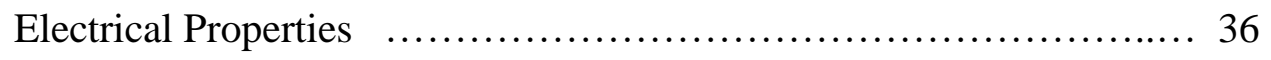

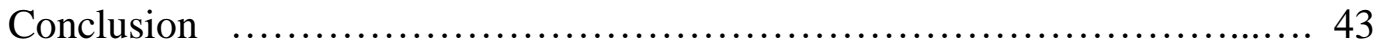

CHAPTER 3. UNRAVELING THE ROLE OF STRUCTURAL ORDER IN TRANSFORMATION OF ELECTRICAL CONDUCTIVITY IN $\mathrm{Ca}_{2} \mathrm{FeCoO}_{6-\delta}$,

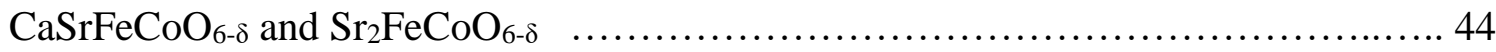




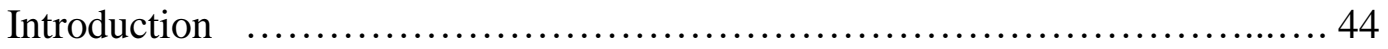

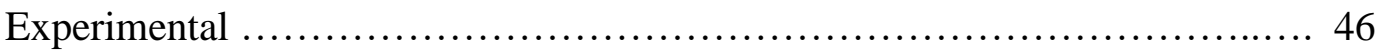

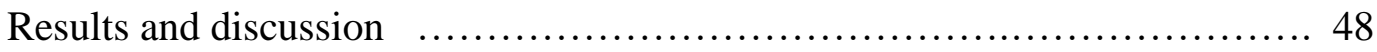

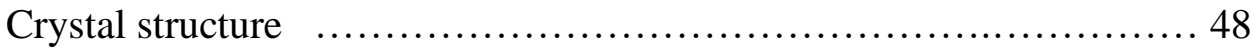

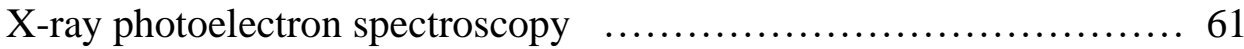

Electrical conductivities $\quad$............................................. 65

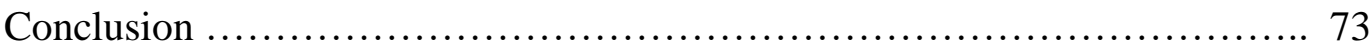

CHAPTER 4. MAGNETIC STRIUCTURE OF $\mathrm{CaSrFeCoO}_{5} \ldots \ldots \ldots \ldots \ldots \ldots \ldots \ldots . \ldots \ldots$

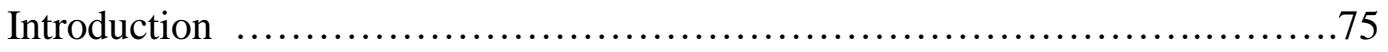

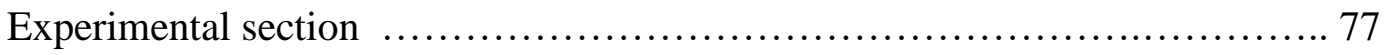

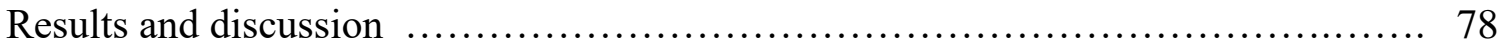

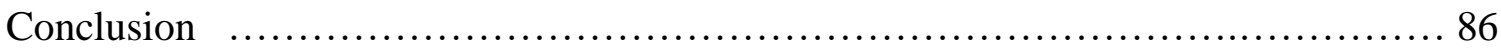

CHAPTER 5. ELECTRICAL PROPERTIES OF ORDERED OXYGEN-DEFICIENT

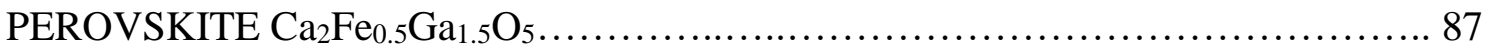

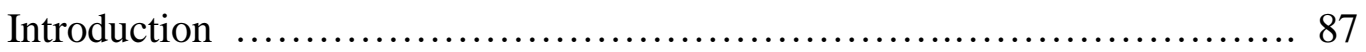

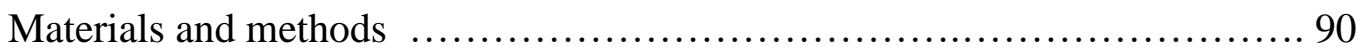

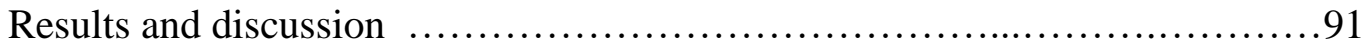

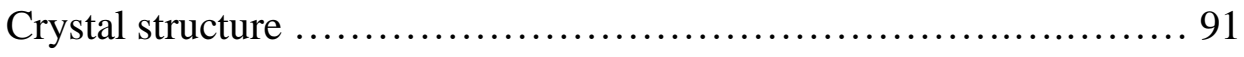


Conclusion

CHAPTER 6. ENHANCED ELECTRICAL PROPERTIES OIN $\mathrm{BaSrFe}_{2} \mathrm{O}_{6-\delta}(\delta=0.5)$ : A DISORDERED DEFECT-PEROVSKITE............................................ 100

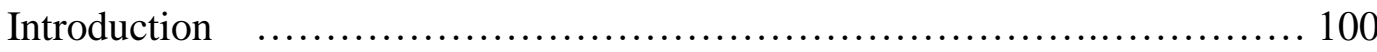

Experimental............................................................ 102

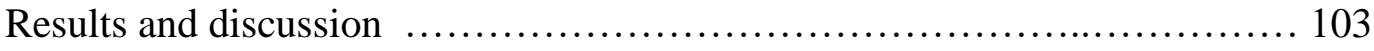

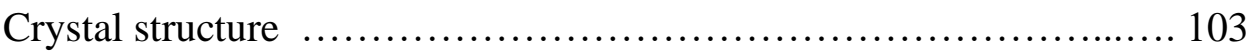

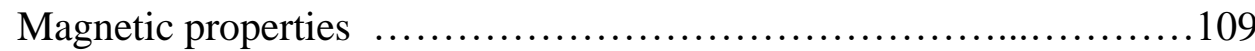

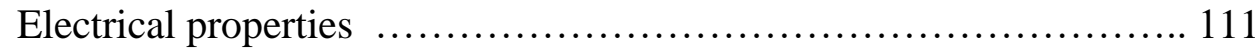

Conclusion …............................................................... 115

CHAPTER 7. CHARGE-TRANSPORT PROPERTIES OF $\mathrm{Ca}_{2} \mathrm{FeGaO}_{6-\delta}$ AND

$\mathrm{CaSrFeGaO}_{6-8}$ : THE EFFECT OF DIFECT-ORDERED ............................... 117

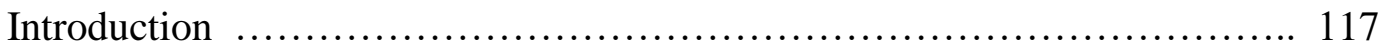

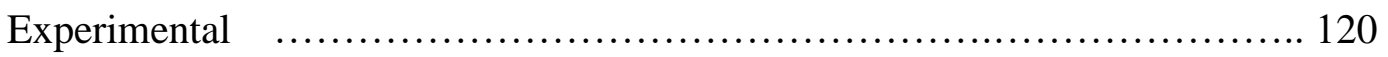

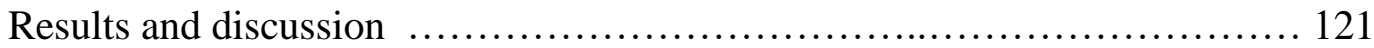

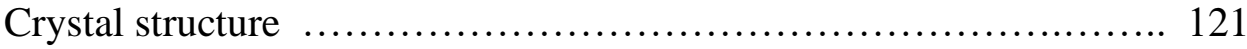

Microstructure and X-ray photoelectron spectroscopy studies...........125

Electrical properties ............................................. 127

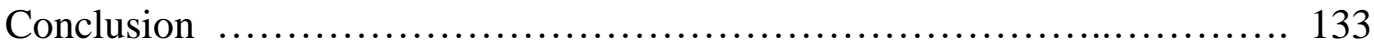


CHAPTER 8. DISPARITY IN ELECTRICAL AND MAGNETIC PROPERTIES OF ISOSTRUCTURAL OXYGEN-DEFICIENT PEROVSKITES $\mathrm{BaSrCo}_{2} \mathrm{O}_{6-\delta}$ AND

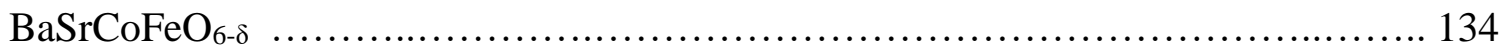

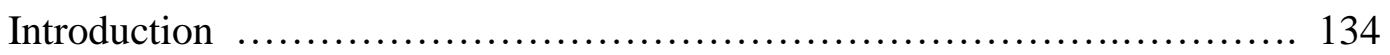

Experimental.................................................. 136

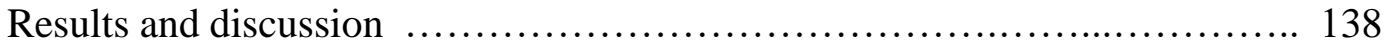

Crystal structure and crystallite morphology ..................... 138

X-ray photoelectron spectroscopy (XPS) and iodometric titration .......141

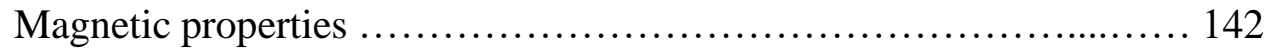

Electrical conductivities ................................... 147

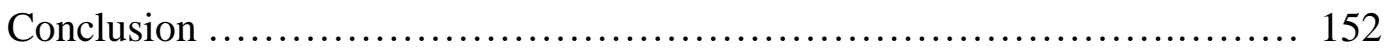

CHAPTER 9. STRUCTURE-DEPEDANCE OF ELECTRICAL CONDUCTIVITY AND ELECTROCATALYTIC PROPERTIES OF $\mathrm{Sr}_{2} \mathrm{Mn}_{2} \mathrm{O}_{6}$ and $\mathrm{CaSrMn}_{2} \mathrm{O}_{6} \ldots \ldots \ldots \ldots . . .154$

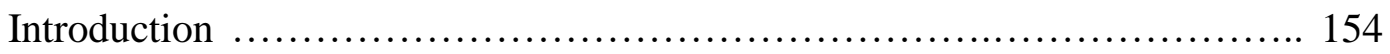

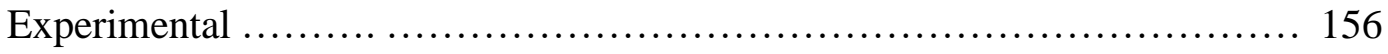

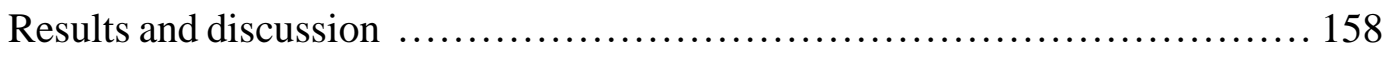

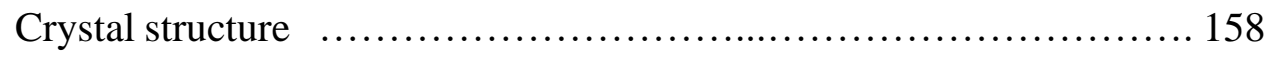

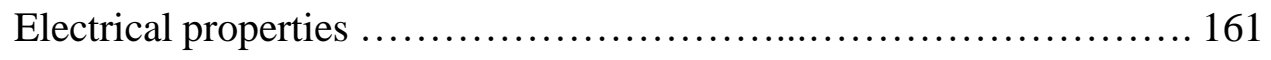

Correlation between electrocatalytic activity and conductivity .........164

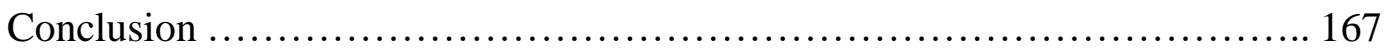


CHAPTER 10. VARIATION IN ELECTRICAL CONDUCTIVITY OF $\mathrm{A}_{2} \mathrm{Fe}_{2} \mathrm{O}_{5}(\mathrm{~A}=\mathrm{Sr}$,

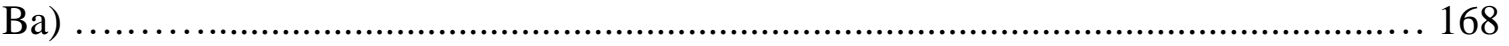

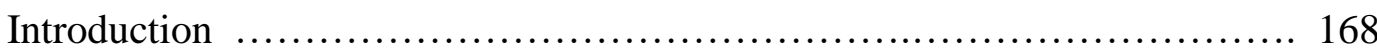

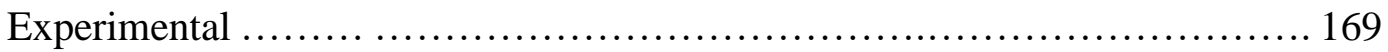

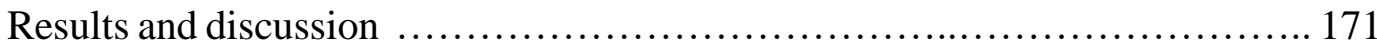

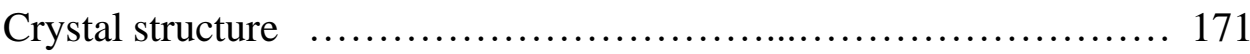

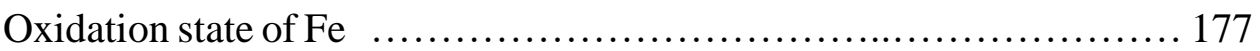

Magnetic properties ….......................................... 178

Electrical conductivity ......................................... 180

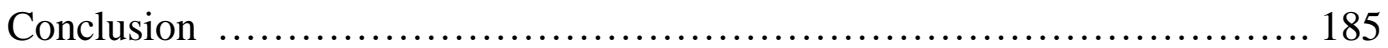

CHAPTER 11. STUDY OF $\mathrm{Sr}_{2} \mathrm{Fe}_{2} \mathrm{O}_{6-\delta}$ AS ANODE ELECTRODE IN Li-ION

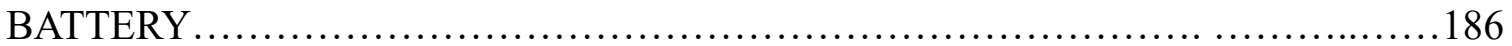

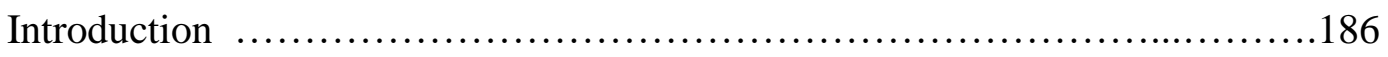

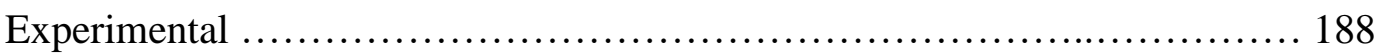

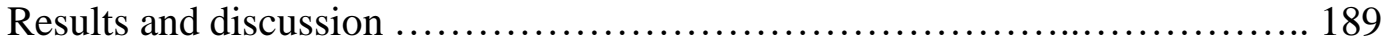

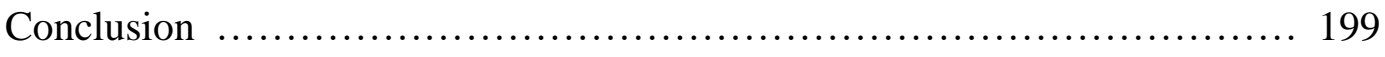

CHAPTER 12. REMARKABLE OXYGEN-EVOLUTION ACTIVITY OF $\mathrm{Ca}_{2}$ -

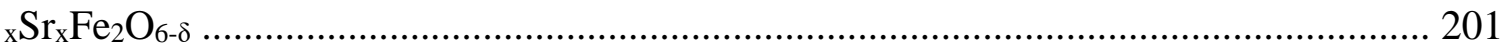

Supporting information ………………......................................................211

Material synthesis and characterization..................................211

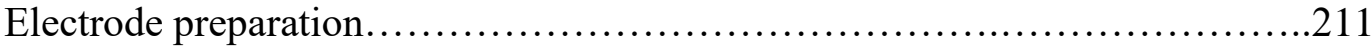

Measurements of OER activity .........................................212 
REFERENCES...

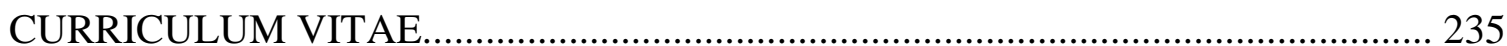




\section{LIST OF FIGURES}

Figure 1.1.1. Lattice planes formed by regular arrays of atoms in a crystal..............

Figure 1.1.2. Bragg's law in diffraction....................................... 8

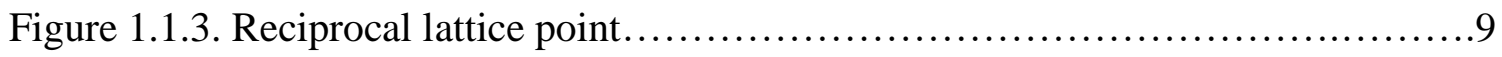

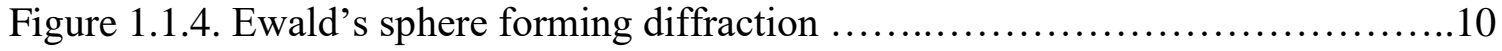

Figure 1.2.1. Crystal structure of perovskite oxide. (a) Crystallographic unit cell and corner-sharing $\mathrm{BO}_{6}$ octahedra (cyan) are highlighted. The large white spheres are the A atoms. (b) View along the unit cell axis. Because of the cubic symmetry, the three axes are identical. (c) Coordination geometry around the A atom, which is 12-coordinated.Figure 2.2. Bragg's law in diffraction .11

Figure 1.2.2. Crystal structure of $\mathrm{CaSrFeCoO}_{6-\delta}$ (a) crystal structure showing unit cell with alternating $(\mathrm{Fe} / \mathrm{Co}) \mathrm{O}_{6}$ octahedra (cyan) and $(\mathrm{Fe} / \mathrm{Co}) \mathrm{O}_{4}$ tetra (pink) layers. The large white spheres are $\mathrm{Sr}$ atoms, the green spheres inside octahedra and tetrahedra are Fe and Co atoms and small red spheres are oxygen atoms. (b) A view along b axis to show the orientation of tetra next to each other in each layer. All atoms are removed for clarity. (c) shows the coordination geometry around the $\mathrm{Sr}$ atoms. Note that the $\mathrm{Sr}$ is 8coordinated.............................................................. 14

Figure 1.2.3. Representation of electron hopping through $\mathrm{Fe}^{3+}-\mathrm{O}-\mathrm{Fe}^{4+}$ bond system

Figure 1.2.4. Super exchange by coupling two metals with unpaired d-electrons with an

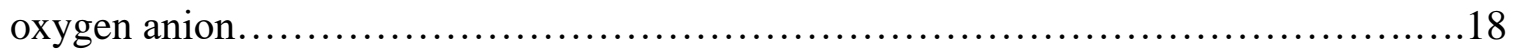

Figure 1.2.5. Three different ordering schemes in antiferromagnetic perovskite oxides..18

Figure 2.1. Rietveld refinement profile for powder X-ray diffraction data of $\mathrm{Sr}_{2} \mathrm{Fe}_{2} \mathrm{O}_{6-\delta}$ in I4/mmm space group. Stars represent experimental data, red solid is the model, vertical tick marks show Bragg peak positions, and the blue line represents the difference plot........26

Figure 2.2. Crystal structure of $\mathrm{Sr}_{2} \mathrm{Fe}_{2} \mathrm{O}_{6-\delta}$. (a) and (b) show the alternating $\mathrm{FeO}_{6}$ octahedra (purple) and $\mathrm{FeO}_{5}$ square pyramids (green), viewed along the $a$ and $c$ axes, respectively. The large grey spheres are $\mathrm{Sr}$ atoms. (c) shows the coordination geometry around the $\mathrm{Sr}$ atoms. Note the presence of both 11 and 12-coordinated $\mathrm{Sr}$ atoms. 
Figure 2.3. Crystal structure of $\mathrm{CaSrFe}_{2} \mathrm{O}_{6-\delta}$. (a) The octahedral $\mathrm{FeO}_{6}$ (purple) and tetrahedral $\mathrm{FeO}_{4}$ (green) layers. Grey spheres represent Sr. (b) View from top to highlight the chain formation in the tetrahedral layer. The $\mathrm{Sr}$ atoms are omitted for clarity. (c) Coordination geometry of $\mathrm{Sr}$ atoms. Note that $\mathrm{Sr}$ is 8 -coordinated. ..................28

Figure 2.4. Rietveld refinement profile for powder X-ray diffraction data of $\mathrm{CaSrFe}_{2} \mathrm{O}_{6-\delta}$, space group Ibm2. Black stars, red line, vertical tick marks and lower blue line represent the experimental data, structural model, Bragg peak positions and difference plot, respectively. .28

Figure 2.5. Scanning electron microscopy images of (a) $\mathrm{Sr}_{2} \mathrm{Fe}_{2} \mathrm{O}_{6-\delta}$ and (b) $\mathrm{CaSrFe}_{2} \mathrm{O}_{6}$ -

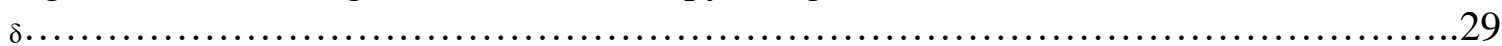

Figure 2.6. X-ray photoelectron spectroscopy data for $\mathrm{CaSrFe}_{2} \mathrm{O}_{6-\delta} \ldots \ldots \ldots \ldots \ldots \ldots \ldots . \ldots \ldots$

Figure 2.7. Powder X-ray diffraction data for the series of materials with varying $\mathrm{Ca} / \mathrm{Sr}$ ratios. 32

Figure 2.8. Refinement of the crystal and magnetic structures using neutron diffraction data for $\mathrm{CaSrFe}_{2} \mathrm{O}_{6-\delta}$. The upper and lower tick marks represent the peak positions for crystal and magnetic structures, respectively. .34

Figure 2.9. The G-type antiferromagnetic order in $\mathrm{CaSrFe}_{2} \mathrm{O}_{6-\delta}$. Note that magnetic moments on each Fe site are aligned opposite to all nearest neighbors. The moments are oriented along the $c$-axis. .35

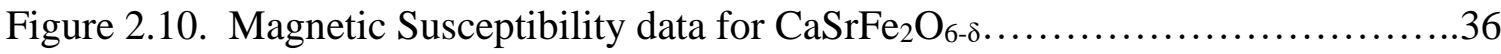

Figure 2.11. (a) Total conductivity of $\mathrm{Sr}_{2} \mathrm{Fe}_{2} \mathrm{O}_{6-\delta}$ and $\mathrm{CaSrFe}_{2} \mathrm{O}_{6-\delta}$ as a function of temperature. For $\mathrm{Sr}_{2} \mathrm{Fe}_{2} \mathrm{O}_{6-\delta}$, the heating and cooling data (red and green) overlap. For $\mathrm{CaSrFe}_{2} \mathrm{O}_{6-\delta}$ hysteresis is observed between heating (black squares) and cooling data (blue circles). (b) Arrhenius plot of the total conductivity for $\mathrm{Sr}_{2} \mathrm{Fe}_{2} \mathrm{O}_{6-\delta}$ (red stars) and

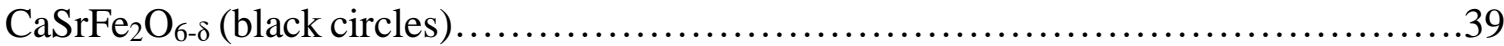

Figure 2.12. Thermogravimetric analysis data for $\mathrm{CaSrFe}_{2} \mathrm{O}_{6-\delta}$ in air....... 
Figure 3.1. Crystal structure of $\mathrm{CaSrFeCoO}_{6-\delta}$. (a) The crystallographic unit cell. The alternating $(\mathrm{Fe} / \mathrm{Co}) \mathrm{O}_{6}$ octahedra (cyan) and $(\mathrm{Fe} / \mathrm{Co}) \mathrm{O}_{4}$ tetra (pink) are highlighted. The large grey spheres are the A-site cations, $\mathrm{Ca}^{2+} / \mathrm{Sr}^{2+}$. (b) A view along the $b$ axis (longest axis) to show the uniform orientation of tetrahedral chains. The A-site cations are omitted for clarity. (c) The coordination geometry around the A-site cation. There are 8 oxygens that are close enough to the A-site cation to be in its coordination sphere................46

Figure 3.2. Rietveld refinement profile for powder X-ray diffraction data of $\mathrm{CaSrFeCoO}_{6}$ $\delta$. Crosses represent experimental data, solid red line is the Ibm2 model, vertical tick marks show Bragg peak positions, and the lower line represents the difference plot............50

Figure 3.3. Neutron diffraction Rietveld refinement profile for $\mathrm{CaSrFeCoO}_{6-\delta}$. Magnetic reflections have been omitted. Crosses represent experimental data, solid red line is the Ibm 2 model, vertical tick marks show Bragg peak positions, and the lower line represents the difference plot.

Figure 3.4. Crystal structure of $\mathrm{Sr}_{2} \mathrm{FeCoO}_{6-\delta}$. (a) The crystallographic unit cell and cornersharing $(\mathrm{Fe} / \mathrm{Co}) \mathrm{O}_{6}$ octahedra (cyan) are highlighted. The large grey spheres are the $\mathrm{Sr}$ atoms. (b) A view along the unit cell axis. Due to cubic symmetry, the three axes are identical. (c) The coordination geometry around the Sr atom, which is 12-coordinated....55

Figure 3.5. Rietveld refinement profile for powder X-ray diffraction data of $\mathrm{Sr}_{2} \mathrm{FeCoO}_{6-\delta}$ refined in Pm-3m space group. Crosses represent experimental data, solid red line is the model, vertical tick marks show Bragg peak positions, and the lower blue line represents the difference plot........................................................56

Figure 3.6. Crystal structure of $\mathrm{Ca}_{2} \mathrm{FeCoO}_{6-\delta}$ featuring alternating $(\mathrm{Fe} / \mathrm{Co}) \mathrm{O}_{6}$ octahedra (cyan) and $(\mathrm{Fe} / \mathrm{Co}) \mathrm{O}_{4}$ tetrahedra (pink). The large grey spheres are $\mathrm{Ca}$ atoms. The crystallographic unit cell is highlighted using yellow lines. Note that the unit cell here is 2times larger than that for the $\mathrm{CaSr}$ analogue.

Figure 3.7. Rietveld refinement profile for powder X-ray diffraction data of $\mathrm{Ca}_{2} \mathrm{FeCoO}_{6-\delta}$ refined in $\mathrm{Pbcm}$ space group. Crosses represent experimental data, red solid line is the model, vertical tick marks show Bragg peak positions, and the blue line represents the

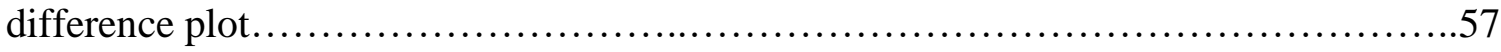

Figure 3.8. Scanning electron microscopy images for $\mathrm{Sr}_{2} \mathrm{FeCoO}_{6-\delta}, \mathrm{CaSrFeCoO}_{6-\delta}$

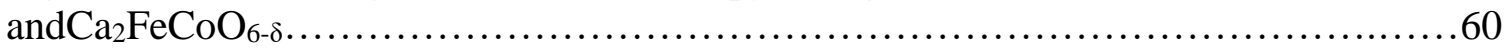


Figure 3.9. Powder XRD data for the series $\mathrm{Sr}_{2-\mathrm{x}} \mathrm{Ca}_{\mathrm{x}} \mathrm{FeCoO}_{6-\delta}, \mathrm{x}=0-1$. The structural transition occurs above $\mathrm{x}=0.3$.

Figure 3.10. Powder XRD data for the series $\mathrm{Sr}_{2-\mathrm{x}} \mathrm{Ca}_{\mathrm{x}} \mathrm{FeCoO}_{6-\delta}, \mathrm{x}=1-2$. The structural

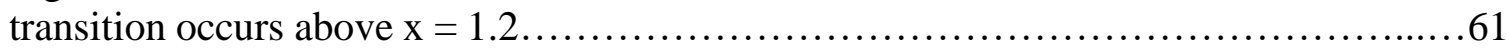

Figure 3.11. The Fe XPS spectra for $\mathrm{Ca}_{2} \mathrm{FeCoO}_{6-\delta}, \mathrm{CaSrFeCoO}_{6-\delta}$ and $\mathrm{Sr}_{2} \mathrm{FeCoO}_{6-\delta} \ldots . .63$

Figure 3.12. The cobalt XPS spectra for $\mathrm{Ca}_{2} \mathrm{FeCoO}_{6-\delta}$, $\mathrm{CaSrFeCoO}_{6-\delta}$ and $\mathrm{Sr}_{2} \mathrm{FeCoO}_{6-}$

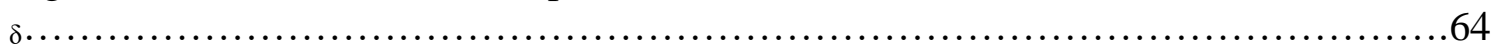

Figure 3.13. Total conductivity of $\mathrm{Sr}_{2} \mathrm{FeCoO}_{6-\delta}, \mathrm{CaSrFeCoO}_{6-\delta}$ and $\mathrm{Ca}_{2} \mathrm{FeCoO}_{6-\delta}$ as a function of temperature. For $\mathrm{Sr}_{2} \mathrm{Fe}_{2} \mathrm{O}_{6-\delta}$, the heating (red) and cooling (black) data overlap. For $\mathrm{CaSrFeCoO}_{6-\delta}$, the heating data are shown in green and cooling data in blue. For $\mathrm{Ca}_{2} \mathrm{FeCoO}_{6-\delta}$, the heating and cooling data are shown in pink and cyan, respectively....67

Figure 3.14. Thermogravimetric analysis of $\mathrm{CaSrFeCoO}_{6-\delta}$ in air. An inflection in the data appears in the temperature range between the dashed lines. The inset shows the derivative plot. .70

Figure 3.15. Arrhenius plot of the total conductivity for $\mathrm{Sr}_{2} \mathrm{FeCoO}_{6-\delta}$ (black triangles),

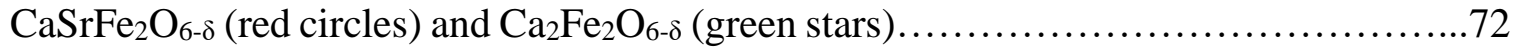

Figure 4.1. Comparison between (a) perovskite and (b) brownmillerite structure. In brownmillerite, the oxygen vacancies are ordered. The difference in transition metal coordination geometry is highlighted. The A-site cations that reside in spaces between polyhedra have been omitted for clarity....

Figure 4.2. Neutron diffraction refinement profile at $10 \mathrm{~K}$ for $\mathrm{CaSrFeCoO}_{5}$. The crosses are experimental data, the red line is the model and the lower grey line represents the difference plot. The upper and lower vertical tick marks locate the crystal and magnetic structure peak positions. .79

Figure 4.3. G-type antiferromagnetic order in $\mathrm{CaSrFeCoO}_{5}$ 79

Figure 4.4. Gaussian fit for (021) and (120) magnetic peaks, indicating (120)/(021) ratio of $\sim 2.96$. . . .81 
Figure 4.5. Fits for (021) and (120) magnetic peaks in neutron diffraction data using three different models with magnetic moments along $a$ (intermediate), $b$ (longest), or $c$ (shortest) axis. As shown here, the model with moments along the $c$-axis leads to an excellent fit..82

Figure 4.6. Neutron diffraction refinement profile at $300 \mathrm{~K}$ for $\mathrm{CaSrFeCoO}_{5}$. The crosses are experimental data, the red line is the model and the lower grey line represents the difference plot. The upper and lower vertical tick marks locate the crystal and magnetic structure peak positions 83

Figure 4.7. Magnetic susceptibility data for $\mathrm{CaSrFeCoO}_{5}$, as well as its $\mathrm{Ca}_{2}$ and $\mathrm{Sr}_{2}$ analogues. Images in a, b and $\mathrm{c}$ show the $\mathrm{Ca}_{2}, \mathrm{CaSr}$ and $\mathrm{Sr}_{2}$ compounds, respectively. Black squares show zero-field-cooled and red circles represent field-cooled data. .85

Figure 5.1 Brownmillerite structure of $\mathrm{Ca}_{2} \mathrm{Fe}_{0.5} \mathrm{Ga}_{1.5} \mathrm{O}_{5}$ .88

Figure 5.2 Refinement profiles for $\mathrm{Ca}_{2} \mathrm{Fe}_{0.5} \mathrm{Ga}_{1.5} \mathrm{O}_{5}$ using (a) X-ray and (b) neutron diffraction. 92

Figure 5.3 SEM images of $\mathrm{Ca}_{2} \mathrm{Fe}_{0.5} \mathrm{Ga}_{1.5} \mathrm{O}_{5}$, showing good contact between the crystallites.............................................................. 93

Figure 5.4 X-ray photoelectron spectroscopy data for $\mathrm{Ca}_{2} \mathrm{Fe}_{0.5} \mathrm{Ga}_{1.5} \mathrm{O}_{5} \ldots \ldots \ldots \ldots \ldots . .94$

Figure 5.5 (a) Electrical conductivity of $\mathrm{Ca}_{2} \mathrm{Fe}_{0.5} \mathrm{Ga}_{1.5} \mathrm{O}_{5}$ as a function of temperature. (b) Arrhenius plot and activation energies for $\mathrm{Ca}_{2} \mathrm{Fe}_{0.5} \mathrm{Ga}_{1.5} \mathrm{O}_{5}$

Figure 5.6 AC impedance data for $\mathrm{Ca}_{2} \mathrm{Fe}_{0.5} \mathrm{Ga}_{1.5} \mathrm{O}_{5}$ at $200{ }^{\circ} \mathrm{C}$. Three resistance-capacitance units indicate the bulk (left), grain boundary (middle) and electrode interface (right) resistance

Figure 5.7 Thermogravimetric analysis for $\mathrm{Ca}_{2} \mathrm{Fe}_{0.5} \mathrm{Ga}_{1.5} \mathrm{O}_{5}$ .97

Figure 6.1. Rietveld refinement profiles for $\mathrm{BaSrFe}_{2} \mathrm{O}_{6-\delta}(\delta=0.5)$ : (a) X-ray and (b) neutron diffraction data. .104

Figure 6.2. Crystal structures of (a) $\mathrm{BaSrFe}_{2} \mathrm{O}_{6-\delta}(\delta=0.5)$ and (b) $\mathrm{CaSrFe}_{2} \mathrm{O}_{6-\delta}(\delta=1) \ldots 105$ 
Figure 6.3. Scanning electron microscopy images of sintered pellets of (a) $\mathrm{BaSrFe}_{2} \mathrm{O}_{6-\delta}$

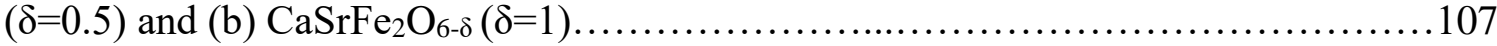

Figure 6.4. X-ray photoelectron spectroscopy data for $\mathrm{BaSrFe}_{2} \mathrm{O}_{6-\delta}(\delta=0.5) \ldots \ldots \ldots \ldots 108$

Figure 6.5. Bulk magnetization data of $\mathrm{BaSrFe}_{2} \mathrm{O}_{6-\delta}(\delta=0.5)$ (a) ZFCFC magnetic susceptibility data. The inset shows inverse of susceptibility versus temperature. (b)

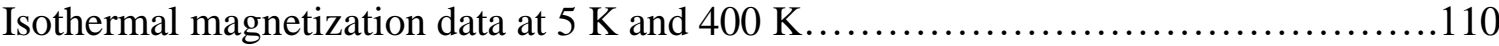

Figure 6.6. Electrical conductivity as a function of temperature for $\mathrm{BaSrFe}_{2} \mathrm{O}_{6-\delta}(\delta=0.5)$

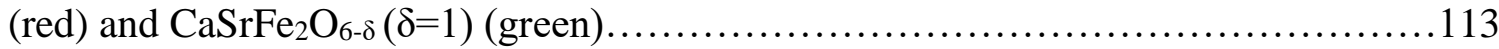

Figure 6.7. Thermogravimetric analysis data for $\mathrm{BaSrFe}_{2} \mathrm{O}_{6-\delta}(\delta=0.5) \ldots \ldots \ldots \ldots \ldots \ldots 114$

Figure 6.8. Arrhenius plot of the electrical conductivity for $\mathrm{BaSrFe}_{2} \mathrm{O}_{6-\delta}(\delta=0.5)$ (red) and

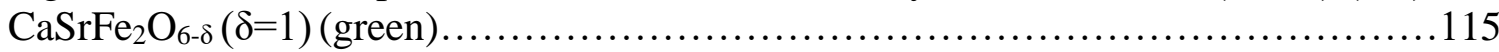

Figure 7.1. Rietveld refinement profiles for powder X-ray diffraction data of (a) $\mathrm{Ca}_{2} \mathrm{FeGaO}_{6-\delta}$ in Pnma, and (b) $\mathrm{CaSrFeGaO}_{6-\delta}$ in $\mathrm{Ibm} 2$ space group. Black crosses represent experimental data, the solid red line is the model, pink vertical tick marks show Bragg peak positions, and the lower blue line represents the difference plot. The arrows show the position of 131 peak, which is present for $\mathrm{Ca}_{2} \mathrm{FeGaO}_{6-\delta}$ and absent for $\mathrm{CaSrFeGaO}_{6-\delta}$...119

Figure 7.2. Rietveld refinement profiles for powder neutron diffraction data of (a) $\mathrm{Ca}_{2} \mathrm{FeGaO}_{6-\delta}$ in Pnma, and (b) $\mathrm{CaSrFeGaO}_{6-\delta}$ in $\mathrm{Ibm} 2$ space group. Black crosses represent experimental data, the solid red line is the model, pink vertical tick marks show Bragg peak positions, and the lower blue line represents the difference plot.

Figure 7.3. (a) Unit cell of $\mathrm{Ca}_{2} \mathrm{FeGaO}_{6-\delta}$, Pnma. (b) Alternating octahedral and tetrahedral coordination geometry in $\mathrm{Ca}_{2} \mathrm{FeGaO}_{6-\delta}$. The $\mathrm{Ca}$ atoms, located in spaces between polyhedra, are omitted for clarity. (c) Orientation of tetrahedral chains in two neighboring layers, which are opposite to each other in $\mathrm{Ca}_{2} \mathrm{FeGaO}_{6-\delta}$ (top view). The tetrahedral sites are primarily occupied by $\mathrm{Ga}$ and octahedral sites by Fe. The red and blue colors represent the two different orientations of tetrahedral chains. .123

Figure 7.4. (a) Unit cell of $\mathrm{CaSrFeGaO}_{6-\delta}, \mathrm{Ibm2}$. (b) Alternating octahedral and tetrahedral coordination geometry in $\mathrm{CaSrFeGaO}_{6-\delta}$. The $\mathrm{Ca} / \mathrm{Sr}$ atoms, located in spaces between 
polyhedra, are omitted for clarity. (c) Orientation of tetrahedral chains in two neighboring layers (top view). All tetrahedral chains have the same orientation in $\mathrm{CaSrFeGaO}_{6-\delta} . . .124$

Figure 7.5. Scanning electron microscopy images of $\mathrm{Ca}_{2} \mathrm{FeGaO}_{6-\delta}$ and $\mathrm{CaSrFeGaO}_{6-}$

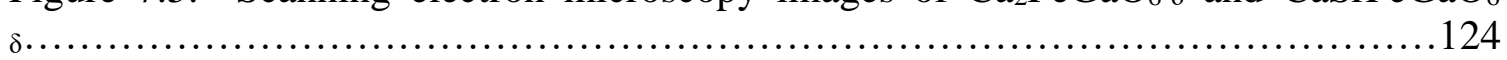

Figure 7.6. X-ray photoelectron spectroscopy data for (a) $\mathrm{Ca}_{2} \mathrm{FeGaO}_{6-\delta}$ and (b) $\mathrm{CaSrFeGaO}_{6-\delta}$. The data for both materials show the same binding energies for $\mathrm{Fe}$ peaks................................................................. 126

Figure 7.7. Representative example of the Nyquist plot for $\mathrm{CaSrFeGaO}_{6-\delta}$ at $25^{\circ} \mathrm{C}$. The semicircle can be fitted using two resistance-capacitance (RC) units, corresponding to the bulk $\left(\mathrm{R} 1-766 \Omega, \mathrm{CPE} 1=2.6 \times 10^{-8} \mathrm{~F}\right)$ and grain boundary $\left(\mathrm{R} 2=3397 \Omega, \mathrm{CPE} 2=1.310^{-7}\right.$ F) 128

Figure 7.8. (a) Electrical conductivity of $\mathrm{Ca}_{2} \mathrm{FeGaO}_{6-\delta}$ (red) and $\mathrm{CaSrFeGaO}_{6-\delta}$ (blue) as a function of temperature. (b) Arrhenius plots for electrical conductivity of $\mathrm{Ca}_{2} \mathrm{FeGaO}_{6-\delta}$

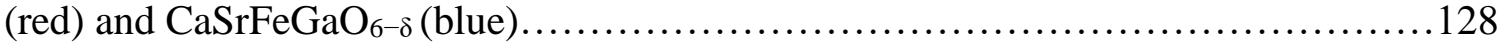

Figure 8.1. Rietveld refinement profile for powder XRD data of a) $\mathrm{BaSrCo}_{2} \mathrm{O}_{6-\delta}$ and b) $\mathrm{BaSrCoFeO}_{6-\delta}$. Black crosses represent experimental data, the solid red line is the cubic $P m-3 m$ model, pink vertical tick marks show Bragg peak positions, and the lower blue line represents the difference plot....................................................

Figure 8.2. Scanning electron microscopy images of $\mathrm{BaSrCoFeO}_{6-\delta}$ (top) and $\mathrm{BaSrCo}_{2} \mathrm{O}_{6-}$ $\delta$ (bottom) 140

Figure 8.3. X-ray photoelectron spectroscopy data. (a) shows the Co peaks for $\mathrm{BaSrCo}_{2} \mathrm{O}_{6}$ $\delta$. (b) and (c) show the $\mathrm{Fe}$ and $\mathrm{Co}$ peaks for $\mathrm{BaSrCoFeO}_{6-\delta}$, respectively. 143

Figure 8.4. Bulk magnetization data for $\mathrm{BaSrCo}_{2} \mathrm{O}_{6-8}$ : (a) $\mathrm{ZFC}$ and $\mathrm{FC}$ magnetic susceptibility data. The inset shows inverse of susceptibility plotted against temperature. (b) Isothermal magnetization versus field, at $2 \mathrm{~K}$ and $400 \mathrm{~K}$. .144

Figure 8.5. Bulk magnetization data for $\mathrm{BaSrCoFeO}_{6-\delta}$ : (a) $\mathrm{ZFC} / \mathrm{FC}$ magnetic susceptibility data. The inset shows inverse of susceptibility versus temperature. (b) Isothermal magnetization data at $2 \mathrm{~K}$ and $400 \mathrm{~K}$ 145 
Figure 8.6. Electrical conductivity of (a) $\mathrm{BaSrCo}_{2} \mathrm{O}_{6-\delta}$ and (b) $\mathrm{BaSrCoFeO}_{6-\delta}$. The measurements were done in the same environment used for synthesis, namely in argon for $\mathrm{BaSrCo}_{2} \mathrm{O}_{6-\delta}$ and in air $\mathrm{BaSrCoFeO}_{6-\delta}$. Heating data are shown in green and the cooling data in red.

Figure 8.7. comparison of the electrical conductivity for $\mathrm{BaSrCo}_{2} \mathrm{O}_{6-\delta}$ and $\mathrm{BaSrCoFeO}_{6-\delta}$ both obtained in argon atmosphere

Figure 8.8. Arrhenius plot for electrical conductivity of (a) $\mathrm{BaSrCo}_{2} \mathrm{O}_{6-\delta}$ and (b)

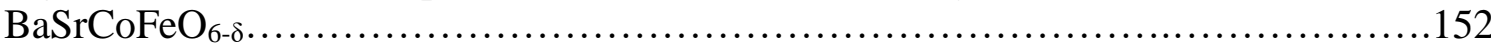

Figure 9.1. (a) Crystal structure and (b) Rietveld refinement profile from X-ray diffraction data for $\mathrm{Sr}_{2} \mathrm{Mn}_{2} \mathrm{O}_{6}, P 6_{3} / m m c$. Black crosses represent experimental data, the solid red line is the model, pink vertical tick marks show Bragg peak positions, and the lower grey line represents the difference plot.

Figure 9.2. (a) Crystal structure and (b) Rietveld refinement profile from X-ray diffraction data for $\mathrm{CaSrMn}_{2} \mathrm{O}_{6}, P m-3 m$. Black crosses represent experimental data, the solid red line is the model, pink vertical tick marks show Bragg peak positions, and the lower grey line represents the difference plot. 160

Figure 9.3. (a) Temperature dependent electrical conductivity and (b) Arrhenius plots for $\mathrm{Sr}_{2} \mathrm{Mn}_{2} \mathrm{O}_{6}$ (blue circles) and $\mathrm{CaSrMn}_{2} \mathrm{O}_{6}$ (green triangles). The inset in (a) is a zoomed view of the increase in conductivity of $\mathrm{Sr}_{2} \mathrm{Mn}_{2} \mathrm{O}_{6}$ .163

Figure 9.4. (a) Polarization curves for OER with mass activities for $\mathrm{Sr}_{2} \mathrm{Mn}_{2} \mathrm{O}_{6}$ (blue) and $\mathrm{CaSrMn}_{2} \mathrm{O}_{6}$ (green). (b) Tafel slopes for $\mathrm{Sr}_{2} \mathrm{Mn}_{2} \mathrm{O}_{6}$ (blue) and $\mathrm{CaSrMn}_{2} \mathrm{O}_{6}$ (green) .....165

Figure 10.1. Rietveld refinement profile for powder XRD data of (a) $\mathrm{Sr}_{2} \mathrm{Fe}_{2} \mathrm{O}_{5}$, with $I b m 2$ space group and (b) $\mathrm{Ba}_{2} \mathrm{Fe}_{2} \mathrm{O}_{5}$ with monoclinic $\mathrm{P} 2{ }_{1} / \mathrm{c}$ space group. The solid red line shows the refinement model, black crosses represent experimental data, pink vertical tick marks show Bragg peak positions, and the lower blue line represents the difference plot.......271

Figure 10.2. Crystal structures of (a) $\mathrm{Sr}_{2} \mathrm{Fe}_{2} \mathrm{O}_{5}$ and (b) $\mathrm{Ba}_{2} \mathrm{Fe}_{2} \mathrm{O}_{5}$ 172

Figure 10.3. The coordination geometry of 7 crystallographically distinct Fe atoms and the connectivity of polyhedra in $\mathrm{Ba}_{2} \mathrm{Fe}_{2} \mathrm{O}_{5}$. Red, green, blue and yellow polyhedra represent 
tetrahedral, square pyramidal, pseudo square pyramidal and octahedral geometry,

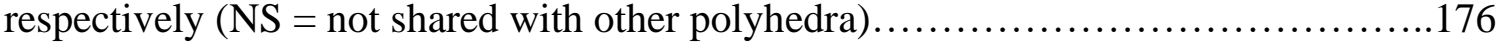

Figure 10.4. Scanning electron microscopy images of $\mathrm{Sr}_{2} \mathrm{Fe}_{2} \mathrm{O}_{5}$ (top) and $\mathrm{Ba}_{2} \mathrm{Fe}_{2} \mathrm{O}_{5}$ (bottom)

Figure 10.5. X-ray photoelectron spectroscopy data for (a) $\mathrm{Sr}_{2} \mathrm{Fe}_{2} \mathrm{O}_{5}$ and (b)

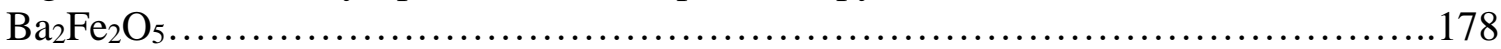

Figure 10.6. (a) Isothermal magnetization data, where black and red show $\mathrm{Sr}_{2} \mathrm{Fe}_{2} \mathrm{O}_{5}$ magnetization at $5 \mathrm{~K}$ and $300 \mathrm{~K}$, and blue and green represent the corresponding data for

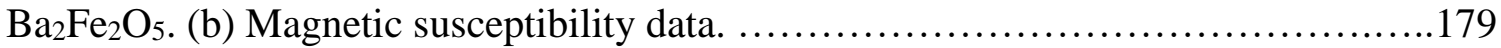

Figure 10.7. Electrical conductivity of (a) $\mathrm{Sr}_{2} \mathrm{Fe}_{2} \mathrm{O}_{5}$ and (b) $\mathrm{Ba}_{2} \mathrm{Fe}_{2} \mathrm{O}_{5}$ in inert atmosphere. Blue circles and red stars represent the data obtained while heating and cooling, respectively. The inset in (b) magnifies the data in the temperature range $600-900{ }^{\circ} \mathrm{C}$ for $\mathrm{Ba}_{2} \mathrm{Fe}_{2} \mathrm{O}_{5}$

Figure 10.8. Thermogravimetric analysis data for (a) $\mathrm{Sr}_{2} \mathrm{Fe}_{2} \mathrm{O}_{5}$ and (b) $\mathrm{Ba}_{2} \mathrm{Fe}_{2} \mathrm{O}_{5} \ldots \ldots .182$

Figure 10.9. Arrhenius plots for electrical conductivity of (a) $\mathrm{Sr}_{2} \mathrm{Fe}_{2} \mathrm{O}_{5}$ and (b) $\mathrm{Ba}_{2} \mathrm{Fe}_{2} \mathrm{O}_{5}$

Figure 11.1. Crystal structure of $\mathrm{Sr}_{2} \mathrm{Fe}_{2} \mathrm{O}_{6-\delta}$. (a) unit cell (b) side view and (c) Top view of the crystallographic structure which show the alternating $\mathrm{FeO}_{6}$ octahedra (cyan) and $\mathrm{FeO}_{5}$ square pyramids (purple). Sr atoms are removed for clarity.

Figure 11.2. Rietveld refinement profile for powder X-ray diffraction data of $\mathrm{Sr}_{2} \mathrm{Fe}_{2} \mathrm{O}_{6-\delta}$ in $14 / \mathrm{mmm}$ space group. Cross symbols represent experimental data, red solid is the model, vertical tick marks show Bragg peak positions, and the blue line represents the difference plot. Inset shows the peak bifurcation at higher $2 \theta$ angle.

Figure 11.3. SEM images of $\mathrm{Sr}_{2} \mathrm{Fe}_{2} \mathrm{O}_{6-\delta}$

Figure 11.4. X-ray photoelectron spectroscopy data for $\mathrm{Sr}_{2} \mathrm{Fe}_{2} \mathrm{O}_{6-\delta}$ 193 
Figure 11.5. Galvanostatic charge-discharge profiles of $\mathrm{Sr}_{2} \mathrm{Fe}_{2} \mathrm{O}_{6-\delta}$ anode electrode at the voltage range of $3.0-0.005 \mathrm{~V}$ with a current density of $25 \mathrm{~mA} / \mathrm{g}$ for $1^{\text {st }}, 2^{\text {nd }}, 10^{\text {th }}, 20^{\text {th }}, 30^{\text {th }}$, $40^{\text {th }}$ and $50^{\text {th }}$ cycles 195

Figure 11.6. Capacities versus cycle numbers of $\mathrm{Sr}_{2} \mathrm{Fe}_{2} \mathrm{O}_{6-\delta}$ anode electrode at the voltage range of $3.0-0.005 \mathrm{~V}$ with a current density of $25 \mathrm{~mA} / \mathrm{g}$.

196

Figure 11.7. C-rate test of $\mathrm{Sr}_{2} \mathrm{Fe}_{2} \mathrm{O}_{6-\delta}$ anode electrode at the voltage range of $3.0-0.005 \mathrm{~V}$ and different current densities of $25,50,100,200$, and $500 \mathrm{~mA} \mathrm{~g}^{-1}$, respectively

Figure 11.8. Cyclic voltammetry of $\mathrm{Sr}_{2} \mathrm{Fe}_{2} \mathrm{O}_{6-\delta}$ anode electrode scanned at the voltage range of $3.0-0.005 \mathrm{~V}$ with a scan speed of $1 \mathrm{mV} \mathrm{s}^{-1}$ 198

Figure 12.1. Crystal structures of (a) $\mathrm{Ca}_{2} \mathrm{Fe}_{2} \mathrm{O}_{6-\delta}$, (b) $\mathrm{CaSrFe}_{2} \mathrm{O}_{6-\delta}$, and (c) $\mathrm{Sr}_{2} \mathrm{Fe}_{2} \mathrm{O}_{6-\delta}$. In (a) and (b), different colors represent different orientations of tetrahedral chains 203

Figure 12.2. (a) Polarization curves showing the OER activities, obtained using the conventional glassy carbon electrode setup. (b) Comparison of the activity of $\mathrm{Sr}_{2} \mathrm{Fe}_{2} \mathrm{O}_{6}$ $\delta$ with state of the art materials $\mathrm{RuO}_{2}$ and $\mathrm{Ba}_{0.5} \mathrm{Sr}_{0.5} \mathrm{Co}_{0.8} \mathrm{Fe}_{0.2} \mathrm{O}_{3-\delta}(\mathrm{BSCF}) \ldots \ldots \ldots \ldots \ldots . . .205$

Figure 12.3. Tafel slopes indicating the OER kinetics .207

Figure 12.4. Plot of $\Delta j=j_{\text {anodic }}-j_{\text {catodic }}$ as a function of scan rate. The $C_{d l}$ for each material is equivalent to half of the slope 208

Figure 12.5. (a) Polarization curves showing the OER activities of pure disks of catalysts. (b) Stability test for $\mathrm{Sr}_{2} \mathrm{Fe}_{2} \mathrm{O}_{6-\delta}$ by performing 500 cycles. .209

Figure S1. Rietveld refinement profiles using powder X-ray diffraction for (a) $\mathrm{Ca}_{2} \mathrm{Fe}_{2} \mathrm{O}_{6-\delta}$, space group Pnma, (b) $\mathrm{CaSrFe}_{2} \mathrm{O}_{6-\delta}$, space group Ibm2, and (c) $\mathrm{Sr}_{2} \mathrm{Fe}_{2} \mathrm{O}_{6-\delta}$, space group $14 / \mathrm{mmm}$. Black symbols are the experimental data, red line represents the model, vertical tick marks show Bragg peak positions, and the lower blue line represents the difference plot. .213 
Figure S2. (a) The setup for testing the OER activity of catalysts disks, without glassy carbon electrode. (b) The entire measurement setup.

Figure S3. Cyclic voltammetry in non-faradic region to obtain double layer capacitance (Figure 4) for the three compounds (a) $\mathrm{Ca}_{2} \mathrm{Fe}_{2} \mathrm{O}_{6-\delta}$, (b) $\mathrm{CaSrFe}_{2} \mathrm{O}_{6-\delta}$, and (c) $\mathrm{Sr}_{2} \mathrm{Fe}_{2} \mathrm{O}_{6-}$

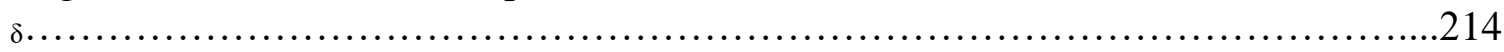

Figure S4. Polarization curve for $\mathrm{Sr}_{2} \mathrm{Fe}_{2} \mathrm{O}_{6-\delta}$, obtained using the conventional glassy carbon setup, and with the addition of carbon black to the catalyst. Comparison to Figure 2 indicates that carbon black does not improve the OER activity. ....................215

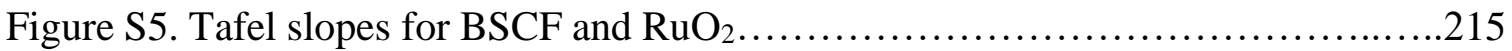

Figure S6. Plot of $\Delta \mathrm{j}=\mathrm{j}_{\text {anodic }}-\mathrm{j}_{\text {cathodic }}$ as a function of scan rate for (a) BSCF and (b) $\mathrm{RuO}_{2}$. The $\mathrm{C}_{\mathrm{dl}}$ for each material is equivalent to half of the slope........................216 


\section{LIST OF TABLES}

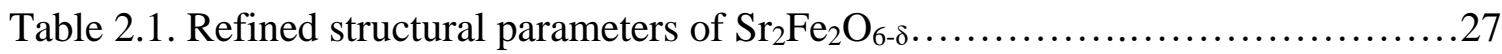

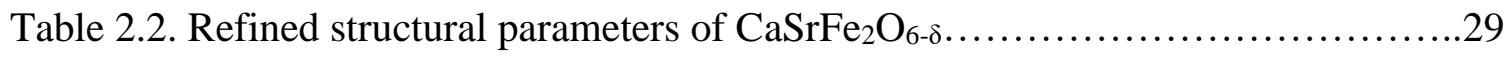

Table 2.3. Room temperature conductivity and activation energies....................37

Table 3.1. Refined structural parameters of $\mathrm{CaSrFeCoO}_{6-\delta}$ using powder X-ray

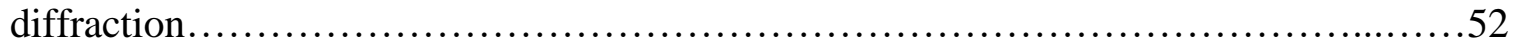

Table 3.2. Refined atomic parameters of $\mathrm{CaSrFeCoO}_{6-\delta}$ in $\mathrm{Ibm} 2$ space group using neutron

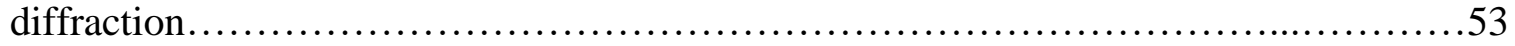

Table 3.3. Comparison of space groups and unit cell parameters for $\mathrm{Ca}_{2} \mathrm{FeCoO}_{6-\delta}$,

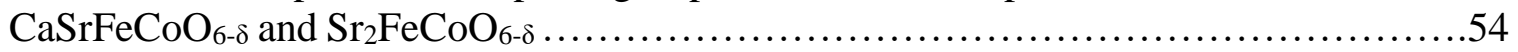

Table 3.4. Refined structural parameters of $\mathrm{Sr}_{2} \mathrm{FeCoO}_{6-\delta} \ldots \ldots \ldots \ldots \ldots \ldots \ldots \ldots \ldots \ldots$

Table 3.5. Refined structural parameters of $\mathrm{Ca}_{2} \mathrm{FeCoO}_{6-\delta} \ldots \ldots \ldots \ldots \ldots \ldots \ldots \ldots \ldots . \ldots . \ldots \ldots$

Table 3.6. Room temperature conductivity and activation energies.....................73

Table 5.1 The refined structural parameters for $\mathrm{Ca}_{2} \mathrm{Fe}_{0.5} \mathrm{Ga}_{1.5} \mathrm{O}_{5}$ from neutron diffraction. Space group: Pnma, $a=5.3669(2), b=14.6214(5), c=5.5927(2), R_{p}=0.0744, w R_{p}=$

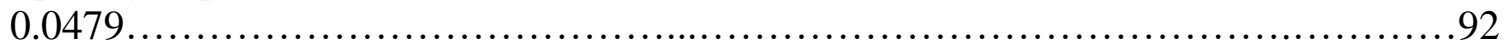

Table 6.1. Refined structural parameters from neutron diffraction data for $\mathrm{BaSrFe}_{2} \mathrm{O}_{6-\delta}$ $(\delta=0.5)$, space group $P m-3 m, \mathrm{a}=3.92851(4), R_{\mathrm{p}}=0.041, \mathrm{w} R_{\mathrm{p}}=0.033$. 105 
Table 6.2. Electrical conductivity, $\sigma\left(\mathrm{S} \mathrm{cm}^{-1}\right)$, and activation energies, $\mathrm{E}_{\mathrm{a}}(\mathrm{eV})$, for increase in electrical conductivity up to $400{ }^{\circ} \mathrm{C}$.

Table 7.1. Refined structural parameters for $\mathrm{Ca}_{2} \mathrm{FeGaO}_{6-\delta}$ from powder neutron diffraction. Space group Pnma, $a=5.38902(9) \AA, b=14.65164(22) \AA, c=5.60081(9) \AA, R_{p}=0.0405$, $w R_{p}=0.0208$.

Table 7.2. Refined structural parameters for $\mathrm{CaSrFeGaO}_{6-\delta}$ from powder neutron diffraction. Space group Ibm2, $a=5.6437(1) \AA, b=15.0577(4) \AA, c=5.4458(1) \AA, R_{p}=$ $0.0450, w R_{p}=0.0216$.

Table 7.3. Room Temperature Conductivity and Activation Energies

Table 8.1. Refined Structural Parameters for $\mathrm{BaSrCoFeO}_{6-\delta}$ using powder X-ray diffraction data..... 139

Table 8.2. Refined Structural Parameters for $\mathrm{BaSrCo}_{2} \mathrm{O}_{6-\delta}$ using powder X-ray diffraction data.

Table 8.3. Room Temperature Conductivity and Activation Energies 148

Table 9.1. Refined structural parameters for $\mathrm{Sr}_{2} \mathrm{Mn}_{2} \mathrm{O}_{6}$ using powder X-ray diffraction. Space group: $P 6_{3} / m m c, a=b=5.45233(8) \AA$, c $=9.0856(1) \AA, R_{\mathrm{p}}=0.030, \mathrm{w} R_{\mathrm{p}}=0.038, \chi^{2}=$ 1.790 159

Table 9.2. Refined structural parameters for $\mathrm{CaSrMn}_{2} \mathrm{O}_{6}$ using powder X-ray diffraction data. Space group: $P m-3 m, a=3.7772(1) \AA, R_{\mathrm{p}}=0.051, \mathrm{w} R_{\mathrm{p}}=0.064, \chi^{2}=1.406 \ldots \ldots .160$

Table 9.3. Room temperature conductivity and activation energies .163

Table 10.1. Refined structural parameters for $\mathrm{Sr}_{2} \mathrm{Fe}_{2} \mathrm{O}_{5}$ using powder X-ray powder diffraction. Space group is $I b m 2$, and unit cell parameters are $a=5.68023(6), b=$ $15.5862(2), c=5.53425(6) \AA$.

Table 10.2. Refined structural parameters for $\mathrm{Ba}_{2} \mathrm{Fe}_{2} \mathrm{O}_{5}$ using powder X-ray powder diffraction. Space group is $P 2{ }_{1} / c$ and unit cell parameters are $a=6.9772(1), b=11.7376(2)$, $c=23.4575(4) \AA$, and $\beta=98.7540(8)^{\circ}$. 174

Table 10.3. Different coordination geometry and bond distances of $\mathrm{Fe}$ in $\mathrm{Ba}_{2} \mathrm{Fe}_{2} \mathrm{O}_{5} \ldots 175$ xxvi 
Table 10.4. The connectivity of polyhedra in $\mathrm{Ba}_{2} \mathrm{Fe}_{2} \mathrm{O}_{5}$.

Table 10.5. Room temperature conductivity and activation energies.

Table 11.1. Refined structural parameters for $\mathrm{Sr}_{2} \mathrm{Fe}_{2} \mathrm{O}_{6-\delta}$ from powder X-ray diffraction. Space group I4/mmm, $a=10.9301(2) \AA, b=10.9301(2) \AA, c=7.6958(2) \AA, R_{p}=0.0211$, $w R_{p}=0.0299$.

Table S1. The refined structural parameters for $\mathrm{Ca}_{2} \mathrm{Fe}_{2} \mathrm{O}_{6-\delta}$ from powder X-ray diffraction. Space group: Pnma, $a=5.40238(5), b=14.7018(1), c=5.57261(5), R_{p}=0.013, w R_{p}=$ $0.018, \chi^{2}=1.941$

Table S2. The refined structural parameters for $\mathrm{CaSrFe}_{2} \mathrm{O}_{6-\delta}$ from powder $\mathrm{X}$-ray diffraction. Space group: Ibm2, $a=5.6313(4), b=15.181(1), c=5.4695(4), R_{p}=0.018$, $w R_{p}=0.026, \chi^{2}=1.604$

Table S3. The refined structural parameters for $\mathrm{Sr}_{2} \mathrm{Fe}_{2} \mathrm{O}_{6-\delta}$ from powder X-ray diffraction. Space group: $14 / \mathrm{mmm}, a=10.9345(7), b=10.9345(7), c=7.6988(5), R_{p}=0.022, w R_{p}=$ $0.031, \chi^{2}=1.505$. 


\section{CHAPTER 1}

\section{INTRODUCTION}

\subsection{Introduction to solid states chemistry}

\section{Solid state chemistry}

Solid-state chemistry, also known as material chemistry, is the study of the synthesis, structure, chemical, and physical properties and the application of solid materials particularly non-molecular solids. In general, the chemistry, structure and properties of the solid materials are interrelated. Thus, for a specific solid state chemistry, the study should be aimed at getting detailed knowledge of the three factors such as how the chemistry affects in the structure of a material and then how the structure affects the physical properties. One example of study of solid state chemistry is study of structure-property relation in perovskite oxide materials. Richard J. D. Tilley has well collected the works of structure-property relation in perovskite oxides. ${ }^{1}$ They have been intensively studied for their dielectric, piezoelectric, and ferroelectric nature. Now the range of property-study has been extended to magnetic ordering, multiferroic properties, electronic conductivity, superconductivity, thermal and optical properties. ${ }^{1}$ Perovskite oxides have wide range of structures from cubic $\mathrm{SrTiO}_{3}$ to cation and anion deficient phases to hexagonal perovskites related to $\mathrm{SrMnO}_{3}{ }^{1}$ The chemical and physical properties of any members of these structural forms can be tuned to wide ranges by simply substituting A or B site cations. Next good example of structure-property relation can be found in a literature ${ }^{2}$ which states the correlation between anti-ferromagnetic Neel temperature and structural destruction in 
the series of rare earth ortho-ferrites $(\mathrm{LnFeO} 3, \mathrm{Ln}=\mathrm{La}-\mathrm{Lu})$. The $\mathrm{t}$ value is below 1 for all $\mathrm{LnFeO}_{3}$ leading to perovskite superstructure due to tilting of octahedra. The crystal structure will experience increasing tilt of the octahedra due to the decreasing effect of A cation radius down the lanthanide series. The interaction between B cations coupled with intervening oxygen ions results Antiferromagnetism. ${ }^{3}$ The effect of super-exchange leads to antiparallel alignment of the spins. The Neel temperature will become lower as the distortion increases causing more tilt. Thus, the Neel temperature can be correlated with the tilt of the octahedra. Thus, structural-property in solid state material can be revealed. So, a solid state chemist requires to have knowledge of structural - property relation to design a material of desired structure, property and application.

\section{Synthesis of solid state materials}

As is clear from the title "solid state chemistry", generally no solution but hihg temperature (heat) is used to propare a desired material. However, in some cases such as in coprecipatipitaiton and solgel method, solutions are used in the pre-stage of the synthesis and in subsequent steps, high heat is used to decompose the precurser compounds after drying. For perovskite oxides, convensional solid state method can normally be employed. In this process, the precurser compounds are mixed in stoichiometric proportions and mixed thoroughly. Since the solid state precursers require complete decomposition to form a completely new compound, multiple and high heating is required. In this case, the reaction takes place by ion diffusion at high temperature which is very slow process. Generally, peroskite oxides are synthesized from nitrates or carbonates. So, two stages of heating namely, calcination (low tempetature) and sintering (high temperature) are acomplished to get a material of pure phase. This technique is commonly used to 
investigate the chemistry induced structural changes and thermal stability of the strucutres. The detailed procedure for this method can be obtained in this thesis based articles.

III. Characterization methods of solid-state mateerials

i. Structure and phase purity identification

Once a sample is synthesized, its phase identification is first job to go ahead with other characterization procedures. A phase is a crystalline solid with a regular 3-dimensional arrangement of the atoms. Powder X-ray diffraction method is employed as a fast phase identification technique in solid state chemistry. The measured diffraction peak positions and intensities are like a fingerprint for a particular phase. The spacing of powder diffraction lines are dependent on the unit cell parameters for a crystalline compound. Phase purity is checked by comparison of the measured pattern with the entries in reference databases using a search-match algorithm. This is also known as qualitative phase analysis. The peak indexing is simplified by the computer programming during the comparison with the structure of another known compound. The powder pattern of a phase is confirmed by refinement process using certain computer programs such as Rietveld refinement using GSAS with EXPIGUI interface, Full prof fitting and Topaz fitting programs, where a known phase model is used for XRD data fitting.

The diffraction data may also be collected using neutrons. Neutron diffraction is a result of interaction of neutrons with nuclei and therefore scatters strongly with light atoms as well as hevy atoms and can easily differentiate between isotopes. X-rays intereact with electron clouds and therefore scatter strongly from heavier elements with larger electron clouds. So, If a compound with elements of nearly similar atomic mass or with light elements is to be identified with exact composition and atomic positions, then neutron 
diffraction is suggesed. For example, we used neutron diffraction for $\mathrm{Ca}_{2} \mathrm{Fe}_{1.5} \mathrm{Ga}_{0.5} \mathrm{O}_{5}$ compound to find the atomic position of $\mathrm{Fe}$ and $\mathrm{Ga}$ elements in the crystal structure which X-ray diffraction could not explain.

\section{ii. Magnetic measurement}

Most materials have no permanent magnetic moment but a moment is induced in the presence of a field. The response is called magnetic susceptibility $(\chi)$. Usually magnetic susceptibility is measured to find the degree of material magnetization in an applied magnetic field. Mathematically, it is the ratio of magnetization $\mathrm{M}$ (magnetic moment per unit volume) to the applied magnetizing field intensity $\mathrm{H}$.

$$
\chi=\frac{M}{H}
$$

Magnetic susceptibility, $\chi$, is a function of temperature, T. So, it is commonly measured over a range of temperature. Magnetic field is also applied during susceptibility measurement. Generally, two measurements are performed for $\chi$ versus T: (a) Zero Field Cooled (ZFC) measurement where sample is first cooled in the absence of a field and then susceptibility is measured in the applied field as temperature rises. (b) Field Cooled (FC) measurement where sample is first cooled in the presence of a field and then susceptibility is measured in the same field as temperature rises. The data obtained from these two measurements, $\chi$ versus $\mathrm{T}$ may overlap or diverge from each other. The divergence shows the formation of magnetic domains induced by the applied field. the $\chi$ versus $\mathrm{T}$ plot appears as a smooth curve at low temperature region for a paramagnet. Any deviation from this behavior is an indication for the presence of a magnetically ordered state and the 
temperature at which the deviation occurs is the magnetic transition temperature. Measurement of magnetization of a material as a function of the applied filed, $\mathrm{H}$, at constant temperature is also common. The magnetization is recorded during $\mathrm{H}$ rising and falling, both times. the $\mathrm{M}$ versus $\mathrm{H}$ graph is linear for a paramagnet, (as long as the saturation at high field and low $\mathrm{T}$ is not reached) and the data collected during both times, $\mathrm{H}$ value rising and falling will overlap. However, if there is any uncompensated magnetic moment in the system, divergence is observed for the data obtained for increase and decrease of $\mathrm{H}$.

\section{iii. Other techniques}

Other techniues for characterization include scanning electron microscopy for micro structure analysis, X-ray Photoelectron Spectroscopy for oxidation state analysis, iodometric titration for oxygen content calculation.

iv. Physico-chemical property measurement

Temperature dependent oxygen absorption and desorption behavior of the materials is measured by thermogravimetric analysis using Ar gas. DC measurement and impedance spectroscopy study are performed for temperature dependent conductivity behavior analysis and conductivity mechanismin of a material, respectively. CV measurement is performed for oxygen evolution reaction.

\section{Basics of diffraction}

i. Bragg's law

$\mathrm{X}$-rays are electromagnetic waves with a much shorter wavelength than visible light, typically on the order of $1 \AA\left(1 \times 10^{-10} \mathrm{~m}\right)$. When a beam of X-rays fall on a single particle, it scatters the incident beam uniformly in all directions. These scattered beams can add 
together in a few directions to reinforce each other resulting a diffraction. The regular arrangement of atomic particles in a crystal system is responsible for the diffraction of the beams. X-ray diffractions were used in the identification of our material's crystal structures.

Crystals are regular arrays of atoms. These arrays form imaginary planes in the crystal system called crystal lattice. Lattice planes are crystallographic planes, characterized by the index triplet hkl, the so-called Miller indices. Parallel planes have the same indices and are equally spaced, separated by the distance $\mathrm{d}_{\mathrm{hkl}}$, (figure 1.1.1). There can be a number of sets of planes running in different directions in a crystal system, and they are named according to their orientation relative to the axes of the unit cell, the smallest repeated unit in the crystal system. For example, a set of planes, in an ideal cubic crystal system, running parallel to bc plane intercepts a axis but do not intercept $\mathrm{b}$ and $\mathrm{c}$ axes and can be expressed by Miller indices, hkl as $(1,0,0)$ planes. These planes are responsible for the constructive interference. 


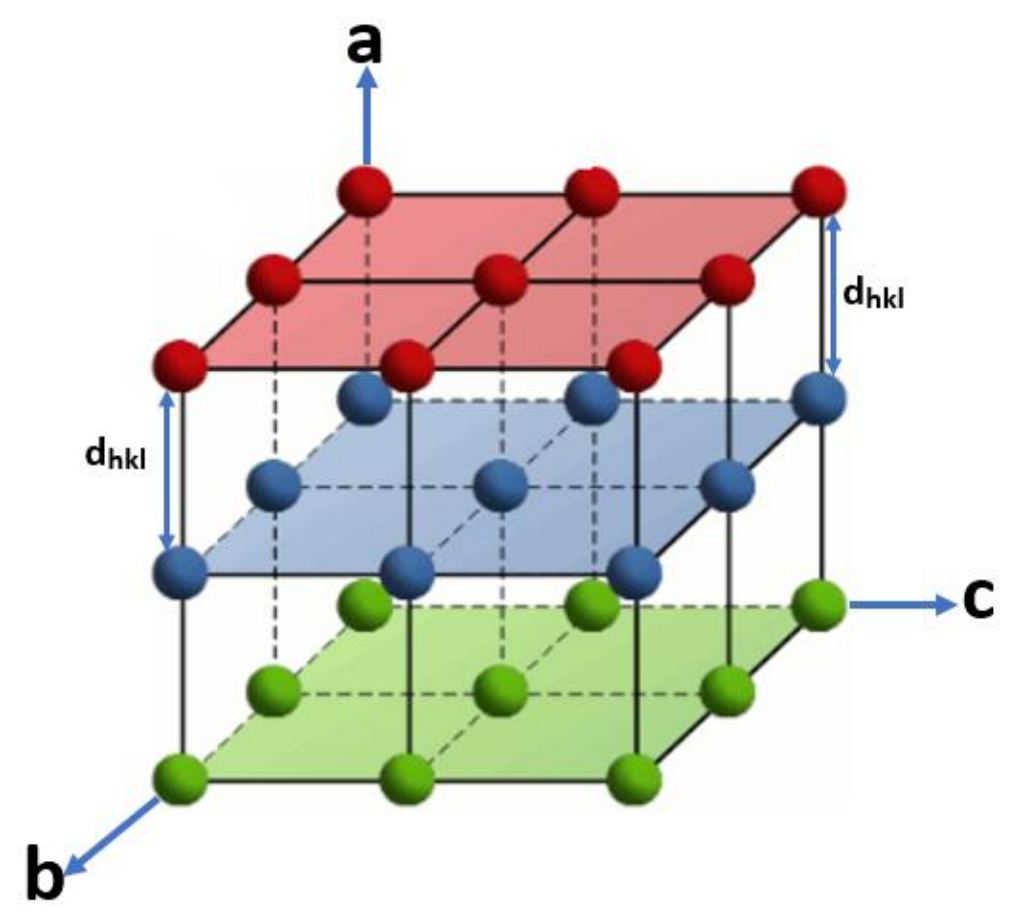

Figure 1.1.1. Lattice planes formed by regular arrays of atoms in a crystal

According to Bragg's law, when the waves are scattered from lattice planes separated by the interplanar distance $d$, the scattered waves interfere constructively and they remain in phase when the difference between the path lengths of the two waves is equal to an integer multiple of the wavelength. The path difference between two waves undergoing interference is given by $2 \mathrm{~d} \sin \theta$, where $\theta$ is the scattering angle (as shown in figure 1.1.2). Mathematically, it can be expressed as

$$
\mathrm{n} \lambda=2 \mathrm{~d} \sin \theta
$$

When the diffraction meets the above condition, a constructive interference between the diffracted beams becomes possible to generate a diffraction. The diffraction data are commonly expressed as a function of $2 \theta$ where the diffraction peak at smallest $2 \theta$ corresponds to the largest $d$ spacing. 


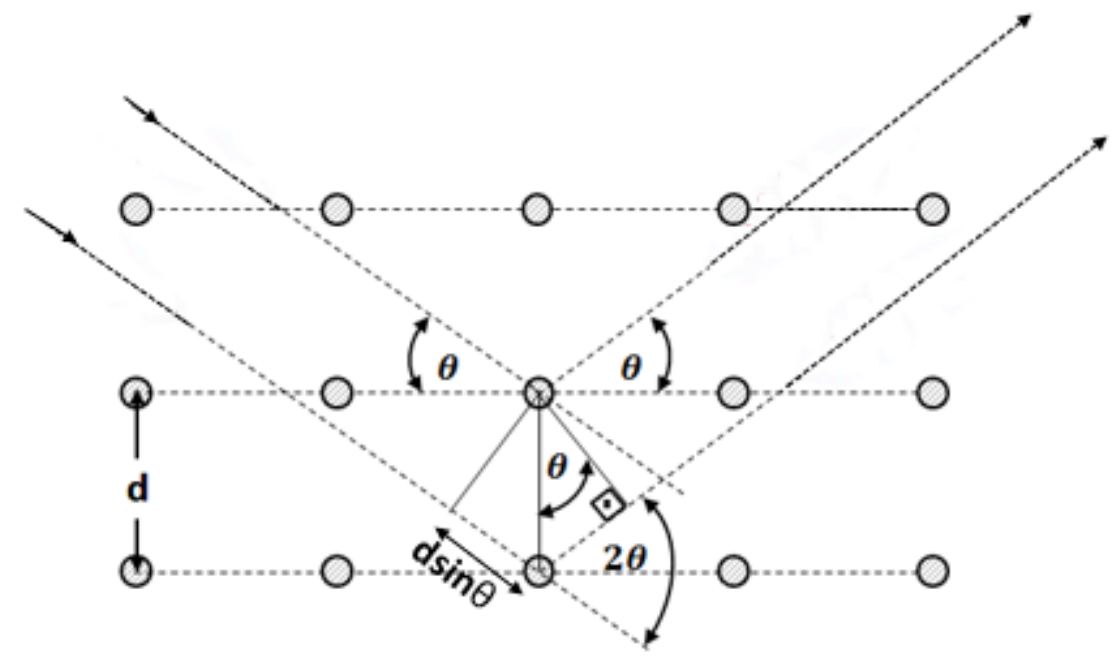

Figure 1.1.2. Bragg's law in diffraction

\section{ii. Reciprocal lattices}

To understand the reciprocal lattice, let us consider a unit cell of a cubic crystal (figure 1.1.3). Let us consider an arbitrary point $\mathrm{A}$ from which a normal $\overrightarrow{A B}$ (vector) is drawn to a plane "bc". The distance of $\overrightarrow{A B}$ should be reciprocal of $\mathrm{d}_{\mathrm{hkl}}$ (distance between two bc planes) i.e. $\overrightarrow{A B}=\frac{1}{d_{h k l}}$. Every line drawn normal to all planes in all directions must have the distance equal to $\frac{1}{d_{h k l}}$. The planes can also be characterized by a vector $\left(\underline{\sigma}_{h k l}\right)$ perpendicular to the normal vector $\overrightarrow{A B}$. Now, the lengths of the perpendicular vectors $(\overrightarrow{A B})$ are reciprocal to the interplanar spacings. The end points of these vectors (blue arrows in figure) also produce a periodic lattice that is known as the reciprocal lattice of the original direct lattice. 


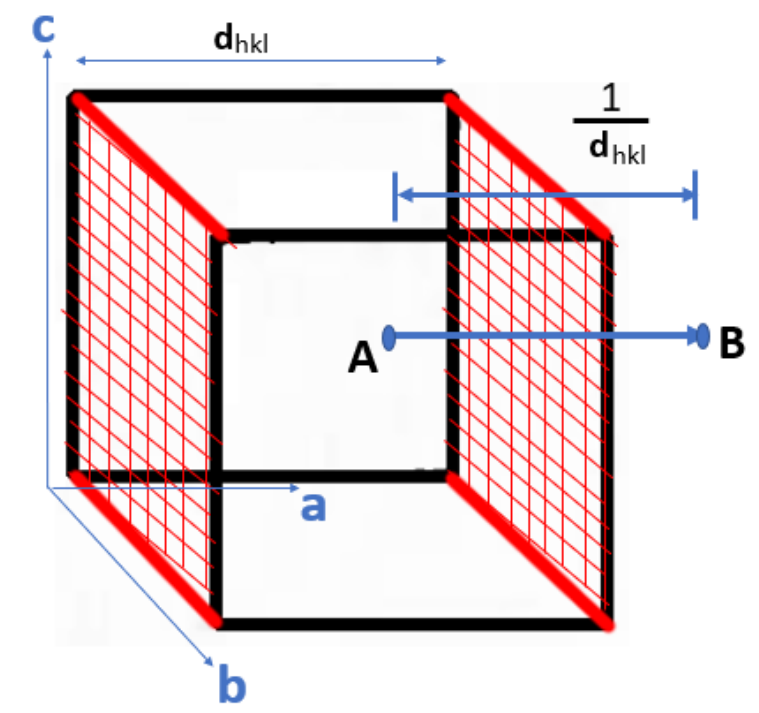

Figure 1.1.3. Reciprocal lattice point

If we consider a set of parallel planes hkl with the interplanar distance $\mathrm{d}_{\mathrm{hkl}}$ and if we take one ( $\left.\underline{\sigma}_{h k l}\right)$ with length $1 / d_{h k l}$ from a set of vectors normal to the planes' family, then, $\underline{\sigma_{h k l}}$ represents the whole family of hkl planes having an interplanar spacing given by $\mathrm{d}_{\mathrm{hkl}}$. iii. Ewald's sphere

The Ewald's sphere is the most useful tool to understand the occurrence of diffraction spots. It helps to visualize the properties of Bragg's law, $\mathrm{n} \lambda=2 \mathrm{~d} \sin \theta$. It is an imaginary sphere of radius $1 / \lambda$ surrounding a crystal ( shown in figure 1.1.4). If we consider a real crystal in the center of the sphere, the origin of the reciprocal lattice lies in the transmitted beam at the edge of the Ewald sphere. Diffraction maxima (reflections, diffraction spots) occur only when the Bragg equation is satisfied and the Bragg's condition, $\mathrm{n} \lambda=2 \mathrm{~d} \sin \theta$ is satisfied by the diffraction only when a reciprocal lattice point lies exactly on the Ewald sphere. 


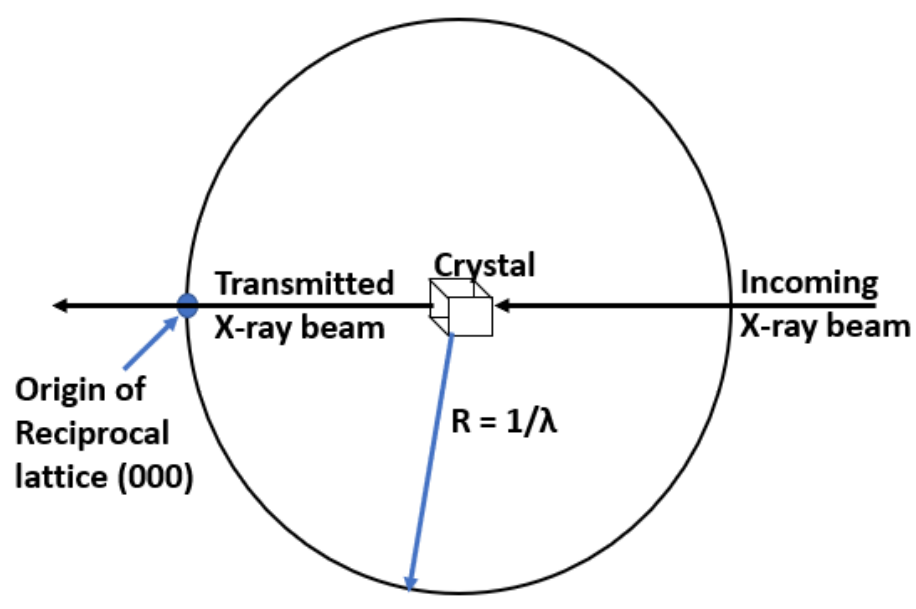

Figure 1.1.4. Ewald's sphere forming diffraction

\subsection{Introduction to oxygen-deficient perovskites}

Perovskites are a class of materials with similar structure and a myriad of exciting properties like superconductivity, magnetoresistance, catalysis and many more. They are easy to synthesize and are considered as the future of solar cells because their attractive structure makes them perfect for enabling low-cost, efficient photovoltaics. Since they have characteristics of mixed ionic and electronic conductivity, they can be applied as electrodes for fuel cells and hence they are also considered as future energy materials. They are predicted to play a role in next-gen electric vehicle batteries, sensors, lasers and much more.

\section{Perovskite oxides}

Oxide groups containing two or more different cations are called complex or mixed oxides. Many types of crystal structures are known for the oxides. One of them is perovskite structure. Perovskite oxides have the crystal structure similar to $\mathrm{CaTiO}_{3}$ and it 
is expressed in general chemical formula $\mathrm{ABO}_{3}$ where $\mathrm{A}$ is generally alkaline earth metal or rare earth metal cations and $\mathrm{B}$ is transition metal or main $\mathrm{p}$ block metal cations (specifically group 13 metals). The ideal cubic-symmetry of perovskite structure has the B cation in 6-fold coordination surrounded by six oxide anions forming an octahedron. The A cation is surrounded by 8 octahedra with 12 -fold cuboctahedral coordination. The size of A cations is bigger than B cations. The ideal structure of perovskite, which is illustrated in Fig. 1.1, is a cubic lattice.

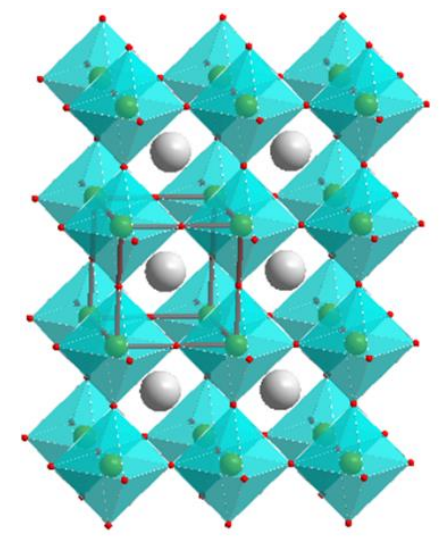

(a)

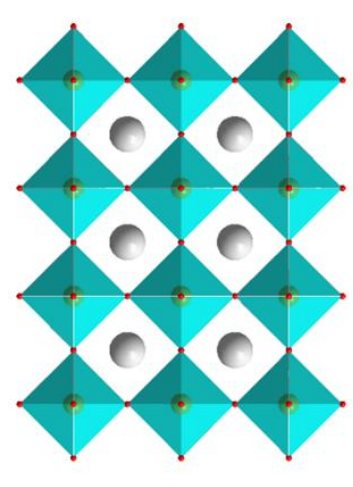

(b)

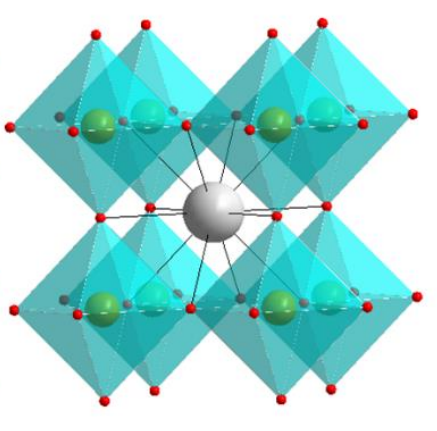

(c)

Figure 1.2.1. Crystal structure of perovskite oxide. (a) Crystallographic unit cell and corner-sharing $\mathrm{BO}_{6}$ octahedra (cyan) are highlighted. The large white spheres are the A atoms. (b) View along the unit cell axis. Because of the cubic symmetry, the three axes are identical. (c) Coordination geometry around the A atom, which is 12-coordinated.

These $\mathrm{ABO}_{3}$ oxides are regarded as purely ionic crystals where the following relationship between the radii of the $\mathrm{A}, \mathrm{B}$, and $\mathrm{O}^{2-}$ ions holds true for a cell axis ( a ) in the ideal cubic structure, ${ }^{1}$

$$
a=\sqrt{2}\left(r_{A}+r_{O}\right)=2\left(r_{B}+r_{O}\right)
$$


Although few compounds have this ideal cubic structure, many oxides have slightly distorted variants with lower symmetry (e. g., hexagonal or orthorhombic). There are various types of distortions in the perovskite structure that are strongly related to their properties. Tolerance factor $(t)$ can be applied in order to understand the deviations from the ideal cubic structure. tolerance factor $(t)$ can be found out from the following equation. ${ }^{1}$

$$
t=\frac{\left(r_{A}+r_{O}\right)}{\sqrt{2}\left(r_{B}+r_{O}\right)}
$$

In perovskite-type compounds, the value of $t$ lies between approximately 0.80 and 1.10 and the ideal cubic structure has the value of $t$ close to 1 . It has been found that the oxides with the lower $t$ values $(0.85)$ crystallize in the distorted variant of cubic form such as orthorhombic or rhombohedral while greater than 1 gives hexagonal or tetragonal structure including units of face sharing BO6 octahedra. Thus, the crystal structure can be transformed by substituting A or B cation by another cation of different ionic radius which alters the Tolerance factor.

\section{Oxygen deficient perovskites}

Oxygen deficient perovskite (ODP) oxides are a class of compounds having less oxygens than in perovskite oxides and it is represented by general formula $\mathrm{ABO}_{3-\mathrm{x}}$ or $\mathrm{A}_{2} \mathrm{~B}_{2} \mathrm{O}_{6}$ $\delta$ where $\mathrm{x}$ or $\delta$ represent oxygen deficiency. Figure 1.2.2 demonstrates a structure of ODP. If $\mathrm{A}$ and $\mathrm{B}$ have different cations (where $\mathrm{A}$ and $\mathrm{A}^{\prime}$ are alkaline earth metal ions and $\mathrm{B}$ and B' are transition metal ions) like in $\mathrm{CaSrFeMnO}_{5}$, the formula can be expressed as AA'BB' ${ }_{6-\delta}$. In oxygen deficient perovskites, the oxygen vacancy (also called as defect) can transform the coordination geometry around B cation from octahedra to tetrahedra ${ }^{4}$ or 
square pyramidal ${ }^{4}$ geometry. There are many ways of vacancy ordering/disordering which make oxygen deficient perovskites possible to have structural flexibility ${ }^{5-6}$. If tetrahedral geometries are formed, $\mathrm{BO}_{4}$ tetrahedra can share corners with other $\mathrm{BO}_{4}$ tetrahedra or $\mathrm{BO}_{6}$ octahedra. This corner sharing tetrahedra can arrange in a regular pattern forming a long chain. This chain may form layer alternating with the layer of $\mathrm{BO}_{6}$ octahedra. These alternating layers are demonstrated in figure 1.2.2. A number of different space group symmetries arise due to difference in the ordering of the tetrahedral chains within the unit cell. ${ }^{7}$ The space group is determined by the relative orientation of the tetrahedral chains. There are two possible orientations, which are arbitrarily called right-handed and lefthanded. If all tetrahedral chains have the same orientation, the space group $I b m 2$ is obtained. If the tetrahedral chains have the same orientation within each layer but are oriented opposite to the chains in the next tetrahedral layer, the resulting space group is Pnma. The random orientation of tetrahedral chains leads to the space group Icmm. A less common space group is $\mathrm{Pbcm}$, where each tetrahedral chain is oriented opposite to all of its nearest neighbors within the same layer and in the neighboring layers. These space groups belong to brownmillerite structures. 


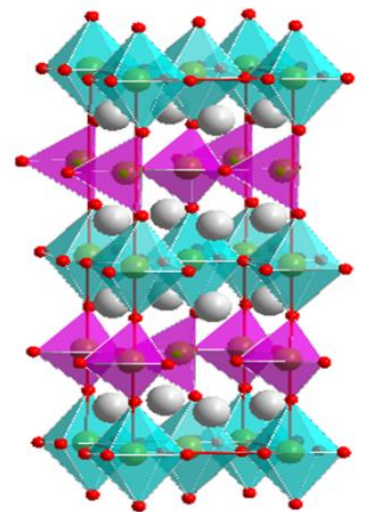

(a)

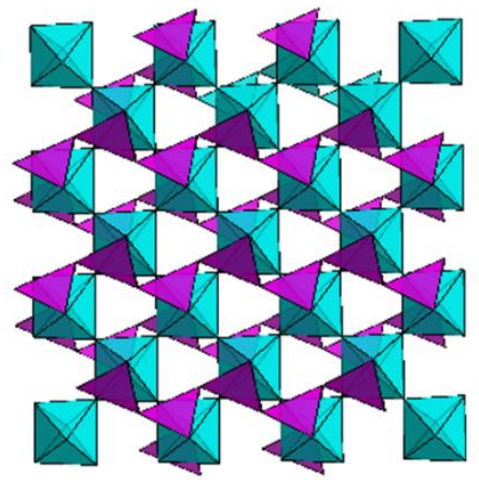

(b)

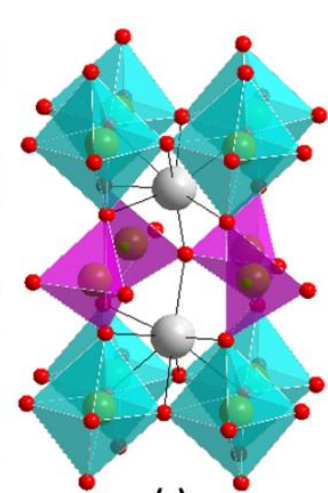

(c)

Figure 1.2.2. Crystal structure of $\mathrm{CaSrFeCoO}_{6-\delta}$ (a) crystal structure showing unit cell with alternating $(\mathrm{Fe} / \mathrm{Co}) \mathrm{O}_{6}$ octahedra (cyan) and $(\mathrm{Fe} / \mathrm{Co}) \mathrm{O}_{4}$ tetra (pink) layers. The large white spheres are Sr atoms, the green spheres inside octahedra and tetrahedra are Fe and Co atoms and small red spheres are oxygen atoms. (b) A view along b axis to show the orientation of tetra next to each other in each layer. All atoms are removed for clarity. (c) shows the coordination geometry around the $\mathrm{Sr}$ atoms. Note that the $\mathrm{Sr}$ is 8-coordinated.

III. Physical properties and their possible applications.

The oxygen deficient perovskites contain $\mathrm{BO}_{6}$ octahedra, $\mathrm{BO}_{4}$ tetrahedra and/or $\mathrm{BO}_{5}$ square pyramids. In such a case, the $\mathrm{B}$ cation can have multiple oxidation states like $\mathrm{Fe}^{3+}$ and $\mathrm{Fe}^{4+}$ which lead to charge transport. ${ }^{4}$ Sometimes these structures are distorted due to some kind of structural strains leading to the creation of structure induced small polarons. ${ }^{8-}$ ${ }^{9}$ This also makes the materials conductive.

B cations in perovskite oxides are transition metals. Transition metal complexes with unpaired d-electrons in transition metal are magnetic. The spin of a single electron is $+(1 / 2)$ or $-(1 / 2)$. when two electrons are paired with each other, the magnetic moments are counter balanced due to opposite spins but when the electron is unpaired, it creates a weak magnetic 
field. More unpaired electrons increase the paramagnetic effects. When a transition metal is in coordination complex, its electron configuration changes due to the repulsive forces between electrons in the ligands and electrons in the metal. Depending on the strength of the ligand, the compound may be paramagnetic or diamagnetic.

In recent years, oxygen deficient perovskites have been studied for the application in energy production and storage such as oxygen evolution reaction, hydrogen evolution reaction and battery materials.

\section{Roll of A- and B-site cations}

When A-site or B-site cation is substituted in a material, it may result in the transformation of crystal structure in the material due to the radii variation. This structural transformation can affect properties of these materials. When B-site cation is substituted, the crystal structure changes due to the change in the B-site ionic radius or the charge on the cation. $\mathrm{Sr}_{2} \mathrm{Fe}_{2} \mathrm{O}_{5}$ has been reported to have a vacancy-ordered structure containing $\mathrm{FeO}_{6}$ octahedra and $\mathrm{FeO}_{4}$ tetrahedra ${ }^{10}$. when one of the $\mathrm{Fe}$ atoms (which is $\mathrm{B}$-site cation) is substituted with $\mathrm{Mn}$, the resulting compound, $\mathrm{Sr}_{2} \mathrm{FeMnO}_{5}$, contains vacancies distributed randomly, without any type of ordering. ${ }^{11}$ Similarly, tetragonal compound, $\mathrm{Sr}_{2} \mathrm{Fe}_{2} \mathrm{O}_{6-\delta}$ with magnetic moments in spin-density wave state, transforms to cubic $\mathrm{Pm}-3 m$ structure of $\mathrm{Sr}_{2} \mathrm{FeMnO}_{6-\delta}$ when one of the $\mathrm{Fe}$ atoms is replaced by $\mathrm{Mn}$. The resulting material, $\mathrm{Sr}_{2} \mathrm{FeMnO}_{6-\delta}$, has inhomogeneous magnetic ground state, where the majority of the sample contains fluctuating spins. ${ }^{11}$ Even minor changes to the B-site cations can sometimes lead to major changes, as highlighted by the difference between $\mathrm{Sr}_{2} \mathrm{Fe}_{1.9} \mathrm{Cr}_{0.1} \mathrm{O}_{6-\delta}$, cubic $P m-3 m$, and $\mathrm{Sr}_{2} \mathrm{Fe}_{1.9} \mathrm{Co}_{0.1} \mathrm{O}_{6-\delta}$, orthorhombic $\mathrm{Cmmm} .{ }^{12}$ Again this leads to significant variation of magnetic properties in these two materials. ${ }^{12}$ 
The material properties can also be affected by changes in the A-site cation. An example is the difference between the two compounds $\mathrm{Ca}_{2} \mathrm{Fe}_{2} \mathrm{O}_{5}$ and $\mathrm{Sr}_{2} \mathrm{Fe}_{2} \mathrm{O}_{5} .{ }^{13-14}$ While both of these materials have ordered structures, where Fe atoms have both octahedral and tetrahedral geometry, the change in the A-site cation leads to different space groups. $\mathrm{Ca}_{2} \mathrm{Fe}_{2} \mathrm{O}_{5}$ crystallizes in the primitive space group $P n m a,{ }^{15}$ whereas $\mathrm{Sr}_{2} \mathrm{Fe}_{2} \mathrm{O}_{5}$ has a bodycentered space group Icmm. ${ }^{10}$ A similar effect is observed for $\mathrm{Ca}_{2} \mathrm{GaMnO}_{5}$ and $\mathrm{Sr}_{2} \mathrm{GaMnO}_{5}$, where the change in the A site cation results in changes in the space group. ${ }^{16}$

\section{Electrical conductivity of oxygen deficient perovskites}

Many ODPs are mixed oxygen ion and electron conducting materials. Electronic transport in ODPs takes place through holes. ${ }^{13,17}$ It is also called as polaron mechanism. For this mechanism, materials should have elements with multiple oxidation states at B-site. ${ }^{18-19}$ For example, $\mathrm{Sr}_{2} \mathrm{Fe}_{2} \mathrm{O}_{6-\delta}$, a well-known ODP, has the oxidation states of $\mathrm{Fe}^{3+}$ and $\mathrm{Fe}^{4+}{ }^{40} \mathrm{In}$ such materials, the electrons hop through M-O-M bond system. In the case of $\mathrm{Sr}_{2} \mathrm{Fe}_{2} \mathrm{O}_{6-\delta}$, the electrons hop through $\mathrm{Fe}^{3+}-\mathrm{O}-\mathrm{Fe}^{4+}$ where the $\mathrm{Fe}^{3+}$ converts to $\mathrm{Fe}^{4+}$ and vice versa after electron hopping. The speed of electron hopping is fast and hence it looks like the positive charge is moving during the electron hopping. So, it is considered as positive charge (also called hole) movement or P-type conductivity and it is called polaron mechanism. The schematic representation of polaron mechanism is shown in figure 1.2.3. The electronic transport in ODPs is governed by various factors. If the hole concentration (polarons) is higher in a material, the electronic transport and electrical conductivity is higher. ${ }^{13,17}$ It has been shown that changes in the electrical conductivity correlate with changes in the bond lengths and angles. ${ }^{21-22}$ The shorter the M-O bond length and the larger the M-O-M bond angle, the better is the orbital overlap and the electronic transport and the conductivity 
becomes better. Thus, the electrical conductivity of ODP does not depend on only one factor. The conductivity is p-type in metallic ODPs and the polaron mechanism is temperature activated. ${ }^{23-24}$

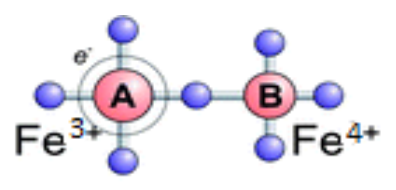

Initial

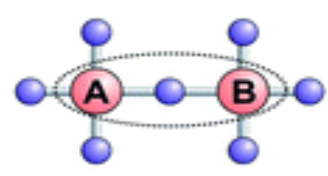

$\mathrm{Fe}^{4+} \mathrm{C}$

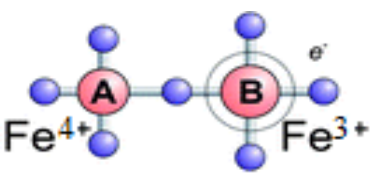

End

Figure 1.2.3. Representation of electron hopping through $\mathrm{Fe}^{3+}-\mathrm{O}-\mathrm{Fe}^{4+}$ bond system

VI. Magnetism in perovskite oxides

Magnetic moment of a system shows the strength and the direction of its magnetism. Magnetism results due to uneven interaction of the magnetic dipole moments. An electron has an electron magnetic dipole moment generated by spinning electric charge. There are many different magnetic behaviors such as paramagnetism, diamagnetism, and ferromagnetism. In perovskite oxides with B cations having unpaired d-electrons such as $\mathrm{Fe}^{3+}, \mathrm{Mn}^{3+}, \mathrm{Co}^{3+}$, magnetic ordering can take place. However, in most of the cases antiferromagnetism and ferromagnetism take place due to super- and double- exchange, respectively. ${ }^{1}$ The energy of the antiferromagnetic system is lower due to the antiparallel alignment of the spins. This system is established by coupling the unpaired d- electrons of two B cations by p-electrons of an intervening oxygen between them as shown in figure 
below. The presence of vacancy can affect in the magnetism of perovskite oxides. ${ }^{1}$

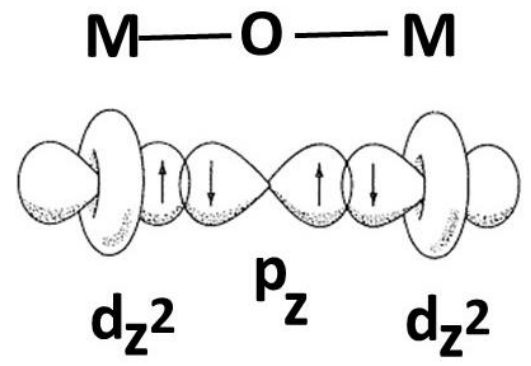

Figure 1.2.4. Super exchange by coupling two metals with unpaired d-electrons with an oxygen anion.

There are different possible anti-ferromagnetic ordering schemes in perovskite oxides such as A-, C- and G-type. A-type AFM has the atoms with opposite moments in adjacent layers. C -type AFM has neighboring atoms in the layers with opposite spins and $\mathrm{G}$ - type AFM has all neighboring B ions with opposite spins. The different schemes are shown below in figure 1.2.5.

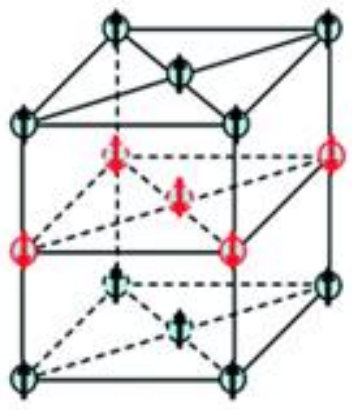

A - AFM

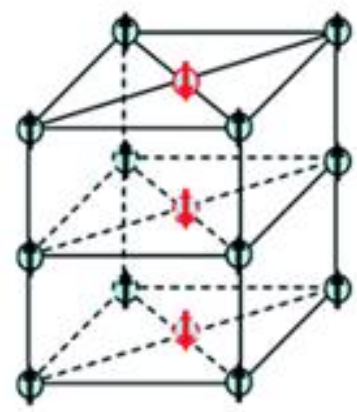

C - AFM

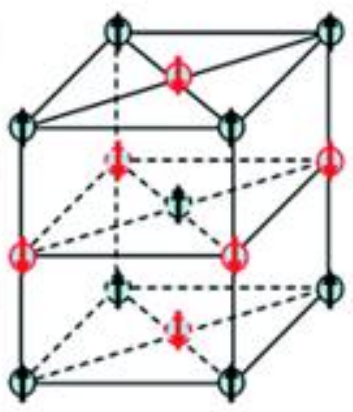

G - AFM

Figure 1.2.5. Three different ordering schemes in antiferromagnetic perovskite oxides 


\section{Oxygen evolution reaction}

Oxygen evolution reaction deals with electrolysis of water. Water electrolysis is the process of electrically splitting water into oxygen and hydrogen. The reaction can be expressed as $2 \mathrm{H}_{2} \mathrm{O} \rightarrow 2 \mathrm{H}_{2}+\mathrm{O}_{2}$

Here, $\mathrm{H}_{2}$ is evolved at cathode and $\mathrm{O} 2$ is evolved at anode. The reaction is associate with $1.23 \mathrm{~V}$ of potential in all media at standard condition. ${ }^{25}$ The efficiency of electrolyzer system is limited by the kinetic overpotential losses associated with the oxygen evolution reaction (OER) at the anode in both acidic and basic medium.

Overpotential is the potential difference between the potentials achieving a specific current density and $1.23 \mathrm{~V}$. Usually it is measured in $\mathrm{mV}$, as an example if a catalyst achieves $\mathrm{E}$ $=1.53 \mathrm{~V}$, then it bears an overpotential of $300 \mathrm{mV}$. Different electrocatalysts have been studied to reduce the overpotential for the oxygen evolution reaction. Recently, ODPs have attracted attention toward this research. Different mechanisms have been proposed for the OER in alkaline media for ODPs. ${ }^{26-27}$ The commonly accepted mechanism in alkaline solution involves four steps, where there is a single electron transfer in each step. In the first step, the reaction initiates by the adsorption of $\mathrm{OH}^{-}$on the active site of the catalyst, i.e., metal site, M. In the second step, a hydroxide from the electrolyte abstracts a proton from $\mathrm{M}-\mathrm{OH}$ to form $\mathrm{M}-\mathrm{O}$ and water. In the third step, $\mathrm{M}-\mathrm{O}$ combines with a hydroxide to form a peroxide. Finally, in the fourth step, the peroxide intermediate reacts with $\mathrm{OH}^{-}$to give an oxygen and water and regenerate the catalyst.

1. $\mathrm{M}+\mathrm{OH}^{-} \rightarrow \mathrm{M}-\mathrm{OH}+\mathrm{e}^{-}$

2. $\mathrm{M}-\mathrm{OH}+\mathrm{OH}^{-} \rightarrow \mathrm{M}-\mathrm{O}+\mathrm{H}_{2} \mathrm{O}+\mathrm{e}^{-}$ 
3. $\mathrm{M}-\mathrm{O}+\mathrm{OH}^{-} \rightarrow \mathrm{M}-\mathrm{OOH}+\mathrm{e}^{-}$

4. $\mathrm{M}-\mathrm{OOH}+\mathrm{OH}^{-} \rightarrow \mathrm{M}+\mathrm{H}_{2} \mathrm{O}+\mathrm{O}_{2}+\mathrm{e}^{-}$ 
CHAPTER 2

TRANSFORMATION OF STRUCTURE, ELECTRICAL CONDUCTIVITY AND

MAGNETISM IN AA' $\mathrm{Fe}_{2} \mathrm{O}_{6-\delta}, \mathrm{A}=\mathrm{Sr}, \mathrm{Ca}$ and $\mathrm{A}^{\prime}=\mathrm{Sr}^{1}$

\section{INTRODUCTION}

The applications of oxygen-deficient perovskites in areas such as solid oxide fuel cells (SOFCs), superconductors, magnetoresistants, and gas diffusion membranes indicate the importance of this family of materials. ${ }^{28-31}$ The general formula for oxygen-deficient perovskites can be represented as $\mathrm{ABO}_{3-\mathrm{x}}$ or $\mathrm{AA}^{\prime} \mathrm{BB}^{\prime} \mathrm{O}_{6-\delta}$, where the $\mathrm{B} / \mathrm{B}^{\prime}$ cations (which can be the same or different) have octahedral, tetrahedral, or square-pyramidal coordination geometries. The A/A' cations (which again can be the same or different) occupy the free spaces in between the above polyhedra. The vacant sites, that are created due to oxygen deficiency, can have a random distribution in the crystal structure. In such cases, the average structure retains the perovskite-type atomic arrangement, but with partial siteoccupancy (as opposed to full occupancy) on oxygen positions. ${ }^{32}$ An alternative scenario involves an ordered arrangement of vacant sites. There can be different types of vacancyordering depending on various parameters, including the degree of oxygen deficiency. For

${ }^{1}$ The work described in this chapter was published in Inorganic Chemistry ( 2017, vol. 56, p. 9716-9724) 
example, materials with large concentration of defects, i.e., $\delta \approx 1$, usually form structures where the oxide deficiency appears in alternating layers, where corner-sharing $\mathrm{B}^{\prime} \mathrm{O}_{4}$ tetrahedra are formed. This leads to a structure that contains layers of $\mathrm{BO}_{6}$ octahedra separated by B' $\mathrm{O}_{4}$ tetrahedra. This is called brownmillerite-type structure. ${ }^{6,33-34}$ Another type of ordering involves the formation of square pyramidal geometry around the B-site cations..$^{35}$ In materials with smaller degree of oxygen deficiency, other schemes of vacancyordering are observed, involving the formation of both square-pyramidal and octahedral coordination geometries. ${ }^{36}$

Understanding various parameters that determine the crystal structure of oxygen-deficient perovskites is important, as there is a direct correlation between their structure and functional properties. The manipulation of cations on the B-site is known to affect the structure of oxygen-deficient perovskites. For example, despite structural similarities between $\mathrm{Ca}_{2} \mathrm{Fe} \mathrm{AlO}_{5}$ and $\mathrm{Ca}_{2} \mathrm{FeGaO}_{5}$, they have different space groups, Ibm2 (Ima2) for the former and Pcmn (Pnma) for the latter. ${ }^{37}$ Both materials feature the brownmilleritetype structure, described above, containing octahedral and tetrahedral layers. The cornersharing tetrahedra form chains that run parallel to the octahedral layers, and have two possible orientations, called right handed (R) and left handed (L). In $\mathrm{Ca}_{2} \mathrm{FeAlO}_{5}$, all tetrahedral chains have the same orientation (either $\mathrm{R}$ or $\mathrm{L}$ ), leading to space group $\mathrm{Ibm} 2$ (Ima2). In $\mathrm{Ca}_{2} \mathrm{FeGaO}_{5}$ however, the $\mathrm{R}$ and $\mathrm{L}$ orientations appear alternately from one tetrahedral layer to the next, resulting in space group Pcmn (Pnma). ${ }^{37}$

The effect of the A-site cation on the crystal structure is also important. For example, $\mathrm{Sr}_{2} \mathrm{GaMnO}_{5}$ has a body-centered structure described by Ima2 or Imcm space group, ${ }^{38}$ whereas $\mathrm{Ca}_{2} \mathrm{GaMnO}_{5}$ has a primitive structure with space group Pnma ${ }^{38}$ However, both of 
these materials have a similar structure-type, containing octahedra and tetrahedra, but with different space group symmetries.

In this article, we study materials with formula, $\mathrm{AA}^{\prime} \mathrm{Fe}_{2} \mathrm{O}_{6-\delta}$, where $\mathrm{A}=\mathrm{Sr}, \mathrm{Ca}$ and $\mathrm{A}^{\prime}=\mathrm{Sr}$. The Sr-containing phase, $\mathrm{Sr}_{2} \mathrm{Fe}_{2} \mathrm{O}_{6-\delta}$, is known to have various oxygen contents. The fully oxidized material, $\mathrm{Sr}_{2} \mathrm{Fe}_{2} \mathrm{O}_{6}$, can only be obtained if the initial air-synthesis is followed by heating under $30 \mathrm{MPa}$ of pure oxygen. ${ }^{36}$ Products with different degrees of oxygen deficiencies can be synthesized if samples are heated under different gas atmospheres. ${ }^{36}$ However, the direct synthesis in air at $1250 \mathrm{C}$, without any additional gas treatment, leads to a product with formula $\mathrm{Sr}_{2} \mathrm{Fe}_{2} \mathrm{O}_{5.75}$, where $\delta \approx 0.25 .{ }^{36}$ Regarding the Ca-containing analogue, $\mathrm{CaSrFe}_{2} \mathrm{O}_{6-\delta}$, little information is known. The formation of an orthorhombic structure with similar composition through liquid nitrogen quenching and vacuum treatment has been reported. ${ }^{39}$ However, the magnetic structure and electrical transport properties of this material are not known. Here, we show that $\mathrm{CaSrFe}_{2} \mathrm{O}_{6-\delta}$ can be synthesized under the same conditions as the $\mathrm{Sr}_{2}$-ananlogue. We have performed neutron diffraction experiments to examine the crystal structure and explore the long-range magnetic order in the CaSr-material. We have also conducted extensive charge transport studies on both $\mathrm{Sr}_{2}$ and $\mathrm{CaSr}$ compounds. These studies have revealed the sharp contrast between these two materials and demonstrated the dramatic transformation of magnetism and electrical conductivity in $\mathrm{AA}^{\prime} \mathrm{Fe}_{2} \mathrm{O}_{6-\delta}$ as a function of A-site cation.

\section{EXPERIMENTAL}

Both materials were synthesized under the same synthesis conditions. Stoichiometric proportions of the precursors, $\mathrm{SrCO}_{3}$ (Sigma Aldrich, 99.9\%), $\mathrm{CaCO}_{3}(\mathrm{Alfa}$ Aesar, 99.95\%), and $\mathrm{Fe}_{2} \mathrm{O}_{3}$ (Alfa Aesar, 99.998\%) were used for solid-state syntheses. The 
mixtures of precursor powders were ground using agate mortar and pestle, pressed into pellets and heated in air at $1000^{\circ} \mathrm{C}$ for 24 hours. The pellets were then ground and refired in air at $1250{ }^{\circ} \mathrm{C}$ for 24 hours. In all cases, the furnace heating and cooling rates were set at $100{ }^{\circ} \mathrm{C} / \mathrm{h}$.

The phase purity and structure of polycrystalline samples were examined by powder X-ray diffraction at room temperature using a Bruker D8 Discover diffractometer with $\mathrm{CuK}_{\alpha}$ radiation and a PANalytical Empyrean diffractometer with $\mathrm{CuK}_{\alpha 1}$ radiation $\left(\lambda=1.54056 \AA\right.$ A). The Rietveld refinements were carried out using GSAS software ${ }^{40}$ and EXPGUI interface. ${ }^{41}$ The morphological analyses were performed using a high resolution field-emission scanning electron microscope (SEM). The electrical properties of the polycrystalline samples were investigated using electrochemical impedance spectroscopy (EIS). AC impedance measurements were performed in the frequency range $0.1 \mathrm{~Hz}-1$ MHz using a computer-controlled frequency response analyzer. Similarly, 2-probe DC measurements were carried out by measuring the output current by applying constant voltage of $1 \mathrm{mV}$. X-ray photoelectron spectroscopy data were obtained using $\mathrm{Mg} \mathrm{K \alpha}$ radiation $(1253.6 \mathrm{eV})$ at room temperature. Thermogravimetric analysis was done from 25 to $800{ }^{\circ} \mathrm{C}$ in air. Magnetic susceptibility data were obtained by applying magnetic field of $1000 \mathrm{Oe}$ in the temperature range $2 \mathrm{~K}$ to $400 \mathrm{~K}$. Neutron diffraction experiments were performed on POWGEN diffractometer at Oak Ridge National Laboratory, with center wavelength of $1.333 \AA$, covering the d-spacing range $0.4142-6.1363 \AA$. 


\section{RESULTS AND DISCUSSION}

Crystal structure

The crystal structure is transformed as a result of replacing one of the $\mathrm{Sr}$ atoms with $\mathrm{Ca}$ on the A-site. The $\mathrm{Sr}_{2}$-compound is known to have a tetragonal crystal structure ${ }^{36}$ consisting of corner sharing $\mathrm{FeO}_{6}$ octahedra and $\mathrm{FeO}_{5}$ square-pyramids. Figures 2.1 and 2.2 show the Rietveld refinement profile and crystal structure of this material. The refined structural parameters are listed in Table 2.1. The $\mathrm{FeO}_{5}$ square-pyramids form dimers that are separated by $\mathrm{FeO}_{6}$ octahedra. There is no connectivity between different dimers in the structure. The octahedral and square-pyramidal Fe sites alternate within each layer. As seen in Figure 2.2c and Table 2.1, there are two distinct crystallographic positions where A-site cations $\left(\mathrm{Sr}^{2+}\right)$ reside. These two sites have coordination numbers 11 and 12 .

The substitution of one $\mathrm{Ca}$ for $\mathrm{Sr}$ leads to a dramatic change in the crystal structure. Our neutron and X-ray diffraction experiments show that the CaSr-compound has an orthorhombic structure, consisting of alternating layers of octahedra and tetrahedra, as shown in Figure 2.3. The $\mathrm{FeO}_{6}$ octahedra share corners with other octahedra within the same layer, and with the tetrahedra in the layers above and below. The tetrahedral layer actually consists of chains of $\mathrm{FeO}_{4}$ tetrahedra that run parallel to the octahedral layers. The A-site cations in this material have coordination number 8 (Figure 2.3c). This is the socalled brownmillerite-type structure. Materials with this structure-type usually have orthorhombic Ibm2, Pnma, Pbcm or Icmm space groups depending on the relative orientation of tetrahedral chains (Figure 2.3b). ${ }^{33-34}$ As mentioned before, the tetrahedral 


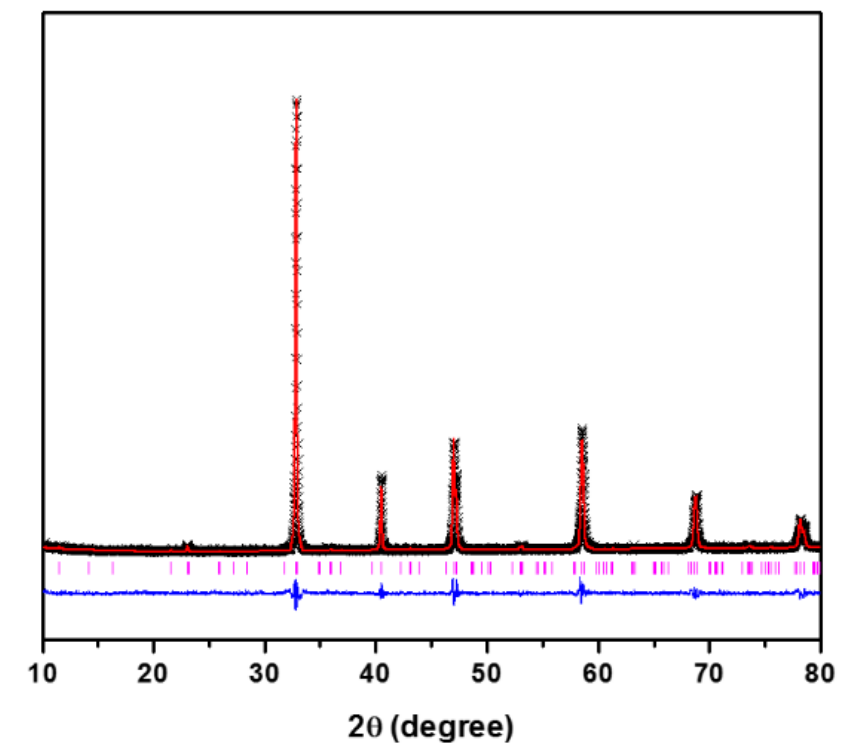

Figure 2.1. Rietveld refinement profile for powder X-ray diffraction data of $\mathrm{Sr}_{2} \mathrm{Fe}_{2} \mathrm{O}_{6-\delta}$ in $14 / \mathrm{mmm}$ space group. Stars represent experimental data, red solid is the model, vertical tick marks show Bragg peak positions, and the blue line represents the difference plot.

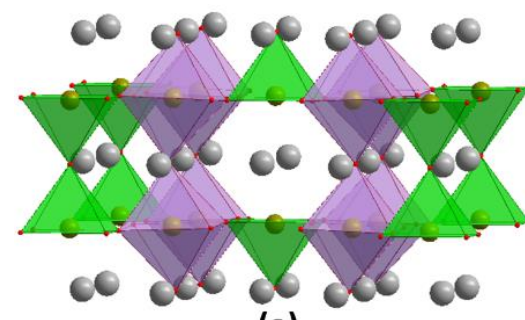

(a)

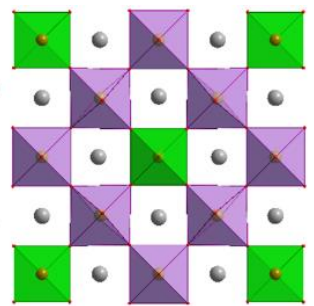

(b)

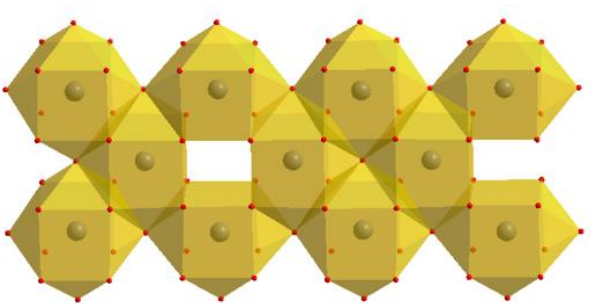

(c)

Figure 2.2. Crystal structure of $\mathrm{Sr}_{2} \mathrm{Fe}_{2} \mathrm{O}_{6-\delta}$. (a) and (b) show the alternating $\mathrm{FeO}_{6}$ octahedra (purple) and $\mathrm{FeO}_{5}$ square pyramids (green), viewed along the $a$ and $c$ axes, respectively. The large grey spheres are $\mathrm{Sr}$ atoms. (c) shows the coordination geometry around the $\mathrm{Sr}$ atoms. Note the presence of both 11 and 12-coordinated $\mathrm{Sr}$ atoms.

chains have two possible orientations, right handed (R) and left handed (L). The space group $I b m 2$ is obtained when all tetrahedral chains have the same orientation. However, if the $\mathrm{R}$ and $\mathrm{L}$ orientations appear alternately from one tetrahedral layer to the next, the space group Pnma is obtained. The Pbcm space group is less common, ${ }^{33-34,42}$ where the 
orientation of each tetrahedral chain is opposite to all nearest neighbors within the same layer and in the neighboring layers above and below. Finally, the space group Icmm is a result of random orientation of tetrahedral chains in the structure. For our CaSr-material, the $\mathrm{Pbcm}$ space group was readily ruled out, because it requires the formation of a large unit cell with distinct supercell peaks. ${ }^{33-34,42}$ These peaks are absent in neutron and X-ray diffraction data. The Pnma structure can be identified by the presence of 131 and 151 peaks, which are also absent in our data, ruling out this space group. The Icmm and Ibm2 models were then examined by Rietveld refinements, leading to a poor fit for Icmm, but an excellent fit for $I b m 2$ space group (Figure 2.4). The refined structural parameters are listed in Table 2.2.

Table 2.1. Refined structural parameters of $\mathrm{Sr}_{2} \mathrm{Fe}_{2} \mathrm{O}_{6-\delta}$.

\begin{tabular}{|c|c|c|c|c|c|c|}
\hline \multicolumn{7}{|c|}{ Space group: $I 4 / \mathrm{mmm}$} \\
\hline \multicolumn{2}{|c|}{$a=10.9343(6) \AA$} & $b=10.9343 \AA$ & $c=7.6988(4) \AA$ & \multicolumn{2}{|c|}{$\mathrm{Rp}=0.0216$} & \multirow{2}{*}{$\frac{w R p=0.0312}{\text { Multiplicity }}$} \\
\hline Element & $\mathrm{x}$ & $\mathrm{y}$ & $\mathrm{z}$ & Occupancy & $\mathrm{U}_{\text {iso }}$ & \\
\hline Sr1 & $0.2601(5)$ & 0 & 0 & 1 & $0.015(2)$ & 8 \\
\hline $\mathrm{Sr} 2$ & $0.2478(4)$ & 0 & 0.5 & 1 & $0.014(2)$ & 8 \\
\hline $\mathrm{Fe} 1$ & 0 & 0 & 0.25 & 1 & $0.024(7)$ & 4 \\
\hline $\mathrm{Fe} 2$ & 0.25 & 0.25 & 0.25 & 1 & $0.007(4)$ & 8 \\
\hline $\mathrm{Fe} 3$ & 0.5 & 0 & 0.25 & 1 & $0.033(7)$ & 4 \\
\hline $\mathrm{O} 1$ & 0 & 0 & 0.5 & 1 & $0.017(1)$ & 2 \\
\hline $\mathrm{O} 2$ & $0.123(2)$ & $0.123(2)$ & $0.225(2)$ & 1 & $0.017(1)$ & 16 \\
\hline $\mathrm{O} 3$ & $0.253(2)$ & $0.253(2)$ & 0.5 & 1 & $0.017(1)$ & 8 \\
\hline $\mathrm{O} 4$ & $0.126(2)$ & $0.626(2)$ & 0.25 & 1 & $0.017(1)$ & 16 \\
\hline O5 & 0.5 & 0 & 0 & 1 & $0.017(1)$ & 4 \\
\hline
\end{tabular}




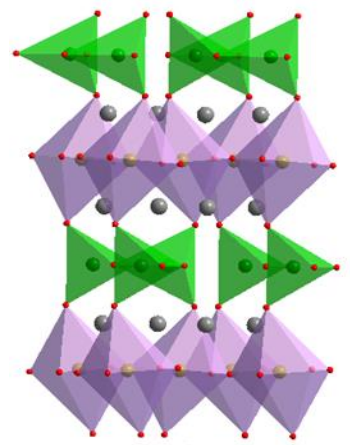

(a)

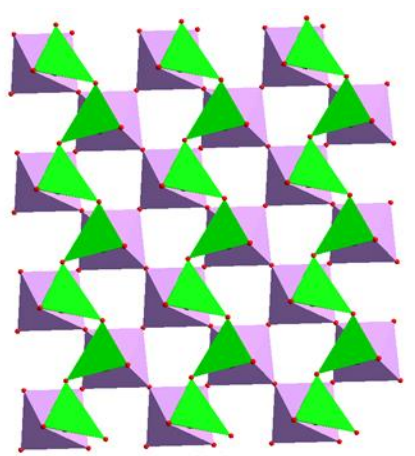

(b)

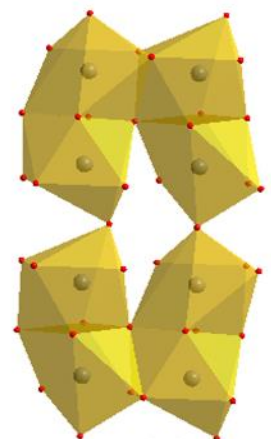

(c)

Figure 2.3. Crystal structure of $\mathrm{CaSrFe}_{2} \mathrm{O}_{6-\delta}$. (a) The octahedral $\mathrm{FeO}_{6}$ (purple) and tetrahedral $\mathrm{FeO}_{4}$ (green) layers. Grey spheres represent Sr. (b) View from top to highlight the chain formation in the tetrahedral layer. The $\mathrm{Sr}$ atoms are omitted for clarity. (c) Coordination geometry of $\mathrm{Sr}$ atoms. Note that $\mathrm{Sr}$ is 8-coordinated.

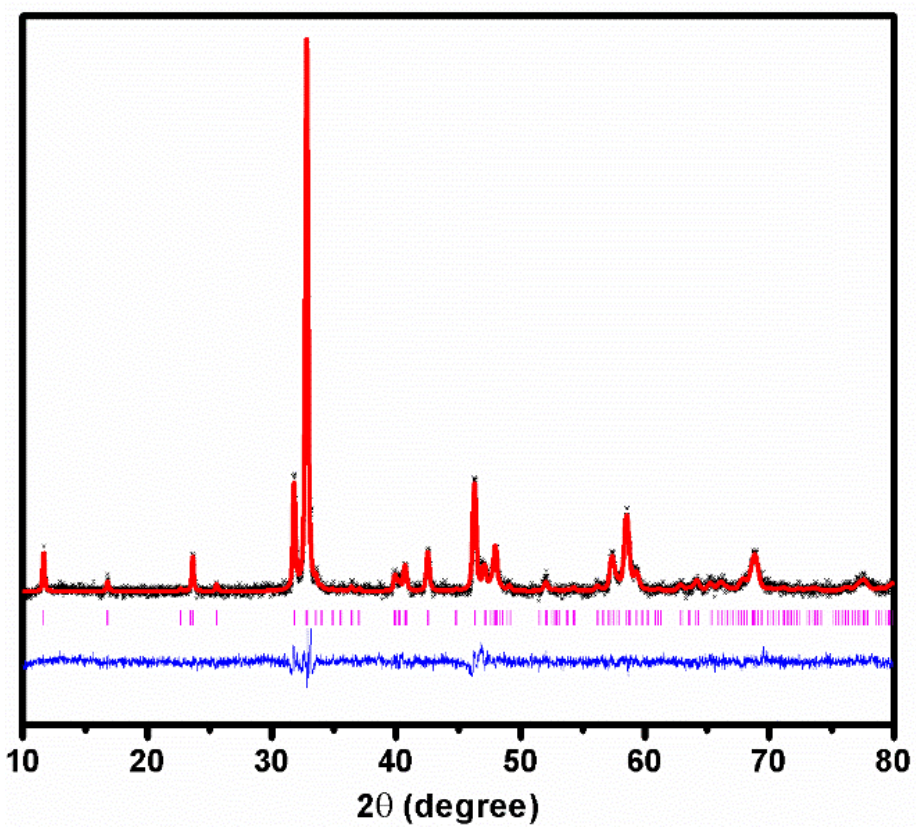

Figure 2.4. Rietveld refinement profile for powder X-ray diffraction data of $\mathrm{CaSrFe}_{2} \mathrm{O}_{6-\delta}$, space group Ibm2. Black stars, red line, vertical tick marks and lower blue line represent the experimental data, structural model, Bragg peak positions and difference plot, respectively. 
Table 2.2. Refined structural parameters of $\mathrm{CaSrFe}_{2} \mathrm{O}_{6-\delta}$.

\begin{tabular}{|c|c|c|c|c|c|c|}
\hline \multicolumn{7}{|c|}{ Space group: $I b m 2$} \\
\hline $\begin{array}{l}a= \\
5.6314(3) \AA\end{array}$ & \multicolumn{2}{|c|}{$\begin{array}{c}b= \\
15.1807(8) \AA\end{array}$} & \multirow{2}{*}{$\begin{array}{c}c= \\
\frac{5.4695(3) \AA}{\mathrm{z}}\end{array}$} & \multirow{2}{*}{$\begin{array}{c}\mathrm{Rp}= \\
0.0174 \\
\text { Occupancy }\end{array}$} & \multicolumn{2}{|c|}{$\begin{array}{l}w R p= \\
0.0237\end{array}$} \\
\hline Elements & $\mathrm{x}$ & $\mathrm{y}$ & & & $\mathrm{U}_{\text {iso }}$ & Multiplicity \\
\hline Ca1 & $0.5127(4)$ & $0.1108(1)$ & $0.009(4)$ & 0.5 & $0.013(1)$ & 8 \\
\hline Sr1 & $0.5127(4)$ & $0.1108(1)$ & $0.009(4)$ & 0.5 & $0.013(1)$ & 8 \\
\hline $\mathrm{Fe} 1$ & $0.0763(6)$ & 0.25 & $-0.003(6)$ & 1 & $0.010(2)$ & 4 \\
\hline $\mathrm{Fe} 2$ & 0 & 0 & 0 & 1 & $0.020(2)$ & 4 \\
\hline O1 & $0.227(3)$ & $0.0067(5)$ & $0.295(5)$ & 1 & $0.015(3)$ & 8 \\
\hline $\mathrm{O} 2$ & $0.0820(1)$ & $0.1485(4)$ & $0.002(8)$ & 1 & $0.015(3)$ & 8 \\
\hline $\mathrm{O} 3$ & $0.382(3)$ & 0.25 & $0.891(6)$ & 1 & $0.015(3)$ & 4 \\
\hline
\end{tabular}

The morphology and crystallite sizes of both $\mathrm{Sr}_{2}$ and $\mathrm{CaSr}$ materials were also examined using scanning electron microscopy. Figure 2.5 shows the surfaces of sintered pellets for both materials. The crystallite size decreases as a result of replacing one $\mathrm{Sr}$ with $\mathrm{Ca}$. In addition, the crystallites seem to be packed more densely and have more contact with each other in the CaSr-material.
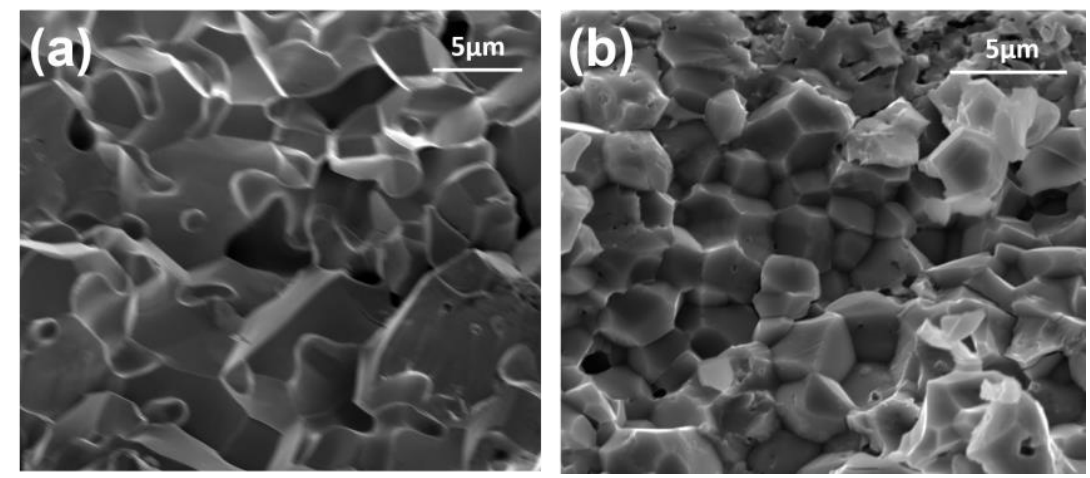

Figure 2.5. Scanning electron microscopy images of (a) $\mathrm{Sr}_{2} \mathrm{Fe}_{2} \mathrm{O}_{6-\delta}$ and (b) $\mathrm{CaSrFe}_{2} \mathrm{O}_{6-\delta}$. 
Some comments on the oxygen contents of these compounds are in order. Note that for both compounds, $\mathrm{Fe}_{2} \mathrm{O}_{3}$ was used as starting material and the synthesis conditions were identical. If iron retains its +3 oxidation state, the oxygen stoichiometry in $\mathrm{AA}^{\prime} \mathrm{Fe}_{2} \mathrm{O}_{6-\delta}$ formula should be 5 , resulting in $\delta=1$. We have performed X-ray photoelectron spectroscopy experiments on the CaSr-compound, to determine the oxidation state of Fe. As shown in Figure 2.6, the satellite peak at $\sim 8 \mathrm{eV}$ higher than the $\mathrm{Fe} 2 \mathrm{p}_{3 / 2}$ peak is a signature of $\mathrm{Fe}^{3+} \cdot{ }^{43-44}$ Therefore, the oxygen content of the CaSr-compound should be very close to 5. This is consistent with the crystal structure of the CaSr-compound, and the formation of brownmillerite-type structure, which has oxygen stoichiometry of 5 . This behavior, namely the retention of +3 oxidation state in perovskite-based oxides synthesized at high temperature, has been observed before. ${ }^{32}$ This is in sharp contrast to the $\mathrm{Sr}_{2}$ compound, where the tetragonal structure implies the oxygen stoichiometry of 5.75 , i.e., $\delta$ $=0.25$, which has also been confirmed by thermogravimetric analyses ${ }^{36}$ This indicates that a considerable amount of iron in the $\mathrm{Sr}_{2}$-compound has been oxidized during the synthesis. Note that both $\mathrm{Sr}_{2}$ and $\mathrm{CaSr}$ materials are synthesized under the same condition. Therefore, the difference in the B-site cation oxidation state as a result of variation in the ionic radius of the A-site cation is remarkable. 


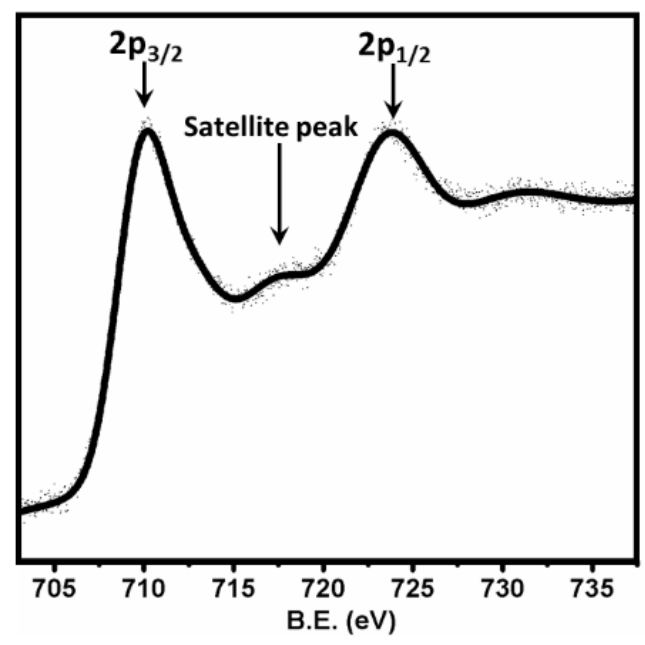

Figure 2.6. X-ray photoelectron spectroscopy data for $\mathrm{CaSrFe}_{2} \mathrm{O}_{6-\delta}$.

The transformation of the crystal structure upon changing the A-site, from $\mathrm{Sr}_{2}$ to $\mathrm{CaSr}$, is clearly related to the average cation size. Note that $\mathrm{Ca}$ and $\mathrm{Sr}$ share the same crystallographic site. This structural change may be explained in terms of the relationship between ionic radii and the coordination geometry of the A-site cations in the two structure types. When only $\mathrm{Sr}^{2+}$ cation is present, the tetragonal structure is stabilized, where this large cation is accommodated in 11 and 12-coordinated A-sites. However, when the average ionic radius on the A-site is decreased (due to the presence of $\mathrm{Ca}^{2+}$ ), the orthorhombic structure, featuring 8-coordinated A-sites, is preferred. At the other extreme, namely in a material with only $\mathrm{Ca}$ on $\mathrm{A} / \mathrm{A}^{\prime}$ sites, the structure remains ordered, as observed in $\mathrm{Ca}_{2} \mathrm{Fe}_{2} \mathrm{O}_{5}$, which also features the brownmillerite-type structure but a different space group. $^{45}$ 


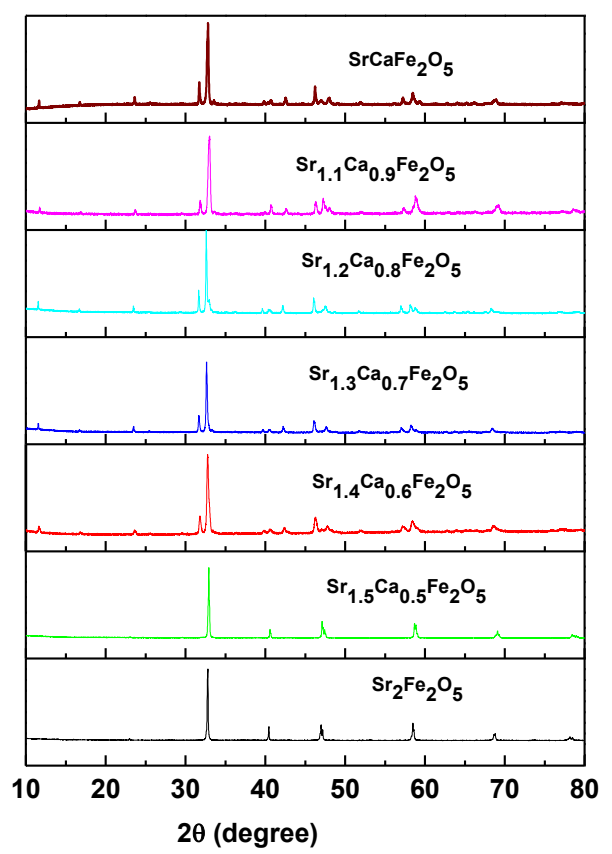

Figure 2.7. Powder X-ray diffraction data for the series of materials with varying $\mathrm{Ca} / \mathrm{Sr}$ ratios.

To investigate this structural transformation further, we set out to determine the A-site ionic radius that prompts this structural change. We synthesized a series of compounds, listed in Figure 2.7, where the $\mathrm{Sr} / \mathrm{Ca}$ ratio was varied systematically. These experiments showed that transformation from tetragonal structure (with octahedral and square pyramidal geometry) to orthorhombic structure (with octahedral and tetrahedral geometry) takes place at $\mathrm{Sr} / \mathrm{Ca}$ ratio of $1.4 / 0.6$. Using this ratio, the average cation size on the A-site can be calculated. As mentioned before, the A-site cations in the tetragonal phase have coordination numbers $(\mathrm{CN}) 11$ and 12 . Ionic radii for $\mathrm{CN}=12$ have been tabulated ${ }^{46}$ for $\mathrm{Sr}^{2+}, 1.44 \AA$, and $\mathrm{Ca}^{2+}, 1.34 \AA$. Using these radii, one can calculate the average ionic radius required for the structural transformation to be $\sim 1.41 \AA$. 


\section{Magnetic structure}

The change in the A-site cation and subsequent alteration of the crystal structure leads to significant changes in the magnetic order. The $\mathrm{Sr}_{2}$ compound is known to have an incommensurate magnetic structure with propagation vector $\mathrm{k}=(0.687,0,0.326)$, where magnetic moments are in "spin-density wave" state. ${ }^{47}$ The magnetic moment value has been found to be $2.54(4) \mu_{\mathrm{B}}$ at $11 \mathrm{~K}$. All magnetic moments are tilted by -35.3 degrees with respect to the $\mathrm{c}$ axis. The moments are aligned within planes perpendicular to the body diagonal of the unit cell, i.e., [111] direction. The magnetic transition temperature is 75 $\mathrm{K}^{47}$

We have shown that the magnetic structure is transformed upon replacing $\mathrm{CaSr}$ for $\mathrm{Sr}_{2}$ on the A-site. We studied the magnetic structure of the CaSr-compound using neutron diffraction. Figure 2.8 shows the Rietveld refinement profile for simultaneous refinement of crystal and magnetic structures of $\mathrm{CaSr}$-material. Neutron experiments at $10 \mathrm{~K}$ indicated that the CaSr-compound is antiferromagnetically ordered, as evident from strong magnetic reflections. In materials with this type of crystal structure, the relative intensities of the two main magnetic peaks is indicative of the orientation of magnetic moments. ${ }^{42}$ If this ratio is close to 1 , the magnetic moments are oriented along the longest unit cell axis. However, if the intensity ratio is close to 3 , the magnetic moments are aligned along the shortest axis. In our neutron diffraction data, these peaks appear at $\mathrm{d} \approx 4.43 \AA$ and $4.51 \AA$ (Figure 2.8) with relative intensity close to 3 , indicating that the magnetic moment orientation should be parallel to the shortest axis. Magnetic structure refinements with neutron diffraction data showed that magnetic moments are indeed parallel to the shortest axis, c. These 


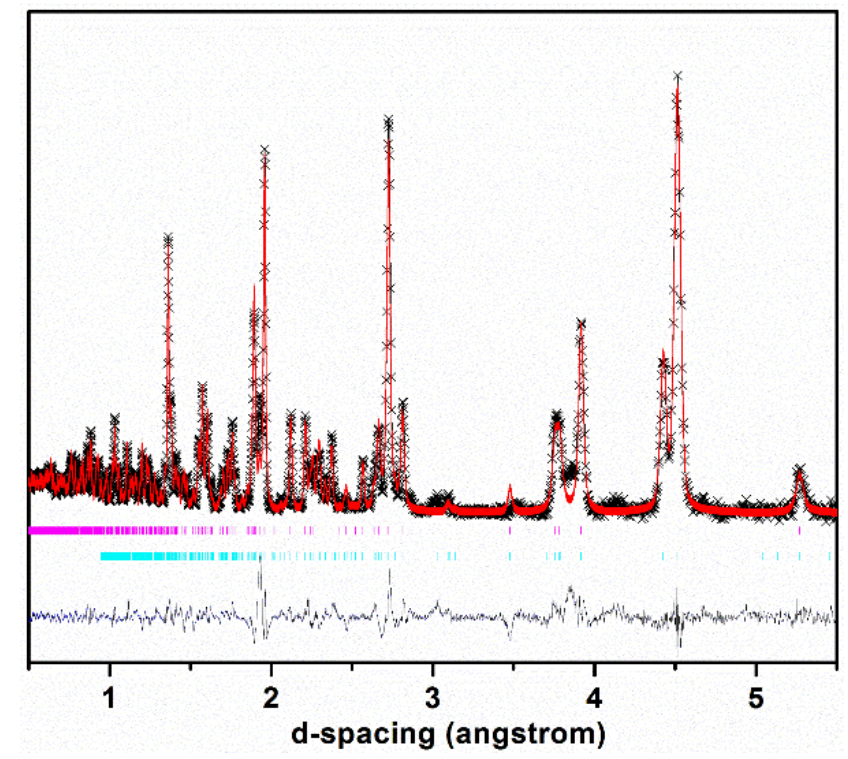

Figure 2.8. Refinement of the crystal and magnetic structures using neutron diffraction data for $\mathrm{CaSrFe}_{2} \mathrm{O}_{6-\delta}$. The upper and lower tick marks represent the peak positions for crystal and magnetic structures, respectively.

refinements also revealed that each magnetic moment is aligned anti-parallel to all of its nearest neighbors, forming the so-called G-type antiferromagnetic structure, as shown in Figure 2.9. The magnetic unit cell has the same size as the crystallographic unit cell. The magnitude of magnetic moments of the octahedral and tetrahedral Fe atoms were also determined. The magnetic moment values at $10 \mathrm{~K}$ are $4.6(2) \mu_{\mathrm{B}}$ and $3.9(2) \mu_{\mathrm{B}}$, for octahedral and tetrahedral sites, respectively. In addition, we performed neutron diffraction experiments at $300 \mathrm{~K}$. These experiments indicated that the G-type antiferromagnetic order in the CaSr-material persists even at room temperature. The magnetic moments are still oriented along the c-axis and the magnitudes of moments at $300 \mathrm{~K}$ are $4.0(2) \mu_{\mathrm{B}}$ and $3.5(2)$ $\mu_{\mathrm{B}}$ for the octahedral and tetrahedral sites, respectively. 


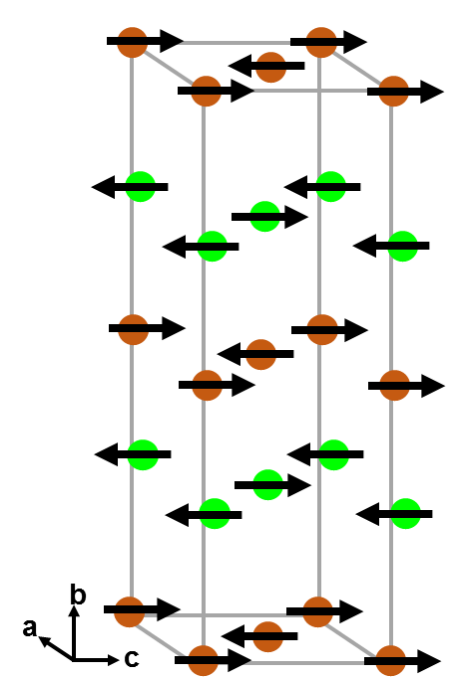

Figure 2.9. The G-type antiferromagnetic order in $\mathrm{CaSrFe}_{2} \mathrm{O}_{6-\delta}$. Note that magnetic moments on each Fe site are aligned opposite to all nearest neighbors. The moments are oriented along the $c$-axis.

Note the sharp contrast between the CaSr-compound, featuring antiferromagnetic order even at room temperature, and the $\mathrm{Sr}_{2}$-material, where the spin-density wave state occurs below $75 \mathrm{~K}$. We also performed magnetic susceptibility measurements on the CaSrmaterial in the temperature range $2-400 \mathrm{~K}$, as shown in Figure 2.10. A broad feature and divergence between zero-field-cooled and field-cooled data were observed at about $52 \mathrm{~K}$. This behavior has been observed before for antiferromagnetic materials at temperatures far below their Neel temperature, ${ }^{33}$ and corresponds to short-range magnetic domains or possible magnetic side product. ${ }^{33}$ However, the absence of any sharp transition combined with the neutron diffraction results that show long-range magnetic order at $300 \mathrm{~K}$, indicate that the Neel temperature for this material should be higher than $400 \mathrm{~K}$. 


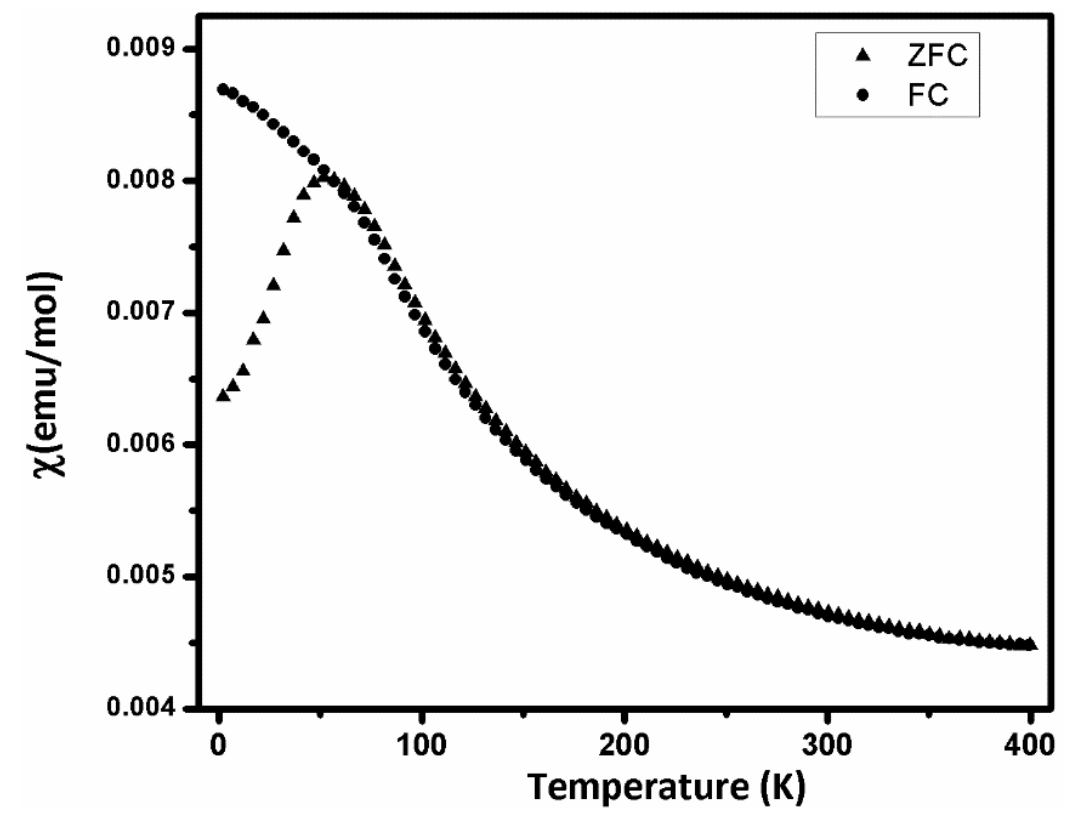

Figure 2.10. Magnetic Susceptibility data for $\mathrm{CaSrFe}_{2} \mathrm{O}_{6-\delta}$.

\section{Electrical properties}

The change in the crystal structure has a pronounced effect on electrical transport properties. The electrical conductivity measurements determine the resistance, $\mathrm{R}$, of each material, and the resistivity, $\rho$, is calculated from $\rho=\mathrm{RA} / \mathrm{L}$, where $\mathrm{L}$ and A are the length and cross sectional area of cylindrical samples, respectively. Conductivity, $\sigma$, is then calculated from the inverse of resistivity. The total conductivities of $\mathrm{Sr}_{2} \mathrm{Fe}_{2} \mathrm{O}_{6-\delta}$ and $\mathrm{CaSrFe}_{2} \mathrm{O}_{6-\delta}$ were obtained using both DC and AC methods. In each case both DC and AC techniques led to very similar total conductivity values, as shown in Table 2.3. Note that various parameters contribute to the total conductivity, including electrode reactions, bulk and grain-boundary resistances, ionic and/or electron transport phenomena. In perovskite-based oxides, heterovalent atoms having more than one stable oxidation state 
(e.g., $\mathrm{Fe}^{3+} / \mathrm{Fe}^{4+}$ ) are needed on the B-site for electronic conductivity. The metal (M) $3 d$ and oxygen $2 p$ orbitals overlap and electron hopping occurs through $\mathrm{M}-\mathrm{O}-\mathrm{M}$ pathways.

Table 2.3. Room temperature conductivity and activation energies.

\begin{tabular}{lcll}
\hline & \multicolumn{2}{c}{ Total conductivity, $\sigma\left(\mathrm{Scm}^{-1}\right)$} & Activation energy $\left(E_{a}\right)$ in eV \\
& $\mathrm{AC}$ & $\mathrm{DC}$ & \\
\hline $\mathrm{Sr}_{2} \mathrm{Fe}_{2} \mathrm{O}_{6-\delta}$ & $7.698 \times 10^{-1}$ & $8.540 \times 10^{-1}$ & 0.118 \\
$\mathrm{CaSrFe}_{2} \mathrm{O}_{6-\delta}$ & $1.201 \times 10^{-1}$ & $1.616 \times 10^{-1}$ & 0.653 for $298-673 \mathrm{~K}\left(25-400{ }^{\circ} \mathrm{C}\right)$ \\
& & & 0.201 for $673-1073 \mathrm{~K}$ \\
& & & $\left(400-800{ }^{\circ} \mathrm{C}\right)$ \\
\hline
\end{tabular}

The $\mathrm{M}-\mathrm{O}$ bond distance and $\mathrm{M}-\mathrm{O}-\mathrm{M}$ angle determine the degree of overlap. Shorter bonds and larger angles are associated with greater orbital overlap and higher conductivity. ${ }^{48}$ Due to the presence of corner-sharing octahedra and tetrahedra in the $\mathrm{CaSr}$ compound, the average $\mathrm{Fe}-\mathrm{O}-\mathrm{Fe}$ bond angle $\left(138.8^{\circ}\right)$ in this material is smaller than that for the $\mathrm{Sr}_{2}$ compound $\left(177.2^{\circ}\right)$. Therefore, smaller degree of orbital overlap is expected in the CaSrcompound. The corner-sharing of octahedra and tetrahedra leads to distortions in the coordination geometry of $\mathrm{Fe}$ atoms, resulting in a wide range of $\mathrm{Fe}-\mathrm{O}$ bond lengths in the CaSr-material, from 1.780(7) $\AA$ to $2.302(7) \AA$. However, the Fe-O bond lengths in the $\mathrm{Sr}_{2}$ compound are close to each other and range from 1.92(3) ̊ to 1.97(3) $\AA$.

As observed in Table 2.3, at room temperature, there is nearly one order of magnitude difference between the total conductivity of the $\mathrm{Sr}_{2}$-material and that of the $\mathrm{CaSr}$ compound, indicating the significant effect of crystal structure on the electrical transport properties. 
To operate any device that works based on conductive oxides over a wide temperature range, knowledge of the charge transport properties as a function of temperature is required. Therefore, variable-temperature DC conductivity studies were performed for both materials at the temperature range of $298 \mathrm{~K}-1073 \mathrm{~K}\left(25^{\circ} \mathrm{C}-800{ }^{\circ} \mathrm{C}\right)$. From these experiments the activation energies were obtained, as shown in Table 2.3. The conductivity trends during heating and cooling cycles and the Arrhenius plots for both materials are shown in Figure 2.11. The plot in Figure 2.11b was used for fitting with the Arrhenius equation for thermally activated conductivity, ${ }^{49-51}$ which helped to find the activation energy of total conductivity.

$$
\sigma \mathrm{T}=\sigma^{\circ} \mathrm{e}^{\frac{-\mathrm{Ea}}{\mathrm{KT}}}
$$

where $\sigma^{\circ}$ is a pre-exponential factor and a characteristic of a material, and $E_{a}, K$ and $T$ are the activation energy for the conductivity, Boltzmann constant and absolute temperature, respectively. The activation energy for the total conductivity $\left(E_{a}\right)$ can be calculated from slope of the line of best fit in log $\sigma \mathrm{T}$ vs $1000 / \mathrm{T}$ plot. As shown in Table 2.3, $E_{a}$ value for the total conductivity of $\mathrm{Sr}_{2} \mathrm{Fe}_{2} \mathrm{O}_{6-\delta}$ is $0.118 \mathrm{eV}$. For $\mathrm{CaSrFe}_{2} \mathrm{O}_{6-\delta}$ two $E_{a}$ values are obtained, $0.653 \mathrm{eV}$ for $25-400{ }^{\circ} \mathrm{C}(298-673 \mathrm{~K})$ and $0.201 \mathrm{eV}$ for $400-800{ }^{\circ} \mathrm{C}(673-$ $1073 \mathrm{~K})$.

There is a sharp contrast between the $\mathrm{CaSr}$ and $\mathrm{Sr}_{2}$ compounds with regards to their conductivity trends as a function of temperature. The $\mathrm{Sr}_{2}$ compound shows metallic properties, ${ }^{52}$ while the CaSr-material is a semiconductor. As mentioned above, the average $\mathrm{Fe}-\mathrm{O}-\mathrm{Fe}$ bond angle in the $\mathrm{Sr}_{2}$-compound is greater than that in the CaSr-material. Several authors have discussed the correlation between increase in bond angles and the broadening 
of valence and conduction bands in perovskites, leading to changes in properties from insulator or semiconductor to metallic systems. ${ }^{48,53-54}$ The broadening of bands and overlap between the metal $3 d$ and oxygen $2 p$ bands closes the band gap completely, leading to the formation of hybrid $\mathrm{M}-\mathrm{O}-\mathrm{M}$ bands, and metallic properties. The charge transfer occurs through these hybrid bands. ${ }^{48,53-54}$

As observed in Figure 2.11, $\mathrm{Sr}_{2} \mathrm{Fe}_{2} \mathrm{O}_{6-\delta}$ displays a trend where conductivity decreases gradually with respect to temperature in the entire range from $298 \mathrm{~K}$ to $1073 \mathrm{~K}$, indicative

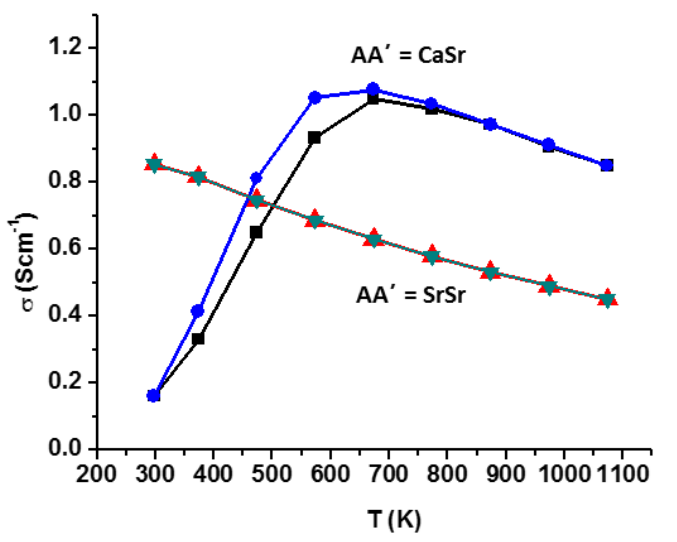

(a)

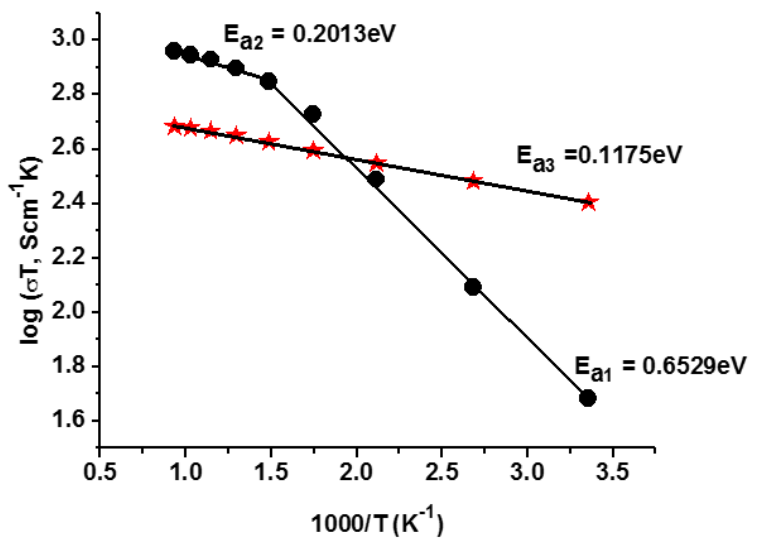

(b)

Figure 2.11. (a) Total conductivity of $\mathrm{Sr}_{2} \mathrm{Fe}_{2} \mathrm{O}_{6-\delta}$ and $\mathrm{CaSrFe}_{2} \mathrm{O}_{6-\delta}$ as a function of temperature. For $\mathrm{Sr}_{2} \mathrm{Fe}_{2} \mathrm{O}_{6-\delta}$, the heating and cooling data (red and green) overlap. For $\mathrm{CaSrFe}_{2} \mathrm{O}_{6-\delta}$ hysteresis is observed between heating (black squares) and cooling data (blue circles). (b) Arrhenius plot of the total conductivity for $\mathrm{Sr}_{2} \mathrm{Fe}_{2} \mathrm{O}_{6-\delta}$ (red stars) and $\mathrm{CaSrFe}_{2} \mathrm{O}_{6-\delta}$ (black circles).

of metallic properties. The decrease in conductivity as a function of temperature in a metallic system is a result of increase in the frequency of collisions between charge carriers and phonons. ${ }^{53}$ 
On the contrary, the conductivity of $\mathrm{CaSrFe}_{2} \mathrm{O}_{6-\delta}$ increases gradually with the rise in temperature, a behavior typical of a semiconductor. The conductivity then decreases after $\sim 673 \mathrm{~K}$. In materials that feature the same structure type as $\mathrm{CaSr}$-compound, i.e., alternating layers of octahedra and tetrahedra, increase in electrical conductivity as a function of temperature is usually observed. ${ }^{55}$

The conductivity mechanism is through the formation of polarons. The different bond lengths of corner-sharing tetrahedra and octahedra in this structure-type introduce structural distortions, resulting in lattice polarizations which favor polaronic charge transport mechanism ${ }^{56-57}$. The dependence of conductivity on oxygen partial pressure has been observed in these materials indicating the $p$-type semiconductivity. ${ }^{55}$ Also, the dominance of electronic conductivity above room temperature in high oxygen partial pressure, e.g., $\mathrm{O}_{2}$ partial pressure in air, has been shown for perovskite-type systems. In these cases, the main charge carriers are electron holes, leading to $p$-type semiconductivity. ${ }^{57-61}$ The extrinsic holes are formed through the absorption of oxygen molecules on the surface of the sample, facilitated by the presence of oxygen vacancies. The process can be described using the following idealized equations ${ }^{57,59}$ :

$$
1 / 2 \mathrm{O}_{2} \rightarrow \mathrm{O}^{2-}+2 \mathrm{~h}^{\cdot}
$$

In ferrites, the variable valence of iron is essential for charge compensation and electronic conductivity.

$$
\mathrm{Fe}^{3+}+\mathrm{h}^{\cdot} \rightarrow \mathrm{Fe}^{4+}
$$

The formation of $\mathrm{Fe}^{4+}$ ions allows the polaronic electronic conductivity to occur through $\mathrm{Fe}^{3+}-\mathrm{O}^{2-}-\mathrm{Fe}^{4+}$ pathway. ${ }^{57,61-62}$ 
The increase in conductivity as a function of temperature ${ }^{50,55,57}$ is explained in terms of temperature-activated hole formation and increased polaron mobility. ${ }^{50}$ Another feature observed in Figure 2.11 is a change in the electrical conductivity trend for the CaSrcompound above $\sim 673 \mathrm{~K}$. This behavior has been observed in similar materials before. ${ }^{55}$, 57, 63-64 Some researchers have observed greater loss of oxygen in thermogravimetric analysis (TGA) above a certain temperature close to the temperature of the conductivity transition. ${ }^{63-64}$ They have assigned the decrease in conductivity above a particular temperature to the loss of oxygen and disruption of the $\mathrm{Fe}^{3+}-\mathrm{O}^{2-}-\mathrm{Fe}^{4+}$ conduction pathways. ${ }^{63-64}$ Our TGA data (Figure 2.12) show a feature between $370-440{ }^{\circ} \mathrm{C}(643-$ $713 \mathrm{~K}$ ), where the weight loss is interrupted by a plateau and a slight weight-gain, before the descending trend in weight continues above $440{ }^{\circ} \mathrm{C}$. The feature in TGA data matches the temperature where the change in conductivity trend occurs. The weight loss is somewhat accelerated above $440{ }^{\circ} \mathrm{C}$, as seen from the slope of the TGA plot. (Figure 2.12) Overall, the total weight loss for the material is less than $1 \%$.

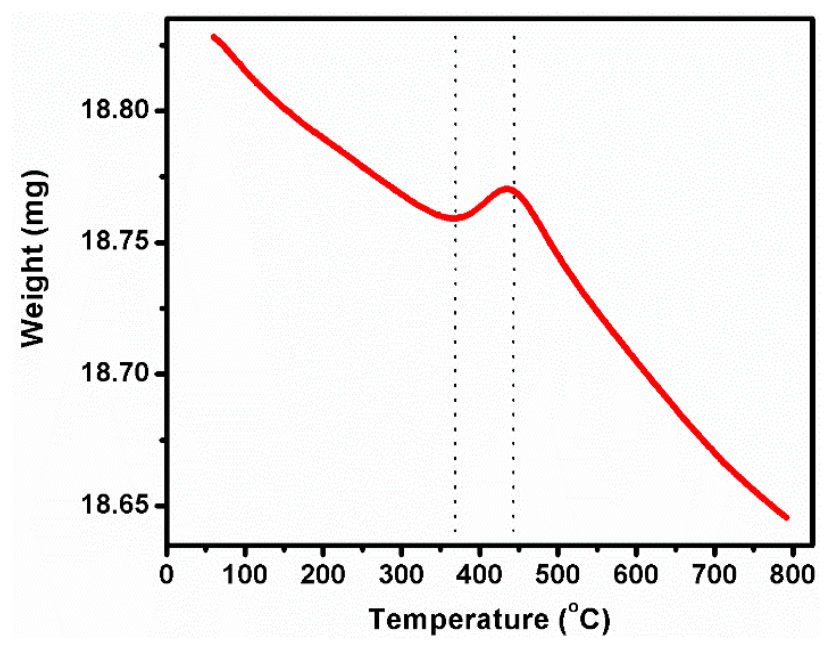

Figure 2.12. Thermogravimetric analysis data for $\mathrm{CaSrFe}_{2} \mathrm{O}_{6-\delta}$ in air 
It is worth noting that the release of oxygen results in more oxygen vacancies, which enhances the oxide ion conductivity. The decrease in total conductivity is observed because the increase in ionic conductivity is less significant than the decrease in electronic conductivity. ${ }^{64}$

We also examined the possibility of any structural phase change at high temperature, which could contribute to the decrease in conductivity above $\sim 673 \mathrm{~K}$. We heated a sample of CaSr-compound to $1073 \mathrm{~K}\left(800{ }^{\circ} \mathrm{C}\right)$, followed by quenching into liquid nitrogen to trap the potential high temperature phases. The XRD data indicated that the crystal structure had remained intact and no structural changes had occurred.

Some researchers have noted that the discontinuity in conductivity trend sometimes occurs close to the Neel temperature. ${ }^{57}$ Some authors have even used electrical conductivity to determine the Neel temperature. ${ }^{65}$ We note that our CaSr-material is antiferromagnetically ordered at room temperature. A comparison between the refined magnetic moments, 4.6 (2) $\mu_{\mathrm{B}}$ and 3.9 (2) $\mu_{\mathrm{B}}$, at $10 \mathrm{~K}$ and the corresponding values at $300 \mathrm{~K}, 4.0$ (2) $\mu_{\mathrm{B}}$ and 3.5 (2) $\mu_{\mathrm{B}}$, indicates that the magnetic moments magnitude remains considerably high at room temperature. Also, the magnetic susceptibility data indicate that the Neel temperature should be higher than $400 \mathrm{~K}$.

Another interesting feature of the electrical conductivity of CaSr-compound is the pronounced hysteresis in the conductivity data obtained during heating and cooling cycles below $\sim 673 \mathrm{~K}$. The conductivity values during cooling are greater than those obtained during heating. The observation of this feature confirms the contribution of oxide ion conductivity to total conductivity of this material, making it a mixed electronic-ionic conductor, as described before for some mixed conductors. ${ }^{62}$, 66 The oxygen 
absorption/desorption phenomena, which are slower than electron diffusion, are responsible for the observation of these hystereses. ${ }^{62,66}$ This also indicates that oxygen loss has a positive impact on overall conductivity and cannot be responsible for the change in conductivity trend above $673 \mathrm{~K}$. The temperature-activated mobility of polarons seems to reach a maximum, where no further increase in mobility occurs with increasing temperature. The collisions between charge carriers lead to decrease in conductivity as temperature increases further, similar to the behavior observed in metallic systems.

\section{CONCLUSION}

The alteration of electrical properties and magnetism of oxygen-deficient perovskites $\mathrm{AA}^{\prime} \mathrm{Fe}_{2} \mathrm{O}_{6-\delta}, \mathrm{A}=\mathrm{Sr}, \mathrm{Ca} ; \mathrm{A}^{\prime}=\mathrm{Sr}$, as a result of changing the crystal structure, due to the effect of the A-site cation, has been demonstrated. It has been shown that the incommensurate magnetic structure of the $\mathrm{Sr}_{2}$ compound, featuring magnetic moments in spin-density wave state which are perpendicular to the body diagonal of the unit cell, can be transformed into a long-range G-type antiferromagnetic system upon changing the A-site cations into CaSr. This occurs as a consequence of a structural alteration. The structure with alternating $\mathrm{FeO}_{5}$ square-pyramidal dimers and $\mathrm{FeO}_{6}$ octahedra is converted into a structure featuring alternating layers of tetrahedra and octahedra. The electrical properties are also transformed by changes in the A-site cation, where a metallic system is converted into a semiconductor featuring mixed ionic-electronic conductivity, as evident form charge transport studies in the temperature range $298 \mathrm{~K}-1073 \mathrm{~K}$. 


\section{CHAPTER 3}

UNRAVELING THE ROLE OF STRUCTURAL ORDER IN TRANSFORMATION OFF ELECTRICAL CONDUCTIVITY IN $\mathrm{Ca}_{2} \mathrm{FeCoO}_{6-\delta}, \mathrm{CaSrFeCoO}_{6-\delta} \mathrm{AND} \mathrm{Sr}_{2} \mathrm{FeCoO}_{6-\delta}$

\section{INTRODUCTION}

Oxygen deficient perovskite oxides possess unique properties that make them ideal candidates for application in devices such as gas diffusion membranes ${ }^{1}$, ceramic membranes for oxygen separation ${ }^{2}$, sensors $^{3}$, solid oxide fuel cells (SOFCs $)^{4}$, superconductors and colossal magnetoresistants. ${ }^{5}$

The oxygen deficient perovskite oxides are represented by general formula $\mathrm{ABO}_{3-\mathrm{x}}$ or $\mathrm{A}_{2} \mathrm{~B}_{2} \mathrm{O}_{6-\delta}$. The $\mathrm{A}$ and $\mathrm{B}$ sites can contain more than one type of cation. Oxygen-deficient perovskites can have a variety of structures depending on several factors including the extent of anion deficiency and vacancy order/disorder. While disordered vacancies in oxygen-deficient perovskites are common, there are a number of ways for the vacancies, created due to oxygen deficiency, to order. This leads to great diversity and variation in structure and properties of this family of compounds ${ }^{6-7}$. The vacancies result in different coordination geometries such as tetrahedral or square pyramidal. ${ }^{8-10}$ One of the structure-

${ }^{2}$ The work described in this chapter was published in Inorganic Chemistry ( 2017, vol. 56, p. 14494-14505) 
types containing tetrahedral geometry comprises corner-sharing tetrahedra which form chains that run almost perpendicular to the longest unit cell axis (Figure 3.1). The tetrahedra in these chains also share apexes with octahedral layers above and below them. Therefore, the overall structure appears as a combination of octahedral and tetrahedral layers as shown in Figure 3.1. This is called the brownmillerite-type structure. In situations like this, where there is more than one unique crystallographic site for the A or B cations, the general formula can be expressed as $\mathrm{AA}^{\prime} \mathrm{BB}^{\prime} \mathrm{O}_{6-\delta}$. The variation in the type of cations on A or B sites, affects the structure and properties of these materials. For example, $\mathrm{Sr}_{2} \mathrm{Fe}_{2} \mathrm{O}_{5}$ has a vacancy-ordered structure containing $\mathrm{FeO}_{6}$ octahedra and $\mathrm{FeO}_{4}$ tetrahedra. ${ }^{11}$ Replacing one of the Fe atoms for Mn leads to a change in crystal structure. The resulting material, $\mathrm{Sr}_{2} \mathrm{FeMnO}_{5}$, contains vacancies that are distributed randomly, without any type of ordering. ${ }^{12}$ Another example is the difference between $\mathrm{Ca}_{2} \mathrm{FeAlO}_{5}$ and $\mathrm{Ca}_{2} \mathrm{FeGaO}_{5}{ }^{13}$ These two materials have the same structure-type, but the change in the B-site cation results in a change in the space group. The Al-containing compound crystalizes in Ibm2 space group, while the Ga-containing material has space group Pcmn. ${ }^{13}$

The material properties can also be affected by changes in the A-site cation. An example is the differences between the two compounds $\mathrm{Ca}_{2} \mathrm{Fe}_{2} \mathrm{O}_{5}$ and $\mathrm{Sr}_{2} \mathrm{Fe}_{2} \mathrm{O}_{5}{ }^{14-15}$ While both of these materials have ordered structures, where $\mathrm{Fe}$ atoms have both octahedral and tetrahedral geometry, the change in the A-site cation leads to different space groups. $\mathrm{Ca}_{2} \mathrm{Fe}_{2} \mathrm{O}_{5}$ crystallizes in the primitive space group $\mathrm{Pnma}^{16}$, whereas $\mathrm{Sr}_{2} \mathrm{Fe}_{2} \mathrm{O}_{5}$ has a bodycenter space group Icmm ${ }^{11}$. A similar effect is observed for $\mathrm{Ca}_{2} \mathrm{GaMnO}_{5}$ and $\mathrm{Sr}_{2} \mathrm{GaMnO}_{5}$, where the change in the A-site cation results in changes in the space group. ${ }^{17}$ 


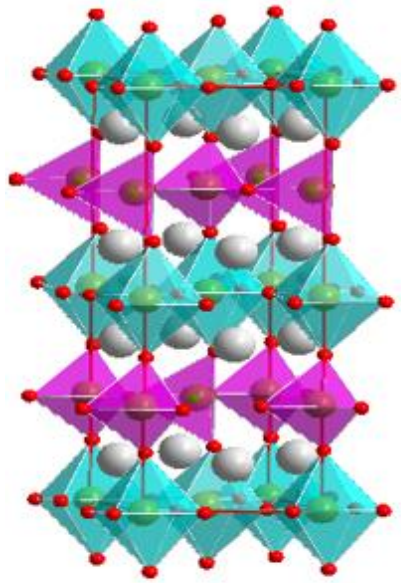

(a)

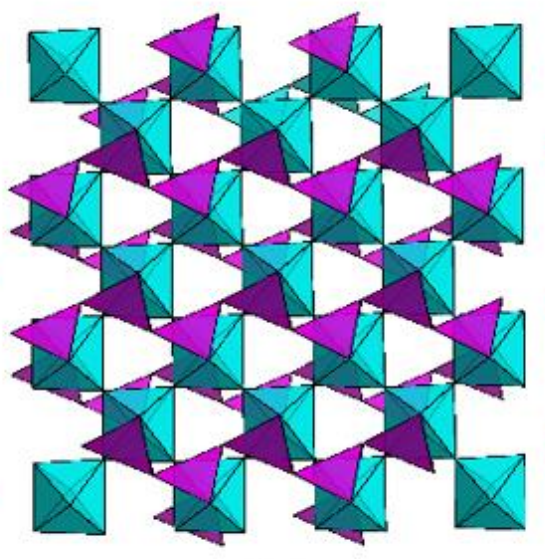

(b)

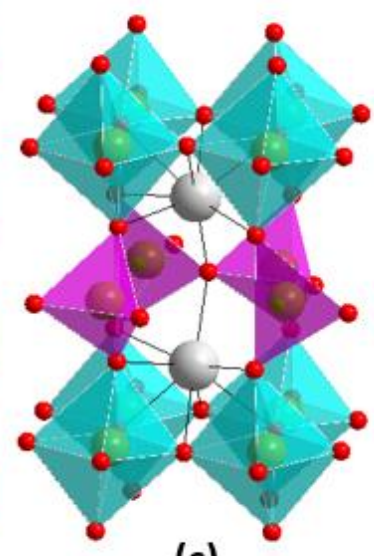

(c)

Figure 3.1. Crystal structure of $\mathrm{CaSrFeCoO}_{6-\delta}$. (a) The crystallographic unit cell. The alternating $(\mathrm{Fe} / \mathrm{Co}) \mathrm{O}_{6}$ octahedra (cyan) and $(\mathrm{Fe} / \mathrm{Co}) \mathrm{O}_{4}$ tetra (pink) are highlighted. The large grey spheres are the A-site cations, $\mathrm{Ca}^{2+} / \mathrm{Sr}^{2+}$. (b) A view along the $b$ axis (longest axis) to show the uniform orientation of tetrahedral chains. The A-site cations are omitted for clarity. (c) The coordination geometry around the A-site cation. There are 8 oxygens that are close enough to the A-site cation to be in its coordination sphere.

In the above examples, the change in the A-site cation only affects the crystal symmetry, and the overall structure-type remains the same. In this article, we show significant changes in structure and electrical properties due to the variation in the A-site cation. We demonstrate that changes to the structural order have major consequences with regard to the electrical charge transport in three oxygen-deficient perovskites, $\mathrm{Ca}_{2} \mathrm{FeCoO}_{6-\delta}$, $\mathrm{CaSrFeCoO}_{6-\delta}$ and $\mathrm{Sr}_{2} \mathrm{FeCoO}_{6-\delta}$.

\section{EXPERIMENTAL}

All three materials, $\mathrm{Ca}_{2} \mathrm{FeCoO}_{6-\delta}, \mathrm{CaSrFCoO}_{6-\delta}$ and $\mathrm{Sr}_{2} \mathrm{FeCoO}_{6-\delta}$, were synthesized under the same condition. The precursor compounds $\mathrm{CaCO}_{3}$ (Alfa Aesar, 99.95\%), $\mathrm{Fe}_{2} \mathrm{O}_{3}$ (Alfa Aesar, 99.998\%), $\mathrm{Co}_{3} \mathrm{O}_{4}$ (Alfa Aesar, 99.7\%) and $\mathrm{SrCO}_{3}$ (Aldrich, 99.9\%) were used in stoichiometric proportions for solid state syntheses. The precursor powders were mixed and ground together using agate mortar and pestle, pressed into a pellet and heated in air 
at $1000^{\circ} \mathrm{C}$ for 24 hours. Then the sample was reground and refired in air at $1200^{\circ} \mathrm{C}$ for 24 hours followed by slow cooling. In all cases, the heating and cooling rates were $100^{\circ} \mathrm{C} / \mathrm{h}$. The phase purity and structure of the polycrystalline samples were studied by powder Xray diffraction at room temperature using $\mathrm{CuK} \alpha 1$ radiation $(\lambda=1.54056 \AA)$, and step-size $0.008^{\circ}$. The GSAS software ${ }^{18}$ and EXPEGUI interface ${ }^{19}$ were used for Rietveld refinements. The sample morphologies were examined using a high-resolution fieldemission scanning electron microscope (SEM). X-ray photoelectron spectroscopy was performed at room temperature using $\mathrm{Al} \mathrm{K} \alpha$ radiation $(1486.7 \mathrm{eV})$. The electrical properties were investigated by $\mathrm{DC}$ and $\mathrm{AC}$ conductivity measurements on pressed pellets that had been sintered at $1200{ }^{\circ} \mathrm{C}$. The densities of sintered pellets were $\sim 50 \%, \sim 27 \%$ and $\sim 76 \%$ of the theoretical densities for $\mathrm{Ca}_{2} \mathrm{FeCoO}_{6-\delta}, \mathrm{CaSrFCoO}_{6-\delta}$ and $\mathrm{Sr}_{2} \mathrm{FeCoO}_{6-\delta}$, respectively. The relative densities are consistent with SEM results, as described in the next section. Electrochemical impedance spectroscopy (EIS) was performed in the frequency range $0.1 \mathrm{~Hz}-1 \mathrm{MHz}$ using a computer-controlled frequency response analyzer at room temperature. The 2-probe DC measurements were performed in the temperature range 298 to $1073 \mathrm{~K}\left(25-800{ }^{\circ} \mathrm{C}\right)$ by applying a constant voltage, $10 \mathrm{mV}$, and collecting the output current. Variable temperature electrical conductivity measurements were carried out during both heating and cooling cycles. The rate of heating and cooling for conductivity measurements was $3{ }^{\circ} \mathrm{C} \min ^{-1}$. Iodometric titrations were performed by dissolving about $50 \mathrm{mg}$ of sample and excess $\mathrm{KI}(\sim 2 \mathrm{~g})$ in $100 \mathrm{~mL}$ of $1 \mathrm{M} \mathrm{HCl} .5 \mathrm{~mL}$ of the solution was then pipetted out, and the iodine that had been generated in the solution was titrated using $0.025 \mathrm{M} \mathrm{Na}_{2} \mathrm{~S}_{2} \mathrm{O}_{3}$. Near the end point of the titration, 10 drops of starch solution were added to act as indicator. All steps were performed under argon atmosphere. Neutron diffraction 
experiments with center wavelength of $1.333 \AA$ were performed on powder samples $(\sim 4 \mathrm{~g})$ in vanadium sample holders at $300 \mathrm{~K}$ on POWGEN diffractometer at Oak Ridge National Laboratory.

\section{RESULTS AND DISCUSSION}

Crystal structure

We have determined the crystal structure of $\mathrm{CaSrFCoO}_{6-\delta}$ and demonstrated the sharp contrast between this material and $\mathrm{Ca}_{2} \mathrm{FeCoO}_{6-\delta}{ }^{20}$ and $\mathrm{Sr}_{2} \mathrm{FeCoO}_{6-\delta}{ }^{21}$

Researchers studying $\mathrm{CO}_{2}$ absorption ${ }^{21}$ have previously identified a phase with a composition similar to $\mathrm{CaSrFCoO}_{6-\delta}$. However, they could only determine the cell dimensions, and no other structural information is available for this material. We examined the structure of this material through a series of Rietveld refinement analyses using monochromatic X-ray and time-of-flight neutron diffraction. The data indicate that this material has an orthorhombic structure featuring tetrahedral chains that are sandwiched between octahedral layers. As shown in Figure 3.1, the tetrahedral chains run parallel to the octahedral layers. Each tetrahedron in the chain shares corners with the octahedral layers above and below. This material is the brownmillerite-type structure that was described above.

Materials with this structure-type usually crystalize in space groups Pnma, Ibm2, Icmm or Pbcm. ${ }^{22-23}$ The space group is determined by relative orientation of tetrahedral chains. There are two possible orientations, which are arbitrarily called right-handed and lefthanded. If all tetrahedral chains have the same orientation, the space group $I b m 2$ is obtained. If the tetrahedral chains have the same orientation within each layer but are oriented opposite to the chains in the next tetrahedral layer, the resulting space group will 
be Pnma. The random orientation of tetrahedral chains leads to space group Icmm. A less common space group is $\mathrm{Pbcm},{ }^{20}$ where each tetrahedral chain is oriented opposite to all of its nearest neighbors within the same layer and in the neighboring layers. Materials that crystalize in space group Pbcm have a large unit cell, that has twice the volume of the unit cell for other space groups mentioned above. The large unit cell is represented by supercell reflections in powder diffraction data, making this type of structure easily identifiable. The absence of Pbcm supercell reflections in the powder X-ray diffraction data of $\mathrm{CaSrFCoO}_{6}$ $\delta$ rules out this space group. The other primitive space group, Pnma, is identified by the presence of 131 and 151 peaks in the powder diffraction data, which are absent in the bodycentered systems. The 131 peak is especially prominent when present. The absence of these peaks in the PXRD data of $\mathrm{CaSrFCoO}_{6-\delta}$ indicates that this material does not have a primitive unit cell and crystallizes in one of the body-centered space groups, Icmm or Ibm2. Multiple Rietveld refinements with these two space groups were performed using highresolution $\mathrm{K}_{1}$ XRD datasets on different $\mathrm{CaSrFCoO}_{6-\delta}$ samples to ensure the reproducibility of the results. With Icmm space group, the atomic parameters could only be refined individually, while simultaneous refinement of all atomic parameters led to the divergence of the Rietveld refinements. This was observed consistently for multiple samples and 


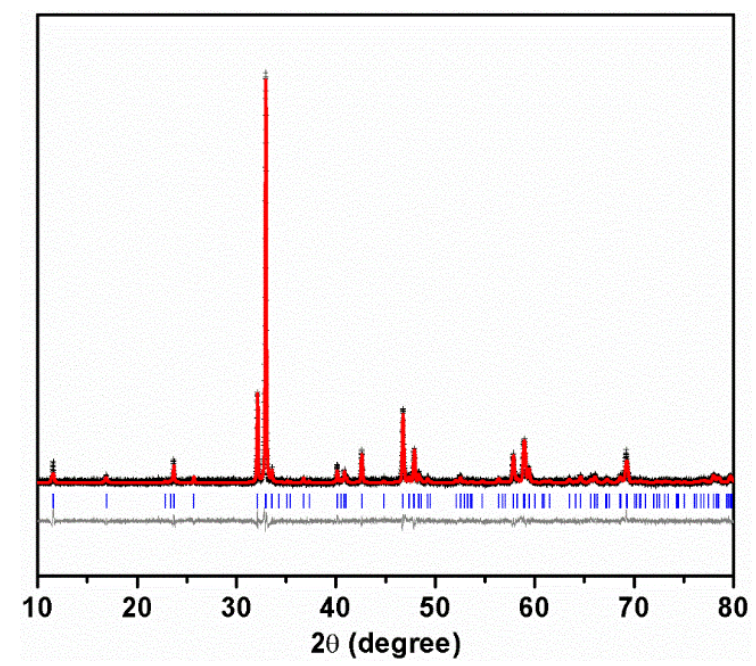

Figure 3.2. Rietveld refinement profile for powder X-ray diffraction data of $\mathrm{CaSrFeCoO}_{6}$ $\delta$. Crosses represent experimental data, solid red line is the $I b m 2$ model, vertical tick marks show Bragg peak positions, and the lower line represents the difference plot.

refinement trials. The Ibm2 space group, however, always led to an excellent fit. All profile parameters, background, unit cell dimensions, atomic positions, and thermal displacement factors were refined simultaneously, giving an excellent fit to Ibm2 space group. The Rietveld refinement profile is shown in Figure 3.2 and the refined atomic parameters are listed in Table 3.1. Given the inherent limitation of laboratory X-ray diffraction with regard to differentiating elements with similar atomic numbers, such as $\mathrm{Fe}$ and $\mathrm{Co}$, neutron diffraction experiments were undertaken to study the distribution of Fe and Co on different positions in this material. Given the large difference between the neutron scattering lengths of $\mathrm{Fe}, 9.45$, and $\mathrm{Co}, 2.49$, these two nuclei are readily distinguishable by neutrons. The neutron diffraction results and Rietveld refinement profile are shown in Table 3.2 and Figure 3.3, respectively. Initially two models were tested where Fe and Co were placed exclusively on tetrahedral and octahedral sites, and vice versa. However, the refined thermal displacement factors on tetrahedral and octahedral sites became unusually large on 
one site and negative on the other. Therefore, the site occupancies on these two sites were refined, leading to an excellent fit and atomic parameters that are shown in Table 3.2. As observed here, $\mathrm{Fe}$ and $\mathrm{Co}$ are distributed nearly evenly over the octahedral and tetrahedral sites.

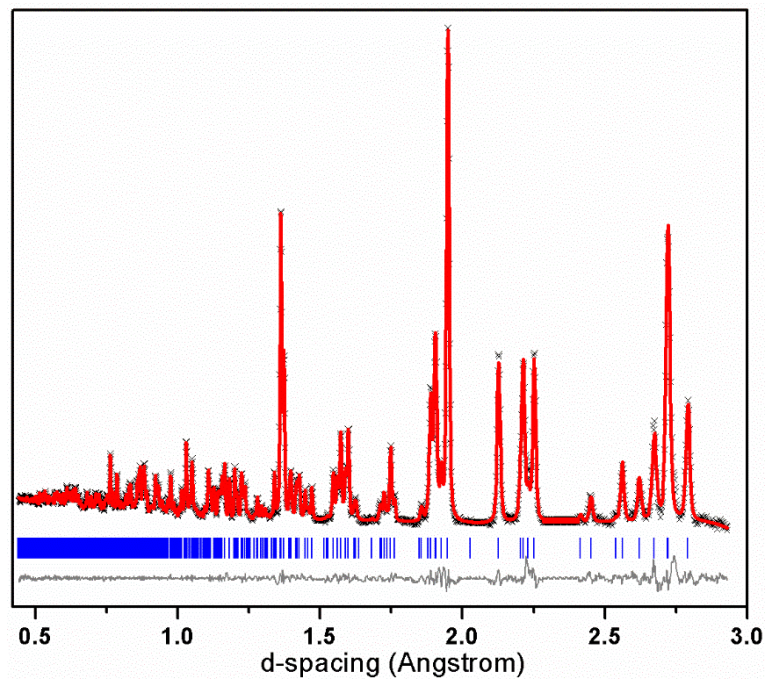

Figure 3.3. Neutron diffraction Rietveld refinement profile for $\mathrm{CaSrFeCoO}_{6-\delta}$. Magnetic reflections have been omitted. Crosses represent experimental data, solid red line is the $\mathrm{Ibm} 2$ model, vertical tick marks show Bragg peak positions, and the lower line represents the difference plot. 
Table 3.1. Refined structural parameters of $\mathrm{CaSrFeCoO}_{6-\delta}$ using powder $\mathrm{X}$-ray diffraction.

Space group: $\operatorname{Ibm} 2$

\begin{tabular}{ccccccc}
\multicolumn{5}{c}{$a=5.5576(2) \AA, b=$} & $15.1658(5) \AA, c=5.4141(2) \AA ⿻ \mathrm{\AA}, \mathrm{Rp}=0.0164$, wRp $=0.0220$ \\
\hline Elements & $\mathrm{x}$ & $\mathrm{y}$ & $\mathrm{z}$ & Occupancy & $\mathrm{U}_{\text {ISO }}$ & Multiplicity \\
\hline $\mathrm{Ca} 1$ & $0.5103(6)$ & $0.1116(2)$ & $-0.004(6)$ & 0.5 & $0.024(1)$ & 8 \\
$\mathrm{Sr} 1$ & $0.5103(6)$ & $0.1116(2)$ & $-0.004(6)$ & 0.5 & $0.024(1)$ & 8 \\
$\mathrm{Fe} 1$ & $0.0776(9)$ & 0.25 & $-0.011(7)$ & 1 & $0.030(3)$ & 4 \\
$\mathrm{Co} 1$ & 0 & 0 & 0 & 1 & $0.031(2)$ & 4 \\
$\mathrm{O} 1$ & $0.25(1)$ & $0.0008(8)$ & $0.268(7)$ & 1 & $0.042(3)$ & 8 \\
$\mathrm{O} 2$ & $-0.075(2)$ & $0.1544(6)$ & $-0.005(1)$ & 1 & $0.042(3)$ & 8 \\
$\mathrm{O} 3$ & $0.372(4)$ & 0.25 & $0.85(1)$ & 1 & $0.042(3)$ & 4 \\
\hline
\end{tabular}

Therefore, both tetrahedral and octahedral layers contain almost equal quantities of Fe and Co. These findings highlight the strength of neutron diffraction in tackling problems that cannot be resolved by X-rays. To study the correlation between crystal structure and electrical conductivity, we also synthesized $\mathrm{Ca}_{2} \mathrm{FeCoO}_{6-\delta}$ and $\mathrm{Sr}_{2} \mathrm{FeCoO}_{6-\delta}$ (hereafter referred to as $\mathrm{Ca}_{2}$ and $\mathrm{Sr}_{2}$ analogues) under the same conditions as $\mathrm{CaSrFeCoO}_{6-\delta}$ (hereafter CaSr-compound). Table 3.3 compares the space groups and unit cell parameters for all three compounds. The $\mathrm{Sr}_{2}$ material crystallizes in the cubic space group $P m-3 m .^{21,24}$ The formation of the cubic structure with space group $P m-3 m$ was confirmed by our Rietveld 
Table 3.2. Refined atomic parameters of $\mathrm{CaSrFeCoO}_{6-\delta}$ in $\mathrm{Ibm} 2$ space group using neutron diffraction.

\begin{tabular}{cccccc}
\hline Elements & $\mathrm{x}$ & $\mathrm{y}$ & $\mathrm{z}$ & Occupancy & $\mathrm{U}_{\text {iso }}$ \\
\hline $\mathrm{Ca} 1$ & $0.5125(6)$ & $0.1110(2)$ & $0.010(2)$ & $0.48(5)$ & $0.0140(8)$ \\
$\mathrm{Sr} 1$ & $0.5125(6)$ & $0.1110(2)$ & $0.010(2)$ & $0.52(5)$ & $0.0140(8)$ \\
$\mathrm{Fe} 1$ & $0.0664(9)$ & 0.25 & $-0.031(2)$ & $0.46(3)$ & $0.022(2)$ \\
$\mathrm{Co} 1$ & $0.0664(9)$ & 0.25 & $-0.031(2)$ & $0.54(3)$ & $0.022(2)$ \\
$\mathrm{Fe} 2$ & 0 & 0 & 0 & $0.52(2)$ & $0.009(1)$ \\
$\mathrm{Co} 2$ & 0 & 0 & 0 & $0.48(2)$ & $0.009(1)$ \\
$\mathrm{O} 1$ & $0.2393(8)$ & $0.0094(2)$ & $0.266(2)$ & 1 & $0.0106(6)$ \\
$\mathrm{O} 2$ & $-0.057(1)$ & $0.1406(2)$ & $0.008(3)$ & 1 & $0.0204(7)$ \\
$\mathrm{O} 3$ & $0.370(1)$ & 0.25 & $0.889(2)$ & 1 & $0.027(1)$ \\
\hline
\end{tabular}

refinement results. Figures 3.4 and 3.5 show the crystal structure and Rietveld refinement profile of the $\mathrm{Sr}_{2}$ compound. The refined atomic parameters are listed in Table 3.4. The $\mathrm{Ca}_{2}$ compound has a brownmillerite-type $\mathrm{Pbcm}$ structure ${ }^{20}$ (Figure 3.6) with a large unit cell, which is double the size of that for a typical brownmillerite, and ordered arrangement of tetrahedral chains, where each chain is oriented opposite to all of its nearest-neighbors. Our Rietveld refinements confirm the formation of the $\mathrm{Pbcm}$ structure ${ }^{20}$ (Figure 3.7) under the same synthesis conditions as the other two materials. Table 3.5 lists the refined atomic parameters for the $\mathrm{Ca}_{2}$ compound. 
Table 3.3. Comparison of space groups and unit cell parameters for $\mathrm{Ca}_{2} \mathrm{FeCoO}_{6-\delta}$, $\mathrm{CaSrFeCoO}_{6-\delta}$ and $\mathrm{Sr}_{2} \mathrm{FeCoO}_{6-\delta}$

\begin{tabular}{|cccc|}
\hline & $\mathrm{Sr} 2 \mathrm{FeCoO} 5$ & $\mathrm{CaSrFeCOO} 5$ & $\mathrm{Ca} 2 \mathrm{FeCoO} 5$ \\
\hline Space group & $P m-3 m$ & $\mathrm{Ibm2}$ & $P b c m$ \\
\hline Lattice & & & \\
parameters & & & \\
$\mathrm{a}(\AA)$ & $3.86469(3)$ & $5.5576(2)$ & $5.36854(8)$ \\
$\mathrm{b}(\AA)$ & $3.86469(3)$ & $15.1658(5)$ & $11.1063(2)$ \\
$\mathrm{c}(\AA)$ & $3.86469(3)$ & $5.4141(2)$ & $14.8079(2)$ \\
$\mathrm{V}\left(\AA^{3}\right)$ & $57.722(1)$ & $456.33(4)$ & $882.92(3)$ \\
\hline
\end{tabular}

The trend in the structural order in progression from $\mathrm{Sr}_{2}$-compound to $\mathrm{CaSr}$ and $\mathrm{Ca}_{2}$ materials is remarkable. In the $\mathrm{Sr}_{2}$ material, the vacant sites, created due to oxygen deficiency, are distributed randomly. In the CaSr compound the vacancies are ordered. The vacant sites only appear in alternating layers, forming tetrahedral chains, instead of octahedral geometry that is commonly observed in perovskites. All tetrahedral chains in this material have the same orientation, as evident from its space group. The $\mathrm{Ca}_{2}$ material has the same type of vacancy order. However, an additional type of ordering is also present, namely the alternating orientation of tetrahedral chains within and between layers. Therefore, it is evident that as the $\mathrm{Ca}$ content increases, the degree of ordering also increases. 


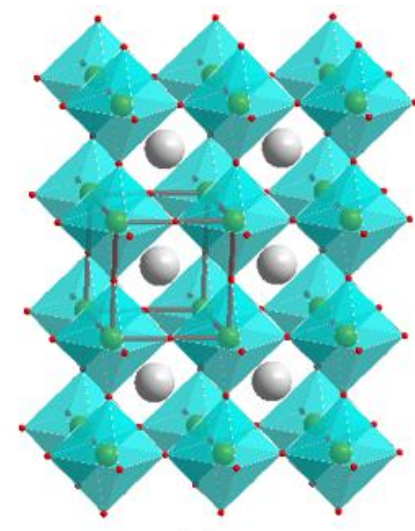

(a)

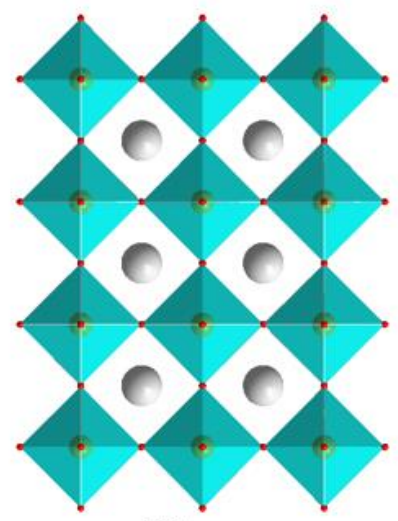

(b)

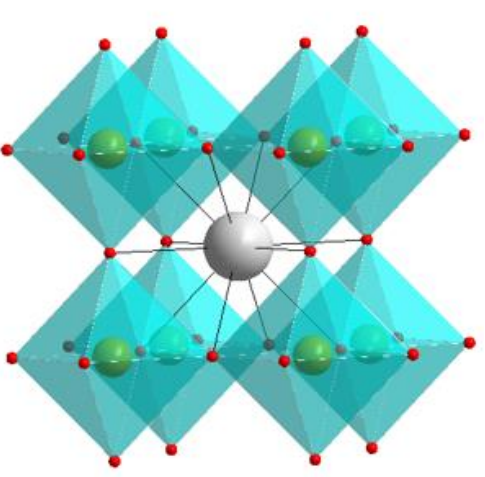

(c)

Figure 3.4. Crystal structure of $\mathrm{Sr}_{2} \mathrm{FeCoO}_{6-\delta}$. (a) The crystallographic unit cell and cornersharing $(\mathrm{Fe} / \mathrm{Co}) \mathrm{O}_{6}$ octahedra (cyan) are highlighted. The large grey spheres are the $\mathrm{Sr}$ atoms. (b) A view along the unit cell axis. Due to cubic symmetry, the three axes are identical. (c) The coordination geometry around the $\mathrm{Sr}$ atom, which is 12-coordinated.

We investigated the oxygen contents of all three materials using iodometric titration, which showed that the formulae for the three compounds can be described as $\operatorname{Sr}_{2} \mathrm{FeCoO}_{6-\delta}(\delta=$ 0.5), $\mathrm{CaSrFeCoO}_{6-\delta}(\delta=0.8)$ and $\mathrm{Ca}_{2} \mathrm{FeCoO}_{6-\delta}(\delta=0.9)$. Note the greater oxygen content of the disordered $\mathrm{Sr}_{2}$ material. We have also confirmed these results by monitoring the oxygen loss due to the heating of samples in argon at temperatures up to $1200{ }^{\circ} \mathrm{C}$, which usually leads to oxygen stoichiometry of 5 , i.e., $\delta=0$, in oxygen-deficient perovskites. ${ }^{10,23}$ We also explored the correlation between the A-site cation and the morphology and crystallite size by performing scanning electron microscopy studies on all three materials. Interestingly, the CaSr compound has the smallest crystallite size, as shown in Figure 3.8. The $\mathrm{Sr}_{2}$ and $\mathrm{Ca}_{2}$ compounds have comparable crystallite sizes. However, the contact between crystallites is enhanced in the $\mathrm{Sr}_{2}$ material compared to the other compounds. 


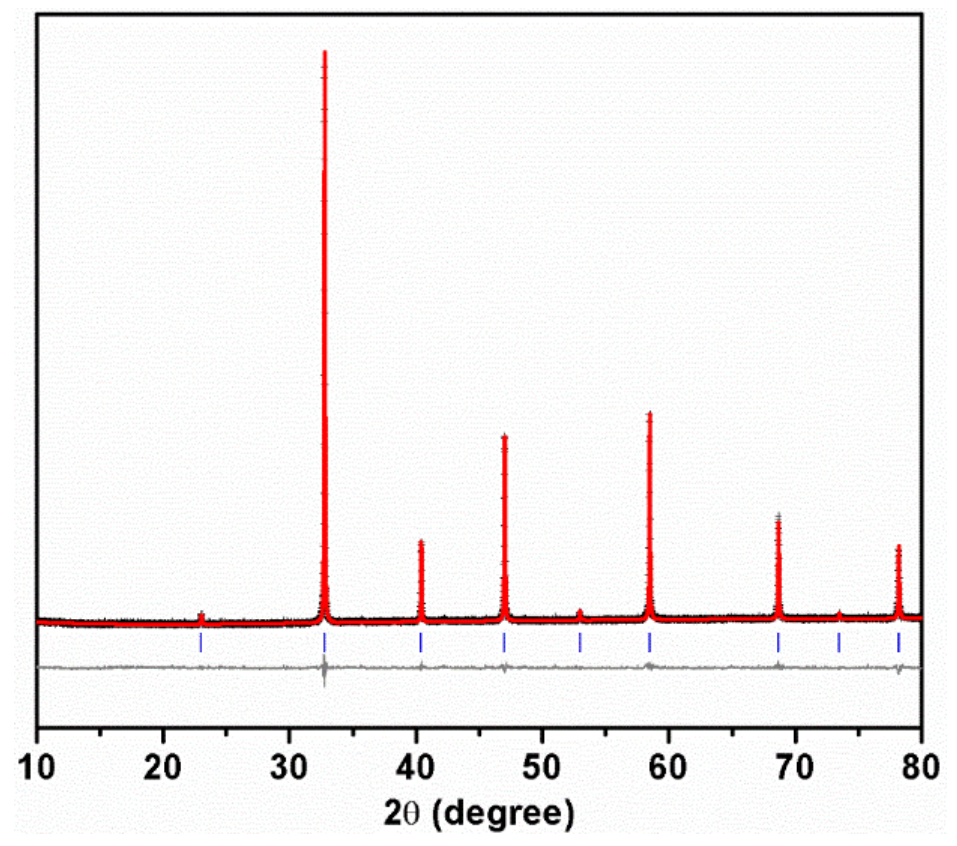

Figure 3.5. Rietveld refinement profile for powder X-ray diffraction data of $\mathrm{Sr}_{2} \mathrm{FeCoO}_{6-\delta}$ refined in Pm-3m space group. Crosses represent experimental data, solid red line is the model, vertical tick marks show Bragg peak positions, and the lower blue line represents the difference plot.

Table 3.4. Refined structural parameters of $\mathrm{Sr}_{2} \mathrm{FeCoO}_{6-\delta}$.

\begin{tabular}{|c|c|c|c|c|c|c|}
\hline \multicolumn{7}{|c|}{$\begin{array}{l}\text { Space group: } P m-3 m \\
a=3.86469(3) \AA, R_{p}=0.0142, w R_{p}=0.0192\end{array}$} \\
\hline Element & $\mathrm{x}$ & $\mathrm{y}$ & $\mathrm{z}$ & Occupancy & $\mathrm{U}_{\text {iso }}$ & Multiplicity \\
\hline Sr1 & 0.5 & 0.5 & 0.5 & 1 & $0.0183(3)$ & 1 \\
\hline $\mathrm{Fe} 1$ & 0 & 0 & 0 & 0.5 & $0.0177(4)$ & 1 \\
\hline Co1 & 0 & 0 & 0 & 0.5 & $0.0177(4)$ & 1 \\
\hline $\mathrm{O} 1$ & 0 & 0 & 0.5 & 0.92 & $0.0220(6)$ & 3 \\
\hline
\end{tabular}




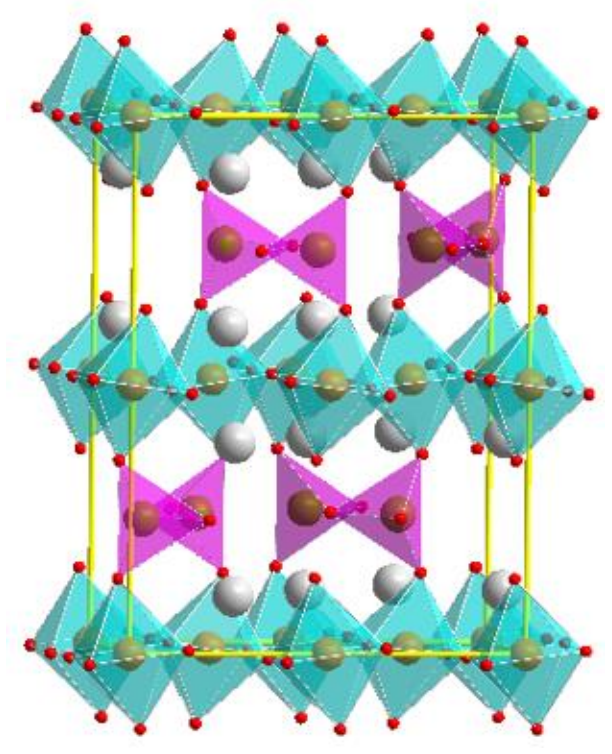

Figure 3.6. Crystal structure of $\mathrm{Ca}_{2} \mathrm{FeCoO}_{6-\delta}$ featuring alternating $(\mathrm{Fe} / \mathrm{Co}) \mathrm{O}_{6}$ octahedra (cyan) and $(\mathrm{Fe} / \mathrm{Co}) \mathrm{O}_{4}$ tetrahedra (pink). The large grey spheres are $\mathrm{Ca}$ atoms. The crystallographic unit cell is highlighted using yellow lines. Note that the unit cell here is 2times larger than that for the $\mathrm{CaSr}$ analogue.

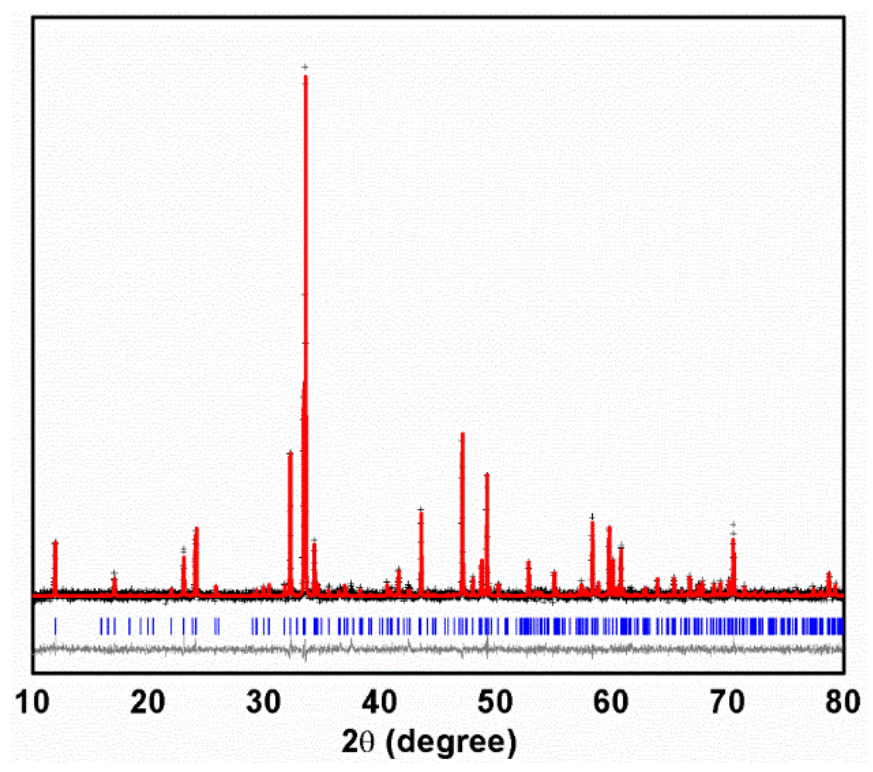

Figure 3.7. Rietveld refinement profile for powder X-ray diffraction data of $\mathrm{Ca}_{2} \mathrm{FeCoO}_{6-\delta}$ refined in $\mathrm{Pbcm}$ space group. Crosses represent experimental data, red solid line is the model, vertical tick marks show Bragg peak positions, and the blue line represents the difference plot. 
To investigate these structural transitions further, we carried out a series of experiments and synthesized 21 different materials by systematically varying the $\mathrm{Ca} / \mathrm{Sr}$ ratio to determine the average ionic radius that is required on the A-site to promote these structural transformations. The ionic radii play an essential role in the formation of different perovskite-type structures. ${ }^{25}$ For our materials, the transition from disordered structure to the ordered system occurs when the $\mathrm{Ca} / \mathrm{Sr}$ ratio is greater than $0.3 / 1.7$. (Figure 3.9) We also determined the $\mathrm{Ca} / \mathrm{Sr}$ ratio that is required for transition from the $\mathrm{Ibm} 2$ structure (where all tetrahedral chains have the same orientation) to the Pbcm system (with alternating orientation of tetrahedral chains). This transition was found to occur at $\mathrm{Ca} / \mathrm{Sr}$ ratio $1.2 / 0.8$, as indicated by the appearance of $(120)$ peak at $2 \theta \approx 23^{\circ}$ in Figure 3.10. Using the ionic radii for 12 -coordinated $\mathrm{Sr}^{2+}, 1.44 \AA$, and $\mathrm{Ca}^{2+}, 1.34 \AA,{ }^{26}$ one can calculate the average ionic radius that prompts each of these phase transitions. The transition from a disordered to ordered system occurs when the average ionic radius on the A-site is greater than $\sim 1.42$ $\AA$. The transition from the ordered system with uniform orientation of chains to the moreordered system, with alternating chain orientation, takes place when the average ionic radius is larger than $1.39 \AA$. 
Table 3.5. Refined structural parameters of $\mathrm{Ca}_{2} \mathrm{FeCoO}_{6-\delta}$.

\begin{tabular}{|c|c|c|c|c|c|c|}
\hline \multicolumn{3}{|c|}{$\begin{array}{l}\text { Space group: } P b c m \\
a=5.36854(8) \AA, \quad b=11.1063(2) \AA,\end{array}$} & \multicolumn{4}{|c|}{$c=14.8079(2) \AA, R_{p}=0.0167, w R_{p}=0.0215$} \\
\hline Element & $\mathrm{x}$ & $\mathrm{y}$ & $\mathrm{z}$ & Occupancy & $\mathrm{U}_{\text {iso }}$ & Multiplicity \\
\hline $\mathrm{Ca} 1$ & $-0.006(2)$ & $0.757(2)$ & $0.393(1)$ & 1 & $0.038(8)$ & 8 \\
\hline $\mathrm{Ca} 2$ & $-0.491(3)$ & $0.516(2)$ & $0.609(1)$ & 1 & $0.035(7)$ & 8 \\
\hline $\mathrm{Fe} 1$ & $0.439(3)$ & $0.719(1)$ & 0.25 & 0.5 & $0.032(7)$ & 4 \\
\hline Co1 & $0.439(3)$ & $0.719(1)$ & 0.25 & 0.5 & $0.032(7)$ & 4 \\
\hline $\mathrm{Fe} 2$ & $-0.054(3)$ & $0.539(2)$ & 0.25 & 0.5 & $0.042(8)$ & 4 \\
\hline $\mathrm{Co} 2$ & $-0.054(3)$ & $0.539(2)$ & 0.25 & 0.5 & $0.042(8)$ & 4 \\
\hline $\mathrm{Fe} 3$ & $-0.504(3)$ & 0.75 & 0.5 & 0.5 & $0.026(8)$ & 4 \\
\hline $\mathrm{Co} 3$ & $-0.504(3)$ & 0.75 & 0.5 & 0.5 & $0.026(8)$ & 4 \\
\hline $\mathrm{Fe} 4$ & 0.0 & 0.0 & 0.0 & 0.5 & $0.043(9)$ & 4 \\
\hline $\mathrm{Co} 4$ & 0.0 & 0.0 & 0.0 & 0.5 & $0.043(9)$ & 4 \\
\hline $\mathrm{O} 1$ & $0.114(8)$ & $0.662(4)$ & 0.25 & 1 & 0.04 & 4 \\
\hline $\mathrm{O} 2$ & $0.604(9)$ & $0.552(4)$ & 0.25 & 1 & 0.04 & 4 \\
\hline $\mathrm{O} 3$ & $-0.213(7)$ & $0.612(4)$ & $0.489(2)$ & 1 & 0.04 & 8 \\
\hline $\mathrm{O} 4$ & $-0.760(9)$ & $0.609(4)$ & $0.490(2)$ & 1 & 0.04 & 8 \\
\hline O5 & $0.042(7)$ & $0.459(4)$ & $0.359(3)$ & 1 & 0.04 & 8 \\
\hline O6 & $0.527(6)$ & $0.781(4)$ & $0.365(4)$ & 1 & 0.04 & 8 \\
\hline
\end{tabular}



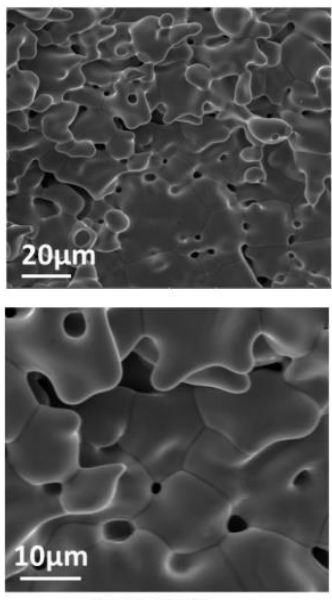

$\mathrm{Sr}_{2} \mathrm{FeCoO}_{6-\delta}$
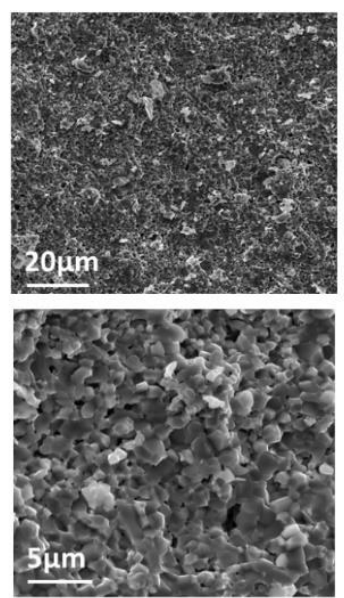

$\mathrm{CaSrFeCoO}_{5-\delta}$
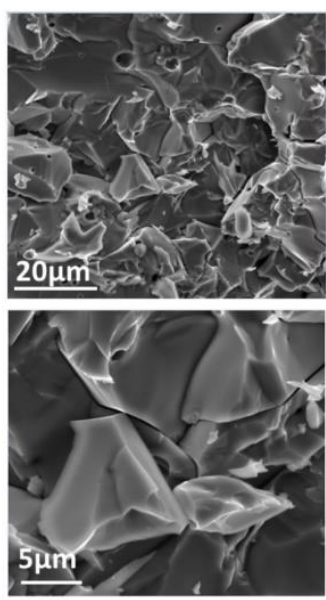

$\mathrm{Ca}_{2} \mathrm{FeCoO}_{5-\delta}$

Figure 3.8. Scanning electron microscopy images for $\mathrm{Sr}_{2} \mathrm{FeCoO}_{6-\delta}, \mathrm{CaSrFeCoO}_{6-\delta}$ and $\mathrm{Ca}_{2} \mathrm{FeCoO}_{6-\delta}$

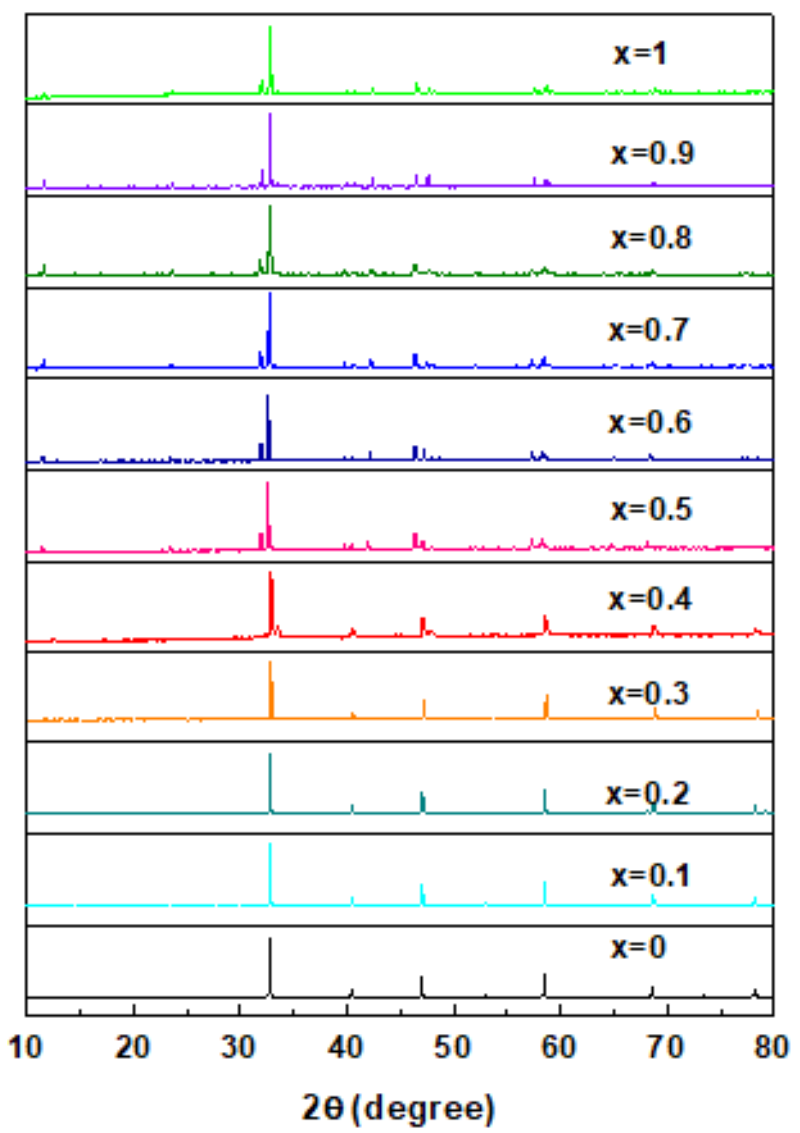

Figure 3.9. Powder XRD data for the series $\mathrm{Sr}_{2-\mathrm{x}} \mathrm{Ca}_{x} \mathrm{FeCoO}_{6-\delta}, \mathrm{x}=0-1$. The structural transition occurs above $\mathrm{x}=0.3$. 


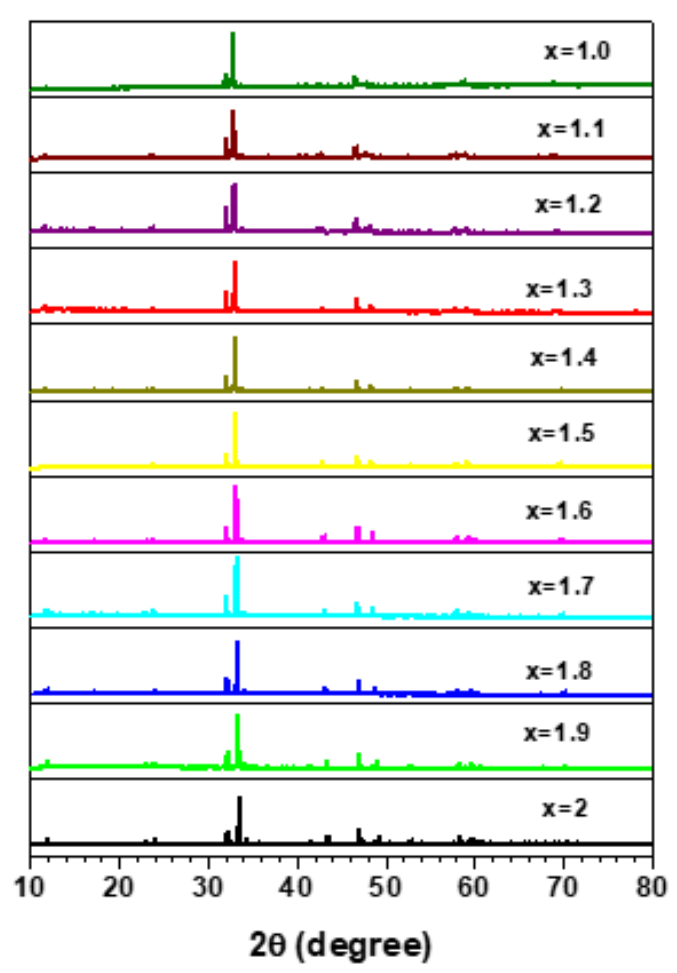

(a)

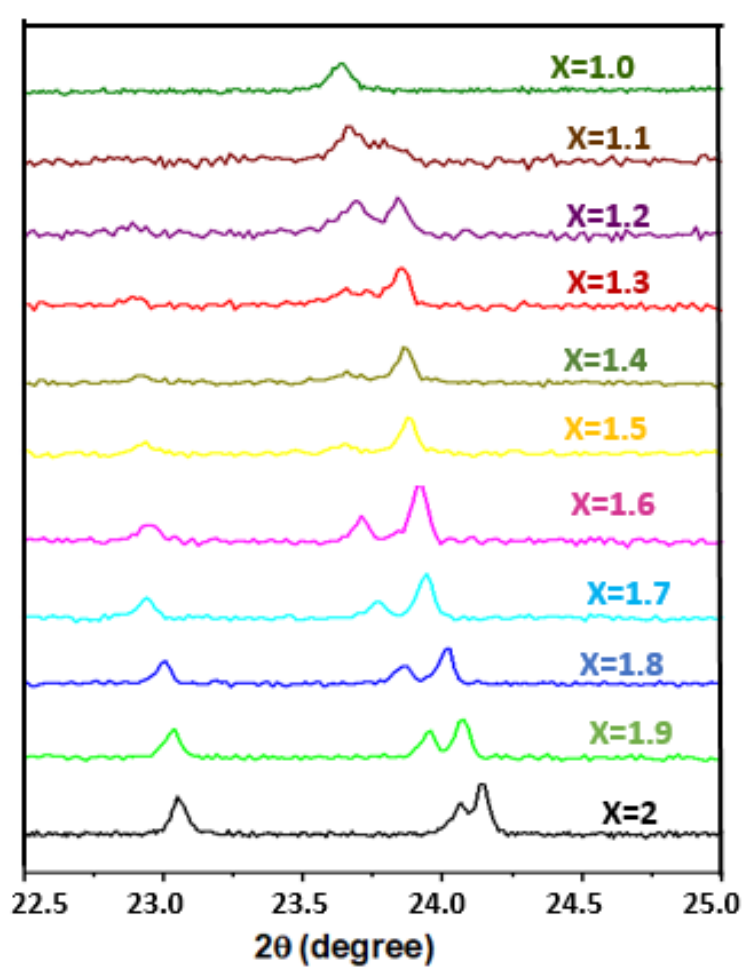

(b)

Figure 3.10. Powder XRD data for the series $\mathrm{Sr}_{2-\mathrm{x}} \mathrm{Ca}_{\mathrm{x}} \mathrm{FeCoO}_{6-\delta}, \mathrm{x}=1-2$. The structural transition occurs above $\mathrm{x}=1.2$.

X-ray photoelectron spectroscopy (XPS)

The oxidation states of iron and cobalt in all three materials were explored using XPS analyses, which revealed an interesting trend with regard to the cation oxidation states in these three compounds. We note that the starting materials used in the syntheses, namely $\mathrm{Fe}_{2} \mathrm{O}_{3}$ and $\mathrm{Co}_{3} \mathrm{O}_{4}$, contained $\mathrm{Fe}^{3+}, \mathrm{Co}^{2+}$ and $\mathrm{Co}^{3+}$. The main XPS peak for $\mathrm{Fe}^{3+}$ is the $2 \mathrm{p}_{3 / 2}$ peak, which appears at about $710-711.5 \mathrm{eV} \cdot{ }^{27-29}$ In our spectra this peak is present. However, additional features are also observed in the spectra indicating that other oxidation states are present as well, as discussed further below. Similar observations are made for 
Co, where the dominant $\mathrm{Co}^{3+}$ peak, i.e., the $2 \mathrm{p}_{3 / 2}$ peak, ${ }^{30-31}$ is observed at $\sim 780 \mathrm{eV}$, but additional features signify the presence of other cobalt oxidation states as well.

For Fe, the position and width of the $2 \mathrm{p}_{3 / 2}$ peak, as well as the positions of satellite peaks, which appear at higher energy than the $2 \mathrm{p}_{3 / 2}$ peak, are indicative of oxidation states. ${ }^{27-29} \mathrm{In}$ the XPS spectra of our materials, two satellite peaks are observed for Fe, one at $\sim 4 \mathrm{eV}$ higher and another at $\sim 6.5-7.5 \mathrm{eV}$ higher than the center of the $2 \mathrm{p}_{3 / 2}$ peak. The first satellite peak, located at $\sim 4 \mathrm{eV}$ higher than the $2 \mathrm{p}_{3 / 2}$ peak, belongs to $\mathrm{Fe}^{2+.28-29}$ The second satellite peak, at $\sim 6.5-7.5 \mathrm{eV}$ higher than the $2 \mathrm{p}_{3 / 2}$ peak, is the signature of $\mathrm{Fe}^{3+.28-29,32}$ Therefore, the XPS data show that these materials contain Fe in both divalent and trivalent states. Figure 3.11 shows the Fe XPS spectra for all three materials.

Unlike the Fe spectra that are quite similar for all three compounds, the Co spectra show an interesting variation in oxidation states. The most striking difference is the low energy shoulder on the cobalt $2 \mathrm{p}_{3 / 2}$ peak for the $\mathrm{Ca}_{2}$ and CaSr-compounds, which is absent for the $\mathrm{Sr}_{2}$-material, as seen in Figure 3.12. The low energy side of the cobalt $2 \mathrm{p}_{3 / 2}$ peak for $\mathrm{Ca}_{2}$ and CaSr-materials is much wider and is stretched further into the low-energy region, showing a distinct shoulder, which is not present in $\mathrm{Sr}_{2}$-material spectrum. 


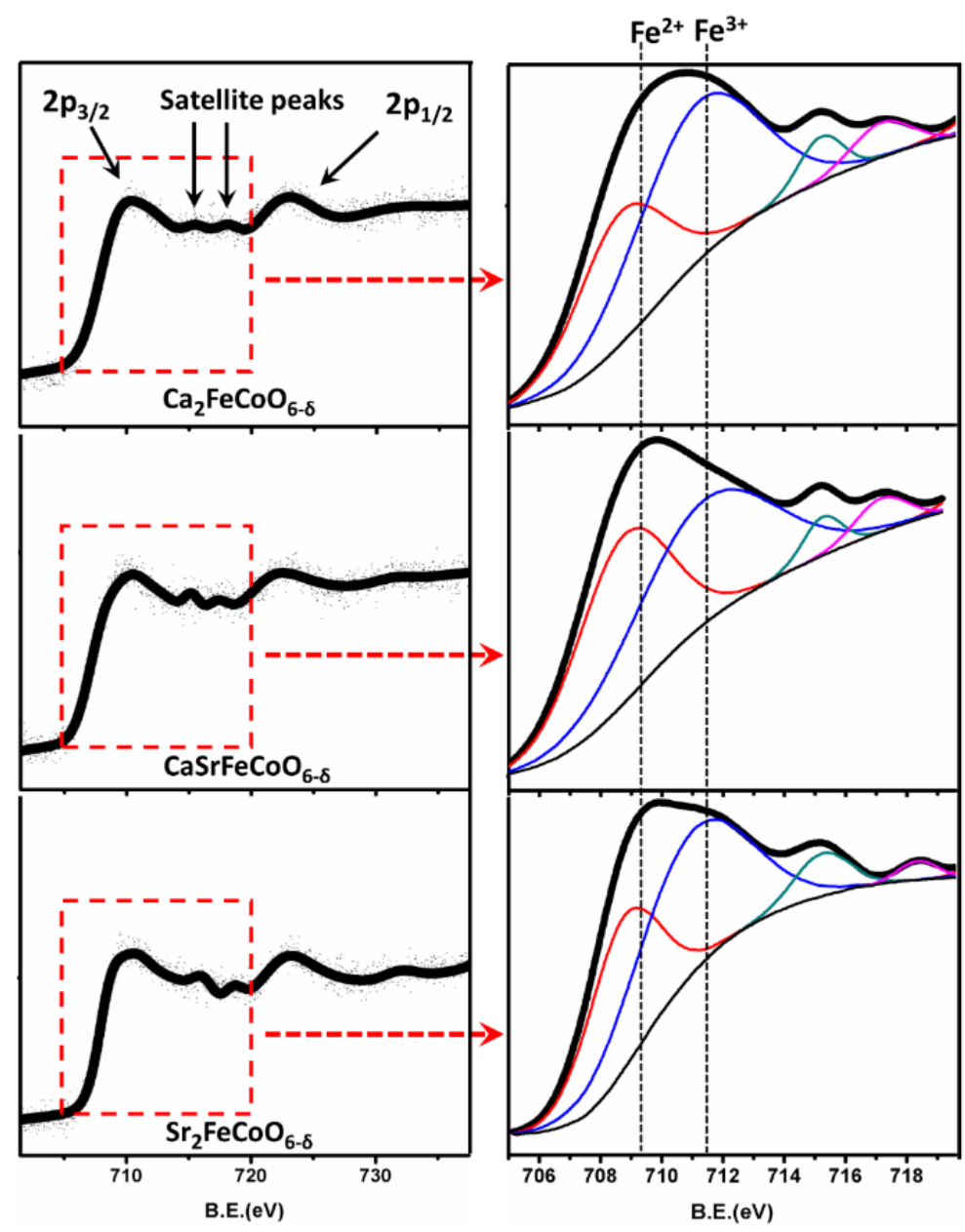

Figure 3.11. The Fe XPS spectra for $\mathrm{Ca}_{2} \mathrm{FeCoO}_{6-\delta}, \mathrm{CaSrFeCoO}_{6-\delta}$ and $\mathrm{Sr}_{2} \mathrm{FeCoO}_{6-\delta}$

The low energy shoulder is indicative of $\mathrm{Co}^{2+} \cdot 30,33$ There is also a shoulder on the high energy side of the $2 \mathrm{p}_{3 / 2}$ peak for all three compounds, which represents $\mathrm{Co}^{4+} \cdot 30-31$ Two satellite peaks are present in the cobalt spectra for all three materials, including the $\mathrm{Sr}_{2}$ compound that lacks $\mathrm{Co}^{2+}$. The first satellite peak appears at about $785 \mathrm{eV}-787 \mathrm{eV}$, while the second satellite peak is observed at about $788 \mathrm{eV}-789 \mathrm{eV}$. The first satellite peak represents tetravalent cobalt, ${ }^{31}$ and is located $\sim 3.5-5 \mathrm{eV}$ higher than the $\mathrm{Co}^{4+}$ peak, as expected. ${ }^{31}$ 


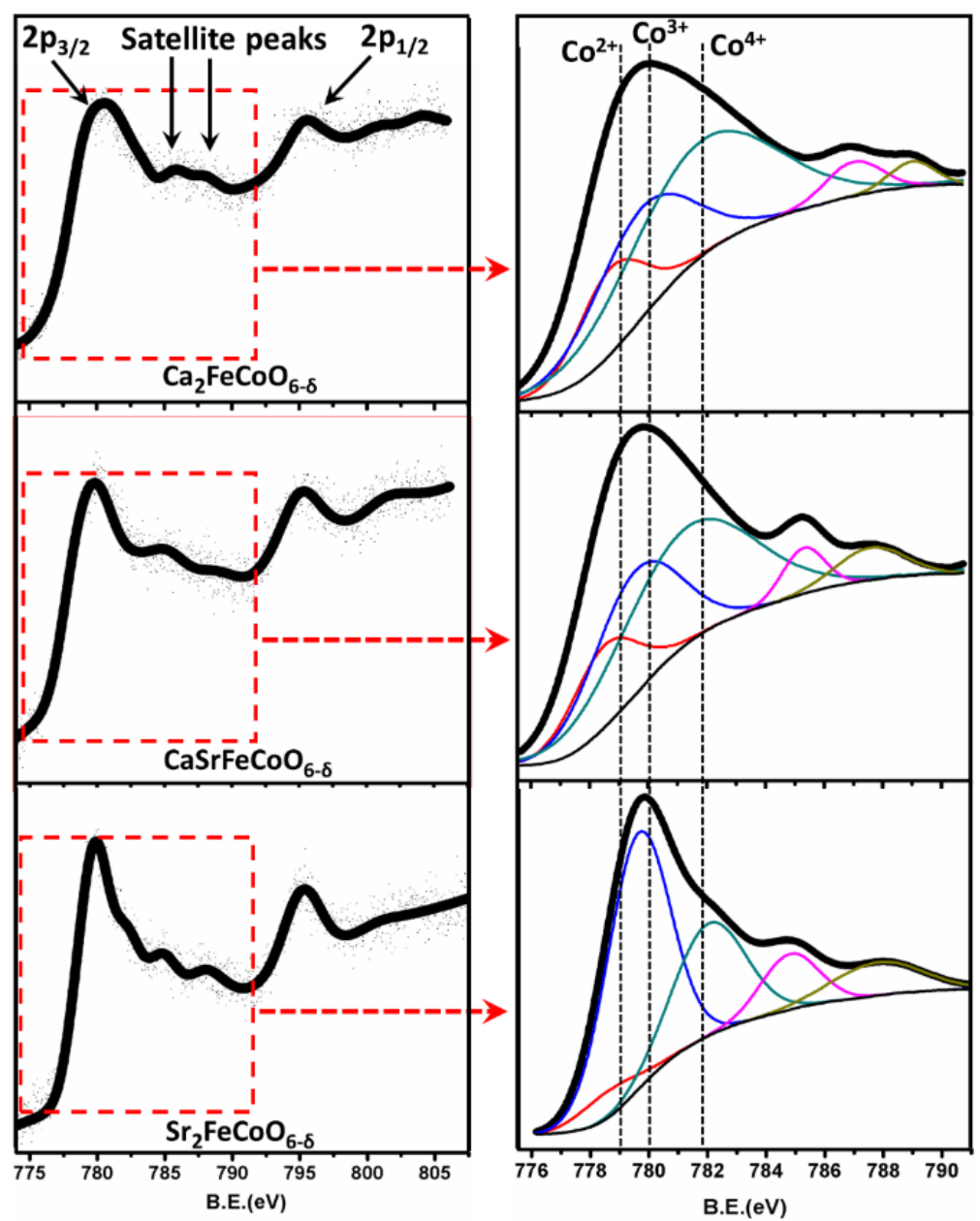

Figure 3.12. The cobalt XPS spectra for $\mathrm{Ca}_{2} \mathrm{FeCoO}_{6-\delta}, \mathrm{CaSrFeCoO}_{6-\delta}$ and $\mathrm{Sr}_{2} \mathrm{FeCoO}_{6-\delta}$

This satellite peak is especially pronounced in the $\mathrm{CaSr}$ and $\mathrm{Sr}_{2}$-materials. The second satellite peak belongs to $\mathrm{Co}^{3+}$, located at $\sim 8-9 \mathrm{eV}$ higher than the $2 \mathrm{p}_{3 / 2}$ peak, as expected for trivalent cobalt. ${ }^{31}$ Note that the relative binding energy of satellite peaks for different oxidation states of cobalt does not follow the same trend as the relative binding energy of the main $2 \mathrm{p}_{3 / 2}$ and $2 \mathrm{p}_{1 / 2}$ peaks, which have a different origin compared to the satellite peaks. The satellite peak for $\mathrm{Co}^{4+}$ appears at lower binding energy than that for $\mathrm{Co}^{3+} \cdot{ }^{31} \mathrm{In}$ general, some degree of cobalt oxidation is expected at high temperature in air. ${ }^{24}$ However, the above observations point to an interesting redox phenomenon in these materials, where 
further oxidation of cobalt becomes possible through the reduction of iron. In addition, it is remarkable that the disordered $\mathrm{Sr}_{2}$ compound lacks $\mathrm{Co}^{2+}$, while the ordered $\mathrm{Ca}_{2}$ and $\mathrm{CaSr}$ materials contain divalent cobalt. It appears that the disordered structure encourages the oxidation of $\mathrm{Co}^{2+}$ to $\mathrm{Co}^{3+}$.

\section{Electrical conductivity}

The electrical properties of these three materials were studied by DC and AC methods. The total conductivity was found by first obtaining the resistance from the intercept of the data with the real axis (Z') of the Nyquist plot at high frequency in the AC method. Similar values were obtained using DC method by applying constant voltage or current and measuring the output current or voltage. The resistance values obtained using the above methods, can be used to calculate the conductivity $(\sigma)$ using the following equation:

$$
\sigma=\mathrm{L} / \mathrm{RA}
$$

where $\mathrm{L}$ and $\mathrm{A}$ represent the thickness and cross sectional area of the cylindrical pellet, respectively. The electrical conductivity values for the three compounds, $\mathrm{Ca}_{2} \mathrm{FeCoO}_{6-\delta}$, $\mathrm{CaSrFCoO}_{6-\delta}$ and $\mathrm{Sr}_{2} \mathrm{FeCoO}_{6-\delta}$ were obtained by $\mathrm{AC}$ method at room temperature and by DC method from 298 to $1073 \mathrm{~K}$. The room temperature conductivity values are listed in Table 3.6. These results show the order of the total conductivity for the three compounds at room temperature:

$$
\mathrm{Ca}_{2} \mathrm{FeCoO}_{6-\delta}<\mathrm{CaSrFeCoO}_{6-\delta}<\mathrm{Sr}_{2} \mathrm{FeCoO}_{6-\delta}
$$

The electrical conductivity increases as the $\mathrm{Sr}$ content and structural disorder increase. Note that the $\mathrm{Sr}_{2}$ compound is the most disordered phase, where oxygen vacancies have random distribution. The $\mathrm{Ca}_{2}$ material is the most ordered phase featuring vacancy order and also tetrahedral chain order. The bond angles may play a role as well. It has been shown that 
changes in electrical conductivity correlate with changes in bond lengths and angles. ${ }^{34-35}$ Some researchers have used density-functional theory calculations to show that when the $\mathrm{Co}-\mathrm{O}-\mathrm{Co}$ bond angles in $\mathrm{La}_{1-\mathrm{x}} \mathrm{Sr}_{\mathrm{x}} \mathrm{CoO}_{3}$ get closer to $180^{\circ}$, the overlap between the unoccupied cobalt $3 \mathrm{~d}$ conduction band and the occupied oxygen $2 \mathrm{p}$ valence band is enhanced, leading to improvement in electrical conductivity. ${ }^{36}$ Metallic conductivity can be obtained due to the formation of a hybrid band as a result of the enhanced overlap between metal $3 \mathrm{~d}$ and oxygen $2 \mathrm{p}$ bands. ${ }^{36-38} \mathrm{~A}$ correlation between conductivity and bond angle is also observed when the $\mathrm{x}$-value is varied in $\mathrm{La}_{1-\mathrm{x}} \mathrm{Sr}_{\mathrm{x}} \mathrm{CoO}_{3} \cdot{ }^{34-35} \mathrm{~A}$ change from semiconductivity to metallic conductivity is observed at $x=0.25$. This change has been explained by the introduction of doped states within the band gap and broadening of these states into a band as $\mathrm{x}$ increases, which leads to band overlap and transition from semiconductor to metal. It has been observed that at around $\mathrm{x}=0.25$, where this transition occurs, there is an abrupt increase in the $\mathrm{Co}-\mathrm{O}-\mathrm{Co}$ bond angle. ${ }^{34-35}$ For our materials, the $\mathrm{Fe}(\mathrm{Co})-\mathrm{O}-\mathrm{Fe}(\mathrm{Co})$ bond angles in the $\mathrm{Sr}_{2}$ compound are $180^{\circ}$, while those angles in the $\mathrm{Ca}_{2}$ material can be as small as $\sim 123^{\circ} .{ }^{20}$ The small angles are a consequence of the vacancyordered structure, where each tetrahedron shares corners with two tetrahedra as well as two octahedra. In order for the structure to accommodate these simultaneous corner-sharing, the bond angles distort from the ideal perovskite angle $\left(180^{\circ}\right)$. Large bond angles in the $\mathrm{Sr}_{2}$ compound and smaller angles in the $\mathrm{Ca}_{2}$ material correlate well with the relative conductivity of these materials at room temperature.

To obtain a more in-depth understanding of the electrical conductivity, variable temperature studies were performed on all three materials. Figure 3.13 shows the conductivity of the three samples during heating and cooling cycles in the temperature 
range $298-1073 \mathrm{~K}$. The conductivity of $\mathrm{Ca}_{2} \mathrm{FeCoO}_{6-\delta}$ and $\mathrm{CaSrFeCoO}_{6-\delta}$ increases with temperature indicating the semiconducting nature of these materials. However, the conductivity of $\mathrm{Sr}_{2} \mathrm{FeCoO}_{6-\delta}$ decreases as the temperature increases, exhibiting metallic behavior.

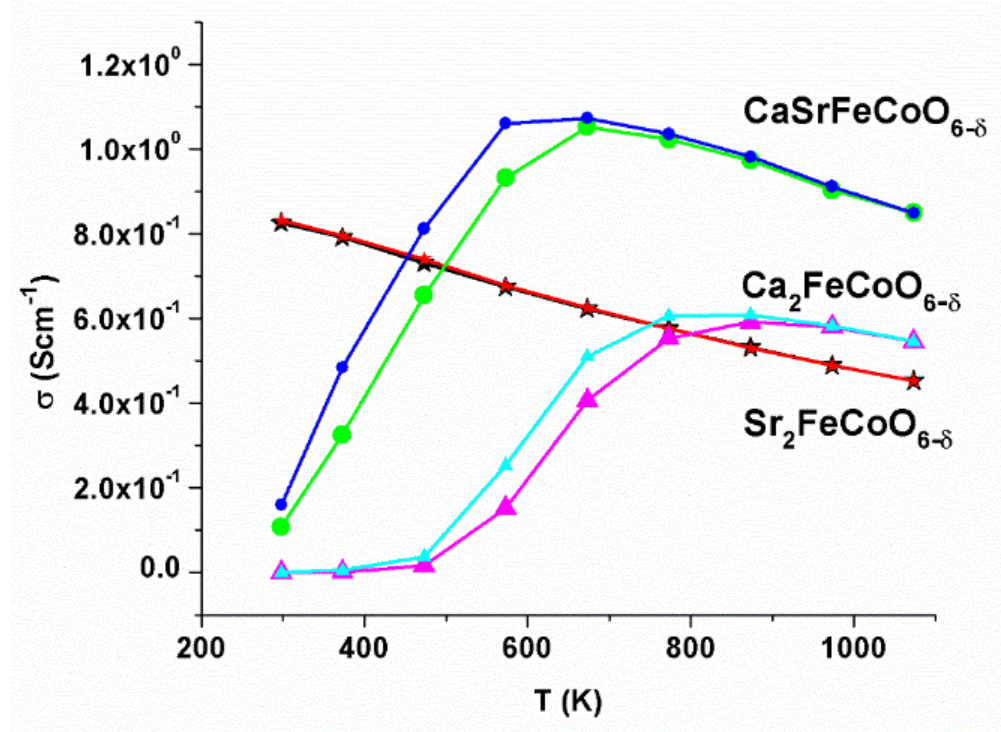

Figure 3.13. Total conductivity of $\mathrm{Sr}_{2} \mathrm{FeCoO}_{6-\delta}, \mathrm{CaSrFeCoO}_{6-\delta}$ and $\mathrm{Ca}_{2} \mathrm{FeCoO}_{6-\delta}$ as a function of temperature. For $\mathrm{Sr}_{2} \mathrm{Fe}_{2} \mathrm{O}_{6-\delta}$, the heating (red) and cooling (black) data overlap. For $\mathrm{CaSrFeCoO}_{6-\delta}$, the heating data are shown in green and cooling data in blue. For $\mathrm{Ca}_{2} \mathrm{FeCoO}_{6-\delta}$, the heating and cooling data are shown in pink and cyan, respectively.

The semiconducting properties, observed in the $\mathrm{Ca}_{2}$ and $\mathrm{CaSr}$ compounds, have been observed for other oxygen-deficient perovskites (ODP) before. In general, when oxygen partial pressure is high, e.g., the oxygen partial pressure in air, the electronic conductivity is usually dominant in ODPs. ${ }^{39-41}$ In semiconducting ODPs, the dependency of conductivity on the oxygen partial pressure indicates the p-type semiconductivity. ${ }^{42-44}$ In these p-type semiconductors, the primary charge carriers are electron holes ${ }^{39-41,45-46}$ The 
holes are generated extrinsically due to the absorption of oxygen molecules on the surface, which is aided by the oxide vacancies present in the material. This leads to the following processes: $:^{40,45}$

$$
\begin{aligned}
& 1 / 2 \mathrm{O}_{2} \rightarrow \mathrm{O}^{2-}+2 \mathrm{~h}^{\bullet} \\
& \mathrm{M}^{3+}+\mathrm{h}^{\bullet} \rightarrow \mathrm{M}^{4+}
\end{aligned}
$$

The presence of a variable valence metal (M) is essential, as it leads to the formation of small polarons, and electronic transport through the $\mathrm{M}^{3+}-\mathrm{O}-\mathrm{M}^{4+}$ network. ${ }^{45}$ The polaronic conductivity in the $\mathrm{Ca}_{2}$ and $\mathrm{CaSr}$ compounds is a result of the structural properties, where structural distortions occur to accommodate the corner-sharing between octahedra and tetrahedra. These distortions lead to lattice polarization that promotes polaronic charge transport. ${ }^{45}$ The polaron mobility is enhanced by rising temperature. This temperatureactivated mobility results in an increase in total conductivity, ${ }^{47}$ which can be expressed by the equation

$$
\sigma=\mathrm{n} \text { e } \mu
$$

where $\sigma, \mathrm{n}$, e and $\mu$ are conductivity, concentration of electrons/holes, charge of electron and mobility of charge carriers, respectively.

The observed differences in the conductivity of the ordered $\mathrm{Ca}_{2}$ and $\mathrm{CaSr}$ materials compared to the disordered $\mathrm{Sr}_{2}$ compound are clearly related to the crystal structure, which seem to lead to different conduction mechanisms. The large bond angles $\left(180^{\circ}\right)$ in the $\mathrm{Sr}_{2}$ compound lead to the enhancement of overlap between metal $3 \mathrm{~d}$ and oxygen $2 \mathrm{p}$ orbitals, leading to metallic conductivity, similar to the situation observed in some other materials, such as $\mathrm{La}_{1-\mathrm{x}} \mathrm{Sr}_{\mathrm{x}} \mathrm{CoO}_{3}{ }^{36}$ In the ordered $\mathrm{Ca}_{2}$ and $\mathrm{CaSr}$ compounds, the distorted angles do 
not allow for good orbital overlap and broadening of bands. The conduction in these materials is through polaronic mechanism, which is activated further at high temperature, leading to enhanced conductivity at elevated temperatures.

For the $\mathrm{Sr}_{2}$ compound, the decrease in electrical conductivity as a function of temperature occurs due to the increase in collisions between phonons and charge carriers as the temperature increases. ${ }^{37}$ In addition, for this material, the conductivity as a function of temperature has the same descending trend in the entire temperature range, $298 \mathrm{~K}-1073$ $\mathrm{K}$. Unlike the disordered $\mathrm{Sr}_{2}$ material, the ordered $\mathrm{Ca}_{2}$ and $\mathrm{CaSr}$ compounds exhibit a change in the conductivity trend at high temperature. For the $\mathrm{Ca}_{2}$ compound, the increase in conductivity continues up to $\sim 500{ }^{\circ} \mathrm{C}(773 \mathrm{~K})$, and then plateaus and even decreases slightly. Similar trend is observed for the $\mathrm{CaSr}$ compound, where the increase in conductivity continues up to $\sim 300{ }^{\circ} \mathrm{C}(573 \mathrm{~K})$ and then a plateau and decrease in conductivity is observed. The downturn in conductivity as a function of temperature is indicative of a metal-like conductivity, pointing to a semiconductor to metal transition. There appears to be a limit to the temperature-activated increase in the polaron mobility, beyond which the collisions between phonons and charge-carriers lead to decrease in conductivity and metal-like temperature-dependent behavior. It should be noted that the ionic conductivity is expected to increase at high temperature, due to some oxygen-loss and creation of more vacancies. However, the decrease in electrical conductivity is greater than the increase in ionic conductivity, leading to an overall decrease in total conductivity. 48 
A small degree of oxygen loss at high temperature has been observed through thermogravimetric analysis (TGA) of the CaSr compound in the temperature range, $25-$ $800{ }^{\circ} \mathrm{C}$. As shown in Figure 3.14, there is about $1 \%$ weight loss as a result of heating up to $800{ }^{\circ} \mathrm{C}$. More interestingly there is an inflection in the TGA data between $300-400{ }^{\circ} \mathrm{C}$, matching the temperature where the transition in electrical conductivity occurs.

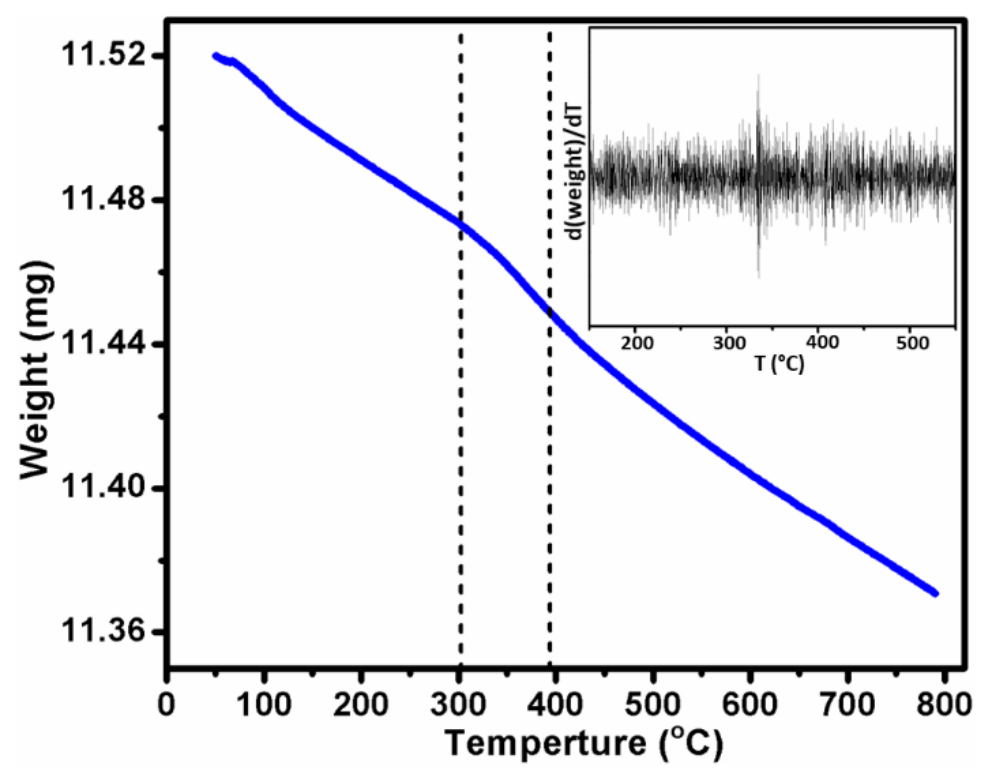

Figure 3.14. Thermogravimetric analysis of $\mathrm{CaSrFeCoO}_{6-\delta}$ in air. An inflection in the data appears in the temperature range between the dashed lines. The inset shows the derivative plot.

In the entire temperature range, the highest conductivity among the three materials is observed for the $\mathrm{CaSr}$-compound at $\sim 400{ }^{\circ} \mathrm{C}$. There appears to be an optimum degree of structural order that leads to the highest conductivity at high temperature. Both the disordered $\mathrm{Sr}_{2}$-compound and the highly ordered $\mathrm{Ca}_{2}$-material have lower conductivities at high temperature compared to the CaSr-compound. 
We also tested the possibility of any structural transition at high temperature. This was done by heating the $\mathrm{CaSr}$ compound to $800^{\circ} \mathrm{C}$, followed by quenching in liquid nitrogen. However, the XRD data showed no changes in the crystal structure.

Another remarkable property was found when we examined the materials' conductivity during both heating and cooling cycles. For the $\mathrm{Sr}_{2}$ compound, the conductivity values during both heating and cooling cycles were the same. However, for the $\mathrm{Ca}_{2}$ and $\mathrm{CaSr}$ compounds, the conductivity was greater during the cooling cycle. The observation of these hystereses confirms the contribution of ionic conductivity to the total conductivity as described for other mixed ionic-electronic conductors. ${ }^{49-50}$ The oxygen absorption/desorption phenomena are relatively slow compared to the electronic transport processes. The oxygen vacancies created at high temperature are not immediately filled when the temperature is lowered. Therefore, the high ionic conductivity persists at lower temperatures, leading to an increase in total conductivity compared to the values obtained during heating. Interestingly, the divergence between the heating and cooling data is only observed below the transition temperature, i.e., in the semiconducting region, for $\mathrm{Ca}_{2}$ and $\mathrm{CaSr}$ compounds. However, above the transition temperature, where the $\mathrm{Ca}_{2}$ and $\mathrm{CaSr}$ materials exhibit metallic behavior, the heating and cooling data overlap. This overlap between heating and cooling data is the same behavior observed for the metallic $\mathrm{Sr}_{2}$ material in the entire temperature range, $298-1073 \mathrm{~K}$.

The Arrhenius equation can be applied to the variation of conductivity with temperature, from which activation energy for conductivity can be obtained. ${ }^{45}$ Figure 3.15 shows the Arrhenius plot for conductivity in the temperature range, $298-1073 \mathrm{~K}$. 


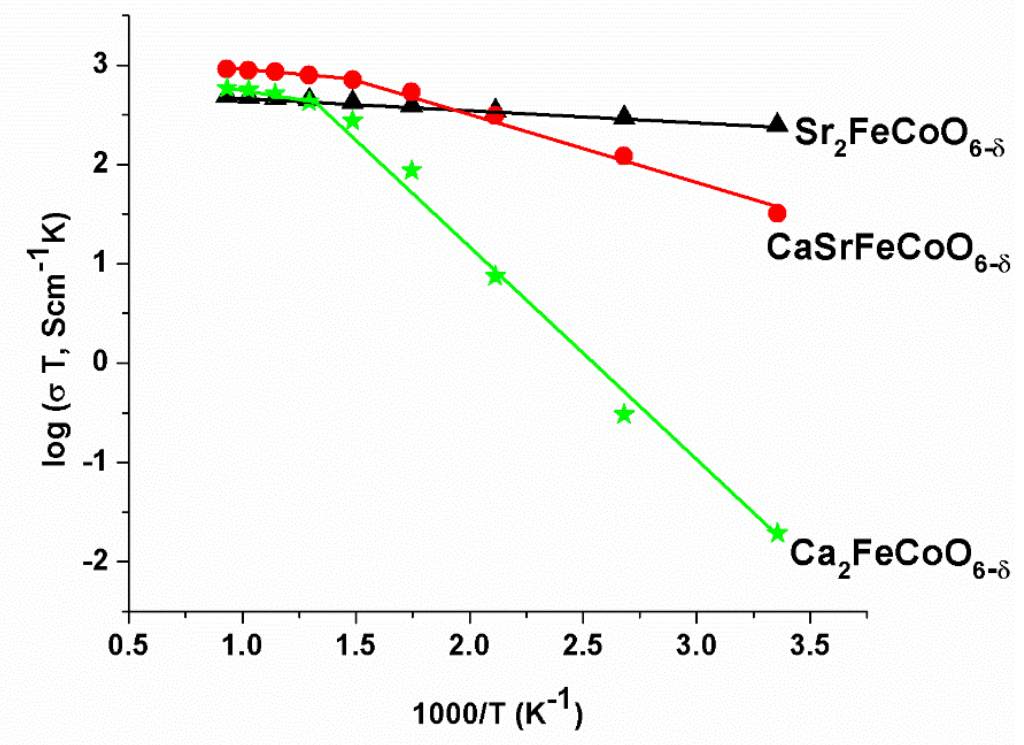

Figure 3.15. Arrhenius plot of the total conductivity for $\mathrm{Sr}_{2} \mathrm{FeCoO}_{6-\delta}$ (black triangles), $\mathrm{CaSrFe}_{2} \mathrm{O}_{6-\delta}$ (red circles) and $\mathrm{Ca}_{2} \mathrm{Fe}_{2} \mathrm{O}_{6-\delta}$ (green stars).

The straight lines represent the fit using the Arrhenius equation for thermally activated conductivity : $:^{47} 51-52$

$$
\sigma \mathrm{T}=\sigma^{\circ} \mathrm{e}^{\frac{-E_{a}}{\mathrm{KT}}}
$$

where $\sigma^{\circ}$ is a pre-exponential factor and a characteristic of a material. $E_{a}, K$ and $T$ are the activation energy for the electrical conductivity, Boltzmann constant and absolute temperature, respectively. The activation energy $\left(E_{a}\right)$ can be calculated from the slope of the line of best fit in the $\log \sigma T$ vs $1000 / T$ plot. These values are listed in Table 3.6. As evident from Figure 3.15 and Table 3.6, the slope of the Arrhenius plot changes at high temperature for the $\mathrm{Ca}_{2}$ and $\mathrm{CaSr}$ compounds, consistent with the change in the conductivity trend. This leads to two different $E_{a}$ values, where the activation energy at high temperature is smaller than that at low temperature for both $\mathrm{Ca}_{2}$ and $\mathrm{CaSr}$ compounds. 
Table 3.6. Room temperature conductivity and activation energies

\begin{tabular}{llll}
\hline \multirow{2}{*}{$\begin{array}{l}\text { Sample } \\
\end{array}$} & \multicolumn{2}{l}{$\begin{array}{l}\text { Total conductivity at room } \\
\text { temperature }\left(\mathrm{Scm}^{-1}\right)\end{array}$} & $\begin{array}{l}\text { Activation energy of total } \\
\text { conductivity }\left(\mathrm{E}_{\mathrm{a}}\right) \mathrm{in} \mathrm{eV}\end{array}$ \\
\cline { 2 - 3 } & $\mathrm{AC}$ & $\mathrm{DC}$ & \\
\hline $\mathrm{Ca}_{2} \mathrm{FeCoO}_{6-\delta}$ & $6.5 \times 10^{-5}$ & $6.49 \times 10^{-5}$ & $\begin{array}{l}298 \mathrm{~K}-800 \mathrm{~K}: 0.2225 \\
800 \mathrm{~K}-1073 \mathrm{~K}: 0.2587\end{array}$ \\
$\mathrm{CaSrFeCoO}_{6-\delta}$ & $1.184 \times 10^{-1}$ & $1.077 \times 10^{-1}$ & $298 \mathrm{~K}-600 \mathrm{~K}: 0.72365$ \\
$\mathrm{Sr}_{2} \mathrm{FeCoO}_{6-\delta}$ & $8.270 \times 10^{-1}$ & $8.256 \times 10^{-1}$ & $600 \mathrm{~K}-1073 \mathrm{~K}: 0.16669$ \\
\hline
\end{tabular}

\section{CONCLUSION}

Considering the importance of solid-state oxides in energy industry, especially for the development of advanced fuel cells, the investigation of methods to tune and control their electrical conductivity is essential. In this article, the correlation between electrical conductivity and structural order has been studied in a series of oxygen-deficient perovskites. Our findings indicate that, transition from disordered to ordered systems can be controlled by controlling the average ionic radius of the A-site cation. The conductivity at room temperature has an inverse correlation with ordering of oxygen vacancies. Furthermore, the least ordered compound exhibits metallic behavior, while the ordered materials are semiconductors. In addition, it appears that high temperature conductivity requires an intermediate level of vacancy-order. At high temperature, the highly ordered and highly disordered systems do not conduct as well as a material with an intermediate degree of ordering. Additionally, structural order leads to a semiconductor-to-metal transition at high temperature, which is absent in the disordered compound. Another effect of ordering is the mixed ionic-electronic conductivity manifested in hysteresis in 
conductivity data during heating and cooling cycles. These findings indicate that electrical properties of oxygen-deficient perovskites can be tuned by modifying the structural order in these materials. 


\section{CHAPTER 4}

\section{MAGNETIC STRUCTURE OF CaSrFeCoO 5 : CORRELATION WITH STRUCTURAL}

\section{ORDER $^{3}$}

\section{INTRODUCTION}

The study of magnetic properties and materials that exhibit various types of magnetism is motivated by scientific curiosity as well as the important applications of magnetic materials in various areas, such as spintronics and magnetic memories. ${ }^{67-69}$ Among magnetic systems, solid-state oxides are particularly fascinating due to the diversity of properties observed in this series of compounds. Oxygen acts as an effective bridge for magnetic coupling, leading to strong magnetic interactions in many transition-metal oxides. Perovskite-type systems, with general formula $\mathrm{ABO}_{3}$, are especially interesting, where $\mathrm{A}$ is usually an alkaline-earth metal or lanthanide and B is usually a transition metal. (Figure 4.1) The large A cations are located in spaces between corner-sharing $\mathrm{BO}_{6}$ octahedra. It is possible to form perovskite materials with some degree of oxygen-deficiency. The vacant sites that are created as a result of oxygen-deficiency can spread in the structure arbitrarily,

${ }^{3}$ The work described in this chapter was published in Material Research Bulletin ( 2018, vol. 106, p. 131-136) 
forming a disordered system. ${ }^{11,70} \mathrm{An}$ example is $\mathrm{Sr}_{2} \mathrm{FeMnO}_{5},{ }^{11}$ where oxide vacancies are distributed randomly, forming a disordered cubic structure. ${ }^{11}$

However, it is also possible to form oxygen-deficient perovskites, in which vacancies have an ordered distribution. The ordering of vacant sites can lead to the formation of new structure-types derived from the perovskite structure. An example is $\mathrm{Ba}_{2} \mathrm{In}_{2} \mathrm{O}_{5},{ }^{71}$ in which the vacancies lead to the formation of tetrahedral coordination geometry in alternating layers. The layers, where the tetrahedral geometry appears, contain chains of cornersharing tetrahedra, that run parallel to the octahedral layers, as shown in Figure 4.1. This arrangement, which is derived from the perovskite system, is called brownmillerite-type structure. ${ }^{12,72-75}$ The magnetic properties of oxygen-deficient perovskites have a strong correlation with the arrangement of vacant sites and the structure of materials. If the material composition contains magnetic cations, the magnetic order usually occurs when structural order is present. ${ }^{72}$ The lack of structural order usually leads to the absence of magnetic order. ${ }^{72}$ However, there are exceptions, such as $\mathrm{Sr}_{2} \mathrm{Fe}_{1.5} \mathrm{Cr}_{0.5} \mathrm{O}_{5}{ }^{76}$ where the vacancies are spread randomly in the structure, forming a disordered system, but the magnetic order is still present, and a long-range antiferromagnetic order is observed. ${ }^{76}$

In this article, we describe the magnetic structure of $\mathrm{CaSrFeCoO}_{5},{ }^{77}$ determined using neutron diffraction experiments. The magnetic order has been discussed in the context of materials with similar compositions, to highlight the magnetic properties that are unique to this compound. 


\section{EXPERIMENTAL}

$\mathrm{CaSrFeCoO}_{5}$ was synthesized using $\mathrm{CaCO}_{3}, \mathrm{SrCO}_{3}, \mathrm{Fe}_{2} \mathrm{O}_{3}$, and $\mathrm{Co}_{3} \mathrm{O}_{4}$ as starting materials. The powders of the precursors were ground, thoroughly mixed, and then pelletized. The pellets were heated at $1000^{\circ} \mathrm{C}$ in air for 24 hours, followed by regrinding and refiring at $1200{ }^{\circ} \mathrm{C}$ and $1250{ }^{\circ} \mathrm{C}$, for 24 hours each. The same synthesis procedure was also used to synthesize the $\mathrm{Ca}_{2}$ analogue, $\mathrm{Ca}_{2} \mathrm{FeCoO}_{5}$ using $\mathrm{CaCO}_{3}, \mathrm{Fe}_{2} \mathrm{O}_{3}$, and $\mathrm{Co}_{3} \mathrm{O}_{4}$. Similarly, the $\mathrm{Sr}_{2}$ analogue was also synthesized under the same conditions.

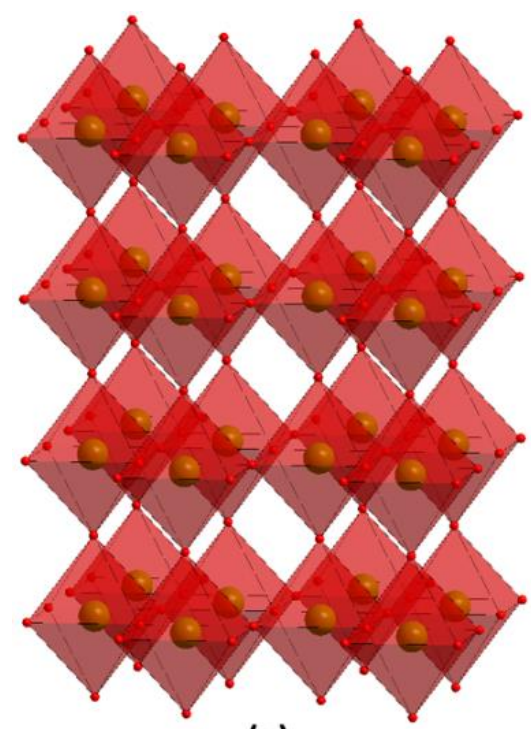

(a)

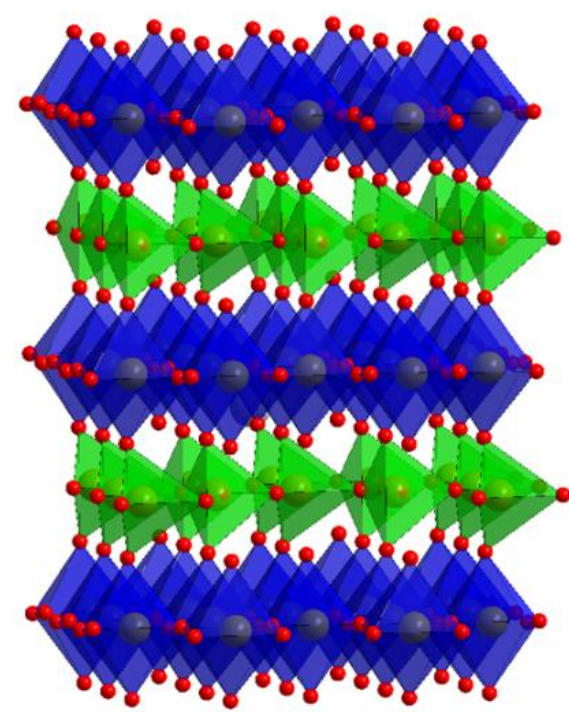

(b)

Figure 4.1. Comparison between (a) perovskite and (b) brownmillerite structure. In brownmillerite, the oxygen vacancies are ordered. The difference in transition metal coordination geometry is highlighted. The A-site cations that reside in spaces between polyhedra have been omitted for clarity.

Neutron diffraction experiments at $10 \mathrm{~K}$ and $300 \mathrm{~K}$ were performed on POWGEN diffractometer at Oak Ridge National Laboratory. The center wavelength of neutrons in 
these experiments was $1.333 \AA$, and the d-spacing range was $0.4142-6.1363 \AA$. Rietveld refinements were performed using GSAS program ${ }^{40}$ and EXPEGUI interface. ${ }^{41}$ Magnetic susceptibility data were obtained in the temperature range $2 \mathrm{~K}-400 \mathrm{~K}$, using the magnetic field of $0.1 \mathrm{~T}$.

\section{RESULTS AND DISCUSSION}

To determine the magnetic structure of $\mathrm{CaSrFeCoO}_{5}$, neutron diffraction experiments were performed at $10 \mathrm{~K}$. The neutron data showed strong magnetic peaks at $\mathrm{d}=4.42 \AA$ and 4.50 $\AA$, corresponding to 021 and 120 reflections. The presence of these peaks indicates longrange magnetic order.

The crystal structure at $10 \mathrm{~K}$ also features structural order, where the vacant sites, created due to oxygen deficiency, are ordered. The space group is $I b m 2$, and unit cell parameters are $a=5.5870(2) \AA, b=15.1684(6) \AA, c=5.4370(2) \AA$. This structure belongs to the brownmillerite family, (Figure 4.1) where chains of corner-sharing $(\mathrm{Fe} / \mathrm{Co}) \mathrm{O}_{4}$ tetrahedra are sandwiched between layers of corner-sharing $(\mathrm{Fe} / \mathrm{Co}) \mathrm{O}_{6}$ octahedra. The crystal structure at $10 \mathrm{~K}$ was confirmed by Rietveld refinements with neutron diffraction data as shown in Figure 4.2.

The magnetic structure is G-type antiferromagnetic, where the magnetic moment of each transition metal is aligned antiparallel to all nearest neighbors, as depicted in Figure 4.3. The magnetic and crystal structures are commensurate. In materials with brownmilleritetype structure, if the magnetic order occurs, the ratio of intensities of the main magnetic peaks is indicative of the direction of the magnetic moments. ${ }^{72,74}$ When the $(120) /(021)$ peak ratio is close to 1 , the magnetic moments are aligned along the longest unit cell axis. 
However, if that ratio is close to 3 , the magnetic moments are oriented along the shortest axis.

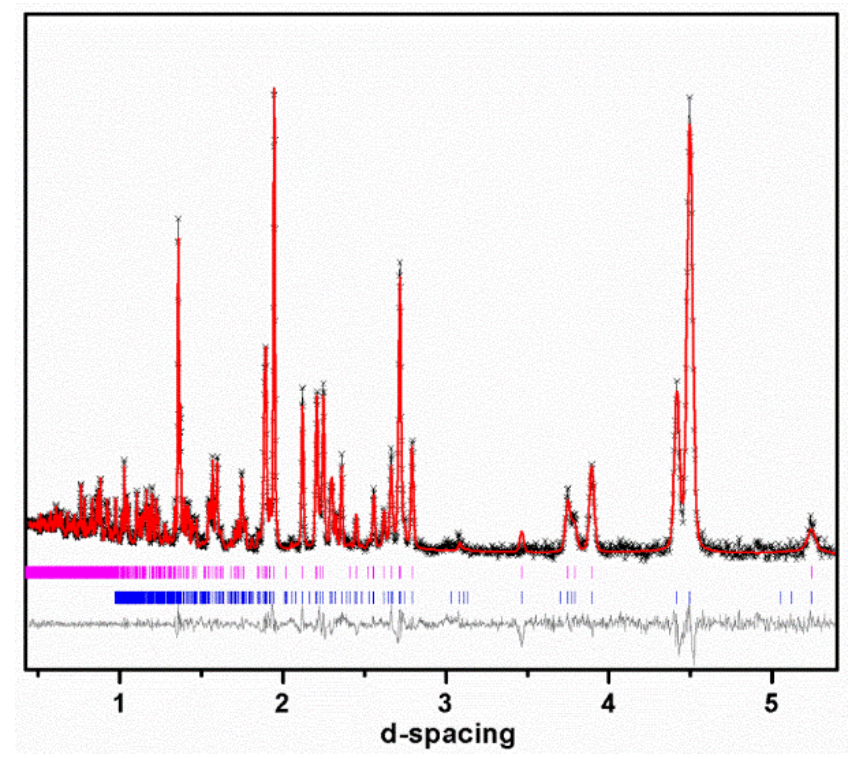

Figure 4.2. Neutron diffraction refinement profile at $10 \mathrm{~K}$ for $\mathrm{CaSrFeCoO}_{5}$. The crosses are experimental data, the red line is the model and the lower grey line represents the difference plot. The upper and lower vertical tick marks locate the crystal and magnetic structure peak positions.

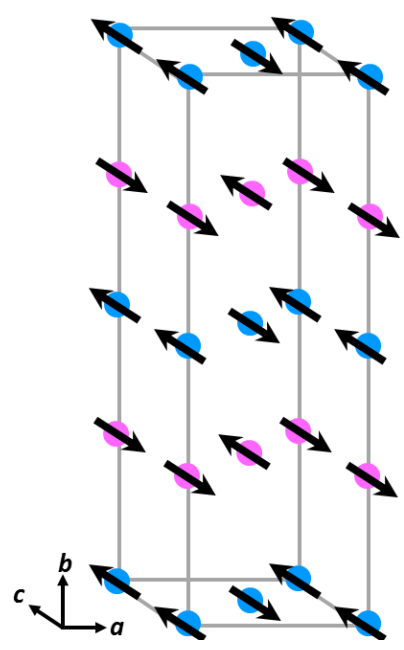

Figure 4.3. G-type antiferromagnetic order in $\mathrm{CaSrFeCoO}_{5}$ 
As demonstrated in Figure 4.4, Gaussian fits indicate that the $(120) /(021)$ peaks ratio for $\mathrm{CaSrFeCoO}_{5}$ is $\sim 2.96$, indicating that the magnetic moments should be along the shortest unit cell axis. We then confirmed the orientation of the moments by a series of Rietveld refinements. Initially, we compared three models, by orienting the magnetic moments along the three unit-cell axes and determining their fit to the data. Figure 4.5 shows three different situations where magnetic structure models with moments along the shortest, intermediate and longest unit cell axes were used. As observed here, the model featuring magnetic moments along the longest axis does not match the data, as it requires the two major magnetic peaks, 021 and 120, to have almost the same intensity. The model where magnetic moments are oriented along the intermediate axis is also ruled out, as it requires the magnetic peaks ratio to be $(120) /(021) \approx \frac{1}{3}$. (Figure 4.5$)$ However, the model with magnetic moments along the shortest axis, which requires a 3/1 ratio for $(120) /(021)$ intensities, leads to an excellent fit, as shown in Figure 4.5. The magnetic moments magnitude was also refined giving 3.6(2) $\mu_{\mathrm{B}}$ at $10 \mathrm{~K}$. There is a sharp contrast between the magnetic structure of $\mathrm{CaSrFeCoO}_{5}$ and that of the $\mathrm{Ca}$-analogue, $\mathrm{Ca}_{2} \mathrm{FeCoO}_{5}$, which has a magnetic structure with moments oriented along the longest axis at $10 \mathrm{~K} \cdot{ }^{74}$ The stark difference between the magnetic structures of the $\mathrm{CaSr}$ and $\mathrm{Ca}_{2}$ compounds is remarkable. The presence of $\mathrm{Sr}$ on the A-site appears to direct the magnetic moments along the shortest axis. In addition, the G-type antiferromagnetic order in $\mathrm{CaSrFeCoO}_{5}$ is retained up to room temperature, as demonstrated by neutron diffraction experiments at $300 \mathrm{~K}$, shown in Figure 4.6. The magnetic structure and orientation of magnetic moments remain unchanged compared to those at $10 \mathrm{~K}$. However, as expected, the magnetic moment magnitude at 300 $\mathrm{K}, 3.2(1) \mu_{\mathrm{B}}$, is smaller than that at $10 \mathrm{~K}, 3.6(2) \mu_{\mathrm{B}}$. 


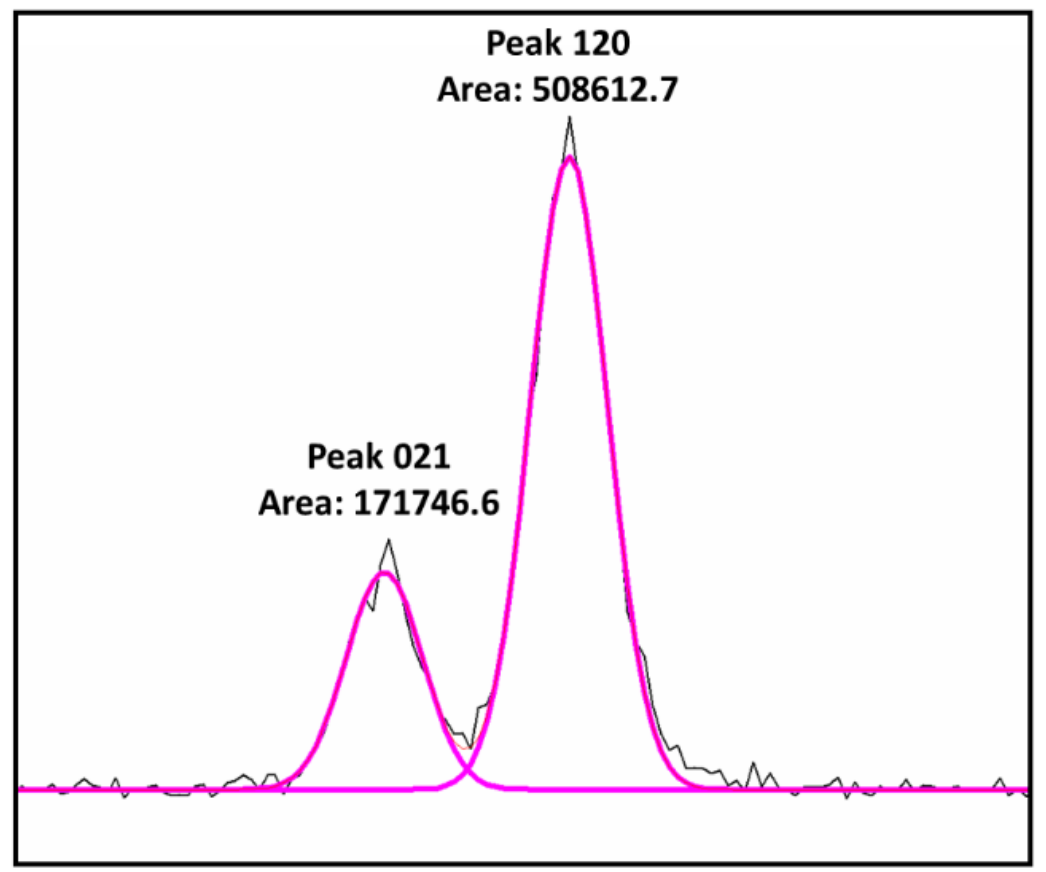

Figure 4.4. Gaussian fit for (021) and (120) magnetic peaks, indicating (120)/(021) ratio of $\sim 2.96$. 


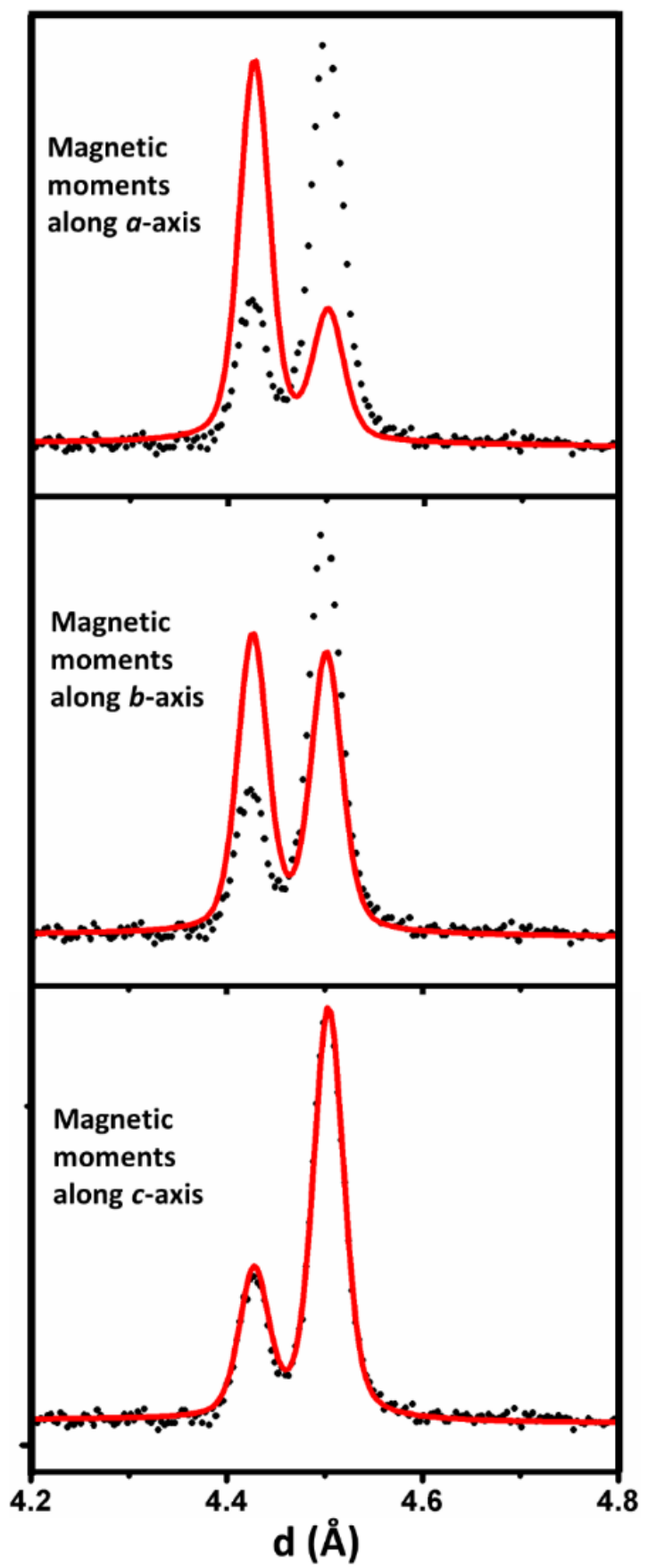

Figure 4.5. Fits for (021) and (120) magnetic peaks in neutron diffraction data using three different models with magnetic moments along $a$ (intermediate), $b$ (longest), or $c$ (shortest) axis. As shown here, the model with moments along the $c$-axis leads to an excellent fit. 
The neutron diffraction data at both $10 \mathrm{~K}$ and $300 \mathrm{~K}$ highlight the role of Sr in directing the magnetic moments in a particular orientation, i.e. along the shortest unit cell axis, regardless of the temperature. This demonstrates another significant difference compared to the $\mathrm{Ca}_{2}$ analogue. In $\mathrm{Ca}_{2} \mathrm{FeCoO}_{5}$, the direction of magnetic moments changes as temperature increases. ${ }^{74}$ The moments in the $\mathrm{Ca}_{2}$ compound are oriented along the longest unit axis at $10 \mathrm{~K}$, and along the shortest axis at $300 \mathrm{~K} .{ }^{74}$

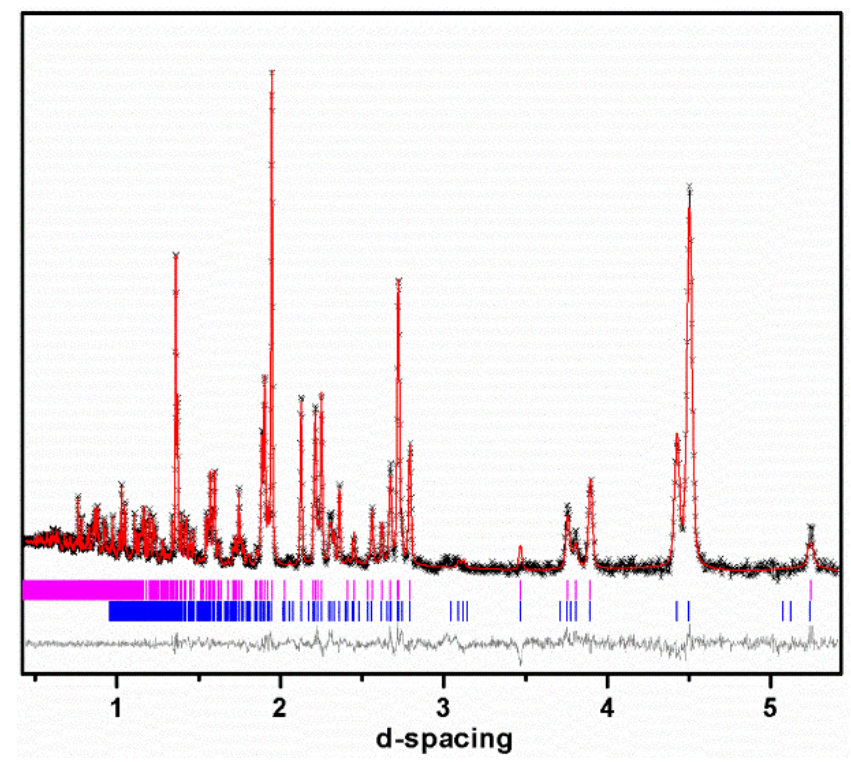

Figure 4.6. Neutron diffraction refinement profile at $300 \mathrm{~K}$ for $\mathrm{CaSrFeCoO}_{5}$. The crosses are experimental data, the red line is the model and the lower grey line represents the difference plot. The upper and lower vertical tick marks locate the crystal and magnetic structure peak positions.

A comparison to the $\mathrm{Mn}$ analogue $\mathrm{e}^{72}$ further highlights the interesting magnetic properties of $\mathrm{CaSrFeCoO}_{5}$. If a composition containing $\mathrm{Mn}$ instead of $\mathrm{Co}$ is synthesized under the same conditions, i.e., $1250{ }^{\circ} \mathrm{C}$ in air, a disordered structure is formed that lacks magnetic order. $^{72}$ This material undergoes a transition to a spin-glass state below $\sim 30 \mathrm{~K}$, and does 
not have any long-range magnetic order. ${ }^{72}$ This observation indicates that the presence of cobalt in the material composition encourages the G-type magnetic order along the shortest unit-cell axis in $\mathrm{CaSrFeCoO}_{5}$. However, the presence of cobalt is not the only factor governing the formation of this magnetic structure. An optimum A-site cation combination is also required, as evident from comparison to the $\mathrm{Ca}_{2}$ analogue. ${ }^{74}$ Therefore, $\mathrm{CaSrFeCoO}_{5}$ appears to be an optimum composition for this type of magnetic order to occur.

The magnetic susceptibility data were also obtained in the temperature range $2 \mathrm{~K}-400 \mathrm{~K}$, as demonstrated in Figure 4.7. $\mathrm{Ca}_{2} \mathrm{FeCoO}_{5}$ shows a divergence between zero-field-cooled (ZFC) and field-cooled (FC) data below $\sim 220 \mathrm{~K}$, as observed in Figure 4.7a. This temperature is where the re-orientation of magnetic moments in the $\mathrm{Ca}_{2}$ compound is completed, as described previously. ${ }^{74}$

For $\mathrm{CaSrFeCoO}_{5}$, there is divergence between the zero-field-cooled and field-cooled data below $\sim 100 \mathrm{~K}$, and the magnetic susceptibility trend for ZFC becomes opposite to that of FC data below $100 \mathrm{~K}$, down to $50 \mathrm{~K}$ (Figure 4.7b). Below $50 \mathrm{~K}$, the upward magnetic susceptibility trend is the same for both ZFC and FC data, but the separation between them persists. Both features at $50 \mathrm{~K}$ and $100 \mathrm{~K}$ are only observed in the ZFC data and corresponded to short-range domains. The material has long-range magnetic order at $10 \mathrm{~K}$ and $300 \mathrm{~K}$, as observed in neutron diffraction data.

To our knowledge the magnetic properties of the $\mathrm{Sr}_{2}$ analogue ${ }^{78}$ synthesized under the same condition as the $\mathrm{CaSr}$-compound, i.e., $1250{ }^{\circ} \mathrm{C}$ in air, has not been studied previously. We synthesized the $\mathrm{Sr}_{2}$ analogue, which has a cubic disordered strcuture ${ }^{78}$ and obtained magnetic 

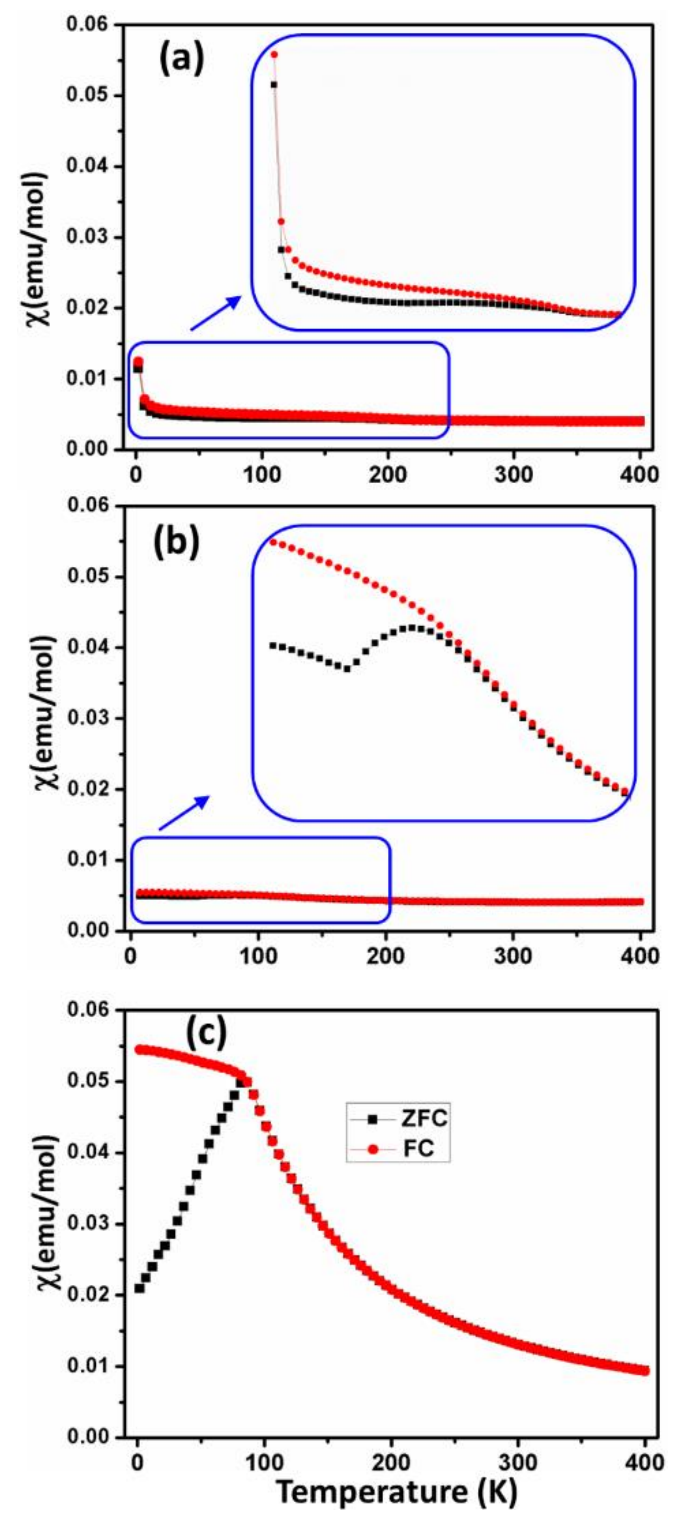

Figure 4.7. Magnetic susceptibility data for $\mathrm{CaSrFeCoO}_{5}$, as well as its $\mathrm{Ca}_{2}$ and $\mathrm{Sr}_{2}$ analogues. Images in a, b and c show the $\mathrm{Ca}_{2}, \mathrm{CaSr}$ and $\mathrm{Sr}_{2}$ compounds, respectively. Black squares show zero-field-cooled and red circles represent field-cooled data.

susceptibility data for this material as well. In the zero-field-cooled data, the magnetic susceptibility increases as the temperature decreases down to $\sim 80 \mathrm{~K}$, where a sudden downturn in susceptibility begins. The field-cooled data shows a different trend, where the slope of the susceptibility data changes drastically below $87 \mathrm{~K}$, but the upturn trend 
continue. This feature, demonstrated in Figure 4.7c, has been previously observed for some other oxygen-deficient perovskites. ${ }^{11,72}$ This is usually indicative of spin-glass magnetic behavior, where the disorder in magnetic moments is quenched. This observation further confirms the important effect of the A-site cation on magnetic properties, where changing $\mathrm{Ca}_{2}$ to $\mathrm{CaSr}$ and $\mathrm{Sr}_{2}$, leads to significant changes in magnetism of these material.

\section{CONCLUSION}

The magnetic structure of $\mathrm{CaSrFeCoO}_{5}$ consists of antiferromagnetically ordered moments that are oriented antiparallel to their nearest neighbors, as determined by neutron diffraction at $10 \mathrm{~K}$ and $300 \mathrm{~K}$. This ordering arrangement is the so-called G-type antiferromagnetic structure. The magnetic moment orientation is along the shortest unit cell axis. The G-type antiferromagnetic order persists up to room temperature and the orientation of magnetic moments also remains unchanged. Comparisons have been made between $\mathrm{CaSrFeCoO}_{5}$ and materials with similar compositions, where $\mathrm{CaSr}$ is replaced by $\mathrm{Ca}_{2}$ and $\mathrm{Sr}_{2}$, or $\mathrm{Co}$ is replaced by $\mathrm{Mn}$. These analogue materials show significantly different magnetic properties, from spin-glass to ordered moments that reorient if heated to room temperature. These observations indicate that $\mathrm{CaSrFeCoO}_{5}$ is an optimum composition for this G-type antiferromagnetic order, where the arrangement and orientation of magnetic moments remain unchanged up to room temperature. 


\section{CHAPTER 5}

\section{ELECTRICAL PROPERTIES OF ORDERED OXYGEN-DEFICIENT PEROVSKITE $\mathrm{Ca}_{2} \mathrm{Fe}_{0.5} \mathrm{Ga}_{1.5} \mathrm{O}_{5}^{4}$}

\section{INTRODUCTION}

Many oxygen-deficient perovskite oxides exhibit mixed ionic-electronic conductivity. ${ }^{23}$ Mixed conducting materials are important in different areas, such as gas sensing devices, ${ }^{79}$ electrodes for solid-oxide fuel cells ${ }^{80}$, and electrocatalysts. ${ }^{81}$

Oxygen deficient perovskites have general formula $\mathrm{ABO}_{3-\delta}$, where $\delta$ represents the oxygen deficiency. The $\mathrm{B}$-site cations usually form $\mathrm{BO}_{6}, \mathrm{BO}_{5}$, or $\mathrm{BO}_{4}$ polyhedra depending on the structure, while the A-site cations reside in spaces between the polyhedra. The crystal structure can vary depending on different parameters including the magnitude of $\delta$. For example, a series of structures have been observed for $\mathrm{SrMnO}_{3-\delta}{ }^{82}$ and $\mathrm{Sr}_{2} \mathrm{Fe}_{2} \mathrm{O}_{3-\delta}{ }^{83}$ due to the variation of oxygen stoichiometry. The crystal structure of oxygen-deficient perovskites also depends on the arrangement of defects that are generated due to oxygendeficiency. ${ }^{4,84-87}$ The defects can be distributed in a disordered ${ }^{76,88-89}$ or ordered fashion. ${ }^{4}$

${ }^{4}$ The work described in this chapter was published in IONICS ( 2019, vol. 25, p. 13151321) 
$12,73-75,84-87,90$ One of the common structures, resulting from the ordering of defects, is brownmillerite-type structure. ${ }^{4,12,73-75,84-87,90}$ In brownmillerite materials, the ordering of defects results in tetrahedral coordination geometry in alternating layers. As shown in Figure 5.1, the $\mathrm{BO}_{4}$ tetrahedra and $\mathrm{BO}_{6}$ octahedra alternate in the crystal lattice. The tetrahedra form chains that run parallel to the octahedral layers. The orientation of tetrahedral chains with respect to each other can be different, leading to different space groups in brownmillerite compounds.

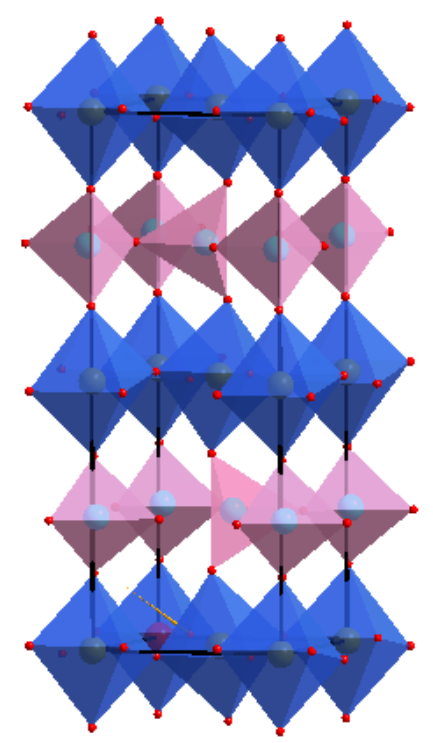

Figure 5.1. Brownmillerite structure of $\mathrm{Ca}_{2} \mathrm{Fe}_{0.5} \mathrm{Ga}_{1.5} \mathrm{O}_{5}$

Changes in the $\mathrm{A}$ or $\mathrm{B}$-site cations in oxygen-deficient perovskites, $\mathrm{ABO}_{3-\delta}$, can change the crystal structure. One example is the significant difference between $\mathrm{Sr}_{2} \mathrm{Fe}_{2} \mathrm{O}_{5}$, which has a brownmillerite-type structure, and $\mathrm{Ba}_{2} \mathrm{Fe}_{2} \mathrm{O}_{5}$, that features a complex structure containing tetrahedral, square-pyramidal and octahedral geometry. ${ }^{86}$ The change in the crystal structure leads to significant differences in electrical properties of $\mathrm{Sr}_{2} \mathrm{Fe}_{2} \mathrm{O}_{5}$ and $\mathrm{Ba}_{2} \mathrm{Fe}_{2} \mathrm{O}_{5} .{ }^{86}$ Another example is the phase transitions in $\mathrm{La}_{1-\mathrm{x}} \mathrm{Sr}_{\mathrm{x}} \mathrm{FeO}_{3-\delta}$ as a result of $\mathrm{Sr}-$ substitution on the A-site, leading to variation in physical properties. ${ }^{91}$ The effect of the B- 
site cation is demonstrated by phase transitions in $\mathrm{SrFe}_{1-\mathrm{x}} \mathrm{Nb}_{\mathrm{x}} \mathrm{O}_{3-\delta}(\mathrm{x}=0.05,0.1,0.2,0.3$, and 0.4) where an $I 4 / \mathrm{mmm}$ tetragonal structure transforms into a cubic structure with Pm$3 m$ space group. ${ }^{92}$ The same study also reported a structural transition between cubic and orthorhombic due to the variation of the B-site cation. ${ }^{92}$

Most oxygen-deficient perovskites contain transition metals on the B-site. However, main group metals, particularly those from group 13, can also be incorporated into these compounds, and occupy some of the B-sites. ${ }^{93-95}$ Nevertheless, in most cases, the majority of cations on the B-site are still transition metal cations. ${ }^{93-95}$ Brownmillerite materials, where the transition metals are eliminated from the structure, are interesting because they can exhibit predominantly ionic conductivity. Thus, they can be used in applications such as fuel cell electrolytes, where ionic conductivity is needed, but electronic conductivity is undesirable. One prominent example of a brownmillerite compound, which possesses only main group elements on the $\mathrm{B}$-site, is the In-containing material $\mathrm{Ba}_{2} \mathrm{In}_{2} \mathrm{O}_{5}{ }^{71}$ and its doped analogues. ${ }^{96-97}$ This material can be synthesized by solid-state method at high temperature. ${ }^{71}$ However, the situation is different for Ga-only brownmillerite compounds, which seem to require high pressure to from. ${ }^{98}$ For example, $\mathrm{Ca}_{2} \mathrm{Ga}_{2} \mathrm{O}_{5}$ has been made under $2.5 \mathrm{GPa}$ of pressure. ${ }^{98}$ In addition, $\mathrm{Sr}_{2} \mathrm{Ga}_{2} \mathrm{O}_{5}$ has been synthesized under $1.5 \mathrm{GPa}$ of pressure, but this material does not form a brownmillerite structure. ${ }^{99}$

There is one report on the crystal structure of $\mathrm{Ca}_{2} \mathrm{Fe}_{0.5} \mathrm{Ga}_{1.5} \mathrm{O}_{5}$, synthesized using standard solid-state synthesis method. ${ }^{98}$ However, that study only reports the crystal structure using X-ray diffraction, and no other information regarding properties of $\mathrm{Ca}_{2} \mathrm{Fe}_{0.5} \mathrm{Ga}_{1.5} \mathrm{O}_{5}$ has been reported. In the current study, we have shown that $\mathrm{Ca}_{2} \mathrm{Fe}_{0.5} \mathrm{Ga}_{1.5} \mathrm{O}_{5}$ represents the maximum level of Ga-doping in $\mathrm{Ca}_{2} \mathrm{Fe}_{2} \mathrm{O}_{5}$ system, which can be achieved by solid-state 
synthesis method. We have examined the possibility of magnetic order in $\mathrm{Ca}_{2} \mathrm{Fe}_{0.5} \mathrm{Ga}_{1.5} \mathrm{O}_{5}$ using neutron diffraction and bulk magnetometry, and have also studied electrical properties of this compound in a wide temperature range, $25-800{ }^{\circ} \mathrm{C}$.

\section{MATERIALS AND METHODS}

Solid-state synthesis method was employed to prepare $\mathrm{Ca}_{2} \mathrm{Fe}_{0.5} \mathrm{Ga}_{1.5} \mathrm{O}_{5}$. The powders of the precursors $\mathrm{CaCO}_{3}$ (Alfa Aesar, 99.95\%), $\mathrm{Fe}_{2} \mathrm{O}_{3}$ (Alfa Aesar, 99.998\%), and $\mathrm{Ga}_{2} \mathrm{O}_{3}$ (Sigma Aldrich, 99.99\%) were mixed, pressed into a pellet, and heated at $1000{ }^{\circ} \mathrm{C}$ for 24 $\mathrm{h}$ in air. The samples were then reground and refired at $1200{ }^{\circ} \mathrm{C}$ for $24 \mathrm{~h}$ in air, followed by slow cooling. The heating and cooling rates were $100{ }^{\circ} \mathrm{C} / \mathrm{h}$. Synthesis of compositions containing higher Ga-content was also attempted. However, single phase products could only be obtained at the maximum Ga-concentration of 1.5 per formula unit. Iodometric titrations were performed under argon atmosphere, as described previously. ${ }^{86}$

High resolution field-emission scanning electron microscopy (SEM) was used to study the micro-structure. X-ray photoelectron spectroscopy (XPS) was carries out at room temperature using $\mathrm{Al} \mathrm{K} \alpha$ radiation $(1486.7 \mathrm{eV})$. The electrical properties were investigated by direct-current (DC) and alternating-current (AC) on pellets that had been sintered at $1200{ }^{\circ} \mathrm{C}$. The AC electrochemical impedance spectroscopy measurements were performed in the frequency range of $0.1 \mathrm{~Hz}$ to $1 \mathrm{MHz}$ using a computer-controlled frequency response analyzer. The DC measurements were done by applying a constant voltage of $10 \mathrm{mV}$ and collecting the output current. Variable-temperature electrical conductivity measurements were carried out during both heating and cooling cycles with $10{ }^{\circ} \mathrm{C}$ intervals. At each measurement temperature, enough time was given for conductivity equilibrium to be achieved before moving to the next temperature. Bulk magnetization data were obtained 
on a vibrating-sample magnetometer using a quartz sample holder in the temperature range $2 \mathrm{~K}-400 \mathrm{~K}$. Powder X-ray diffraction measurements were done at room temperature using $\mathrm{Cu}$ K $\alpha 1$ radiation $(\lambda=1.54056 \AA)$. Neutron diffraction experiments were performed on POWGEN diffractometer at Oak Ridge National Laboratory, with center wavelength of 0.7 Å. Rietveld refinements were done using GSAS software ${ }^{100}$ and EXPEGUI interface. ${ }^{101}$

\section{RESULTS AND DISCUSSION}

Crystal structure

As discussed in the experimental section, $\mathrm{Ca}_{2} \mathrm{Fe}_{0.5} \mathrm{Ga}_{1.5} \mathrm{O}_{5}$ represents the highest degree of Ga-doping in the brownmillerite compound, $\mathrm{Ca}_{2} \mathrm{Fe}_{2} \mathrm{O}_{5}$, which can be achieved by solidstate synthesis method. Higher degree of Ga-doping is only possible if the synthesis is done in high pressure, $2.5 \mathrm{GPa} .{ }^{98}$ The only report on $\mathrm{Ca}_{2} \mathrm{Fe}_{0.5} \mathrm{Ga}_{1.5} \mathrm{O}_{5}$ describes X-ray diffraction data of this material, indicating Pcmn space group. ${ }^{102}$ Here, we study the structure using a combination of neutron and X-ray diffraction. The common space groups for brownmillerite compounds are Imma, Ima2, Pnma (Pcmn) and Pbcm. . $74-75,84,87^{-10}$

The $P b c m$ space group is recognized by the presence of superstructure peaks, given that materials crystalizing in this space group have unit cells that are double the size of those for typical brownmillerites. ${ }^{4}, 74-75,84,87$ These superstructure peaks do not appear in the diffraction data for $\mathrm{Ca}_{2} \mathrm{Fe}_{0.5} \mathrm{Ga}_{1.5} \mathrm{O}_{5}$, ruling out the Pbcm space group. The Pnma space group is identified by the presence of the 131 peak, which is absent in I-centered space

groups. ${ }^{4}$, 74-75, 84, 87 The powder X-ray and neutron diffraction data for $\mathrm{Ca}_{2} \mathrm{Fe}_{0.5} \mathrm{Ga}_{1.5} \mathrm{O}_{5}$ show the 131 peak, indicative of space group Pnma. This was then confirmed by Rietveld refinements. Table 5.1 lists the refined structural parameters, and Figure 5.2 shows the Xray and neutron refinement profiles. In these refinements, initially Fe and Ga were mixed 
on both octahedral and tetrahedral sites. Refining the site-occupancies on these sites indicates that $\mathrm{Fe}$ is exclusively located on the octahedral site, while Ga occupies all of the tetrahedral sites and half of the octahedral positions.

Table 5.1 The refined structural parameters for $\mathrm{Ca}_{2} \mathrm{Fe}_{0.5} \mathrm{Ga}_{1.5} \mathrm{O}_{5}$ from neutron diffraction. Space group: Pnma, $a=5.3669(2), b=14.6214(5), c=5.5927(2), R_{p}=0.0744, w R_{p}=$ 0.0479

\begin{tabular}{ccccccc}
\hline Elements & $\mathrm{x}$ & $\mathrm{y}$ & $\mathrm{z}$ & Multiplicity & Occupancy & $\mathrm{U}_{\text {iso }}$ \\
\hline $\mathrm{Ca}$ & $0.0076(6)$ & $0.1075(1)$ & $0.5281(3)$ & 8 & 1 & $0.0013(2)$ \\
$\mathrm{Ga} 1$ & $0.0463(4)$ & 0.25 & $0.0700(4)$ & 4 & 1 & $0.0025(3)$ \\
$\mathrm{Ga} 2$ & 0.0 & 0.0 & 0.0 & 4 & 0.5 & $0.0004(2)$ \\
$\mathrm{Fe}$ & 0.0 & 0.0 & 0.0 & 4 & 0.5 & $0.0004(2)$ \\
$\mathrm{O} 1$ & $0.3991(7)$ & 0.25 & $0.1279(6)$ & 4 & 1 & $0.0052(4)$ \\
$\mathrm{O} 2$ & $0.0193(5)$ & $0.6412(2)$ & $0.0710(4)$ & 8 & 1 & $0.0063(3)$ \\
$\mathrm{O} 3$ & $0.2513(6)$ & $0.0140(1)$ & $0.2489(7)$ & 8 & 1 & $0.0020(2)$ \\
\hline
\end{tabular}

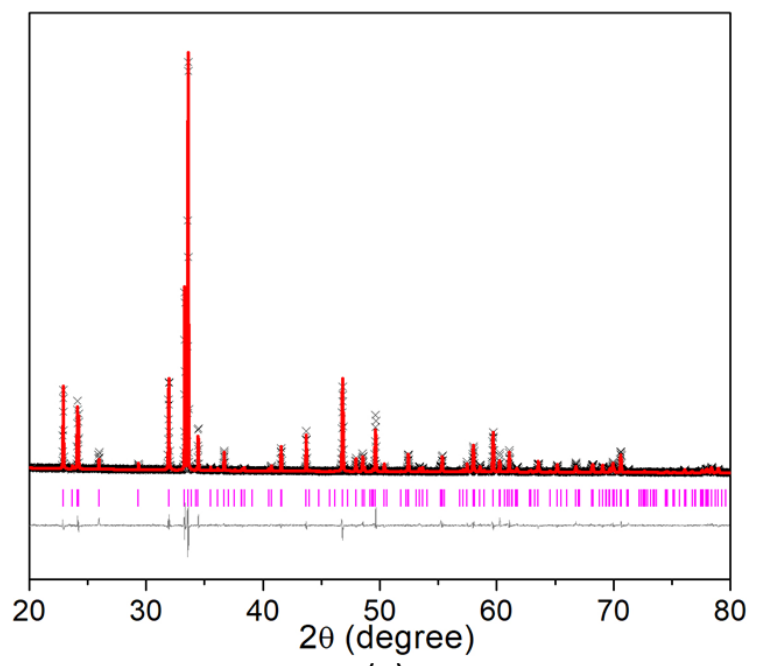

(a)

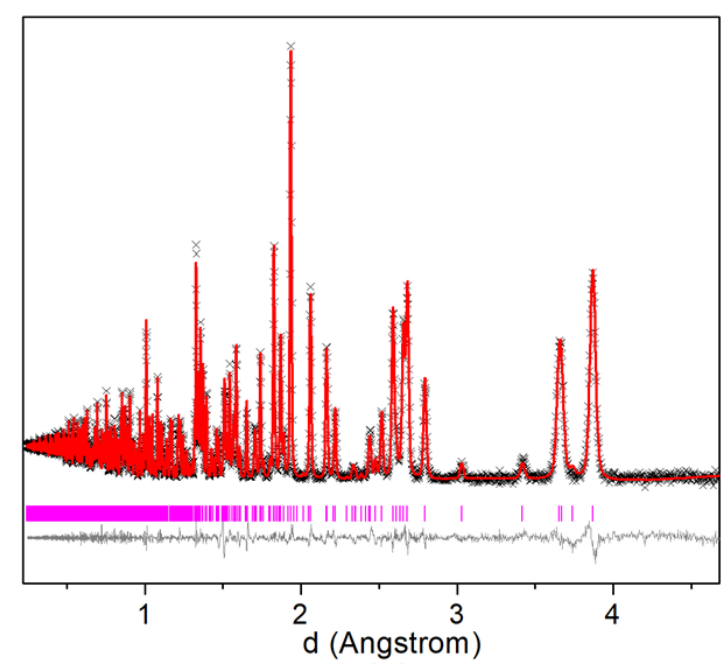

(b)

Figure 5.2 Refinement profiles for $\mathrm{Ca}_{2} \mathrm{Fe}_{0.5} \mathrm{Ga}_{1.5} \mathrm{O}_{5}$ using (a) $\mathrm{X}$-ray and (b) neutron diffraction 
Scanning electron microscopy data show high sinterability and good contact between crystallites of $\mathrm{Ca}_{2} \mathrm{Fe}_{0.5} \mathrm{Ga}_{1.5} \mathrm{O}_{5}$, as shown in Figure 5.3. Oxidation state of $\mathrm{Fe}$ was investigated by X-ray photoelectron spectroscopy (XPS). The $2 \mathrm{P}_{3 / 2}$ peaks for trivalent and tetravalent $\mathrm{Fe}$ are expected to appear at $\sim 710-711 \mathrm{eV}^{103-104}$ and $\sim 712-713 \mathrm{eV},{ }^{44,} 105$ respectively. In addition, a satellite peak appearing at about $7-9 \mathrm{eV}$ higher than the $2 \mathrm{P}_{3 / 2}$ peak is the signature of trivalent Fe. ${ }^{103-104}$ The XPS data for $\mathrm{Ca}_{2} \mathrm{Fe}_{0.5} \mathrm{Ga}_{1.5} \mathrm{O}_{5}$ (Figure 5.4) show the $2 \mathrm{P}_{3 / 2}$ peak for $\mathrm{Fe}$ at $\sim 710 \mathrm{eV}$ followed by a satellite peak at $\sim 718 \mathrm{eV}$, confirming that $\mathrm{Fe}$ is primarily in trivalent state. There is also a sight shoulder on the right side of the $\mathrm{Fe} 2 \mathrm{P}_{2 / 3}$ peak, which indicates the presence of a small amount of tetravalent $\mathrm{Fe}$. These findings were then confirmed by iodometric titrations, which showed the accurate oxygen stoichiometry to be 5.07 moles per formula unit, i.e., $\mathrm{Ca}_{2} \mathrm{Fe}_{0.5} \mathrm{Ga}_{1.5} \mathrm{O}_{5.07}$. These results confirm the XPS findings that the oxidation state of $\mathrm{Fe}$ is primarily $3+$, along with a small amount of tetravalent Fe.
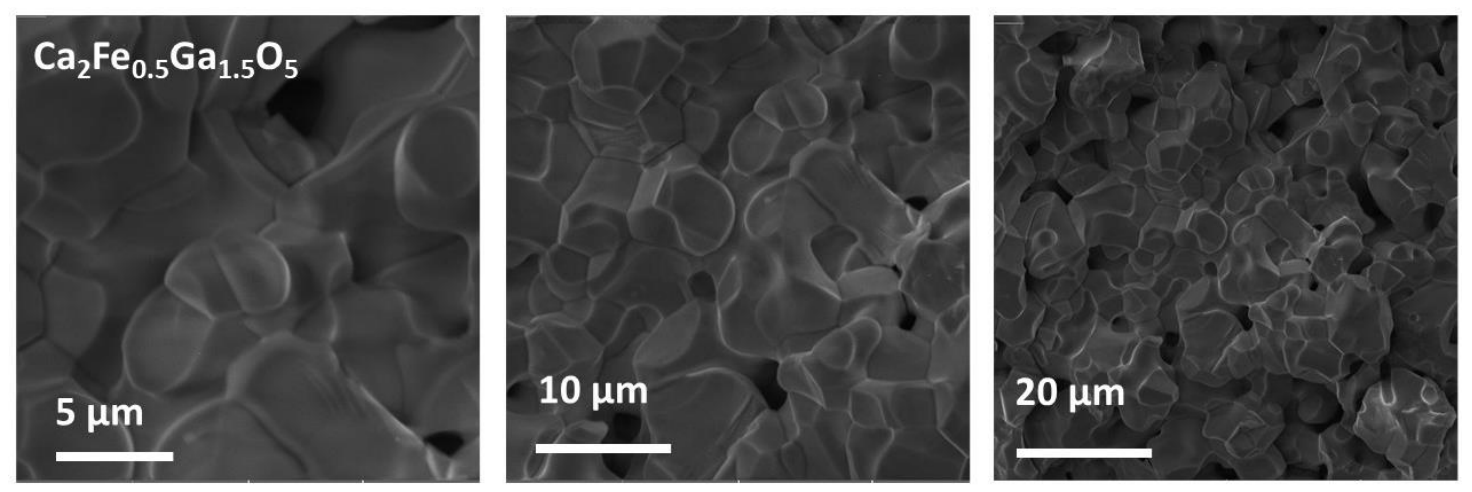

Figure 5.3 SEM images of $\mathrm{Ca}_{2} \mathrm{Fe}_{0.5} \mathrm{Ga}_{1.5} \mathrm{O}_{5}$, showing good contact between the crystallites 


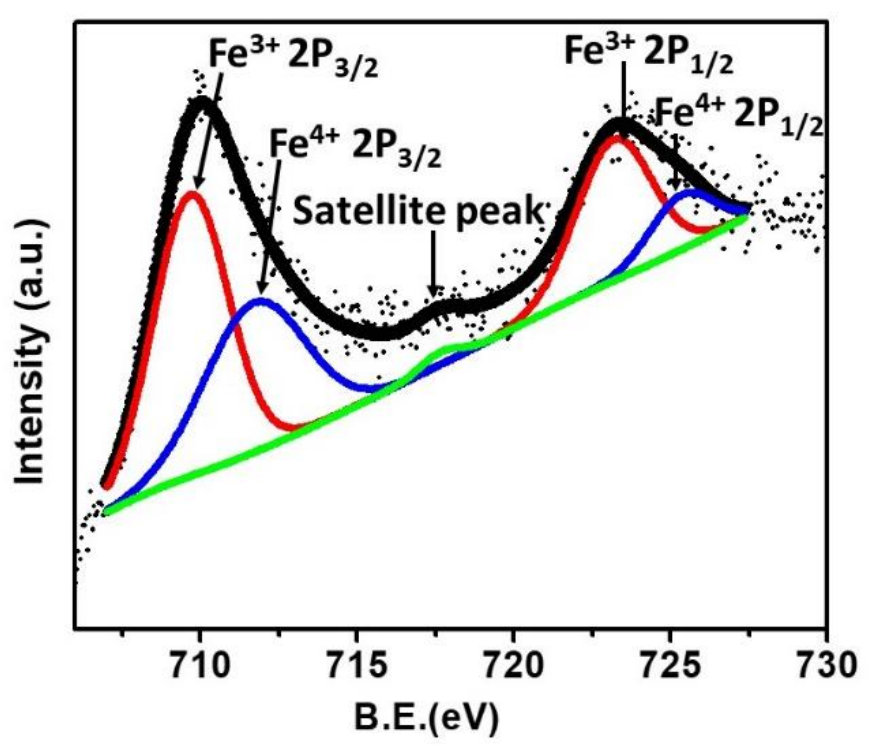

Figure 5.4 X-ray photoelectron spectroscopy data for $\mathrm{Ca}_{2} \mathrm{Fe}_{0.5} \mathrm{Ga}_{1.5} \mathrm{O}_{5}$

\section{Electrical properties}

The electrical properties of $\mathrm{Ca}_{2} \mathrm{Fe}_{0.5} \mathrm{Ga}_{1.5} \mathrm{O}_{5}$ were studied by alternating current (AC) and direct current (DC) methods. In DC method, the output current (I) is measured while applying a constant voltage. This current is then converted into resistance using Ohm's law. In AC impedance spectroscopy, the resistance is determined from the intercept of the data with the real axis $\left(Z^{\prime}\right)$ of the Nyquist plot at low frequency. The resistance values (R) are then used to calculate the conductivity $(\sigma)$ using the following equation:

$$
\sigma=L / R A
$$

where $\mathrm{L}$ and $\mathrm{A}$ represent the thickness and cross-sectional area of the sample, respectively. The electrical conductivity was also obtained at variable temperatures from 25 to $800{ }^{\circ} \mathrm{C}$ (Figure 5.5). In brownmillerite compounds, the electronic conductivity is achieved due to the presence of cations that have multiple stable oxidation states such as $\mathrm{Fe}^{2+} / \mathrm{Fe}^{3+} / \mathrm{Fe}^{4+}$. Variable oxidation states can also be created due to oxygen loss, i.e., partial reduction of 
cations, ${ }^{106}$ or oxygen absorption, leading to partial oxidation. ${ }^{50,107}$ These processes result in the formation of small polarons. ${ }^{50}$ The electrons hop through $\mathrm{M}^{\mathrm{m+}}-\mathrm{O}-\mathrm{M}^{\mathrm{n+}}$ conduction pathways, which are created due the presence

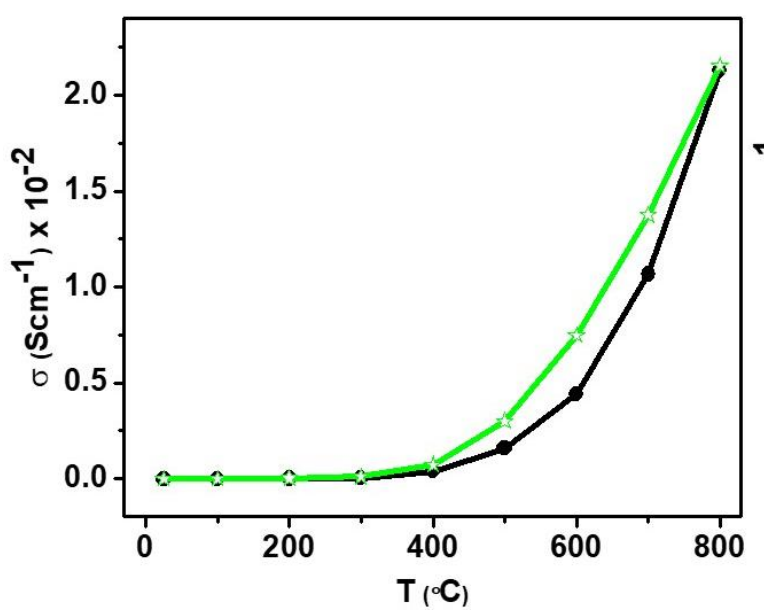

(a)

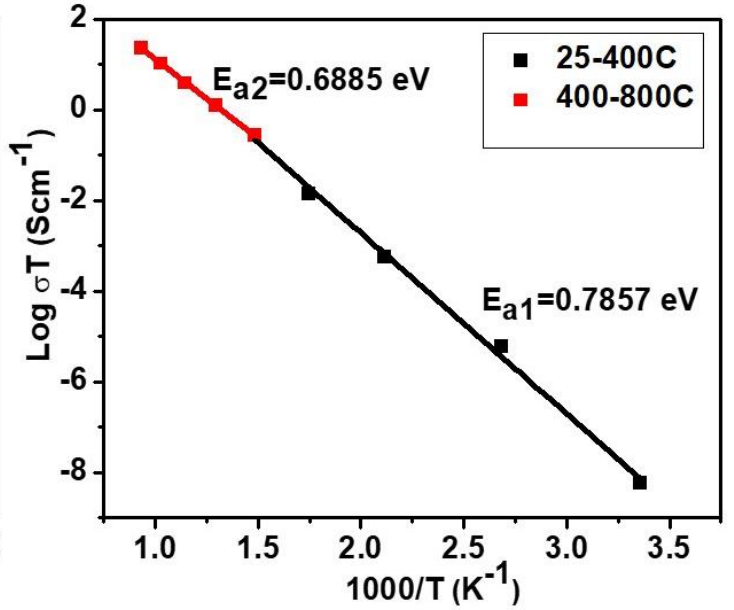

(b)

Figure 5.5 (a) Electrical conductivity of $\mathrm{Ca}_{2} \mathrm{Fe}_{0.5} \mathrm{Ga}_{1.5} \mathrm{O}_{5}$ as a function of temperature. (b) Arrhenius plot and activation energies for $\mathrm{Ca}_{2} \mathrm{Fe}_{0.5} \mathrm{Ga}_{1.5} \mathrm{O}_{5}$

variable oxidation states. ${ }^{108-109}$ However, many brownmillerite materials are mixedconductors, where the total conductivity includes contribution from both electron and oxide-ion conductivity. The latter can become dominant when the transition metals are eliminated from the material composition. ${ }^{71}$

In $\mathrm{Ca}_{2} \mathrm{Fe}_{0.5} \mathrm{Ga}_{1.5} \mathrm{O}_{5}$, contributions from both ionic and electronic conductivity are expected, considering the oxygen-vacancies and the presence of variable oxidation states of $\mathrm{Fe}$ in the material composition. The conductivity of $\mathrm{Ca}_{2} \mathrm{Fe}_{0.5} \mathrm{Ga}_{1.5} \mathrm{O}_{5}$, can be compared to a predominantly ionic conductor, $\mathrm{Ba}_{2} \operatorname{In}_{2} \mathrm{O}_{5}$, which shows electrical conductivity values, that are less than $\sim 10^{-3} \mathrm{Scm}^{-1}$ at $700{ }^{\circ} \mathrm{C}^{71}$ Whereas the conductivity of $\mathrm{Ca}_{2} \mathrm{Fe}_{0.5} \mathrm{Ga}_{1.5} \mathrm{O}_{5}$ at the same temperature is more than one order of magnitude higher, $\sim 10^{-2} \mathrm{Scm}^{-1}$. The higher 
conductivity of $\mathrm{Ca}_{2} \mathrm{Fe}_{0.5} \mathrm{Ga}_{1.5} \mathrm{O}_{5}$ may be attributed to the contribution of the electronic conductivity in addition to the ionic conductivity.

In oxide materials, the bulk, grain-boundary and electrode-interface resistance can be determined using AC impedance spectroscopy. The observation of semicircles in the Nyquist plot of impedance spectroscopy indicates significant contribution form ionic conductivity, as described by other researchers. ${ }^{110}$ The fit to the impedance data for $\mathrm{Ca}_{2} \mathrm{Fe}_{0.5} \mathrm{Ga}_{1.5} \mathrm{O}_{5}$ at $200{ }^{\circ} \mathrm{C}$ is shown in Figure 5.6. Three resistance-capacitance (RC) units were used for this fit, where the RC unit at highest frequency (left) corresponds to the bulk resistance. The semicircle in the middle shows the grain-boundary resistance and the semicircle at the lowest frequency (right) represents the electrode interface resistance.

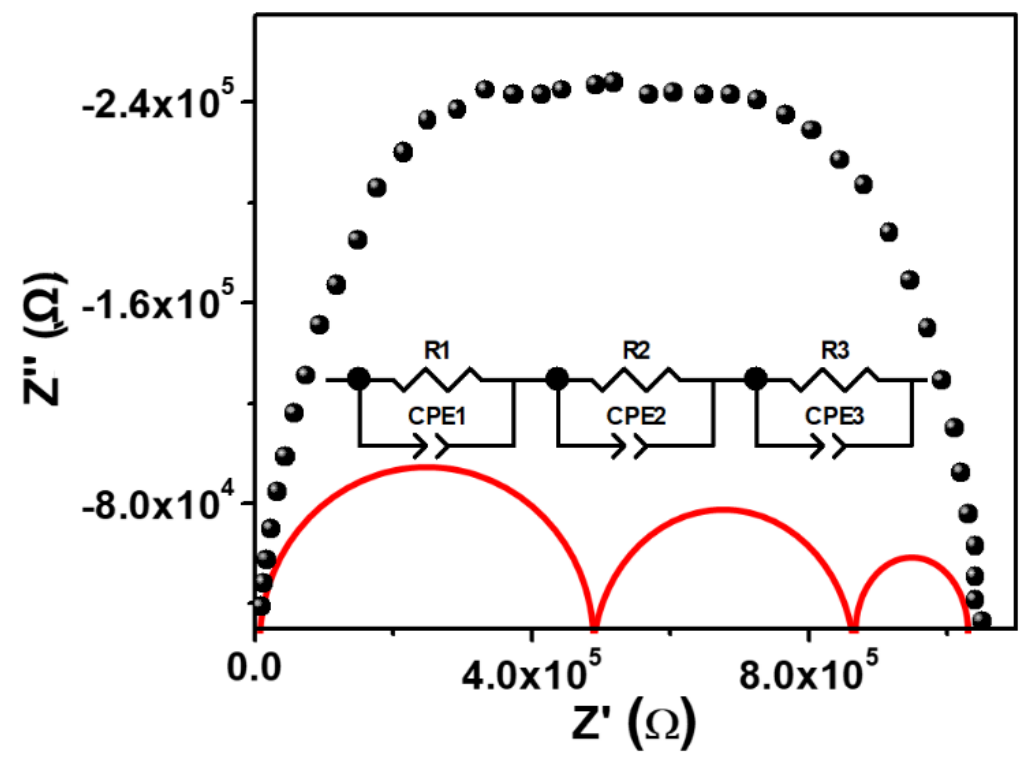

Figure 5.6 AC impedance data for $\mathrm{Ca}_{2} \mathrm{Fe}_{0.5} \mathrm{Ga}_{1.5} \mathrm{O}_{5}$ at $200^{\circ} \mathrm{C}$. Three resistance-capacitance units indicate the bulk (left), grain boundary (middle) and electrode interface (right) resistance. 
In some oxide materials, an increase in conductivity as a function of temperature is observed. This behavior is seen in $\mathrm{Ca}_{2} \mathrm{Fe}_{0.5} \mathrm{Ga}_{1.5} \mathrm{O}_{5}$. The temperature-dependent increase in electrical conductivity is expected, due to an increase in the mobility of charge carriers, ${ }^{9}$ according to the following relation:

$$
\sigma=\text { ne } \mu
$$

where $\sigma, \mathrm{n}, \mathrm{e}$, and $\mu$ are the conductivity, concentration of charge carriers, charge of electron, and mobility of the charge carriers, respectively. In addition, the loss of oxygen at higher temperature, as shown by thermogravimetric analysis (Figure 5.7), can enhance the ionic conductivity, due to the increase in concentration of defects. It is known that oxide ion conductivity is enhanced above $500{ }^{\circ} \mathrm{C} .{ }^{111-112}$ Figure 5.6 shows a sharp increase in conductivity of $\mathrm{Ca}_{2} \mathrm{Fe}_{0.5} \mathrm{Ga}_{1.5} \mathrm{O}_{5}$ above $500{ }^{\circ} \mathrm{C}$.

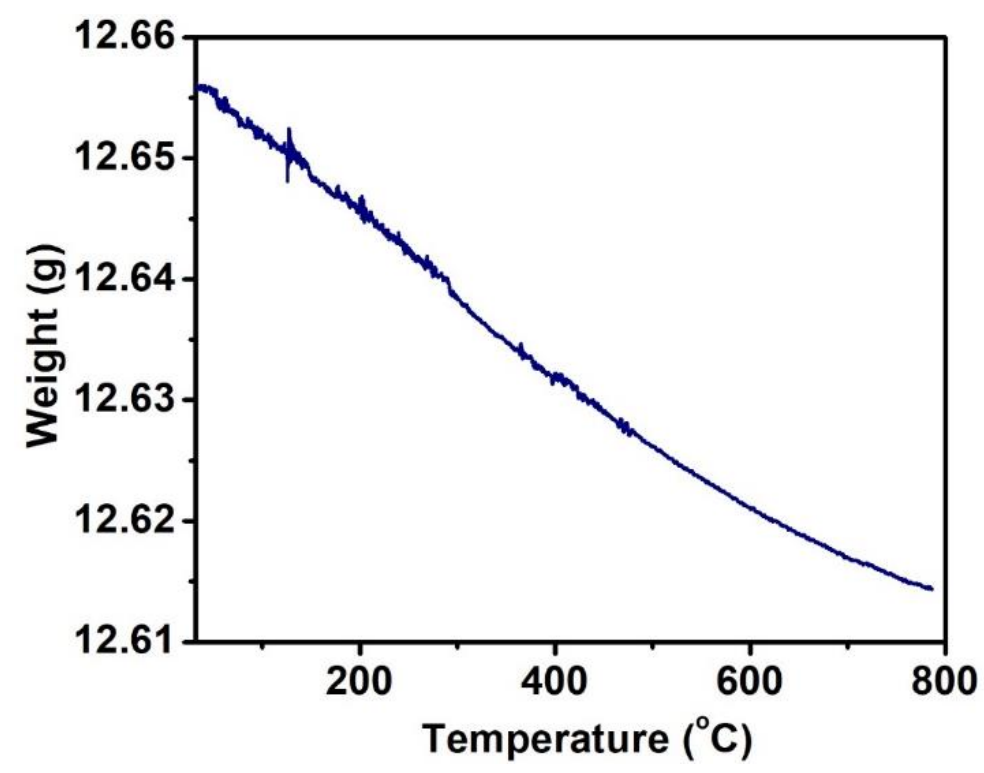

Figure 5.7 Thermogravimetric analysis for $\mathrm{Ca}_{2} \mathrm{Fe}_{0.5} \mathrm{Ga}_{1.5} \mathrm{O}_{5}$ 
We performed the variable-temperature conductivity measurements during both heating and cooling cycles. As noted in the experimental section, at each measurement temperature, enough time was given ( 40 minutes) for the conductivity to plateau, i.e., equilibrate, before moving to the next temperature. An interesting observation is the presence of small hysteresis, where the conductivity values obtained during cooling have higher values than those obtained during heating. This behavior has been observed in some other materials before. ${ }^{113-114}$ Serval researchers have reported such hysteresis, ${ }^{113-114}$ which have been attributed to oxygen-desorption, ${ }^{113}$ or defect-mediate ion mobility. ${ }^{114}$ In $\mathrm{Ca}_{2} \mathrm{Fe}_{0.5} \mathrm{Ga}_{1.5} \mathrm{O}_{5}$, the hysteresis is very small, but it indicates the effect of oxide ion conductivity. As shown by TGA (Figure 5.7) this material loses oxygen as temperature increases. The loss of oxygen can lead to enhanced ionic mobility, resulting in a small increase in total conductivity. The re-absorption of the lost oxygen upon cooling is a very slow process compared to the electronic transport phenomena. As a result, the enhanced ionic conductivity is retained after cooling the material, leading to hysteresis. It is noted that the hysteresis is observed at $500{ }^{\circ} \mathrm{C}$ and higher, where the contribution of ionic conductivity is expected to be more significant. ${ }^{111-112}$

We have also calculated the activation energies for the increase in electrical conductivity as a function of temperature. For thermally activated conductivity, activation energy can be calculated using the Arrhenius equation: ${ }^{49-51}$

$$
\sigma T=\sigma^{0} e^{\frac{-E_{a}}{K T}}
$$

where $\sigma^{\circ}$ is a preexponential factor and a characteristic of the material. $\mathrm{E}_{\mathrm{a}}, \mathrm{K}$, and $\mathrm{T}$ are the activation energy for the electrical conductivity, Boltzmann constant, and absolute temperature, respectively. The activation energy $\left(E_{a}\right)$ is calculated from the slope of the 
line of best fit in the $\log \sigma \mathrm{T}$ versus 1000/T plot. The Arrhenius plot and activation energies for $\mathrm{Ca}_{2} \mathrm{Fe}_{0.5} \mathrm{Ga}_{1.5} \mathrm{O}_{5}$ are presented in Figure 5.6, showing values close to those typically observed in this class of compounds.

\section{CONCLUSIONS}

$\mathrm{Ca}_{2} \mathrm{Fe}_{0.5} \mathrm{Ga}_{1.5} \mathrm{O}_{5}$ represents the highest degree of Ga-doping in the brownmillerite compound, $\mathrm{Ca}_{2} \mathrm{Fe}_{2} \mathrm{O}_{5}$, which can be reached through solid-state synthesis method. The non-magnetic $\mathrm{Ga}^{3+}$ cation leads to the absence of long range magnetic order at temperatures where the parent material shows antiferromagnetic order. The bulk magnetization studies indicate that this material exhibits paramagnetic behavior above $250 \mathrm{~K}$, and short-range magnetic interactions below this temperature. The electrical conductivity of $\mathrm{Ca}_{2} \mathrm{Fe}_{0.5} \mathrm{Ga}_{1.5} \mathrm{O}_{5}$ indicates semiconducting behavior, and mixed ionic-electronic conductivity. The properties of $\mathrm{Ca}_{2} \mathrm{Fe}_{0.5} \mathrm{Ga}_{1.5} \mathrm{O}_{5}$ are consistent with results from XPS and iodometric titrations, which indicate that Fe is primarily in trivalent state, accompanied by a small amount of tetravalent Fe. 


\section{CHAPTER 6}

\section{ENHANCED ELECTRICAL PROPERTIES IN $\mathrm{BaSrFe}_{2} \mathrm{O}_{6-\delta}(\delta=0.5)$ : A DISORDERED DEFECT- PEROVSKITE ${ }^{5}$}

\section{INTRODUCTION}

Oxygen deficient perovskites have emerged as an interesting class of functional materials for application in areas such as oxygen separation membranes ${ }^{115-116}$, electrodes in solid oxide fuel cells ${ }^{117}$, and promoters in Lithium sulfur batteries. ${ }^{118}$ Oxygen-deficient perovskites have general formula $\mathrm{ABO}_{3-\delta}$ or $\mathrm{A}_{2} \mathrm{~B}_{2} \mathrm{O}_{6-\delta}$ where $\mathrm{A}$ is an alkaline-earth or rareearth metal and $\mathrm{B}$ is usually a transition metal with octahedral coordination. Here, $\delta$ represents the degree of oxygen-deficiency, which results in the formaiton of defects in the crystal structure. These vacant sites can be distributed in a radom or ordered manner in the structure. ${ }^{72,84}$ The defects convert the octahedral $\mathrm{BO}_{6}$ units into other types of plolyhedra, such as square pyrmids ${ }^{119-120}$ or tetrahera. ${ }^{89}$ The type of structure in this class of materials depends on several factors, including the concentraiton of defects, i.e., $\delta$ value, as well as the nature of the A- and B-site cations. For example, $\mathrm{Sr}_{2} \mathrm{Mn}_{2} \mathrm{O}_{6-\delta}$ can have different space

\footnotetext{
${ }^{5}$ The work described in this chapter was published in Inorganic Chemistry ( 2019, vol.
} 167, p. 69-74) 
groups depending on the amount of oxygen-deficiency. ${ }^{121}$ Similarly, $\mathrm{Sr}_{2} \mathrm{Fe}_{2} \mathrm{O}_{6-\delta}$ shows a series of structures as a function of variation in $\delta{ }^{83}$

Not surprisingly, the nature of the B-site cation also affects the structure and properties. ${ }^{75}$, ${ }^{88}$ For example, both $\mathrm{Ca}_{2} \mathrm{Mn}_{2} \mathrm{O}_{5}{ }^{119}$ and $\mathrm{Ca}_{2} \mathrm{Cr}_{2} \mathrm{O}_{5}{ }^{122}$ have defect-ordered structures, but the type of ordering in these two compounds is different. $\mathrm{Ca}_{2} \mathrm{Mn}_{2} \mathrm{O}_{5}$ contains only one type of B-site coordination, namely square pyramidal $\mathrm{MnO}_{5}$ units. ${ }^{119}$ However, $\mathrm{Ca}_{2} \mathrm{Cr}_{2} \mathrm{O}_{5}$ contains alternating layers of $\mathrm{CrO}_{4}$ tetrahedra and $\mathrm{CrO}_{6}$ octahedra, ${ }^{122}$ forming the so-called brownmillerite-type structure. These structural differences result in very different magnetic ${ }^{122-123}$ and electrical properties. ${ }^{110,}{ }^{124}$ In some cases, even small variation, 5\%, of the B-site cation can cause significant changes to the distribution of defects in the structure, as observed for $\mathrm{Sr}_{2} \mathrm{Fe}_{1.9} \mathrm{Cr}_{0.1} \mathrm{O}_{6-\delta}{ }^{12}$ as compared to its parent compound $\mathrm{Sr}_{2} \mathrm{Fe}_{2} \mathrm{O}_{6}$ $\delta .{ }^{4}$

Similar situation applies to the role of A-site cation. ${ }^{85,90}$ For example, partial substitution of $\mathrm{Ca}$ in $\mathrm{Ca}_{2} \mathrm{FeCoO}_{6-\delta}$ results in $\mathrm{CaSrFeCoO}_{6-\delta}$, which has a different space group, leading to different magnetic and electrical properties.$^{84}$ Note that in this case, the substitution on the A-site leads to the change in the space group, but the overall structural framework in both compounds is the same. Both materials have defect-ordered brownmillerite-type structure.

In this article, we demonstrate drastic changes in the distribution of defects as a result of increase in the average ionic radius of the A-site cations. We report $\mathrm{BaSrFe}_{2} \mathrm{O}_{6-\delta}(\delta=0.5)$ which has a disoreded cubic strcuture, whereas the Ca-analogue, $\mathrm{CaSrFe}_{2} \mathrm{O}_{6-\delta}(\delta=1),{ }^{4}$ is known to be a defect-ordered material with orthorhombic structure. The remarkably higher 
electrical condcutivty of $\mathrm{BaSrFe}_{2} \mathrm{O}_{6-\delta}(\delta=0.5)$ and the transformation of magnetic properties have been demonstrated.

\section{EXPERIMENTAL}

Both compounds $\mathrm{CaSrFe}_{2} \mathrm{O}_{6-\delta}(\delta=1)$ and $\mathrm{BaSrFe}_{2} \mathrm{O}_{6-\delta}(\delta=0.5)$ were synthesized under identical conditions by solid state method. The powders of the precursor compounds $\mathrm{CaCO}_{3}$ (Alfa Aesar, 99.95\%), $\mathrm{SrCO}_{3}$ (Alfa Aesar, 99.95\%), $\mathrm{BaCO}_{3}$ (Alfa Aesar, 99.95\%), and $\mathrm{Fe}_{2} \mathrm{O}_{3}$ (Alfa Aesar,99.998\%) were mixed in stoichiometric proportions and ground together using agate mortar and pestle. The powder was then pressed into a pellet and heated for $24 \mathrm{~h}$ at $1000{ }^{\circ} \mathrm{C}$ in air. Subsequently, the samples were reground and refired at $1200{ }^{\circ} \mathrm{C}$ for $24 \mathrm{~h}$ in air. The heating and cooling rates were $100{ }^{\circ} \mathrm{C} / \mathrm{h}$. The phase purity and structure of the polycrystalline samples were determined by powder X-ray diffraction at room temperature using $\mathrm{Cu} \mathrm{K \alpha l}$ radiation $(\lambda=1.54056 \AA)$. Neutron diffraction experiments were performed on POWGEN diffractometer at Oak Ridge National Laboratory. The GSAS software ${ }^{40}$ and EXPEGUI interface ${ }^{125}$ were used for Rietveld refinements. In these refinements, the peak profile parameters, background, sample displacement, unit-cell parameters, atomic coordinates and thermal displacement factors were refined simultaneously. The neutron diffraction data were also used to refine the site occupancy of oxygen and show that it matched the observed results from XPS and iodometric titrations.

The microstructures were studied using high resolution field-emission scanning electron microscopy (SEM). The electrical properties were investigated by direct-current (dc) method on pellets that had been sintered at $1200{ }^{\circ} \mathrm{C}$. Alternating current (ac) measurements of resistance were also performed. As expected from a material with predominantly 
electronic conductivity, no semicircle was observed. ${ }^{126-127}$ The resistance obtained from the intercept with the real axis in the Nyquist plot was the same as that obtained from dc measurements. The 4-probe conductivity measurements were performed in the temperature-range from 25 to $800{ }^{\circ} \mathrm{C}$. The sample was kept at each temperature until a plateau in conductivity was reached, before increasing the temperature. The rate of heating between different measurement temperatures was $3{ }^{\circ} \mathrm{C} / \mathrm{min}$. Iodometric titrations were performed by dissolving about $50 \mathrm{mg}$ of sample and excess $\mathrm{KI}(\sim 2 \mathrm{~g})$ in $100 \mathrm{~mL}$ of $1 \mathrm{M}$ $\mathrm{HCl}$. A total of $5 \mathrm{~mL}$ of the solution was then pipetted out, and iodine that had been generated in the solution was titrated against $0.025 \mathrm{M} \mathrm{Na}_{2} \mathrm{~S}_{2} \mathrm{O}_{3}$. Near the end point of the titration, $0.2 \mathrm{~mL}$ of a starch solution was added to act as an indicator. All steps were performed under argon atmosphere. X-ray photoelectron spectroscopy (XPS) was done at room temperature using $\mathrm{Al} \mathrm{K} \alpha$ radiation $(1486.7 \mathrm{eV})$ to study the oxidation states of Fe.

\section{RESULT AND DISCUSSIONS}

\subsection{Crystal structure}

The crystal structures are determined by powder X-ray and neutron diffraction. Also, the degree of oxygen-deficiency $(\delta)$ and the Fe oxidation states have been studied by iodometric titrations and X-ray photoelectron spectroscopy. A phase change is observed between $\mathrm{CaSrFe}_{2} \mathrm{O}_{6-\delta}(\delta=1)$ and $\mathrm{BaSrFe}_{2} \mathrm{O}_{6-\delta}(\delta=0.5)$, where the arrangement of defects changes from ordered to disordered. This transformation has significant consequences in terms of charge transport properties, as discussed in the next section. Note that both materials were synthesized under identical conditions. For $\mathrm{BaSrFe}_{2} \mathrm{O}_{6-\delta}(\delta=0.5)$ all 
diffraction peaks can be indexed on cubic $P m-3 m$ space group, as confirmed by Rietveld refinements using X-ray and neutron diffraction data, shown in Figure 6.1.

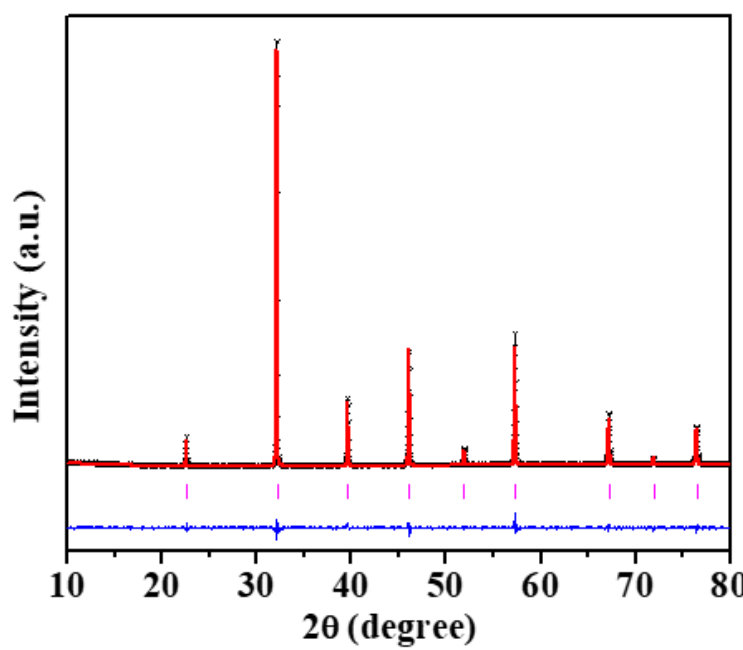

(a)

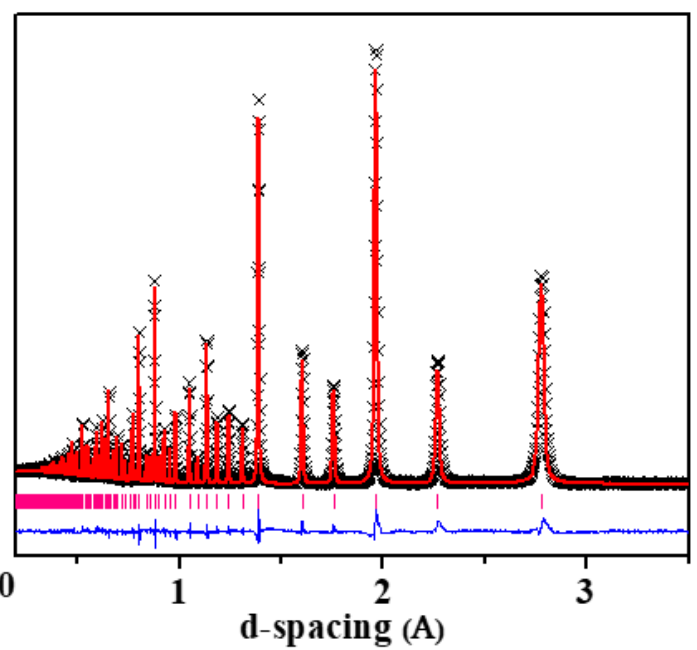

(b)

Figure 6.1. Rietveld refinement profiles for $\mathrm{BaSrFe}_{2} \mathrm{O}_{6-\delta}(\delta=0.5)$ : (a) X-ray and (b) neutron diffraction data.

In this structure, the defects, created due to oxygen-deficiency, have a random distribution. As a result, the average structure looks similar to that of a typical perovskite ${ }^{70,88}$ (Figure 6.2), but with partial occupancy on the oxygen positions (Table 6.1). This is in sharp contrast to the structure of $\mathrm{CaSrFe}_{2} \mathrm{O}_{6-\delta}(\delta=1),{ }^{4}$ (Figure 6.2) which is known to contain an ordered array of defects, that appear in alternating layers, converting the octahedral geometry into tetrahedra in those layers. The outcome is a structure with alternating $\mathrm{FeO}_{6}$ octahedra and $\mathrm{FeO}_{4}$ tetrahedra, ${ }^{4}$ the so called brownmillerite-type structure. ${ }^{72-74}$ Clearly, the average ionic radius on the A-site of the oxygen-deficient perovskites $\mathrm{A}_{2} \mathrm{Fe}_{2} \mathrm{O}_{6-8}$ is the 
driving force behind the structural transformation from defect-ordered to disordered. The average A-site ionic radius for $\mathrm{CaSrFe}_{2} \mathrm{O}_{6-\delta}(\delta=1)$ is $1.39 \AA$,

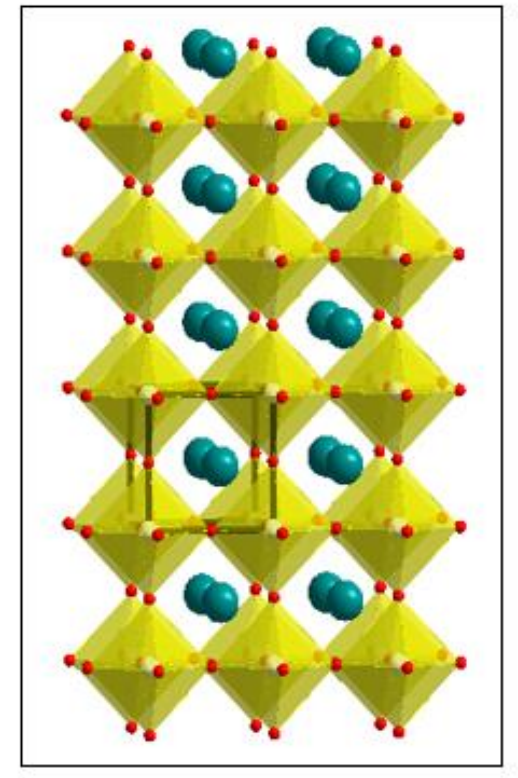

(a)

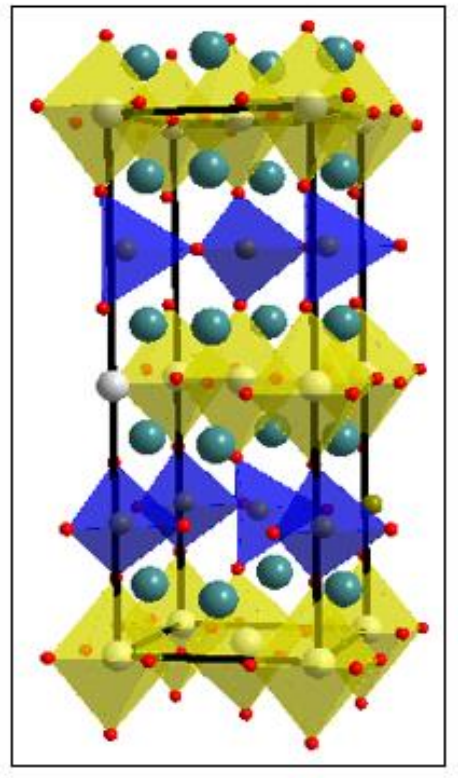

(b)

Figure 6.2. Crystal structures of (a) $\mathrm{BaSrFe}_{2} \mathrm{O}_{6-\delta}(\delta=0.5)$ and (b) $\mathrm{CaSrFe}_{2} \mathrm{O}_{6-\delta}(\delta=1)$.

Table 6.1. Refined structural parameters from neutron diffraction data for $\mathrm{BaSrFe}_{2} \mathrm{O}_{6-\delta}$ $(\delta=0.5)$, space group $P m-3 m, \mathrm{a}=3.92851(4), R_{\mathrm{p}}=0.041, \mathrm{w} R_{\mathrm{p}}=0.033$

\begin{tabular}{ccccccc}
\hline & $\mathrm{x}$ & $\mathrm{y}$ & $\mathrm{z}$ & Uiso & occupancy & multiplicity \\
\hline $\mathrm{Ba} 1$ & 0 & 0 & 0 & $0.0056(7)$ & 0.5 & 1 \\
$\mathrm{Sr} 1$ & 0 & 0 & 0 & $0.0056(7)$ & 0.5 & 1 \\
$\mathrm{Fe} 1$ & 0.5 & 0.5 & 0.5 & $0.0072(6)$ & 1.0 & 1 \\
$\mathrm{O} 1$ & 0 & 0.5 & 0.5 & $0.0145(1)$ & $0.902(3)$ & 3 \\
\hline
\end{tabular}


while for $\mathrm{BaSrFe}_{2} \mathrm{O}_{6-\delta}(\delta=0.5)$, it is $1.52 \AA .{ }^{128}$ It is noted that the coordination environment of the A-site cation is different in the two materials. In $\mathrm{CaSrFe}_{2} \mathrm{O}_{6-\delta}(\delta=1)$, the $\mathrm{Ca}^{2+} / \mathrm{Sr}^{2+}$ ions are 8-coordinated, whereas $\mathrm{BaSrFe}_{2} \mathrm{O}_{6-\delta}(\delta=0.5)$ contains 12-coordinated $\mathrm{Ba}^{2+} / \mathrm{Sr}^{2+}$ cations.

Given the structural distortions in $\mathrm{CaSrFe}_{2} \mathrm{O}_{6-\delta}(\delta=1)$, which occur in order to accommodate the simultaneous corner-sharing between octahedra and tetrahedra, the $\mathrm{Fe}-\mathrm{O}-\mathrm{Fe}$ bond angles deviate significantly from $180^{\circ}$ and can be as small as $146.2(2)^{\circ}$. However, the bond angles in $\mathrm{BaSrFe}_{2} \mathrm{O}_{6-\delta}(\delta=0.5)$ are $180^{\circ}$, as expected from a cubic structure. The $\mathrm{Fe}-\mathrm{O}$ bond distances are also different, ranging from 1.842(4) $\AA$ to $2.150(4) \AA$ for $\mathrm{CaSrFe}_{2} \mathrm{O}_{6-\delta}(\delta=1)$, and 1.964(2) $\AA$ for $\mathrm{BaSrFe}_{2} \mathrm{O}_{6-\delta}(\delta=0.5)$.

The morphology and crystallite sizes of both materials were studied using scanning electron microscopy (SEM). Figure 6.3 shows the SEM images of sintered pellets. There is a significant increase in grain size in transition from $\mathrm{CaSrFe}_{2} \mathrm{O}_{6-\delta}(\delta=1)$ to $\mathrm{BaSrFe}_{2} \mathrm{O}_{6-\delta}$ $(\delta=0.5)$ 


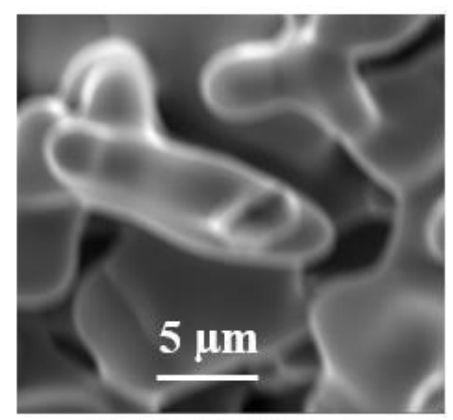

$\mathrm{BaSrFe}_{2} \mathrm{O}_{6-\delta}$

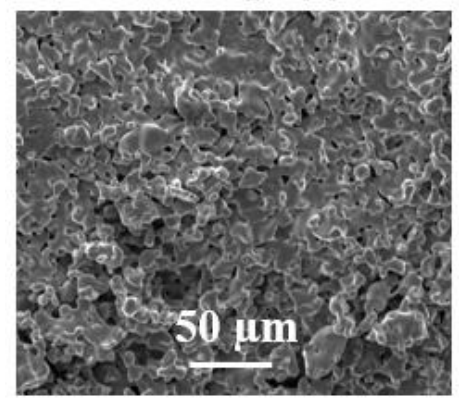

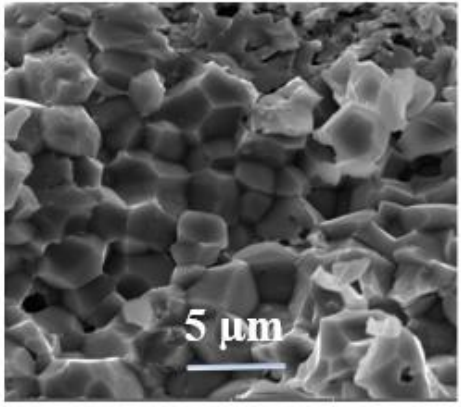

$\mathrm{CaSrFe}_{2} \mathrm{O}_{6-\delta}$

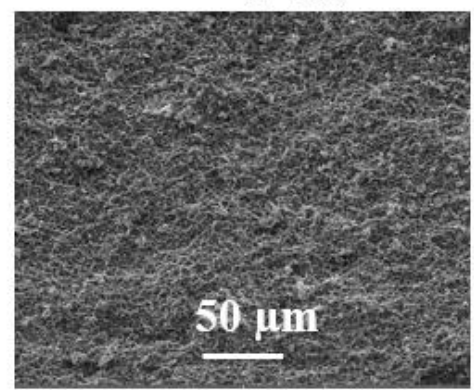

Figure 6.3. Scanning electron microscopy images of sintered pellets of (a) $\mathrm{BaSrFe}_{2} \mathrm{O}_{6-\delta}$ $(\delta=0.5)$ and (b) $\mathrm{CaSrFe}_{2} \mathrm{O}_{6-\delta}(\delta=1)$.

The degree of oxygen-deficiency and oxidation state of Fe cations were also investigated using X-ray photoelectron spectroscopy (XPS) study and iodometric titrations. Note that $\mathrm{Fe}_{2} \mathrm{O}_{3}$ was used as starting material in the synthesis of both compounds, and the synthesis conditions were identical. If iron preserves its +3 oxidation state, the number of oxygens per formula unit in $\mathrm{AA}^{\prime} \mathrm{Fe}_{2} \mathrm{O}_{6-\delta}$ should be 5, resulting in $\delta=1$. Oxidation state of $\mathrm{Fe}$ is determined based on the position of the $2 \mathrm{P}_{3 / 2}$ peak as well as the satellite peak associated with $2 \mathrm{P}_{3 / 2}$. As shown in the XPS data (Figure 6.4), the $2 \mathrm{P}_{3 / 2}$ peak appears at $\sim 710 \mathrm{eV}$, followed by a satellite peak at $\sim 718 \mathrm{eV}$, indicating the presence of $\mathrm{Fe}^{3+}$ in $\mathrm{BaSrFe}_{2} \mathrm{O}_{6-\delta}$ $(\delta=0.5){ }^{44,129-130}$ The shoulder on the high energy side of the $2 \mathrm{P}_{3 / 2}$ peak indicates that $\mathrm{Fe}^{4+}$ is also present in $\mathrm{BaSrFe}_{2} \mathrm{O}_{6-\delta}(\delta=0.5) .{ }^{130}$ These results were also confirmed by iodometric titrations, which provided a quantitative understanding of the 


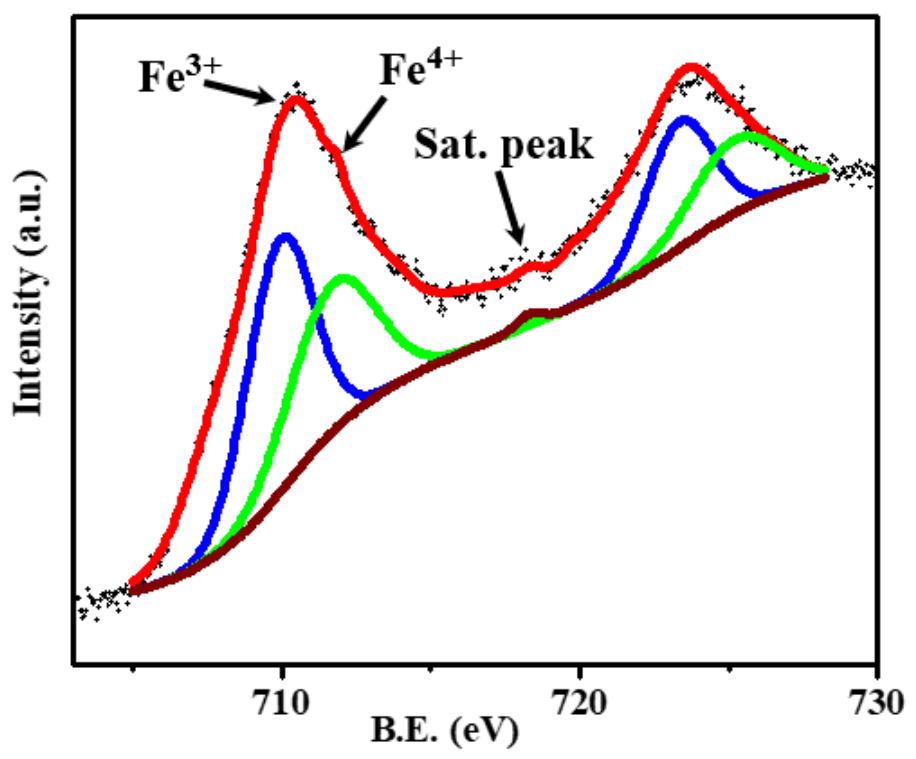

Figure 6.4. X-ray photoelectron spectroscopy data for $\mathrm{BaSrFe}_{2} \mathrm{O}_{6-\delta}(\delta=0.5)$.

concentration of oxygen-defects. Iodometric titration indicated that the oxygen stoichiometry in $\mathrm{BaSrFe}_{2} \mathrm{O}_{6-\delta}(\delta=0.5)$ is 5.5 per formula unit, corresponding to $\delta=0.5$ and confirming the presence of both $\mathrm{Fe}^{3+}$ and $\mathrm{Fe}^{4+}$. This is also consistent with the oxygen siteoccupancy obtained from Rietveld refinement using neutron diffraction data (Table 6.1). Similar analyses, using XPS and iodometric titrations, have shown that $\mathrm{CaSrFe}_{2} \mathrm{O}_{6-\delta}(\delta=1)$ contains only $\mathrm{Fe}^{3+}$, with 5 oxygens per formula unit, i.e., $\delta=1 .{ }^{4}$ It is interesting that the increase in ionic radius on the A-site facilitates the oxidation of $\mathrm{Fe}$ in $\mathrm{BaSrFe}_{2} \mathrm{O}_{6-\delta}(\delta=0.5)$, leading to greater oxygen-content and smaller concentration of defects compared to $\mathrm{CaSrFe}_{2} \mathrm{O}_{6-\delta}(\delta=1)$. These findings are consistent with the crystal structures determined using Rietveld refinements. The structure of $\mathrm{BaSrFe}_{2} \mathrm{O}_{6-\delta}(\delta=0.5)$ can accommodate a greater amount of oxygen on lattice sites, whereas, the brownmillerite-type framework of $\mathrm{CaSrFe}_{2} \mathrm{O}_{6-\delta}(\delta=1)$ can accommodate only up to 5 oxygens per formula unit, and any additional oxygen would need to be located on interstitial sites. Therefore, the structural 
differences between the two compounds lead to more facile oxidation of $\mathrm{Fe}$ in one material compared to the other.

\subsection{Magnetic properties}

The change in the distribution of defects and the subsequent changes in the crystal structure have a major impact on the magnetic properties. $\mathrm{CaSrFe}_{2} \mathrm{O}_{6-\delta}(\delta=1)$ is known to feature antiferromagnetic order. ${ }^{4}$ Previous studies using neutron diffraction at $10 \mathrm{~K}$ have shown an antiferromagnetic system where the magnetic moment on each Fe site is oriented opposite to all of its nearest neighbors. ${ }^{4}$ We therefore, performed neutron diffraction on $\mathrm{BaSrFe}_{2} \mathrm{O}_{6}$ $\delta(\delta=0.5)$ at $10 \mathrm{~K}$ to be able to make direct comparison between the two materials. In addition, the bulk magnetization data for $\mathrm{BaSrFe}_{2} \mathrm{O}_{6-\delta}(\delta=0.5)$ show a transition at $\sim 50 \mathrm{~K}$. Thus, the neutron experiments at $10 \mathrm{~K}$ can shed light on the magnetic state below the transition temperature for $\mathrm{BaSrFe}_{2} \mathrm{O}_{6-\delta}(\delta=0.5)$. Given the large magnetic moments associated with $\mathrm{Fe}$ atoms, any long-range magnetic order can be readily identified. However, neutron diffraction experiments (Figure 6.1) show no magnetic peaks for $\mathrm{BaSrFe}_{2} \mathrm{O}_{6-\delta}(\delta=0.5)$, indicating the absence of long-range magnetic order, in sharp contrast to the situation in $\mathrm{CaSrFe}_{2} \mathrm{O}_{6-\delta}(\delta=1) .{ }^{4}$

As stated above, the bulk magnetization data (Figure 6.5) for $\mathrm{BaSrFe}_{2} \mathrm{O}_{6-\delta}(\delta=0.5)$ feature a transition at $\sim 50 \mathrm{~K}$, where the zero-field-cooled and field-cooled data diverge. This type of transition has been observed in other oxygen-deficient perovskites before and has been attributed to transition into a spin-glass state. ${ }^{12,72}$ The inverse susceptibility plot versus temperature indicates that the Currie-Weiss behavior does not occur below $\sim 300 \mathrm{~K}$. The linear paramagnetic trend is observed above this temperature. The fit to this linear region results in the Currie constant of 6.64(4) and Weiss constant of -254(4). The negative value 
of the Weiss constant indicates that short-range antiferromagnetic interactions are present. The isothermal magnetization data for $\mathrm{BaSrFe}_{2} \mathrm{O}_{6-\delta}(\delta=0.5)$ at $5 \mathrm{~K}$ show small hysteresis implying the presence of a small uncompensated moment. The isothermal data at $300 \mathrm{~K}$ is linear without any hysteresis, as expected from a typical

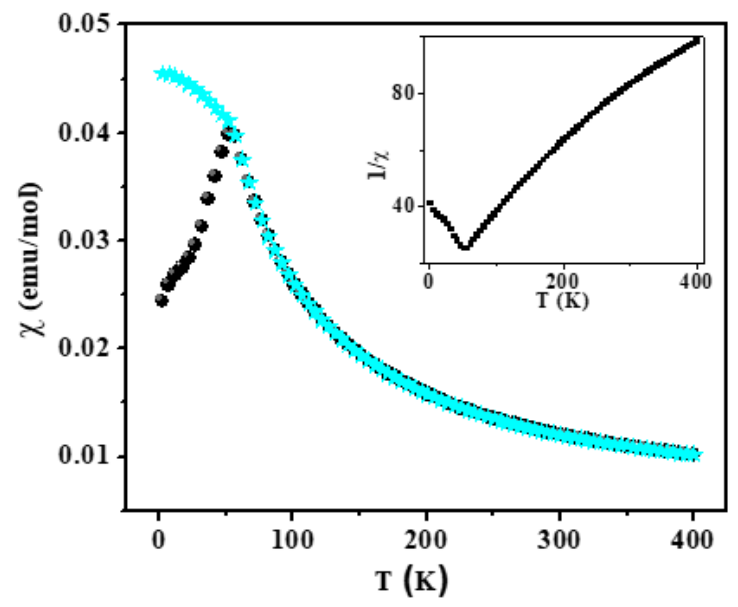

(a)

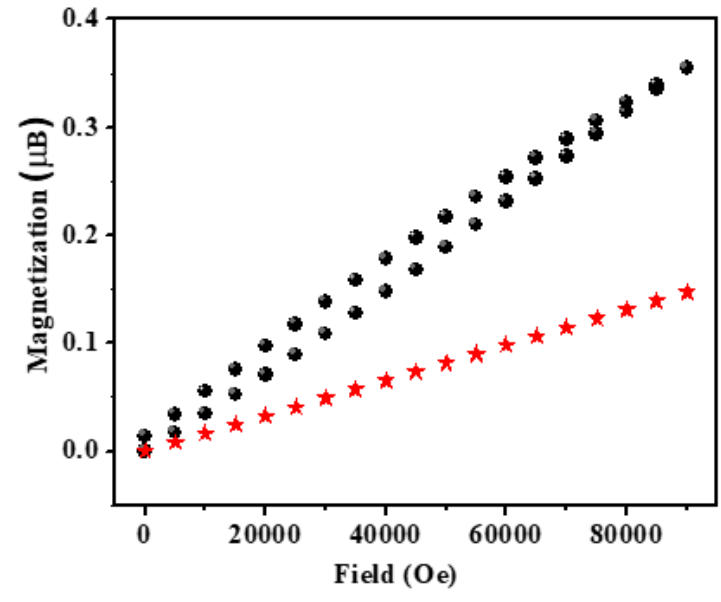

(b)

Figure 6.5. Bulk magnetization data of $\mathrm{BaSrFe}_{2} \mathrm{O}_{6-\delta}(\delta=0.5)$ (a) $\mathrm{ZFCFC}$ magnetic susceptibility data. The inset shows inverse of susceptibility versus temperature. (b) Isothermal magnetization data at $5 \mathrm{~K}$ and $400 \mathrm{~K}$.

paramagnetic system. This is consistent with the observation from inverse susceptibility plot, which shows paramagnetic behavior above $300 \mathrm{~K}$.

Overall, the transformation of magnetic properties as a result of changes in the distribution of defects is remarkable. The transition from defect-order in $\mathrm{CaSrFe}_{2} \mathrm{O}_{6-\delta}(\delta=1)$ to disorder in $\mathrm{BaSrFe}_{2} \mathrm{O}_{6-\delta}(\delta=0.5)$ results in the change from antiferromagnetic order to spin-glass magnetic state. 


\subsection{Electrical properties}

The resistance values $(\mathrm{R})$ obtained from 4-probe measurements were used to calculate the conductivity $(\sigma)$ using the following equation:

$$
\sigma=L / R A
$$

where $\mathrm{L}$ represents the distance between the voltage probes and $\mathrm{A}$ indicates the crosssectional area of the pellet through which the current is applied. The electrical conductivity was obtained at variable temperatures from 25 to $800{ }^{\circ} \mathrm{C}$. As shown in Figure 6.6, the conductivity of $\mathrm{BaSrFe}_{2} \mathrm{O}_{6-\delta}(\delta=0.5)$ is considerably higher than that of $\mathrm{CaSrFe}_{2} \mathrm{O}_{6-\delta}(\delta=1)$ in the entire temperature range. Therefore, the increase in the average ionic radius of the A-site, and the subsequent transformation of the crystal structure, lead to a significant increase in the electrical conductivity.

Oxygen-deficient perovskites can show mixed electronic and oxide-ion conductivity. ${ }^{29,}{ }^{131-}$ ${ }^{133}$ In situations where the B-site cations have more than one stable oxidation state, such as $\mathrm{Fe}^{3+} / \mathrm{Fe}^{4+}$, the electronic conductivity is usually dominant in ambient condition. ${ }^{134}$ The electron transport occurs through hopping of electrons through Metal-Oxygen-Metal bond system. ${ }^{108-109}$ The degree of electrical conductivity in oxygen deficient perovskites depends on a number of factors. Conductivity is higher for shorter $\mathrm{M}-\mathrm{O}$ bond lengths and for larger $\mathrm{M}^{\mathrm{m}+}-\mathrm{O}-\mathrm{M}^{\mathrm{n}+}$ bond angles. ${ }^{135}$ As mentioned in the previous section, the simultaneous corner-sharing between tetrahedra and octahedra leads to distortions in the structure of $\mathrm{CaSrFe}_{2} \mathrm{O}_{6-\delta}(\delta=1)$, which results in a range of $\mathrm{Fe}-\mathrm{O}$ bond distances, from 1.842(4) $\AA$ to 2.150(4) $\AA$. Due to these distortions, the average bond distance in $\mathrm{CaSrFe}_{2} \mathrm{O}_{6-\delta}(\delta=1)$ is similar to that of $\mathrm{BaSrFe}_{2} \mathrm{O}_{6-\delta}(\delta=0.5)$, which has a cubic structure, and $\mathrm{Fe}-\mathrm{O}=1.964(2)$ $\AA$. More importantly, the bond angles in $\mathrm{CaSrFe}_{2} \mathrm{O}_{6-\delta}(\delta=1)$ are significantly deviated from 
linearity and can be as small as $146^{\circ}$. Whereas, the bond angles in $\mathrm{BaSrFe}_{2} \mathrm{O}_{6-\delta}(\delta=0.5)$ are $180^{\circ}$, which facilitate the electronic conductivity through $\mathrm{M}^{\mathrm{m}+}-\mathrm{O}-\mathrm{M}^{\mathrm{n}+}$ pathways. In addition, as demonstrated by XPS and iodometric titration, nearly all of the Fe atoms in $\mathrm{CaSrFe}_{2} \mathrm{O}_{6-\delta}(\delta=1)$ are in trivalent state, whereas in $\mathrm{BaSrFe}_{2} \mathrm{O}_{6-\delta}(\delta=0.5)$ both trivalent and tetravalent $\mathrm{Fe}$ cations are present. As a result, there is a significant number of $\mathrm{Fe}^{3+}-\mathrm{O}-\mathrm{Fe}^{4+}$ conduction pathways in $\mathrm{BaSrFe}_{2} \mathrm{O}_{6-\delta}(\delta=0.5)$, leading to the enhanced conductivity.

To study the behavior of these materials in a wide temperature-range, the electrical conductivity was examined from 25 to $800{ }^{\circ} \mathrm{C}$. For both compounds, the conductivity initially increases with temperature, indicating semiconducting behavior. Multiple studies have shown p-type semiconductivity in oxygen-deficient perovskites, ${ }^{24,136-137}$ due to the dependence on oxygen partial pressure, ${ }^{58-59,138}$ and the formation of holes as charge carriers. $9,23,58-59$

As shown in Figure 6.6, the increase in conductivity of $\mathrm{BaSrFe}_{2} \mathrm{O}_{6-\delta}(\delta=0.5)$ is significantly greater than that of $\mathrm{CaSrFe}_{2} \mathrm{O}_{6-\delta}(\delta=1)$. At $400{ }^{\circ} \mathrm{C}$ the difference between the conductivity of the two materials is more than one order of magnitude. The rise in conductivity continues up to $400{ }^{\circ} \mathrm{C}$, where an inflection point is observed for both materials. Above this temperature, the conductivity 


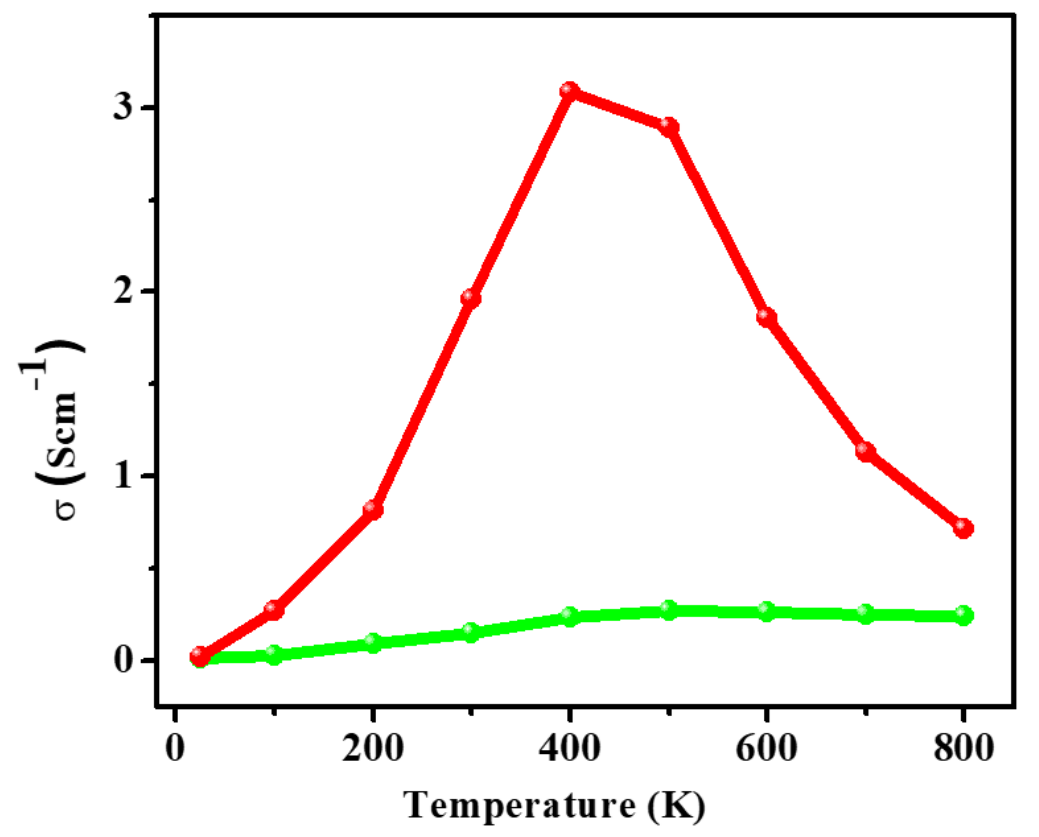

Figure 6.6. Electrical conductivity as a function of temperature for $\mathrm{BaSrFe}_{2} \mathrm{O}_{6-\delta}(\delta=0.5)$ (red) and $\mathrm{CaSrFe}_{2} \mathrm{O}_{6-\delta}(\delta=1)$ (green).

of $\mathrm{CaSrFe}_{2} \mathrm{O}_{6-\delta}(\delta=1)$ nearly plateaus. The change is more drastic for $\mathrm{BaSrFe}_{2} \mathrm{O}_{6-\delta}(\delta=0.5)$, where a sharp decrease in conductivity occurs above $400{ }^{\circ} \mathrm{C}$. The drastic change in conductivity seems to correlate with the degree of oxygen loss, as demonstrated by the thermogravimetric analysis (TGA) data in Figure 6.7. Above $400{ }^{\circ} \mathrm{C}$, there is a sudden decline in the TGA data, indicating the onset of significant oxygen loss, as has been observed before. ${ }^{106,139-140}$ This can result in a decrease in the concentration of $\mathrm{Fe}^{3+}-\mathrm{O}-\mathrm{Fe}^{4+}$ conduction pathways, leading to a decrease in conductivity. ${ }^{134,141-142}$

The mechanism of oxygen vacancy creation can be expressed by following equations, using Kroger-Vink notation. ${ }^{134,139-140,143}$

$$
\begin{aligned}
& O_{o}^{\prime \prime} \rightarrow \frac{1}{2} \mathrm{O} 2+V_{o}^{\bullet \bullet}+2 e^{\prime} \\
& 2 \mathrm{Fe}^{4+}+2 e^{\prime} \rightarrow 2 \mathrm{Fe}^{3+}
\end{aligned}
$$

Where $O_{o}^{\prime \prime}, V_{o}^{\bullet \bullet}$ and $e^{\prime}$ represent lattice oxygen, vacancy and electron respectively. 
The change in conductivity as a function of temperature can be described using the Arrhenius equation for thermally activated conductivity: ${ }^{49-51}$

$$
\sigma T=\sigma^{o} e^{\frac{-E_{a}}{K T}}
$$

where $\sigma^{\circ}$ is a pre-exponential factor and a characteristic of the material. $\mathrm{E}_{\mathrm{a}}, \mathrm{K}$, and $\mathrm{T}$ are the activation energy for the electrical conductivity, Boltzmann constant, and absolute temperature, respectively.

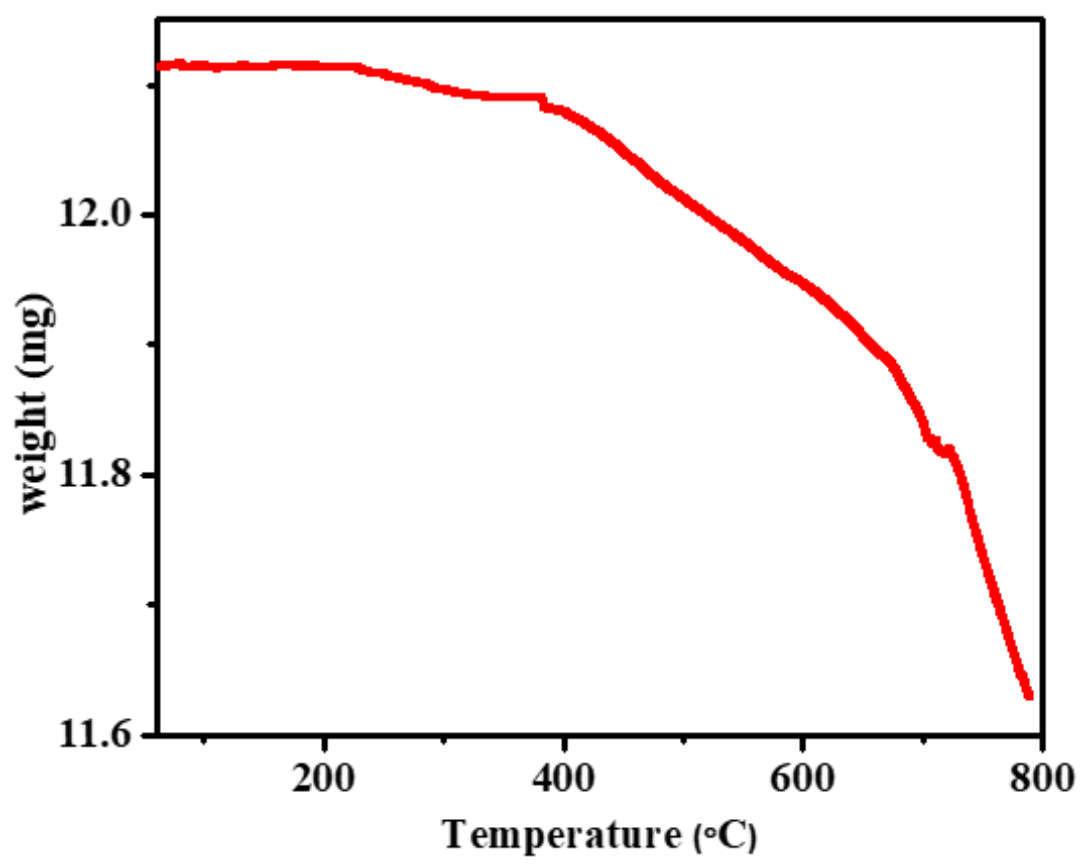

Figure 6.7. Thermogravimetric analysis data for $\mathrm{BaSrFe}_{2} \mathrm{O}_{6-\delta}(\delta=0.5)$.

Table 6.2. Electrical conductivity, $\sigma\left(\mathrm{S} \mathrm{cm}^{-1}\right)$, and activation energies, $\mathrm{E}_{\mathrm{a}}(\mathrm{eV})$, for increase in electrical conductivity up to $400{ }^{\circ} \mathrm{C}$.

\begin{tabular}{lcccc}
\hline & $25{ }^{\circ} \mathrm{C}$ & $400{ }^{\circ} \mathrm{C}$ & $800{ }^{\circ} \mathrm{C}$ & $\mathrm{E}_{\mathrm{a}}(\mathrm{eV})$ \\
\hline $\mathrm{BaSrFe}_{2} \mathrm{O}_{6-\delta}(\delta=0.5)$ & $2.22 \times 10^{-2}$ & 3.08 & $7.13 \times 10^{-1}$ & 0.241 \\
$\mathrm{CaSrFe}_{2} \mathrm{O}_{6-\delta}(\delta=1)$ & $1.27 \times 10^{-2}$ & $2.3 \times 10^{-1}$ & $2.4 \times 10^{-1}$ & 0.174 \\
\hline
\end{tabular}


The activation energy $\left(E_{a}\right)$ for the change in conductivity as a function of temperature can be determined from the slope of the line of best fit in the log $\sigma \mathrm{T}$ versus 1000/T plot, ${ }^{49-51}$ as shown in Figure 6.8. The activation energies for the increase in conductivity between 25 to $400{ }^{\circ} \mathrm{C}$ are $0.174 \mathrm{eV}$ and $0.241 \mathrm{eV}$, for $\mathrm{CaSrFe}_{2} \mathrm{O}_{6-\delta}(\delta=1)$ and $\mathrm{BaSrFe}_{2} \mathrm{O}_{6-\delta}(\delta=0.5)$, respectively.

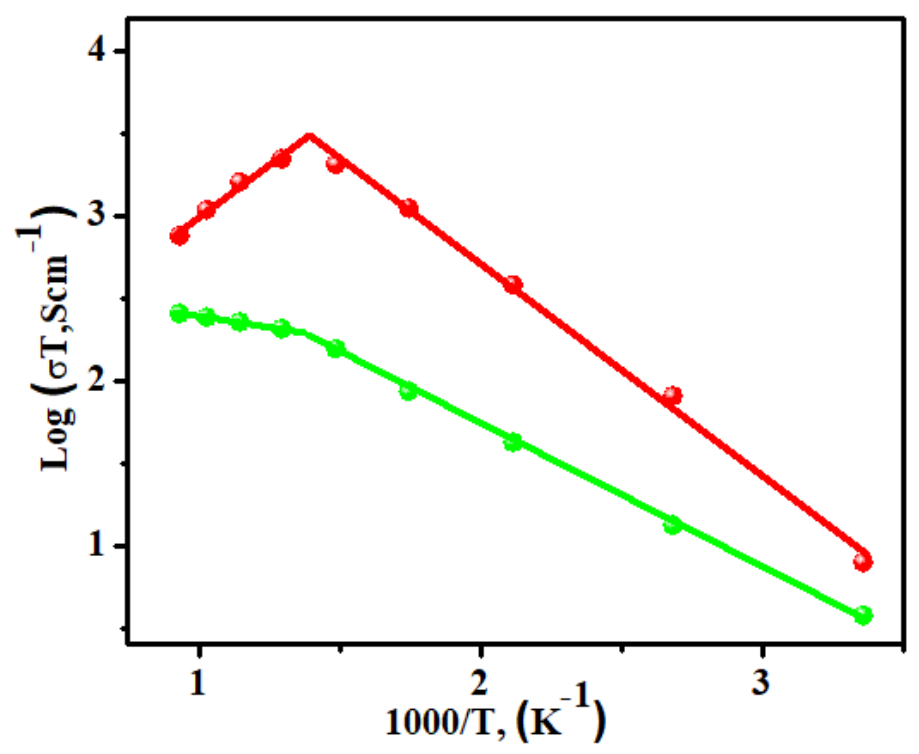

Figure 6.8. Arrhenius plot of the electrical conductivity for $\mathrm{BaSrFe}_{2} \mathrm{O}_{6-\delta}(\delta=0.5)$ (red) and $\mathrm{CaSrFe}_{2} \mathrm{O}_{6-\delta}(\delta=1)$ (green).

\section{CONCLUSIONS}

The increase in the average ionic radius of the A-site in $\mathrm{A}_{2} \mathrm{Fe}_{2} \mathrm{O}_{6-\delta}$ leads to structural transformation between $\mathrm{CaSrFe}_{2} \mathrm{O}_{6-\delta}(\delta=1)$ and $\mathrm{BaSrFe}_{2} \mathrm{O}_{6-\delta}(\delta=0.5)$. The latter features disordered distribution of defect sites, that are created due to oxygen deficiency, whereas the former is known to contain defect-order. The change in the structure results in the 
variation of magnetic properties, where one material is antiferromagnetically ordered, while the other shows spin-glass behavior. The structural changes also lead to the transformation of electrical properties, where the electrical conductivity of $\mathrm{BaSrFe}_{2} \mathrm{O}_{6-\delta}$ $(\delta=0.5)$ is over one order of magnitude greater than that of $\mathrm{CaSrFe}_{2} \mathrm{O}_{6-\delta}(\delta=1)$. These results demonstrate that in oxygen-deficient perovskites, the change in the defect arrangement can be used as an effective tool to manipulate the electrical properties. 


\section{CHAPTER 7}

\section{CHARGE-TRANSPORT PROPERTIES OF $\mathrm{Ca}_{2} \mathrm{FeGaO}_{6-\delta}$ and $\mathrm{CaSrFeGaO}_{6-\delta}$ : THE EFFECT OF DEFECT-ORDERED ${ }^{6}$}

\section{INTRODUCTION}

Oxygen-deficient perovskites have been studied for their various properties, such as mixed ion and electronic conductivity, ${ }^{23}$ which is essential to the operation of solid oxide fuel cells. ${ }^{117}$ These materials have also been extensively studied as oxygen transport membranes, given that oxide ion diffusion through an ion conductive material produces nearly pure oxygen. ${ }^{144}$ Usually, the ion transport in perovskite oxides takes place at high temperature, ${ }^{145}$ but some oxygen-deficient perovskites such as $\mathrm{SrFeO}_{3-x}$ or $\mathrm{SrCoO}_{3-x}$ exhibit oxygen mobility at room temperature. ${ }^{132,146}$ Given the attractive properties of this class of compounds, it is important to explore the parameters that control those properties. The structure-property relationships are particularly important.

The general formula of oxygen-deficient perovskites is $\mathrm{ABO}_{3-\delta}$. Here, $\mathrm{A}$ is usually a lanthanide or alkaline-earth metal and B is a transition metal. A-site or B-site cations can have more than one type of element. In such cases, the general formula can be written as

${ }^{6}$ The work described in this chapter was published in Material Chemistry and Physics ( 2019, vol. 238, p. 121924) 
$\mathrm{AA}^{\prime} \mathrm{BB}^{\prime} \mathrm{O}_{6-\delta}$. The degree of oxygen-deficiency can affect the structural order. ${ }^{12}$ For example, $\mathrm{SrMnO}_{3-\delta}$ shows a four-layered (4L) hexagonal structure for $0 \leq \delta \leq 0.12 .{ }^{147}$ This structure transforms into a cubic perovskite structure if the degree of oxygendeficiency changes to $0.25 \leq \delta \leq 0.38 .{ }^{147-148,82}$ Another example is $\mathrm{Sr}_{2} \mathrm{Fe}_{2} \mathrm{O}_{6-\delta}$, where a series of different phases have been reported, depending on the variation of the $\delta$ value. ${ }^{83}$ The arrangement of oxygen-vacancies in the crystal lattice is also important. The defects, generated due to oxygen deficiency, can be arranged in ordered ${ }^{4,72,86,89}$ or disordered $^{88-89}$ manner, leading to different structure and properties. ${ }^{4,}{ }^{90}$ One of the common types of defect-order results in the brownmillerite structure, which contains alternating $\mathrm{BO}_{6}$ octahedra and $\mathrm{B}^{\prime} \mathrm{O}_{4}$ tetrahedra. The formation of brownmillerite-type structure can be controlled by altering the A- or B-site cations. For example, the substitution of A-site cations in $\mathrm{Sr}_{2} \mathrm{FeMnO}_{5}$, which has a disordered cubic structure, leads to $\mathrm{Ca}_{2} \mathrm{FeMnO}_{5}$, which features brownmillerite-type ordering. ${ }^{85}$ The changes in the arrangement of defects leads to significant changes in electrical properties. The electrical conductivity of the ordered $\mathrm{Ca}_{2}$ compound is almost unaffected by the change in atmosphere from argon to air, while the conductivity of the disordered $\mathrm{Sr}_{2}$ compound increases significantly in air compared to the argon atmosphere. ${ }^{85}$ Another example is the differences in electrical properties of $\mathrm{Ca}_{2} \mathrm{FeCoO}_{6-\delta}$, which has brownmillerite-type structure, ${ }^{74}$ and $\mathrm{Sr}_{2} \mathrm{FeCoO}_{6-\delta}$, which lacks the ordering of defects. ${ }^{84}$ The latter exhibits metallic properties, whereas the former displays semiconducting behavior. ${ }^{84}$

The above examples are related to situations where the properties of an ordered material are compared to those of a disordered system. In this article, we show that the electrical 


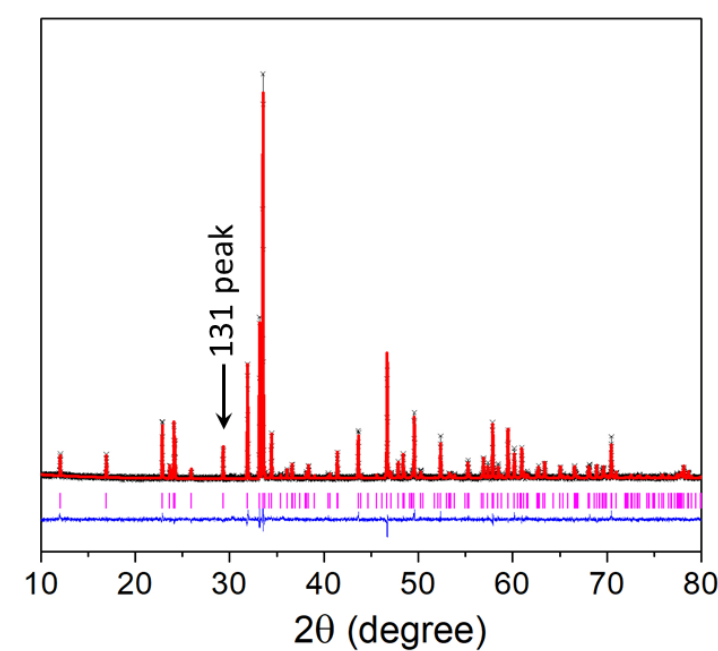

(a)

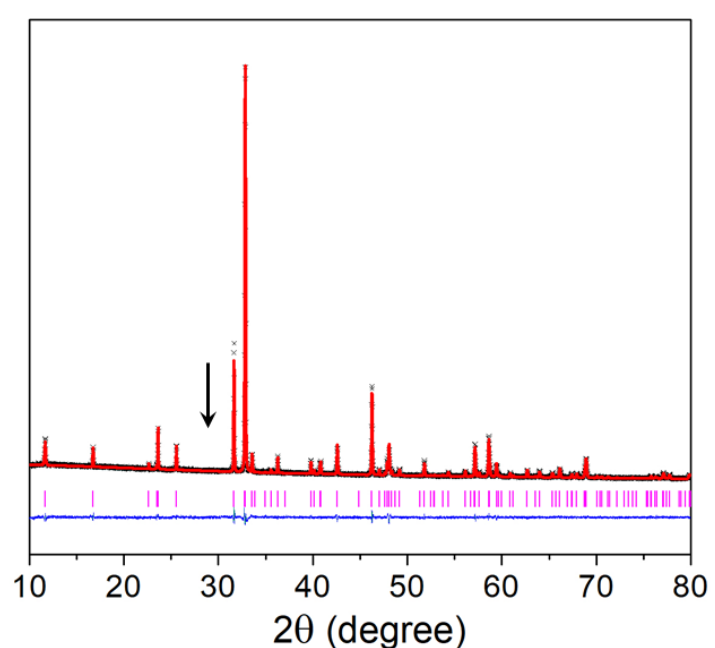

(b)

Figure 7.1. Rietveld refinement profiles for powder X-ray diffraction data of (a) $\mathrm{Ca}_{2} \mathrm{FeGaO}_{6-\delta}$ in $\mathrm{Pnma}$, and (b) $\mathrm{CaSrFeGaO}_{6-\delta}$ in $\mathrm{Ibm} 2$ space group. Black crosses represent experimental data, the solid red line is the model, pink vertical tick marks show Bragg peak positions, and the lower blue line represents the difference plot. The arrows show the position of 131 peak, which is present for $\mathrm{Ca}_{2} \mathrm{FeGaO}_{6-\delta}$ and absent for $\mathrm{CaSrFeGaO}_{6-\delta}$.

properties can be manipulated by subtle changes to the arrangement of defects, while maintaining the defect-order in the system. We report a new oxygen-deficient perovskite, $\mathrm{CaSrFeGaO}_{6-\delta}$, which unlike its $\mathrm{Ca}$-only analogue, $\mathrm{Ca}_{2} \mathrm{FeGaO}_{6-\delta}$, has a noncentrosymmetric structure. Little information is known about the Ca-only analogue, with only magnetodielectric properties reported. ${ }^{37}$ Here, we perform neutron diffraction, X-ray photoelectron spectroscopy, and charge-transport studies on both $\mathrm{Ca}_{2} \mathrm{FeGaO}_{6-\delta}$ and $\mathrm{CaSrFeGaO}_{6-\delta}$, to demonstrate that the manipulation of defect-order can be used as a tool to control the electrical properties of oxygen-deficient perovskites. 


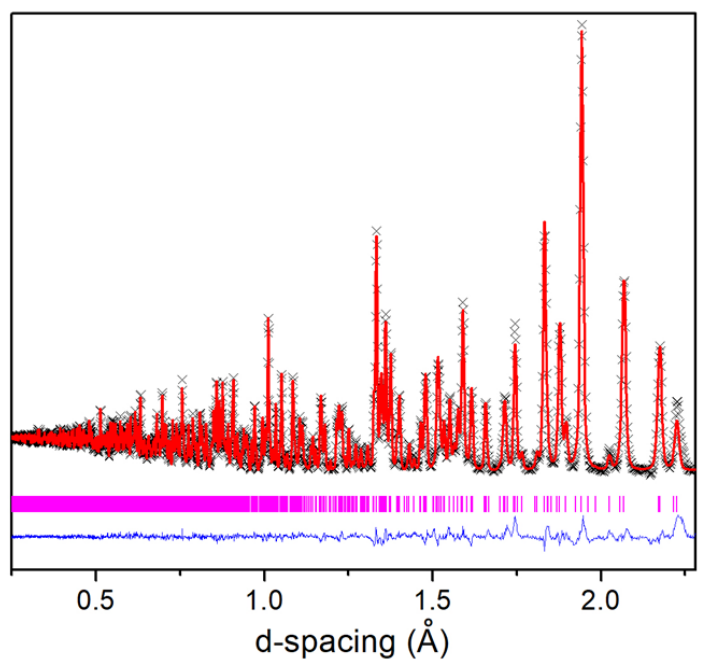

(a)

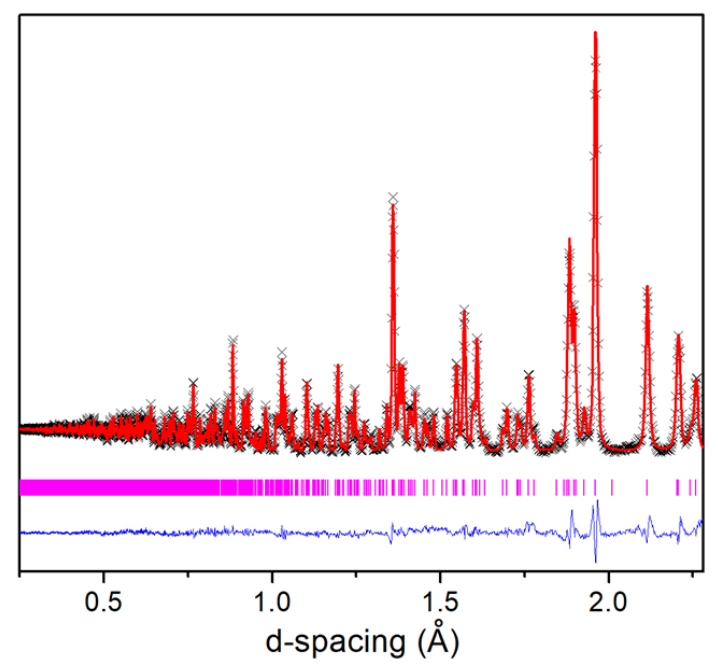

(b)

Figure 7.2. Rietveld refinement profiles for powder neutron diffraction data of (a) $\mathrm{Ca}_{2} \mathrm{FeGaO}_{6-\delta}$ in Pnma, and (b) $\mathrm{CaSrFeGaO}_{6-\delta}$ in Ibm2 space group. Black crosses represent experimental data, the solid red line is the model, pink vertical tick marks show Bragg peak positions, and the lower blue line represents the difference plot.

\section{EXPERIMENTAL}

Solid-state synthesis method was used to synthesize the materials $\mathrm{CaSrFeGaO}_{6-\delta}$ and $\mathrm{Ca}_{2} \mathrm{FeGaO}_{6-\delta}$. The synthesis of the $\mathrm{Sr}_{2}$-analogue was also attempted, but it consistently led to multiphase products. The powders of the precursor compounds, $\mathrm{CaCO}_{3}$ (Alfa Aesar, 99.95\%), $\mathrm{SrCO}_{3}$ (Alfa Aesar, 99.95\%), $\mathrm{Fe}_{2} \mathrm{O}_{3}$ (Alfa Aesar,99.998\%) and $\mathrm{Ga}_{2} \mathrm{O}_{3}$ (Sigma Aldrich,99.99\%) were mixed in stoichiometric proportions and ground together using an agate mortar and pestle, pressed into a pellet, and calcined in air at $1000{ }^{\circ} \mathrm{C}$ for $24 \mathrm{~h}$. The samples were then reground and sintered at $1200{ }^{\circ} \mathrm{C}$ for $24 \mathrm{~h}$ in the same environment, followed by slow cooling. The heating and cooling rates were $100{ }^{\circ} \mathrm{C} / \mathrm{h}$. The phase purity and structure of the polycrystalline samples were determined by powder X-ray diffraction

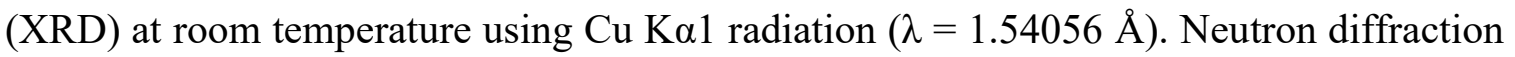
experiments were performed on POWGEN beamline at Oak Ridge National Laboratory, 
with center wavelength of $0.7 \AA$. The GSAS software ${ }^{100}$ and EXPGUI interface ${ }^{101}$ were used for Rietveld refinements. The microstructures were studied using high resolution field-emission scanning electron microscopy (SEM). X-ray photoelectron spectroscopy (XPS) was performed at room temperature using $\mathrm{Al} \mathrm{K} \alpha$ radiation $(1486.7 \mathrm{eV})$ to study the oxidation states of Fe. The electrical properties were investigated by direct-current (DC) and alternating-current (AC) methods, on samples that had been sintered at $1200{ }^{\circ} \mathrm{C}$. The $\mathrm{AC}$ electrochemical impedance spectroscopy measurements were done in the frequency range $0.1 \mathrm{~Hz}$ to $1 \mathrm{MHz}$ using a computer-controlled frequency response analyzer. The DC measurements were performed at constant voltage of $10 \mathrm{mV}$, in the temperature range 25

$-800{ }^{\circ} \mathrm{C}$. The conductivity measurements were performed at $100{ }^{\circ} \mathrm{C}$ intervals, and equilibrium was reached at each temperature of measurement before moving to the next temperature. The rate of heating between measurement-temperatures was $3{ }^{\circ} \mathrm{C} / \mathrm{min}$. Iodometric titrations were performed by dissolving about $50 \mathrm{mg}$ of the sample and excess $\mathrm{KI}(\sim 2 \mathrm{~g})$ in $100 \mathrm{~mL}$ of $1 \mathrm{M} \mathrm{HCl}$. A total of $5 \mathrm{~mL}$ of the solution was then pipetted out, and the iodine that had been generated in the solution was titrated against $0.025 \mathrm{M} \mathrm{Na}_{2} \mathrm{~S}_{2} \mathrm{O}_{3}$. Near the end point of the titration, $0.2 \mathrm{~mL}$ of a starch solution was added to act as an indicator. All steps were performed under argon atmosphere.

\section{RESULTS AND DISCUSSION}

\subsection{Crystal structure}

Structural characterizations involved Rietveld refinements with powder X-ray and neutron diffraction data based on different crystallographic models. Both $\mathrm{Ca}_{2} \mathrm{FeGaO}_{6-\delta}$ and $\mathrm{CaSrFeGaO}_{6-\delta}$ are defect-ordered oxygen-deficient perovskites with brownmillerite-type 
structure. X-ray diffraction data for $\mathrm{Ca}_{2} \mathrm{FeGaO}_{6-\delta}$ has been reported, ${ }^{149}$ showing Pcmn (Pnma) space group. Here, we study the crystal structures using neutron diffraction, and show the structural transformation upon changing the average ionic-radius on the A-site. Brownmillerite compounds usually crystalize in one of the four space groups Imma, Ibm2, Pnma, or $\mathrm{Pbcm},{ }^{4,72,84}$ depending on the relative orientation of tetrahedral chains. There are two possible chain-orientations, which are arbitrarily called right-handed and left-handed. If the tetrahedral chains are oriented randomly relative to each other, the space group will be Imma $(\mathrm{Icmm}) .{ }^{150}$ If all tetrahedral chains have the same orientation in all layers, the space group will be $\operatorname{Ibm} 2$ (Ima2). ${ }^{4,72,84}$ If the tetrahedral chains have the same orientation within each layer but are oriented opposite to the chains in the next tetrahedral layer, the space group will be Pnma. ${ }^{4,72,84}$ Finally, if each tetrahedral chain is oriented opposite to its nearest neighbors within the same layer and the next layer, the space group will be Pbcm. $4,72,74,84$

The two primitive space groups are readily identifiable by their characteristic peaks. Space group $\mathrm{Pbcm}$ can be easily distinguished from others due to the presence of superstructure peaks in its powder diffraction data, considering that its unit cell is double the size of those of the other three space groups. ${ }^{4,72,74,84}$ Space group Pnma is recognized by the presence of 131 and 151 peaks, which are absent in the body-centered systems. The 131 peak is 


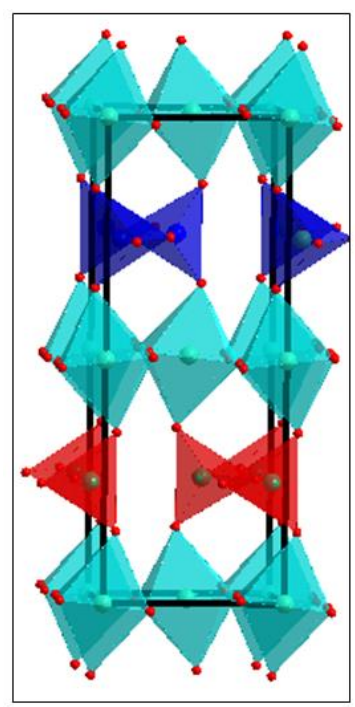

(a)

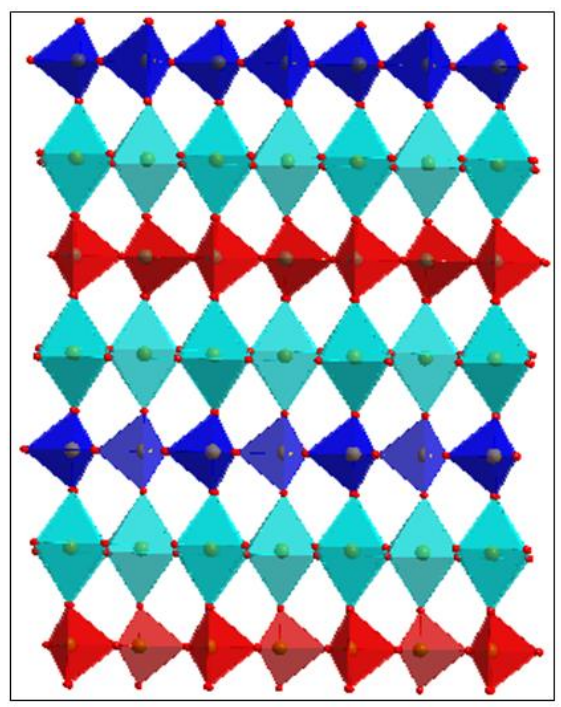

(b)

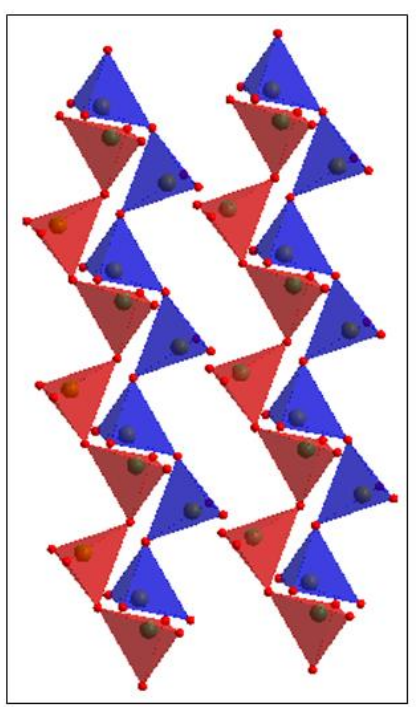

(c)

Figure 7.3. (a) Unit cell of $\mathrm{Ca}_{2} \mathrm{FeGaO}_{6-\delta}$, Pnma. (b) Alternating octahedral and tetrahedral coordination geometry in $\mathrm{Ca}_{2} \mathrm{FeGaO}_{6-\delta}$. The $\mathrm{Ca}$ atoms, located in spaces between polyhedra, are omitted for clarity. (c) Orientation of tetrahedral chains in two neighboring layers, which are opposite to each other in $\mathrm{Ca}_{2} \mathrm{FeGaO}_{6-\delta}$ (top view). The tetrahedral sites are primarily occupied by $\mathrm{Ga}$ and octahedral sites by Fe. The red and blue colors represent the two different orientations of tetrahedral chains.

particularly prominent. ${ }^{4,} 72,84 \mathrm{We}$ examined all four space groups in our Rietveld refinements. All peaks can be indexed to orthorhombic unit cell for both compounds. The Pbcm space group was readily ruled out for both materials, as the superstructure peaks associated with this space group were absent. For $\mathrm{Ca}_{2} \mathrm{FeGaO}_{6-\delta}$, the presence of the 131 peak indicted that the space group should be primitive Pnma, which was confirmed by Rietveld refinements with both X-ray (Figure 7.1a) and neutron diffraction data (Figure 7.2a). These refinements also showed that the octahedral sites are primarily occupied by 


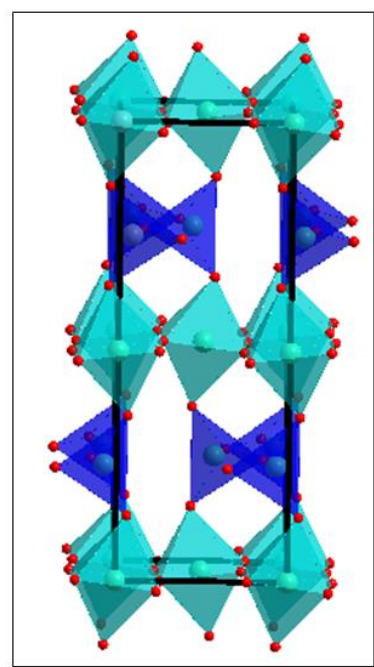

(a)

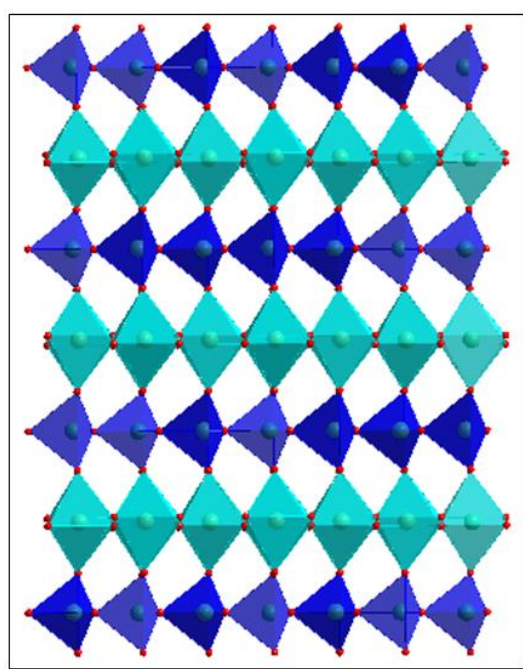

(b)

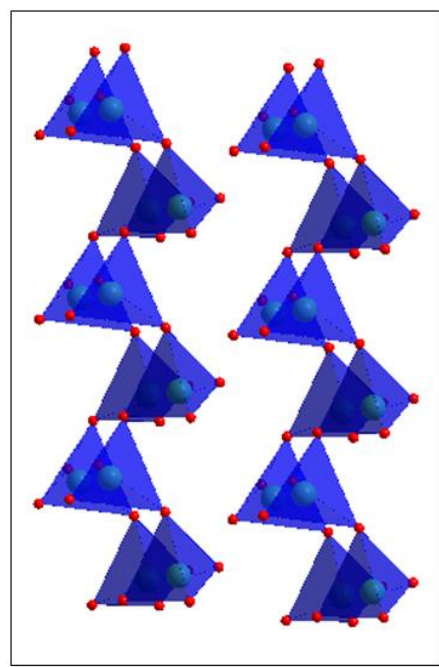

(c)

Figure 7.4. (a) Unit cell of $\mathrm{CaSrFeGaO}_{6-\delta}, \mathrm{Ibm2}$. (b) Alternating octahedral and tetrahedral coordination geometry in $\mathrm{CaSrFeGaO}_{6-\delta}$. The $\mathrm{Ca} / \mathrm{Sr}$ atoms, located in spaces between polyhedra, are omitted for clarity. (c) Orientation of tetrahedral chains in two neighboring layers (top view). All tetrahedral chains have the same orientation in $\mathrm{CaSrFeGaO}_{6-\delta}$.
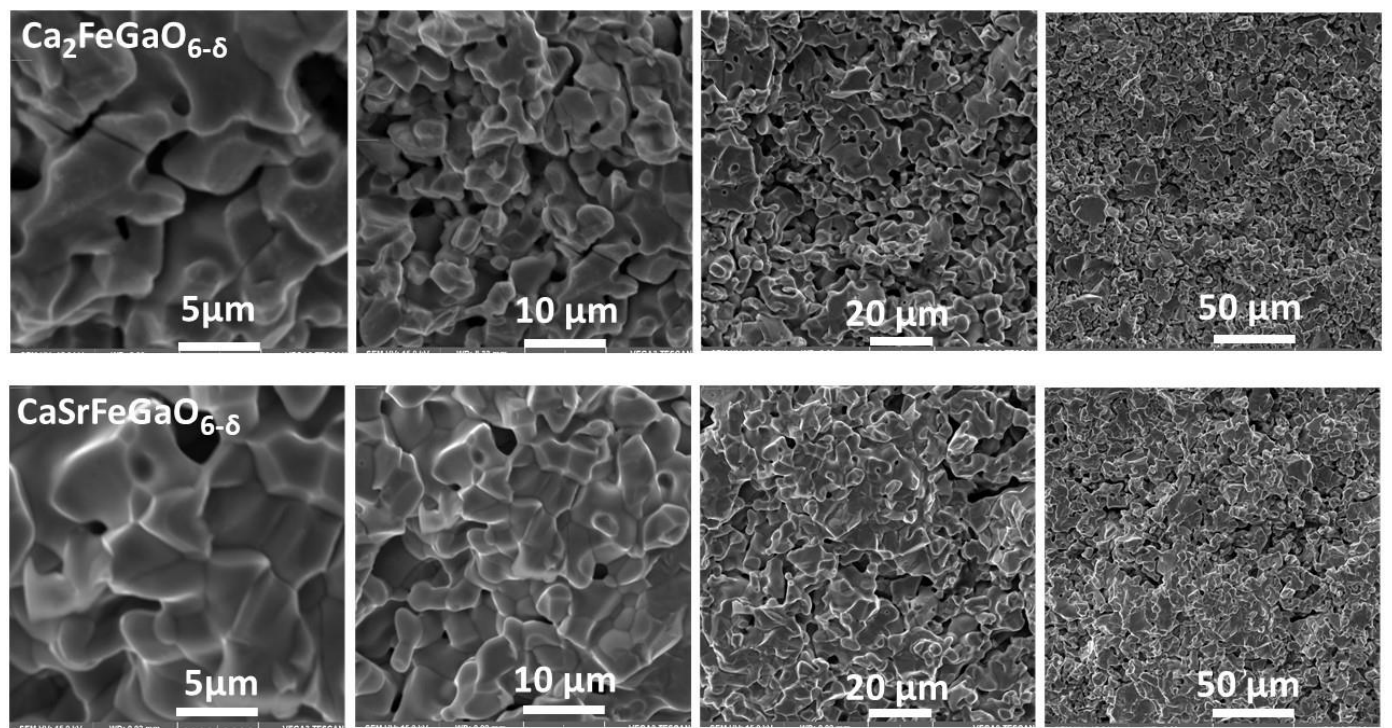

Figure 7.5. Scanning electron microscopy images of $\mathrm{Ca}_{2} \mathrm{FeGaO}_{6-\delta}$ and $\mathrm{CaSrFeGaO}_{6-\delta}$. 
Fe and tetrahedral sites by Ga. Table 7.1 shows the refined structural parameters for $\mathrm{Ca}_{2} \mathrm{FeGaO}_{6-\delta}$.

Interestingly, $\mathrm{CaSrFeGaO}_{6-\delta}$ has a different structure, where the superstructure peaks of Pbcm, and the 131 peak of Pnma are absent, indicating the formation of a body-centered structure. Among the body-centered space groups, Imma and Ibm2, the latter showed the best fit for this material. The Rietveld refinement profiles for X-ray and neutron diffraction data of $\mathrm{CaSrFeGaO}_{6-\delta}$ are shown in Figures 7.1b and 7.2b, respectively. The distribution of $\mathrm{Fe}$ and $\mathrm{Ga}$ was also determined from neutron diffraction, which indicated that Fe mostly occupies the octahedral positions, while Ga resides mainly on the tetrahedral sites. Table 7.2 shows the refined structural parameters for $\mathrm{CaSrFeGaO}_{6-\delta}$.

These results demonstrate the important effect of the average ionic radius of the A-site cations on the orientation of tetrahedral chains. Smaller ionic radius in $\mathrm{Ca}_{2} \mathrm{FeGaO}_{6-\delta}$ favors a more ordered arrangement, where tetrahedral chains in each layer are oriented opposite to the neighboring layers (Figure 7.3), leading to a centrosymmetric structure. Whereas, larger ionic radius in $\mathrm{CaSrFeGaO}_{6-\delta}$ leads to a non-centrosymmetric structure, where all tetrahedral chains have the same orientation (Figure 7.4).

\subsection{Microstructure and X-ray photoelectron spectroscopy studies}

The microstructure and crystallite size for $\mathrm{Ca}_{2} \mathrm{FeGaO}_{6-\delta}$ and $\mathrm{CaSrFeGaO}_{6-\delta}$ were studied using scanning electron microscopy (SEM). Figure 7.5 shows the images of sintered pellets for both materials. As observed in this figure, the two compounds contain crystallites with similar size and similar degree of contact. The SEM images also show the uniformity and homogeneity of the crystallites in both $\mathrm{Ca}_{2} \mathrm{FeGaO}_{6-\delta}$ and $\mathrm{CaSrFeGaO}_{6-\delta}$. 

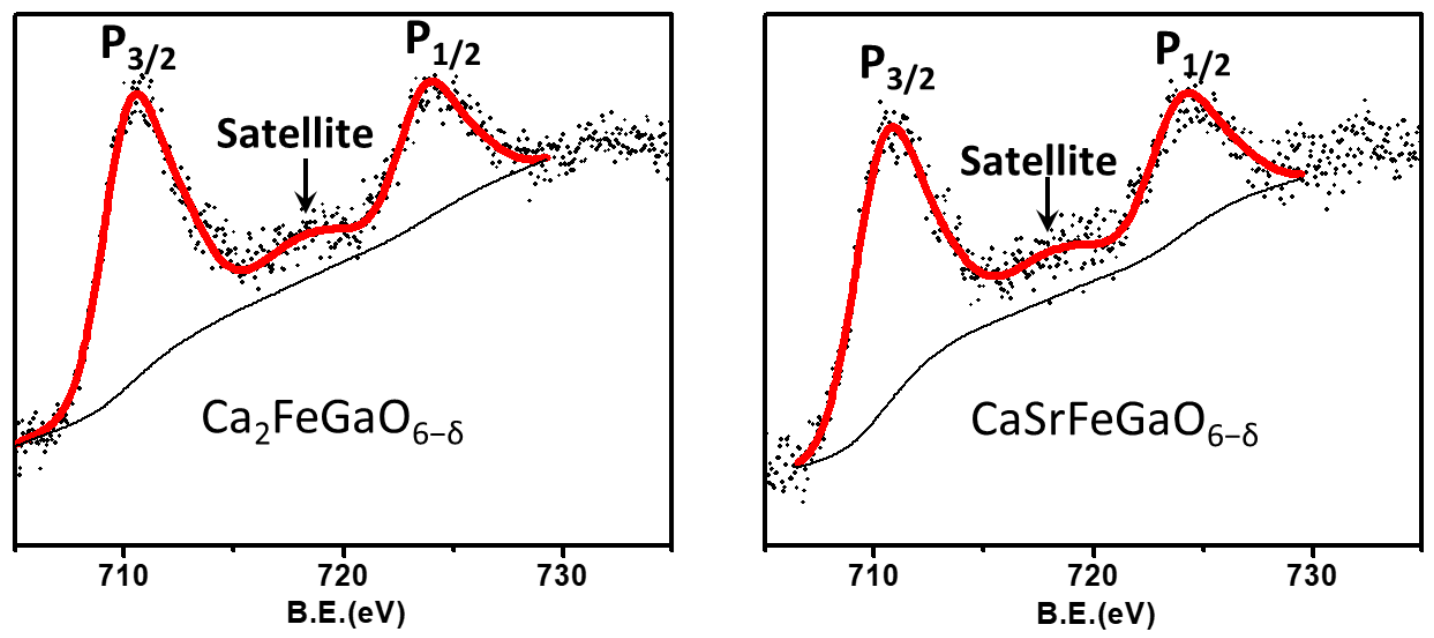

Figure 7.6. X-ray photoelectron spectroscopy data for $\mathrm{Ca}_{2} \mathrm{FeGaO}_{6-\delta}$ and $\mathrm{CaSrFeGaO}_{6-\delta}$. The data for both materials show the same binding energies for Fe peaks.

We have also performed X-ray photoelectron spectroscopy (XPS) to investigate the oxidation state of iron in both compounds. It should be noted that these materials were synthesized in air using $\mathrm{Fe}_{2} \mathrm{O}_{3}$ as starting material. Therefore, $\mathrm{Fe}$ is expected to be trivalent, but the possibility of its oxidation in air should also be considered. The position of the $2 \mathrm{P}_{3 / 2}$ peaks as well as satellite peaks are indicative of the oxidation states. The $2 \mathrm{P}_{3 / 2}$ peak for trivalent $\mathrm{Fe}$ is expected to appear at about $710-711.5 \mathrm{eV}$ followed by its satellite peak at about $8-9 \mathrm{eV}$ higher in binding energy. ${ }^{43-44,104-105}$ The satellite peak position is particularly important, as it is the signature of trivalent Fe. The XPS data are shown in Figure 7.6. For both $\mathrm{Ca}_{2} \mathrm{FeGaO}_{6-\delta}$ and $\mathrm{CaSrFeGaO}_{6-\delta}$, the $2 \mathrm{P}_{3 / 2}$ main peak appears at $710.6 \mathrm{eV}$ followed by a satellite peak at about $8.6 \mathrm{eV}$ higher in energy, namely at $719.2 \mathrm{eV}$. This indicates that Fe is in trivalent state in both compounds. However, even without the satellite peaks, which can sometimes have low intensity and be hard to detect, it is evident that tetravalent $\mathrm{Fe}$ in these compounds can be ruled out, as it should show the $2 \mathrm{P}_{3 / 2}$ peaks at $\sim 712-713 \mathrm{eV} .{ }^{43-44}$, 
104-105 We also performed iodometric titration analysis to confirm the results of XPS measurements. These analyses showed $\delta \approx 1$ for both $\mathrm{CaSrFeGaO}_{6-\delta}$ and $\mathrm{Ca}_{2} \mathrm{FeGaO}_{6-\delta}$, consistent with primarily trivalent state for Fe, as also demonstrated by XPS. The degree of oxygen deficiency indicates that the oxygen stoichiometry is very close to that expected for ideal brownmillerite, i.e. 5 oxygens per formula unit.

\subsection{Electrical properties}

The electrical conductivities of these materials were investigated in air by both direct current (DC) and alternating current (AC) methods. In DC technique, the Ohm's law is employed to obtain the resistance $(\mathrm{R})$ using the output current upon applying a certain voltage. For AC impedance spectroscopy, ${ }^{151}$ the total resistance is measured from the intercept with the real axis $\left(Z^{\prime}\right)$ of the Nyquist plot at low frequency. Figure 7.7 shows a representative example of the Nyquist plot and the corresponding fit. The resistance values from both DC and AC methods are similar. The resistance values (R) obtained using the above methods are then used to calculate the conductivity $(\sigma)$ using the following equation:

$$
\sigma=L / R A
$$

where $\mathrm{L}$ and A represent the thickness and cross-sectional area of the sample, respectively. The room temperature conductivity values, listed in Table 7.3, reveal the interesting effect of the structural order on electrical conductivity. There is an inverse correlation between the degree of order and the magnitude of conductivity. $\mathrm{CaSrFeGaO}_{6-\delta}$, in which all tetrahedral chains have the same orientation, exhibits significantly greater conductivity than $\mathrm{Ca}_{2} \mathrm{FeGaO}_{6-\delta}$, where the tetrahedral chains have alternating orientations in neighboring layers. 


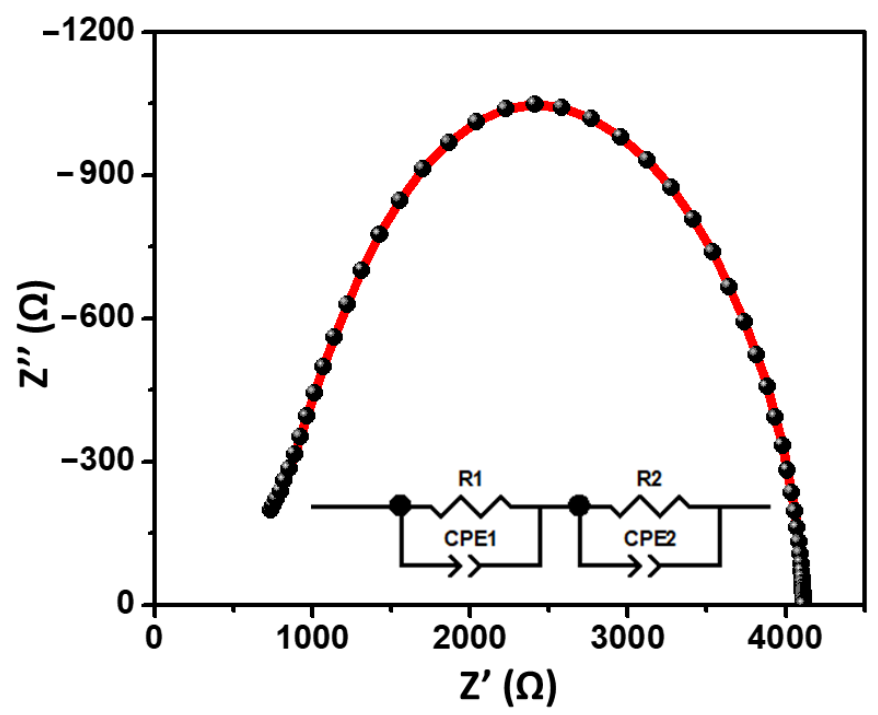

Figure 7.7. Representative example of the Nyquist plot for $\mathrm{CaSrFeGaO}_{6-\delta}$ at $25{ }^{\circ} \mathrm{C}$. The semicircle can be fitted using two resistance-capacitance (RC) units, corresponding to the bulk $\left(\mathrm{R} 1=766 \Omega, \mathrm{CPE} 1=2.6 \times 10^{-8} \mathrm{~F}\right)$ and grain boundary $(\mathrm{R} 2=3397 \Omega, \mathrm{CPE} 2=1.3 \times$ $\left.10^{-7} \mathrm{~F}\right)$.

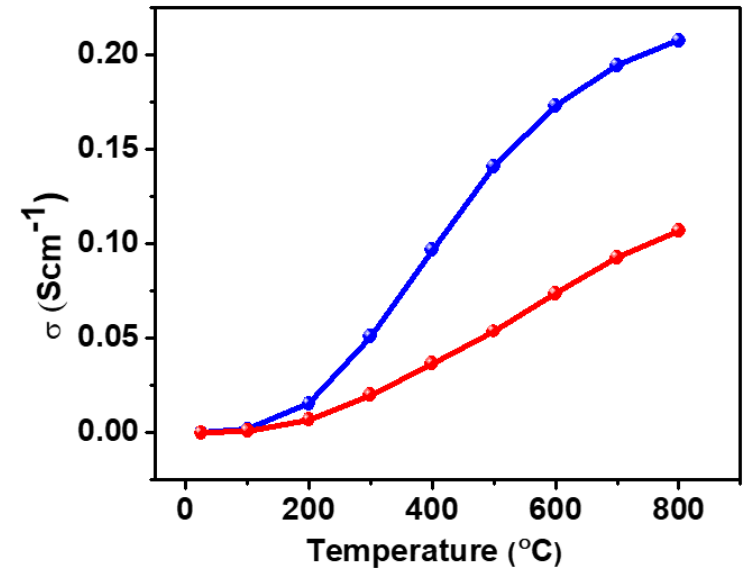

(a)

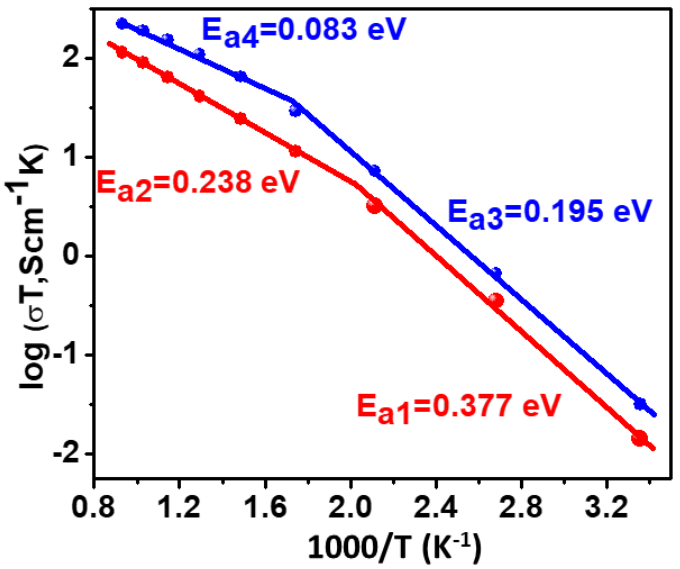

(b)

Figure 7.8. (a) Electrical conductivity of $\mathrm{Ca}_{2} \mathrm{FeGaO}_{6-\delta}$ (red) and $\mathrm{CaSrFeGaO}_{6-\delta}$ (blue) as a function of temperature. (b) Arrhenius plots for electrical conductivity of $\mathrm{Ca}_{2} \mathrm{FeGaO}_{6-\delta}$ (red) and $\mathrm{CaSrFeGaO}_{6-\delta}$ (blue). 
The conductivity mechanism in oxygen-deficient perovskites is based on the hopping of charge carriers through metal-oxygen-metal (M-O-M) pathways. ${ }^{108-109,}$ 152-153 Oxygen absorption or desorption leads to the formation of conduction pathways such as $\mathrm{M}^{2+}-\mathrm{O}-$ $\mathrm{M}^{3+}$ and $\mathrm{M}^{3+}-\mathrm{O}-\mathrm{M}^{4+}$, i.e., small polarons, as observed in other oxygen-deficient perovskites. ${ }^{9}$ 109, $154-156$ The presence of elements with multiple stable oxidation states are needed for the formation of small polarons. ${ }^{9}$ Both $\mathrm{Ca}_{2} \mathrm{FeGaO}_{6-\delta}$ and $\mathrm{CaSrFeGaO}_{6-\delta}$ have $\mathrm{Fe}$ and Ga on B-site, where only Fe can have flexible oxidation states. During the conduction process through $\mathrm{M}^{\mathrm{m}+}-\mathrm{O}-\mathrm{M}^{\mathrm{n}+}$ pathway, the charge carriers hop between $\mathrm{M}^{\mathrm{m}+}$ and $\mathrm{M}^{\mathrm{n}+}$, resulting in change of oxidation states of cations. ${ }^{152-153}$

The degree of electrical conductivity depends strongly on the structural parameters, such as the $\mathrm{M}-\mathrm{O}$ bond lengths and $\mathrm{M}-\mathrm{O}-\mathrm{M}$ bond angles. Higher conductivity is usually associated with shorter M-O distances or larger M-O-M bond angles. For example, the changes in electrical conductivity of $\mathrm{La}_{1-\mathrm{x}} \mathrm{Sr}_{\mathrm{x}} \mathrm{CoO}_{3}$ correlate with variation in bond angles. ${ }^{21,157} \mathrm{~A}$ change from semiconductivity to metallic conductivity in $\mathrm{La}_{1-\mathrm{x}} \mathrm{Sr}_{\mathrm{x}} \mathrm{CoO}_{3}$ has been observed at $\mathrm{x}=0.25$, where there is an abrupt increase in the $\mathrm{Co}-\mathrm{O}-\mathrm{Co}$ bond angle. ${ }^{21}$, ${ }^{157}$ For our materials, both $\mathrm{Ca}_{2} \mathrm{FeGaO}_{6-\delta}$ and $\mathrm{CaSrFeGaO}_{6-\delta}$, have similar oxygen content, as well as similar oxidation state for Fe, as shown by XPS and iodometric titrations. The greater electrical conductivity of $\mathrm{CaSrFeGaO}_{6-\delta}$ can be due to larger bond angles in this compound, which enhance the hopping of charge carriers through M-O-M conduction pathways. In $\mathrm{CaSrFeGaO}_{6-\delta}$, the $\mathrm{M}-\mathrm{O}-\mathrm{M}$ bond angles are $127.6(2)^{\circ}$ (between two tetrahedral sites), $143.1(1)^{\circ}$ (between a tetrahedral and an octahedral site), and $170.68(8)^{\circ}$ 
Table 7.1. Refined structural parameters for $\mathrm{Ca}_{2} \mathrm{FeGaO}_{6-\delta}$ from powder neutron diffraction. Space group Pnma, $a=5.38903(8) \AA, b=14.6517(2) \AA, c=5.60081(8) \AA, R_{p}=0.0400$, $w R_{p}=0.0209$.

\begin{tabular}{ccccccc}
\hline Elements & $\mathrm{x}$ & $\mathrm{y}$ & $\mathrm{z}$ & Occupancy & $\mathrm{U}_{\text {iso }}$ & $\begin{array}{c}\text { Multip } \\
\text { licity }\end{array}$ \\
\hline $\mathrm{Ca}$ & $0.0178(2)$ & $0.10796(6)$ & $0.5255(2)$ & 1 & $0.0018(1)$ & 8 \\
$\mathrm{Ga} 1$ & $0.0496(2)$ & 0.25 & $0.0682(2)$ & $0.82(1)$ & $0.0011(1)$ & 4 \\
$\mathrm{Fe} 1$ & $0.0496(2)$ & 0.25 & $0.0682(2)$ & $0.18(1)$ & $0.0011(1)$ & 4 \\
$\mathrm{Ga} 2$ & 0.0 & 0.0 & 0.0 & $0.18(1)$ & $0.00114(7)$ & 4 \\
$\mathrm{Fe} 2$ & 0.0 & 0.0 & 0.0 & $0.82(1)$ & $0.00114(7)$ & 4 \\
$\mathrm{O} 1$ & $0.3979(3)$ & 0.25 & $0.1275(2)$ & 1 & $0.0030(2)$ & 4 \\
$\mathrm{O} 2$ & $0.0279(2)$ & $0.64164(6)$ & $0.0733(2)$ & 1 & $0.0035(1)$ & 8 \\
$\mathrm{O} 3$ & $0.2599(2)$ & $0.01528(6)$ & $0.2401(2)$ & 1 & $0.00275(9)$ & 8
\end{tabular}

(between two octahedral sites). The corresponding angles in $\mathrm{Ca}_{2} \mathrm{FeGaO}_{6-\delta}$ are smaller, $125.65(8)^{\circ}, 138.75(5)^{\circ}$ and $166.11(5)^{\circ}$, respectively.

The electrical conductivity for $\mathrm{Ca}_{2} \mathrm{FeGaO}_{6-\delta}$ and $\mathrm{CaSrFeGaO}_{6-\delta}$ were also investigated at variable temperatures from $25{ }^{\circ} \mathrm{C}$ to $800{ }^{\circ} \mathrm{C}$. Figure 7.8 shows the conductivity data at different temperatures. For both materials, there is an increase in conductivity as a function of temperature, indicating the semiconducting nature of these compounds. A visible rise in conductivity begins above $100{ }^{\circ} \mathrm{C}$, where a slow increase is observed up to $200{ }^{\circ} \mathrm{C}$, followed by a steep increase above this temperature. The increase in conductivity of 
Table 7.2. Refined structural parameters for $\mathrm{CaSrFeGaO}_{6-\delta}$ from powder neutron diffraction. Space group Ibm2, $a=5.6437(1) \AA, b=15.0577(4) \AA, c=5.4458(1) \AA, R_{p}=$ $0.0450, w R_{p}=0.0216$.

\begin{tabular}{ccccccc}
\hline $\begin{array}{c}\text { Elem } \\
\text { ents }\end{array}$ & $\mathrm{x}$ & $\mathrm{y}$ & $\mathrm{z}$ & $\begin{array}{c}\text { Occupa } \\
\text { ncy }\end{array}$ & $\mathrm{U}_{\text {iso }}$ & $\begin{array}{c}\text { Multip } \\
\text { licity }\end{array}$ \\
\hline $\mathrm{Ca}$ & $0.5202(3)$ & $0.10910(9)$ & $0.0076(5)$ & 0.5 & $0.0041(2)$ & 8 \\
$\mathrm{Sr}$ & $0.5202(3)$ & $0.10910(9)$ & $0.0076(5)$ & 0.5 & $0.0041(2)$ & 8 \\
$\mathrm{Ga} 1$ & $0.0713(3)$ & 0.25 & $0.0429(4)$ & $0.79(4)$ & $0.0020(3)$ & 4 \\
$\mathrm{Fe} 1$ & $0.0713(3)$ & 0.25 & $0.0429(4)$ & $0.21(4)$ & $0.0020(3)$ & 4 \\
$\mathrm{Ga} 2$ & 0.0 & 0.0 & 0.0 & $0.20(3)$ & $0.019(2)$ & 4 \\
$\mathrm{Fe} 2$ & 0.0 & 0.0 & 0.0 & $0.80(3)$ & $0.019(2)$ & 4 \\
$\mathrm{O} 1$ & $0.7545(4)$ & $-0.01045(9)$ & $0.2526(5)$ & 1 & $0.0029(2)$ & 4 \\
$\mathrm{O} 2$ & $-0.0618(3)$ & $0.1416(1)$ & $-0.0237(5)$ & 1 & $0.0063(3)$ & 8 \\
$\mathrm{O} 3$ & $0.3685(6)$ & 0.25 & $0.8843(7)$ & 1 & $0.0077(4)$ & 8 \\
\hline
\end{tabular}

$\mathrm{CaSrFeGaO}_{6-\delta}$ is much sharper compared to that of $\mathrm{Ca}_{2} \mathrm{FeGaO}_{6-\delta}$. The rise in temperature leads to an increase in the mobility of polarons. This temperature-activated mobility results in enhanced electrical conductivity, ${ }^{50}$ according to the following relation

$$
\sigma=\text { ne } \mu \quad(21)
$$

where $\sigma, \mathrm{n}, \mathrm{e}$, and $\mu$ are the conductivity, concentration of electrons/holes, charge of the electron, and mobility of the charge carriers, respectively. An additional contribution can be the rise in ionic conductivity due to the increase in temperature. Some studies ${ }^{145}$ indicate that oxide ion transport in perovskites starts at $\sim 500{ }^{\circ} \mathrm{C}$, although some reports suggest that it can also occur at lower temperatures. ${ }^{132,146}$ However, it has been shown that at high oxygen partial-pressure, such as in atmospheric air, the electronic conductivity can be dominant over the ionic conductivity in perovskite-based systems. ${ }^{9}, 23,58-59,138,141$ 
The activation energy for the increase in conductivity as a function of temperature can be obtained using Arrhenius equation for thermally activated conductivity ${ }^{49-51}$ as follows:

$$
\sigma T=\sigma^{0} e^{\frac{-E_{a}}{K T}}
$$

where $\sigma^{\circ}$ is a pre-exponential factor and characteristic of the material. $\mathrm{E}_{\mathrm{a}}, \mathrm{K}$, and $\mathrm{T}$ are the activation energy for the electrical conductivity, Boltzmann constant, and absolute temperature, respectively. The activation energy $\left(\mathrm{E}_{\mathrm{a}}\right)$ can be calculated from the slope of the line of best fit in the $\log \sigma \mathrm{T}$ versus $1000 / \mathrm{T}$ plot. Figure $7.8 \mathrm{~b}$ shows the Arrhenius plot for both materials in the temperature range $25^{\circ} \mathrm{C}-800{ }^{\circ} \mathrm{C}(298 \mathrm{~K}-1073 \mathrm{~K})$. The activation energies are lower for $\mathrm{CaSrFeGaO}_{6-\delta}$ compared to $\mathrm{Ca}_{2} \mathrm{FeGaO}_{6-\delta}$, as shown in Table 7.3. The sharp differences between the electrical properties of these two materials indicate the pronounced impact of structural order on charge-transport in oxygen-deficient perovskites.

Table 7.3. Room Temperature Conductivity and Activation Energies.

\begin{tabular}{|c|c|c|c|}
\hline \multirow[b]{2}{*}{ compounds } & \multicolumn{2}{|c|}{ total conductivity, $\sigma\left(\mathrm{S} \mathrm{cm}^{-1}\right)$} & \multirow[b]{2}{*}{ Activation energy, $E_{a}(e V)$} \\
\hline & $\mathrm{AC}$ & $\mathrm{DC}$ & \\
\hline \multirow[t]{2}{*}{$\mathrm{CaSrFeGaO}_{5}$} & $1.00 \times 10^{-4}$ & $1.07 \times 10^{-4}$ & $0.195 \mathrm{eV}$ for 25 to $300^{\circ} \mathrm{C}$ \\
\hline & & & $0.083 \mathrm{eV}$ for 300 to $800^{\circ} \mathrm{C}$ \\
\hline \multirow[t]{2}{*}{$\mathrm{Ca}_{2} \mathrm{FeGaO}_{5}$} & $4.80 \times 10^{-5}$ & $4.79 \times 10^{-5}$ & $0.377 \mathrm{eV}$ for 25 to $220^{\circ} \mathrm{C}$ \\
\hline & & & $0.238 \mathrm{eV}$ for 220 to $800^{\circ} \mathrm{C}$ \\
\hline
\end{tabular}




\section{CONCLUSIONS}

Subtle changes in defect-order can have a significant impact on the electrical conductivity of oxygen-deficient perovskites. Different distribution of defects in $\mathrm{CaSrFeGaO}_{6-\delta}$ leads to different orientation of tetrahedral chains compared to that in $\mathrm{Ca}_{2} \mathrm{FeGaO}_{6-\delta}$. Importantly, these changes lead to enhanced electrical conductivity in $\mathrm{CaSrFeGaO}_{6-\delta}$, which exhibits significantly greater conductivity than $\mathrm{Ca}_{2} \mathrm{FeGaO}_{6-\delta}$. Variable-temperature conductivity studies show that the enhanced electrical conductivity of $\mathrm{CaSrFeGaO}_{6-\delta}$ persists at high temperature, up to $800{ }^{\circ} \mathrm{C}$. The XPS and iodometric titration studies show the same degree of oxygen deficiency for both materials. They also indicate similar oxidation states of $\mathrm{Fe}$ in both compounds. Therefore, the difference in relative orientation of tetrahedral chains appears to be the main factor that leads to the changes in electrical conductivity between these materials. This indicates that controlling the structural parameters can be used as a tool to control the charge-transport properties of oxygen-deficient perovskites. 


\section{CHAPTER 8}

\section{DISPARITY IN ELECTRICAL AND MAGNETIC PROPERTIES OF ISOSTRUCTURAL OXYGEN DEFICIENT PEROVSKITES $\mathrm{BaSrCo}_{2} \mathrm{O}_{6-\delta}$ AND $\mathrm{BaSrCoFeO}_{6-\delta}{ }^{7}$}

\section{INTRODUCTION}

We have recently studied ${ }^{20,158}$ the structure-property relationships in a series of oxygen deficient perovskites (ODPs). These materials have general formula $\mathrm{ABO}_{3-\mathrm{x}}$ where $\mathrm{A}$ is usually an alkaline earth metal or lanthanide, and B is a smaller ion, typically a transition metal, although some main group elements could also reside on the B-site. The ODPs feature interesting properties, from superconductivity ${ }^{159}$, to magnetoresistant, ${ }^{160}$ and have been considered for different applications including gas sensors ${ }^{161}$, gas diffusion membranes for gas separation ${ }^{162}$, and electrodes in solid oxide fuel cells ${ }^{163}$. In typical perovskites, oxygen atoms form octahedral geometry around the $\mathrm{B}$-site metal, forming $\mathrm{BO}_{6}$ octahedra. In ODPs, the absence of some oxygen atoms from the structure creates vacancies which may lead to the formation of different coordination geometries, such as

${ }^{7}$ The work described in this chapter was published in Journal of Materials Science: Materials in Electronics ( 2018, vol. 29, p. 13464-13473) 
tetrahedral $\left(\mathrm{BO}_{4}\right)$ or square pyramidal $\left(\mathrm{BO}_{5}\right){ }^{20}$ Considering the various coordination geometries that can be formed due to the presence of oxygen vacancies, a high degree of structural diversity is observed in ODPs. ${ }^{11,72-76,164}$

Structural changes can sometimes occur through substitution on the A or B-sites. For example, the coordination around the B-site cation can change when the A site cation is substituted in $\mathrm{Sr}_{2-\mathrm{x}} \mathrm{Ca}_{\mathrm{x}} \mathrm{Fe}_{2} \mathrm{O}_{6-\delta} .{ }^{20} \mathrm{Here}, \mathrm{Sr}_{2} \mathrm{Fe}_{2} \mathrm{O}_{6-\delta}$ has a tetragonal structure and contains alternating octahedral and square pyramidal coordination of the B-site cations. When one $\mathrm{Sr}$ is substituted by $\mathrm{Ca}$, i.e., $\mathrm{SrCaFe}_{2} \mathrm{O}_{6-\delta}$, the structure changes into orthorhombic, and the coordination geometry transforms into alternating tetrahedral and octahedral. ${ }^{20}$ These structural changes lead to the transformation of electrical properties from metallic to semiconductor. The magnetic structure also changes, where the spin-density wave state in $\mathrm{Sr}_{2} \mathrm{Fe}_{2} \mathrm{O}_{6-\delta}$ is converted into long-range G-type antiferromagnetic order in $\mathrm{SrCaFe}_{2} \mathrm{O}_{6-\delta}{ }^{20}$ Similar changes in structure and electrical conductivity as a result of substitution on the Asite have been observed for $\mathrm{Sr}_{2-\mathrm{x}} \mathrm{Ca}_{\mathrm{x}} \mathrm{FeCoO}_{6-\delta}{ }^{20}$

Substitution on the B-site can also lead to changes in crystal structure and material properties. For example, the above mentioned tetragonal compound, $\mathrm{Sr}_{2} \mathrm{Fe}_{2} \mathrm{O}_{6-\delta}$, which has magnetic moments in spin-density wave state, can be modified by replacing one of the $\mathrm{Fe}$ atoms with $\mathrm{Mn}$. The resulting material, $\mathrm{Sr}_{2} \mathrm{FeMnO}_{6-\delta}$, has a cubic $\mathrm{Pm}-3 m$ structure and inhomogeneous magnetic ground state, where the majority of the sample contains fluctuating spins at $4 \mathrm{~K}$, but a small fraction is magnetically ordered. ${ }^{11}$ Even minor changes to the B-site cations can sometimes lead to major changes, as highlighted by the difference between $\mathrm{Sr}_{2} \mathrm{Fe}_{1.9} \mathrm{Cr}_{0.1} \mathrm{O}_{6-\delta}$, cubic Pm-3m, and $\mathrm{Sr}_{2} \mathrm{Fe}_{1.9} \mathrm{Co}_{0.1} \mathrm{O}_{6-\delta}$, orthorhombic Cmmm. ${ }^{164}$ Again this leads to significant variation of magnetic properties in these two materials. ${ }^{164}$ 
The above examples describe changes in material properties as a consequence of structural modifications due to B-site cation substitution. However, circumstances where cation substitution leads to isostructural materials, which have significantly different electrical and magnetic properties are less common. In the present work, two isostructural oxygen deficient perovskites, $\mathrm{BaSrCo}_{2} \mathrm{O}_{6-\delta}(\delta=1.35)$ and $\mathrm{BaSrCoFeO}_{6-\delta}(\delta=0.73)$, have been investigated and the significant contrast between their electrical and magnetic properties has been demonstrated. Cubic, Pm-3m structure has been reported for a material with similar composition to $\mathrm{BaSrCo}_{2} \mathrm{O}_{6-\delta}(\delta=1.35)$, but with greater oxygen content. Also, some information has been reported on bulk magnetization and electrical conductivity in the temperature span of $\sim 135$ degrees Celsius. ${ }^{165} \mathrm{BaSrCo}_{2} \mathrm{O}_{6-\delta}$ discussed in the current manuscript has about $18 \%$ more vacancies, and has been investigated in detail using X-ray photoelectron spectroscopy, SEM, bulk magnetization, and DC and AC electrical conductivity studies in a wide temperature range, $25^{\circ} \mathrm{C}$ to $900{ }^{\circ} \mathrm{C}$. Regarding $\mathrm{BaSrCoFeO}_{6}$ $\delta$, its crystal structure has been reported to be cubic $P m-3 m,{ }^{166}$ but no information is available on magnetism and electrical conductivity of this material. In this article, an array of characterization methods has been employed to show remarkable differences between the two isostructural materials, $\mathrm{BaSrCo}_{2} \mathrm{O}_{6-\delta}$ and $\mathrm{BaSrCoFeO}_{6-\delta}$, due to the difference in their oxygen stoichiometry. Most significantly, the disparities between electrical properties and temperature-dependent electrical conductivities are reported here.

\section{EXPERIMENTAL}

Syntheses at different temperatures and under argon or air atmosphere were attempted. The powders of precursors $\mathrm{BaCO}_{3}$ (Alfa Aesar, 99.95\%), $\mathrm{SrCO}_{3}$ (Aldrich, 99.9\%), $\mathrm{Fe}_{2} \mathrm{O}_{3}$ (Alfa Aesar,99.998\%), and $\mathrm{Co}_{3} \mathrm{O}_{4}$ (Alfa Aesar, 99.7\%) were mixed and ground together using 
an agate mortar and pestle, pressed into a pellet, and heated at $1100{ }^{\circ} \mathrm{C}$ for $24 \mathrm{~h}$. Then the samples were reground and refired at $1100{ }^{\circ} \mathrm{C}$ for $24 \mathrm{~h}$, followed by slow cooling. In all cases, the heating and cooling rates were $100{ }^{\circ} \mathrm{C} / \mathrm{h} . \mathrm{BaSrCo}_{2} \mathrm{O}_{6-\delta}$ could only be synthesized in argon atmosphere, while $\mathrm{BaSrCoFeO}_{6-\delta}$ could only be made in air. Syntheses under other conditions led to the formation of multiphase products. The phase purity and structure of the polycrystalline samples were determined by powder X-ray diffraction (XRD) at room temperature using $\mathrm{Cu} \mathrm{K \alpha 1}$ radiation $(\lambda=1.54056 \AA)$. The GSAS software ${ }^{40}$ and EXPEGUI ${ }^{41}$ interface were used for Rietveld refinements. The sample morphologies were studied using high resolution field-emission scanning electron microscopy (SEM). X-ray photoelectron spectroscopy (XPS) was performed at room temperature using $\mathrm{Al} \mathrm{K} \alpha$ radiation $(1486.7 \mathrm{eV})$ to study the oxidation states of Fe and Co. The electrical properties were investigated by direct-current (DC) and alternating-current (AC) conductivity measurements on pressed pellets that had been sintered at $1100^{\circ} \mathrm{C}$. Magnetic susceptibility data were obtained by cooling each material to $2 \mathrm{~K}$, then applying magnetic field of 1000 Oe and measuring the magnetization up to $400 \mathrm{~K}$ to obtain the zero-field-cooled (ZFC) data. The process was then repeated by cooling the material in the presence of the field and measuring the magnetization to obtain the field-cooled (FC) data. Electrochemical impedance spectroscopy was performed in the frequency range of $0.1 \mathrm{~Hz}$ to $1 \mathrm{MHz}$ using a computer-controlled frequency response analyzer at room temperature. Two-probe dc measurements were performed in the temperature range $25-900^{\circ} \mathrm{C}$ by applying a constant voltage of $10 \mathrm{mV}$ and collecting the output current. Variable-temperature electrical conductivity measurements were carried out during both heating and cooling cycles. The rate of heating and cooling for conductivity measurements was $3{ }^{\circ} \mathrm{C} / \mathrm{min}$. Iodometric 
titrations were performed by dissolving about $50 \mathrm{mg}$ of the sample and excess $\mathrm{KI}(\sim 2 \mathrm{~g})$ in $100 \mathrm{~mL}$ of $1 \mathrm{M} \mathrm{HCl}$. A total of $5 \mathrm{~mL}$ of the solution was then pipetted out, and the iodine that had been generated in the solution was titrated against $0.025 \mathrm{M} \mathrm{Na}_{2} \mathrm{~S}_{2} \mathrm{O}_{3}$. Near the end point of the titration, $0.2 \mathrm{~mL}$ of a starch solution was added to act as an indicator. All steps were performed under argon atmosphere.

\section{RESULTS AND DISCUSSION}

Crystal structure and crystallite morphology

As mentioned in the experimental section, single phase cubic structure could only be obtained when syntheses were done in argon for $\mathrm{BaSrCo}_{2} \mathrm{O}_{6-\delta}$, and in air for $\mathrm{BaSrCoFeO}_{6}$ $\delta$. This led to the formation of isostructural phases that have different oxygen stoichiometry and presented a great opportunity to study the effect of oxygen content on material properties.

The crystal structures of these materials were characterized by powder X-ray diffraction (PXRD). Figure 8.1 shows the Rietveld refinement profiles for both materials, which have cubic structure with $P m-3 m$ space group as previously reported ${ }^{165-166}$. The refined unit cell parameters and atomic positions for both materials are listed in Tables 8.1 and 8.2. $\mathrm{BaSrCoFeO}_{6-\delta}$ has a slightly smaller unit cell, which is also evident from the systematic shift of X-ray diffraction peaks to the right, compared to the data for $\mathrm{BaSrCo}_{2} \mathrm{O}_{6-\delta}$. This is consistent with the cation oxidation states in the two materials. As described in the next section, $\mathrm{BaSrCoFeO}_{6-\delta}$ contains tri- and tetravalent $\mathrm{Fe}$ and $\mathrm{Co}$, whereas $\mathrm{BaSrCo}_{2} \mathrm{O}_{6-\delta}$

contains $\mathrm{Co}^{2+} / \mathrm{Co}^{3+}$. Therefore, the average ionic radius in the latter is greater than that in the former, leading to the difference in the unit cell dimensions. 


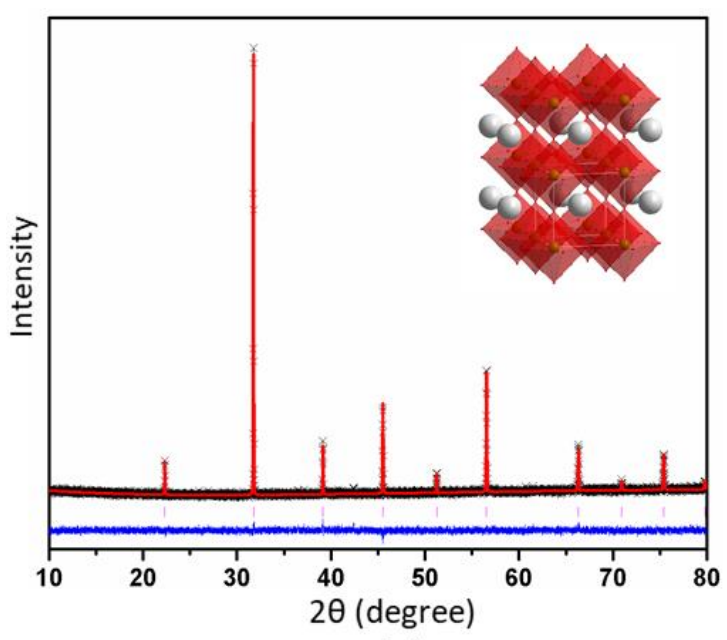

(a)

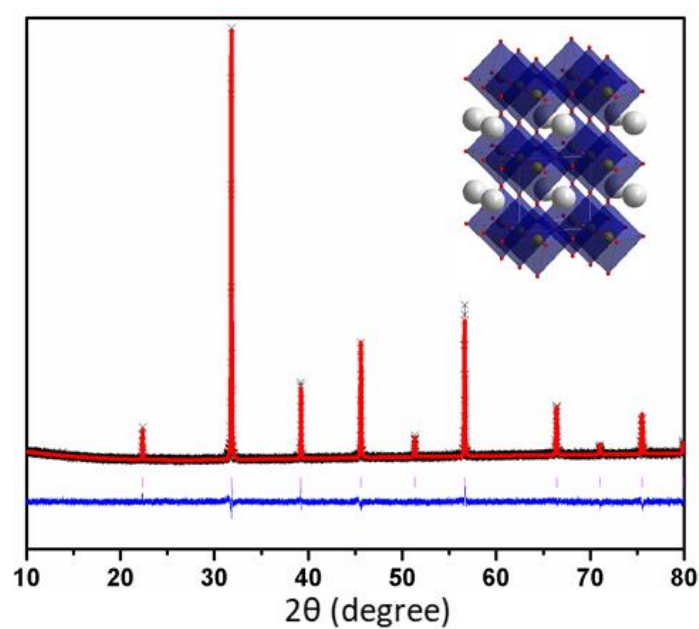

(b)

Figure 8.1. Rietveld refinement profile for powder XRD data of a) $\mathrm{BaSrCo}_{2} \mathrm{O}_{6-\delta}$ and b) $\mathrm{BaSrCoFeO}_{6-\delta}$. Black crosses represent experimental data, the solid red line is the cubic Pm-3m model, pink vertical tick marks show Bragg peak positions, and the lower blue line represents the difference plot.

Table 8.1. Refined Structural Parameters for $\mathrm{BaSrCoFeO}_{6-\delta}$ using powder X-ray diffraction data.

\begin{tabular}{|c|c|c|c|c|c|}
\hline \multicolumn{6}{|c|}{$\begin{array}{l}\text { Space group: } P m-3 m, a=3.9804(2) \AA, V=63.066(1) \AA^{3}, R p=0.0323 \text {, } \\
w R p=0.0419\end{array}$} \\
\hline Elements & $\mathrm{X}$ & $\mathrm{y}$ & $\mathrm{Z}$ & Occupancy & Uiso \\
\hline $\mathrm{Fe}$ & 0 & 0 & 0 & 0.5 & $0.035(1)$ \\
\hline $\mathrm{Co}$ & 0 & 0 & 0 & 0.5 & $0.035(1)$ \\
\hline $\mathrm{Ba}$ & 0.5 & 0.5 & 0.5 & 0.5 & $0.0202(7)$ \\
\hline $\mathrm{Sr}$ & 0.5 & 0.5 & 0.5 & 0.5 & $0.0202(7)$ \\
\hline $\mathrm{O}$ & 0.5 & 0 & 0 & 0.88 & $0.056(3)$ \\
\hline
\end{tabular}


Table 8.2. Refined Structural Parameters for $\mathrm{BaSrCo}_{2} \mathrm{O}_{6-\delta}$ using powder X-ray diffraction data.

\begin{tabular}{|c|c|c|c|c|c|}
\hline \multicolumn{6}{|c|}{$\begin{array}{l}\text { Space group: } P m-3 m, \mathrm{a}=3.9839(2) \AA, \mathrm{V}=63.23(1) \AA^{3}, \\
\mathrm{Rp}=0.0385, \mathrm{wRp}=0.0492\end{array}$} \\
\hline Elements & $\mathrm{X}$ & $\mathrm{y}$ & $\mathrm{z}$ & Occupancy & Uiso \\
\hline Co & 0 & 0 & 0 & 1 & $0.026(2)$ \\
\hline $\mathrm{Ba}$ & 0.5 & 0.5 & 0.5 & 0.5 & $0.0152(9)$ \\
\hline $\mathrm{Sr}$ & 0.5 & 0.5 & 0.5 & 0.5 & $0.0152(9)$ \\
\hline $\mathrm{O}$ & 0.5 & 0 & 0 & 0.77 & $0.084(5)$ \\
\hline
\end{tabular}

The morphology and crystallite sizes of both materials were also studied using scanning electron microscopy. Despite having the same crystal structure and same space group, the crystallite morphology for the two materials is significantly different. Figure 8.2 shows the sintered pellets for both materials. As seen in this figure, the crystallite size is larger in $\mathrm{BaSrCo}_{2} \mathrm{O}_{6-\delta}$. In addition, the crystallites appear to be more densely packed in $\mathrm{BaSrCo}_{2} \mathrm{O}_{6-}$ $\delta$
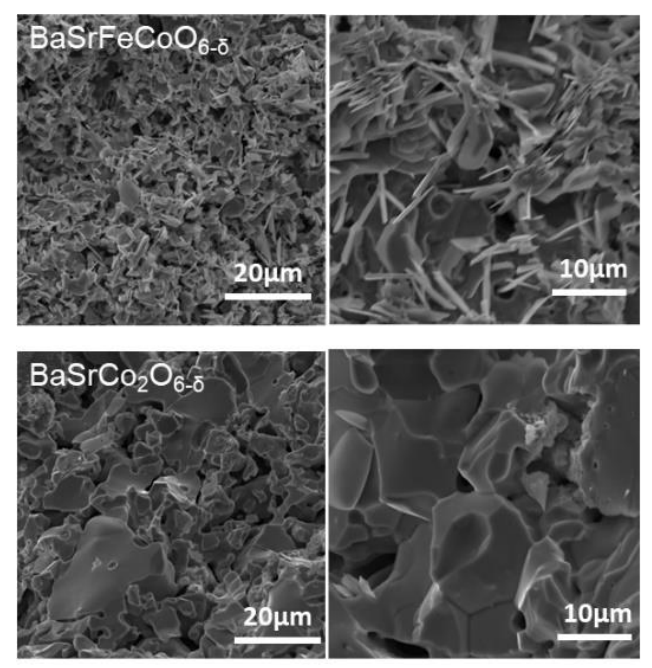

Figure 8.2. Scanning electron microscopy images of $\mathrm{BaSrCoFeO}_{6-\delta}$ (top) and $\mathrm{BaSrCo}_{2} \mathrm{O}_{6-}$ $\delta$ (bottom) 
X-ray photoelectron spectroscopy (XPS) and iodometric titration

The XPS studies were used to analyze the oxidation states of Fe and Co in both materials. The XPS analyses were guided by the information regarding synthesis conditions, which affect the oxygen content, as well as the iodometric titration results that show the oxygen stoichiometry in each material. Since $\mathrm{BaSrCoFeO}_{6-\delta}$ was synthesized in air, the oxygen content in this compound is expected to be greater than that of $\mathrm{BaSrCo}_{2} \mathrm{O}_{6-\delta}$, which was made in argon. This is confirmed by iodometric titrations results, which indicate the oxygen stoichiometry for the two compounds to be 5.27 and 4.65 , corresponding to $\mathrm{BaSrCoFeO}_{6}$ $\delta(\delta=0.73)$ and $\mathrm{BaSrCo}_{2} \mathrm{O}_{6-\delta}(\delta=1.35)$, respectively. Note that the starting materials for syntheses were $\mathrm{Co}_{3} \mathrm{O}_{4}$ and $\mathrm{Fe}_{2} \mathrm{O}_{3}$, which contain divalent and trivalent cobalt, as well as trivalent iron. The oxygen stoichiometry of $\mathrm{BaSrCoFeO}_{6-\delta}$ indicates that there should be some tetravalent $\mathrm{Fe}$ and/or $\mathrm{Co}$ in this material. On the other hand, the low oxygen stoichiometry of $\mathrm{BaSrCo}_{2} \mathrm{O}_{6-\delta}$ indicates that the oxidation state of cobalt should be a combination of divalent and trivalent.

We discuss the XPS data for $\mathrm{BaSrCo}_{2} \mathrm{O}_{6-\delta}$ first. The $\mathrm{Co} 2 \mathrm{P}_{3 / 2}$ peak appears at $\sim 780 \mathrm{eV}$, and two satellite peaks are present at $\sim 4.5 \mathrm{eV}$ and $10 \mathrm{eV}$ higher than the $2 \mathrm{P}_{3 / 2}$ peak (Figure 8.3a). $\mathrm{Co}^{2+}$ and $\mathrm{Co}^{4+}$ are both expected to show satellite peaks at about $5 \mathrm{eV}$ higher than the $2 \mathrm{P}_{3 / 2}$ peak. ${ }^{167-168}$ Considering that $\mathrm{BaSrCo}_{2} \mathrm{O}_{6-\delta}$ was synthesized in argon atmosphere, the oxidation of cobalt to tetravalent state is not expected. In addition, the oxygen stoichiometry determined by iodometric titration indicates $\delta=1.35$ for $\mathrm{BaSrCo}_{2} \mathrm{O}_{6-\delta}$, which rules out the possibility of the presence of tetravalent cobalt. Therefore, for this material, the satellite peak at $\sim 4.5 \mathrm{eV}$ higher than the $2 \mathrm{P}_{3 / 2}$ peak belongs to $\mathrm{Co}^{2+}$. The satellite peak at $\sim 10 \mathrm{eV}$ higher than the $2 \mathrm{P}_{3 / 2}$ peak is signature of $\mathrm{Co}^{3+} .{ }^{167}$ 
The XPS spectra for $\mathrm{BaSrCoFeO}_{6-\delta}$ are shown in Figures $8.3 \mathrm{~b}$ and c. The $\mathrm{Fe}^{3+} 2 \mathrm{P}_{3 / 2}$ peak is expected to appear at $\sim 710-711 \mathrm{eV}$ with a satellite peak at $\sim 7-9 \mathrm{eV}$ higher than the $2 \mathrm{P}_{3 / 2}$ peak. ${ }^{169} 170-172$ Compared to $\mathrm{Fe}^{3+}$, the $\mathrm{Fe}^{4+} 2 \mathrm{P}_{3 / 2}$ peak is expected to have higher binding energy, appearing at $\sim 712-713 \mathrm{eV} \cdot{ }^{169,171}$ In the XPS spectrum for $\mathrm{BaSrCoFeO}_{6-\delta}$ (Figure 8.3b) the $\mathrm{Fe} 2 \mathrm{P}_{3 / 2}$ peak appears at $\sim 710 \mathrm{eV}$ with a shoulder at $\sim 713 \mathrm{eV}$, indicating the presence $\mathrm{Fe}^{3+}(\sim 710 \mathrm{eV})$ and $\mathrm{Fe}^{4+}(\sim 713 \mathrm{eV})$ in this material. The satellite peak for $\mathrm{Fe}^{3+}$ is present at about $718 \mathrm{eV}$, as expected.

The XPS spectrum for cobalt in $\mathrm{BaSrCoFeO}_{6-\delta}$ (Figure 8.3c) is very similar to that of $\mathrm{BaSrCo}_{2} \mathrm{O}_{6-\delta}$. The $\mathrm{Co} 2 \mathrm{P}_{3 / 2}$ peak appears at $\sim 780 \mathrm{eV}$, along with two satellite peaks at $\sim 5.5$ $\mathrm{eV}$ and $10 \mathrm{eV}$ higher than the $2 \mathrm{P}_{3 / 2}$ peak. Again, these indicate the presence of $\mathrm{Co}^{2+}$ and $\mathrm{Co}^{3+}$, as described above. Therefore, iodometric titration and XPS indicate that $\mathrm{Fe}$ in $\mathrm{BaSrCoFeO}_{6-\delta}$ is in tri- and tetravalent states, whereas $\mathrm{Co}$ is in di- and trivalent states in both materials.

Magnetic properties.

Magnetic susceptibility as a function of temperature shows another sharp contrast between these two isostructural materials. Both $\mathrm{ZFC}$ and $\mathrm{FC}$ data were obtained in the temperature range $2 \mathrm{~K}-400 \mathrm{~K}$. The magnetic susceptibility data for $\mathrm{BaSrCo}_{2} \mathrm{O}_{6-\delta}$ (Figure 8.4a) shows ZFC-FC divergence below $300 \mathrm{~K}$. There is no sharp magnetic transition in the temperature range $2 \mathrm{~K}-400 \mathrm{~K}$. As shown in the inset of Figure 8.4a, the inverse susceptibility plot 


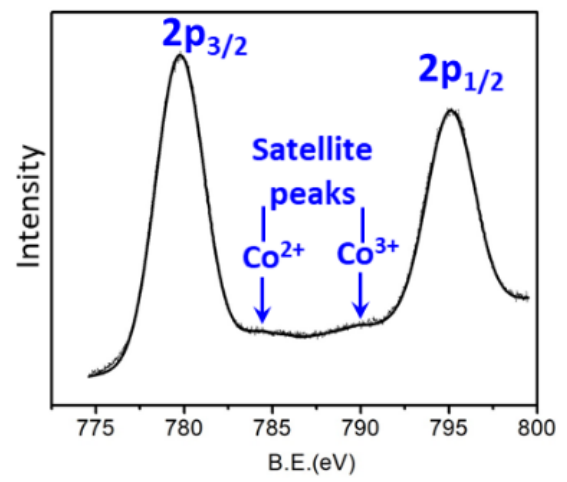

(a)

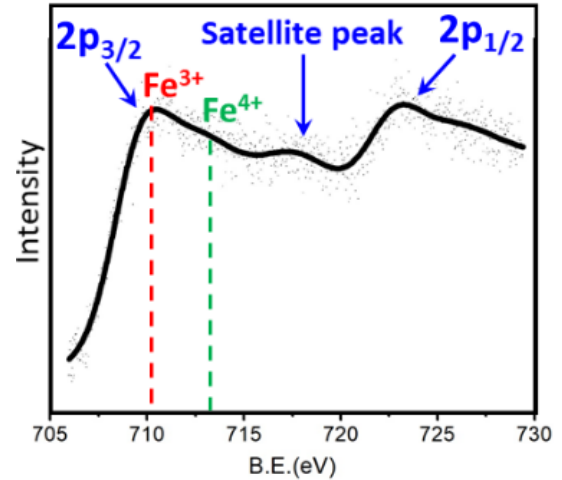

(b)

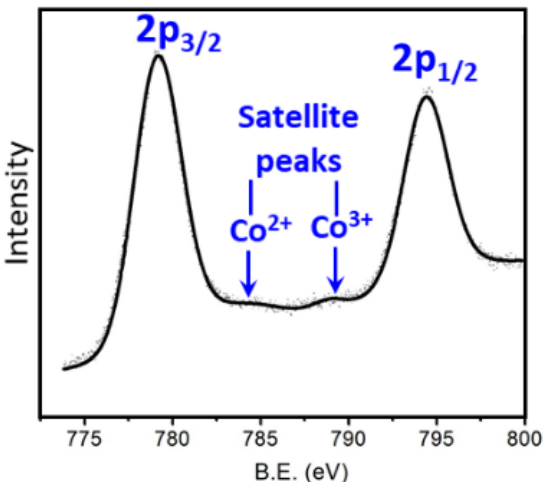

(c)

Figure 8.3. X-ray photoelectron spectroscopy data. (a) shows the Co peaks for $\mathrm{BaSrCo}_{2} \mathrm{O}_{6}$ $\delta$. (b) and (c) show the $\mathrm{Fe}$ and $\mathrm{Co}$ peaks for $\mathrm{BaSrCoFeO}_{6-\delta}$, respectively.

shows deviation from linear paramagnetic behavior. The very low magnitude of magnetic susceptibility in the entire temperature range for $\mathrm{BaSrCo}_{2} \mathrm{O}_{6-\delta}$ indicates that there is little uncompensated moment in this material. This is further confirmed by isothermal magnetization data (Figure 8.4b) that show very low magnetization even at $2 \mathrm{~K}$, where the magnetization value reaches a maximum of $0.16 \mu_{\mathrm{B}}$ at magnetic field of $9 \mathrm{~T}$. As evident from the inverse susceptibility plot, $\mathrm{BaSrCo}_{2} \mathrm{O}_{6-\delta}$ is not paramagnetic at $2 \mathrm{~K}$. The low magnetization value could be due to antiferromagnetic order, with a transition temperature higher than the measured temperature range. The antiferromagnetic order is consistent with that reported for a similar composition, which has $18 \%$ less oxygen-vacancies than our 
material and shows $\mathrm{T}_{\mathrm{N}} \approx 525 \mathrm{~K} .{ }^{165}$ For that composition, only FC data has been reported. ${ }^{165}$ The ZFC-FC divergence observed in Figure 8.4a can be indicative of reorientation of magnetic moments while the overall antiferromagnetic order is retained and the low magnetization value persists. This has been observed before for another oxygen-deficient perovskite with formula $\mathrm{Ca}_{2} \mathrm{FeCoO}_{5},{ }^{74}$ where neutron diffraction data showed reorientation of magnetic moments from along the a-axis to $b$-axis while the antiferromagnetic order was retained. ${ }^{74}$ The magnetic susceptibly of $\mathrm{Ca}_{2} \mathrm{FeCoO}_{5}$ below the magnetic transition temperature showed ZFC-FC divergence, occurring at the temperature where the magnetic moment reorientation was completed, similar to the situation observed in $\mathrm{BaSrCo}_{2} \mathrm{O}_{6-\delta} .{ }^{74}$

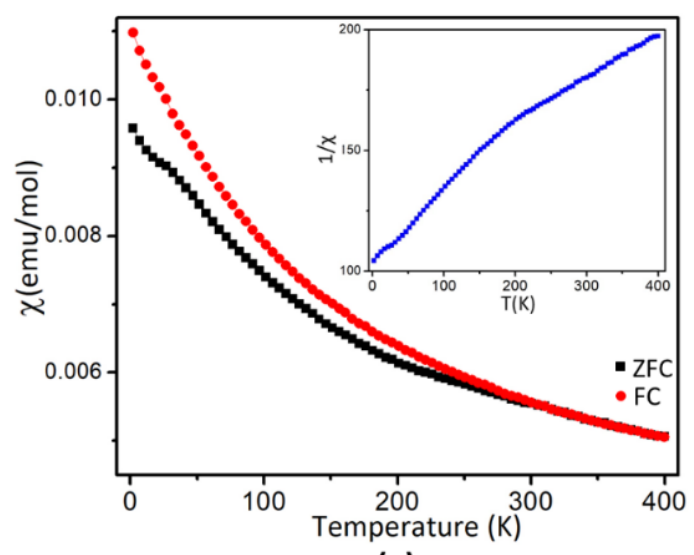

(a)

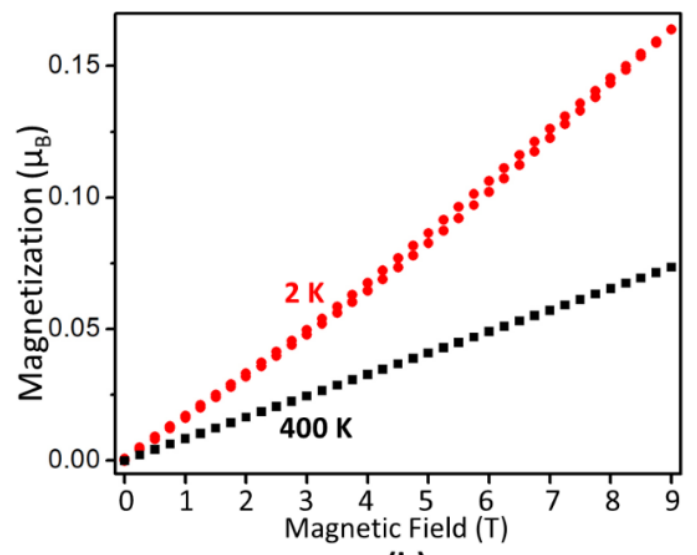

(b)

Figure 8.4. Bulk magnetization data for $\mathrm{BaSrCo}_{2} \mathrm{O}_{6-8}$ : (a) $\mathrm{ZFC}$ and $\mathrm{FC}$ magnetic susceptibility data. The inset shows inverse of susceptibility plotted against temperature. (b) Isothermal magnetization versus field, at $2 \mathrm{~K}$ and $400 \mathrm{~K}$.

For $\mathrm{BaSrCoFeO}_{6-\delta}$, there is no $\mathrm{ZFC}-\mathrm{FC}$ divergence in the magnetic susceptibility data, as shown in Figure 8.5a. However, the susceptibility deviates significantly from paramagnetic behavior, showing a sharp increase at low temperature, indicative of uncompensated 
moments due to ferro- or ferrimagnetic coupling. ${ }^{173-174,175-176}$ This is also evident when inverse susceptibility is plotted as a function of temperature, where the only linear region is in the temperature range $300 \mathrm{~K}-400 \mathrm{~K}$. The linear paramagnetic region can be fitted using the inverse Curie-Weiss equation:

$$
\frac{1}{\chi}=\frac{T-\theta}{C}
$$

The fit values are $\mathrm{C}=4.49(3)$ and $\theta=-110(3)$. The negative $\theta$ value confirms the presence of ferrimagnetic interactions. Below $300 \mathrm{~K}$, the inverse susceptibility deviates from that of a paramagnetic system. There is no sharp magnetic transition, and the changes are gradual.

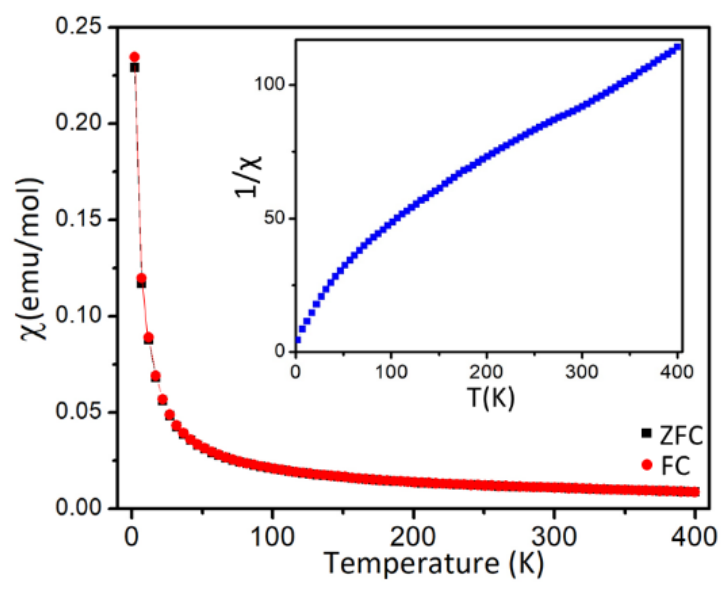

(a)

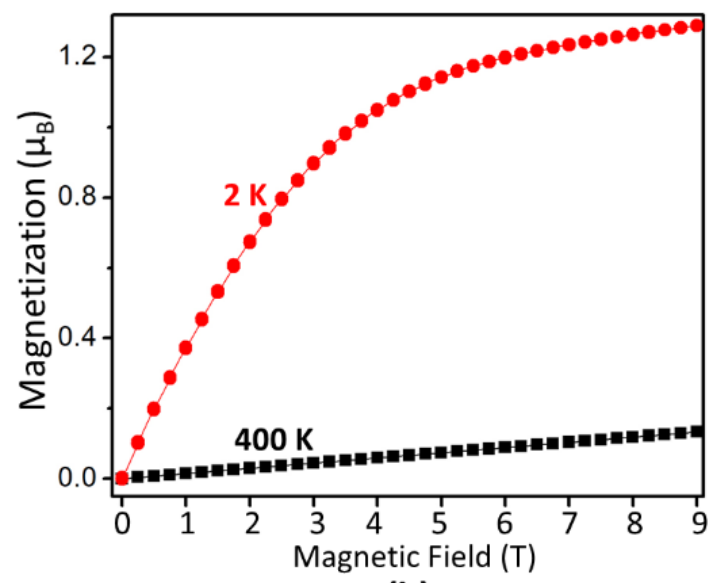

(b)

Figure 8.5. Bulk magnetization data for $\mathrm{BaSrCoFeO}_{6-\delta}$ : (a) $\mathrm{ZFC} / \mathrm{FC}$ magnetic susceptibility data. The inset shows inverse of susceptibility versus temperature. (b) Isothermal magnetization data at $2 \mathrm{~K}$ and $400 \mathrm{~K}$.

The isothermal magnetization data as a function of field further confirm the ferrimagnetic behavior at low temperature. As shown in Figure 8.5b, isothermal magnetization at $400 \mathrm{~K}$ has a typical paramagnetic behavior. However, at $2 \mathrm{~K}$, the isothermal data shows a sharp increase in magnetization followed by a semi-plateau. However, even at high field of $\sim 9$ 
$\mathrm{T}$, the magnetization continues to increase gradually and does not reach saturation. In addition, the maximum magnetization at $9 \mathrm{~T}$ is $\sim 1.28 \mu_{\mathrm{B}}$, much lower than that anticipated for ferromagnetic coupling of $\mathrm{Fe}$ and $\mathrm{Co}$ in their trivalent and tetravalent states. This again indicates that the magnetic interactions are ferrimagnetic. Note that as far as electronic configurations are concerned, there are two types of magnetic ions in $\mathrm{BaSrCoFeO}_{6-\delta}$, as suggested by XPS. They are spin 5/2 ions $\left(\mathrm{Fe}^{3+}\right.$ and $\left.\mathrm{Co}^{4+}\right)$ and spin $4 / 2$ ions $\left(\mathrm{Fe}^{4+}\right.$ and $\left.\mathrm{Co}^{3+}\right)$, for high-spin configurations which are common in perovskite-based oxides. If ferrimagnetic coupling occurs between equal concentrations of spin $5 / 2$ and spin $4 / 2$ ions, the expected saturation moment will be $\sim 1 \mu_{\mathrm{B}}$, close to the value obtained experimentally,

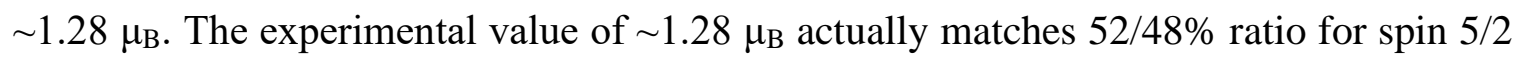
to spin $4 / 2$ ions. Another observation is that there is no hysteresis in the isothermal magnetization data at $2 \mathrm{~K}$ or $400 \mathrm{~K}$. The data collected while increasing the magnetic field from $0 \mathrm{~T}-9 \mathrm{~T}$ overlap with the data obtained while decreasing the field from $9 \mathrm{~T}-0 \mathrm{~T}$. The absence of hysteresis in the isothermal magnetization at $2 \mathrm{~K}$ indicates that the ferrimagnetic interactions are not long-range, and occur in short-range scale, similar to the situation in superparamagnetic systems. ${ }^{177}$

The significantly different magnetic properties between the two isostructural materials is interesting. The magnetic susceptibility of $\mathrm{BaSrCo}_{2} \mathrm{O}_{6-\delta}$ deviates from paramagnetism in the entire temperature range, $2-400 \mathrm{~K}$. The very low magnetization values even at $2 \mathrm{~K}$ and $9 \mathrm{~T}$, indicate antiferromagnetic order in $\mathrm{BaSrCo}_{2} \mathrm{O}_{6-\delta}$, consistent with a report on a similar material. On the other hand, $\mathrm{BaSrCoFeO}_{6-\delta}$ shows ferrimagnetic properties with large uncompensated moments and isothermal magnetization similar to that observed in superparamagnetic systems, indicating the presence of ferrimagnetic clusters. 


\section{Electrical conductivity}

The electrical behavior of our samples was studied using DC method, as well as AC electrochemical impedance spectroscopy (EIS). In both methods, the resistance of a material is analyzed to get total conductivity. In AC impedance spectroscopy, the total impedance is generally measured as the low frequency intercept of arc on the real axis (Z') of a Nyquist plot. Whereas DC method requires a constant applied current or voltage to measure the output voltage or current, respectively. Consequently, the total conductivity $(\sigma)$ is obtained by using the relation $\sigma=\mathrm{L} / \mathrm{RA}$, where L, R and A represent the thickness, resistance and cross-sectional area of the cylindrical pellet, respectively. The total conductivity values for $\mathrm{BaSrCo}_{2} \mathrm{O}_{6-\delta}$ and $\mathrm{BaSrCoFeO}_{6-\delta}$ measured using both AC and DC methods at room temperature are listed in Table 8.3. The conductivity values measured by AC and DC methods are the same. Several interesting observations were made with regard to the electrical conductivity of these materials, as follows.

At room temperature, $\mathrm{BaSrCoFeO}_{6-\delta}$ has higher electrical conductivity than $\mathrm{BaSrCo}_{2} \mathrm{O}_{6-\delta}$. The electrical conductivity in perovskite-based oxides occurs by electron hopping through M-O-M (metal-oxygen-metal) bonds. For this process to happen, metals with multiple oxidation states such as $\mathrm{Fe}^{2+} / \mathrm{Fe}^{3+} / \mathrm{Fe}^{4+}$ or $\mathrm{Co}^{2+} / \mathrm{Co}^{3+} / \mathrm{Co}^{4+}$ are required on the octahedrally coordinated B-site. In our materials, the metal $3 \mathrm{~d}$ orbitals overlap with oxygen $2 \mathrm{p}$ orbitals, and electron hopping occurs through the $(\mathrm{Fe} / \mathrm{Co})-\mathrm{O}-(\mathrm{Fe} / \mathrm{Co})$ or $\mathrm{Co}-\mathrm{O}-\mathrm{Co}$ pathways. The extent of electronic conductivity is affected by the degree of $\mathrm{M}-\mathrm{O}$ orbital overlap. Shorter $\mathrm{M}-\mathrm{O}$ bond distance and larger $\mathrm{M}-\mathrm{O}-\mathrm{M}$ bond angles lead to better orbital overlap and higher conductivity. ${ }^{178}$ 
In situations where structural transitions take place, the bond distances and angles are affected leading to changes in electrical conductivity. ${ }^{20}$ An example is the structural transition between $\mathrm{Sr}_{2} \mathrm{Fe}_{2} \mathrm{O}_{6-\delta}$ and $\mathrm{CaSrFe}_{2} \mathrm{O}_{6-\delta}$, where some bond angles in the latter become significantly small, leading to smaller degree of orbital overlap and lower conductivity at room temperature. ${ }^{20}$

Table 8.3. Room Temperature Conductivity and Activation Energies.

\begin{tabular}{|c|c|c|c|}
\hline \multirow[t]{2}{*}{ compounds } & \multicolumn{2}{|c|}{$\begin{array}{l}\text { total conductivity, } \sigma(\mathrm{S} \\
\left.\mathrm{cm}^{-1}\right)\end{array}$} & \multirow[t]{2}{*}{ Activation energy $\left(\mathrm{E}_{\mathrm{a}}\right)$} \\
\hline & $\mathrm{AC}$ & $\mathrm{DC}$ & \\
\hline $\mathrm{BaSrFeCoO}_{6-\delta}$ & 0.07368 & 0.07365 & $\begin{array}{l}0.144 \mathrm{eV} \text { for } 298-473 \mathrm{~K}\left(25-200^{\circ} \mathrm{C}\right) \\
0.010 \mathrm{eV} \text { for } 473-1173 \mathrm{~K}\left(200-900^{\circ} \mathrm{C}\right)\end{array}$ \\
\hline $\mathrm{BaSrCo}_{2} \mathrm{O}_{6-\delta}$ & 0.000134 & 0.000133 & $\begin{array}{l}0.218 \mathrm{eV} \text { for } 298-673 \mathrm{~K}\left(25-400^{\circ} \mathrm{C}\right) \\
0.571 \mathrm{eV} \text { for } 673-1173 \mathrm{~K}\left(400-900^{\circ} \mathrm{C}\right)\end{array}$ \\
\hline
\end{tabular}

However, the two materials investigated in the present study, $\mathrm{BaSrCo}_{2} \mathrm{O}_{6-\delta}$ and $\mathrm{BaSrCoFeO}_{6-\delta}$, have the same crystal structure. The $\mathrm{M}-\mathrm{O}-\mathrm{M}$ bond angles are $180^{\circ}$ in both compounds. Nevertheless, the size of the unit cell and bond lengths are slightly different between these two materials. $\mathrm{BaSrCoFeO}_{6-\delta}$ has smaller unit cell and slightly shorter $\mathrm{Fe}(\mathrm{Co})-\mathrm{O}$ bond distances, $1.9902(1) \AA$, as compared to those in $\mathrm{BaSrCo}_{2} \mathrm{O}_{6-\delta}, 1.9920(1)$ A. The improvement in conductivity as a result of decrease in unit cell parameters is expected, as observed in other materials such as $\mathrm{SrFe}_{1-\mathrm{x}} \mathrm{Al}_{\mathrm{x}} \mathrm{O}_{3-\delta} \cdot{ }^{136} \mathrm{In}$ addition, the degree of oxygen deficiency and cation combinations that form the conduction pathways in the two materials are different. Higher degree of oxygen deficiency is expected to decrease the number of conduction pathways. As outlined in the XPS section, $\mathrm{BaSrCoFeO}_{6-\delta}$ has higher oxygen content and contains $\mathrm{Fe}^{3+} / \mathrm{Fe}^{4+}$ and $\mathrm{Co}^{3+} / \mathrm{Co}^{4+}$, while $\mathrm{BaSrCo}_{2} \mathrm{O}_{6-\delta}$ contains 
$\mathrm{Co}^{2+} / \mathrm{Co}^{3+}$. The difference in the room temperature conductivity of these two materials is due to the combination of three factors, namely the subtle difference in bond distances, difference in concentration of oxygen vacancies and the dissimilarity of conduction pathways.

Variable-temperature conductivity studies in the temperature range $25-900{ }^{\circ} \mathrm{C}$ provide further insight into the properties of these two materials. The measurements were performed under the same conditions used for the synthesis of these materials, namely in air for $\mathrm{BaSrCoFeO}_{6-\delta}$ and in argon for $\mathrm{BaSrCo}_{2} \mathrm{O}_{6-\delta}$ (Figure 8.6) However, we also performed conductivity measurements in argon atmosphere for $\mathrm{BaSrCoFeO}_{6-\delta}$, which showed the same temperature-dependent trend as the air-measurement, but with lower overall conductivity (Figure 8.7).

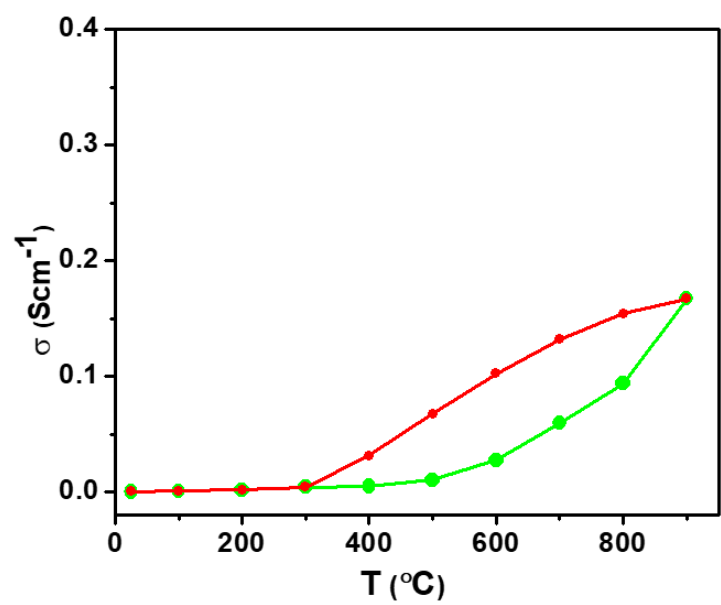

(a)

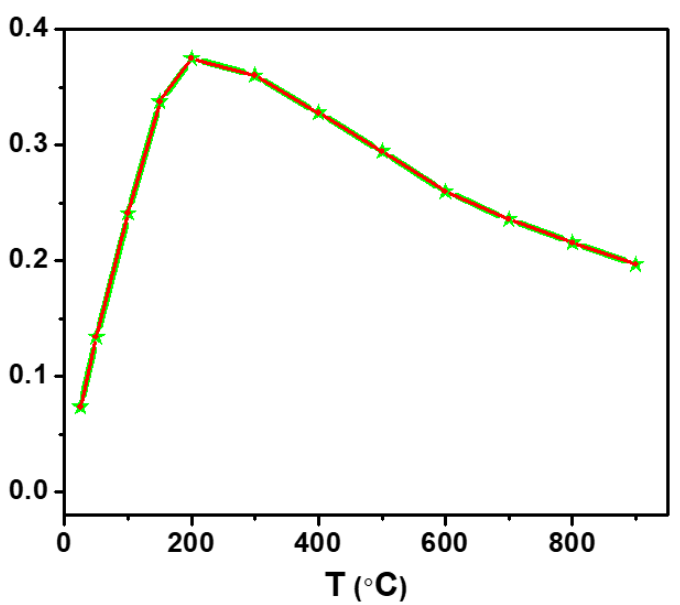

(b)

Figure 8.6. Electrical conductivity of (a) $\mathrm{BaSrCo}_{2} \mathrm{O}_{6-\delta}$ and (b) $\mathrm{BaSrCoFeO}_{6-\delta}$. The measurements were done in the same environment used for synthesis, namely in argon for $\mathrm{BaSrCo}_{2} \mathrm{O}_{6-\delta}$ and in air $\mathrm{BaSrCoFeO}_{6-\delta}$. Heating data are shown in green and the cooling data in red. 


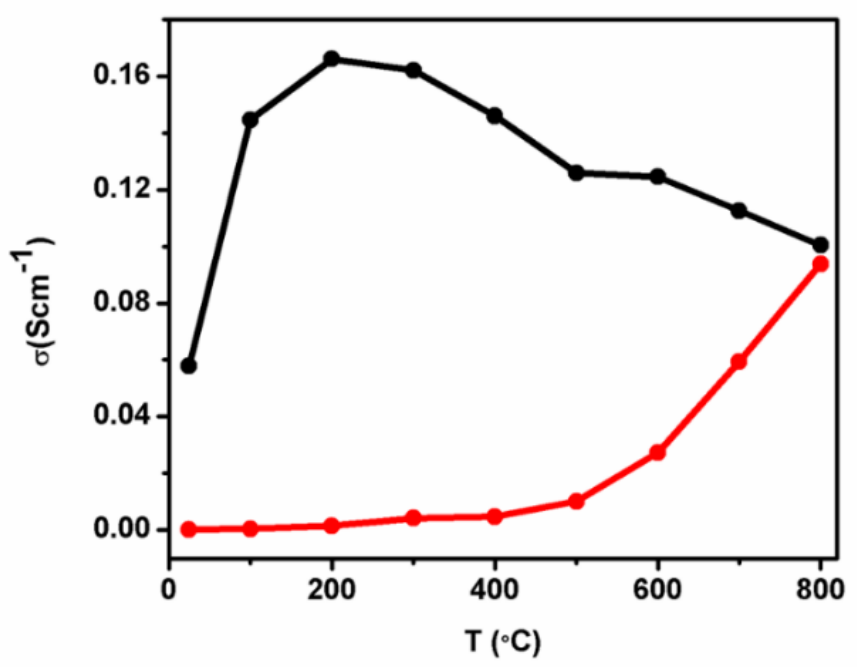

Figure 8.7. comparison of the electrical conductivity for $\mathrm{BaSrCo}_{2} \mathrm{O}_{6-\delta}$ and $\mathrm{BaSrCoFeO}_{6-\delta}$ both obtained in argon atmosphere.

There is a sharp contrast between the $\mathrm{BaSrCo}_{2} \mathrm{O}_{6-\delta}$ and $\mathrm{BaSrCoFeO}_{6-\delta}$ with regard to their electrical conductivity as a function of temperature. Comparison between the two materials reveals significant increase in conductivity as a function of temperature for $\mathrm{BaSrCoFeO}_{6-\delta}$ up to $200{ }^{\circ} \mathrm{C}$, whereas $\mathrm{BaSrCo}_{2} \mathrm{O}_{6-\delta}$ shows electrical conductivity which is nearly independent of temperature up to $400{ }^{\circ} \mathrm{C}$. The retention of the same level of conductivity over a range of nearly 400 degrees can be a very useful property, especially in applications such as sensors where the change in conductivity should only be induced by the analyte and not temperature.

Above $400{ }^{\circ} \mathrm{C}, \mathrm{BaSrCo}_{2} \mathrm{O}_{6-\delta}$ shows increase in conductivity, a typical behavior of semiconductors. However, $\mathrm{BaSrCoFeO}_{6-\delta}$ exhibits a transition above $200{ }^{\circ} \mathrm{C}$, where a downturn in conductivity begins and continues to $900{ }^{\circ} \mathrm{C}$ (Figure 8.6).

Another interesting feature of the electrical conductivity of $\mathrm{BaSrCo}_{2} \mathrm{O}_{6-\delta}$ is the distinct hysteresis between the conductivity data obtained during heating and cooling cycles. 
(Figure 8.6) Whereas, $\mathrm{BaSrCoFeO}_{6-\delta}$ shows nearly identical conductivity values during heating and cooling cycles. The temperature-activated enhancement of conductivity in $\mathrm{BaSrCo}_{2} \mathrm{O}_{6-\delta}$ is retained upon cooling the material, leading to the observation of hysteresis. The activation energies for electrical conductivity of both materials were also calculated using Arrhenius plots. The plots in Figure 8.8 were used for fitting with the Arrhenius equation for thermally activated conductivity, ${ }^{51,179-180}$ as shown below:

$$
\sigma T=\sigma^{\circ} \mathrm{e}^{-E \mathrm{a} / k T}
$$

where $\sigma^{\circ}$ is a pre-exponential factor and a characteristic of a material, and $E_{a}, \mathrm{k}$, and $\mathrm{T}$ are the activation energy for the conductivity, Boltzmann constant, and absolute temperature, respectively. The activation energy for the total conductivity $\left(E_{a}\right)$ can be obtained from the slope of the line of best fit in the $\log \sigma \mathrm{T}$ vs $1000 / \mathrm{T}$ plot. As shown in Table 8.3, the $E_{a}$ values are higher for $\mathrm{BaSrCo}_{2} \mathrm{O}_{6-\delta}$ compared to $\mathrm{BaSrCoFeO}_{6-\delta}$. Note that activation energies are dependent on the change in conductivity as a function of temperature. $\mathrm{BaSrCo}_{2} \mathrm{O}_{6-\delta}$ has very small conductivity at low temperature (in the order of $10^{-4} \mathrm{Scm}^{-1}$ ), and its conductivity increases to $0.167 \mathrm{Scm}^{-1}$ at high 


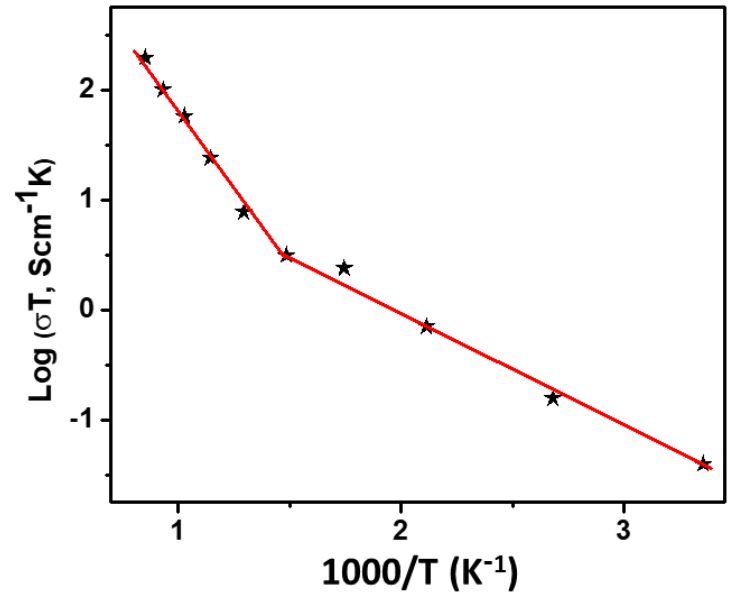

(a)

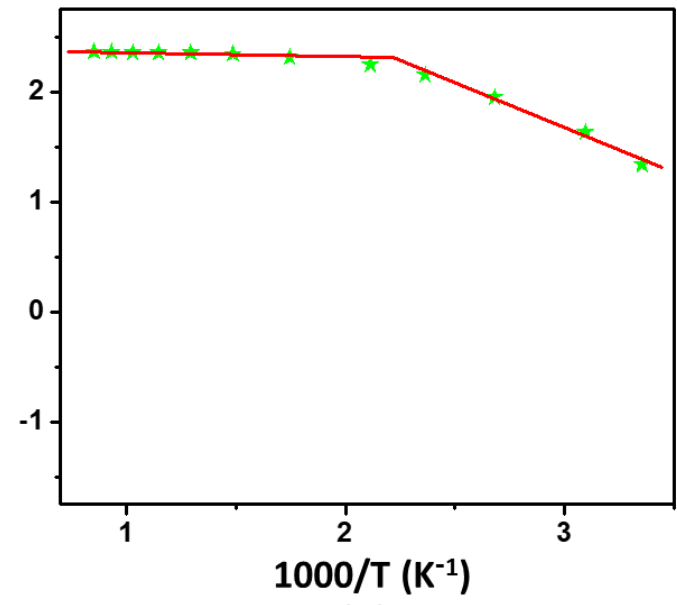

(b)

Figure 8.8. Arrhenius plot for electrical conductivity of (a) $\mathrm{BaSrCo}_{2} \mathrm{O}_{6-\delta}$ and (b) $\mathrm{BaSrCoFeO}_{6-\delta}$.

temperature. Therefore, the change in conductivity as a function of temperature is large for this material. This leads to a large slope in the Arrhenius plot and higher activation energy for $\mathrm{BaSrCo}_{2} \mathrm{O}_{6-\delta}$. For $\mathrm{BaSrCoFeO}_{6-\delta}$, the conductivity is higher, but the difference between the conductivity values at low and high temperature is not as significant as that of $\mathrm{BaSrCo}_{2} \mathrm{O}_{6-\delta}$

\section{CONCLUSIONS}

The isostructural oxygen-deficient perovskites $\mathrm{BaSrCo}_{2} \mathrm{O}_{6-\delta}$ and $\mathrm{BaSrCoFeO}_{6-\delta}$ show significant disparity in magnetic and electrical properties. The magnetic susceptibility of $\mathrm{BaSrCo}_{2} \mathrm{O}_{6-\delta}$ deviates from paramagnetism in the entire temperature range, $2-400 \mathrm{~K}$. The very low magnetization values even at $2 \mathrm{~K}$ and $9 \mathrm{~T}$, indicate antiferromagnetic order in $\mathrm{BaSrCo}_{2} \mathrm{O}_{6-\delta}$, consistent with a report on a similar material. On the other hand, $\mathrm{BaSrCoFeO}_{6-\delta}$ shows ferrimagnetic properties with large uncompensated moments and isothermal magnetization reminiscent of superparamagnetic systems, indicating the 
presence of ferrimagnetic clusters. The differences in electrical conductivity are also remarkable. $\mathrm{BaSrCo}_{2} \mathrm{O}_{6-\delta}$ shows nearly constant conductivity up to $400 \mathrm{C}$, followed by semiconducting properties above this temperature. Whereas the $\mathrm{BaSrCoFeO}_{6-\delta}$ shows semiconducting properties up to $200{ }^{\circ} \mathrm{C}$, above which a downturn in conductivity is observed, similar to metallic conductors. 


\section{CHAPTER 9}

\section{STRUCTURE DEPENDENCE OF ELECTRICAL CONDUCTIVITY AND ELECTROCATALYTIC PROPERTIES OF $\mathrm{Sr}_{2} \mathrm{Mn}_{2} \mathrm{O}_{6}$ AND CaSrMn $2 \mathrm{O}_{6}{ }^{8}$}

\section{INTRODUCTION}

Perovskite oxides exhibit a wide range of structural, electrical and magnetic properties. ${ }^{82}$, 147-148, 181 They have been studied for their potential applications in different technological fields, such as fuel cells, thermoelectric devices and sensors. ${ }^{148,182-183}$ The structure and properties of perovskites can vary significantly ${ }^{4,84-85,92,120,184-186}$ upon variation of the Aor B-site cations in the $\mathrm{ABO}_{3}$ formula, where the A-cations occupy the 12-coordinated sites located between the $\mathrm{BO}_{6}$ octahedra.

Given the wide range of transition metal or main group cations that can reside on the Bsite, the structural transformation upon B-site doping are frequently observed. Various parameters, such as ionic radius, oxidation state and electronic structure lead to these changes. For example, when $\mathrm{Mn}$ is substituted by Fe, the orthorhombic structure of $\mathrm{CaMnO}_{3}{ }^{124}$ transforms into the orthorhombic structure of a double perovskite, $\mathrm{Ca}_{2} \mathrm{FeMnO}_{6}$, with layered ordering. ${ }^{187-188}$ Similarly, $\mathrm{Sr}_{2} \mathrm{FeMoO}_{6}$ has a tetragonal structure, while the B-

\footnotetext{
${ }^{8}$ The work described in this chapter was published in journal of Chemical Sciences (
} 2019, vol 131, p 109) 
site substituted $\mathrm{Sr}_{2} \mathrm{FeWO}_{6}$ is orthorhombic. ${ }^{189}$ The structural transformation leads to significant variation in properties as well, where $\mathrm{Sr}_{2} \mathrm{FeMoO}_{6}$ behaves as a half metal and a ferromagnetic material, whereas $\mathrm{Sr}_{2} \mathrm{FeWO}_{6}$ shows insulating and antiferromagnetic properties. ${ }^{189}$ Note that there are two B-site cations in these materials. In situations, where there are two crystallographically distinct positions for the A- or B-site cations, the general formula can be represented by $\mathrm{AA}^{\prime} \mathrm{B}_{2} \mathrm{O}_{6}$ and $\mathrm{A}_{2} \mathrm{BB}^{\prime} \mathrm{O}_{6}$, respectively.

There are also oxide materials where the general formula is similar to that of a typical perovskite, but the connectivity in their crystal lattice is different. An example is $\mathrm{SrMnO}_{3}$, better described as $\mathrm{Sr}_{2} \mathrm{Mn}_{2} \mathrm{O}_{6}$ since it has two crystallographically distinct positions for $\mathrm{Sr}$ and two for Mn. ${ }^{181}$ Unlike typical perovskites that contain corner-sharing octahedra, this material has dimeric units of face-sharing $\mathrm{MnO}_{6}$ octahedra. ${ }^{181}$ This compound has been of interest in recent years, given the potential of the Mn-based oxides for applications in areas such as sensing, ${ }^{182}$ spintronics ${ }^{190}$ and solid oxide fuel cells. ${ }^{183}$ Several substituted derivatives have been investigated, showing variations in structure and properties. For example, when $50 \%$ of $\mathrm{Mn}$ in $\mathrm{Sr}_{2} \mathrm{Mn}_{2} \mathrm{O}_{6}$ is substituted by Fe, the crystal structure changes to a perovskite type system with corner-sharing octahedra. ${ }^{191}$ The structural transformation also leads to changes in magnetic properties, where the antiferromagnetic arrangement of $\mathrm{Mn}^{4+}$ magnetic moments ${ }^{181}$ in $\mathrm{Sr}_{2} \mathrm{Mn}_{2} \mathrm{O}_{6}$ transforms into ferromagnetic arrangement upon partial substitution of Mn by Fe. ${ }^{191}$

It is also possible to partially or completely replace the A-site cation in $\mathrm{Sr}_{2} \mathrm{Mn}_{2} \mathrm{O}_{6}$. For example, the $\mathrm{Ba}$ substitution on the A-site results in orthorhombic and rhombohedral structures for $50 \%$ and $100 \%$ substitutions, respectively. ${ }^{192}$ The structural transformation upon Ba-doping is followed by the change in electrical properties. ${ }^{192}$ It is also possible to 
replace $\mathrm{Sr}^{2+}$ by a smaller cation, namely $\mathrm{Ca}^{2+}$, to obtain $\mathrm{CaSrMn}_{2} \mathrm{O}_{6}$, which has a perovskite-type structure ${ }^{193}$ unlike the parent $\mathrm{Sr}_{2}$ compound. To our knowledge, the effect of doping a smaller A-site cation, i.e., $\mathrm{Ca}^{2+}$, on charge transport properties of $\mathrm{Sr}_{2} \mathrm{Mn}_{2} \mathrm{O}_{6}$ has not been investigated. In this paper, we report the remarkable structure-property relationships, where the structural changes prompted by the replacement of the A-site cation lead to significant enhancement of the electrical charge-transport and electrocatalytic activity. Dramatic increase in the electrical conductivity is observed over a wide range of temperature, due to Ca-doping. Furthermore, we have studied the electrocatalytic activity of both $\mathrm{Sr}_{2} \mathrm{Mn}_{2} \mathrm{O}_{6}$ and $\mathrm{CaSrMn}_{2} \mathrm{O}_{6}$ for oxygen-evolution reaction, demonstrating the enhancement of catalytic properties in the latter compound.

\section{EXPERIMENTAL}

The materials $\mathrm{Sr}_{2} \mathrm{Mn}_{2} \mathrm{O}_{6}$ and $\mathrm{CaSrMn}_{2} \mathrm{O}_{6}$ were synthesized in air by solid state method. Stoichiometric amounts of the powders of the precursor compounds $\mathrm{CaCO}_{3}$ (Alfa Aesar, 99.95\%), $\mathrm{Mn}_{2} \mathrm{O}_{3}$ (Alfa Aesar,99.998\%) and $\mathrm{SrCO}_{3}$ (Sigma Aldrich,99.99\%) were mixed together using an agate mortar and pestle and pressed into a pellet which was calcined in air at $1000{ }^{\circ} \mathrm{C}$ for $24 \mathrm{~h}$ in order to decompose the carbonates and start the reaction. The samples were then ground and sintered at $1200{ }^{\circ} \mathrm{C}$ for $24 \mathrm{~h}$ in the same environment to complete the reaction and form pure products. Both heat treatments were followed by slow cooling. The heating and cooling rates were $100{ }^{\circ} \mathrm{C} / \mathrm{h}$. The phase purity and structure of the polycrystalline samples were examined by powder X-ray diffraction at room temperature using $\mathrm{Cu} \mathrm{K} \alpha 1$ radiation $(\lambda=1.54056 \AA)$. The GSAS software ${ }^{100}$ and EXPEGUI interface ${ }^{125}$ were used for Rietveld refinements. The sample morphologies were studied using high resolution field-emission scanning electron microscopy (SEM). The 
electrical properties were investigated by 4-point probe measurements. Electrical conductivity was measured at $100{ }^{\circ} \mathrm{C}$ intervals from 25 to $800{ }^{\circ} \mathrm{C}$ during both heating and cooling cycles. At each measurement point, the temperature was maintained constant until a plateau in conductivity was observed, before changing the temperature. The rate of heating and cooling for conductivity measurements was $3{ }^{\circ} \mathrm{C} / \mathrm{min}$. Oxygen contents of each material was determined by iodometric titration as explained elsewhere. ${ }^{88,120,130}$

Electrocatalytic performances of the materials were measured in a three-electrode electrochemical workstation using a rotating disc electrode at $1600 \mathrm{rpm}$. A glassy carbon electrode loaded with catalysts, a commercial platinum electrode and $\mathrm{Ag} / \mathrm{AgCl}$ (in $3 \mathrm{M}$ $\mathrm{NaCl}$ ) were used as working, counter and reference electrodes, respectively. For working electrode preparation, $35 \mathrm{mg}$ of the sample and $20 \mu \mathrm{L}$ of nafion $(5 \% \mathrm{w} / \mathrm{w}$ in water/1propanol) were mixed in $7 \mathrm{~mL}$ of THF and sonicated for 5 minutes. The catalyst ink was loaded onto the glassy carbon electrode (diameter $5 \mathrm{~mm}$, area $0.196 \mathrm{~cm}^{2}$ ) by four subsequent coatings (each coating with $10 \mu \mathrm{L}$ ). OER was performed in $0.1 \mathrm{M} \mathrm{KOH}$ electrolyte which was deaerated by bubbling argon gas for at least $30 \mathrm{~min}$ before the measurement started. The cyclic voltammetry $(\mathrm{CV})$ measurements were performed at a scan rate of $10 \mathrm{mV} \mathrm{s}^{-1}$ from 0 to $0.8 \mathrm{~V}$ vs $\mathrm{Ag} / \mathrm{AgCl}$. The potential versus reversible hydrogen electrode (RHE) was calculated according to Nernst equation:

$\mathrm{E}_{\mathrm{RHE}}=\mathrm{E}_{\mathrm{Ag} / \mathrm{AgCl}}+0.059 \mathrm{pH}+\mathrm{E}_{\mathrm{Ag} / \mathrm{AgCl}}^{0}$ 


\section{RESULT AND DISCUSION}

\subsection{Crystal structure}

The X-ray diffraction studies demonstrate the structural changes due to the variation of the A-site cation in these $\mathrm{A}_{2} \mathrm{~B}_{2} \mathrm{O}_{6}$ compounds $\left(\mathrm{A}_{2}=\mathrm{Sr}_{2}, \mathrm{CaSr} ; \mathrm{B}=\mathrm{Mn}\right)$. The crystal structure of $\mathrm{Sr}_{2} \mathrm{Mn}_{2} \mathrm{O}_{6}$ can be indexed on a hexagonal cell with space group $P 6_{3} / m m c$, consistent with previous reports. ${ }^{181}$ Rietveld refinement profile and the refined structural parameters from powder X-ray diffraction data are shown in Figure 9.1 and Table 9.1, respectively. As seen in Figure 9.1, the structure of $\mathrm{Sr}_{2} \mathrm{Mn}_{2} \mathrm{O}_{6}$ consists of dimeric units of face-sharing $\mathrm{MnO}_{6}$ octahedra. Each dimer is connected to other dimers through corner-sharing, leading to a 3-dimensional network. As shown in Table 9.1, there are two crystallographically distinct $\mathrm{Sr}$ sites and two distinct $\mathrm{Mn}$ positions, hence the formula $\mathrm{Sr}_{2} \mathrm{Mn}_{2} \mathrm{O}_{6}$. The crystal structure is transformed upon changing the A-site cations from $\mathrm{Sr}_{2}$ to $\mathrm{CaSr}$, as demonstrated in Figure 9.2. The Ca-containing compound, $\mathrm{CaSrMn}_{2} \mathrm{O}_{6}$, features a cubic $P m-3 m$ structure, where individual $\mathrm{MnO}_{6}$ octahedra are all connected to each other through corner-sharing, consistent with a previous report. ${ }^{193}$ There are no dimeric units in $\mathrm{CaSrMn}_{2} \mathrm{O}_{6}$, as shown in Figure 9.2. There is only one Mn position, and one A-site, jointly occupied by both $\mathrm{Ca}$ and $\mathrm{Sr}$, as shown in Table 9.2 that lists the refined structural parameters for $\mathrm{CaSrMn}_{2} \mathrm{O}_{6}$. 


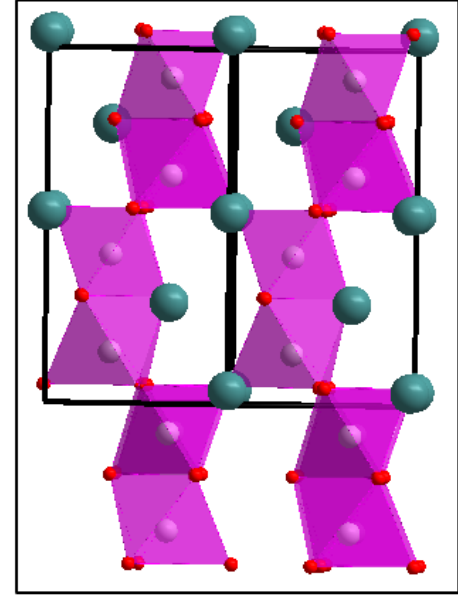

(a)

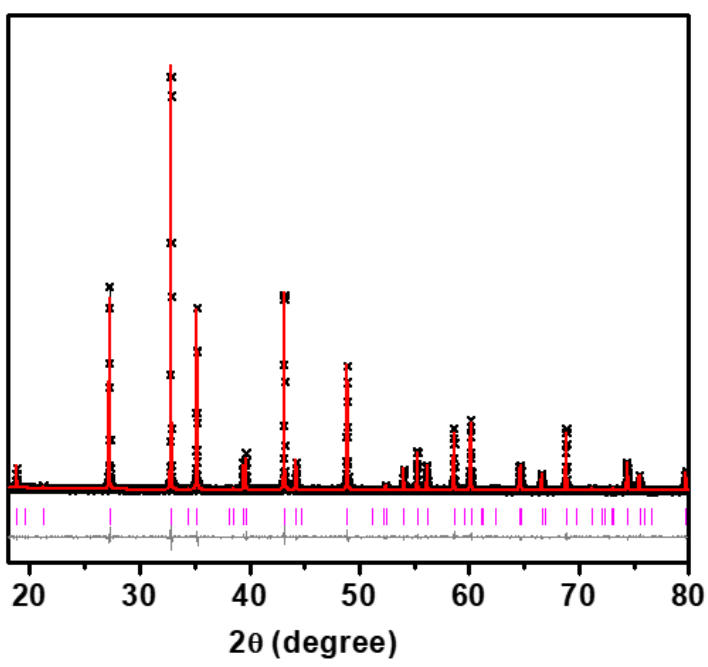

(b)

Figure 9.1. (a) Crystal structure and (b) Rietveld refinement profile from X-ray diffraction data for $\mathrm{Sr}_{2} \mathrm{Mn}_{2} \mathrm{O}_{6}, P 6_{3} / m m c$. Black crosses represent experimental data, the solid red line is the model, pink vertical tick marks show Bragg peak positions, and the lower grey line represents the difference plot.

Table 9.1. Refined structural parameters for $\mathrm{Sr}_{2} \mathrm{Mn}_{2} \mathrm{O}_{6}$ using powder X-ray diffraction. Space group: $P 6_{3} / m m c, a=b=5.45233(8) \AA, \mathrm{c}=9.0856(1) \AA, R_{\mathrm{p}}=0.030, \mathrm{w} R_{\mathrm{p}}=0.038, \chi^{2}$ $=1.790$

\begin{tabular}{ccccccc}
\hline element & $\mathrm{x}$ & $\mathrm{y}$ & $\mathrm{z}$ & $\mathrm{U}_{\text {iso }}$ & occupancy & multiplicity \\
\hline $\mathrm{O} 1$ & $0.191(8)$ & $0.359(1)$ & $0.298(2)$ & $0.020(3)$ & 1 & 6 \\
$\mathrm{O} 2$ & $-0.021(3)$ & $0.507(5)$ & $0.049(2)$ & $0.021(3)$ & 1 & 6 \\
$\mathrm{Mn} 1$ & $1 / 3$ & $2 / 3$ & $0.4160(8)$ & $0.013(3)$ & 1 & 2 \\
$\mathrm{Mn} 2$ & $1 / 3$ & $2 / 3$ & $0.1412(8$ & $0.046(4)$ & 1 & 2 \\
$\mathrm{Sr} 1$ & $1 / 3$ & $2 / 3$ & $0.7850(9)$ & $0.0271(7)$ & 1 & 2 \\
$\mathrm{Sr} 2$ & 0.0 & 0.0 & $0.039(1)$ & $0.0298(7)$ & 1 & 2 \\
\hline
\end{tabular}




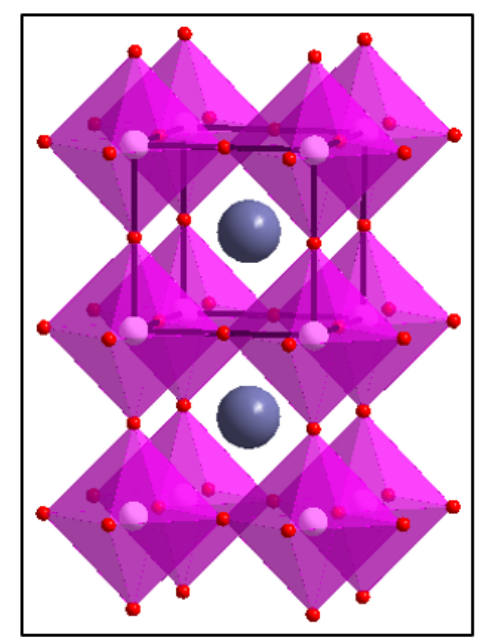

(a)

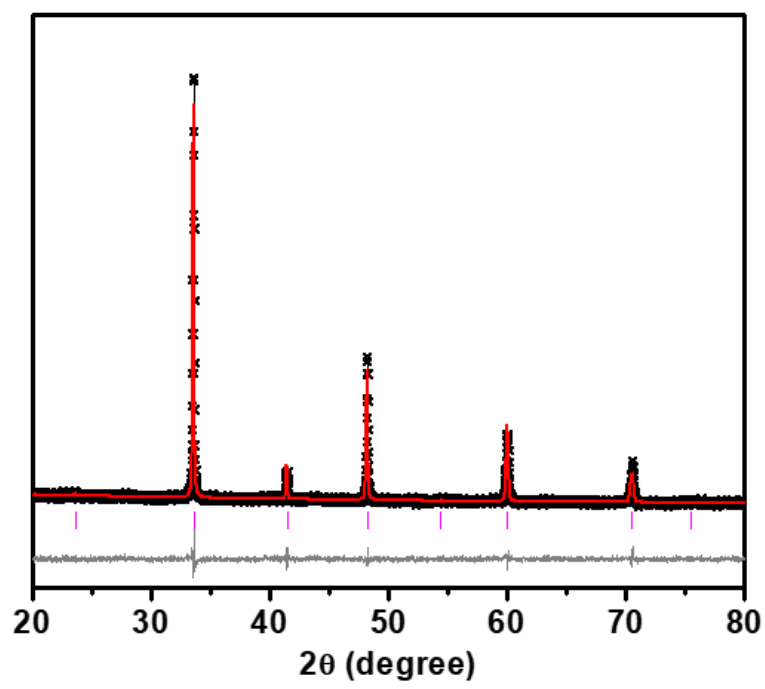

(b)

Figure 9.2. (a) Crystal structure and (b) Rietveld refinement profile from X-ray diffraction data for $\mathrm{CaSrMn}_{2} \mathrm{O}_{6}, P m-3 m$. Black crosses represent experimental data, the solid red line is the model, pink vertical tick marks show Bragg peak positions, and the lower grey line represents the difference plot.

Table 9.2. Refined structural parameters for $\mathrm{CaSrMn}_{2} \mathrm{O}_{6}$ using powder X-ray diffraction data. Space group: $P m-3 m, a=3.7772(1) \AA, R_{\mathrm{p}}=0.051, \mathrm{w} R_{\mathrm{p}}=0.064, \chi^{2}=1.406$

\begin{tabular}{ccccccc}
\hline & $\mathrm{x}$ & $\mathrm{y}$ & $\mathrm{z}$ & $\mathrm{U}_{\text {iso }}$ & occupancy & multiplicity \\
\hline $\mathrm{O} 1$ & 0.5 & 0.0 & 0.0 & $0.043(2)$ & 1 & 3 \\
$\mathrm{Mn} 1$ & 0.0 & 0.0 & 0.0 & $0.007(1)$ & 1 & 1 \\
$\mathrm{Ca} 1$ & 0.5 & 0.5 & 0.5 & $0.024(2)$ & 0.5 & 1 \\
$\mathrm{Sr} 1$ & 0.5 & 0.5 & 0.5 & $0.024(2)$ & 0.5 & 1 \\
\hline
\end{tabular}

We note that further substitution of $\mathrm{Sr}$ by $\mathrm{Ca}$, beyond $\mathrm{CaSrMn}_{2} \mathrm{O}_{6}$, does not change the polyhedral connectivity. The completely substituted product, that contains only $\mathrm{Ca}$ on the A-site, contains the same type of corner-sharing connectivity as $\mathrm{CaSrMn}_{2} \mathrm{O}_{6}$, although the corner-sharing $\mathrm{MnO}_{6}$ octahedra are somewhat distorted, leading to orthorhombic symmetry. ${ }^{194}$ Nevertheless, the overall picture that emerges is that the transition from 
$\mathrm{Sr}_{2} \mathrm{Mn}_{2} \mathrm{O}_{6}$ to $\mathrm{CaSrMn}_{2} \mathrm{O}_{6}$ results in significant structural transformation, but further substitution of $\mathrm{Sr}$ by $\mathrm{Ca}$ does not have a substantial effect on the structure-type.

The Mn-O bond distances in $\mathrm{Sr}_{2} \mathrm{Mn}_{2} \mathrm{O}_{6}$ vary widely, 1.81(2), 1.87(2), 1.91(2), and 2.03(2) $\AA$, due to the existence of two types of polyhedral connectivity, namely face-sharing and corner-sharing. Given the structural differences between the two compounds, direct comparison of the bond distances is not warranted. However, the average $\mathrm{Mn}-\mathrm{O}$ bond distance in $\mathrm{Sr}_{2} \mathrm{Mn}_{2} \mathrm{O}_{6}, 1.91 \AA$, is longer than that of $\mathrm{CaSrMn}_{2} \mathrm{O}_{6}, 1.89 \AA$. Furthermore, the Mn-O-Mn bond angles in $\mathrm{Sr}_{2} \mathrm{Mn}_{2} \mathrm{O}_{6}$ can be as small as $80.9(3)^{\circ}$ due to the face-sharing in octahedral dimers. The angle between the corner-sharing octahedra in $\mathrm{Sr}_{2} \mathrm{Mn}_{2} \mathrm{O}_{6}$ is $\mathrm{Mn}-\mathrm{O}-$ $\mathrm{Mn}=166(1)^{\circ}$. However, $\mathrm{CaSrMn}_{2} \mathrm{O}_{6}$ contains only one type of Mn-O-Mn angle, which is $180^{\circ}$, as expected from a cubic perovskite structure.

\subsection{Electrical properties}

Changes in composition, and the subsequent variation of the crystal structure lead to significant changes in the electrical properties, which were studied by four probe technique. The electrical conductivity $(\sigma)$ is obtained from the measured resistance $(R)$, using the following equation: ${ }^{195}$

$$
\sigma=L / R A
$$

where $\mathrm{L}$ is the voltage probe spacing and $\mathrm{A}$ represents the cross-sectional area of the rectangular pellet where the current probes are connected. The remarkable effect of structural transformation is immediately clear from the improvement in the electrical conductivity by five orders of magnitude, $4.5 \times 10^{-1} \mathrm{~S} \mathrm{~cm}^{-1}$ for $\mathrm{CaSrMn}_{2} \mathrm{O}_{6}$, compared to 
$1.0 \times 10^{-6} \mathrm{~S} \mathrm{~cm}^{-1}$ for $\mathrm{Sr}_{2} \mathrm{Mn}_{2} \mathrm{O}_{6}$ at room temperature. The electrical conductivity in oxide materials occurs through $\mathrm{M}-\mathrm{O}-\mathrm{M}$ pathways, where $\mathrm{M}$ is the transition metal. It has been demonstrated that larger bond angles lead to enhanced electrical conductivity due to the improved overlap between the metal $3 \mathrm{~d}$ band and oxygen $2 \mathrm{p}$ band. ${ }^{135}$ The change in crystal structure between $\mathrm{Sr}_{2} \mathrm{Mn}_{2} \mathrm{O}_{6}$ and $\mathrm{CaSrMn}_{2} \mathrm{O}_{6}$ clearly leads to the enhancement of the $\mathrm{Mn}-$ O-Mn bond angles. The face sharing of $\mathrm{MnO}_{6}$ octahedra in $\mathrm{Sr}_{2} \mathrm{Mn}_{2} \mathrm{O}_{6}$ results in bond angles as small as $80.9(3)^{\circ}$, as described in the previous section. However, the $\mathrm{Mn}-\mathrm{O}-\mathrm{Mn}$ bond angles in $\mathrm{CaSrMn}_{2} \mathrm{O}_{6}$ are $180.0^{\circ}$, due to the cubic structure and corner-sharing between the octahedra.

The superior electrical conductivity of $\mathrm{CaSrMn}_{2} \mathrm{O}_{6}$ persists at higher temperature, as evident from the results of conductivity studies in a wide temperature range, $25-800{ }^{\circ} \mathrm{C}$, shown in Figure 9.3. Both compounds show increase in conductivity as a function of temperature, a behavior typical of semiconductors. The increase in temperature results in the loss of oxygen and reduction of some of $\mathrm{Mn}^{4+}$ ions into $\mathrm{Mn}^{3+}$, leading to facile charge transport through the resultant $\mathrm{Mn}^{4+}-\mathrm{O}-\mathrm{Mn}^{3+}$ pathways. In addition, the oxide ion conductivity is enhanced due to the creation of lattice defects upon oxygen loss. Furthermore, the rise in temperature leads to an increase in the mobility of charge carriers, which in turn results in the enhancement of the electrical conductivity as described by the equation: 7

$$
\sigma=\text { ne } \mu
$$

where $\sigma, \mathrm{n}, \mathrm{e}$, and $\mu$ are the conductivity, concentration of charge carriers (electrons/holes), charge of the electron, and mobility of the charge carriers, respectively. 
The activation energy for the increase in electrical conductivity as a function of temperature can be found using the Arrhenius equation for thermally activated conductivity: ${ }^{49-51}$

$$
\sigma T=\sigma^{0} e^{\frac{-E_{a}}{K T}}
$$

where $\sigma^{0}$ is a preexponential factor and a characteristic of the material. Ea, K, and T are the activation energy for the increase in conductivity, Boltzmann constant, and absolute temperature, respectively. The activation energy $(\mathrm{Ea})$ is calculated from the slope of the line of best fit in the $\log \sigma \mathrm{T}$ versus 1000/T plot (Figure 9.3). The Ea values for the sharp rise in conductivity above $500{ }^{\circ} \mathrm{C}$ are $0.687 \mathrm{eV}$ for $\mathrm{Sr}_{2} \mathrm{Mn}_{2} \mathrm{O}_{6}$ and $0.449 \mathrm{eV}$ for $\mathrm{CaSrMn}{ }_{2} \mathrm{O}_{6}$.

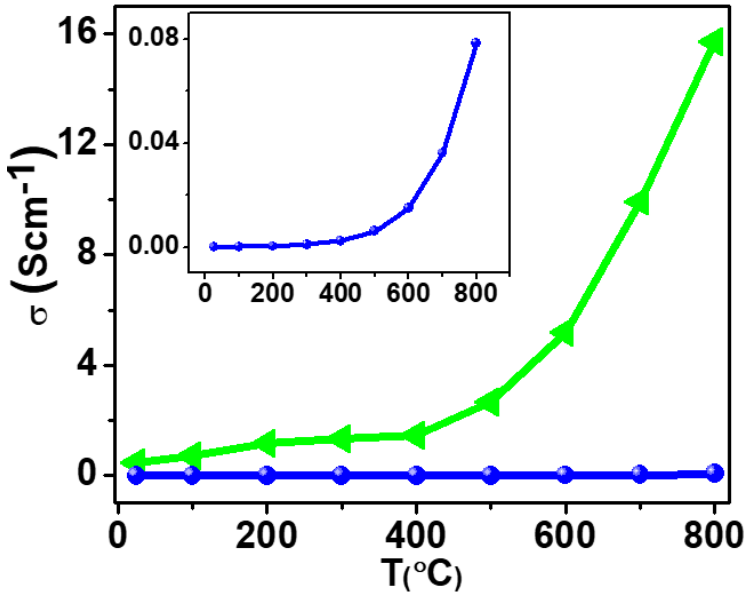

(a)

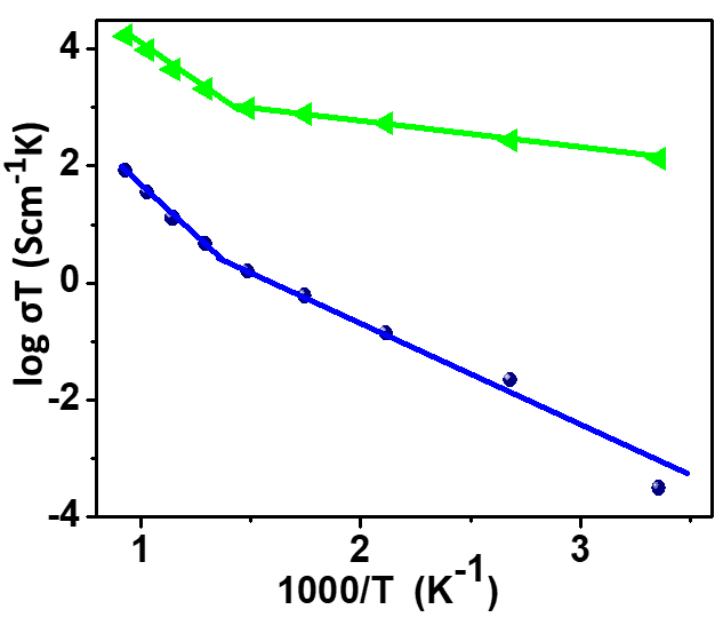

(b)

Figure 9.3. (a) Temperature dependent electrical conductivity and (b) Arrhenius plots for $\mathrm{Sr}_{2} \mathrm{Mn}_{2} \mathrm{O}_{6}$ (blue circles) and $\mathrm{CaSrMn}_{2} \mathrm{O}_{6}$ (green triangles). The inset in (a) is a zoomed view of the increase in conductivity of $\mathrm{Sr}_{2} \mathrm{Mn}_{2} \mathrm{O}_{6}$.

Table 9.3. Room temperature conductivity and activation energies.

\begin{tabular}{ccl}
\hline & Conductivity, $\sigma\left(\mathrm{Scm}^{-1}\right)$ & Activation energy, $\mathrm{E}_{\mathrm{a}}(\mathrm{eV})$ \\
\hline $\mathrm{Sr}_{2} \mathrm{Mn}_{2} \mathrm{O}_{6}$ & $1.0 \times 10^{-6}$ & $0.382 \mathrm{eV}$ for 25 to $500^{\circ} \mathrm{C}$ \\
& $0.687 \mathrm{eV}$ for 500 to $800^{\circ} \mathrm{C}$ \\
& & \\
$\mathrm{CaSrMn}_{2} \mathrm{O}_{6}$ & $4.5 \times 10^{-1}$ & $0.093 \mathrm{eV}$ for 25 to $400^{\circ} \mathrm{C}$ \\
& & $0.449 \mathrm{eV}$ for 400 to $800^{\circ} \mathrm{C}$ \\
\hline
\end{tabular}




\subsection{Correlation between electrocatalytic activity and conductivity}

The electrocatalytic activity of these materials for oxygen evolution reaction (OER) was studied by cyclic voltammetry in alkaline medium. The OER mechanism in alkaline solution has been examined by several researchers. ${ }^{26-27,} 196$ The commonly accepted mechanism involves four steps, where there is a single electron transfer in each step. ${ }^{26-27}$, ${ }^{196}$ In the first step, the reaction initiates by the adsorption of $\mathrm{OH}^{-}$on the active site of the catalyst, i.e., metal site, M. ${ }^{27,}{ }^{196}$ In the second step, a hydroxide from the electrolyte abstracts a proton from $\mathrm{M}-\mathrm{OH}$ to form $\mathrm{M}-\mathrm{O}$ and water. In the third step, $\mathrm{M}-\mathrm{O}$ combines with a hydroxide to form a peroxide. Finally, in the fourth step, the peroxide intermediate reacts with $\mathrm{OH}^{-}$to give an oxygen and water and regenerate the catalyst.

5. $\mathrm{M}+\mathrm{OH}^{-} \rightarrow \mathrm{M}-\mathrm{OH}+\mathrm{e}^{-}$

6. $\mathrm{M}-\mathrm{OH}+\mathrm{OH}^{-} \rightarrow \mathrm{M}-\mathrm{O}+\mathrm{H}_{2} \mathrm{O}+\mathrm{e}^{-}$

7. $\mathrm{M}-\mathrm{O}+\mathrm{OH}^{-} \rightarrow \mathrm{M}-\mathrm{OOH}+\mathrm{e}^{-}$

\section{8. $\mathrm{M}-\mathrm{OOH}+\mathrm{OH}^{-} \rightarrow \mathrm{M}+\mathrm{H}_{2} \mathrm{O}+\mathrm{O}_{2}+\mathrm{e}^{-}$}

The conventional technique for investigation of OER activity involves the addition of carbon black to the electrode composition in order to enhance the electrical conductivity within the electrode and maximize the utilization of the catalyst. ${ }^{197-199}$ However, recent studies have shown that the role of carbon is more complex than a simple enhancement of conductivity. ${ }^{200-201}$ For example, it has been shown that cobalt in an OER catalyst is reduced by carbon during the composite preparation process. ${ }^{202}$ Therefore, OER 
experiments without carbon black are prefered, ${ }^{135}$ in order to examine the intrinsic catalytic activity of a material, without interference from carbon. ${ }^{203}$ The two materials studied in this work demonstrate the effect of crystal structure and electrical conductivity on OER activity. As observed from Figure 9.4, $\mathrm{CaSrMn}_{2} \mathrm{O}_{6}$ shows significantly enhanced onset potential, $\sim 1.55 \mathrm{~V}$, compared to $\mathrm{Sr}_{2} \mathrm{Mn}_{2} \mathrm{O}_{6}, \sim 1.65 \mathrm{~V}$. While these onset potentials are not as good as those of the state of the art catalysts materials, such as $\mathrm{RuO}_{2}, \sim 1.45 \mathrm{~V},{ }^{119} \mathrm{IrO}_{2}$, $\sim 1.50 \mathrm{~V},{ }^{119}$ and BSCF, $\sim 1.53 \mathrm{~V},{ }^{204}$ they are comparable to or better than those of several other reported catalysts, such as $\mathrm{CaMnO}_{3} / \mathrm{C}(1.6 \mathrm{~V}),{ }^{119}$ Co-based microporous polymers, $1.57 \mathrm{~V},{ }^{205}$ and Co-phthalocyanine, $1.70 \mathrm{~V} .{ }^{205}$ We have also determined the mass activity of each catalyst, using the catalyst loading mass on the electrode surface $\left(0.2 \mathrm{mg} \mathrm{cm}^{-2}\right)$ and the measured current density for that electrode $\left.\mathrm{J}(\mathrm{mA} \mathrm{cm})^{-2}\right)$. The inset in Figure 9.4 compares the OER mass activity of the two materials at $1.80 \mathrm{~V}$ vs RHE. As observed in this figure, $\mathrm{CaSrMn}_{2} \mathrm{O}_{6}$ shows mass activity of $0.42 \mathrm{~A} / \mathrm{g}$, compared to $0.28 \mathrm{~A} / \mathrm{g}$ for $\mathrm{Sr}_{2} \mathrm{Mn}_{2} \mathrm{O}_{6}$.

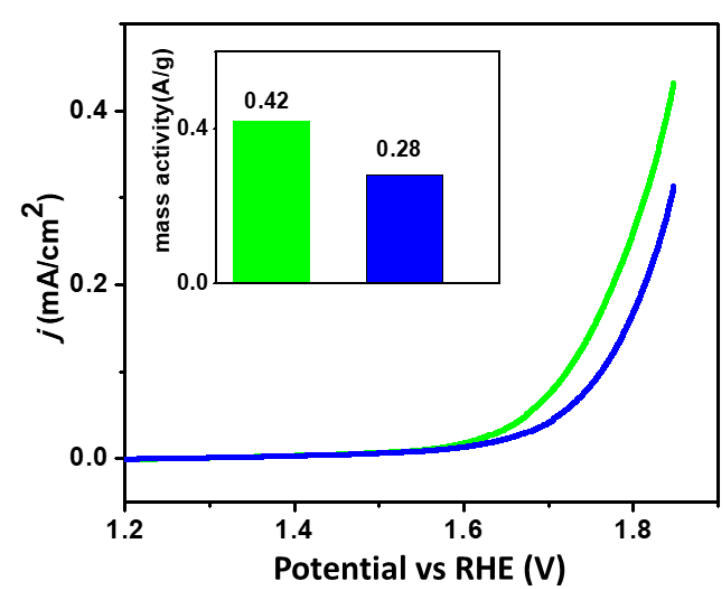

(a)

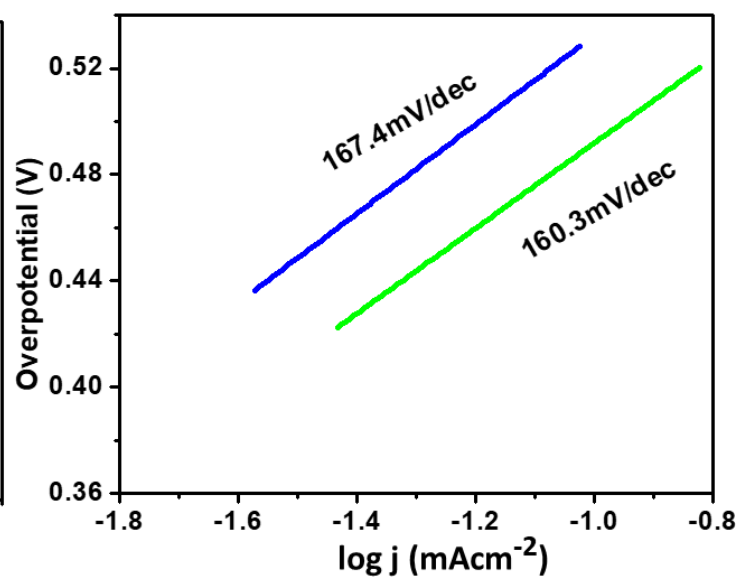

(b)

Figure 9.4. (a) Polarization curves for OER with mass activities for $\mathrm{Sr}_{2} \mathrm{Mn}_{2} \mathrm{O}_{6}$ (blue) and $\mathrm{CaSrMn}_{2} \mathrm{O}_{6}$ (green). (b) Tafel slopes for $\mathrm{Sr}_{2} \mathrm{Mn}_{2} \mathrm{O}_{6}$ (blue) and $\mathrm{CaSrMn}_{2} \mathrm{O}_{6}$ (green). 
The kinetics of OER is usually examined using the Tafel equation $\eta=a+b \log j$ where $\eta$ is the overpotential, and $j$ is the current density. ${ }^{206-207}$ The plot is obtained from the curved portion of the OER cyclic voltammogram, as commonly done in OER analysis. ${ }^{208-209}$ The slope of the Tafel plot, $\eta$ vs. $\log j$, is used as a measure for the reaction kinetics and is influenced by electron and mass transport ability of the catalyst. ${ }^{210-211}$ Smaller slope is associated with faster reaction, and indicates that once the reaction commences, it proceeds quickly without the need for significant increase in potential. ${ }^{212}$ In other words, small increase in potential leads to the generation of significant current by OER process due to the fast kinetics of the reaction. Small change in potential accompanied by large increase in current results in a small slope in the plot of $\eta$ vs. $\log j$. The Tafel slopes (Figure 9.4) for $\mathrm{CaSrMn}_{2} \mathrm{O}_{6}$ and $\mathrm{Sr}_{2} \mathrm{Mn}_{2} \mathrm{O}_{6}$ are $160.3 \mathrm{mV} / \mathrm{dec}$ and $167.4 \mathrm{mV} / \mathrm{dec}$, respectively, consistent with the greater OER activity of $\mathrm{CaSrMn}_{2} \mathrm{O}_{6}$ and the improved charge transport in this compound. These Tafel slopes are higher than those observed for highly active catalysts such as BSCF $(87 \mathrm{mv} / \mathrm{dec}){ }^{203}$ However, they are lower than the Tafel slopes for several other OER catalysts reported in the literature, with values ranging from 220 $\mathrm{mV} / \mathrm{dec},{ }^{213}$ to $184 \mathrm{mV} / \mathrm{dec},{ }^{214}$ and $169 \mathrm{mV} / \mathrm{dec} .{ }^{215}$ The relationship between the electrical conductivity and OER activity is remarkable. $\mathrm{CaSrMn}_{2} \mathrm{O}_{6}$, which has superior electrical conductivity, also shows higher OER activity compared to $\mathrm{Sr}_{2} \mathrm{Mn}_{2} \mathrm{O}_{6}$. These findings demonstrate an interesting correlation between crystal structure, electrical conductivity and electrocatalytic properties. The modification of the crystal structure can be used as a tool to enhance the electrical conductivity, which in turn can have an impact on the electrocatalytic properties. 


\section{CONCLUSION}

The substitution of $50 \%$ of the A-site cations in $\mathrm{Sr}_{2} \mathrm{Mn}_{2} \mathrm{O}_{6}$ results in the formation of $\mathrm{CaSrMn}_{2} \mathrm{O}_{6}$, which features a different crystal structure and polyhedral connectivity. The change in the crystal structure leads to a significantly improved electrical conductivity in $\mathrm{CaSrMn}_{2} \mathrm{O}_{6}$. Variable-temperature electrical conductivity measurements indicate that the higher conductivity of $\mathrm{CaSrMn}_{2} \mathrm{O}_{6}$ persists up to $800{ }^{\circ} \mathrm{C}$. The enhanced conductivity in turn results in superior catalytic activity of $\mathrm{CaSrMn}_{2} \mathrm{O}_{6}$ for oxygen evolution reaction. These findings indicate the dependence of the electrical conductivity on crystal structure, and the correlation between structure, charge-transport and electrocatalytic activity. 


\section{CHAPTER 10}

\section{VARIATION IN ELECTRICAL CONDUCTIVITY OF $\mathrm{A}_{2} \mathrm{Fe}_{2} \mathrm{O}_{5}(\mathrm{~A}=\mathrm{Sr}, \mathrm{Ba})^{9}$}

\section{INTRODUCTION}

Oxygen deficient perovskites (ODPs) are an important component of solid oxide fuel cells. ${ }^{163}$ They have also been studied for application as gas sensors, ${ }^{161}$ gas diffusion membranes, ${ }^{162}$ and photocatalysts. ${ }^{216}$ Given the structure-property relationship in ODPs, it is important to study the variation in structural order and its impact on the properties of this class of materials. ODPs have the general formula $\mathrm{ABO}_{3-\mathrm{x}}\left(\mathrm{A}_{2} \mathrm{~B}_{2} \mathrm{O}_{6-\mathrm{y}}\right)$ where $\mathrm{A}$ is usually an alkaline earth metal or lanthanide, and B is a transition metal. As a result of oxygen deficiency, some vacancies are created and some of the $\mathrm{BO}_{6}$ octahedra are converted into other coordination geometries such as $\mathrm{BO}_{4}$ tetrahedra or $\mathrm{BO}_{5}$ square pyramids. ${ }^{4}$ In some cases, the vacant sites appear in random locations within the crystal lattice, and no structural order is present. ${ }^{12,76,87,89}$

However, the ordered arrangement of the vacant sites is also common. ${ }^{4,72-75,84}$ The structural order can be manipulated by substitution on the B-site. For example, $\mathrm{Sr}_{2} \mathrm{Fe}_{2} \mathrm{O}_{6}$ $\delta^{4,83}$ and $\mathrm{Sr}_{2} \mathrm{FeMnO}_{6-\delta}{ }^{89}$ synthesized under the same condition, i.e., $1250{ }^{\circ} \mathrm{C}$ in air, have

${ }^{9}$ The work described in this chapter was published in Material Research Express ( 2018, vol. 5, p. 9076307) 
remarkably different structure and properties. $\mathrm{Sr}_{2} \mathrm{Fe}_{2} \mathrm{O}_{6-\delta}$ has a tetragonal $14 / \mathrm{mmm}$ structure, where vacancy-order leads to the formation of alternating $\mathrm{FeO}_{6}$ octahedra and $\mathrm{FeO}_{5}$ square pyramids. ${ }^{4,}{ }^{83}$ In $\mathrm{Sr}_{2} \mathrm{FeMnO}_{6-\delta}$, on the other hand, the vacancy order is eliminated and a cubic $P m-3 m$ structure is formed. ${ }^{89}$ These structural changes affect the magnetic properties, where the spin-density wave state in $\mathrm{Sr}_{2} \mathrm{Fe}_{2} \mathrm{O}_{5.75}{ }^{217}$ changes into an inhomogeneous magnetic ground state. ${ }^{89}$

Changes in structural order can also occur due to substitution on the A-site. For example, $\mathrm{Sr}_{2} \mathrm{FeCoO}_{5}, \mathrm{CaSrFeCoO}_{5}$, and $\mathrm{Ca}_{2} \mathrm{FeCoO}_{5}$ have significantly different crystal structures. ${ }^{84}$ $\mathrm{Sr}_{2} \mathrm{FeCoO}_{5}$ lacks any long-range order and has a cubic $\mathrm{Pm}-3 m$ structure, whereas $\mathrm{CaSrFeCoO}_{5}$ exhibits structural order with orthorhombic Ibm2 space group. ${ }^{84} \mathrm{Ca}_{2} \mathrm{FeCoO}_{5}$ has an even greater degree of ordering and crystalizes in Pbcm space group. ${ }^{73-74,84}$ These variations in crystal structure lead to differences in electrical conductivity, highlighting the strong structure-property correlations in these compounds. ${ }^{84}$

In this paper, we have studied the electrical properties of $\mathrm{Sr}_{2} \mathrm{Fe}_{2} \mathrm{O}_{5}$ and $\mathrm{Ba}_{2} \mathrm{Fe}_{2} \mathrm{O}_{5}$, that feature drastic differences in structural order and electrical charge transport, as a result of variation in A-site cation.

\section{EXPERIMENTAL}

The materials $\mathrm{Ba}_{2} \mathrm{Fe}_{2} \mathrm{O}_{5}$ and $\mathrm{Sr}_{2} \mathrm{Fe}_{2} \mathrm{O}_{5}$ were synthesized under identical conditions through solid state method. The powders of the precursor compounds $\mathrm{BaCO}_{3}$ (Alfa Aesar, 99.95\%), $\mathrm{SrCO}_{3}$ (Aldrich, 99.9\%), and $\mathrm{Fe}_{2} \mathrm{O}_{3}$ (Alfa Aesar,99.998\%) were mixed and ground together using agate mortar and pestle, pressed into a pellet, and heated in argon at $1200{ }^{\circ} \mathrm{C}$ for 24 h. The samples were then reground and refired at $1200{ }^{\circ} \mathrm{C}$ for $24 \mathrm{~h}$ under the same condition, followed by slow cooling. The heating and cooling rates were $100{ }^{\circ} \mathrm{C} / \mathrm{h}$. The 
phase purity and structure of the polycrystalline samples were determined by powder Xray diffraction $(\mathrm{XRD})$ using $\mathrm{Cu} \mathrm{K} \alpha 1$ radiation $(\lambda=1.54056 \AA)$. The GSAS software ${ }^{100}$ EXPEGUI interface ${ }^{101}$ were used for Rietveld refinements. The sample morphologies were studied using high resolution field-emission scanning electron microscopy (SEM). X-ray photoelectron spectroscopy (XPS) was performed at room temperature using $\mathrm{Al} \mathrm{K} \alpha$ radiation $(1486.7 \mathrm{eV})$ to study the oxidation states of Fe. The electrical properties were investigated by direct-current (dc) and alternating-current (ac) conductivity measurements on as-prepared pellets that had been sintered at $1100{ }^{\circ} \mathrm{C}$. Electrochemical impedance spectroscopy was performed in the frequency range of $0.1 \mathrm{~Hz}$ to $1.0 \mathrm{MHz}$ using a computercontrolled frequency response analyzer at room temperature. The two-probe dc measurements were performed in the temperature range of $25-900{ }^{\circ} \mathrm{C}$ by applying a constant voltage of $10 \mathrm{mV}$ and collecting the output current. Variable-temperature electrical conductivity measurements were carried out during both heating and cooling cycles. The rate of heating and cooling for conductivity measurements was $3{ }^{\circ} \mathrm{C} / \mathrm{min}$. Iodometric titrations were performed by dissolving about $50 \mathrm{mg}$ of the sample and excess $\mathrm{KI}(\sim 2 \mathrm{~g})$ in $100 \mathrm{~mL}$ of $1 \mathrm{M} \mathrm{HCl}$. A total of $5 \mathrm{~mL}$ of the solution was then pipetted out, and the iodine that had been generated in the solution was titrated against $0.025 \mathrm{M} \mathrm{Na}_{2} \mathrm{~S}_{2} \mathrm{O}_{3}$. Near the end point of the titration, $0.2 \mathrm{~mL}$ of a starch solution was added to act as an indicator. All steps were performed under argon atmosphere. Magnetic susceptibility data were obtained using vibrating sample magnetometry by applying magnetic field of $0.1 \mathrm{~T}$ in the temperature range $2-400 \mathrm{~K}$. Isothermal magnetization data were obtained at $5 \mathrm{~K}$ and $300 \mathrm{~K}$ using the magnetic field of $0-9 \mathrm{~T}$. 


\section{RESULTS AND DISCUSSION}

Crystal structure

The crystal structure of the two compounds were confirmed using X-ray diffraction and were consistent with reported structures of these materials. ${ }^{218-219,14}$ Despite being synthesized under identical conditions, $\mathrm{Sr}_{2} \mathrm{Fe}_{2} \mathrm{O}_{5}$ and $\mathrm{Ba}_{2} \mathrm{Fe}_{2} \mathrm{O}_{5}$ have remarkably different crystal structures. Figures 10.1 and 10.2 show Rietveld refinement profiles and crystal structures of the two compounds. $\mathrm{Sr}_{2} \mathrm{Fe}_{2} \mathrm{O}_{5}$ features alternating layers of $\mathrm{FeO}_{6}$ octahedra and $\mathrm{FeO}_{4}$ tetrahedra, as shown in Figure 10.1. This is the brownmillerite-type structure, which is one of the common variations of oxygen-deficient perovskites. The refined structural parameters for $\mathrm{Sr}_{2} \mathrm{Fe}_{2} \mathrm{O}_{5}$ are listed in Table 10.1 .

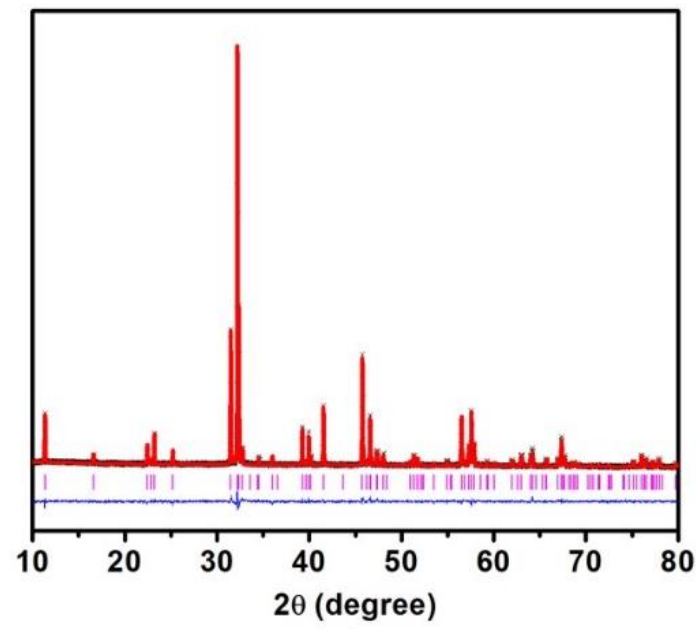

(a)

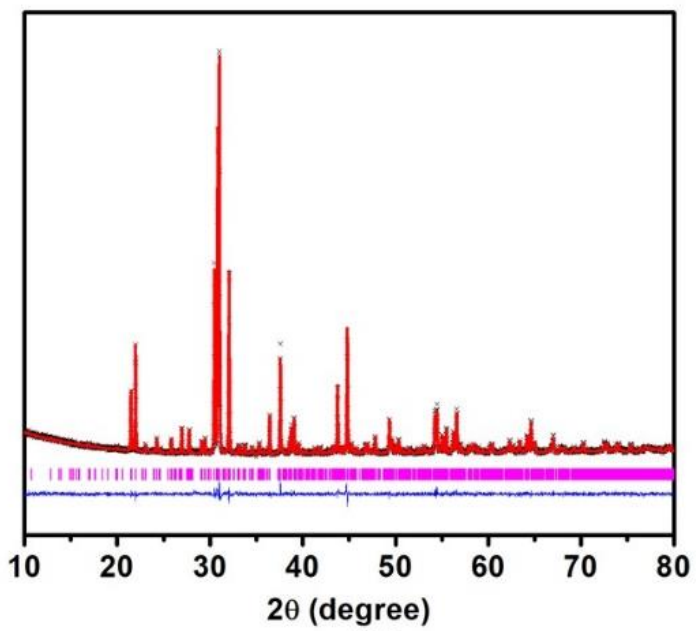

(b)

Figure 10.1. Rietveld refinement profile for powder XRD data of (a) $\mathrm{Sr}_{2} \mathrm{Fe}_{2} \mathrm{O}_{5}$, with $\mathrm{Ibm} 2$ space group and (b) $\mathrm{Ba}_{2} \mathrm{Fe}_{2} \mathrm{O}_{5}$ with monoclinic $\mathrm{P} 2{ }_{1} / \mathrm{c}$ space group. The solid red line shows the refinement model, black crosses represent experimental data, pink vertical tick marks show Bragg peak positions, and the lower blue line represents the difference plot. 
Table 10.1. Refined structural parameters for $\mathrm{Sr}_{2} \mathrm{Fe}_{2} \mathrm{O}_{5}$ using powder X-ray powder diffraction. Space group is $\mathrm{Ibm} 2$, and unit cell parameters are $a=5.68023(6), b=$ $15.5862(2), c=5.53425(6) \AA$.

\begin{tabular}{ccccccc}
\hline Element & $x$ & $y$ & $z$ & $\mathrm{U}_{\text {iso }}$ & Occupancy & Multiplicity \\
\hline $\mathrm{Sr} 1$ & $0.0159(5)$ & $0.1094(1)$ & $0.498(3)$ & $0.0130(9)$ & 1 & 8 \\
$\mathrm{Fe} 1$ & 0.0 & 0.0 & 0.0 & $0.0128(2)$ & 1 & 4 \\
$\mathrm{Fe} 2$ & $0.9356(9)$ & 0.25 & $0.962(3)$ & $0.021(3)$ & 1 & 4 \\
$\mathrm{O} 1$ & $0.268(4)$ & $0.9904(6)$ & $0.260(9)$ & 0.03 & 1 & 8 \\
$\mathrm{O} 2$ & $0.051(2)$ & $0.1396(4)$ & $0.028(4)$ & 0.03 & 1 & 8 \\
$\mathrm{O} 3$ & $0.855(3)$ & 0.25 & $0.622(4)$ & 0.03 & 1 & 4 \\
\hline
\end{tabular}

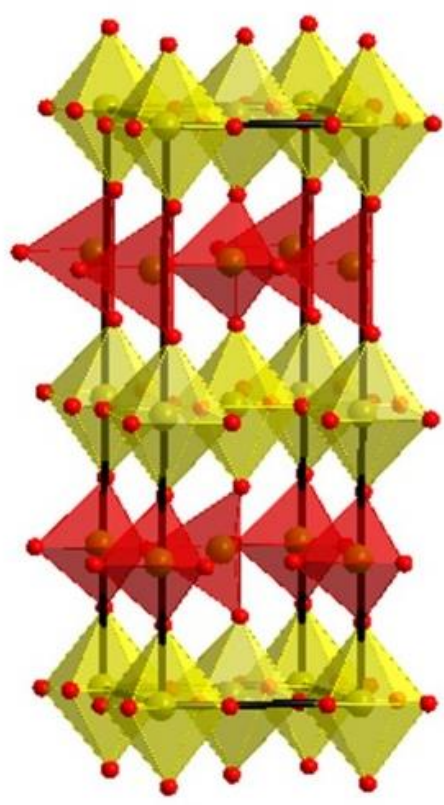

(a)

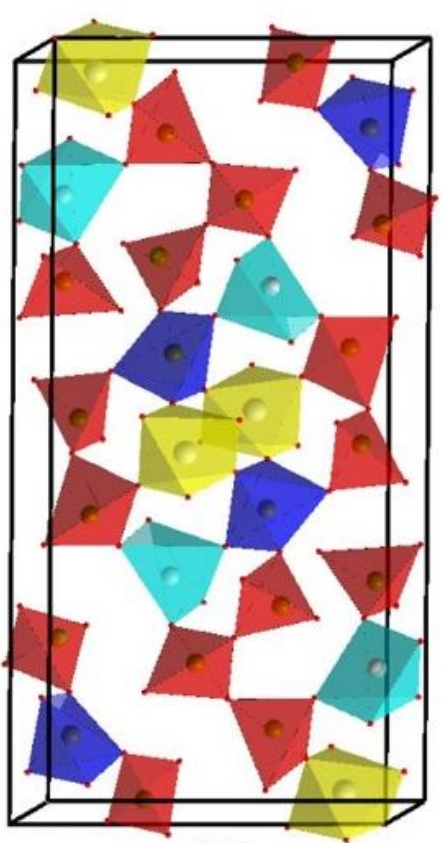

(b)

Figure 10.2. Crystal structures of (a) $\mathrm{Sr}_{2} \mathrm{Fe}_{2} \mathrm{O}_{5}$ and (b) $\mathrm{Ba}_{2} \mathrm{Fe}_{2} \mathrm{O}_{5}$. 
The crystal structure of $\mathrm{Ba}_{2} \mathrm{Fe}_{2} \mathrm{O}_{5}$ is entirely different and contains a complex array of tetrahedral, square-pyramidal and octahedral coordination geometries around Fe atoms. ${ }^{218-219}$ Our Rietveld refinement results confirm this complex structure. Tables 10.2 and 10.3 list the refined atomic positions, coordination geometries and bond distances for Fe atoms. There are seven crystallographically distinct $\mathrm{Fe}$ sites in $\mathrm{Ba}_{2} \mathrm{Fe}_{2} \mathrm{O}_{5}$. They include four tetrahedral, two square-pyramidal and one octahedral Fe position.

Each $\mathrm{FeO}_{\mathrm{n}}$ polyhedron $(\mathrm{n}=4,5,6)$ shares corners with other types of polyhedra, as well as polyhedra of the same type, as shown in Figures 10.2 and 10.3. For example, each $\mathrm{FeO}_{6}$ octahedron shares corners with one $\mathrm{FeO}_{6}$ octahedron, two $\mathrm{FeO}_{4}$ tetrahedra and three $\mathrm{FeO}_{5}$ square-pyramids. In the crystal lattice, the $\mathrm{FeO}_{6}$ octahedra and $\mathrm{FeO}_{5}$ square-pyramids share all of their apexes with other polyhedra, whereas some of the $\mathrm{FeO}_{4}$ tetrahedra have one unshared corner. Table 10.4 lists the connectivity between different polyhedra. 
Table 10.2. Refined structural parameters for $\mathrm{Ba}_{2} \mathrm{Fe}_{2} \mathrm{O}_{5}$ using powder X-ray powder diffraction. Space group is $P 2_{1} / c$ and unit cell parameters are $a=6.9772(1), b=11.7376(2)$, $c=23.4575(4) \AA$, and $\beta=98.7540(8)^{\circ}$.

\begin{tabular}{ccccccc}
\hline Element & $\mathrm{x}$ & $\mathrm{y}$ & $\mathrm{z}$ & $\mathrm{U}_{\text {iso }}$ & Occupancy & Multiplicity \\
\hline $\mathrm{Ba} 1$ & $0.054(2)$ & $0.354(1)$ & $0.1148(5)$ & $0.008(4)$ & 1 & 4 \\
$\mathrm{Ba} 2$ & $0.250(2)$ & $0.614(1)$ & $0.3290(6)$ & $0.027(5)$ & 1 & 4 \\
$\mathrm{Ba3}$ & $0.133(2)$ & $0.131(1)$ & $0.3219(5)$ & $0.018(5)$ & 1 & 4 \\
$\mathrm{Ba} 4$ & $0.322(2)$ & $0.633(1)$ & $0.0391(7)$ & $0.034(5)$ & 1 & 4 \\
$\mathrm{Ba5}$ & $0.052(2)$ & $0.615(1)$ & $0.6014(7)$ & $0.012(4)$ & 1 & 4 \\
$\mathrm{Ba} 6$ & $0.555(2)$ & $0.351(1)$ & $0.2575(6)$ & $0.025(5)$ & 1 & 4 \\
$\mathrm{Ba} 7$ & $0.351(2)$ & $0.110(1)$ & $0.0357(8)$ & $0.017(4)$ & 1 & 4 \\
$\mathrm{Fe} 1$ & $0.532(4)$ & $0.364(3)$ & $0.100(2)$ & $0.024(8)$ & 1 & 4 \\
$\mathrm{Fe} 2$ & $0.385(4)$ & $0.580(3)$ & $0.188(2)$ & $0.024(8)$ & 1 & 4 \\
$\mathrm{Fe3}$ & $0.074(4)$ & $0.395(3)$ & $0.258(2)$ & $0.014(7)$ & 1 & 4 \\
$\mathrm{Fe} 4$ & $0.179(5)$ & $0.155(3)$ & $0.472(2)$ & $0.03(1)$ & 1 & 4 \\
$\mathrm{Fe} 5$ & $0.427(5)$ & $0.384(3)$ & $0.399(2)$ & $0.014(7)$ & 1 & 4 \\
$\mathrm{Fe6}$ & $0.269(5)$ & $0.102(3)$ & $0.176(2)$ & $0.030(7)$ & 1 & 4 \\
$\mathrm{Fe} 7$ & $0.135(6)$ & $0.624(3)$ & $0.466(2)$ & $0.030(7)$ & 1 & 4 \\
O1 & 0 & 0 & 0 & 0.03 & 1 & 2 \\
O2 & $0.28(2)$ & $0.721(9)$ & $0.152(5)$ & 0.03 & 1 & 4 \\
O3 & $-0.04(1)$ & $0.272(9)$ & $0.236(5)$ & 0.03 & 1 & 4 \\
O4 & $0.04(1)$ & $0.046(7)$ & $0.419(4)$ & 0.03 & 1 & 4 \\
O5 & $-0.00(2)$ & $0.25(1)$ & $-0.006(5)$ & 0.03 & 1 & 4 \\
O6 & $0.28(2)$ & $0.51(1)$ & $0.426(6)$ & 0.03 & 1 & 4 \\
O7 & $0.72(2)$ & $0.273(9)$ & $0.069(5)$ & 0.03 & 1 & 4 \\
O8 & $0.05(2)$ & $0.12(1)$ & $0.109(7)$ & 0.03 & 1 & 4 \\
O9 & $0.40(2)$ & $-0.022(9)$ & $0.136(5)$ & 0.03 & 1 & 4 \\
O10 & $0.40(2)$ & $0.261(8)$ & $0.150(5)$ & 0.03 & 1 & 4 \\
O11 & $0.13(2)$ & $-0.001(9)$ & $0.207(5)$ & 0.03 & 1 & 4 \\
O12 & $0.48(1)$ & $0.13(1)$ & $0.253(5)$ & 0.03 & 1 & 4 \\
O13 & $0.17(1)$ & $0.490(9)$ & $0.207(5)$ & 0.03 & 1 & 4 \\
O14 & $0.55(1)$ & $0.491(9)$ & $0.146(4)$ & 0.03 & 1 & 4 \\
O15 & $0.28(2)$ & $0.26(1)$ & $0.418(6)$ & 0.03 & 1 & 4 \\
O16 & $0.37(2)$ & $0.390(8)$ & $0.034(7)$ & 0.03 & 1 & 4 \\
O17 & $0.26(1)$ & $0.372(9)$ & $0.311(5)$ & 0.03 & 1 & 4 \\
O18 & $0.67(2)$ & $0.353(8)$ & $0.465(7)$ & 0.03 & 1 & 4 \\
\hline & & & & & & \\
& & & & & & 4 \\
\end{tabular}


Table 10.3. Different coordination geometry and bond distances of $\mathrm{Fe}$ in $\mathrm{Ba}_{2} \mathrm{Fe}_{2} \mathrm{O}_{5}$.

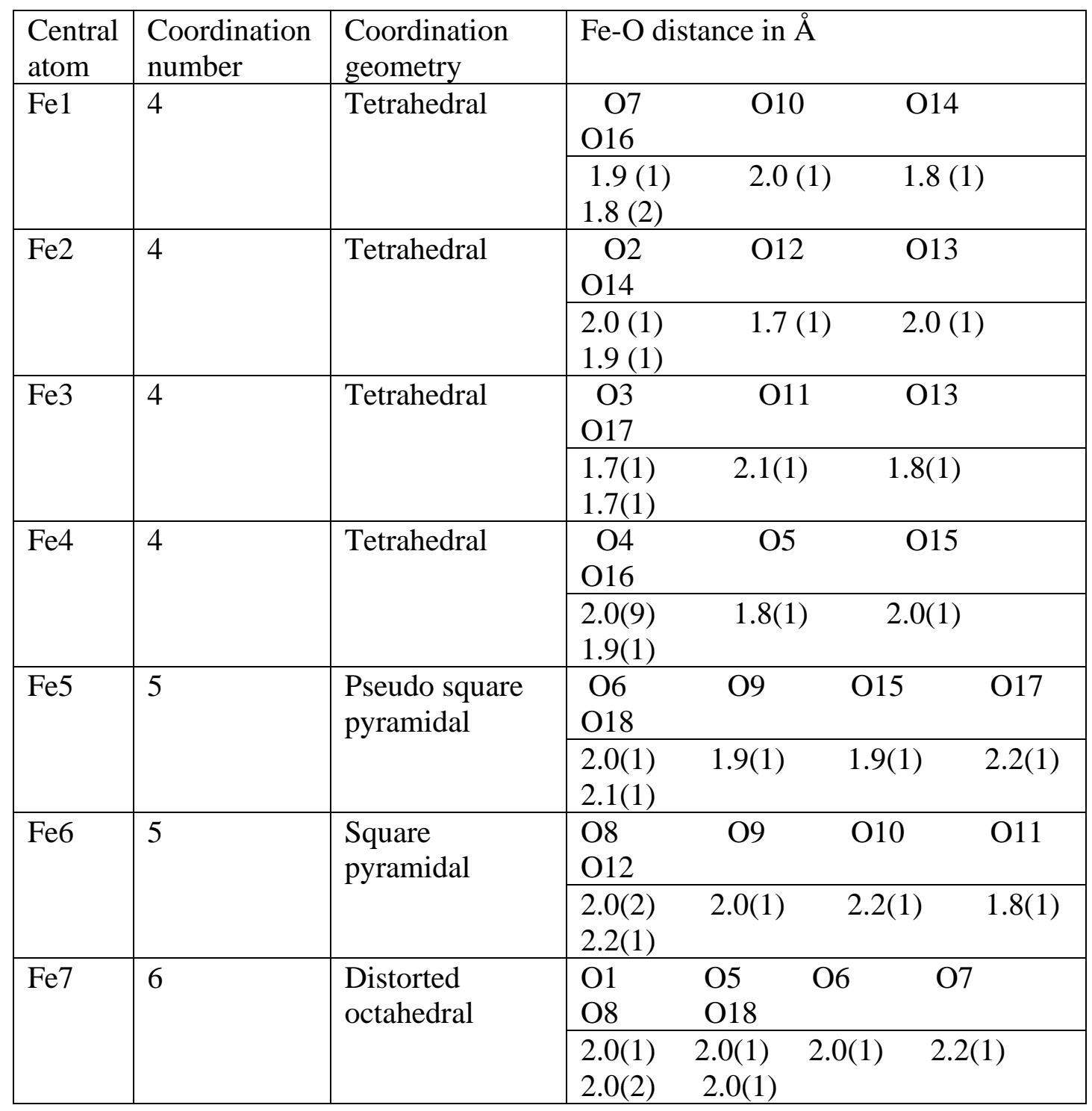


Table 10.4. The connectivity of polyhedra in $\mathrm{Ba}_{2} \mathrm{Fe}_{2} \mathrm{O}_{5}$.

\begin{tabular}{|l|l|}
\hline Polyhedron & Corner shared to polyhedra of: \\
\hline Fe1 (tetrahedron) & $\mathrm{Fe}$, $\mathrm{Fe} 4, \mathrm{Fe} 6, \mathrm{Fe} 7$ \\
\hline Fe2 (tetrahedron) & $\mathrm{Fe} 1, \mathrm{Fe} 3, \mathrm{Fe} 6$ \\
\hline Fe3 (tetrahedron) & $\mathrm{Fe}$, Fe5, Fe6 \\
\hline Fe4 (tetrahedron) & $\mathrm{Fe} 1, \mathrm{Fe} 5, \mathrm{Fe} 7$ \\
\hline Fe5 (pseudo square pyramid) & $\mathrm{Fe} 6, \mathrm{Fe} 7, \mathrm{Fe} 7, \mathrm{Fe} 4, \mathrm{Fe} 3$ \\
\hline Fe6 (square pyramid) & $\mathrm{Fe} 1, \mathrm{Fe} 2, \mathrm{Fe} 3, \mathrm{Fe} 5, \mathrm{Fe} 7$ \\
\hline Fe7 (distorted octahedron) & $\mathrm{Fe} 1, \mathrm{Fe} 4, \mathrm{Fe} 5, \mathrm{Fe} 5, \mathrm{Fe} 6, \mathrm{Fe} 7$ \\
\hline
\end{tabular}

(a) Fe1

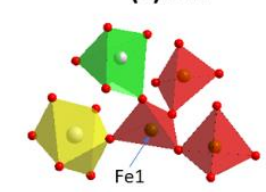

(e) Fe5

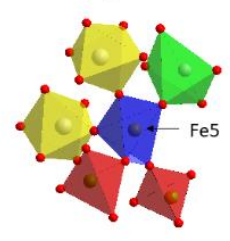

(b) Fe2

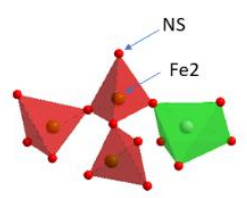

(f) Fe6
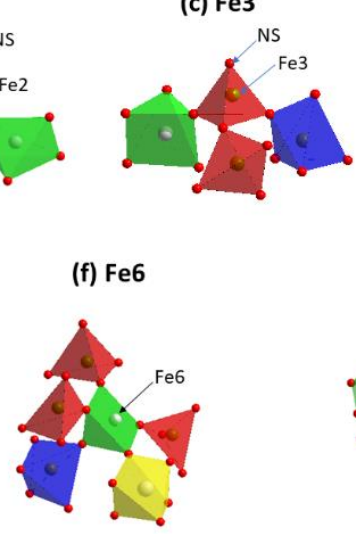

(d) $\mathrm{Fe} 4$

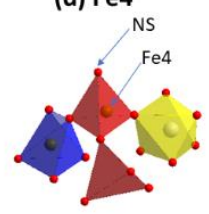

(g) Fe7

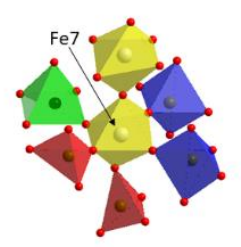

Figure 10.3. The coordination geometry of 7 crystallographically distinct Fe atoms and the connectivity of polyhedra in $\mathrm{Ba}_{2} \mathrm{Fe}_{2} \mathrm{O}_{5}$. Red, green, blue and yellow polyhedra represent tetrahedral, square pyramidal, pseudo square pyramidal and octahedral geometry, respectively (NS $=$ not shared with other polyhedra). 
The morphologies of sintered pellets of the two materials were studied by scanning electron microscope (SEM). Figure 10.4 compares the SEM images of $\mathrm{Ba}_{2} \mathrm{Fe}_{2} \mathrm{O}_{5}$ and $\mathrm{Sr}_{2} \mathrm{Fe}_{2} \mathrm{O}_{5}$. As seen here, $\mathrm{Ba}_{2} \mathrm{Fe}_{2} \mathrm{O}_{5}$ is more porous and has smaller crystallite size compared to $\mathrm{Sr}_{2} \mathrm{Fe}_{2} \mathrm{O}_{5}$. The crystallites are more densely packed in $\mathrm{Sr}_{2} \mathrm{Fe}_{2} \mathrm{O}_{5}$.
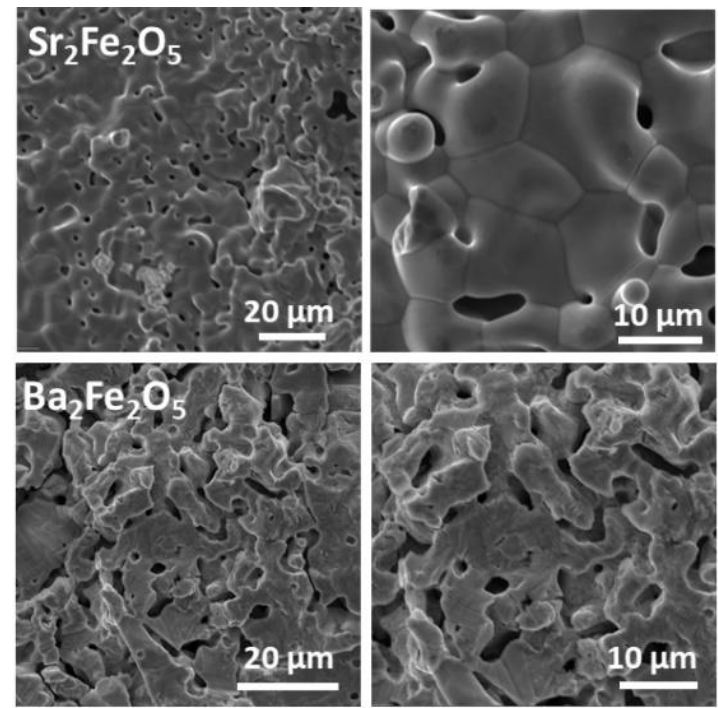

Figure 10.4. Scanning electron microscopy images of $\mathrm{Sr}_{2} \mathrm{Fe}_{2} \mathrm{O}_{5}$ (top) and $\mathrm{Ba}_{2} \mathrm{Fe}_{2} \mathrm{O}_{5}$ (bottom)

\section{Oxidation state of Fe}

The oxidation state of $\mathrm{Fe}$ in both compounds was analyzed by X-ray Photoelectron Spectroscopy (XPS). The $2 \mathrm{P}_{3 / 2}$ peak for $\mathrm{Fe}^{3+}$ usually appears at $\sim 710-711 \mathrm{eV}$, and a satellite peak is usually present at about $7-9 \mathrm{eV}$ higher than the $2 \mathrm{P}_{3 / 2}$ peak. ${ }^{43-44,103-104,220}$ Both $\mathrm{Ba}_{2} \mathrm{Fe}_{2} \mathrm{O}_{5}$ and $\mathrm{Sr}_{2} \mathrm{Fe}_{2} \mathrm{O}_{5}$ show the $2 \mathrm{P}_{3 / 2}$ peak at $\sim 710 \mathrm{eV}$ and the signature satellite peak at about $\sim 718 \mathrm{eV}$, indicative of $\mathrm{Fe}^{3+}$ (Figure 10.5). This is expected, as both compounds were synthesized in argon atmosphere with $\mathrm{Fe}_{2} \mathrm{O}_{3}$ as starting material. Given the inert synthesis condition, the retention of the trivalent state for iron was anticipated. 
We also confirmed these findings by performing iodometric titrations, which showed oxygen stoichiometry of 5.06 and 5.02 per formula unit of $\mathrm{Sr}_{2} \mathrm{Fe}_{2} \mathrm{O}_{5}$ and $\mathrm{Ba}_{2} \mathrm{Fe}_{2} \mathrm{O}_{5}$, respectively, consistent with $\mathrm{Fe}$ in trivalent oxidation state.

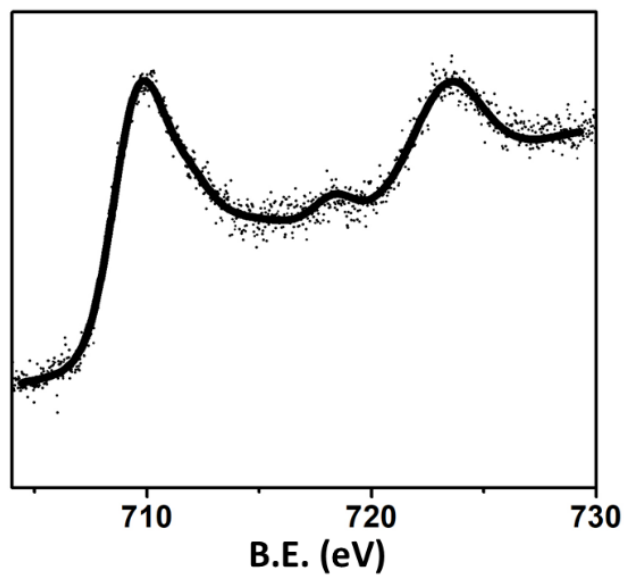

(a)

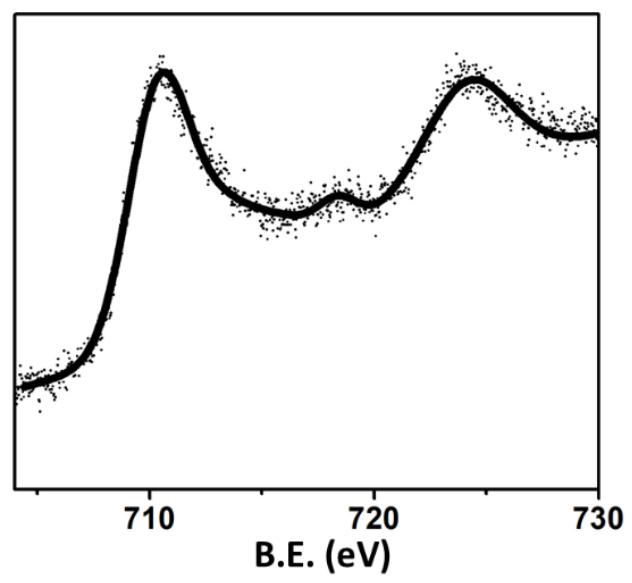

(b)

Figure 10.5. X-ray photoelectron spectroscopy data for (a) $\mathrm{Sr}_{2} \mathrm{Fe}_{2} \mathrm{O}_{5}$ and (b) $\mathrm{Ba}_{2} \mathrm{Fe}_{2} \mathrm{O}_{5}$.

\section{Magnetic properties}

Previous reports ${ }^{219,} 221$ have shown that both of these materials have antiferromagnetic order with Neel temperature of $\sim 693 \mathrm{~K}$ and $720 \mathrm{~K}$ for $\mathrm{Sr}_{2} \mathrm{Fe}_{2} \mathrm{O}_{5}{ }^{221}$ and $\mathrm{Ba}_{2} \mathrm{Fe}_{2} \mathrm{O}_{5},{ }^{219}$ respectively. We performed isothermal magnetization measurements using magnetic field of up to $9 \mathrm{~T}$ on both materials. As shown in Figure 10.6, the magnetization of both materials shows linear field-dependent behavior. However, the difference in structural order has an impact on the magnitude of magnetization. At the highest field, $9 \mathrm{~T}$, the magnetization of $\mathrm{Sr}_{2} \mathrm{Fe}_{2} \mathrm{O}_{5}$ reaches a maximum of $0.045 \mu_{\mathrm{B}}$ at $5 \mathrm{~K}$, whereas the magnetization of $\mathrm{Ba}_{2} \mathrm{Fe}_{2} \mathrm{O}_{5}$ is more than $30 \%$ higher, and reaches $\sim 0.06 \mu_{\mathrm{B}}$ at $9 \mathrm{~T}$ and $5 \mathrm{~K}$. In addition, the isothermal 
magnetization of $\mathrm{Sr}_{2} \mathrm{Fe}_{2} \mathrm{O}_{5}$ at $5 \mathrm{~K}$ and $300 \mathrm{~K}$ overlap, whereas $\mathrm{Ba}_{2} \mathrm{Fe}_{2} \mathrm{O}_{5}$ shows a clear decrease in magnetization values at $300 \mathrm{~K}$ compared to $5 \mathrm{~K}$. The effect of structural order on magnetism is also demonstrated by the magnetic susceptibility data shown in Figure 10.6. As seen here, the molar magnetic susceptibility for $\mathrm{Ba}_{2} \mathrm{Fe}_{2} \mathrm{O}_{5}$ is two times greater than that of $\mathrm{Sr}_{2} \mathrm{Fe}_{2} \mathrm{O}_{5}$. However, the overall magnitude of susceptibility is small for both materials as the temperature range of study, $2-400 \mathrm{~K}$, falls in the antiferromagnetic region for both compounds. As stated above, the Neel temperature of $\mathrm{Sr}_{2} \mathrm{Fe}_{2} \mathrm{O}_{5}$ has been previously determined by neutron diffraction to be $693 \mathrm{~K} \cdot{ }^{221}$ Also, the Neel temperature of $\mathrm{Ba}_{2} \mathrm{Fe}_{2} \mathrm{O}_{5}$ has been found using Mossbauer spectroscopy to be $720 \mathrm{~K}^{219}{ }^{222}$ Another difference between the two materials is the ZFC/FC splitting, which occurs below $240 \mathrm{~K}$ for $\mathrm{Sr}_{2} \mathrm{Fe}_{2} \mathrm{O}_{5}$, but at a much higher temperature, $\sim 355 \mathrm{~K}$, for $\mathrm{Ba}_{2} \mathrm{Fe}_{2} \mathrm{O}_{5}$.

While both materials have small magnetic moments in the range $2-400 \mathrm{~K}$, the effect of variation in structural order on magnetic properties is clear.

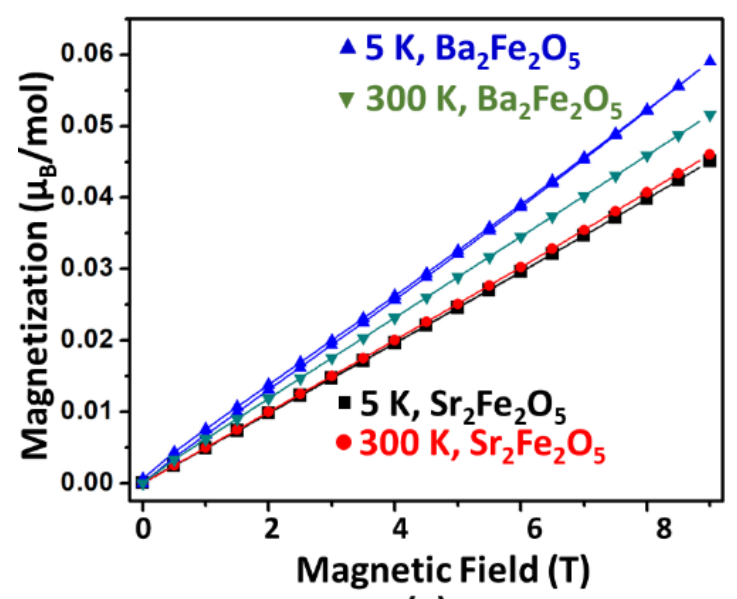

(a)

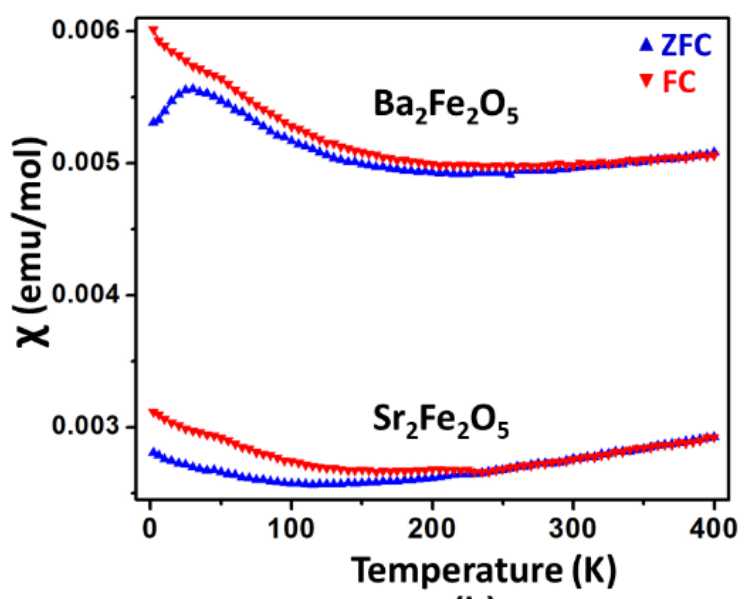

(b)

Figure 10.6. (a) Isothermal magnetization data, where black and red show $\mathrm{Sr}_{2} \mathrm{Fe}_{2} \mathrm{O}_{5}$ magnetization at $5 \mathrm{~K}$ and $300 \mathrm{~K}$, and blue and green represent the corresponding data for $\mathrm{Ba}_{2} \mathrm{Fe}_{2} \mathrm{O}_{5}$. (b) Magnetic susceptibility data. 


\section{Electrical conductivity}

The difference in structural order leads to a sharp difference in electrical conductivity of $\mathrm{Ba}_{2} \mathrm{Fe}_{2} \mathrm{O}_{5}$ and $\mathrm{Sr}_{2} \mathrm{Fe}_{2} \mathrm{O}_{5}$. Their electrical conductivities were measured by both direct current (DC) and alternating current (AC) methods using impedance spectroscopy. In impedance spectroscopy, total resistance is obtained from the intercept of the data with the real axis $\left(Z^{\prime}\right)$ of the Nyquist plot at low frequency. In DC method, the output current is measured while applying constant voltage, which is then converted into resistance. The resistance $(\mathrm{R})$ is used to determine the conductivity $(\sigma)$ of a material using the following equation

$$
\sigma=\mathrm{L} / \mathrm{RA}
$$

where $\mathrm{L}$ and A represent the thickness and cross-sectional area of the cylindrical pellet, respectively. The AC and DC values were similar throughout the whole range of conductivity measurement for both materials. The room temperature conductivity values are listed in Table 10.5, indicating that the room temperature conductivity of $\mathrm{Sr}_{2} \mathrm{Fe}_{2} \mathrm{O}_{5}$ is two orders of magnitude greater than that of $\mathrm{Ba}_{2} \mathrm{Fe}_{2} \mathrm{O}_{5}$. Variable temperature conductivity measurements from $25{ }^{\circ} \mathrm{C}$ to $900{ }^{\circ} \mathrm{C}$ were also performed to study the temperaturedependent behavior of electrical conductivity (Figure 10.7). 


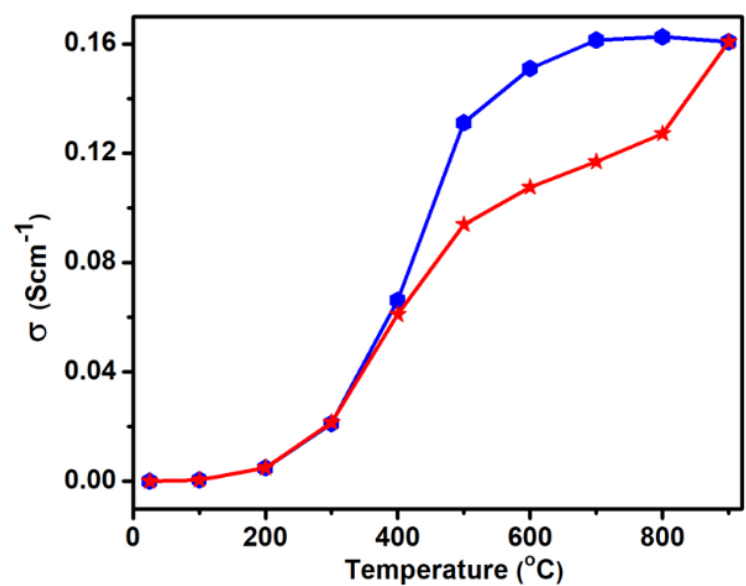

(a)

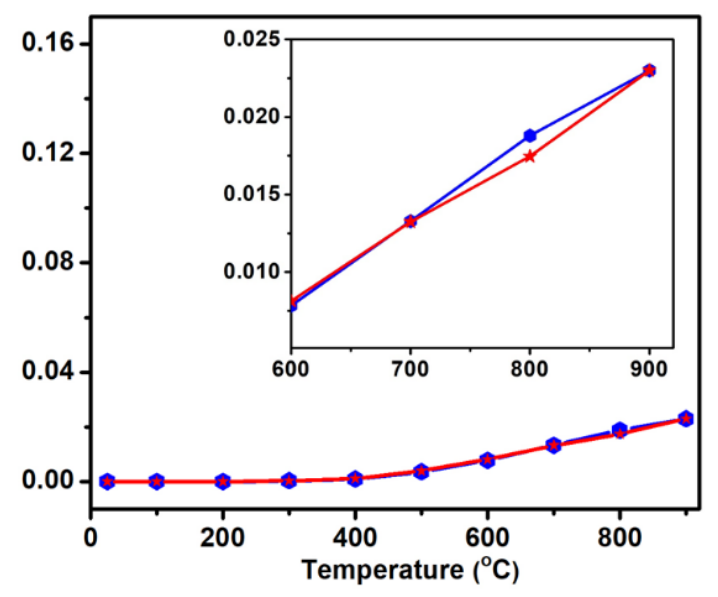

(b)

Figure 10.7. Electrical conductivity of (a) $\mathrm{Sr}_{2} \mathrm{Fe}_{2} \mathrm{O}_{5}$ and (b) $\mathrm{Ba}_{2} \mathrm{Fe}_{2} \mathrm{O}_{5}$ in inert atmosphere. Blue circles and red stars represent the data obtained while heating and cooling, respectively. The inset in (b) magnifies the data in the temperature range $600-900{ }^{\circ} \mathrm{C}$ for $\mathrm{Ba}_{2} \mathrm{Fe}_{2} \mathrm{O}_{5}$.

It is immediately clear that $\mathrm{Sr}_{2} \mathrm{Fe}_{2} \mathrm{O}_{5}$ has greater conductivity than $\mathrm{Ba}_{2} \mathrm{Fe}_{2} \mathrm{O}_{5}$ in the entire temperature range. The temperature-dependent behavior is also drastically different for the two materials. For $\mathrm{Sr}_{2} \mathrm{Fe}_{2} \mathrm{O}_{5}$, there is little change in electrical conductivity up to $\sim 200{ }^{\circ} \mathrm{C}$, whereas for $\mathrm{Ba}_{2} \mathrm{Fe}_{2} \mathrm{O}_{5}$, the conductivity remains nearly unchanged up to $\sim 400{ }^{\circ} \mathrm{C}$. For $\mathrm{Sr}_{2} \mathrm{Fe}_{2} \mathrm{O}_{5}$, there is a sharp increase in conductivity in the range $200-500{ }^{\circ} \mathrm{C}$, a behavior typical of semiconductors. Above $500{ }^{\circ} \mathrm{C}$, the increase in conductivity for $\mathrm{Sr}_{2} \mathrm{Fe}_{2} \mathrm{O}_{5}$ slows down, finally reaching a maximum at $700{ }^{\circ} \mathrm{C}$, above which the electrical conductivity plateaus.

The temperature-dependent behavior of $\mathrm{Ba}_{2} \mathrm{Fe}_{2} \mathrm{O}_{5}$ in different, where the electrical conductivity remains the same up to $400{ }^{\circ} \mathrm{C}$ followed by a mild linear increase between $400-900{ }^{\circ} \mathrm{C}$. The semiconducting behavior persists up to $900{ }^{\circ} \mathrm{C}$ and there are no peaks or plateau regions at high temperature. 
Another interesting feature is the observation of hysteresis, where the conductivity values

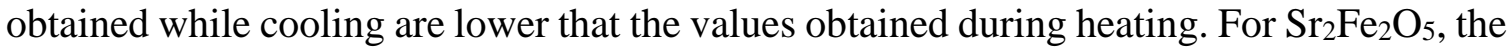
hysteresis is large and readily detectable. Whereas, $\mathrm{Ba}_{2} \mathrm{Fe}_{2} \mathrm{O}_{5}$ shows a small hysteresis at $800{ }^{\circ} \mathrm{C}$, but the heating and cooling data overlap at lower temperatures.

The electrical conductivity in oxides occurs by electron hopping through $\mathrm{M}-\mathrm{O}-\mathrm{M}$ ' (metaloxygen-metal) bond system where $\mathrm{M}$ and $\mathrm{M}$ ' have different oxidation states. Thus, metals with variable oxidation states such as $\mathrm{Fe}^{2+} / \mathrm{Fe}^{3+} / \mathrm{Fe}^{4+}$ are required for this process. The increase in temperature leads to enhanced mobility of charge carriers, resulting in increase in electrical conductivity in semiconductors. However, as observed in the TGA data (Figure 10.8), the increase in temperature also leads to the loss of oxygen, which can have a negative impact on electronic conductivity by disrupting some of the $\mathrm{M}-\mathrm{O}-\mathrm{M}^{\prime}$ conduction pathways. However, it can also have an impact on the ionic conductivity, ${ }^{223}$ as more defects are created due to the loss of oxygen. Therefore, the increase in temperature has multiple consequence that can have positive or negative effects on total conductivity.

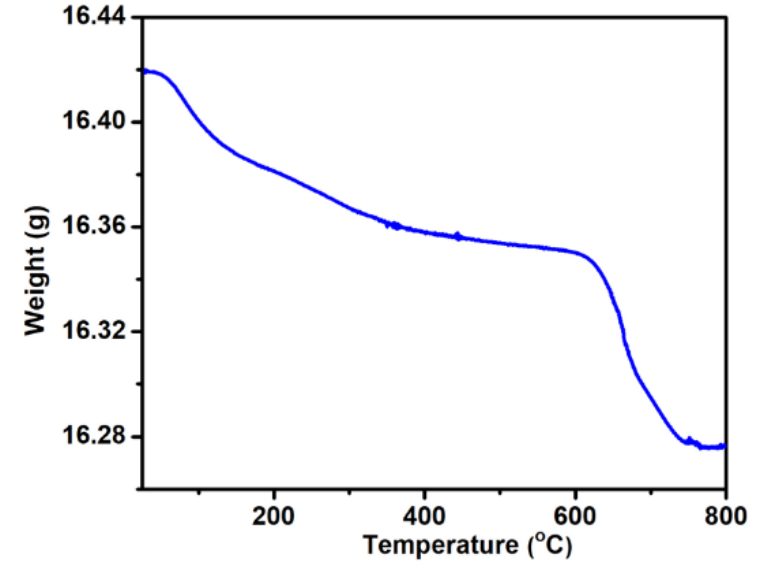

(a)

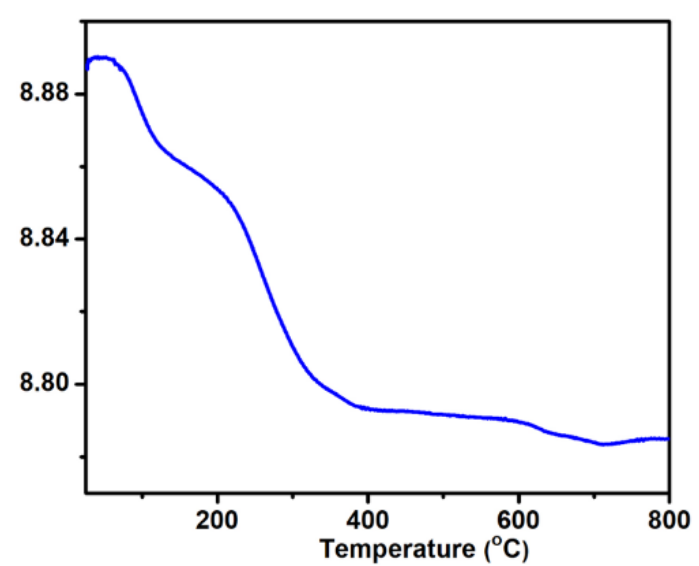

(b)

Figure 10.8. Thermogravimetric analysis data for (a) $\mathrm{Sr}_{2} \mathrm{Fe}_{2} \mathrm{O}_{5}$ and (b) $\mathrm{Ba}_{2} \mathrm{Fe}_{2} \mathrm{O}_{5}$. 
During the heating-cooling cycle, the temperature-dependent increase in mobility of charge carries is usually the dominant effect in semiconductors, leading to the commonly observed upturn in conductivity as temperature increases. During cooling, the charge carrier mobility decreases, leading to downturn in conductivity. In our experiments, since the conductivity measurements are done in inert atmosphere, the defects created during heating remain in the crystal lattice, even after the sample is cooled to lower temperatures. While the concentration of these defects is small, they can still have a negative impact on conductivity, as some of the $\mathrm{M}-\mathrm{O}-\mathrm{M}$ ' conduction pathways are disrupted. Eventually, as the temperature is lowered further, the conductivity value becomes too small for any parameter other than charge carrier mobility to have a visible impact on conductivity. That is why the hysteresis is only observed at high temperature. Note that the high temperature conductivity of $\mathrm{Sr}_{2} \mathrm{Fe}_{2} \mathrm{O}_{5}$ is almost one order of magnitude greater than that of $\mathrm{Ba}_{2} \mathrm{Fe}_{2} \mathrm{O}_{5}$. The hystereses for both materials are present at similar conductivity values. $\mathrm{For}_{\mathrm{Sr}} \mathrm{Fe}_{2} \mathrm{O}_{5}$ the hysteresis disappears when the material is cooled to $300{ }^{\circ} \mathrm{C}$, where electrical conductivity becomes less than $0.021 \mathrm{Scm}^{-1}$, and for $\mathrm{Ba}_{2} \mathrm{Fe}_{2} \mathrm{O}_{5}$ the hysteresis disappears when the material is cooled to $700{ }^{\circ} \mathrm{C}$, where electrical conductivity goes below $0.013 \mathrm{Scm}^{-}$ 1.

The activation energies for electrical conductivity (Table 10.5) can be obtained using Arrhenius plot (Figure 10.9) where the Arrhenius equation for thermally activated conductivity $^{50-51,179}$ is used for fitting:

$$
\sigma T=\sigma^{\circ} \mathrm{e}^{-E \mathrm{a} / k T}
$$


where $\sigma^{\circ}$ is a pre-exponential factor and a characteristic of the material, and $E_{a}, k$, and $T$ are the activation energy for conductivity, Boltzmann constant, and absolute temperature, respectively. The activation energy for the total conductivity $\left(E_{a}\right)$ is obtained from slope of the line of best fit in the $\log \sigma \mathrm{T}$ vs $1000 / \mathrm{T}$ plot. The activation energies are listed in Table 10.5 .

Table 10.5. Room temperature conductivity and activation energies

\begin{tabular}{|c|c|c|c|}
\hline \multirow{2}{*}{ compounds } & \multicolumn{2}{|c|}{$\begin{array}{c}\text { total conductivity, } \\
\sigma\left(\mathrm{S} \mathrm{cm}^{-1}\right)\end{array}$} & \multirow{2}{*}{ Activation energy, $\mathrm{E}_{\mathrm{a}}(\mathrm{eV})$} \\
\hline & $\mathrm{AC}$ & $\mathrm{DC}$ & \\
\hline $\mathrm{Ba}_{2} \mathrm{Fe}_{2} \mathrm{O}_{5}$ & $1.15 \times 10^{-7}$ & $1.15 \times 10^{-7}$ & $\begin{array}{l}0.462 \mathrm{eV} \text { for } 273 \text { to } 1173 \mathrm{~K}\left(25-300^{\circ} \mathrm{C}\right) \\
0.448 \mathrm{eV} \text { for } 273 \text { to } 1173 \mathrm{~K}\left(300-900^{\circ} \mathrm{C}\right)\end{array}$ \\
\hline $\mathrm{Sr}_{2} \mathrm{Fe}_{2} \mathrm{O}_{5}$ & $3.65 \times 10^{-5}$ & $3.65 \times 10^{-5}$ & $\begin{array}{l}0.375 \mathrm{eV} \text { for } 298 \text { to } 773 \mathrm{~K}\left(25-300{ }^{\circ} \mathrm{C}\right) \\
0.280 \mathrm{eV} \text { for } 298 \text { to } 773 \mathrm{~K}\left(300-600^{\circ} \mathrm{C}\right) \\
0.089 \mathrm{eV} \text { for } 773 \text { to } 1173 \mathrm{~K}\left(600-900{ }^{\circ} \mathrm{C}\right)\end{array}$ \\
\hline
\end{tabular}

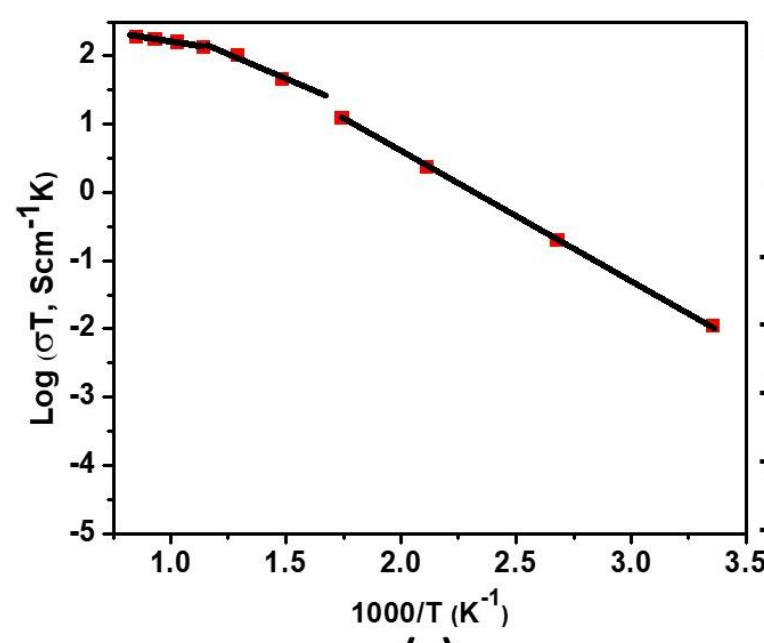

(a)

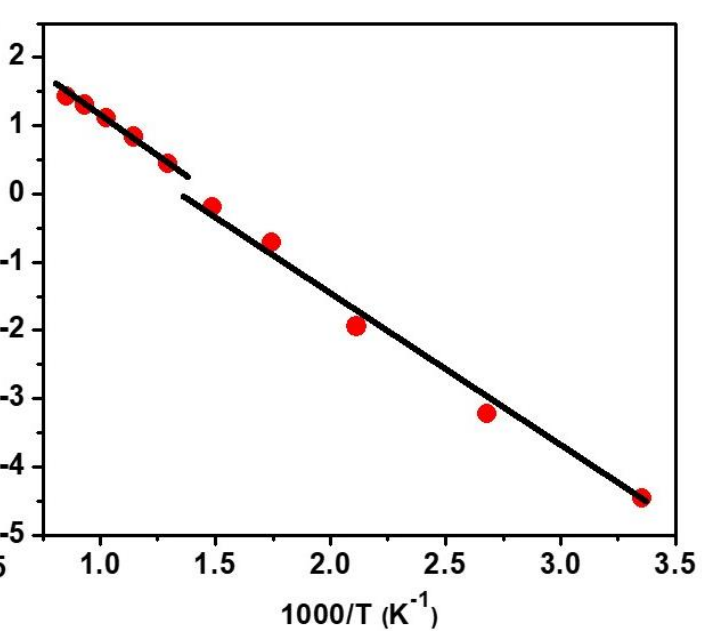

(b)

Figure 10.9. Arrhenius plots for electrical conductivity of (a) $\mathrm{Sr}_{2} \mathrm{Fe}_{2} \mathrm{O}_{5}$ and (b) $\mathrm{Ba}_{2} \mathrm{Fe}_{2} \mathrm{O}_{5}$. 
Overall, the correlation between structural order and electrical conductivity is demonstrated by these two materials, where electrical conductivity varies drastically due to the difference in type of ordering

\section{CONCLUSION}

The variation in the type of ordering in oxygen-deficient perovskites can lead to significant differences in magnetic and electrical properties. This has been demonstrated through investigation of $\mathrm{Sr}_{2} \mathrm{Fe}_{2} \mathrm{O}_{5}$ and $\mathrm{Ba}_{2} \mathrm{Fe}_{2} \mathrm{O}_{5}$, which feature two different types of ordering. $\mathrm{Sr}_{2} \mathrm{Fe}_{2} \mathrm{O}_{5}$ contains alternating $\mathrm{FeO}_{4}$ tetrahedra and $\mathrm{FeO}_{6}$ octahedra, whereas $\mathrm{Ba}_{2} \mathrm{Fe}_{2} \mathrm{O}_{5}$ has a complex structure, where $\mathrm{Fe}$ atoms have tetrahedral, square-pyramidal and octahedral geometry. The effect of these differences on magnetic properties is evident from the considerably higher magnetization of $\mathrm{Ba}_{2} \mathrm{Fe}_{2} \mathrm{O}_{5}$ compared to $\mathrm{Sr}_{2} \mathrm{Fe}_{2} \mathrm{O}_{5}$. In addition, the electrical conductivity of the two compounds are drastically different, with $\mathrm{Sr}_{2} \mathrm{Fe}_{2} \mathrm{O}_{5}$ showing nearly two-orders of magnitude higher conductivity at room temperature. Furthermore, the temperature-dependence of electrical conductivity varies significantly between the two materials, where the conductivity of $\mathrm{Sr}_{2} \mathrm{Fe}_{2} \mathrm{O}_{5}$ reaches saturation at high

temperature. This saturation is absent for $\mathrm{Ba}_{2} \mathrm{Fe}_{2} \mathrm{O}_{5}$, where electrical conductivity shows a gradual increase all the way to $900{ }^{\circ} \mathrm{C}$. These findings show the direct correlations between structure and physical properties of oxygen-deficient perovskites. 


\section{CHAPTER 11}

\section{STUDY OF $\mathrm{Sr}_{2} \mathrm{Fe}_{2} \mathrm{O}_{6-\delta}$ AS ANODE ELECTRODE IN Li-ION BATTERY}

\section{INTRODUCTION}

Lithium ion batteries (LIB) are extensively used in cell phones and laptops. The performance of the LIB is basically rooted on the efficiency of its electrodes and electrolyte. Since LIB has been used in devices like cell phones and laptops, which have become a part of our daily life activities, researchers have been committed to improving the durability and performance of the electrodes and electrolyte of LIB. ${ }^{224-225}$ Anode is one of the electrodes that researchers have been showing deep interest in improving the efficiency and durability. ${ }^{226-229}$

There are certain criteria that need to be fulfilled by a material to be a good and efficient anode in LIB such as a low atomic weight, low cost, and low standard potential as well as high ionic and electrical conductivities. ${ }^{230}$ Graphite is one of the primarily commercially used anode material in LIB. ${ }^{231}$ Lithium may intercalate between the graphene layers of graphite until a composition of $\mathrm{LiC}_{6}$ is reached resulting in a theoretical specific capacity of $372 \mathrm{mAh}^{-1} 224,228,230,232$ but practically the capacity is $\sim 300-320 \mathrm{mAh} \mathrm{g}^{-1} .^{230}$ Graphite has a low potential of around $0.1 \mathrm{~V}$ vs. $\mathrm{Li} / \mathrm{Li}+$ for most lithium concentrations. ${ }^{230}$, 233 However, it has some defects such as it reacts with organic electrolyte to form an SEI on its surface in the initial few charge and discharge cycles. ${ }^{230,233-234}$ Many researchers 
have been committed to improving the quality and efficiency of the anode and to develop new material to overcome the shortcomings of the graphite anode. ${ }^{230,}{ }^{235}$ Tin based materials (Sn-oxides, mixed oxides and alloys) and pure and mixed transition metal oxides ${ }^{227-228,236-238}$ were studied as alternative materials for anode to substitute the graphite. The tin based materials have been reported to be high capacity anode materials where the capacity arises from the reversible Li-Sn alloy formation/decomposition during the charge/discharge cycles. ${ }^{228,230,234,236-237}$ The transition metal oxides are non-intercalating and non-alloying oxides. ${ }^{230}$ The reversible capacity in transition metal oxides is believed to develop from reversible oxidation of metal and lithia decomposition/formation. ${ }^{230,239-241}$

Later on, materials with different structures and phases such as spinel phase have been studied for the alternative materials for the anode. ${ }^{230,242-243}$ The reversible capacity in spinel type materials such as $\mathrm{ZnCo}_{2} \mathrm{O}_{4}$ is believed to arise from $\mathrm{Li}$ intercalation into spinel lattice, crystal structure destruction followed by metal particle formation and alloy formation with Zn. ${ }^{230,244-245}$ Recently, $\mathrm{Ca}_{2} \mathrm{Fe}_{2} \mathrm{O}_{5}$ and $\mathrm{Ca}_{2} \mathrm{Co}_{2} \mathrm{O}_{5}$ have been studied for their possibility of a potential candidate for a better and efficient anode material in LIB by Sharma and Chowdari et.al. ${ }^{226} \mathrm{Ca}_{2} \mathrm{Fe}_{2} \mathrm{O}_{5}$ and $\mathrm{Ca}_{2} \mathrm{Co}_{2} \mathrm{O}_{5}$ have the brownmillerite type structures which belong to oxygen deficient perovskite (ODP). ${ }^{226}$ Here, the mechanism or the operation of the electrodes is believed to involve the destruction of the crystal lattice to form the nanoparticles of $\mathrm{Fe}$ and $\mathrm{Co}$ embedded in an amorphous matrix of $\mathrm{Li}_{2} \mathrm{O}$ and $\mathrm{CaO}^{226}$ The nanoparticles react reversibly with $\mathrm{Li}_{2} \mathrm{O}$ contributing to the capacity and $\mathrm{Ca}$ is presumed to act as a beneficial spectator ion helping in the stabilization of the amorphous nanometal oxide matrix. ${ }^{226}$ We have studied similar type of oxygen deficient perovskite material, $\mathrm{Sr}_{2} \mathrm{Fe}_{2} \mathrm{O}_{6-\delta}$ but with different structure and phase, for its potential application as anode in 
LIB. In this paper, we report the synthesis and electrochemical property of $\mathrm{Sr}_{2} \mathrm{Fe}_{2} \mathrm{O}_{6-\delta}$ that is applicable for LIB anode.

\section{EXPERIMENTAL}

Conventional solid-state synthesis method was applied to synthesize the material. Stoichiometric proportions of the precursors, $\mathrm{SrCO}_{3}$ (Sigma Aldrich, 99.9\%), and $\mathrm{Fe}_{2} \mathrm{O}_{3}$ (Alfa Aesar, 99.998\%) were used for the synthesis. The mixtures of precursor powders were ground using agate mortar and pestle, pressed into pellets and calcined at $1000^{\circ} \mathrm{C}$ for 24 hours. The pellets were then ground and refired in air at $1200{ }^{\circ} \mathrm{C}$ for 24 hours. In all cases, the furnace heating and cooling rates were set at $100{ }^{\circ} \mathrm{C} / \mathrm{h}$.

The phase purity and structure of polycrystalline samples were tested by Rietveld refinements of powder X-ray diffraction data taken at room temperature using a PAN analytical Empyrean diffractometer with $\mathrm{CuK}_{\alpha 1}$ radiation $(\lambda=1.54056 \AA)$. The Rietveld refinements were carried out using GSAS software ${ }^{100}$ and EXPEGUI interface. ${ }^{125}$ The micro-structure of the compound was investigated using a high resolution field-emission scanning electron microscope (SEM). X-ray photoelectron spectroscopy (XPS) study and iodometric titration were performed to investigate the oxidation states of $\mathrm{Fe}$. $\mathrm{The} \mathrm{Sr}_{2} \mathrm{Fe}_{2} \mathrm{O}_{6}$ $\delta$ powder of accurately weighed $(5 \mathrm{mg})$ was mixed with $3 \mathrm{mg}$ of teflonized acetylene black binder on an agate mortar with pestle to make an anode electrode. The $\mathrm{Sr}_{2} \mathrm{Fe}_{2} \mathrm{O}_{6-\delta}$ electrode was used as a working electrode and Li foil as counter electrode separated by piece of glass fiber filter (ADVANTEC, GB-100R, Japan) using 2032 coin-type cell. The electrolyte used was $1 \mathrm{M} \mathrm{LiPF}_{6}-\mathrm{EC}$ (ethylene carbonate): DMC (dimethyl carbonate) (1:2 by volume). The 
galvanostatic charge-discharge measurements were carried out using Arbin instrument. Charge-discharge measurement were carried out in the voltage range between $3.0-0.005$ $\mathrm{V}$ with a current density of $25 \mathrm{~mA} \mathrm{~g}^{-1}$. The rate capability test was carried out at different currents of $25,50,100,200$, and $500 \mathrm{~mA} \mathrm{~g}^{-1}$, respectively. The cyclic voltammetry measurement was carried out in the voltage range of $3.0-0.005 \mathrm{~V}$ with a scan speed of 1 $\mathrm{mV} \mathrm{s}^{-1}$ using SP200 Biologic Instrument.

\section{RESULT AND DISCUSSION}

The crystal structure of the compound was determined by the analysis of Powder X-ray diffraction (XRD). $\mathrm{Sr}_{2} \mathrm{Fe}_{2} \mathrm{O}_{6-\delta}$ was found to be tetragonal with space group $14 / \mathrm{mmm}{ }^{4}$ Figure 11.1 shows the crystallographic structure of $\mathrm{Sr}_{2} \mathrm{Fe}_{2} \mathrm{O}_{6-\delta}$. Figure 11.2 shows the Rietveld refinement profile and crystal structure of the material. The inset picture shows the bifurcation of the peak at higher $2 \theta$ angle which helps to distinguish the tetragonal structure (model) from cubic structure. The refined structural parameters are listed in Table 11.1. $\mathrm{Sr}_{2} \mathrm{Fe}_{2} \mathrm{O}_{6-\delta}$ is an oxygen deficient perovskite (ODP) with a general formula, $\mathrm{ABO}_{3-\delta}$ (or $\mathrm{A}_{2} \mathrm{~B}_{2} \mathrm{O}_{6-\delta}$ ) where Sr occupies A site and Fe occupies B site. Oxygen deficiency in ODPs can form tetrahedral or square pyramidal coordination geometry around B site cations. ${ }^{4}$ The crystal structure of $\mathrm{Sr}_{2} \mathrm{Fe}_{2} \mathrm{O}_{6-\delta}$ contains square pyramidal and octahedral geometries $\mathrm{Sr}$ is 11 or 12 coordinated. ${ }^{4}$ The micro-stricture of $\mathrm{Sr}_{2} \mathrm{Fe}_{2} \mathrm{O}_{6-\delta}$ is investigated by high resolution scanning electron microscopy (SEM). The SEM images are shown in figure 11.3. It seems that the grain shapes are irregular, and the grains show well contact though some pores appear in the sample. 


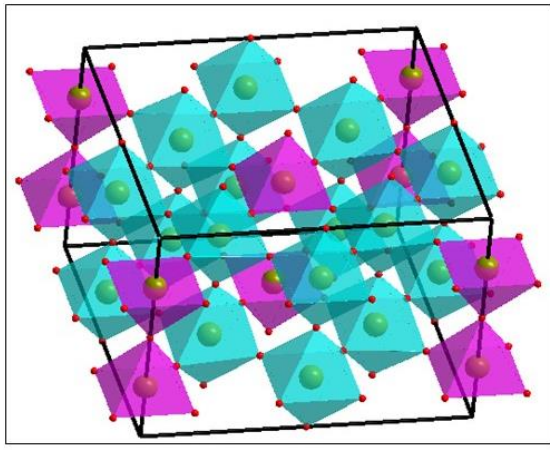

(a)

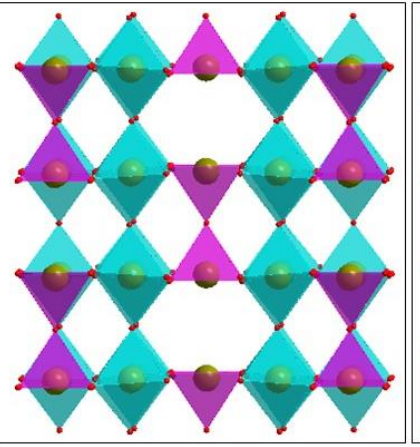

(b)

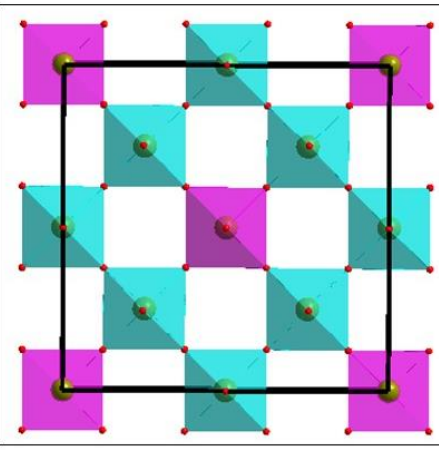

(c)

Figure 11.1. Crystal structure of $\mathrm{Sr}_{2} \mathrm{Fe}_{2} \mathrm{O}_{6-\delta}$. (a) unit cell (b) side view and (c) Top view of the crystallographic structure which show the alternating $\mathrm{FeO}_{6}$ octahedra (cyan) and $\mathrm{FeO}_{5}$ square pyramids (purple). Sr atoms are removed for clarity.

Table 11.1. Refined structural parameters for $\mathrm{Sr}_{2} \mathrm{Fe}_{2} \mathrm{O}_{6-\delta}$ from powder X-ray diffraction. Space group I4/mmm, $a=10.9301(2) \AA, b=10.9301(2) \AA, c=7.6958(2) \AA ⿻$ 还 $R_{p}=0.0211$, $w R_{p}=0.0299$.

\begin{tabular}{ccccccc}
\hline Elements & $\mathrm{x}$ & $\mathrm{y}$ & $\mathrm{z}$ & Occupancy & $\mathrm{U}_{\text {iso }}$ & Multiplicity \\
\hline $\mathrm{Sr} 1$ & $0.2587(6)$ & 0 & 0 & 1 & $0.031(2)$ & 8 \\
$\mathrm{Sr} 2$ & $0.2493(6)$ & 0 & 0.5 & 1 & $0.035(2)$ & 8 \\
$\mathrm{Fe} 1$ & 0.0 & 0.0 & 0.25 & 1 & $0.042(7)$ & 4 \\
$\mathrm{Fe} 2$ & 0.25 & 0.25 & 0.25 & 1 & $0.019(3)$ & 8 \\
$\mathrm{Fe} 3$ & 0.5 & 0 & 0.25 & 1 & $0.060(7)$ & 4 \\
$\mathrm{O} 1$ & 0 & 0 & 0.5 & 1 & $0.02(1)$ & 2 \\
$\mathrm{O} 2$ & $0.124(2)$ & $0.124(2)$ & $0.216(2)$ & 1 & $0.02(1)$ & 16 \\
$\mathrm{O} 3$ & $0.251(2)$ & $0.251(2)$ & 0.5 & 1 & $0.02(1)$ & 8 \\
$\mathrm{O} 4$ & $0.125(2)$ & 0.625 & 0.25 & 1 & $0.04(1)$ & 16 \\
$\mathrm{O} 5$ & 0.5 & 0 & 0 & 1 & $0.04(3)$ & 4 \\
\hline
\end{tabular}




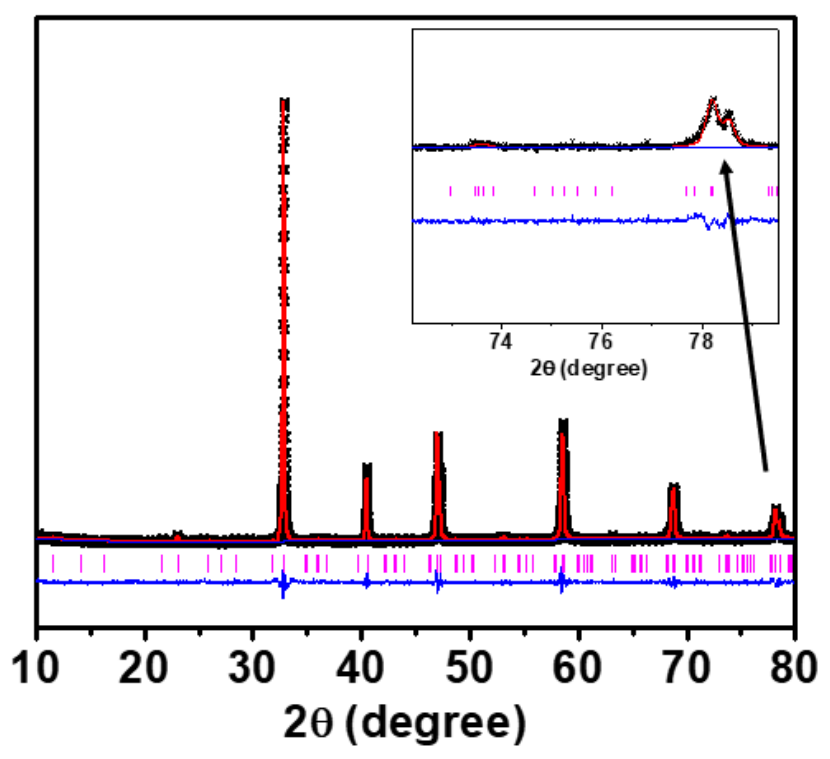

Figure 11.2. Rietveld refinement profile for powder $X$-ray diffraction data of $\mathrm{Sr}_{2} \mathrm{Fe}_{2} \mathrm{O}_{6-\delta}$ in $14 / \mathrm{mmm}$ space group. Cross symbols represent experimental data, red solid is the model, vertical tick marks show Bragg peak positions, and the blue line represents the difference plot. Inset shows the peak bifurcation at higher $2 \theta$ angle.
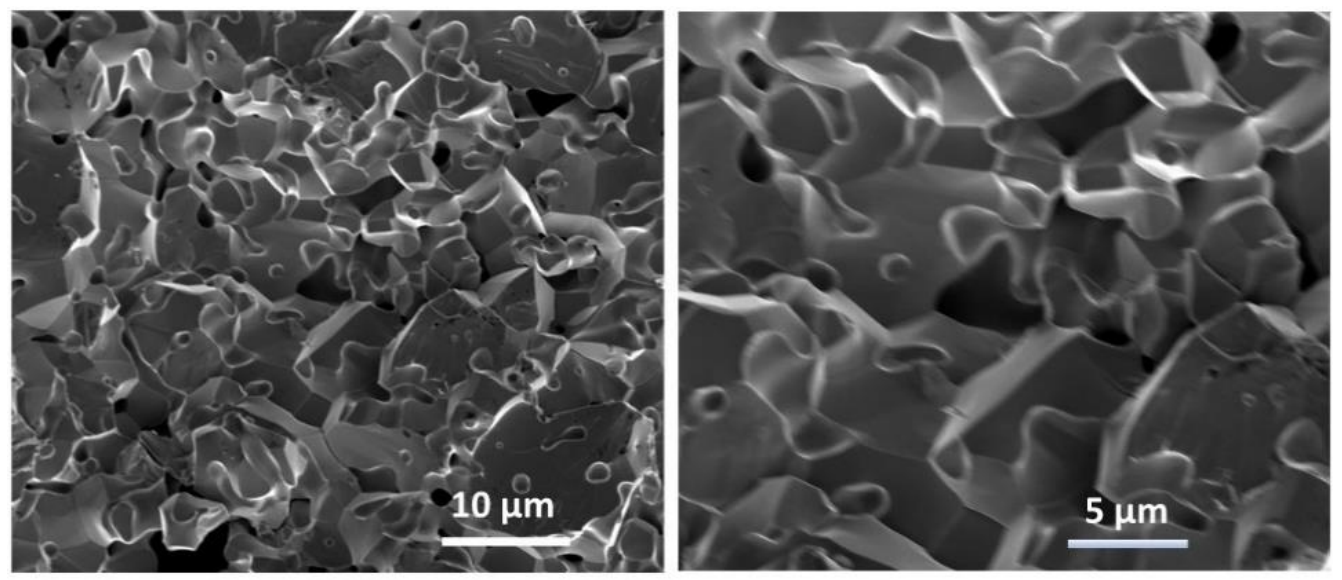

Figure 11.3. SEM images of $\mathrm{Sr}_{2} \mathrm{Fe}_{2} \mathrm{O}_{6-\delta}$. 
Past studies of Fe based ODPs showed the different oxidation states of +3 and +4 for Fe. ${ }^{4}$, ${ }^{246-247}$ So, we performed the X-ray photoelectron spectroscopy (XPS) study and iodometric titration to investigate the oxidation states of $\mathrm{Fe}$ in $\mathrm{Sr}_{2} \mathrm{Fe}_{2} \mathrm{O}_{6-\delta}$. If the compound contains only +3-oxidation state for Fe, the oxygen stoichiometry in $\mathrm{Sr}_{2} \mathrm{Fe}_{2} \mathrm{O}_{6-\delta}$ formula should be 5 , or $\delta=1$. Oxidation state of $\mathrm{Fe}$ in a compound is characterized by its $2 \mathrm{P}_{3 / 2}$ peak position. For $\mathrm{Fe}^{3+}, 2 \mathrm{P}_{3 / 2}$ peak is followed by a satellite peak at around $\sim 8 \mathrm{eV}$. As shown in Figure 11.4 for our material, the $2 \mathrm{P}_{3 / 2}$ peak observed at $\sim 710 \mathrm{eV}$ followed by a satellite peak at $\sim 8 \mathrm{eV}$ higher than the $\mathrm{Fe} 2 \mathrm{P}_{3 / 2}$ peak confirmed the presence of $\mathrm{Fe}^{3+} \cdot 43-44$ The XPS data for early transition metals with high-spin unpaired electrons show multiplet structure for the $2 p$ spectra due to spin-orbital and electrostatic interactions. ${ }^{248-249}$ It has been reported that the high-spin $\mathrm{Fe}^{3+}$ species can be fitted to multiple peaks. ${ }^{43}, 248,250-252$ We have followed the same procedure and the Fe $2 \mathrm{p}$ spectra obtained could be successfully fitted into four multiplets, indicating the presence of $\mathrm{Fe}$ in $3+$ oxidation state in the compound. For +4 oxidation state, a peak (fitted) appears at binding energy higher than that of +3 oxidation state $^{44}$. We can see a small bump raised at around $713 \mathrm{eV}$ as indicated by blue arrow in the figure. The fitting also shows a peak associated with the bump at around $713 \mathrm{eV}$. It indicates the presence of +4 oxidation state. The blue arrows show the peaks in the raw data. It has also been confirmed by iodometric titration, i.e., $\delta=0.25\left(\mathrm{Sr}_{2} \mathrm{Fe}_{2} \mathrm{O}_{6-\delta}\right)$. Thus, the formula becomes $\mathrm{Sr}_{2} \mathrm{Fe}_{2} \mathrm{O}_{5.75}$. To become the oxygen stoichiometry 5.75 (or $\left.\mathrm{Sr}_{2} \mathrm{Fe}_{2} \mathrm{O}_{5.75}\right)$, Fe must have mixed oxidation states $(+3$ and +4$)$. Thus, iodometric titration also indicates that a considerable amount of iron has been oxidized during the synthesis. Note that the starting material was $\mathrm{Fe}_{2} \mathrm{O}_{3}$. 


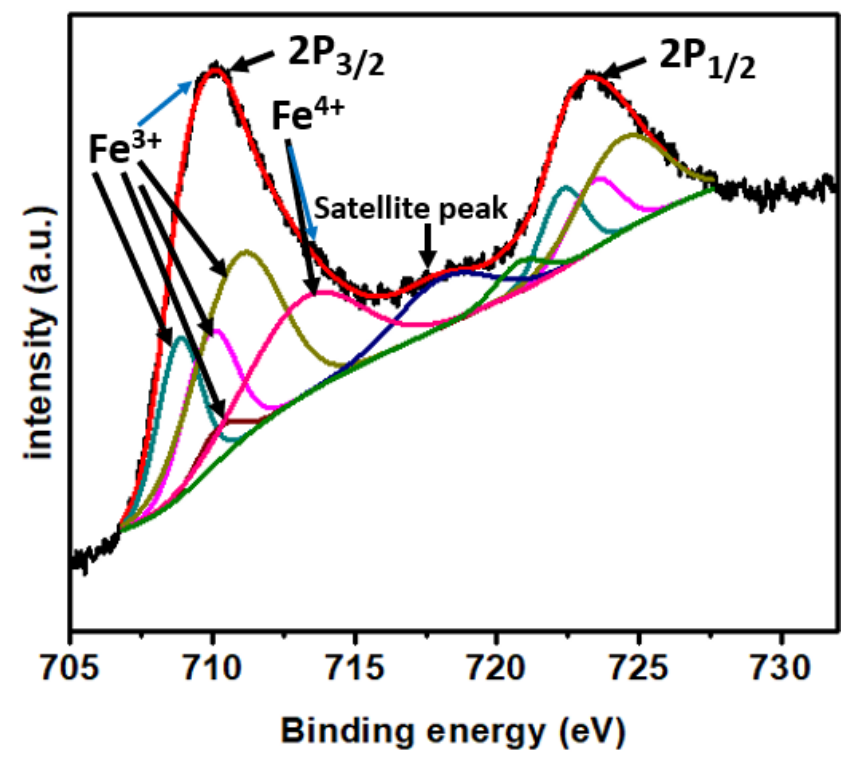

Figure 11.4. X-ray photoelectron spectroscopy data for $\mathrm{Sr}_{2} \mathrm{Fe}_{2} \mathrm{O}_{6-\delta}$.

Figure 11.5 depicts the first, second, tenth, twentieth, thirtieth, fortieth and fiftieth cycles of galvanostatic charge/discharge curves of $\mathrm{Sr}_{2} \mathrm{Fe}_{2} \mathrm{O}_{6-\delta}$ tested at a current density of 25 $\mathrm{mAg}^{-1}$ with voltage range from 3.0 to $0.005 \mathrm{~V}$. It has an initial discharge capacity of 2400 $\mathrm{mAh} \mathrm{g}^{-1}$ during the first initial cycle. Higher capacity could result from higher amount of lithium incorporation at defects within crystal surfaces. ${ }^{229}$ The first cycle discharging has a platue similar as seen in other material at the region between $0.9-0.75 \mathrm{~V} .{ }^{226,}{ }^{253}$ The electrode exhibits a discharge capacity of $840,570,500,490,460$ and $410 \mathrm{mAh} \mathrm{g}^{-1}$ at the 2nd, 10th, $20^{\text {th }}, 30^{\text {th }}, 40^{\text {th }}$ and 50th, cycles, respectively. The first discharge and charge capacities of the as-prepared anode is $2400 \mathrm{mAh} \mathrm{g}^{-1}$ and $680 \mathrm{mAh} \mathrm{g}^{-1}$, respectively; indicating an initial coulombic efficiency of $28.3 \%$. The irreversible capacity is due to the solid/electrolyte interphase (SEI) formation. ${ }^{254-255}$ Therefore, the first discharge irreversible capacity loss of $\sim 1720 \mathrm{mAh} \mathrm{g}^{-1}$ could mainly originate from the reduction of 
electrode and the formation of a SEI on the surface of an electrode. ${ }^{255}$ The voltage profiles for the first discharge shows qualitatively similar to reported data of $\mathrm{Ca}_{2} \mathrm{Fe}_{2} \mathrm{O}_{5}$ and $\mathrm{Ca}_{2} \mathrm{Co}_{2} \mathrm{O}_{5}{ }^{226}$ and quantitatively to that of $\mathrm{Fe}_{2} \mathrm{O}_{3}{ }^{253}$ with a plateau at around $0.9 \mathrm{~V}$ for $\mathrm{Sr}_{2} \mathrm{Fe}_{2} \mathrm{O}_{6-\delta}$ until a capacity of around $1000 \mathrm{mAh} \mathrm{g}^{-1}$ is reached and thereafter the voltage decreases gradually to $0.005 \mathrm{~V}$, suggesting an electrochemical process similar to $\mathrm{Ca}_{2} \mathrm{Fe}_{2} \mathrm{O}_{5}$ and $\mathrm{Ca}_{2} \mathrm{Co}_{2} \mathrm{O}_{5}{ }^{226}$. The comparative plateau voltage position observed for the compound was found to be sensitive to the crystal structure and nature of metal ion involved in electrochemical reaction. ${ }^{226,253}$ Similar observations have also been reported for other compounds with 3-d metal oxides. ${ }^{226,}{ }^{253}$ After the first cycle, the charge and discharge profile shows the analogous curves indicating a reversible insertion/extraction of Li. At the $50^{\text {th }}$ cycle, the average of both the charge and discharge potential is around $\sim 1.5 \mathrm{~V}$, suggesting a low average potential, which means $\mathrm{Sr}_{2} \mathrm{Fe}_{2} \mathrm{O}_{6-\delta}$ anode is a good electrode with almost no charge/discharge hysteresis. ${ }^{229}$ The observed 2nd discharge capacity is around $840 \mathrm{mAh} \mathrm{g}^{-1}$ and the $50^{\text {th }}$ cycle is $410 \mathrm{mAh} \mathrm{g}^{-1}$. The corresponding first, second, third and $50^{\text {th }}$ charge capacities are $660,630,510$ and $350 \mathrm{mAh} \mathrm{g}^{-1}$, respectively. The observed values of the first discharge and charge capacity are higher than the values reported for similar compounds, $\mathrm{Ca}_{2} \mathrm{Co}_{2} \mathrm{O}_{5}$ and $\mathrm{Ca}_{2} \mathrm{Fe}_{2} \mathrm{O}_{5} .{ }^{226}$ 


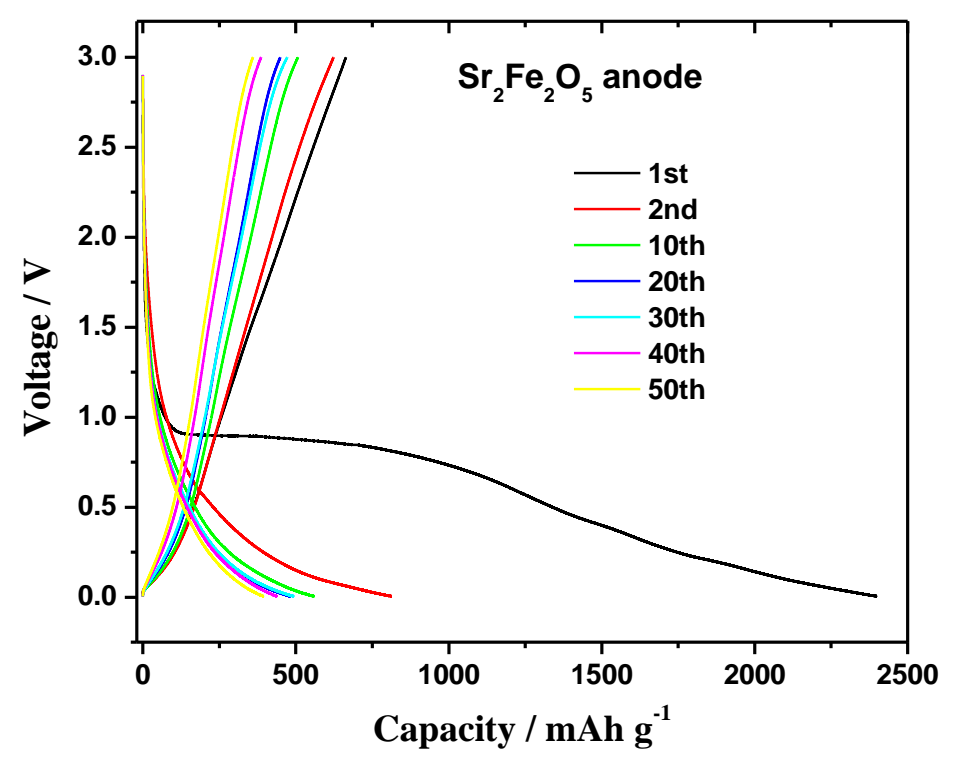

Figure 11.5. Galvanostatic charge-discharge profiles of $\mathrm{Sr}_{2} \mathrm{Fe}_{2} \mathrm{O}_{6-\delta}$ anode electrode at the voltage range of $3.0-0.005 \mathrm{~V}$ with a current density of $25 \mathrm{~mA} / \mathrm{g}$ for $1^{\text {st }}, 2^{\text {nd }}, 10^{\text {th }}, 20^{\text {th }}, 30^{\text {th }}$, $40^{\text {th }}$ and $50^{\text {th }}$ cycles.

Figure 11.6_shows the cycle performance of as prepared $\mathrm{Sr}_{2} \mathrm{Fe}_{2} \mathrm{O}_{6-\delta}$ at the current density of $25 \mathrm{~mA} \mathrm{~g}^{-1}$. The electrode keeps a steady capacity of about $400 \mathrm{mAh} \mathrm{g}^{-1}$ until 50 cycles, which is slightly higher than that of theoretical capacity of graphite anode $372 \mathrm{mAh} \cdot \mathrm{g}^{-1} \cdot 230$ The capacity has been found to be $300-320 \mathrm{mAh} \mathrm{g}^{-1}$ in practice for graphite anode. ${ }^{230}$ Due to the various irreversible processes such as the formation of solid/electrolyte interphase (SEI) film and electrode decomposition, the capacity of $\mathrm{Sr}_{2} \mathrm{Fe}_{2} \mathrm{O}_{6-\delta}$ has a reasonable loss in the first cycle. ${ }^{254-256}$ As shown in the figure, its capacity was maintained up to 50 cycles with an average coulombic efficiency of over $90 \%$ but still we need to increase the coulombic efficiency for practical application. 


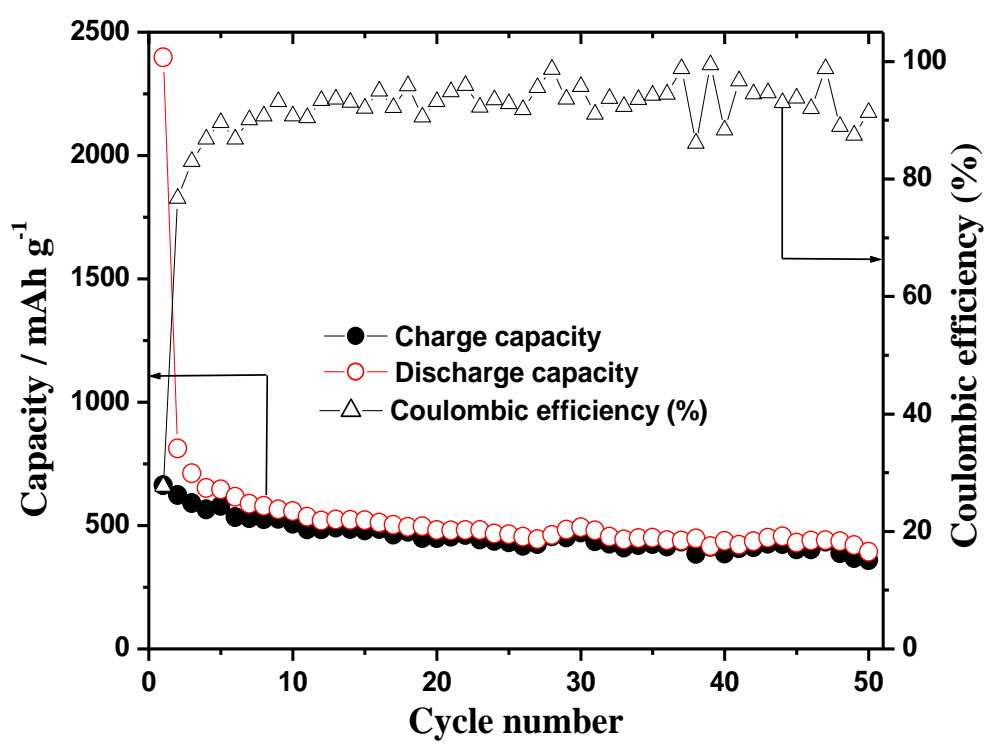

Figure 11.6. Capacities versus cycle numbers of $\mathrm{Sr}_{2} \mathrm{Fe}_{2} \mathrm{O}_{6-\delta}$ anode electrode at the voltage range of $3.0-0.005 \mathrm{~V}$ with a current density of $25 \mathrm{~mA} / \mathrm{g}$.

Figure 11.7 shows the rate capability test of the $\mathrm{Sr}_{2} \mathrm{Fe}_{2} \mathrm{O}_{6-\delta}$ electrode in the voltage range of 3.0-0.005 V under different current densities from 25, 50, 100, 200, and $500 \mathrm{~mA} \mathrm{~g}^{-1}$. With the increase in current densities from 25, 50, 100, 200, and $500 \mathrm{~mA} \mathrm{~g}^{-1}$, the $\mathrm{Sr}_{2} \mathrm{Fe}_{2} \mathrm{O}_{6-\delta}$ electrode delivers discharge capacities of $1885,416,325, \mathrm{v} 261$ and $187 \mathrm{mAh} \mathrm{g}^{-1}$, respectively. On reducing the current rate to the lower value of 50 , and $25 \mathrm{~mA} \mathrm{~g}^{-1}$ after rate performance test, $\mathrm{Sr}_{2} \mathrm{Fe}_{2} \mathrm{O}_{6-\delta}$ electrode still retains the discharge capacities of 340, and $390 \mathrm{mAh} \mathrm{g}^{-1}$, indicating good reversibility and high rate capability. 


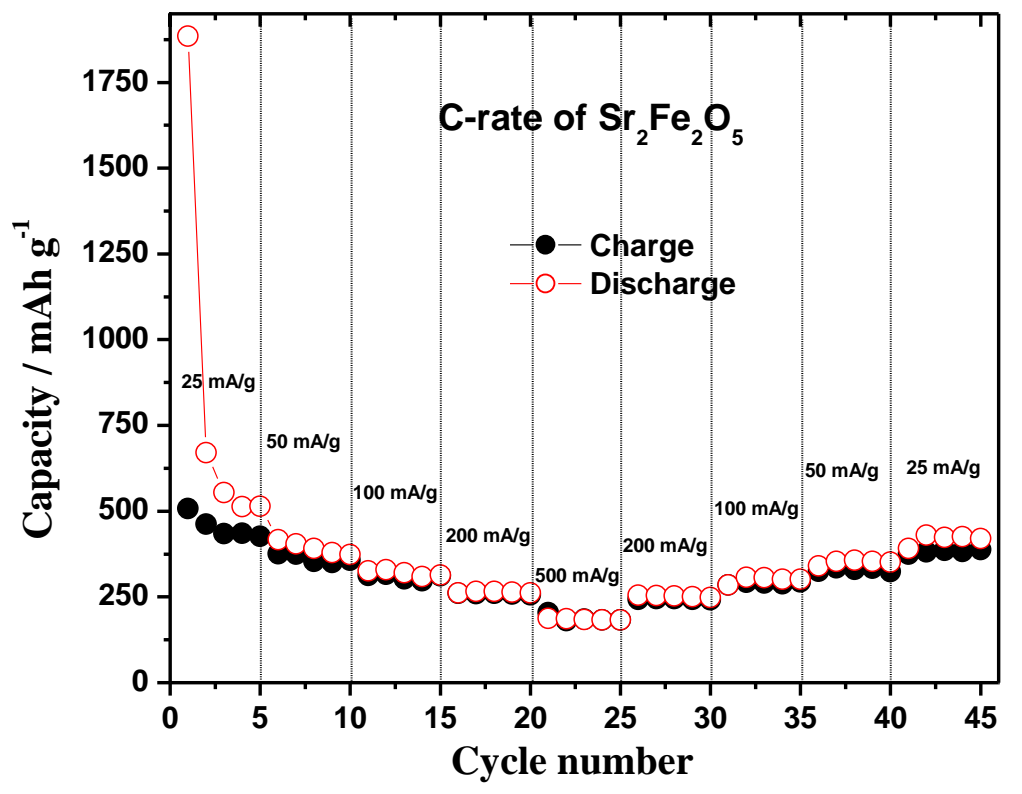

Figure 11.7. C-rate test of $\mathrm{Sr}_{2} \mathrm{Fe}_{2} \mathrm{O}_{6-\delta}$ anode electrode at the voltage range of $3.0-0.005$ $\mathrm{V}$ and different current densities of 25, 50, 100, 200, and $500 \mathrm{~mA} \mathrm{~g}^{-1}$, respectively.

Figure 11.8 shows the cyclic voltammetry of $\mathrm{Sr}_{2} \mathrm{Fe}_{2} \mathrm{O}_{6-\delta}$ anode electrode scanned at the voltage range of $0.005-3.0 \mathrm{~V}\left(\mathrm{vs} \mathrm{Li} / \mathrm{Li}^{+}\right)$with a scan speed of $1 \mathrm{mV} \mathrm{s}^{-1}$ for study of the electrochemical mechanism. For the $\mathrm{CV}$ testing, the anodic scan (oxidation process) is first performed and then followed by cathodic scan (reduction process). Two peaks are observed in the reduction process of the first cycle, but remaining cycles exhibited only one peak. The voltage profile observed was similar to previous report of $\mathrm{Fe}_{2} \mathrm{O}_{3}{ }^{253}$ with a long flat plateau at around $0.9 \mathrm{~V}$ during discharging. A peak observed between $0.8 \mathrm{~V}$ to $1.2 \mathrm{~V}$ in $\mathrm{CV}$ is indication for nonreversible formation of solid/electrolyte interphase (SEI) on the surface of the electrode ${ }^{257-258}$ which led to irreversible capacity loss. ${ }^{254-255}$ A cathodic peaks in the first cycle is observed in $\mathrm{CV}$ at around $0.95 \mathrm{~V}$. This peak is masked by the lines of other CVs. we can see small cathodic peak at around $1.0 \mathrm{~V}$ for cycles 2,3,4 and 5. The 
$2^{\text {nd }}, 3^{\text {rd }}, 4^{\text {th }}$ and 5 th $\mathrm{CV}$ curves of have higher extent of overlapping. Such overlapping is suggested to be the result of good reversibility and structural stability. ${ }^{254}$ The cathodic and anodic peaks can shift left or right for different cycles. ${ }^{254,258-261}$ The peak at around $0.3 \mathrm{~V}$ for the first cycle can be a peak shifted left from those of the other cycles. Thus, the peak at around $0.95 \mathrm{~V}$ for the first cycle may be attributed to the crystal structure destruction. ${ }^{226 \text {, }}$ 258, 262 The other reduction peak at around $0.3 \mathrm{~V}$ for the first cycle and at around $1 \mathrm{~V}$ for other remaining cycles could be attributed to electrochemical reaction. ${ }^{261,} 263$ In the oxidation process, the $\mathrm{CV}$ curves reveal the shifting of the anodic peak from right to left as the cycle number increases. The chemical process for $\mathrm{Fe}^{4+}$ is unknown. The chemical process of $\mathrm{Fe}^{3+}$ can be described as in the previous report. ${ }^{226}$

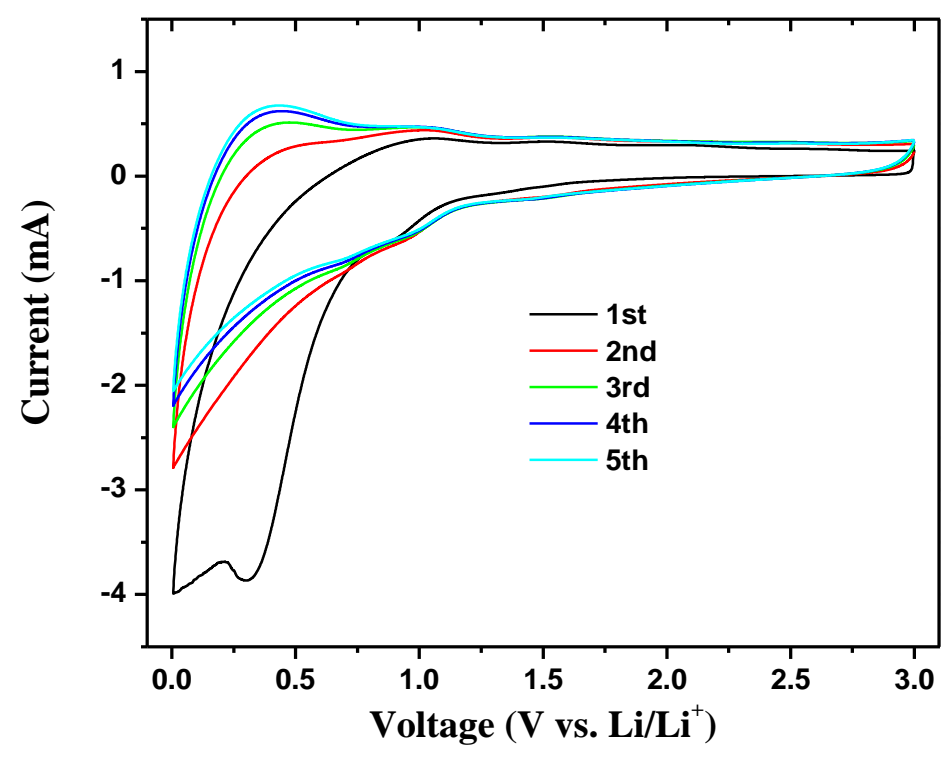

Figure 11.8. Cyclic voltammetry of $\mathrm{Sr}_{2} \mathrm{Fe}_{2} \mathrm{O}_{6-\delta}$ anode electrode scanned at the voltage range of $3.0-0.005 \mathrm{~V}$ with a scan speed of $1 \mathrm{mV} \mathrm{s}^{-1}$. 
The first discharge reaction for the compounds involves the irreversible crystal structure destruction leading to the formation of metal nanoparticles embedded in an amorphous matrix of $\mathrm{SrO}$ and $\mathrm{Li}_{2} \mathrm{O}{ }^{226}$

$\mathrm{Sr}_{2} \mathrm{Fe}_{2}{ }^{3+} \mathrm{O}_{6-\delta}+6 \mathrm{Li}^{+}+6 \mathrm{e}^{-} \rightarrow 2 \mathrm{SrO}+2 \mathrm{Fe}^{0}+3 \mathrm{Li}_{2} \mathrm{O}$

On subsequent charging, these metal particles are converted to their oxides together with the decomposition of $\mathrm{Li}_{2} \mathrm{O}$ which can be written as: ${ }^{226}$

$\mathrm{Fe}^{0}+\mathrm{Li}_{2} \mathrm{O} \leftrightarrow \mathrm{Fe}^{2+} \mathrm{O}+2 \mathrm{Li}^{+}+2 \mathrm{e}^{-}$

This reversible displacement reaction, viz. the formation and decomposition of $\mathrm{Li}_{2} \mathrm{O}$ accompanied by the reduction and oxidation of metal nanoparticles gives rise to reversible capacity in the subsequent charge-discharge cycles. ${ }^{226}$ This is a three-phase reaction between metal oxide, the reduced metal and $\mathrm{Li}_{2} \mathrm{O} \cdot{ }^{226}$ From the second cycle to the fifth cycle, the CV curves have higher extent overlapping suggesting the good reversibility and structural stability of $\mathrm{Sr}_{2} \mathrm{Fe}_{2} \mathrm{O}_{6-\delta} .{ }^{254}$ The way how $\mathrm{Fe}^{4+}$ undergoes in reaction has not been discussed before and is yet to be researched.

\section{CONCLUSION}

Oxygen deficient perovskite, $\mathrm{Sr}_{2} \mathrm{Fe}_{2} \mathrm{O}_{6-\delta}$ has been synthesized by solid state method to study its anodic property for Li ion battery. $\mathrm{Sr}_{2} \mathrm{Fe}_{2} \mathrm{O}_{6-\delta}$ electrode showed an initial discharge capacity of $2400 \mathrm{mAh} \mathrm{g}^{-1}$ with large irreversible capacity and low coulombic efficiency of 28.3\%. Further charge-discharge measurement of $\mathrm{Sr}_{2} \mathrm{Fe}_{2} \mathrm{O}_{6-\delta}$ electrode shows the increase 
in coulombic efficiency of over $90 \% . \mathrm{Sr}_{2} \mathrm{Fe}_{2} \mathrm{O}_{6-\delta}$ electrode shows the stable capacity of over $393 \mathrm{mAh} \mathrm{g}^{-1}$ up to 50 cycles with high reversible capacity at current density of $25 \mathrm{~mA}$ $\mathrm{g}^{-1}$ and superior reversible capacity throughout the whole studied number of cycles. The rate capability test of $\mathrm{Sr}_{2} \mathrm{Fe}_{2} \mathrm{O}_{6-\delta}$ electrode shows good performance in terms of capacity and can be comparable to the present graphitic carbon anode electrode in Li-ion battery. 


\section{CHAPTER 12}

\section{REMARKABLE OXYGEN EVOLUTION ACTIVITY OF Ca $\mathrm{Ca}_{2-\mathrm{x}} \mathrm{Sr}_{\mathrm{x}} \mathrm{Fe}_{2} \mathrm{O}_{6-\delta}{ }^{10}$}

The rise in global energy consumption has intensified the demand for clean and sustainable energy conversion and storage. ${ }^{264}$ The oxygen evolution reaction (OER) during water oxidation is an important electrochemical process for renewable-energy technologies, such as water splitting, rechargeable metal-air batteries, and regenerative fuel cells. ${ }^{265-270}$ However, the intrinsically sluggish kinetics of OER hinders the performance of these advanced energy devices, and results in a considerable overpotential for OER. ${ }^{271-272}$ The overpotential can be lowered by electrocatalysts, such as $\mathrm{IrO}_{2}$ and $\mathrm{RuO}_{2}$, which show high activity, but their scarcity, high cost and limitations in long-term stability in alkaline solutions hamper their practical use..$^{204,273-275}$ Designing inexpensive, earth-abundant and efficient electrocatalysts with low overpotential, which are stable upon prolonged exposure to oxidizing conditions in alkaline solutions, has been a major challenge. ${ }^{81,204,275-276}$

Oxygen-deficient perovskites $\left(\mathrm{ABO}_{3-\delta}\right)$ with alkaline or rare-earth cations on the A-site and 3d-metal cations on the B-site have demonstrated great potential for electrocatalytic

${ }^{10}$ The work described in this chapter was published in Angewandte Chemie International Edition ( 2019, vol. 58, p. 2060-2063) 
activity in alkaline solutions. ${ }^{119,135,199,201,209,266,275,277}$ Much research has been dedicated to understanding the parameters that can improve the OER activity of oxygen-deficient perovskites. ${ }^{119,135,199,201,209,266,275,277}$ One study reported that a near-unity occupancy of the $e_{g}$ orbital of the first-row transition metal in perovskite oxides can enhance the intrinsic activity for OER in alkaline solution. ${ }^{266}$ Based on this descriptor, the oxygen-deficient perovskite $\mathrm{Ba}_{0.5} \mathrm{Sr}_{0.5} \mathrm{Co}_{0.8} \mathrm{Fe}_{0.2} \mathrm{O}_{3-\delta}$ (BSCF) was developed as a highly active catalyst. ${ }^{198,266}$ Since then, various other oxygen-deficient perovskites have been studied, ${ }^{119,}$ 135, 199, 201, 209 , 266, 275, 277 but BSCF remains the most highly regarded catalyst for OER. However, cobalt criticality has emerged as a major concern in recent years, and development of cobalt-free catalysts for electrochemical for electrochemical processes such as OER is a priority. Recently, the qualitative $\mathrm{pH}$-dependence of OER catalysis was reported for some Fe-based perovskites, without any quantitative investigation of the OER activity, overpotential, Tafel kinetics, or electrochemically active surface areas. ${ }^{278}$ In line with efforts to develop inexpensive and earth-abundant electrocatalysts, we report the electrocatalytic activity of a Fe-based oxygen-deficient perovskite, $\mathrm{Sr}_{2} \mathrm{Fe}_{2} \mathrm{O}_{6-\delta},{ }^{4}$ which shows greater intrinsic OER activity than BSCF. We also demonstrate the correlations between the electrocatalytic activity, composition and defect-order as well as electrical conductivity in the series $\mathrm{Ca}_{2} \mathrm{Fe}_{2} \mathrm{O}_{6-\delta}, \mathrm{CaSrFe}_{2} \mathrm{O}_{6-\delta}$ and $\mathrm{Sr}_{2} \mathrm{Fe}_{2} \mathrm{O}_{6-\delta} ., 279$

All compounds were synthesized under identical conditions, and characterized by Rietveld refinements ${ }^{100-101}$ using powder X-ray diffraction. The material synthesis methods, Rietveld refinement profiles and the refined structural parameters are provided in the supplementary information. In this series of oxygen-deficient perovskites, the defects created due to oxygen-deficiency are distributed in an ordered fashion, rather than being 
spread randomly in the structure. In $\mathrm{Ca}_{2} \mathrm{Fe}_{2} \mathrm{O}_{6-\delta}$, the defects only appear in alternating layers, where the coordination number of Fe decreases from 6 to 4 . This leads to the formation of alternating tetrahedral and octahedral layers, ${ }^{279}$ as shown in Figure 12.1a. The tetrahedral layers actually consist of one-dimensional chains, which can have either righthanded or left-handed orientation. In $\mathrm{Ca}_{2} \mathrm{Fe}_{2} \mathrm{O}_{6-\delta}$, the tetrahedral chains in each layer are oriented opposite to those in the neighboring layer. $\mathrm{CaSrFe}_{2} \mathrm{O}_{6-\delta}$ has the same type of defect-order, resulting in alternating tetrahedral and octahedral geometry, but the tetrahedral chains in this compound have the same orientation throughout the crystal lattice (Figure 12.1b). ${ }^{4}$ Finally, $\mathrm{Sr}_{2} \mathrm{Fe}_{2} \mathrm{O}_{6-\delta}$ exhibits a different type of defect order, leading to the formation of alternating square pyramidal and octahedral coordination, as shown in Figure $12.1 \mathrm{c} .^{4}$

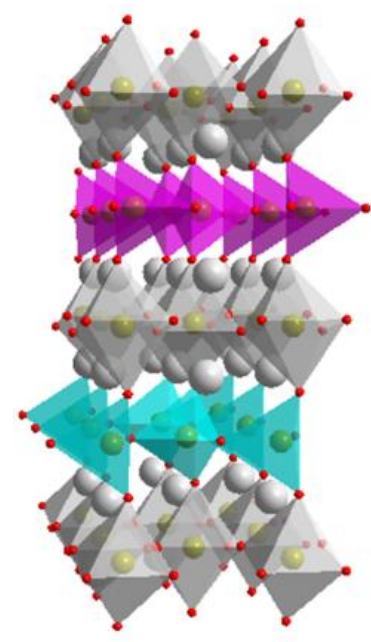

(a)

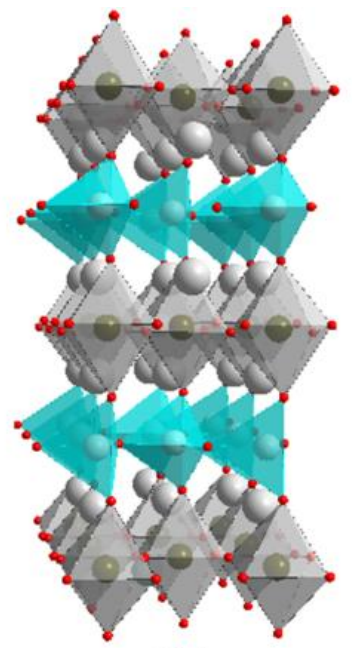

(b)

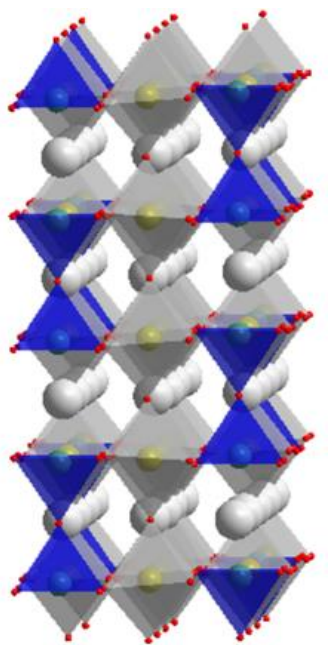

(c)

Figure 12.1. Crystal structures of (a) $\mathrm{Ca}_{2} \mathrm{Fe}_{2} \mathrm{O}_{6-\delta}$, (b) $\mathrm{CaSrFe}_{2} \mathrm{O}_{6-\delta}$, and (c) $\mathrm{Sr}_{2} \mathrm{Fe}_{2} \mathrm{O}_{6-\delta}$. In (a) and (b), different colors represent different orientations of tetrahedral chains. 
The OER activities were studied for all three compounds, $\mathrm{Ca}_{2} \mathrm{Fe}_{2} \mathrm{O}_{6-\delta}, \mathrm{CaSrFe}_{2} \mathrm{O}_{6-\delta}$ and $\mathrm{Sr}_{2} \mathrm{Fe}_{2} \mathrm{O}_{6-\delta}$, as well as the state of the art materials $\mathrm{Ba}_{0.5} \mathrm{Sr}_{0.5} \mathrm{Co}_{0.8} \mathrm{Fe}_{0.2} \mathrm{O}_{3-\delta}{ }^{26,204}$ and $\mathrm{RuO}_{2}{ }^{273}$ The details of electrode preparation and experimental setup are described in the supplementary information. For OER measurements, carbon black is often added to the electrode composition in order to enhance the conductivity within the electrode and maximize the utilization of the catalyst. ${ }^{197-199}$ However, recent studies have shown that carbon plays a more complex role than just a simple conductive support. ${ }^{200-201}$ For example, X-ray absorption near-edge spectroscopy study of $\mathrm{Ba}_{0.5} \mathrm{Sr}_{0.5} \mathrm{Co}_{0.8} \mathrm{Fe}_{0.2} \mathrm{O}_{3-\delta} /$ carbon composite electrode demonstrated the reduction of Co during the composite preparation process. ${ }^{202}$ As a result, some researchers have conducted OER experiments without carbon black. ${ }^{135}$ For the series of materials studied in this work, we performed the OER experiments both with and without carbon black. Both experiments led to similar results, particularly for compounds with higher OER activity, indicating that in these materials carbon black is not essential to the catalytic performance. The experiments without carbon black are preferred, as they demonstrate the intrinsic catalytic activity of each material. The conventional electrode preparation method usually involves drop casting the catalyst on the surface of a glassy carbon (GC) electrode. The GC electrode helps to improve the electrical charge transport. In the present study, the conventional method was initially employed. One of the parameters in the OER activity of a catalyst is the onset potential in polarization curve. ${ }^{275,280-281}$ As shown in Figure 12.2, the onset potential decreases systematically in progression from $\mathrm{Ca}_{2} \mathrm{Fe}_{2} \mathrm{O}_{6-\delta}$ to $\mathrm{CaSrFe}_{2} \mathrm{O}_{6-\delta}$ and $\mathrm{Sr}_{2} \mathrm{Fe}_{2} \mathrm{O}_{6-\delta}$. The latter shows onset potential of $1.5 \mathrm{~V}$, which is similar to those reported for highly active OER 
catalysts, such as $\mathrm{Ba}_{0.5} \mathrm{Sr}_{0.5} \mathrm{Fe}_{0.2} \mathrm{Co}_{0.8} \mathrm{O}_{3-\delta},{ }^{81,} 275 \quad \mathrm{IrO}_{2},{ }^{119,}, 282 \quad \mathrm{SrNb}_{0.1} \mathrm{Co}_{0.7} \mathrm{Fe}_{0.2} \mathrm{O}_{3-\delta},{ }^{204}$ $\mathrm{Ca}_{2} \mathrm{Mn}_{2} \mathrm{O}_{5}{ }^{119}$, and $\mathrm{Co}_{3} \mathrm{O}_{4} /$ carbon nanotubes. ${ }^{281}$

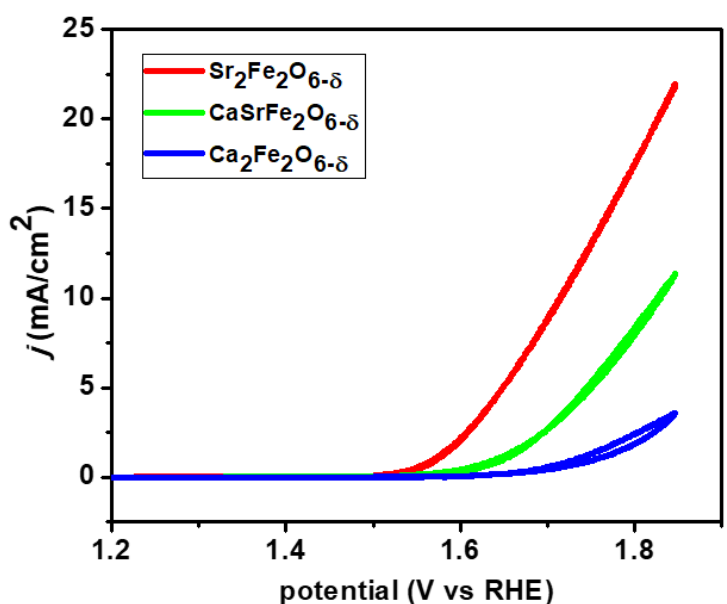

(a)

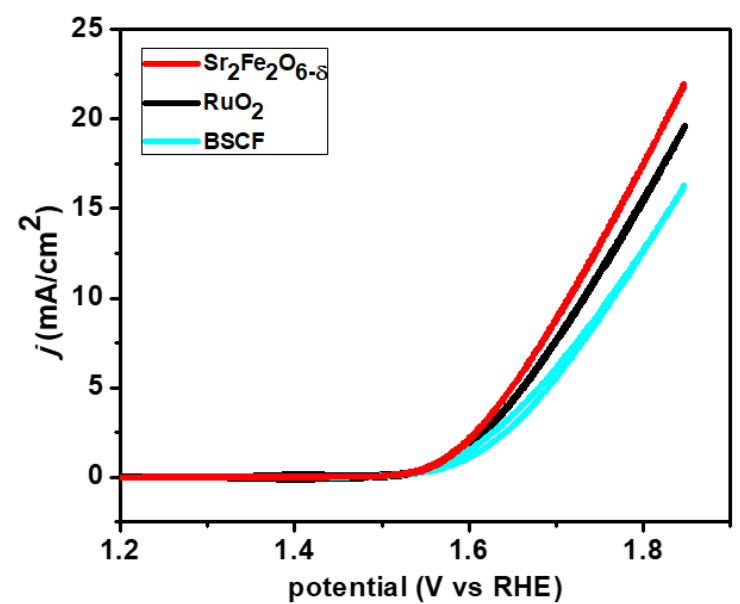

(b)

Figure 12.2. (a) Polarization curves showing the OER activities, obtained using the conventional glassy carbon electrode setup. (b) Comparison of the activity of $\mathrm{Sr}_{2} \mathrm{Fe}_{2} \mathrm{O}_{6}$ $\delta$ with state of the art materials $\mathrm{RuO}_{2}$ and $\mathrm{Ba}_{0.5} \mathrm{Sr}_{0.5} \mathrm{Co}_{0.8} \mathrm{Fe}_{0.2} \mathrm{O}_{3-\delta}(\mathrm{BSCF})$.

The overpotential, beyond the ideal thermodynamic potential of $1.23 \mathrm{~V}$, at $10 \mathrm{~mA} / \mathrm{cm}^{2}$ is an important parameter in describing the OER activity. The current density of $10 \mathrm{~mA} / \mathrm{cm}^{2}$ is related to solar fuel synthesis ${ }^{204,283}$ and the potential at this current density has been adopted as a metric for comparison of different OER catalysts. Among the three compounds, $\mathrm{Ca}_{2} \mathrm{Fe}_{2} \mathrm{O}_{6-\delta}$ shows low current density that does not reach $10 \mathrm{~mA} / \mathrm{cm}^{2}$ in the potentials up to $1.8 \mathrm{~V}$ vs RHE. $\mathrm{CaSrFe}_{2} \mathrm{O}_{6-\delta}$ shows overpotential of $0.60 \mathrm{~V}$, making it a reasonable catalyst. However, the most remarkable OER activity belongs to $\mathrm{Sr}_{2} \mathrm{Fe}_{2} \mathrm{O}_{6-\delta}$, that shows overpotential of $0.48 \mathrm{~V}$ (Figure 12.2a), which is superior to the state of the art 
compound, $\mathrm{Ba}_{0.5} \mathrm{Sr}_{0.5} \mathrm{Co}_{0.8} \mathrm{Fe}_{0.2} \mathrm{O}_{3-\delta} .{ }^{26,204}$ For direct comparison, we examined two of the best OER catalysts, $\mathrm{Ba}_{0.5} \mathrm{Sr}_{0.5} \mathrm{Co}_{0.8} \mathrm{Fe}_{0.2} \mathrm{O}_{3-\delta}(\mathrm{BSCF})^{26,204}$ and $\mathrm{RuO}_{2}{ }^{273}$ under the same conditions. As shown in Figure 12.2b, the OER activity of $\mathrm{Sr}_{2} \mathrm{Fe}_{2} \mathrm{O}_{6-\delta}$ is superior to both of these catalysts.

The OER kinetics is usually evaluated using Tafel equation $\eta=\mathrm{a}+\mathrm{b} \log j^{206-207}$ where $\eta$ is the overpotential, and $j$ is the current density. Tafel plot, $\eta$ vs. $\log j$, is influenced by electron and mass transport. ${ }^{210-211}$ Thus, it can provide information about electronic and geometric enhancement in the activity of electrocatalysts. ${ }^{211}$ In general, a decrease in the slope of the Tafel plot indicates better kinetics of OER, and smaller slopes represent faster reactions. The Tafel slopes for $\mathrm{Ca}_{2} \mathrm{Fe}_{2} \mathrm{O}_{6-\delta}, \mathrm{CaSrFe}_{2} \mathrm{O}_{6-\delta}$ and $\mathrm{Sr}_{2} \mathrm{Fe}_{2} \mathrm{O}_{6-\delta}$ are $185 \mathrm{mV} / \mathrm{dec}, 101$ $\mathrm{mV} / \mathrm{dec}$ and $60 \mathrm{mV} / \mathrm{dec}$, respectively, as shown in Figure 12.3. $\mathrm{Sr}_{2} \mathrm{Fe}_{2} \mathrm{O}_{6-\delta}$ shows the lowest Tafel slope among the three materials, which is consistent with the highest OER activity, and indicates facile charge transport in this compound. ${ }^{210-211,284}$

The electrochemically active surface area (ECSA) of each catalyst was estimated from double layer capacitance, $\mathrm{C}_{\mathrm{dl}}$, in non-faradic region, ${ }^{285}$ where electrode reactions are considered to be negligible and the current is primarily from electrical double layer charge and discharge ${ }^{285-286}$ The relationship between ECSA and $\mathrm{C}_{\mathrm{dl}}$ is described by ECSA $=\mathrm{C}_{\mathrm{d} /} / \mathrm{C}_{\mathrm{s}}$, where $C_{s}$ is specific capacitance. ${ }^{207,285,287}$ Some researchers have treated $C_{s}$ as a constant, $40 \mu \mathrm{F} / \mathrm{cm}^{2}$, when dealing with metal electrodes. ${ }^{207,285}$ Regardless of the value of $\mathrm{C}_{\mathrm{s}}$, the double layer capacitance, $\mathrm{C}_{\mathrm{dl}}$, is clearly proportional to electrochemically active surface area of the electrode (ECSA). ${ }^{206,288-290}$ Therefore $\mathrm{C}_{\mathrm{dl}}$ is commonly taken as representative of the magnitude of ECSA. ${ }^{206,289-290}$ The value of $\mathrm{C}_{\mathrm{dl}}$ can be obtained using the relationship $\mathrm{C}_{\mathrm{dl}}=\Delta \mathrm{j} / v,{ }^{206,289-290}$ where $\Delta \mathrm{j}$ is the absolute value of the difference between $\mathrm{j}_{\text {anodic }}$ and $\mathrm{j}$ catodic 
from cyclic voltammetry, and $v$ is the scan rate. ${ }^{206,289-290}$ The plot of $\Delta \mathrm{j}$ versus $v$ is frequently utilized, where the magnitude of $\mathrm{C}_{\mathrm{dl}}$ is equivalent to half of the slope of this plot. ${ }^{206,289-290}$ Some researchers have plotted javerage, the average of the absolute values of $\mathrm{j}_{\text {anodic }}$ and $\mathrm{j}_{\text {catodic }}$, versus $v .^{26,288} \mathrm{In}$ that case, the slope is directly equivalent to $\mathrm{C}_{\mathrm{dl}}$ and does not need to be divided by $2 .{ }^{288}$ In Figure 12.4, the values of $\Delta \mathrm{j}$ at the middle potential, ${ }^{206}$ $0.977 \mathrm{~V}$, are plotted against their corresponding scan rates from cyclic voltammetry experiments (Figure $\mathrm{S} 3$ ), and $\mathrm{C}_{\mathrm{dl}}$ is obtained as half of the slope of this plot. ${ }^{206,289-290}$ The $\mathrm{C}_{\mathrm{dl}}$ for $\mathrm{Sr}_{2} \mathrm{Fe}_{2} \mathrm{O}_{6-\delta}$ is significantly greater than that for $\mathrm{CaSrFe}_{2} \mathrm{O}_{6-\delta}$ and $\mathrm{Ca}_{2} \mathrm{Fe}_{2} \mathrm{O}_{6-\delta}$. This indicates larger ECSA for $\mathrm{Sr}_{2} \mathrm{Fe}_{2} \mathrm{O}_{6-\delta}$, consistent with its high OER catalytic activity.

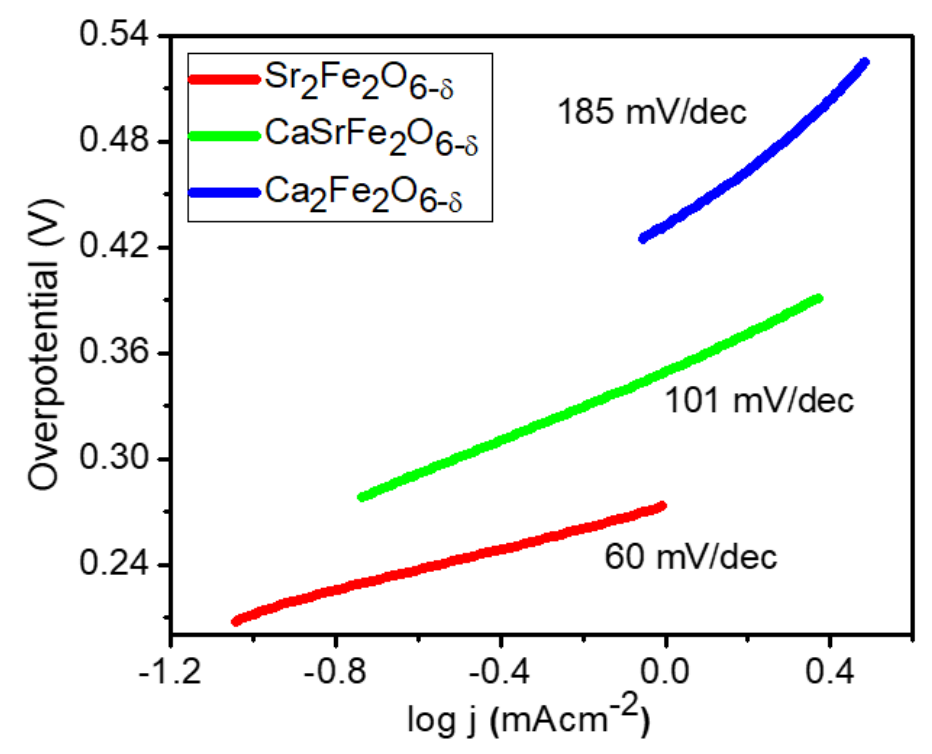

Figure 12.3. Tafel slopes indicating the OER kinetics.

In recent years, in addition to the conventional glassy carbon experiments, some researchers have used pure disks of catalytic oxides, without glassy carbon disk, to examine hydrogen or oxygen evolution activities. ${ }^{291-292}$ The advantage of this method is that it 
provides information about the intrinsic properties of the catalyst without any interference from the glassy carbon disk, which usually helps with the electrical conductivity. In this method, the material itself is responsible for both electrical conductivity and OER activity, and the contribution from glassy carbon disk is eliminated. ${ }^{291-292}$ Considering that this method represents the intrinsic catalytic activity of a material, we performed additional experiments using this technique to further examine the three compounds $\mathrm{Ca}_{2} \mathrm{Fe}_{2} \mathrm{O}_{6-\delta}$, $\mathrm{CaSrFe}_{2} \mathrm{O}_{6-\delta}$, and $\mathrm{Sr}_{2} \mathrm{Fe}_{2} \mathrm{O}_{6-\delta}$, as well as the state-of-the-art compound, $\mathrm{Ba}_{0.5} \mathrm{Sr}_{0.5} \mathrm{Co}_{0.8} \mathrm{Fe}_{0.2} \mathrm{O}_{3-\delta}$, which to our knowledge has not been examined using this method.

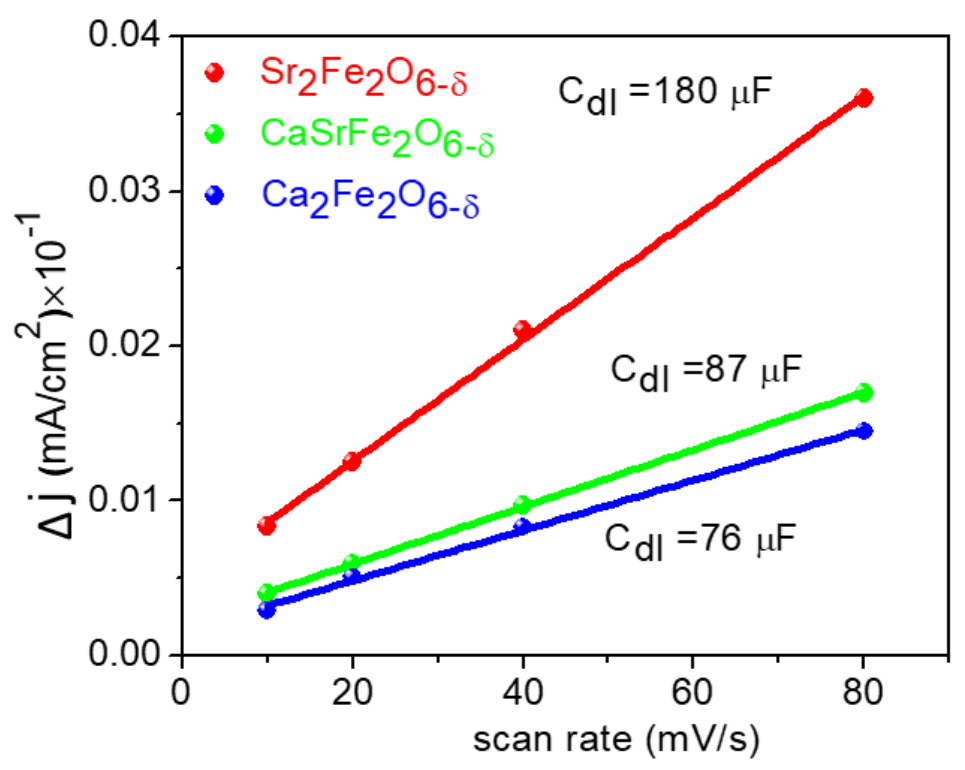

Figure 12.4. Plot of $\Delta \mathrm{j}=\mathrm{j}_{\text {anodic }}-\mathrm{j}_{\text {catodic }}$ as a function of scan rate. The $\mathrm{C}_{\mathrm{dl}}$ for each material is equivalent to half of the slope. 


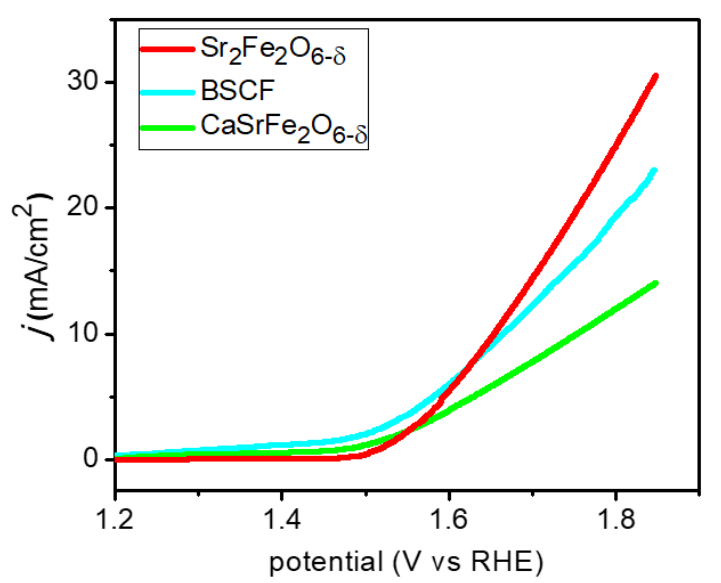

(a)

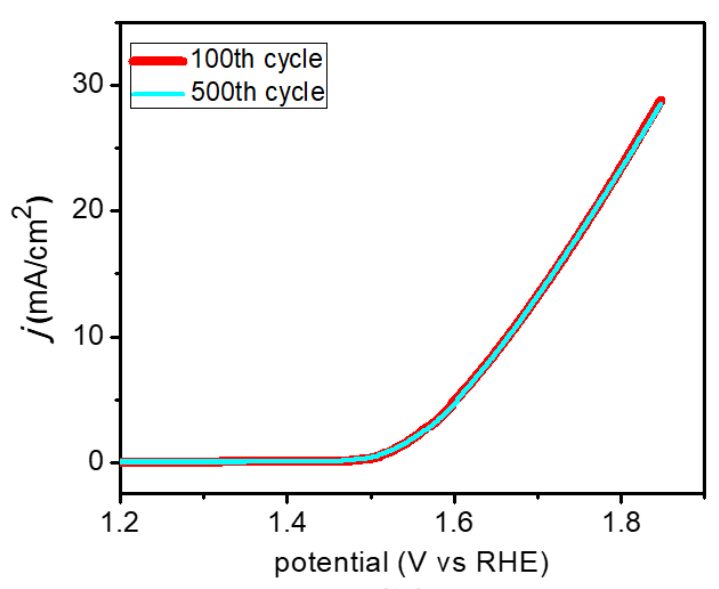

(b)

Figure 12.5. (a) Polarization curves showing the OER activities of pure disks of catalysts. (b) Stability test for $\mathrm{Sr}_{2} \mathrm{Fe}_{2} \mathrm{O}_{6-\delta}$ by performing 500 cycles.

The polarization curves obtained from pure disks indicated almost no OER activity for $\mathrm{Ca}_{2} \mathrm{Fe}_{2} \mathrm{O}_{6-\delta}$, which is expected, given the low electrical conductivity of this compound. Our 4-probe measurements show the room temperature conductivity of $1.13 \times 10^{-9} \mathrm{Scm}^{-1}$ for $\mathrm{Ca}_{2} \mathrm{Fe}_{2} \mathrm{O}_{6-\delta}, 7.78 \times 10^{-3} \mathrm{Scm}^{-1}$ for $\mathrm{CaSrFe}_{2} \mathrm{O}_{6-\delta}, 7.30 \mathrm{Scm}^{-1}$ for $\mathrm{Sr}_{2} \mathrm{Fe}_{2} \mathrm{O}_{6-\delta}$, and $8.17 \times 10^{-3} \mathrm{Scm}^{-}$ ${ }^{1}$ for $\mathrm{Ba}_{0.5} \mathrm{Sr}_{0.5} \mathrm{Co}_{0.8} \mathrm{Fe}_{0.2} \mathrm{O}_{3-\delta}$. The enhanced electrical conductivity in $\mathrm{Sr}_{2} \mathrm{Fe}_{2} \mathrm{O}_{6-\delta}$ can be attributed to two different parameters. The first is the larger average bond angle in $\mathrm{Sr}_{2} \mathrm{Fe}_{2} \mathrm{O}_{6-\delta}\left(\sim 170^{\circ}\right)$ compared to that of $\mathrm{Ca}_{2} \mathrm{Fe}_{2} \mathrm{O}_{6-\delta}$ and $\mathrm{CaSrFe}_{2} \mathrm{O}_{6-\delta}\left(\sim 139^{\circ}\right)$, which enhances the electron transfer through $\mathrm{M}^{\mathrm{n}+}-\mathrm{O}-\mathrm{M}^{\mathrm{m}+}$ pathways, where $\mathrm{M}$ is a metal (such as $\mathrm{Fe}$ ) with variable oxidation states $\mathrm{n}+$ and $\mathrm{m}+$. Previous reports on perovskite-based compounds have demonstrated the improvement of the electrical conductivity as a function of bond angle. ${ }^{[27]}$ The second parameter is the presence of a greater number of $\mathrm{Fe}^{3+}-\mathrm{O}-\mathrm{Fe}^{4+}$ conduction pathways in $\mathrm{Sr}_{2} \mathrm{Fe}_{2} \mathrm{O}_{6-\delta}$. As shown before, ${ }^{[9]}$ the oxygen stoichiometry in this material is 5.75 (i.e., $\delta=0.25$ ), indicating that Fe has both tri- and tetravalent oxidation states. However, $\mathrm{Ca}_{2} \mathrm{Fe}_{2} \mathrm{O}_{6-\delta}$ and $\mathrm{CaSrFe}_{2} \mathrm{O}_{6-\delta}$ have oxygen stoichiometry of 5.0 (i.e., $\delta=$ 
$1.0),{ }^{[9,10]}$ which indicates that $\mathrm{Fe}$ is primarily in +3 oxidation state. We have confirmed the oxygen stoichiometry using iodometric titrations. Note that metals with multiple stable oxidation states are needed for enhanced electronic conductivity. While perovskite-based materials are known to conduct both electrons and ions, ${ }^{[28]}$ the dominance of electronic conductivity has been demonstrated in this class of materials. ${ }^{[28]}$ Therefore, the enhancement of the electrical conductivity in $\mathrm{Sr}_{2} \mathrm{Fe}_{2} \mathrm{O}_{6-\delta}$ is primarily due to the superior electronic charge transport.

The polarization curves for pure disks of $\mathrm{CaSrFe}_{2} \mathrm{O}_{6-\delta}$ and $\mathrm{Sr}_{2} \mathrm{Fe}_{2} \mathrm{O}_{6-\delta}$ (Figure 12.5) show overpotential values of $0.52 \mathrm{~V}$ and $0.42 \mathrm{~V}$, respectively. Considering that the disk is made of pure catalyst, the entire disk surface participates in OER activity, leading to the enhanced overpotential compared to the glassy carbon electrode method. The remarkable overpotential of $\mathrm{Sr}_{2} \mathrm{Fe}_{2} \mathrm{O}_{6-\delta}$ disk is due to a combination of good OER catalytic activity and sufficient intrinsic electrical conductivity. As shown in Figure 12.5, the $\mathrm{Sr}_{2} \mathrm{Fe}_{2} \mathrm{O}_{6-\delta}$ disk shows better OER activity than the disk of the state-of-the-art catalyst, $\mathrm{Ba}_{0.5} \mathrm{Sr}_{0.5} \mathrm{Co}_{0.8} \mathrm{Fe}_{0.2} \mathrm{O}_{3-\delta}$, consistent with the lower electrical conductivity of $\mathrm{Ba}_{0.5} \mathrm{Sr}_{0.5} \mathrm{Co}_{0.8} \mathrm{Fe}_{0.2} \mathrm{O}_{3-\delta}$ compared to $\mathrm{Sr}_{2} \mathrm{Fe}_{2} \mathrm{O}_{6-\delta}$. Also, note the well-defined non-faradaic region and sharp onset for $\mathrm{Sr}_{2} \mathrm{Fe}_{2} \mathrm{O}_{6-\delta}$ disk.

Given the outstanding OER activity of $\mathrm{Sr}_{2} \mathrm{Fe}_{2} \mathrm{O}_{6-\delta}$ disk, we also examined its stability by repeating the polarization curves over 500 cycles. As shown in Figure 12.5b, this material shows remarkable stability and there is little change to its OER activity after 500 cycles.

The findings described in this communication indicate that the OER activity is enhanced as electrical conductivity increases, leading to the outstanding catalytic activity of an ironbased catalyst, $\mathrm{Sr}_{2} \mathrm{Fe}_{2} \mathrm{O}_{6-\delta}$. It has been found for other series of compounds as well. ${ }^{293}$ 


\section{SUPPORTING INFORMATION}

Material synthesis and characterization

All materials used for each type of analysis were synthesized under identical conditions. The catalysts used for standard oxygen evolution reaction (OER) on glassy carbon electrode were prepared by sol-gel method. Stoichiometric amounts of metal nitrates were dissolved in water and equimolar concentration of citric acid was also added to the mixture. The solution was heated to $80{ }^{\circ} \mathrm{C}$ to form a gel. The sample was then heated for 6 hours at $600{ }^{\circ} \mathrm{C}$, followed by 12 hours at $1200{ }^{\circ} \mathrm{C}$. Similar method was used for the preparation of BSCF. $\mathrm{RuO}_{2}$ was purchased from Alfa Aesar. The phase purity and structure of polycrystalline samples were examined by powder X-ray diffraction at room temperature using $\mathrm{Cu} \mathrm{K \alpha} 1$ radiation $(\lambda=1.54056 \AA)$. The Rietveld refinements were carried out using GSAS software and EXPGUI interface. ${ }^{[11]}$ The refinement profiles are shown in Figure S1. Electrode preparation

$35 \mathrm{mg}$ of the sample was added to $40 \mu \mathrm{L}$ of Nafion ${ }^{\circledR} \mathrm{D}-521$ solution and stirred using magnetic stirrer. $7 \mathrm{~mL}$ of THF was then added and stirred for a few more minutes and then sonicated for 10 minutes. This mixture was made both with and without carbon powder to test the effect of carbon on OER activity. For experiments with carbon, $7 \mathrm{mg}$ of carbon powder was added prior to adding THF. The catalyst ink was drop casted on the surface of a glassy electrode with a diameter of $5 \mathrm{~mm}$ and was left to dry in air overnight.

The OER experiments were also performed on disks of pure samples, without the use of glassy carbon electrode or other additives. A pellet of the material with $5.56 \mathrm{~mm}$ diameter and $2.02 \mathrm{~mm}$ thickness was prepared by sintering at $1200{ }^{\circ} \mathrm{C}$. One side of the pellet was painted with gold and dried at $800{ }^{\circ} \mathrm{C}$. The pellet was then fixed inside a plastic tube with 
the gold paint facing the inside of the tube, as shown in Figure S2. The exposed surface of the pellet faced the outside and was aligned with the opening of the tube. The pellet fitted inside the tube tightly, such that there was no leakage of the solvent to the inside of the tube as confirmed by many repeated experiments. The tube was then attached to a PINE Research rotating disk electrode, where the gold-painted side of the pellet was connected to a copper wire, as shown in Figure S2.

Measurements of OER activity

Electrocatalytic activity measurements were carried out on an electrochemical workstation in a three-electrode glass cell system. A glassy carbon electrode coated with the catalyst or the pure disk of the catalytic material were used as the working electrode. A commercial platinum electrode was used as the counter electrode. $\mathrm{Ag} / \mathrm{AgCl}$ (in $3 \mathrm{M} \mathrm{NaCl}$ ) was used as reference electrode and was calibrated against standard hydrogen electrode prior to use. The potential obtained versus $\mathrm{Ag} / \mathrm{AgCl}$ electrode $\left(\mathrm{E}_{\mathrm{Ag} / \mathrm{AgCl}}\right)$ was converted to potential versus reversible hydrogen electrode ( $\left.\mathrm{E}_{\mathrm{RHE}}\right)$ according to the Nernst equation:

$\mathrm{E}_{\mathrm{RHE}}=\mathrm{E}_{\mathrm{Ag} / \mathrm{AgCl}}+0.059 \mathrm{pH}+\mathrm{E}^{\mathbf{0}} \mathrm{Ag} / \mathrm{AgCl}$

where $\mathrm{E}^{0} \mathrm{Ag} / \mathrm{AgCl}$ is the potential of the $\mathrm{Ag} / \mathrm{AgCl}$ electrode versus $\mathrm{RHE}$.

Before starting each measurement, $0.1 \mathrm{M} \mathrm{KOH}$ electrolyte was bubbled with oxygen for at least $30 \mathrm{~min}$. The cyclic voltammetry $(\mathrm{CV})$ profiles were recorded at a scan rate of $10 \mathrm{mV}$ $\mathrm{s}^{-1}$ from 0 to $1.85 \mathrm{~V}$ vs RHE using a rotating disk electrode, which was continuously rotated at $1600 \mathrm{rpm}$. 


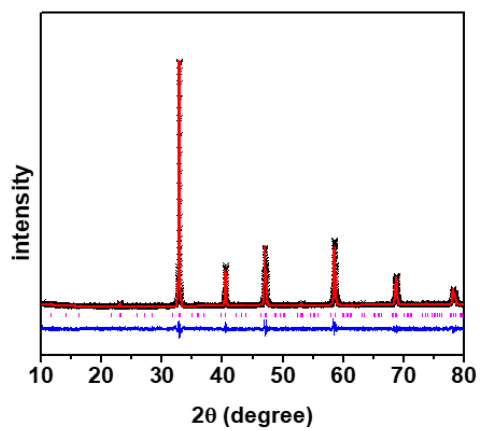

(a)

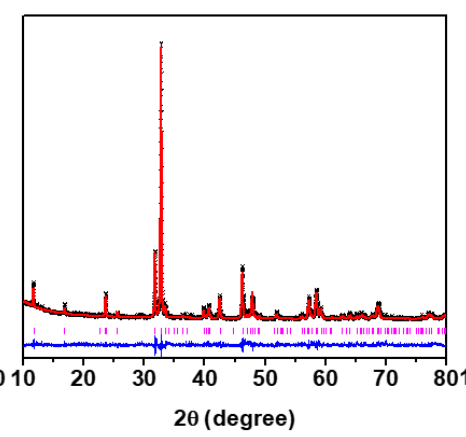

(b)

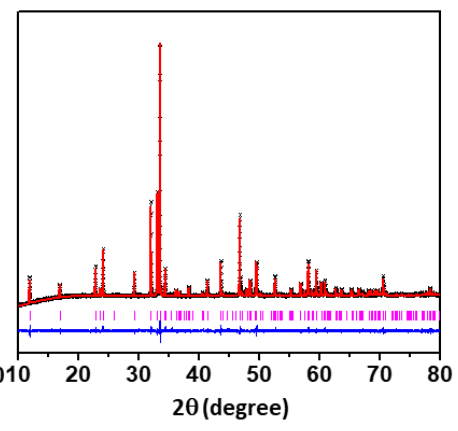

(c)

Figure S1. Rietveld refinement profiles using powder X-ray diffraction for (a) $\mathrm{Ca}_{2} \mathrm{Fe}_{2} \mathrm{O}_{6}$ $\delta$, space group Pnma, (b) $\mathrm{CaSrFe}_{2} \mathrm{O}_{6-\delta}$, space group Ibm2, and (c) $\mathrm{Sr}_{2} \mathrm{Fe}_{2} \mathrm{O}_{6-\delta}$, space group $I 4 / \mathrm{mmm}$. Black symbols are the experimental data, red line represents the model, vertical tick marks show Bragg peak positions, and the lower blue line represents the difference plot.

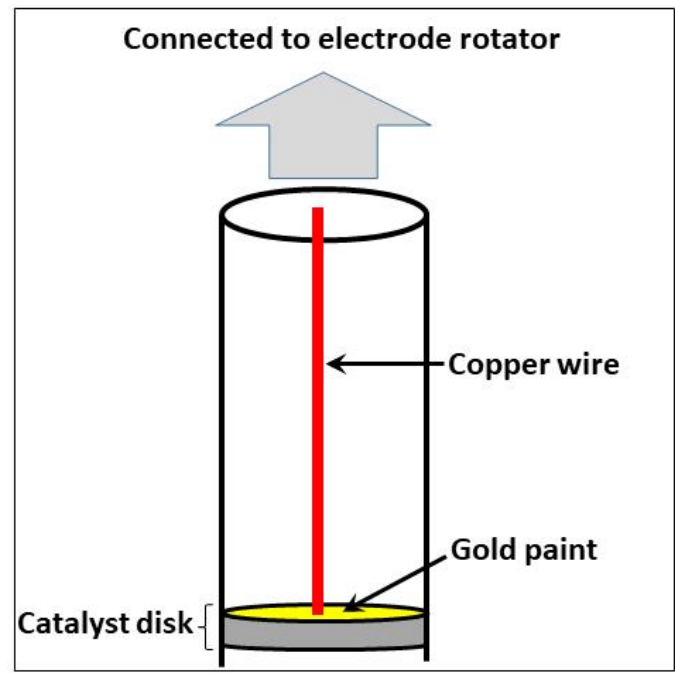

(a)

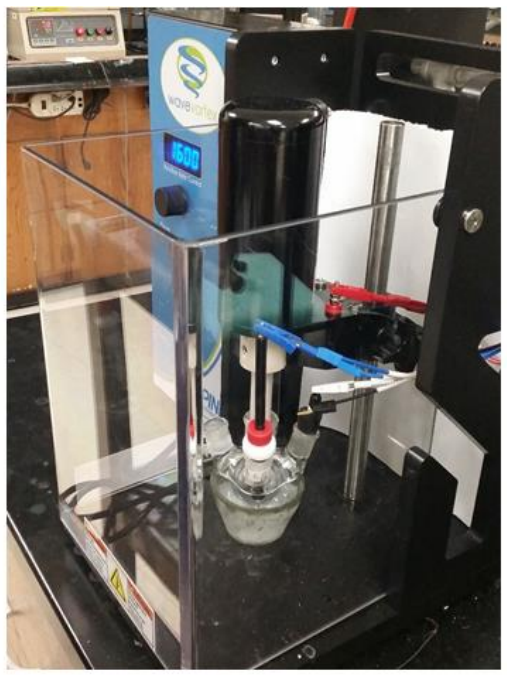

(b)

Figure S2. (a) The setup for testing the OER activity of catalysts disks, without glassy carbon electrode. (b) The entire measurement setup. 


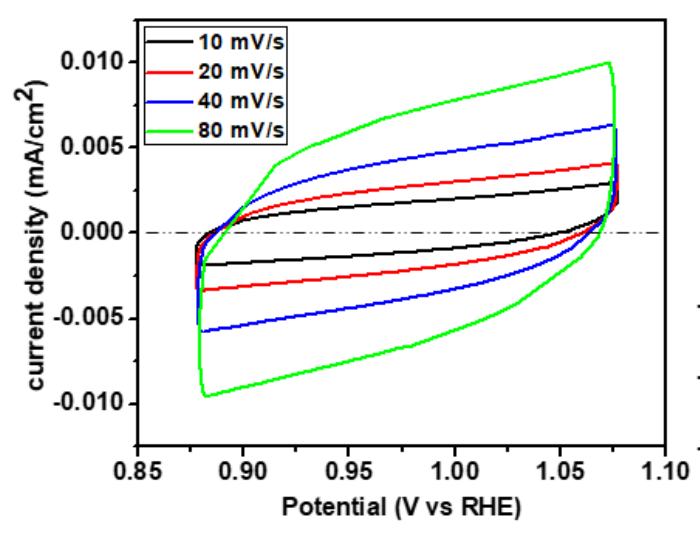

(a)

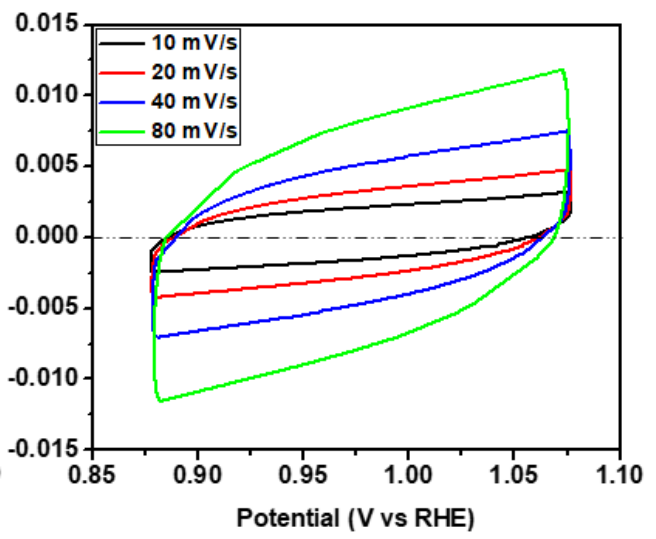

(b)

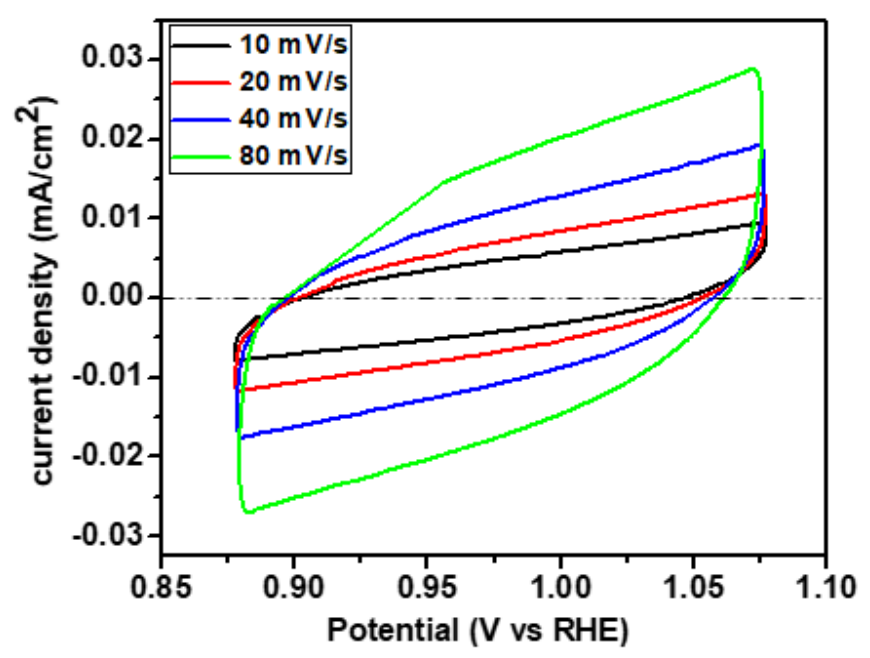

(c)

Figure S3. Cyclic voltammetry in non-faradic region to obtain double layer capacitance (Figure 12.4) for the three compounds (a) $\mathrm{Ca}_{2} \mathrm{Fe}_{2} \mathrm{O}_{6-\delta}$, (b) $\mathrm{CaSrFe}_{2} \mathrm{O}_{6-\delta}$, and (c) $\mathrm{Sr}_{2} \mathrm{Fe}_{2} \mathrm{O}_{6-}$ $\delta$. 


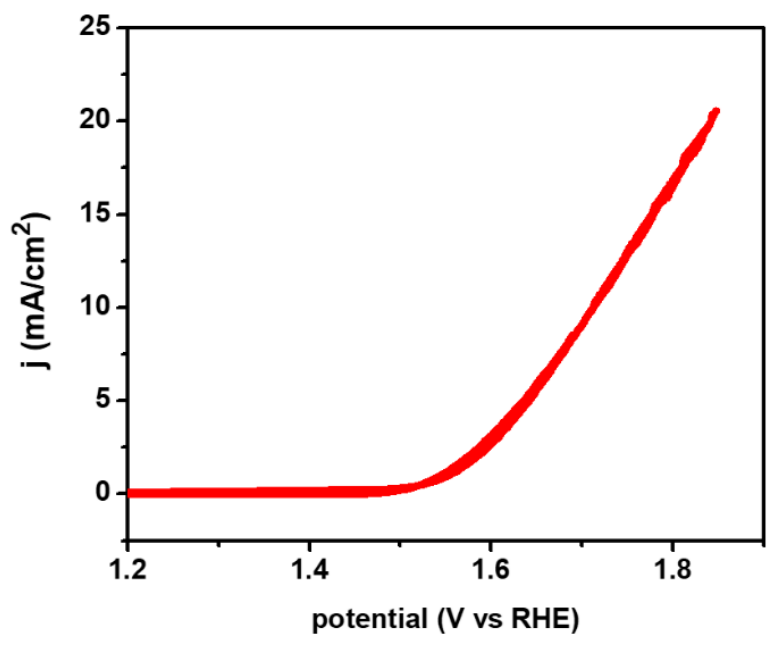

Figure S4. Polarization curve for $\mathrm{Sr}_{2} \mathrm{Fe}_{2} \mathrm{O}_{6-\delta}$, obtained using the conventional glassy carbon setup, and with the addition of carbon black to the catalyst. Comparison to Figure 12.2 indicates that carbon black does not improve the OER activity.

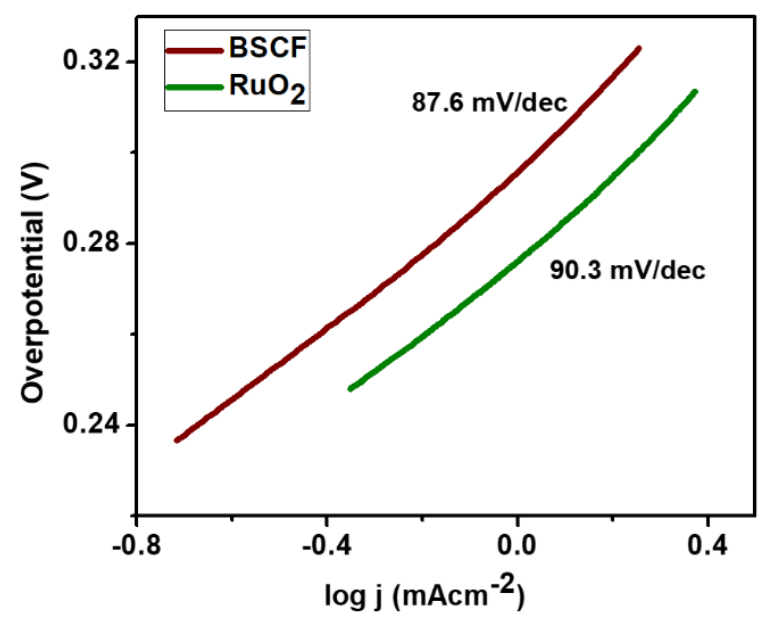

Figure S5. Tafel slopes for BSCF and $\mathrm{RuO}_{2}$. 


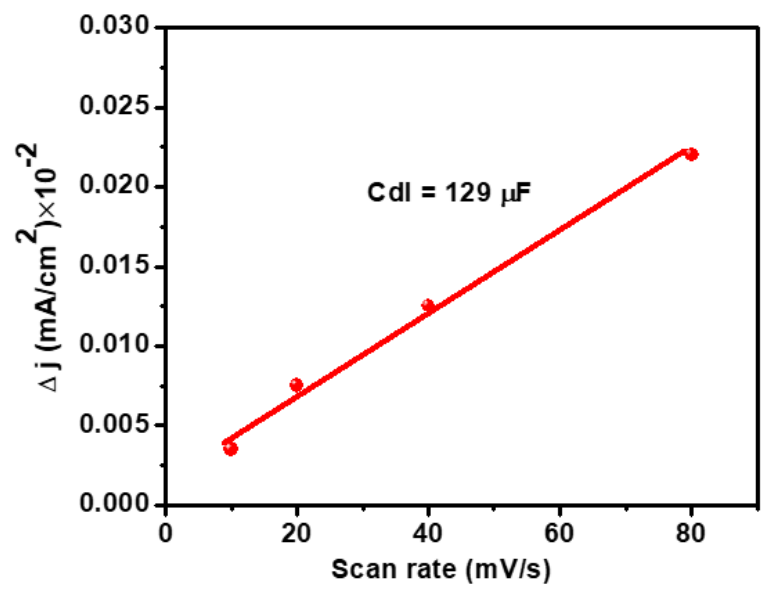

(a)

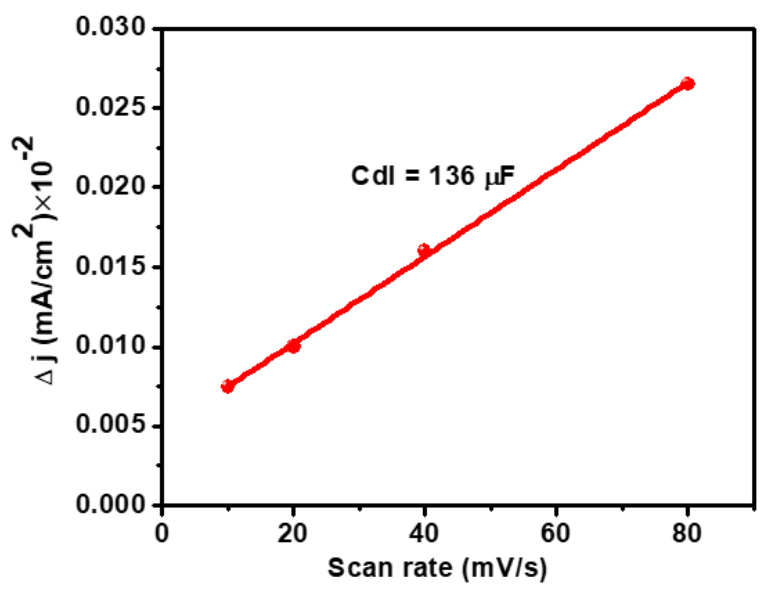

(b)

Figure S6. Plot of $\Delta \mathrm{j}=\mathrm{j}_{\text {anodic }}-\mathrm{j}_{\text {cathodic }}$ as a function of scan rate for (a) BSCF and (b) $\mathrm{RuO}_{2}$. The $\mathrm{C}_{\mathrm{dl}}$ for each material is equivalent to half of the slope.

Table $\mathrm{S} 1$. The refined structural parameters for $\mathrm{Ca}_{2} \mathrm{Fe}_{2} \mathrm{O}_{6-\delta}$ from powder X-ray diffraction. Space group: Pnma, $a=5.40238(5), b=14.7018(1), c=5.57261(5), R_{p}=0.013, w R_{p}=$ $0.018, \chi^{2}=1.941$

\begin{tabular}{llllll}
\hline Elements & $\mathrm{x}$ & $\mathrm{y}$ & $\mathrm{z}$ & occupancy & $\mathrm{U}_{\text {iso }}$ \\
\hline $\mathrm{Ca}$ & $0.4807(5)$ & $0.1080(1)$ & $0.0248(4)$ & 1 & $0.0281(9)$ \\
$\mathrm{Fe} 1$ & 0.0 & 0.0 & 0.0 & 1 & $0.027(1)$ \\
$\mathrm{Fe} 2$ & $-0.0551(6)$ & 0.25 & $-0.0670(4)$ & 1 & $0.030(1)$ \\
$\mathrm{O} 1$ & $0.261(3)$ & $-0.0154(3)$ & $0.234(1)$ & 1 & $0.021(1)$ \\
$\mathrm{O} 2$ & $0.0297(1)$ & $0.1423(3)$ & $0.0773(8)$ & 1 & $0.021(1)$ \\
$\mathrm{O} 3$ & $0.6084(2)$ & 0.25 & $-0.124(2)$ & 1 & $0.021(1)$ \\
\hline
\end{tabular}


Table S2. The refined structural parameters for $\mathrm{CaSrFe}_{2} \mathrm{O}_{6-\delta}$ from powder $\mathrm{X}$-ray diffraction. Space group: $\mathrm{Ibm} 2, a=5.6313(4), b=15.181(1), c=5.4695(4), R_{p}=0.018$, $w R_{p}=0.026, \chi^{2}=1.604$

\begin{tabular}{llllll}
\hline Elements & $\mathrm{x}$ & $\mathrm{y}$ & $\mathrm{z}$ & occupancy & $\mathrm{U}_{\text {iso }}$ \\
\hline $\mathrm{Ca}$ & $0.5127(5)$ & $0.1109(1)$ & $0.008(4)$ & 0.5 & $0.015(1)$ \\
$\mathrm{Sr}$ & $0.5127(5)$ & $0.1109(1)$ & $0.008(4)$ & 0.5 & $0.015(1)$ \\
$\mathrm{Fe} 1$ & 0.0 & 0.0 & 0.0 & 1 & $0.022(2)$ \\
$\mathrm{Fe} 2$ & $0.0760(7)$ & 0.25 & $-0.004(7)$ & 1 & $0.013(2)$ \\
$\mathrm{O} 1$ & $0.229(4)$ & $0.0069(6)$ & $0.292(5)$ & 1 & $0.014(3)$ \\
& & & & & \\
$\mathrm{O} 2$ & $-0.082(1)$ & $0.149(5)$ & $0.001(8)$ & 1 & $0.014(3)$ \\
& & & & & \\
$\mathrm{O} 3$ & $0.382(3)$ & 0.25 & $0.887(7)$ & 1 & $0.014(3)$ \\
& & & & & \\
\hline
\end{tabular}

Table S3. The refined structural parameters for $\mathrm{Sr}_{2} \mathrm{Fe}_{2} \mathrm{O}_{6-\delta}$ from powder X-ray diffraction. Space group: $14 / \mathrm{mmm}, a=10.9345(7), b=10.9345(7), c=7.6988(5), R_{p}=0.022, w R_{p}=$ $0.031, \chi^{2}=1.505$

\begin{tabular}{llllll}
\hline Elements & $\mathrm{x}$ & $\mathrm{y}$ & $\mathrm{z}$ & occupancy & $\mathrm{U}_{\text {iso }}$ \\
\hline $\mathrm{Sr} 1$ & $0.2561(7)$ & 0.0 & 0.0 & 1 & $0.026(2)$ \\
$\mathrm{Sr} 2$ & $0.2488(7)$ & 0.0 & 0.5 & 1 & $0.041(3)$ \\
$\mathrm{Fe} 1$ & 0.0 & 0.0 & 0.25 & 1 & $0.061(9)$ \\
$\mathrm{Fe} 2$ & 0.25 & 0.25 & 0.25 & 1 & $0.023(3)$ \\
$\mathrm{Fe} 3$ & 0.5 & 0.0 & 0.25 & 1 & $0.042(8)$ \\
$\mathrm{O} 1$ & 0.0 & 0.0 & 0.25 & 1 & $0.024(2)$ \\
$\mathrm{O} 2$ & $0.124(2)$ & $0.124(2)$ & $0.208(2)$ & 1 & $0.024(2)$ \\
$\mathrm{O} 3$ & $0.250(2)$ & $0.250(2)$ & 0.5 & 1 & $0.024(2)$ \\
$\mathrm{O} 4$ & $0.131(2)$ & $0.631(2)$ & 0.25 & 1 & $0.024(2)$ \\
$\mathrm{O} 5$ & 0.5 & 0.0 & 0.0 & 1 & $0.024(2)$ \\
\hline
\end{tabular}




\section{REFERENCES}

1. Tilley, R. J. D., Perovskites: Structure-Property Relationships. John Wiley \& Sons, Ltd: 2016.

2. Bombik, A.; Leśniewska, B.; Mayer, J.; Pacyna, A. W., Crystal structure of solid solutions $\mathrm{REFe}_{1-\mathrm{x}}(\mathrm{Al} \text { or } \mathrm{Ga})_{\mathrm{x}} \mathrm{O}_{3}(\mathrm{RE}=\mathrm{Tb}, \mathrm{Er}, \mathrm{Tm})$ and the correlation between superexchange interaction $\mathrm{Fe}^{+3}-$ $\mathrm{O}^{-2}-\mathrm{Fe}^{+3}$ linkage angles and Néel temperature. J. Magn. Magn. Mater. 2003, 257, 206-219.

3. Karki, S. B.; Ramezanipour, F., Magnetic and electrical properties of $\mathrm{BaSrMMoO}_{6}(\mathrm{M}=\mathrm{Mn}$, $\mathrm{Fe}, \mathrm{Co}$, and Ni). Mater. Today Chem. 2019, 13, 25-33.

4. Hona, R. K.; Huq, A.; Mulmi, S.; Ramezanipour, F., Transformation of structure, electrical conductivity, and magnetism in $\mathrm{AA}^{\prime} \mathrm{Fe}_{2} \mathrm{O}_{6-\delta}, \mathrm{A}=\mathrm{Sr}, \mathrm{Ca}$ and $\mathrm{A}^{\prime}=\mathrm{Sr}$. Inorg. Chem. 2017, 56, 97169724.

5. Berastegui, P.; Eriksson, S. G.; Hull, S., A neutron diffraction study of the temperature dependence of $\mathrm{Ca}_{2} \mathrm{Fe}_{2} \mathrm{O}_{5}$. Mater. Res. Bull. 1999, 34, 303-314.

6. Stolen, S.; Bakken, E.; Mohn, C. E., Oxygen-deficient perovskites: linking structure, energetics and ion transport. Phys. Chem. Chem. Phys 2006, 8 (4), 429-447.

7. Ramezanipour, F.; Greedan, J. E.; Grosvenor, A. P.; Britten, J. F.; Cranswick, L. M. D.; Garlea, V. O., Intralayer Cation Ordering in a Brownmillerite Superstructure: Synthesis, Crystal, and Magnetic Structures of $\mathrm{Ca}_{2} \mathrm{FeCoO}_{5}$. Chem. Mater. 2010, 22 (21), 6008-6020.

8. Tilley, R. J. D., defects in solids. John Wiley \& Sons, Inc. 2008.

9. Asenath-Smith, E.; Misture, S. T.; Edwards, D. D., Structural behavior and thermoelectric properties of the brownmillerite system $\mathrm{Ca}_{2}\left(\mathrm{ZnxFe}_{2-\mathrm{x}}\right) \mathrm{O}_{5}$. J. Solid State Chem. 2011, 184, $2167-$ 2177.

10. Auckett, J. E.; Studer, A. J.; Sharma, N.; Ling, C. D., Floating-zone growth of brownmillerite $\mathrm{Sr}_{2} \mathrm{Fe}_{2} \mathrm{O}_{5}$ and the observation of a chain-ordered superstructure by single-crystal neutron diffraction. Solid State lonics 2012, 225, 432-436.

11. Ramezanipour, F.; Greedan, J. E.; Siewenie, J.; Proffen, T.; Ryan, D. H.; Grosvenor, A. P.; Donaberger, R. L., Local and Average Structures and Magnetic Properties of $\mathrm{Sr}_{2} \mathrm{FeMnO}_{5+y}, \mathrm{y}=0.0$, 0.5. Comparisons with $\mathrm{Ca}_{2} \mathrm{FeMnO}_{5}$ and the Effect of the A-Site Cation. Inorg. Chem. 2011, 50 (16), 7779-7791.

12. Ramezanipour, F.; Greedan, J. E.; Cranswick, L. M. D.; Garlea, V. O.; Siewenie, J.; King, G.; Llobet, A.; Donaberger, R. L., The effect of the B-site cation and oxygen stoichiometry on the local and average crystal and magnetic structures of $\mathrm{Sr}_{2} \mathrm{Fe}_{1.9} \mathrm{M}_{0.1} \mathrm{O}_{5+\mathrm{y}}(\mathrm{M}=\mathrm{Mn}, \mathrm{Cr}, \mathrm{Co} ; \mathrm{y}=0,0.5)$. J. Mater. Chem. 2012, 22, 9522-9538.

13. Shaula, A. L.; Kolotygin, V. A.; Naumovich, E. N.; Pivak, Y. V.; Kharton, V. V., Oxygen Ionic Transport in Brownmillerite-Type and Calcium Ferrite-Based Composite Membranes. Solid State Phenom. 2013, 200, 286-292.

14. Greaves, C.; Jacobson, A. J.; Tofield, B. C.; Fender, B. E. F., A powder neutron diffraction investigation of the nuclear and magnetic structure of $\mathrm{Sr}_{2} \mathrm{Fe}_{2} \mathrm{O}_{5}$. Acta Crystallogr., Sect. B 1975, 31, 641-646.

15. Dhankhar, S.; Bhalerao, G.; Ganesamoorthy, S.; Baskar, K.; Singh, S., Growth and comparison of single crystals and polycrystalline brownmillerite $\mathrm{Ca}_{2} \mathrm{Fe}_{2} \mathrm{O}_{5}$. J. Cryst. Growth 2017, $468,311$. 
16. Pomjakushin, V. Y.; Balagurov, A. M.; Elzhov, T. V.; Sheptyakov, D. V.; Fischer, P.; Khomskii, D. I.; Yushankhai, V. Y.; Abakumov, A. M.; Rozova, M. G.; Antipov, E. V.; Lobanov, M. V.; Billinge, S. J. L., Atomic and magnetic structures, disorder effects, and unconventional superexchange interactions in $\mathrm{A}_{2} \mathrm{MnGaO}_{5+d}(\mathrm{~A}=\mathrm{Sr}, \mathrm{Ca})$ oxides of layered brownmillerite-type structure. Phys. Rev. B 2002, 66, 184412.

17. Patrakeev, M. V.; Kharton, V. V.; Bakhteeva, Y. A.; Shaula, A. L.; Leonidov, I. A.; Kozhevnikov, V. L.; Naumovich, E. N.; Yaremchenko, A. A.; Marques, F. M. B., Oxygen nonstoichiometry and mixed conductivity of $\mathrm{SrFe}_{1-x} \mathrm{M}_{\mathrm{x}} \mathrm{O}_{3-\delta}(\mathrm{M}=\mathrm{Al}, \mathrm{Ga})$ : Effects of B-site doping. Solid State Sci. 2006, 8 (5), 476-487.

18. Hona, R. K.; Ramezanipour, F., Enhanced electrical properties in $\mathrm{BaSrFe}_{2} \mathrm{O}_{6-\delta}(\delta=0.5)$ : A disordered defect-perovskite. Polyhedron 2019, 167, 69-74.

19. Hona, R. K.; Huq, A.; Ramezanipour, F., Charge transport properties of $\mathrm{Ca}_{2} \mathrm{FeGaO}_{6-\delta}$ and $\mathrm{CaSrFeGaO}_{6-\delta}$ : The effect of defect-order. Mater. Chem. Phys. 2019, 238, 121924.

20. Hona, R. K.; Huq, A.; Mulmi, S.; Ramezanipour, F., Transformation of Structure, Electrical Conductivity, and Magnetism in $\mathrm{AA}^{\prime} \mathrm{Fe}_{2} \mathrm{O}_{6-\delta}, \mathrm{A}=\mathrm{Sr}, \mathrm{Ca}$ and $\mathrm{A}^{\prime}=$ Sr. Inorganic Chemistry 2017, 56 (16), 9716-9724.

21. Mineshige, A.; Kobune, M.; Fujii, S.; Ogumi, Z.; Inaba, M.; Yao, T.; Kikuchi, K., MetalInsulator Transition and Crystal Structure of $\mathrm{La}_{1-x} \mathrm{Sr}_{x} \mathrm{CoO}_{3}$ as Functions of Sr-Content, Temperature, and Oxygen Partial Pressure. J. Solid State Chem. 1999, 142, 374-381.

22. Mineshige, A.; Inaba, M.; Yao, T.; Ogumi, Z.; Kikuchi, K.; Kawase, M., Crystal Structure and Metal-Insulator Transition of $\mathrm{La}_{1-x} \mathrm{Sr}_{x} \mathrm{CoO}_{3}$. J. Solid State Chem. 1996, 121, 423-429.

23. Patrakeev, M. V.; Kharton, V. V.; Bakhteeva, Y. A.; Shaula, A. L.; Leonidov, I. A.; Kozhevnikov, V. L.; Naumovich, E. N.; Yaremchenko, A. A.; Marques, F. M. B., Oxygen nonstoichiometry and mixed conductivity of $\mathrm{SrFe}_{1-x} \mathrm{M}_{\mathrm{x}} \mathrm{O}_{3-\delta}(\mathrm{M}=\mathrm{Al}, \mathrm{Ga})$ : Effects of B-site doping. Solid State Sci. 2006, 8, 476-487.

24. Kozhevnikov, V. L.; Leonidov, I. A.; Patrakeev, M. V.; Mitberg, E. B.; Poeppelmeier, K. R., Electrical Properties of the Ferrite $\mathrm{SrFeO}_{y}$ at High Temperatures. J. Solid State Chem. 2001, 158, 320-326.

25. Cheng, Y.; Jiang, S. P., Advances in electrocatalysts for oxygen evolution reaction of water electrolysis-from metal oxides to carbon nanotubes. Progress in Natural Science: Materials International 2015, 25, 545-553.

26. Zhu, Y.; Zhou, W.; Sunarso, J.; Zhong, Y.; Shao, Z., Phosphorus-doped perovskite oxide as highly efficient water oxidation electrocatalyst in alkaline solution. Adv. funct. mater. 2016, 26, 5862-5872.

27. Rong, X.; Parolin, J.; Kolpak, A. M., A Fundamental Relationship between Reaction Mechanism and Stability in Metal Oxide Catalysts for Oxygen Evolution. ACS Catal. 2016, 6, 11531158.

28. Sammells, A. F.; Schwartz, M.; Mackay, R. A.; Barton, T. F.; Peterson, D. R., Catalytic membrane reactors for spontaneous synthesis gas production. Catal. Today 2000, 56 (1-3), 325328.

29. Li, Q.; Sun, L.; Zeng, X.; Zhao, H.; Huo, L.; Grenier, J.-C.; Bassat, J.-M.; Mauvy, F., Evaluation of a brownmillerite oxide as cathode for solid oxide fuel cells. J. Power Sources 2013, 238, 11-16.

30. Kobayashi, K. I.; Kimura, T.; Sawada, H.; Terakura, K.; Tokura, Y., Room-temperature magnetoresistance in an oxide material with an ordered double-perovskite structure. Nature 1998, 395 (6703), 677-680.

31. Shunji, T.; Hiroshi, N.; Yoshio, I.; Nobuo, I., The Oxygen-Deficient Perovskite Solid Solution Nd 1+ x Ba 2- x Cu $3 \mathrm{O}$ y and Its Superconductivity. Jpn. J. Appl. Phys. 1987, 26 (12A), L2076. 
32. Ramezanipour, F.; Greedan, J. E.; Siewenie, J.; Proffen, T.; Ryan, D. H.; Grosvenor, A. P.; Donaberger, R. L., Local and Average Structures and Magnetic Properties of $\mathrm{Sr}_{2} \mathrm{FeMnO}_{5+y}, \mathrm{y}=0.0$, 0.5. Comparisons with $\mathrm{Ca}_{2} \mathrm{FeMnO}_{5}$ and the Effect of the A-Site Cation. Inorg Chem. 2011, 50 (16), 7779-7791.

33. Ramezanipour, F.; Cowie, B.; Derakhshan, S.; Greedan, J. E.; M.D., L.; c, C., Crystal and magnetic structures of the brownmillerite compound $\mathrm{Ca}_{2} \mathrm{Fe}_{1.039(8)} \mathrm{Mn}_{0.962(8)} \mathrm{O}_{5}$. J. Solid State Chem. 2009, 182 (153-159), 153-159.

34. Ramezanipour, F.; Greedan, J. E.; Cranswick, L. M. D.; Garlea, V. O.; Donaberger, R. L.; Siewenie, J., Systematic Study of Compositional and Synthetic Control of Vacancy and Magnetic Ordering in Oxygen-Deficient Perovskites $\mathrm{Ca}_{2} \mathrm{Fe}_{2-x} \mathrm{Mn}_{x} \mathrm{O}_{5+y}$ and $\mathrm{CaSrFe}_{2-x} \mathrm{Mn}_{x} \mathrm{O}_{5+y}(\mathrm{x}=1 / 2,2 / 3$, and 1; $y=0-1 / 2$ ). J. Am. Chem. Soc 2012, 134 (6), 3215-3227.

35. Kim, J.; Yin, X.; Tsao, K.-C.; Fang, S.; Yang, H., $\mathrm{Ca}_{2} \mathrm{Mn}_{2} \mathrm{O}_{5}$ as Oxygen-Deficient Perovskite Electrocatalyst for Oxygen Evolution Reaction. J. Am. Chem. Soc 2014, 136 (42), 14646-14649.

36. Hodges, J. P.; Short, S.; Jorgensen, J. D.; Xiong, X.; Dabrowski, B.; Mini, S. M.; Kimball, C. W., Evolution of Oxygen-Vacancy Ordered Crystal Structures in the Perovskite Series $\mathrm{Sr}_{n} \mathrm{Fe}_{n} \mathrm{O}_{3 n-1}$ $(\mathrm{n}=2,4,8$, and $\infty)$, and the Relationship to Electronic and Magnetic Properties. J. Solid State Chem. 2000, 151 (2), 190-209.

37. Wu, J. W.; Wang, J.; Liu, G.; Wu, Y. J.; Liu, X. Q.; Chen, X. M., Giant room-temperature magnetodielectric coupling in spark plasma sintered brownmillerite ceramics. Appl. Phys. Lett. 2014, 105 (22), 222906.

38. Pomjakushin, V. Y.; Balagurov, A. M.; Elzhov, T. V.; Sheptyakov, D. V.; Fischer, P.; Khomskii, D. I.; Yushankhai, V. Y.; Abakumov, A. M.; Rozova, M. G.; Antipov, E. V.; Lobanov, M. V.; Billinge, S. J. L., Atomic and magnetic structures, disorder effects, and unconventional superexchange interactions in $\mathrm{A}_{2} \mathrm{MnGaO}_{5+d}(\mathrm{~A}=\mathrm{Sr}, \mathrm{Ca})$ oxides of layered brownmillerite-type structure. Phys. Rev. B 2002, 66 (18), 184412.

39. Nemudry, A.; Rogatchev, A.; Gainutdinov, I.; Schöllhorn, R., Reactivity of the perovskite system $\mathrm{Ca}_{1-\mathrm{x}} \mathrm{Sr}_{\mathrm{x}} \mathrm{FeO}_{2.5}$ in topotactic electrochemical oxidation at ambient temperature. $J$ Solid State Electrochem. 2001, 5 (7), 450-458.

40. Larson, A. C.; Von Dreele, R. B., General Structure Analysis System (GSAS), Los Alamos National Laboratory Report LAUR. 1994, 86-748.

41. Toby, B. H., J. Appl. Crystallogr. 2001, 34 210-213.

42. Ramezanipour, F.; Greedan, J. E.; Grosvenor, A. P.; Britten, J. F.; Cranswick, L. M. D.; Garlea, V. O., Intralayer Cation Ordering in a Brownmillerite Superstructure: Synthesis, Crystal, and Magnetic Structures of $\mathrm{Ca}_{2} \mathrm{FeCoO}_{5}$. Chemistry of Materials 2010, 22 (21), 6008-6020.

43. Jijil, C. P.; Lokanathan, M.; Chithiravel, S.; Nayak, C.; Bhattacharyya, D.; Jha, S. N.; Babu, P. D.; Kakade, B.; Devi, R. N., Nitrogen doping in oxygen-deficient $\mathrm{Ca}_{2} \mathrm{Fe}_{2} \mathrm{O}_{5}$ : A strategy for efficient oxygen reduction oxide catalysts. ACS Appl. Mater. Interfaces 2016, 8, 34387-34395.

44. Ghaffari, M.; Liu, T.; Huang, H.; Tan, O. K.; Shannon, M., Investigation of local structure effect and X-ray absorption characteristics (EXAFS) of Fe (Ti) K-edge on photocatalyst properties of $\mathrm{SrTi}_{(1-\mathrm{x})} \mathrm{Fe}_{\mathrm{x}} \mathrm{O}_{(3-\delta)}$. Mater. Chem. Phys. 2012, 136, 347-357.

45. Berastegui, P.; Eriksson, S. G.; Hull, S., A neutron diffraction study of the temperature dependence of Ca2Fe2O5. Materials Research Bulletin 1999, 34 (2), 303-314.

46. Shannon, R. D., Revised Effective Ionic Radii and Systematic Studies of Interatomie Distances

in Halides and Chaleogenides. Acta Cryst. 1976, A32, 751.

47. Reehuis, M.; Ulrich, C.; Maljuk, A.; Niedermayer, C.; Ouladdiaf, B.; Hoser, A.; Hofmann, T.; Keimer, B., Neutron diffraction study of spin and charge ordering in $\mathrm{SrFeO}_{3-\delta}$. Phys. Rev. B 2012, 85 (18), 184109. 
48. Cheng, X.; Fabbri, E.; Nachtegaal, M.; Castelli, I. E.; El Kazzi, M.; Haumont, R.; Marzari, N.; Schmidt, T. J., Oxygen Evolution Reaction on $\mathrm{La}_{1-x} \mathrm{Sr}_{x} \mathrm{CoO}_{3}$ Perovskites: A Combined Experimental and Theoretical Study of Their Structural, Electronic, and Electrochemical Properties. Chem. Mater. 2015, 27 (22), 7662-7672.

49. Pizzini, S., Physical chemistry of semiconductor materials and processes John Wiley \& Sons Ltd, West Sussex, United Kingdom 2015.

50. Asenath-Smith, E.; Lokuhewa, I. N.; Misture, S. T.; Edwards, D. D., p-Type thermoelectric properties of the oxygen-deficient perovskite $\mathrm{Ca}_{2} \mathrm{Fe}_{2} \mathrm{O}_{5}$ in the brownmillerite structure. J. Solid State Chem. 2010, 183, 1670-1677.

51. Andoulsi, R.; Horchani-Naifer, K.; Férid, M., Electrical conductivity of $\mathrm{La}_{1-x} \mathrm{Ca}_{x} \mathrm{FeO}_{3-\delta}$ solid solutions. Ceram. Int. 2013, 39, 6527-6531.

52. Patrakeev, M. V.; Leonidov, I. A.; Kozhevnikov, V. L.; Kharton, V. V., Ion-electron transport in strontium ferrites: relationships with structural features and stability. SOLID STATE SCI 2004, 6 (9), 907-913.

53. Yu, H.-C.; Fung, K.-Z., Role of Sr Addition on the Structure Stability and Electrical Conductivity of Sr-Doped Lanthanum Copper Oxide Perovskites. J. Mater. 2004, 19 (3), 943-949.

54. Torrance, J. B.; Lacorre, P.; Nazzal, A. I.; Ansaldo, E. J.; Niedermayer, C., Systematic study of insulator-metal transitions in perovskites $\mathrm{RNiO}_{3}(\mathrm{R}=\mathrm{Pr}, \mathrm{Nd}, \mathrm{Sm}, \mathrm{Eu})$ due to closing of chargetransfer gap. Phy. Rev. B 1992, 45 (14), 8209-8212.

55. Shaula, A. L.; Kharton, V. V.; Patrakeev, M. V.; Waerenborgh, J. C.; Rojas, D. P.; Marques, F. M. B., Defect formation and transport in $\mathrm{SrFe}_{1-\mathrm{x}} \mathrm{Al}_{x} \mathrm{O}_{3-\delta}$. Ionics 2004, 10 (5), 378-384.

56. $\quad$ Tilley, R. J. D., Defects in Solids. John Wiley \& Sons, Hoboken, NJ (2008.

57. Asenath-Smith, E.; Misture, S. T.; Edwards, D. D., Structural behavior and thermoelectric properties of the brownmillerite system $\mathrm{Ca}_{2}\left(\mathrm{Zn}_{x} \mathrm{Fe}_{2-\mathrm{x}}\right) \mathrm{O}_{5}$. J. Solid State Chem. 2011, 184 (8), 21672177.

58. Ren, P.; Maso, N.; Liu, Y.; Ma, L.; Fan, H.; West, A. R., Mixed oxide ion and proton conduction and $\mathrm{p}$-type semiconduction in $\mathrm{BaTi}_{0.98} \mathrm{Ca}_{0.02} \mathrm{O}_{2.98}$ ceramics. J.Mater. Chem. C 2013, 1, 2426-2432.

59. Ren, P.; Maso, N.; West, A. R., Hole conductivity in oxygen-excess $\mathrm{BaTi}_{1-\mathrm{x}} \mathrm{Ca}_{\mathrm{x}} \mathrm{O}_{3-\mathrm{x}+\delta}$. Phy.Chem. Chem. Phys. 2013, 15, 20943-20950.

60. Patrakeev, M. V.; Kharton, V. V.; Bakhteeva, Y. A.; Shaula, A. L.; Leonidov, I. A.; Kozhevnikov, V. L.; Naumovich, E. N.; Yaremchenko, A. A.; Marques, F. M. B., Oxygen nonstoichiometry and mixed conductivity of $\mathrm{SrFe}_{1-x} \mathrm{M}_{\mathrm{x}} \mathrm{O}_{3-\delta}(\mathrm{M}=\mathrm{Al}, \mathrm{Ga})$ : Effects of B-site doping. SOLID STATE SCI 2006, 8 (5), 476-487.

61. Patrakeev, M. V.; Leonidov, I. A.; Kozhevnikov, V. L.; Poeppelmeier, K. R., p-Type electron transport in $\mathrm{La}_{1-x} \mathrm{Sr}_{x} \mathrm{FeO}_{3-\delta}$ at high temperatures. J. Solid State Chem. 2005, 178 (3), 921-927.

62. A. A. Fargali, M. K. Z., M. H. Khedr and A. F. Moustafa, Phase and conductivity dynamics of strontium hexaferrite nanocrystals in a hydrogen gas flow. Int. J. Phys. Sci. 2008, 3 (5), 131.

63. Armstrong, T.; Prado, F.; Manthiram, A., Synthesis, crystal chemistry, and oxygen permeation properties of $\mathrm{LaSr}_{3} \mathrm{Fe}_{3-\mathrm{x}} \mathrm{Co}_{x} \mathrm{O}_{10}(0 \leq x \leq 1.5)$. Solid State lon. 2001, 140 (1-2), 89-96.

64. Chen, Z.; Ran, R.; Zhou, W.; Shao, Z.; Liu, S., Assessment of $\mathrm{Ba}_{0.5} \mathrm{Sr}_{0.5} \mathrm{Co}_{1-y} \mathrm{Fe}_{y} \mathrm{O}_{3-\delta}$ (y = 0.01.0) for prospective application as cathode for IT-SOFCs or oxygen permeating membrane. Electrochim. Acta 2007, 52 (25), 7343-7351.

65. Picard, C.; Gerdanian, P., Determination of the néel temperature in $\mathrm{YBa}_{2} \mathrm{Cu}_{3} \mathrm{O}_{2}$ by electrical conductivity measurements. Solid State Commun. 1993, 87 (9), 775-778.

66. Richardson, G.; O'Kane, S. E. J.; Niemann, R. G.; Peltola, T. A.; Foster, J. M.; Cameron, P. J.; Walker, A. B., Can slow-moving ions explain hysteresis in the current-voltage curves of perovskite solar cells? Energy Environ Sci. 2016, 9 (4), 1476-1485. 
67. Guite, C.; Kerk, I. S.; Sekhar, M. C.; Ramu, M.; Goolaup, S.; Lew, W. S., All-electrical deterministic single domain wall generation for on-chip applications. Sci. Rep. 2014, 4, 7459.

68. Tomasello, R.; Martinez, E.; Zivieri, R.; Torres, L.; Carpentieri, M.; Finocchio, G., A strategy for the design of skyrmion racetrack memories. Sci. Rep. 2014, 4, 6784.

69. Elilarassi, R.; Chandrasekaran, G., Optical, electrical and ferromagnetic studies of ZnO:Fe diluted magnetic semiconductor nanoparticles for spintronic applications. Spectrochim. Acta Mol. Biomol. Spectrosc. 2017, 186, 120-131.

70. Ramezanipour, F.; Greedan, J. E.; Siewenie, J.; Donaberger, R. L.; Turner, S.; Botton, G. A., $A$ vacancy-disordered, oxygen-deficient perovskite with long-range magnetic ordering: Local and average structures and magnetic properties of $\mathrm{Sr}_{2} \mathrm{Fe}_{1.5} \mathrm{Cr}_{0.5} \mathrm{O}_{5}$. Inorg. Chem. 2012, 51, 2638-2644. 71. Zhang, G. B.; Smyth, D. M., Defects and transport of the brownmillerite oxides with high oxygen ion conductivity - $\mathrm{Ba}_{2} \mathrm{In}_{2} \mathrm{O}_{5}$. Solid State lonics 1995, 82 (3), 161-172.

72. Ramezanipour, F.; Greedan, J. E.; Cranswick, L. M. D.; Garlea, V. O.; Donaberger, R. L.; Siewenie, J., Systematic study of compositional and synthetic control of vacancy and magnetic ordering in oxygen-deficient perovskites $\mathrm{Ca}_{2} \mathrm{Fe}_{2-\mathrm{x}} \mathrm{Mn}_{\mathrm{x}} \mathrm{O}_{5+\mathrm{y}}$ and $\mathrm{CaSrFe}_{2-\mathrm{x}} \mathrm{Mn}_{\mathrm{x}} \mathrm{O}_{5+\mathrm{y}}(\mathrm{x}=1 / 2,2 / 3$, and $1 ; y=0-1 / 2)$. J. Am. Chem. Soc. 2012, 134, 3215-3227.

73. Turner, S.; Verbeeck, J.; Ramezanipour, F.; Greedan, J. E.; Van Tendeloo, G.; Botton, G. A., Atomic resolution coordination mapping in $\mathrm{Ca}_{2} \mathrm{FeCoO}_{5}$ brownmillerite by spatially resolved electron energy-loss spectroscopy. Chem. Mater. 2012, 24, 1904-1909.

74. Ramezanipour, F.; Greedan, J. E.; Grosvenor, A. P.; Britten, J. F.; Cranswick, L. M. D.; Garlea, V. O., Intralayer cation ordering in a brownmillerite superstructure: synthesis, crystal, and magnetic structures of $\mathrm{Ca}_{2} \mathrm{FeCoO}_{5}$. Chem. Mater. 2010, 22, 6008-6020.

75. Ramezanipour, F.; Cowie, B.; Derakhshan, S.; Greedan, J. E.; Cranswick, L. M. D., Crystal and magnetic structures of the brownmillerite compound $\mathrm{Ca}_{2} \mathrm{Fe}_{1.039(8)} \mathrm{Mn}_{0.962(8)} \mathrm{O}_{5}$. J. Solid State Chem. 2009, 182, 153-159.

76. Ramezanipour, F.; Greedan, J. E.; Siewenie, J.; Donaberger, R. L.; Turner, S.; Botton, G. A., A Vacancy-Disordered, Oxygen-Deficient Perovskite with Long-Range Magnetic Ordering: Local and Average Structures and Magnetic Properties of $\mathrm{Sr}_{2} \mathrm{Fe}_{1.5} \mathrm{Cr}_{0.5} \mathrm{O}_{5}$. Inorg. Chem. 2012, 51 (4), 2638-2644.

77. Homonnay, Z.; Nomura, K.; Juhász, G.; Kuzmann, E.; Hamakawa, S.; Hayakawa, T.; Vértes, A., Microstructure and $\mathrm{CO}_{2}$-absorption in $\mathrm{Sr}_{0.95} \mathrm{Ca}_{0.05} \mathrm{CO}_{0.5} \mathrm{Fe}_{0.5} \mathrm{O}_{3-8} ; \# 948$; and $\mathrm{Sr}_{0.5} \mathrm{Ca}_{0.5} \mathrm{CO}_{0.5} \mathrm{Fe}_{0.5} \mathrm{O}_{3}-$ ;;\#948; as studied by Emission Mössbauer Spectroscopy. J. Radioanal. Nucl. Chem. 2003, 255 (3), 425-429.

78. Aksenova, T. V. G., L. Ya; Cherepanov, V. A., Phase equilibria and crystal structure of the complex oxides in the Sr-Fe-Co-O system. J. Solid State Chem. 2008, 181 (6), 1480-1484.

79. Gómez, L.; Galeano, V.; Parra, R.; Michel, C. R.; Paucar, C.; Morán, O., Carbon dioxide gas sensing properties of ordered oxygen deficient perovskite $\mathrm{LnBaCO}_{2} \mathrm{O}_{5+\delta}(\mathrm{Ln}=\mathrm{La}, \mathrm{Eu})$. Sensors and Actuators B: Chem. 2015, 221, 1455-1460.

80. Liu, P.; Luo, Z.; Kong, J.; Yang, X.; Liu, Q.; Xu, H., $\mathrm{Ba}_{0.5} \mathrm{Sr}_{0.5} \mathrm{CO}_{0.8} \mathrm{Fe}_{0.2} \mathrm{O}_{3-\delta}$-based dual-gradient cathodes for solid oxide fuel cells. Ceram. Int. 2018, 44 (4), 4516-4519.

81. Chen, G.; Zhou, W.; Guan, D.; Sunarso, J.; Zhu, Y.; Hu, X.; Zhang, W.; Shao, Z., Two orders of magnitude enhancement in oxygen evolution reactivity on amorphous $\mathrm{Ba}_{(0.5)} \mathrm{Sr}_{(0.5)} \mathrm{Co}_{(0.8)} \mathrm{Fe}_{(0.2)} \mathrm{O}_{(3-\delta)}$ nanofilms with tunable oxidation state. Sci. Adv. 2017, 3, e1603206.

82. Suescun, L.; Chmaissem, O.; Mais, J.; Dabrowski, B.; Jorgensen, J. D., Crystal structures, charge and oxygen-vacancy ordering in oxygen deficient perovskites $\mathrm{SrMnO}_{\mathrm{x}}(\mathrm{x}<2.7)$. J.Solid State Chem. 2007, 180, 1698-1707.

83. Hodges, J. P.; Jorgensen, J. D.; Xiong, X.; Dabrowski, B.; Mini, S. M.; Kimball, C. W.; Materials Science, D.; Northern Illinois, U., Evolution of oxygen-vacancy ordered crystal structures 
in the perovskite series $\mathrm{Sr}_{n} \mathrm{Fe}_{n} \mathrm{O}_{3 n-1}(\mathrm{n}=2,4,8$, and \{infinity\}), and the relationship to electronic and magnetic properties. J. Solid State Chem. 2000, 151, 209.

84. Hona, R. K.; Huq, A.; Ramezanipour, F., Unraveling the role of structural order in the transformation of electrical conductivity in $\mathrm{Ca}_{2} \mathrm{FeCoO}_{6-\delta}, \mathrm{CaSrFeCoO}_{6-\delta}$, and $\mathrm{Sr}_{2} \mathrm{FeCoO}_{6-\delta}$. Inorg. Chem. 2017, 56, 14494-14505.

85. Mulmi, S.; Hona, R. K.; Jasinski, J. B.; Ramezanipour, F., Electrical conductivity of $\mathrm{Sr}_{2}$ ${ }_{x} \mathrm{Ca}_{\mathrm{x}} \mathrm{FeMnO}_{5}(\mathrm{x}=0$, 1, 2). J. Solid State Electrochem. 2018, 22, 2329-2338.

86. Hona, R. K.; Ramezanipour, F., Variation in electrical conductivity of $\mathrm{A}_{2} \mathrm{Fe}_{2} \mathrm{O}_{5}(A=\mathrm{Sr}, \mathrm{Ba})$ : the role of structural order. Mater. Res. Express 2018, 5 (7), 076307.

87. Ramezanipour, F.; Greedan, J. E.; Cranswick, L. M. D.; Garlea, V. O.; Donaberger, R. L.; Siewenie, J., Systematic Study of Compositional and Synthetic Control of Vacancy and Magnetic Ordering in Oxygen-Deficient Perovskites $\mathrm{Ca}_{2} \mathrm{Fe}_{2-x} \mathrm{Mn}_{x} \mathrm{O}_{5+y}$ and $\mathrm{CaSrFe}_{2-x} \mathrm{Mn}_{x} \mathrm{O}_{5+y}(x=1 / 2,2 / 3$, and $1 ; \mathrm{y}=0-1 / 2$ ). J. Am. Chem. Soc. 2012, 134 (6), 3215-3227.

88. Hona, R. K.; Ramezanipour, F., Disparity in electrical and magnetic properties of isostructural oxygen-deficient perovskites $\mathrm{BaSrCO}_{2} \mathrm{O}_{6-\delta}$ and $\mathrm{BaSrCoFeO}_{6-\delta}$. J. Mater. Sci. Mater. Electron. 2018.

89. Ramezanipour, F.; Greedan, J. E.; Siewenie, J.; Proffen, T.; Ryan, D. H.; Grosvenor, A. P.; Donaberger, R. L., Local and average structures and magnetic properties of $\mathrm{Sr}_{2} \mathrm{FeMnO}_{5+y}, \mathrm{Y}=0.0$, 0.5. Comparisons with $\mathrm{Ca}_{2} \mathrm{FeMnO}_{5}$ and the effect of the A-site cation. Inorg.Chem. 2011, 50, 77797791.

90. Hona, R. K.; Huq, A.; Ramezanipour, F., Magnetic structure of $\mathrm{CaSrFeCoO}_{6-\delta}$ : Correlations with structural order. Mater. Res. Bull. 2018, 106, 131-136.

91. Fossdal, A.; Menon, M.; Wærnhus, I.; Wiik, K.; Einarsrud, M. A.; Grande, T., Crystal Structure and Thermal Expansion of $\mathrm{La}_{1-\mathrm{x}} \mathrm{Sr}_{x} \mathrm{FeO}_{3-\delta}$ Materials. J. Am. Ceram.Soc. 2005, 87 (10), 1952-1958.

92. Anikina, P. V.; Markov, A. A.; Patrakeev, M. V.; Leonidov, I. A.; Kozhevnikov, V. L., The structure, nonstoichiometry, and thermodynamic characteristics of oxygen in strontium ferrite doped with niobium, $\mathrm{SrFe}_{1-} \mathrm{Nb}_{\times} \mathrm{O}_{3}-\delta$. Russian J. Phys. Chem. A 2009, 83, 699-704.

93. Colville, A. A.; Geller, S., The Crystal Structure of Brownmillerite, $\mathrm{Ca}_{2} \mathrm{FeAlO}_{5}$. Acta Cryst. 1971, (B27), 2311.

94. Hans D'Hondt; JokeHadermann; ArtemM.Abakumov; AnnaS.Kalyuzhnaya; Marina G.Rozova; AlexanderA.Tsirlin; RameshNath; HaiyanTan; JoVerbeeck; EvgenyV.Antipov; GustaafVanTendeloo, Synthesis, crystal structure and magnetic properties of the $\mathrm{Sr}_{2} \mathrm{Al}$ $0.78 \mathrm{Mn}_{1.22} \mathrm{O}_{5.2}$ anion-deficient layered perovskite. J.Solid State Chem. 2009, 182, 356-363.

95. Lindberg, F.; Istomin, S. Y.; Berastegui, P.; Svensson, G.; Kazakov, S. M.; Antipov, E. V., Synthesis and structural studies of $\mathrm{Sr}_{2} \mathrm{Co}_{2-x} \mathrm{Ga}_{x} \mathrm{O}_{5}, 0.3 \leqslant x \leqslant 0.8$. J. Solid State Chem. 2003, 173 (2), 395-406.

96. Didier, C.; Claridge, J.; Rosseinsky, M., Crystal structure of brownmillerite $\mathrm{Ba}_{2} \operatorname{lnGaO_{5}}$. J. Solid State Chem. 2014, 218, 38-43.

97. Mohn, C. E.; Allan, N. L.; Stølen, S., $\mathrm{Sr}$ and Ga substituted $\mathrm{Ba}_{2} \mathrm{In}_{2} \mathrm{O}_{5}$ : Linking ionic conductivity and the potential energy surface. Solid State lonics 2006, 177 (3), 223-228.

98. Kahlenberg, V.; Shaw, C. S. J., $\mathrm{Ca}_{2} \mathrm{Ga}_{2} \mathrm{O}_{5}$ : a new high pressure oxogallate. In Z. Kristallogr. - New Cryst. Struct., 2001; Vol. 216, p 206.

99. Kahlenberg, V.; Goettgens, V.; Mair, P.; Schmidmair, D., High-pressure synthesis and crystal structures of the strontium oxogallates $\mathrm{Sr}_{2} \mathrm{Ga}_{2} \mathrm{O}_{5}$ and $\mathrm{Sr}_{5} \mathrm{Ga}_{6} \mathrm{O}_{14}$. J. Solid State Chem. 2015, 228, 27-35.

100. Larson, A. C.; Von Dreele, A. C., General structure analysis system (GSAS). Los Alamos National Laboratory Report LAUR 1994, 86 - 748. 
101. Toby, B. H., A graphical user interface for GSAS. J. Appl. Crystallogr. 2001, 34, 210-213. 102. Luo, K.; Amano Patino, M.; Hayward, M. A., $\mathrm{Ca}_{2} \mathrm{Cr}_{0.5} \mathrm{Ga}_{1.5} \mathrm{O}_{5}$-An extremely redox-stable brownmillerite phase. J. Solid State Chem. 2015, 222, 71-75.

103. Julián Morales, L. S., Francisco Martín, Frank Berry, and Xiaolin Renc, Synthesis and characterization of nanometric iron and iron-titanium oxides by mechanical milling: Electrochemical properties as anodic materials in lithium cells. J. Electrochem. Soc. 2005, 152, A1748-A1754.

104. Doi, A.; Nomura, M.; Obukuro, Y.; Maeda, R.; Obata, K.; Matsushima, S.; Kobayashi, K., Characterization of Ti-doped $\mathrm{CaFe}_{2} \mathrm{O}_{4}$ prepared from a malic acid complex. J. Ceram. Soc. Jpn. 2014, 122 (2), 175-178.

105. Ruttanapun, C.; Maensiri, S., Effects of spin entropy and lattice strain from mixed-trivalent $\mathrm{Fe}^{3+} / \mathrm{Cr}^{3+}$ on the electronic, thermoelectric and optical properties of delafossite $\mathrm{CuFe}_{1-x} \mathrm{Cr}_{x} \mathrm{O}_{2}(x=$ 0.25, 0.5, 0.75), J. Phys. D: Appl. Phys. 2015, 48, 495103. 2015.

106. Mueller, D. N.; De Souza, R. A.; Yoo, H.-I.; Martin, M., Phase stability and oxygen nonstoichiometry of highly oxygen-deficient perovskite-type oxides: A case study of

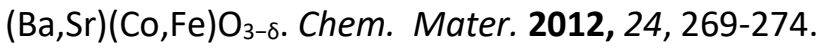

107. Shaula, A.; Pivak, Y.; Waerenborgh, J.; Gaczynski, P.; Yaremchenko, A.; Kharton, V., Ionic conductivity of brownmillerite-type calcium ferrite under oxidizing conditions. Solid State lonics 2006, 177 (33-34), 2923-2930.

108. Zhang, Q.; Xu, Z. F.; Wang, L. F.; Gao, S. H.; Yuan, S. J., Structural and electromagnetic properties driven by oxygen vacancy in $\mathrm{Sr}_{2} \mathrm{FeMoO}_{6-\delta}$ double perovskite. J. Alloys Compd. 2015, 649, 1151-1155.

109. Kozhevnikov, V. L.; Leonidov, I. A.; Mitberg, E. B.; Patrakeev, M. V.; Petrov, A. N.; Poeppelmeier, K. R., Conductivity and carrier traps in $\mathrm{La}_{1-\mathrm{x}} \mathrm{SrxCo}_{1-\mathrm{z}} \mathrm{Mn}_{z} \mathrm{O}_{3-\delta}(\mathrm{x}=0.3 ; z=0$ and 0.25$)$. J. Solid State Chem. 2003, 172, 296-304.

110. Kontoulis, I.; Steele, B. C. H., Fabrication and conductivity of a new compound $\mathrm{Ca}_{2} \mathrm{Cr}_{2} \mathrm{O}_{5}$. J. Eur. Ceram. Soc. 1992, 9, 459-462.

111. Bhosale, D. R.; Yusuf, S. M.; Kumar, A.; Mukadam, M. D.; Patil, S. I., High oxide ion conductivity below $500{ }^{\circ} \mathrm{C}$ in garnets $\mathrm{La}_{x} \mathrm{Y}_{3-x} \mathrm{Fe}_{5} \mathrm{O}_{12+\delta}$. Phys. Rev. Mater. 2017, 1 (1), 015001.

112. Corallini, S.; Ceretti, M.; Cousson, A.; Ritter, C.; Longhin, M.; Papet, P.; Paulus, W., Cubic $\mathrm{Sr}_{2} \mathrm{ScGaO}_{5}$ perovskite: structural stability, oxygen defect structure, and ion conductivity explored on single crystals. Inorg. Chem. 2017, 56, 2977-2984.

113. Fargali, A. A.; Zayed, M. K.; Khedr, M. H.; Moustafa, A. F., Phase and conductivity dynamics of strontium hexaferrite nanocrystals in a hydrogen gas flow. Intl. J. Phys. Sci. 2008, 3, 131-139.

114. Richardson, G.; O'Kane, S. E. J.; Niemann, R. G.; Peltola, T. A.; Foster, J. M.; Cameron, P. J.; Walker, A. B., Can slow-moving ions explain hysteresis in the current-voltage curves of perovskite solar cells? Energy \& Environ. Sci. 2016, 9 (4), 1476-1485.

115. Fabbri, E.; Mohamed, R.; Levecque, P.; Conrad, O.; Kötz, R.; Schmidt, T. J., Composite electrode boosts the activity of $\mathrm{Ba}_{0.5} \mathrm{Sr}_{0.5} \mathrm{CO}_{0.8} \mathrm{Fe}_{0.2} \mathrm{O}_{3-\delta}$ perovskite and carbon toward oxygen reduction in alkaline media. ACS Catal. 2014, 4, 1061-1070.

116. Zeng, P.; Chen, Z.; Zhou, W.; Gu, H.; Shao, Z.; Liu, S., Re-evaluation of $\mathrm{Ba}_{0.5} \mathrm{Sr}_{0.5} \mathrm{Co}_{0.8} \mathrm{Fe}_{0.2} \mathrm{O}_{3-\delta}$ perovskite as oxygen semi-permeable membrane. J. Membr. Sci. 2007, 291, 148-156.

117. Liu, P.; Luo, Z.; Kong, J.; Yang, X.; Liu, Q.; Xu, H., $\mathrm{Ba}_{0.5} \mathrm{Sr}_{0.5} \mathrm{Co}_{0.8} \mathrm{Fe}_{0.2} \mathrm{O}_{3-8}$-based dual-gradient cathodes for solid oxide fuel cells. Ceram. Int. 2018, 44, 4516-4519.

118. Kong, L.; Chen, X.; Li, B. Q.; Peng, H. J.; Huang, J. Q.; Xie, J.; Zhang, Q., A bifunctional perovskite promoter for polysulfide regulation toward stable lithium-sulfur batteries. Adv. Mater. 2018, 30, 1705219. 
119. Kim, J.; Yin, X.; Tsao, K. C.; Fang, S.; Yang, H., $\mathrm{Ca}_{2} \mathrm{Mn}_{2} \mathrm{O}_{5}$ as oxygen-deficient perovskite electrocatalyst for oxygen evolution reaction. J. Am. Chem. Soc. 2014, 136, 14646-14649.

120. Hona, R. K.; Ramezanipour, F., Variation in electrical conductivity of $\mathrm{A}_{2} \mathrm{Fe}_{2} \mathrm{O}_{5}(A=\mathrm{Sr}, \mathrm{Ba})$ : the role of structural order. Mater. Res. Express 2018, 5, 076307.

121. Suescun, L.; Chmaissem, O.; Mais, J.; Dabrowski, B.; Jorgensen, J. D., Crystal structures, charge and oxygen-vacancy ordering in oxygen deficient perovskites $\mathrm{SrMnO}_{\mathrm{x}}(\mathrm{x}<2.7)$. J. Solid State Chem. 2007, 180 (5), 1698-1707.

122. Arevalo-Lopez, A. M.; Attfield, J. P., Crystal and magnetic structures of the brownmillerite $\mathrm{Ca}_{2} \mathrm{Cr}_{2} \mathrm{O}_{5}$. Dalton Trans. 2015, 44, 10661-4.

123. Pan, L.; Wei-Hua, W.; Wei-Chao, W.; Ya-Hui, C.; Feng, L.; Hui, L., D-type anti-ferromagnetic ground state in $\mathrm{Ca}_{2} \mathrm{Mn}_{2} \mathrm{O}_{5}$. Chin. Phys. Lett. 2017, 34, 027101.

124. Molinari, M.; Tompsett, D. A.; Parker, S. C.; Azough, F.; Freer, R., Structural, electronic and thermoelectric behaviour of $\mathrm{CaMnO}_{3}$ and $\mathrm{CaMnO}_{(3-\delta)}$. J. Mater. Chem. A 2014, 2, 14109-14117.

125. Toby, B. H., EXPGUI, a graphical user interface for GSAS. J. Appl. Crystallogr. 2001, 34 , 210-213.

126. Mohd Zaid, N. A.; Idris, N. H., Enhanced Capacitance of Hybrid Layered Graphene/Nickel Nanocomposite for Supercapacitors. Sci. Rep. 2016, 6, 32082.

127. PGR, A.; B, M.; S, S. D.; KS, P., Study of Electrical Properties of Nickel Doped Polyurethane Nanocomposites. Polym. Sci. 2018, 4, No.1:7.

128. Shannon, R. D., Revised effective ionic radii and systematic studies of interatomic distances in halides and chalcogenides. Acta Cryst. 1976, 32, 751-767.

129. Jijil, C. P.; Lokanathan, M.; Chithiravel, S.; Nayak, C.; Bhattacharyya, D.; Jha, S. N.; Babu, P. D.; Kakade, B.; Devi, R. N., Nitrogen Doping in Oxygen-Deficient $\mathrm{Ca}_{2} \mathrm{Fe}_{2} \mathrm{O}_{5}$ : A Strategy for Efficient Oxygen Reduction Oxide Catalysts. ACS Appl. Mater. Interfaces 2016, 8 (50), 34387-34395.

130. Hona, R. K.; Huq, A.; Ramezanipour, F., Electrical properties of the ordered oxygendeficient perovskite $\mathrm{Ca}_{2} \mathrm{Fe}_{0.5} \mathrm{Ga}_{1.5} \mathrm{O}_{5}$. Ionics 2018.

131. Iguchi, E.; Hashimoto, T.; Yokoyama, S., Electrical transports and stability of small polarons of O $2 p$ holes in $\mathrm{Li}_{x} \mathrm{Co}_{1-x} \mathrm{O}$. J. Phys. Soc. Jpn. 1996, 65, 221-229.

132. Inoue, S.; Kawai, M.; Ichikawa, N.; Kageyama, H.; Paulus, W.; Shimakawa, Y., Anisotropic oxygen diffusion at low temperature in perovskite-structure iron oxides. Nat. Chem. 2010, 2, 213.

133. Thangadurai, V.; Schmid Beurmann, P.; Weppner, W., Mixed oxide ion and electronic conductivity in perovskite-type $\mathrm{SrSnO}_{3}$ by Fe substitution. Mater. Sci. Eng., B 2003, 100, 18-22.

134. Shaula, A. L.; Kharton, V. V.; Vyshatko, N. P.; Tsipis, E. V.; Patrakeev, M. V.; Marques, F. M. B.; Frade, J. R., Oxygen ionic transport in $\mathrm{SrFe}_{1-\mathrm{y}} \mathrm{Al}_{\mathrm{y}} \mathrm{O}_{3-\delta}$ and $\mathrm{Sr}_{1-\mathrm{x}} \mathrm{Ca}_{x} \mathrm{Fe}_{0.5} \mathrm{Al}_{0.5} \mathrm{O}_{3-\delta}$ ceramics. J. Eur. Ceram. Soc. 2005, 25, 489-499.

135. Cheng, X.; Fabbri, E.; Nachtegaal, M.; Castelli, I. E.; El Kazzi, M.; Haumont, R.; Marzari, N.; Schmidt, T. J., Oxygen evolution reaction on $\mathrm{La}_{1-x} \mathrm{Sr}_{x} \mathrm{CoO}_{3}$ perovskites: a combined experimental and theoretical study of their structural, electronic, and electrochemical properties. Chem. Mater. 2015, 27, 7662-7672.

136. Shaula, A. L.; Kharton, V. V.; Patrakeev, M. V.; Waerenborgh, J. C.; Rojas, D. P.; Marques, F. M. B., Defect formation and transport in $\mathrm{SrFe}_{1-\mathrm{x}} \mathrm{Al}_{\mathrm{x}} \mathrm{O}_{3-\delta}$. Ionics 2004, 10, 378-384.

137. Leonidov, I. A.; Patrakeev, M. V.; Bahteeva, J. A.; Mitberg, E. B.; Kozhevnikov, V. L.; Colomban, P.; Poeppelmeier, K. R., High-temperature phase equilibria in the oxide systems $\mathrm{SrFe}_{1-\mathrm{x}} \mathrm{Ga}_{x} \mathrm{O}_{2.5}-\mathrm{SrFe}_{1-\mathrm{x}} \mathrm{Ga}_{\mathrm{x}} \mathrm{O} 3$ (x=0, 0.1, 0.2). J. Solid State Chem. 2006, 179, 1093-1099.

138. Waerenborgh, J. C.; Rojas, D. P.; Shaula, A. L.; Mather, G. C.; Patrakeev, M. V.; Kharton, V. V.; Frade, J. R., Phase formation and iron oxidation states in $\mathrm{SrFe}(\mathrm{Al}) \mathrm{O}_{3-\delta}$ perovskites. Mater. Lett. 2005, 59 (13), 1644-1648. 
139. Mizusaki, J.; Yoshihiro, M.; Yamauchi, S.; Fueki, K., Nonstoichiometry and defect structure of the perovskite-type oxides $\mathrm{La}_{1-\mathrm{x}} \mathrm{Sr}_{\mathrm{x}} \mathrm{FeO}_{3-\delta}$. J. Solid State Chem. 1985, 58, 257-266.

140. Garces, D.; Setevich, C. F.; Caneiro, A.; Cuello, G. J.; Mogni, L., Effect of cationic orderdisorder on the transport properties of $\mathrm{LaBaCO}_{2} \mathrm{O}_{6-\delta}$ and $\mathrm{La}_{0.5} \mathrm{Ba}_{0.5} \mathrm{CoO}_{3-\delta}$ perovskites. J. Appl. Cryst. 2014, 47, 325-334.

141. Patrakeev, M. V.; Leonidov, I. A.; Kozhevnikov, V. L.; Poeppelmeier, K. R., p-Type electron transport in $\mathrm{La}_{1-\mathrm{x}} \mathrm{Sr}_{x} \mathrm{FeO}_{3-\delta}$ at high temperatures. J. Solid State Chem. 2005, 178, 921-927.

142. Das, V. D.; Bhat, K. S., Electrical conductivity of air-exposed and unexposed lead telluride thin films-temperature and size effects. J. Phys. D: Appl. Phys. 1989, 22, 162.

143. Bernuy-Lopez, C.; Høydalsvik, K.; Einarsrud, M.-A.; Grande, T., Effect of A-Site Cation Ordering on Chemical Stability, Oxygen Stoichiometry and Electrical Conductivity in Layered $\mathrm{LaBaCO}_{2} \mathrm{O}_{(5+\delta)}$ Double Perovskite. Materials 2016, 9, 154.

144. Balachandran, U.; Dusek, J. T.; Maiya, P. S.; Ma, B.; Mieville, R. L.; Kleefisch, M. S.; Udovich, C. A., Ceramic membrane reactor for converting methane to syngas. Catal. Today 1997, 36 (3), 265-272.

145. Skinner, S. J.; Kilner, J. A., Oxygen ion conductors. Mater.Today 2003, 6 (3), 30-37.

146. Nemudry, A.; Weiss, M.; Gainutdinov, I.; Boldyrev, V.; Schöllhorn, R., Room Temperature Electrochemical Redox Reactions of the Defect Perovskite SrFeO2.5+x. Chem. Mater. 1998, 10 (9), 2403-2411.

147. Lee, K. J.; Iguchi, E., Electronic properties of $\mathrm{SrMnO}_{3 x}$. J. Solid State Chem. 1995, 114, $242-$ 248.

148. Kim, C. M.; Seo, J. W.; Choi, S. M.; Seo, W. S.; Lee, S.; Lim, Y. S.; Park, K., Structural and thermoelectric properties of $\mathrm{n}$-type $\mathrm{Sr}_{1-\mathrm{x}} \mathrm{Ti}_{\mathrm{x}} \mathrm{MnO}_{3-\delta}$ perovskite system. Electron. Mater. Lett. 2015, 11, 276-281.

149. Arpe, R.; Müller-Buschbaum, H.; Schenck, R. V., Über Erdalkalimetalloxogallate. VI. Zur Kenntnis von $\mathrm{Ca}_{2} \mathrm{FeGaO}_{5}$, ein Beitrag zur Kristallchemie von MMeO5-Verbindungen. Z. Anorg. Allg. Chem. 1974, 410 (2), 97-103.

150. Ceretti, M.; Corallini, S.; Paulus, W., Influence of phase transformations on crystal growth of stoichiometric brownmillerite oxides: $\mathrm{Sr}_{2} \mathrm{ScGaO}_{5}$ and $\mathrm{Ca}_{2} \mathrm{Fe}_{2} \mathrm{O}_{5}$. Crystals 2016, 6, 146.

151. Chandra, A.; Roberts, A. J.; Slade, R. C. T., Studies of nanostructures and conductivity in the system V xMo1-xOy. Solid State Commun. 2008, 147 (3), 83-87.

152. Papaioannou, J. C.; Patermarakis, G. S.; Karayianni, H. S., Electron hopping mechanism in hematite $\left(\alpha-\mathrm{Fe}_{2} \mathrm{O}_{3}\right)$. J. Phys. Chem. Solids 2005, 66, 839-844.

153. Rosso, K. M.; Smith, D. M. A.; Dupuis, M., An ab initio model of electron transport in hematite $\left(\alpha-\mathrm{Fe}_{2} \mathrm{O}_{3}\right)$ basal planes. J. Chem. Phys. 2003, 118, 6455-6466.

154. Ellis, B.; Perry, L. K.; Ryan, D. H.; Nazar, L. F., Small Polaron Hopping in $\mathrm{Li}_{x} \mathrm{FePO}_{4}$ Solid Solutions: Coupled Lithium-Ion and Electron Mobility. J. Am. Chem.Soc. 2006, 128 (35), 1141611422.

155. Yoshino, T.; Ito, E.; Katsura, T.; Yamazaki, D.; Shan, S.; Guo, X.; Nishi, M.; Higo, Y.; Funakoshi, K. i., Effect of iron content on electrical conductivity of ferropericlase with implications for the spin transition pressure. J. Geophys. Res. Solid Earth 2011, 116 (B4).

156. Sanson, A.; Zaltron, A.; Argiolas, N.; Sada, C.; Bazzan, M.; Schmidt, W. G.; Sanna, S., Polaronic deformation at the $\mathrm{Fe}^{2+/ 3+}$ impurity site in Fe:LiNbO 3 crystals. Phys. Rev.B 2015, 91 (9), 094109.

157. Mineshige, A.; Inaba, M.; Yao, T.; Ogumi, Z.; Kikuchi, K.; Kawase, M., Crystal Structure and Metal-Insulator Transition of $\mathrm{La}_{1-\mathrm{x}} \mathrm{Sr}_{x} \mathrm{CoO}_{3}$. J.Solid State Chem. 1996, 121, 423-429. 
158. Hona, R. K.; Huq, A.; Ramezanipour, F., Unraveling the Role of Structural Order in the Transformation of Electrical Conductivity in $\mathrm{Ca} 2 \mathrm{FeCoO}-\delta$, CaSrFeCoO6- $\delta$, and Sr2FeCoO6- $\delta$. Inorganic Chemistry 2017, 56 (23), 14494-14505.

159. Shunji, T.; Hiroshi, N.; Yoshio, I.; Nobuo, I., The Oxygen-Deficient Perovskite Solid Solution $\mathrm{Nd}_{1+\times} \mathrm{Ba}_{2-x} \mathrm{Cu}_{3} \mathrm{O}_{y}$ and Its Superconductivity. Jpn. J. Appl. Phys. 1987, 26 (12A), L2076.

160. Maignan, A.; Michel, C.; Hervieu, M.; Raveau, B., A monoclinic manganite, $\mathrm{La}_{0.9} \mathrm{MnO}_{3-\delta}$, with colossal magnetoresistance properties near room temperature. Solid State Commun. 1997, 101, 277-281.

161. Gómez, L.; Galeano, V.; Parra, R.; Michel, C. R.; Paucar, C.; Morán, O., Carbon dioxide gas sensing properties of ordered oxygen deficient perovskite $\mathrm{LnBaCO}_{2} \mathrm{O}_{5+\delta}(\mathrm{Ln}=\mathrm{La}, \mathrm{Eu})$. Sens. Actuators, B: Chemical 2015, 221, 1455-1460.

162. Leo, A.; Liu, S.; Diniz da Costa, J. C.; Shao, Z., Oxygen permeation through perovskite membranes and the improvement of oxygen flux by surface modification. Sci. Tech. Adv. Mater. 2006, 7, 819-825.

163. Skinner, S. J., Recent advances in Perovskite-type materials for solid oxide fuel cell cathodes. Int. J. Inorg. Mater. 2001, 3, 113-121.

164. Ramezanipour, F.; Greedan, J. E.; Cranswick, L. M. D.; Garlea, V. O.; Siewenie, J.; King, G.; Llobet, A.; Donaberger, R. L., The effect of the B-site cation and oxygen stoichiometry on the local and average crystal and magnetic structures of $\mathrm{Sr}_{2} \mathrm{Fe}_{1.9} \mathrm{M}_{0.1} \mathrm{O}_{5+\mathrm{y}}(\mathrm{M}=\mathrm{Mn}, \mathrm{Cr}, \mathrm{Co} ; \mathrm{y}=0,0.5)$. J. Mater. Chem. 2012, 22 (19), 9522-9538.

165. Boulahya, K.; Ruiz-Morales, J. C.; Hernando, M.; González-Calbet, J. M.; Parras, M., Synthesis, Structural, Magnetic, and Electrical Study of $\mathrm{BaSrCO}_{2} \mathrm{O}_{5}$, a Highly Disordered Cubic Perovskite. Chem. Mater. 2009, 21 (10), 2045-2054.

166. Tomkiewicz, A. C.; Tamimi, M. A.; Huq, A.; McIntosh, S., Evidence for the low oxygen stoichiometry of cubic $\mathrm{BaO} .5 \mathrm{~S} r 0.5 \mathrm{Co} 0.5 \mathrm{Fe} 0.5 \mathrm{O}-\delta$ from in-situ neutron diffraction. Solid State lonics 2013, 253, 27-31.

167. Dupin, J. C.; Gonbeau, D.; Benqlilou-Moudden, H.; Vinatier, P.; Levasseur, A., XPS analysis of new lithium cobalt oxide thin-films before and after lithium deintercalation. Thin Solid Films 2001, 384, 23-32.

168. Davison, N.; McWhinnie, W. R.; Hooper, A., X-ray photoelectron spectroscopic study of cobalt(II) and nickel(II) sorbed on hectorite and montmorillonite. Clays Clay Miner. 1991, 39 (1), 22-27.

169. Ghaffari; M. Liu, T.; Huang, H.; Tan, O. K.; Shannon, M., Investigation of local structure effect and X-ray absorption characteristics (EXAFS) of Fe (Ti) K-edge on photocatalyst properties of $\mathrm{SrTi}_{(1-x)} \mathrm{Fe}_{\mathrm{x}} \mathrm{O}_{(3-\delta)}$. Mater. Chem. Phys. 2012, 136, 347-357.

170. Morales, J., Sánchez, L., Martín, F., Berry, F., Renc, X., Synthesis and Characterization of Nanometric Iron and Iron-Titanium Oxides by Mechanical Milling: Electrochemical Properties as Anodic Materials in Lithium Cells. J. Electrochem. Soc. 2005, 1521748.

171. Ruttanapun, C.; S. Maensiri, Effects of spin entropy and lattice strain from mixed-trivalent $\mathrm{Fe}^{3+} / \mathrm{Cr}^{3+}$ on the electronic, thermoelectric and optical properties of delafossite $\mathrm{CuFe}_{1-x} \mathrm{Cr}_{x} \mathrm{O}_{2}(\mathrm{x}=$ 0.25, 0.5, 0.75). J. Phys. D: Appl. Phys. 2015, 48, 16.

172. Doi, A.; Nomura, M.; Obukuro, Y.; Maeda, R.; Obata, K.; Matsushima, S.; Kobayashi, K., Characterization of Ti-doped $\mathrm{CaFe}_{2} \mathrm{O}_{4}$ prepared from a malic acid complex 2014 Ceram Soc Japn 122 (1422):175-178.

173. Pannunzio Miner, E. V.; De Paoli, J. M.; Alonso, J. A.; García-Hernández, M.; Sánchez, R. D.; Carbonio, R. E., Ferrimagnetic order in the insulating $\mathrm{Sr}_{3} \mathrm{Fe}_{2} \mathrm{ReO}_{9}$ double perovskite. Physica $B$ : Condensed Matter 2007, 398 (2), 397-400. 
174. Zhao, Q.; Liu, M.; Dai, J.; Deng, H.; Yin, Y.; Zhou, L.; Yang, J.; Hu, Z.; Agrestini, S.; Chen, K.; Pellegrin, E.; Valvidares, M.; Nataf, L.; Baudelet, F.; Tjeng, L. H.; Yang, Y.-f.; Jin, C.; Long, Y., HighPressure Synthesis and Ferrimagnetic Ordering of the B-Site-Ordered Cubic Perovskite $\mathrm{Pb}_{2} \mathrm{FeOsO}_{6}$. Inorg. Chem. 2016, 55 (19), 9816-9821.

175. Feng, H. L.; Arai, M.; Matsushita, Y.; Tsujimoto, Y.; Guo, Y.; Sathish, C. I.; Wang, X.; Yuan, Y.-H.; Tanaka, M.; Yamaura, K., High-Temperature Ferrimagnetism Driven by Lattice Distortion in Double Perovskite $\mathrm{Ca}_{2} \mathrm{FeOsO}_{6}$. J. Am.Chem. Soc. 2014, 136 (9), 3326-3329.

176. Lin, Q.; Greenblatt, M.; Caspi, E. a. N.; Avdeev, M., Crystallographic and magnetic properties of $\mathrm{CaLaMnMoO}_{6}$ double perovskite. J. Solid State Chem. 2006, 179 (7), 2086-2092.

177. Mezzadri, F.; Delmonte, D.; Orlandi, F.; Pernechele, C.; Calestani, G.; Solzi, M.; Lantieri, M.; Spina, G.; Cabassi, R.; Bolzoni, F.; Fittipaldi, M.; Merlini, M.; Migliori, A.; Manuel, P.; Gilioli, E., Structural and magnetic characterization of the double perovskite $\mathrm{Pb}_{2} \mathrm{FeMoO}_{6}$. J. Mater. Chem. $\mathrm{C}$ 2016, 4 (7), 1533-1542.

178. Cheng, X.; Fabbri, E.; Nachtegaal, M.; Castelli, I. E.; El Kazzi, M.; Haumont, R.; Marzari, N.; Schmidt, T. J., Oxygen Evolution Reaction on $\mathrm{La}_{1-x} \mathrm{Sr}_{x} \mathrm{CoO}_{3}$ Perovskites: A Combined Experimental and Theoretical Study of Their Structural, Electronic, and Electrochemical Properties. Chem. Mater. 2015, 27, 7662-7672.

179. Pizzini, S., Physical Chemistry of Semiconductor Materials and Processes, John Wiley \& Sons Ltd, West Sussex, United Kingdom. 2015.

180. Asenath-Smith, E.; Lokuhewa, I. N.; Misture, S. T.; Edwards, D. D., p-Type thermoelectric properties of the oxygen-deficient perovskite $\mathrm{Ca}_{2} \mathrm{Fe}_{2} \mathrm{O}_{5}$ in the brownmillerite structure. J. Solid State Chem. 2010, 183 (7), 1670-1677.

181. A. Belik, A.; Matsushita, Y.; Katsuya, Y.; Tanaka, M.; Kolodiazhnyi, T.; Isobe, M.; TakayamaMuromachi, E., Crystal structure and magnetic properties of 6H-SrMnO $3.2011,84,094438$.

182. Liu, T.; Yang, X.; Ma, C.; Hao, X.; Liang, X.; Liu, F.; Liu, F.; Yang, C.; Zhu, H.; Lu, G., $\mathrm{CeO}_{2^{-}}$ based mixed potential type acetone sensor using $\mathrm{MMnO}_{3}(\mathrm{M}: \mathrm{Sr}, \mathrm{Ca}$, $\mathrm{La}$ and $\mathrm{Sm})$ sensing electrode. Solid State Ionics 2018, 317, 53-59.

183. Ryu, J.; O'Hayre, R.; Lee, H., Structural analysis and electrochemical properties of cobaltdoped $\mathrm{Sr}_{0.9} \mathrm{Ce}_{0.1} \mathrm{MnO}_{3-\delta}$ cathode for IT-SOFCs. J. Mater. Res. 2014, 29, 2667-2672.

184. Zhu, Z.-L.; Gu, J.-H.; Jia, Y.; Hu, X., A comparative study of electronic structure and magnetic properties of $\mathrm{SrCrO}_{3}$ and $\mathrm{SrMoO}_{3}$. Physica B: Condensed Matter 2012, 407, 1990-1994.

185. Zhang, W.; Meng, J.; Zhang, X.; Zhang, L.; Liu, X.; Meng, J., Co-incorporating enhancement on oxygen vacancy formation energy and electrochemical property of $\mathrm{Sr}_{2} \mathrm{Co}_{1+\times} \mathrm{Mo}_{1-x} \mathrm{O}_{6-\delta}$ cathode for intermediate-temperature solid oxide fuel cell. Solid State lonics 2018, 316, 20-28.

186. Gorodea, I. A., Influence of the B-site cation nature on crystal structure and magnetic properties of $\mathrm{Ca}_{2} \mathrm{BMoO}_{6}(\mathrm{~B}=\mathrm{Cr}$, La, Sm) double perovskite. Acta Chemica lasi 2014, 22, 145-154.

187. Hosaka, Y.; Ichikawa, N.; Saito, T.; Manuel, P.; Khalyavin, D.; Attfield, J. P.; Shimakawa, Y., Two-Dimensional Charge Disproportionation of the Unusual High Valence State $\mathrm{Fe}^{4+}$ in a Layered Double Perovskite. J. Am. Chem. Soc. 2015, 137 (23), 7468-7473.

188. Ganesanpotti, S.; Tassel, C.; Hayashi, N.; Goto, Y.; Bouilly, G.; Yajima, T.; Kobayashi, Y.; Kageyama, H., Charge Disproportionation and Magnetoresistivity in a Double Perovskite with Alternate $\mathrm{Fe}^{4+}\left(\mathrm{d}^{4}\right)$ and $\mathrm{Mn}^{4+}\left(\mathrm{d}^{3}\right)$ Layers. Eur. J. Inorg. Chem. 2014, 2014 (15), 2576-2581.

189. Chan, T. S.; Liu, R. S.; Hu, S. F.; Lin, J. G., Structure and physical properties of double perovskite compounds $\mathrm{Sr}_{2} \mathrm{FeMO}_{6}$ (M=Mo, W). Mater. Chem. Phys. 2005, 93, 314-319.

190. Sayani, M.; Sebastiaan van, D., Pulsed laser deposition of $\mathrm{La}_{1-}{ }_{x} \mathrm{Sr}_{x} \mathrm{MnO}_{3}$ : thin-film properties and spintronic applications. J. Phys. D: Appl. Phys. 2014, 47, 034010.

191. Cui, L.; Yang, L., Magnetic and transport properties of the double perovskite $\mathrm{Sr}_{2} \mathrm{FeMnO}_{6}$. Adv. Mater. Res. 2012, 393-395, 157-160. 
192. Markiewicz, E.; Bujakiewicz-Koronska, R.; Budziak, A.; Kalvane, A.; Nalecz, D. M., Impedance spectroscopy studies of $\mathrm{SrMnO}_{3}, \mathrm{BaMnO}_{3}$ and $\mathrm{Ba}_{0.5} \mathrm{Sr}_{0.5} \mathrm{MnO}_{3}$ ceramics. Phase Transitions 2014, 87, 1060-1072.

193. Töpfer, J.; Pippardt, U.; Voigt, I.; Kriegel, R., Structure, nonstoichiometry and magnetic properties of the perovskites $\mathrm{Sr}_{1-x} \mathrm{Ca}_{\times} \mathrm{MnO}_{3-\delta}$. Solid State Sci. 2004, 6, 647-654.

194. Aschauer, U.; Pfenninger, R.; Selbach, S. M.; Grande, T.; Spaldin, N. A., Strain-controlled oxygen vacancy formation and ordering in $\mathrm{CaMnO}_{3}$. Phys. Rev. B 2013, 88, 054111.

195. Singh, Y., Electrical Resistivity Measurements: a Review. Int. J. Modern Phys.: Conference Series 2013, 22, 745-756.

196. Wang, J.; Gao, Y.; Chen, D.; Liu, J.; Zhang, Z.; Shao, Z.; Ciucci, F., Water Splitting with an Enhanced Bifunctional Double Perovskite. ACS Catal. 2018, 8, 364-371.

197. Jin, C.; Cao, X.; Zhang, L.; Zhang, C.; Yang, R., Preparation and electrochemical properties of urchin-like $\mathrm{La}_{0.8} \mathrm{Sr}_{0.2} \mathrm{MnO}_{3}$ perovskite oxide as a bifunctional catalyst for oxygen reduction and oxygen evolution reaction. J. Power Sources 2013, 241, 225-230.

198. May, K. J.; Carlton, C. E.; Stoerzinger, K. A.; Risch, M.; Suntivich, J.; Lee, Y. L.; Grimaud, A.; Shao Horn, Y., Influence of oxygen evolution during water oxidation on the surface of perovskite oxide catalysts. J. Phys. Chem. Lett. 2012, 3, 3264-3270.

199. Malkhandi, S.; Trinh, P.; Manohar, A. K.; Jayachandrababu, K. C.; Kindler, A.; Surya Prakash, G. K.; Narayanan, S. R., Electrocatalytic activity of transition metal oxide-carbon composites for oxygen reduction in alkaline batteries and fuel cells. J. Electrochem. Soc. 2013, 160, 943-952.

200. Liang, Y.; Li, Y.; Wang, H.; Zhou, J.; Wang, J.; Regier, T.; Dai, H., $\mathrm{Co}_{3} \mathrm{O}_{4}$ nanocrystals on graphene as a synergistic catalyst for oxygen reduction reaction. Nat. Mater. 2011, 10, 780-786.

201. Mohamed, R.; Cheng, X.; Fabbri, E.; Levecque, P.; Kötz, R.; Conrad, O.; Schmidt, T. J., Electrocatalysis of perovskites: the influence of carbon on the oxygen evolution activity. J. Electrochem. Soc. 2015, 162, 579-586.

202. Fabbri, E.; Nachtegaal, M.; Cheng, X.; Schmidt Thomas, J., Superior bifunctional electrocatalytic activity of $\mathrm{Ba}_{0.5} \mathrm{Sr}_{0.5} \mathrm{Co}_{0.8} \mathrm{Fe}_{0.2} \mathrm{O}_{3-\delta} /$ carbon composite electrodes: insight into the local electronic structure. Adv. Energy Mater. 2015, 5, 1402033.

203. Hona, R. K.; Ramezanipour, F., Remarkable Oxygen-Evolution Activity of a Perovskite Oxide from the $\mathrm{Ca}_{2-} \mathrm{Sr}_{x} \mathrm{Fe}_{2} \mathrm{O}_{6-\delta}$ Series. Angew. Chem. Int. Ed. 2019, 58, 2060-2063.

204. Zhu, Y.; Zhou, W.; Chen, Z. G.; Chen, Y.; Su, C.; Tadé Moses, O.; Shao, Z., $\mathrm{SrNb}_{0.1} \mathrm{Co}_{0.7} \mathrm{Fe}_{0.2} \mathrm{O}_{3-\delta}$ perovskite as a next-generation electrocatalyst for oxygen evolution in alkaline solution. Angew. Chem. Int. Ed. 2015, 54, 3897-3901.

205. Singh, A.; Roy, S.; Das, C.; Samanta, D.; Maji, T. K., Metallophthalocyanine-based redox active metal-organic conjugated microporous polymers for OER catalysis. Chem. Commun. 2018, 54, 4465-4468.

206. Pan, Y.; Chen, Y.; Li, X.; Liu, Y.; Liu, C., Nanostructured nickel sulfides: phase evolution, characterization and electrocatalytic properties for the hydrogen evolution reaction. RSC Advances 2015, 5, 104740-104749.

207. Oh, S.; Kim, H.; Kwon, Y.; Kim, M.; Cho, E.; Kwon, H., Porous Co-P foam as an efficient bifunctional electrocatalyst for hydrogen and oxygen evolution reactions. J. Mater.Chem. A 2016, 4, $18272-18277$.

208. Zhang, W.; Zhang, X.; Chen, L.; Dai, J.; Ding, Y.; Ji, L.; Zhao, J.; Yan, M.; Yang, F.; Chang, C.R.; Guo, S., Single-Walled Carbon Nanotube Induced Optimized Electron Polarization of Rhodium Nanocrystals To Develop an Interface Catalyst for Highly Efficient Electrocatalysis. ACS Catal. 2018, 8, 8092-8099. 
209. Zhu, Y.; Zhou, W.; Yu, J.; Chen, Y.; Liu, M.; Shao, Z., Enhancing Electrocatalytic Activity of Perovskite Oxides by Tuning Cation Deficiency for Oxygen Reduction and Evolution Reactions. Chem. Mater. 2016, 28, 1691-1697.

210. Song, F.; Hu, X., Ultrathin cobalt-manganese layered double hydroxide Is an efficient oxygen evolution catalyst. J. Am. Chem. Soc. 2014, 136, 16481-16484.

211. Moir, J.; Soheilnia, N.; O’Brien, P.; Jelle, A.; Grozea, C. M.; Faulkner, D.; Helander, M. G.; Ozin, G. A., Enhanced hematite water electrolysis using a 3D antimony-doped tin oxide electrode. ACS Nano 2013, 7, 4261-4274.

212. Shinagawa, T.; Garcia-Esparza, A. T.; Takanabe, K., Insight on Tafel slopes from a microkinetic analysis of aqueous electrocatalysis for energy conversion. Sci. Rep. 2015, 5, 13801.

213. Ranaweera, C. K.; Zhang, C.; Bhoyate, S.; Kahol, P. K.; Ghimire, M.; Mishra, S. R.; Perez, F.; Gupta, B. K.; Gupta, R. K., Flower-shaped cobalt oxide nano-structures as an efficient, flexible and stable electrocatalyst for the oxygen evolution reaction. Mater. Chem. Front. 2017, 1, 1580-1584. 214. Joya, K. S.; UI Ain Babar, N.; Gilani, S. R.; Yasmeen, F.; Sarfaraz, M.; Ikram, S.; Colak, S. G.; Ocakoglu, K.; Ince, M., Heterogeneous Electrocatalysts for Efficient Water Oxidation Derived from Metal Phthalocyanine. Chem. Select 2018, 3, 11357-11366.

215. Lv, X.; Zhu, Y.; Jiang, H.; Yang, X.; Liu, Y.; Su, Y.; Huang, J.; Yao, Y.; Li, C., Hollow mesoporous $\mathrm{NiCO}_{2} \mathrm{O}_{4}$ nanocages as efficient electrocatalysts for oxygen evolution reaction. Dalton Trans. 2015, 44, 4148-4154.

216. Yang, Y.; Jiang, Y.; Wang, Y.; Sun, Y., Photoinduced decomposition of $\mathrm{BaFeO}_{3}$ during photodegradation of methyl orange. J. Mol. Catal. A: Chem. 2007, 270 (1), 56-60.

217. Reehuis, M.; Ulrich, C.; Maljuk, A.; Niedermayer, C.; Ouladdiaf, B.; Hoser, A.; Hofmann, T.; Keimer, B., Neutron diffraction study of spin and charge ordering in $\mathrm{SrFeO}_{3-\delta}$. Phys. Rev. B 2012, 85 (18), 184109.

218. Zou, X. D.; Hovmoller, S.; Parras, M.; Gongalez-Calbet, J. M.; Vallet-Regi, M.; Grenier, J. C., The Complex Perovskite-Related Superstructure $\mathrm{Ba}_{2} \mathrm{Fe}_{2} \mathrm{O}_{5}$ Solved by HREM and CIP. Acta Cryst. 1993, A49, 27-35.

219. Clemens, O.; Gröting, M.; Witte, R.; Perez-Mato, J. M.; Loho, C.; Berry, F. J.; Kruk, R.; Knight, K. S.; Wright, A. J.; Hahn, H.; Slater, P. R., Crystallographic and Magnetic Structure of the Perovskite-Type Compound $\mathrm{BaFeO}_{2.5}$ : Unrivaled Complexity in Oxygen Vacancy Ordering. Inorg. Chem. 2014, 53 (12), 5911-5921.

220. Ruttanapun, C.; Maensiri, S., Effects of spin entropy and lattice strain from mixed-trivalent $\mathrm{Fe}^{3+} / \mathrm{Cr}^{3+}$ on the electronic, thermoelectric and optical properties of delafossite $\mathrm{CuFe}_{1-\mathrm{x}} \mathrm{Cr}_{x} \mathrm{O}_{2}(\mathrm{x}$ $=0.25,0.5,0.75)$. J.Phys. D: Appl. Phys. 2015, 48 (49), 495103.

221. Schmidt, M.; Campbell, S. J., Crystal and Magnetic Structures of $\mathrm{Sr}_{2} \mathrm{Fe}_{2} \mathrm{O}_{5}$ at Elevated Temperature. J. Solid State Chem. 2001, 156 (2), 292-304.

222. Parras, M.; Fournes, L.; Grenier, J. C.; Pouchard, M.; Vallet, M.; Calbet, J. M.; Hagenmuller, P., Structural aspects and Mössbauer resonance investigation of $\mathrm{Ba}_{2} \mathrm{Fe}_{2} \mathrm{O}_{5}$. J.Solid State Chem. 1990, 88 (1), 261-268.

223. Bhosale, D. R.; Yusuf, S. M.; Kumar, A.; Mukadam, M. D.; Patil, S. I., High oxide ion conductivity below $500^{\circ} \mathrm{C}$ in the garnets $\mathrm{La}_{x} \mathrm{Y}_{3-\mathrm{x}} \mathrm{Fe}_{5} \mathrm{O}_{12+\delta}$. Phys. Rev. Mater. 2017, 1 (1), 015001.

224. Winter, M.; Besenhard, J. O.; Spahr, M. E.; Novák, P., Insertion Electrode Materials for Rechargeable Lithium Batteries. Adv. Mater. 1998, 10, 725-763.

225. Clemm, C.; Sinai, C.; Ferkinghoff, C.; Dethlefs, N.; Nissen, N. F.; Lang, K. In Durability and cycle frequency of smartphone and tablet lithium-ion batteries in the field, Electronics Goes Green (EGG), 2016; pp 1-7.

226. Sharma, N.; Shaju, K. M.; Subba Rao, G. V.; Chowdari, B. V. R., Mixed oxides $\mathrm{Ca}_{2} \mathrm{Fe}_{2} \mathrm{O}_{5}$ and $\mathrm{Ca}_{2} \mathrm{CO}_{2} \mathrm{O}_{5}$ as anode materials for Li-ion batteries. Electrochim. Acta 2004, 49, 1035-1043. 
227. Sharma, N.; Shaju, K. M.; Subba Rao, G. V.; Chowdari, B. V. R., Sol-gel derived nanocrystalline $\mathrm{CaSnO}_{3}$ as high capacity anode material for Li-ion batteries. Electrochem. Commun. 2002, 4, 947-952.

228. Aurbach, D.; Nimberger, A.; Markovsky, B.; Levi, E.; Sominski, E.; Gedanken, A., Nanoparticles of SnO produced by sonochemistry as anode materials for rechargeable lithium batteries. Chem. Mater. 2002, 14, 4155-4163.

229. Carreon, M. L.; Thapa, A. K.; Jasinski, J. B.; Sunkara, M. K., The capacity and durability of amorphous silicon nanotube thin film anode for lithium ion battery applications. ECS Electrochem. Lett. 2015, 4, A124-A128.

230. Reddy, M. V.; Subba Rao, G. V.; Chowdari, B. V. R., Metal oxides and oxysalts as anode materials for Li ion batteries. Chem. Rev. 2013, 113, 5364-5457.

231. Kam, K. C.; Doeff, M. M., Electrode Materials for Lithium Ion Batteries. Material Matters 2012, 7, n4.

232. Flandrois, S.; Simon, B., Carbon materials for lithium-ion rechargeable batteries. Carbon 1999, 37, 165-180.

233. Fu, R.; Zhou, X.; Fan, H.; Blaisdell, D.; Jagadale, A.; Zhang, X.; Xiong, R., Comparison of lithium-ion anode materials using an experimentally verified physics-based electrochemical model. Energies 2017, 10, 2174.

234. Winter, M.; Besenhard, J. O., Electrochemical lithiation of tin and tin-based intermetallics and composites. Electrochim. Acta 1999, 45, 31-50.

235. Mishra, A.; Mehta, A.; Basu, S.; Malode, S. J.; Shetti, N. P.; Shukla, S. S.; Nadagouda, M. N.; Aminabhavi, T. M., Electrode Materials for Lithium-ion Batteries. Mater. Sci. Energy Technol. 2018, 1, 182-187.

236. Courtney, I. A.; McKinnon, W. R.; Dahn, J. R., On the aggregation of tin in SnO composite glasses caused by the reversible reaction with lithium. J. Electrochem. Soc. 1999, 146, 59-68.

237. Mohamedi, M.; Lee, S. J.; Takahashi, D.; Nishizawa, M.; Itoh, T.; Uchida, I., Amorphous tin oxide films: preparation and characterization as an anode active material for lithium ion batteries. Electrochim. Acta 2001, 46, 1161-1168.

238. Behm, M.; Irvine, J. T. S., Influence of structure and composition upon performance of tin phosphate based negative electrodes for lithium batteries. Electrochim. Acta 2002, 47, 17271738.

239. Laruelle, S.; Grugeon, S.; Poizot, P.; Dollé, M.; Dupont, L.; Tarascon, J.-M., On the origin of the extra electrochemical capacity displayed by $\mathrm{MO} / \mathrm{Li}$ cells at low potential. J. Electrochem. Soc. 2002, 149, 627-634.

240. Obrovac, M. N.; Dunlap, R. A.; Sanderson, R. J.; Dahn, J. R., The electrochemical displacement reaction of lithium with metal oxides. J. Electrochem. Soc. 2001, 148, 576-588.

241. Poizot, P.; Laruelle, S.; Grugeon, S.; Dupont, L.; Tarascon, J. M., Nano-sized transitionmetal oxides as negative-electrode materials for lithium-ion batteries. Nature 2000, 407, 496.

242. Yang, Z.; Choi, D.; Kerisit, S.; Rosso, K. M.; Wang, D.; Zhang, J.; Graff, G.; Liu, J., Nanostructures and lithium electrochemical reactivity of lithium titanites and titanium oxides: A review. J. Power Sources 2009, 192, 588-598.

243. Yi, T. F.; Jiang, L. J.; Shu, J.; Yue, C.-B.; Zhu, R. S.; Qiao, H. B., Recent development and application of $\mathrm{Li}_{4} \mathrm{Ti}_{5} \mathrm{O}_{12}$ as anode material of lithium ion battery. J. Phys.Chem. Solids 2010, 71, 1236-1242.

244. Sharma, Y.; Sharma, N.; Subba Rao, G. V.; Chowdari, B. V. R., Nanophase $\mathrm{ZnCo}_{2} \mathrm{O}_{4}$ as a High Performance Anode Material for Li-Ion Batteries. Adv. Funct. Mater. 2007, 17, 2855-2861.

245. Yang, Y.; Zhao, Y.; Xiao, L.; Zhang, L., Nanocrystalline $\mathrm{ZnMn}_{2} \mathrm{O}_{4}$ as a novel lithium-storage material. Electrochem. Commun. 2008, 10, 1117-1120. 
246. Yang, J. B.; Zhou, X. D.; Chu, Z.; Hikal, W. M.; Cai, Q.; Ho, J. C.; Kundaliya, D. C.; Yelon, W. B.; James, W. J.; Anderson, H. U.; Hamdeh, H. H.; Malik, S. K., Charge disproportionation and ordering in $\mathrm{La}_{1 / 3} \mathrm{Sr}_{2 / 3} \mathrm{FeO}_{3-\delta .}$.J. Phy.: Condens. Matter 2003, 15, 5093.

247. Zhao, K.; Chen, J.; Li, H.; Zheng, A.; He, F., Effects of Co-substitution on the reactivity of double perovskite oxides $\mathrm{LaSrFe}_{2-\mathrm{x}} \mathrm{CO}_{\times} \mathrm{O}_{6}$ for the chemical-looping steam methane reforming. $J$. Energy Institute 2018, 1-10.

248. Gupta, R. P.; Sen, S. K., Calculation of multiplet structure of core p -vacancy levels. Phys. Rev.B 1975, 12, 15-19.

249. Gupta, R. P.; Sen, S. K., Calculation of multiplet structure of core p-vacancy Levels. . Phys. Rev. B 1974, 10, 71-77.

250. Grosvenor, A. P.; Kobe, B. A.; Biesinger, M. C.; McIntyre, N. S., Investigation of multiplet splitting of Fe 2p XPS spectra and bonding in iron compounds. Surf. Interface Anal. 2004, 36, 15641574.

251. Biesinger, M. C.; Payne, B. P.; Grosvenor, A. P.; Lau, L. W. M.; Gerson, A. R.; Smart, R. S. C., Resolving surface chemical states in XPS analysis of first row transition metals, oxides and hydroxides: Cr, Mn, Fe, Co and Ni. Appl. Surf. Sci. 2011, 257, 2717-2730.

252. McIntyre, N. S.; Zetaruk, D. G., X-ray photoelectron spectroscopic studies of iron oxides. Anal.Chem. 1977, 49, 1521-1529.

253. Lin, Y.-M.; Abel, P. R.; Heller, A.; Mullins, C. B., $\alpha-\mathrm{Fe}_{2} \mathrm{O}_{3}$ Nanorods as Anode Material for Lithium Ion Batteries. J. Phys. Chem. Lett. 2011, 2, 2885-2891.

254. Zhang, J.; Wang, B.; Zhou, J.; Xia, R.; Chu, Y.; Huang, J., Preparation of advanced CuO nanowires/functionalized graphene composite anode material for lithium ion batteries. Materials 2017, 10, 72.

255. Chen, X.; Bi, Q.; Sajjad, M.; Wang, X.; Ren, Y.; Zhou, X.; Xu, W.; Liu, Z., One-dimensional porous silicon nanowires with large surface area for fast charge-discharge lithium-ion batteries. Nanomaterials 2018, 8, 285.

256. Dharmasena, R.; Thapa, A. K.; Hona, R. K.; Jasinski, J.; Sunkara, M. K.; Sumanasekera, G. U., Mesoporous $\mathrm{TiO}_{2}$ coating on carbon-sulfur cathode for high capacity Li-sulfur battery. RSC Advances 2018, 8, 11622-11632.

257. Kim, I. T.; Magasinski, A.; Jacob, K.; Yushin, G.; Tannenbaum, R., Synthesis and electrochemical performance of reduced graphene oxide/maghemite composite anode for lithium ion batteries. Carbon 2013, 52, 56-64.

258. Xu, X.; Wan, Y.; Sha, Y.; Deng, W.; Xue, G.; Zhou, D., Nanoporous iron oxide@carbon composites with low carbon content as high-performance anodes for lithium-ion batteries. RSC Adv. 2015, 5, 89092-89098.

259. Chaudhari, N. K.; Kim, M.-S.; Bae, T.-S.; Yu, J.-S., Hematite $\left(\alpha-\mathrm{Fe}_{2} \mathrm{O}_{3}\right)$ nanoparticles on vulcan carbon as an ultrahigh capacity anode material in lithium ion battery. Electrochim. Acta 2013, 114, 60-67.

260. Zhang, X.; Liu, H.; Petnikota, S.; Ramakrishna, S.; Fan, H. J., Electrospun $\mathrm{Fe}_{2} \mathrm{O}_{3}-\mathrm{carbon}$ composite nanofibers as durable anode materials for lithium ion batteries. J. Mater. Chem. A 2014, 2, 10835-10841.

261. Liang, J.; Xiao, C.; Chen, X.; Gao, R.; Ding, S., Porousy- $\mathrm{Fe}_{2} \mathrm{O}_{3}$ spheres coated with N-doped carbon from polydopamine as Li-ion battery anode materials. Nanotechnology 2016, 27, 215403. 262. Ping, Q.; iXu, B.; Ma, X.; Tian, J.; Wang, B., An iron oxyborate $\mathrm{Fe}_{3} \mathrm{BO}_{5}$ material as a highperformance anode for lithium-ion and sodium-ion batteries. Dalton Trans. 2019, 48, 5741-5748. 263. Oh, H.-D.; Lee, S.-W.; Kim, S.-O.; Lee, J. K., Facile synthesis of carbon layer-entangled $\mathrm{Fe}_{2} \mathrm{O}_{3}$ clusters as anode materials for improved Li-ion batteries. J. Power Sources 2013, 244, 575-580. 
264. Chu, S.; Majumdar, A., Opportunities and challenges for a sustainable energy future. Nature 2012, 488, 294-303.

265. Mallouk, T. E., Divide and conquer. Nat. Chem. 2013, 5, 362-263.

266. Suntivich, J.; May, K. J.; Gasteiger, H. A.; Goodenough, J. B.; Shao-Horn, Y., A perovskite oxide optimized for oxygen evolution catalysis from molecular orbital principles. Science 2011, 334, 1383-1385.

267. Turner, J. A., Sustainable Hydrogen Production. Science 2004, 305, 972-974.

268. Walter, M. G.; Warren, E. L.; McKone, J. R.; Boettcher, S. W.; Mi, Q.; Santori, E. A.; Lewis, N. S., Solar Water Splitting Cells. Chem. Rev. 2010, 110, 6446-6473.

269. Gray, H. B., Powering the planet with solar fuel. Nat. chem. 2009, 1, 7.

270. Service, R. F., Hydrogen Cars: Fad or the Future? Science 2009, 324, 1257-1259.

271. Hamdani, M.; Singh, R. N.; Chartier, P., $\mathrm{CO}_{3} \mathrm{O}_{4}$ and Co- Based Spinel Oxides Bifunctional Oxygen Electrodes Int. J. Electrochem. Sci. 2010, 5, 556 - 577.

272. Li, Y.; Gong, M.; Liang, Y.; Feng, J.; Kim, J.-E.; Wang, H.; Hong, G.; Zhang, B.; Dai, H., Advanced zinc-air batteries based on high-performance hybrid electrocatalysts. Nat. Commun. 2013, 4, 1805.

273. Lee, Y.; Suntivich, J.; May, K. J.; Perry, E. E.; Shao-Horn, Y., Synthesis and activities of rutile $\mathrm{IrO}_{2}$ and $\mathrm{RuO}_{2}$ nanoparticles for oxygen evolution in acid and alkaline solutions. J. Phys. Chem. Lett. 2012, 3, 399-404.

274. Frame, F. A.; Townsend, T. K.; Chamousis, R. L.; Sabio, E. M.; Dittrich, T.; Browning, N. D.;

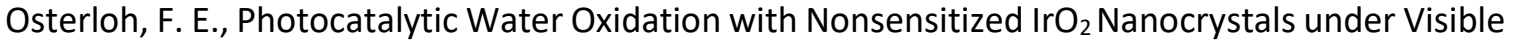
and UV Light. J. Am. Chem. Soc. 2011, 133, 7264-7267.

275. He, D.; He, G.; Jiang, H.; Chen, Z.; Huang, M., Enhanced durability and activity of the perovskite electrocatalyst $\operatorname{Pr}_{0.5} \mathrm{Ba}_{0.5} \mathrm{CoO}_{3-\delta}$ by $\mathrm{Ca}$ doping for the oxygen evolution reaction at room temperature. Chem. Commun. 2017, 53, 5132-5135.

276. Kanan, M. W.; Nocera, D. G., In situ formation of an oxygen-evolving catalyst in neutral water containing phosphate and $\mathrm{Co}^{2+}$. Science 2008, 321, 1072-1075.

277. Park, H. W.; Lee, D. U.; Zamani, P.; Seo, M. H.; Nazar, L. F.; Chen, Z., Electrospun porous nanorod perovskite oxide/nitrogen-doped graphene composite as a bi-functional catalyst for metal air batteries. Nano Energy 2014, 10, 192-200.

278. Han, B.; Grimaud, A.; Giordano, L.; Hong, W. T.; Diaz-Morales, O.; Yueh-Lin, L.; Hwang, J.; Charles, N.; Stoerzinger, K. A.; Yang, W.; Koper, M. T. M.; Shao-Horn, Y., Iron-Based Perovskites for Catalyzing Oxygen Evolution Reaction. J. Phys. Chem. C 2018, 122, 8445-8454.

279. Berggren, J., Refinement of the crystal structure of dicalcium ferrite, $\mathrm{Ca}_{2} \mathrm{Fe}_{2} \mathrm{O}_{5}$. Acta Chem. Scand. 1971, 25, 3616-3624.

280. Tong, X.; Yang, P.; Wang, Y.; Qin, Y.; Guo, X., Enhanced photoelectrochemical water splitting performance of $\mathrm{TiO}_{2}$ nanotube arrays coated with an ultrathin nitrogen-doped carbon film by molecular layer deposition. Nanoscale 2014, 6, 6692-6700.

281. Lu, X.; Zhao, C., Highly efficient and robust oxygen evolution catalysts achieved by anchoring nanocrystalline cobalt oxides onto mildly oxidized multiwalled carbon nanotubes. J. Mater. Chem. A 2013, 1, 12053-12059.

282. Bu, Y.; Gwon, O.; Nam, G.; Jang, H.; Kim, S.; Zhong, Q.; Cho, J.; Kim, G., A highly efficient and robust cation ordered perovskite oxide as a bifunctional catalyst for rechargeable zinc-air batteries. ACS Nano 2017, 11, 11594-11601.

283. Matsumoto, Y.; Sato, E., Electrocatalytic properties of transition metal oxides for oxygen evolution reaction. Mater. Chem. Phys. 1986, 14, 397-426. 
284. Tavakkoli, M.; Kallio, T.; Reynaud, O.; Nasibulin, A. G.; Sainio, J.; Jiang, H.; Kauppinen, E. I.; Laasonen, K., Maghemite nanoparticles decorated on carbon nanotubes as efficient electrocatalysts for the oxygen evolution reaction. J. Mater. Chem. A 2016, 4, 5216-5222.

285. Jung, S.; McCrory, C. C. L.; Ferrer, I. M.; Peters, J. C.; Jaramillo, T. F., Benchmarking nanoparticulate metal oxide electrocatalysts for the alkaline water oxidation reaction. J. Mater.Chem. A 2016, 4, 3068-3076.

286. Lu, B.; Cao, D.; Wang, P.; Wang, G.; Gao, Y., Oxygen evolution reaction on Ni-substituted $\mathrm{Co}_{3} \mathrm{O}_{4}$ nanowire array electrodes. Int. J. Hydrogen Energy 2011, 36, 72-78.

287. Lee, J. G.; Hwang, J.; Hwang, H. J.; Jeon, O. S.; Jang, J.; Kwon, O.; Lee, Y.; Han, B.; Shul, Y.G., A new family of perovskite catalysts for oxygen-evolution reaction in alkaline media: $\mathrm{BaNiO}_{3}$ and $\mathrm{BaNi}_{0.83} \mathrm{O}_{2.5}$. J. Am.Chem. Soc. 2016, 138, 3541-3547.

288. Petrie, J. R.; Cooper, V. R.; Freeland, J. W.; Meyer, T. L.; Zhang, Z.; Lutterman, D. A.; Lee, $\mathrm{H}$. N., Enhanced bifunctional oxygen catalysis in strained $\mathrm{LaNiO}_{3}$ perovskites. J. Am. Chem.Soc. 2016, 138, 2488-2491.

289. Zhang, B.; Lui, Y. H.; Zhou, L.; Tang, X.; Hu, S., An alkaline electro-activated Fe-Ni phosphide nanoparticle-stack array for high-performance oxygen evolution under alkaline and neutral conditions. J. Mater. Chem. A 2017, 5, 13329-13335.

290. Konkena, B.; junge Puring, K.; Sinev, I.; Piontek, S.; Khavryuchenko, O.; Dürholt, J. P.; Schmid, R.; Tüysüz, H.; Muhler, M.; Schuhmann, W.; Apfel, U.-P., Pentlandite rocks as sustainable and stable efficient electrocatalysts for hydrogen generation. Nat. Commun. 2016, 7, 12269.

291. Chen, Y.; Yu, G.; Chen, W.; Liu, Y.; Li, G.-D.; Zhu, P.; Tao, Q.; Li, Q.; Liu, J.; Shen, X.; Li, H.; Huang, X.; Wang, D.; Asefa, T.; Zou, X., Highly Active, Nonprecious Electrocatalyst Comprising Borophene Subunits for the Hydrogen Evolution Reaction. J. Am. Chem. Soc. 2017, 139, 1237012373.

292. Adolphsen, J. Q.; Sudireddy, B. R.; Gil, V.; Chatzichristodoulou, C., Oxygen Evolution Activity and Chemical Stability of $\mathrm{Ni}$ and Fe Based Perovskites in Alkaline Media. J. Electrochem. Soc. 2018, 165, 827-835.

293. Hona, R. K.; Ramezanipour, F., Structure-dependence of electrical conductivity and electrocatalytic properties of $\mathrm{Sr}_{2} \mathrm{Mn}_{2} \mathrm{O}_{6}$ and $\mathrm{CaSrMn}_{2} \mathrm{O}_{6}$. J. Chem. Sci. 2019, 131, 109. 


\section{CURRICULUM VITAE}

\section{Ram Krishna Hona}

Address: 2241 Arthur Ford Ct. Apt 3

Louisville, KY 40217

Phone: 5022917880

Email: ramkhona@gmail.com

Education

School year

GPA

Doctor of Philosophy: Chemistry

2019

3.92

University of Louisville, Louisville, KY, USA

Research: Solid state material science

Master of Science: Chemistry

2014

3.9

Thesis:

New Mexico State University, Las Cruces, NM, USA

ion pair

Reverse micellar effect on the electron transfer reaction within the

Master of Science: Chemistry

1997

$1^{\text {st }}$

Division

Tribhubvan University, Kathmandu, Nepal

Bachelor of Science: Biology and Chemistry

1995

$1^{\text {st }}$ Division

Tribhubvan University, Kathmandu, Nepal

Peer reviewed publications

1. Hona R. K., and Ramezanipour, F., Remarkable oxygen-evolution activity of oxygen-deficient perovskites, $\mathrm{Ca}_{2} \mathrm{Fe}_{2} \mathrm{O}_{6-\delta}, \quad \mathrm{CaSrFe}_{2} \mathrm{O}_{6-\delta}$, and $\mathrm{Sr}_{2} \mathrm{Fe}_{2} \mathrm{O}_{6-\delta}$ : correlations with defect-order and electrical conductivity, Angew. Chem. Int. Ed. 2019, 58, 2060 -2063 (DOI: 10.1002/anie.201813000)

2. Hona RK, Huq A, Mulmi S and Ramezanipour, F., Transformation of structure, electrical conductivity, and magnetism in $\mathrm{AA}^{\prime} \mathrm{Fe}_{2} \mathrm{O}_{6-}, \mathrm{A}=\mathrm{Sr}, \mathrm{Ca}$ and $\mathrm{A}^{\prime}=\mathrm{Sr}$ Inorg. Chem. 2017, 56, 9716-24 (DOI: 10.1021/acs.inorgchem.7b01228)

3. Hona R. K., Huq A. and Ramezanipour, F., Unraveling the role of structural order in the transformation of electrical conductivity in $\mathrm{Ca}_{2} \mathrm{FeCoO}_{6-\delta}, \mathrm{CaSrFeCoO}_{6-\delta}$, 
and $\mathrm{Sr}_{2} \mathrm{FeCoO}_{6-\delta} \quad$ Inorg. Chem. 2017, 56, 14494-505 (DOI: 10.1021/acs.inorgchem.7b02079)

4. Mulmi S., Hona R. K., Jasinski J. B. and Ramezanipour, F., Electrical Conductivity of $\mathrm{Sr}_{2-\mathrm{x}} \mathrm{Ca}_{\mathrm{x}} \mathrm{FeMnO}_{5}(\mathrm{x}=0,1,2)$ J. Solid State Electrochem. 2018, 22, 2329-38 (doi.org/10.1007/s10008-018-3947-6)

5. Hona R. K., Huq A. and Ramezanipour, F., Magnetic structure of $\mathrm{CaSrFeCoO}_{6-\delta}$ : correlations with structural order Mater. Res. Bull. 2018, 106, 131-6 (doi.org/10.1016/j.materresbull.2018.05.030)

6. Hona, R. K.; Ramezanipour, F., Variation in electrical conductivity of $\mathrm{A}_{2} \mathrm{Fe}_{2} \mathrm{O}_{5}$ $(\mathrm{A}=\mathrm{Sr}, \mathrm{Ba})$ : the role of structural order. Mater. Res. Express 2018, 5, 076307 (doi.org/10.1088/2053-1591/aad10e)

7. Hona, R. K.; Ramezanipour, F., Disparity in electrical and magnetic properties of isostructural oxygen-deficient perovskites $\mathrm{BaSrCo}_{2} \mathrm{O}_{6-\delta}$ and $\mathrm{BaSrCoFeO}_{6-\delta}$. $J$. Mater. Sci.- Mater. Electron. 2018. 29, 13464-13473 (doi.org/10.1007/s10854018-9471-8)

8. Dharmasena R., Thapa A. K., Hona R.K., Mesoporous TiO2 coating on carbonsulfur cathode for high capacity Li-sulfur battery RSC Adv., 2018, 8, 11622-11632 (DOI: 10.1039/c8ra01380b)

9. Hona R. K., Huq A. and Ramezanipour, F., Electrical properties of the ordered oxygen-deficient perovskite $\mathrm{Ca}_{2} \mathrm{Fe}_{0.5} \mathrm{Ga}_{1.5} \mathrm{O}_{5}$. IONICS, 2019, 25, 1315-1321, (doi.org/10.1007/s11581-018-2759-0)

10. Hona R. K., and Ramezanipour, F., Improved Electrical Conductivity in a Disordered Oxygen-Deficient Perovskite, $\mathrm{BaSrFe}_{2} \mathrm{O}_{6-\delta}$, J. mater. Sci., 2019, 167, 69-74 (doi.org/10.1016/j.poly.2019.04.018)

11. Hona R. K., and Ramezanipour, F., Charge Transport Properties of OxygenDeficient Perovskites, $\mathrm{Ca}_{2} \mathrm{FeGaO}_{6-\delta}$ and $\mathrm{CaSrFeGaO}_{6-\delta}$ : The Effect of DefectOrder, J. mater. Sci. 2019, 238, 121924, (doi.org/10.1016/j.matchemphys.2019.121924)

12. Hona R. K., and Ramezanipour, F., Electrical Conductivity and Electrocatalytic activity of $\mathrm{Sr}_{2} \mathrm{Mn}_{2} \mathrm{O}_{6}$ and $\mathrm{CaSrMn}_{2} \mathrm{O}_{6}$, J. Chem. Sci. 2019, 131, 109

\section{Conference presentations}

1. Poster presentation: RE3 workshop; renewable energy and energy efficiency 2017 , Louisville KY

Title: Effect of Ca incorporation on crystal structure, magnetism and electrical properties of $\mathrm{Sr}_{2-x} \mathrm{Ca}_{x} \mathrm{Fe}_{2} \mathrm{O}_{6-\delta}$.

2. Poster presentation: SERMACS 2017, Charlotte

Title: Variation in crystal structure, magnetism and electrical conductivity of $\mathrm{Ca}$ doped

$\mathrm{Sr}_{2} \mathrm{Fe}_{2} \mathrm{O}_{6-\delta}$.

3. Oral presentation: 2017 Ohio inorganic week, OSU, Ohio

Title: Study of crystal structure, magnetic and electrical properties of $\mathrm{CaSrFe}_{2} \mathrm{O}_{6}$ $\delta$. 
4. Oral presentation: UofL GRADtalks Brown Bag Series 2018, April 3-5, February, Louisville, KY

Title: Impact of the Ordering of Oxygen Vacancies on the Crystal Structure \& Functional Properties in Oxygen Deficient Perovskites

5. Oral presentation: Graduate Student Regional Research Conference (GSRRC), UofL., Louisville KY 2018

Title: Role of Ca in the Crystal Structure of CaSrFe2O6- $\delta$ and Its Electrical and Magnetic Properties

6. Oral presentation: 2018 Glass City Chemistry Conference (Regional ACS), Toledo, OHIO

(invited speaker)

Title: Effect of Structural order on the electrical conductivity of $\mathrm{Sr}_{2-x} \mathrm{Ca}_{x} \mathrm{Fe}_{2} \mathrm{O}_{5}(x$ $=0$ and 1$)$.

7. Oral presentation: UofL GRADtalks Brown Bag Series 2018, November, Louisville, KY

Title: Oxygen Deficient Perovskites: Effect of vacancy order on structure and conductivity.

8. Oral presentation: UofL (Graduate Student Regional Research Conference) GSRRC 2019 February 27-28.

Title: $\mathrm{Sr}_{2} \mathrm{Fe}_{2} \mathrm{O}_{6-\delta}$ as an anode electrode for Li-ion battery.

9. Oral Presentation: Spring 2019 ACS national meeting, Orlando, Florida (March 31April 4)

Title: Effect of vacancy order on the structure and charge-transport of oxygen deficient perovskites.

Skills/Knowledge

Material synthesis: Design new materials of different composition by conventional solid state synthesis, solgel, co-precipitation method, thermal energy control for the material synthesis.

Material characterization: Scanning electron microscope (SEM), X-ray diffractometer (XRD), Powder Neutron diffraction (PNRD), ultraviolet visible (UV-Vis) spectroscope, thermogravimetric analysis (TGA), differential scanning calorimetry (DSC), stopped flow fluorescence spectroscopy.

Functional Property measurement: 2 probe and 4- probe conductivity measurement, Impedance spectroscopy for conductivity. Physical property measurement system for magnetism and Neutron diffraction analysis for magnetism.

Li-ion battery works: New material synthesis for electrode. Coin cell assembly inside and outside the glove box. Battery testing and analysis.

Computer programs: MS office, GSAS, Origin, EIS, Fullprof, Mercury, Dimond, Zview, Jade, Machine Learning.

Electrocatalytic water splitting: Electrocatalyst synthesis and its activity analysis for oxygen evolution, hydrogen evolution by water splitting.

Pseudo capacitance: measurement and analysis.

Material Sensing property measurement: For different gases at various temperatures.

Reverse micelles: Design the reverse micelles of different sizes and reaction kinetics study. 
Air sensitive glove box reaction: Organo-metallic reaction for crystal growth, CV running.

Rewards and Achievements:

As an undergraduate and graduate student

- Frederick and Eileen Coulston award for Spring with amount of \$ 453 (NMSU) 2012-2013

- Travel Award from GNAS in UofL

As a professional

- Western New Mexico State University, NM, USA 2014

- Principal of Global College (Nepal) 2009

- Principal of Lalitpur College (Nepal) 2008

- Head of the department of mathematics and science 2006 - 2008

Sainik School (Nepal)

- Honor in Sainik Mahavidhyalaya as one of the best Chemistry faculty 2008

- Lecturer in Tribhuvan University (Nepal)

$1997-$ 2006

Reviewer

Science Journal of Chemistry (SJC)

Journal of Materials Science and Chemical Engineering (MSCE)

References:

1. Dr. Farshid Ramezanipour (My research adviser) Assistant professor, chemistry department, University of Louisville KY, USA farshid.ramezanipour@louisville.edu, 502-852-7061 (office)

2. Dr. Arjun Kumar Thapa (He trained me for Li-ion battery work)

Battery R \& D manager, Conn center for renewable energy research, University of Louisville, KY, USA arjun.thapa@louisville.edu, 5025958075 (mobile)

3. Dr. Ming $\mathrm{Yu}$ My research seminar and thesis defense committee member, Professor, Physics Department, University of Louisville, KY, USA m0yu0001@louisville.edu, (502) 852-0931 (office) 